\title{
Spent Nuclear Fuel Discharges from U.S. Reactors 1992
}

\author{
May 1994
}

\section{Energy Information Administration}

Office of Coal, Nuclear, Electric and Alternate Fuels

Survey Management Division

U.S. Department of Energy

Washington, DC 20585 


\section{DISCLAIMER}

This report was prepared as an account of work sponsored by an agency of the United States Government. Neither the United States Government nor any agency thereof, nor any of their employees, make any warranty, express or implied, or assumes any legal liability or responsibility for the accuracy, completeness, or usefulness of any information, apparatus, product, or process disclosed, or represents that its use would not infringe privately owned rights. Reference herein to any specific commercial product, process, or service by trade name, trademark, manufacturer, or otherwise does not necessarily constitute or imply its endorsement, recommendation, or favoring by the United States Government or any agency thereof. The views and opinions of authors expressed herein do not necessarily state or reflect those of the United States Government or any agency thereof. 


\section{DISCLAIMER}

Portions of this document may be illegible in electronic image products. Images are produced from the best available original document. 


\section{Preface}

Spent Nuclear Fuel Discharges from U.S. Reactors 1992 provides current statistical data on every fuel assembly irradiated in commercial nuclear reactors operating in the United States. It also provides data on the current inventories and storage capacities of those reactors to a wide audience, including Congress, Federal and State agencies, the nuclear and electric industries, and the general public. It uses data from the mandatory, "Nuclear Fuel Data" survey, Form RW-859 for 1992 and historical data collected by the Energy Information Administration (EIA) on previous Form RW-859 surveys. The report was prepared by the EIA under a Memorandum of Understanding with the Office of Civilian Radioactive Waste Management. The EIA is the independent agency for data collection and analysis within the U.S. Department of Energy.

Data collected on the "Nuclear Fuel Data" survey provide a comprehensive statistical characterization of the industry's activities for the survey year and include some information about industry plans and commitments for the future. The methodology used in the survey, including data collection procedures and data editing, analysis, and processing, is described in Appendix A. The quantities and characteristics of each specific fuel assembly type discharged by each reactor is described in Appendix B. A series of pictograms showing storage site and pool configurations, capacities, and inventories, are shown in Appendix C. A description of the EIA Quality Assurance reports and a more detailed discussion of EIA Quality Assurance procedures is included in Appendix D. Appendix E contains technical notes providing further details on information contained in the report.

Data on reactor spent fuel discharges and storage activity for 1992, nuclear power plant data, annual discharges and burnup, cladding: enrichment and burnup, and away-from-reactor storage facilities are presented in Chapter 1. Data on site capacities and inventories at utilities and storage facilities, as well as dry storage at utilities, are presented in Chapter 2. Chapter 3 presents data on assembly type charac- teristics, their background, fabricator summary, assembly distribution, and assembly identifiers.

The detailed data collected on Form RW-859 and summarized in this report are directly useful for assessing spent fuel storage requirements. They also constitute one of the inputs to the spent fuel Characteristics Data Base (CDB) developed by Oak Ridge National Laboratory (ORNL). The discharge dates, assembly types, burnups, and initial enrichments are used to calculate the gamma, neutron, and thermal source intensities. These radiological characteristics, along with reported fuel quantities and dimensions, are then used by the Civilian Radioactive Waste Management System (CRWMS) designers for shielding design, thermal design, and sizing of facilities and equipment.

The data are also used in the 1993 Acceptance Priority Ranking (APR) report. The 1993 APR details the order in which DOE will allocate Federal waste acceptance capacity. As required by the Standard Contract for Disposal of Spent Nuclear Fuel and/or High-Level Radioactive Waste (10 CFR 961), the ranking is based on the age of permanently discharged spent nuclear fuel, with the owners of the oldest spent nuclear fuel, on an industry-wide basis, given the highest priority.

The DOE estimates future discharges from U.S. commercial nuclear reactors and the characteristics of those discharges based on trends of historical spent nuclear fuel data provided by the respondents. The need for additional spent fuel storage capacity is based on these estimated cumulative discharges and on the estimated maximum storage capacity of both at-reactor and away-from-reactor storage facilities.

Questions regarding the contents of this report may be directed to:

Survey Management Division, EI-52

Energy Information Administration

U.S. Department of Energy

1000 Independence Ave., S.W.

Washington, DC 20585 
Questions of a general nature should be directed to Howard Walton, Director of the Survey Management Division (202/254-5500), Fred Mayes, Chief of the Renewable \& Financial Data Systems \& Outreach Branch (202/254-5482); or James Disbrow, Team Leader, Renewables \& Outreach (202/254-5558).
Questions of a detailed or technical nature should be directed to the Project Leader, Kathy Gibbard (202/254-5559).

Additional copies may be obtained by returning the order form at the back of this report. 


\section{Contents}

Feature Article: Canisters and Nonfuel Components at Commercial Nuclear Reactors.............. xi

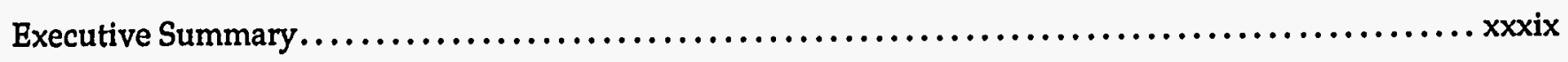

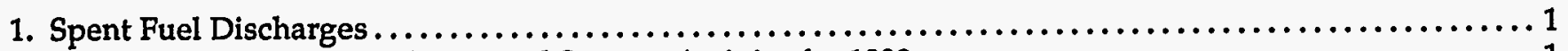

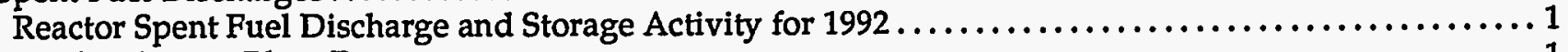

Nuclear Power Plant Data ........................................................ 1

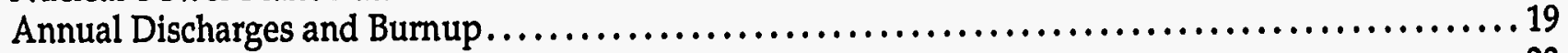

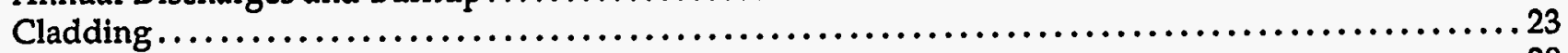

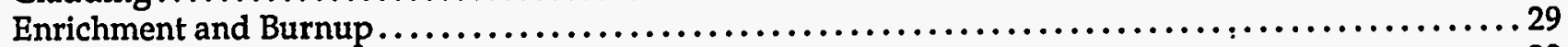

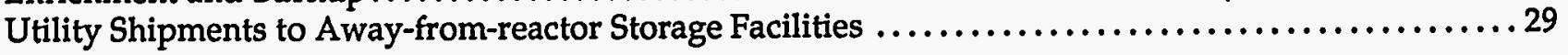

2. Site Capacities, Inventories, and Dry Storage at Utilities and Storage Facilities.................. 33

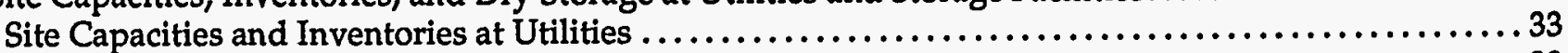

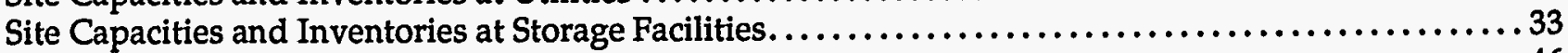

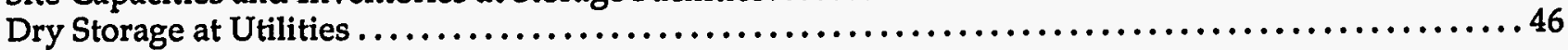

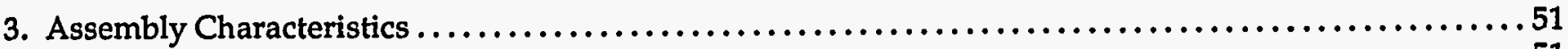

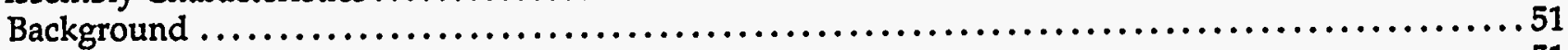

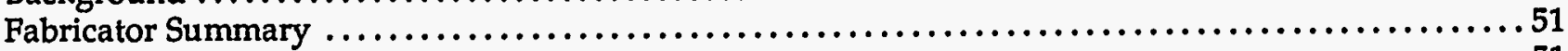

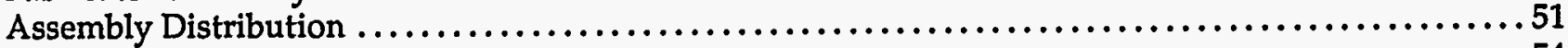

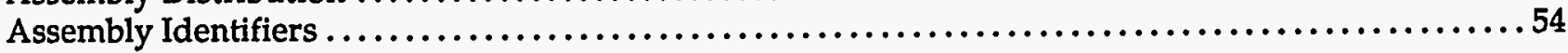

Appendices

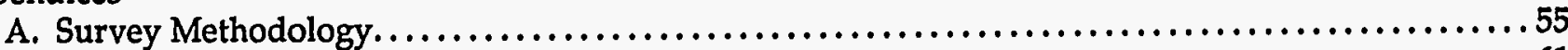

B. Detailed Assembly Type Statistics...........................................61

C. Pictograms Showing Reactor Storage Pool Configurations, Pool

Capacities, and Inventories at Nuclear Power Plant Sites and

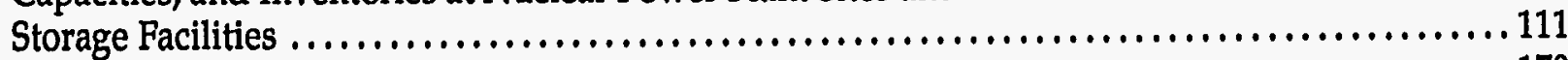

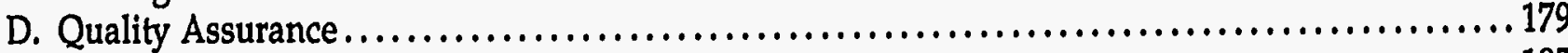

E. Technical Notes................................................... 185

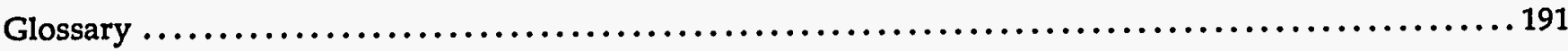

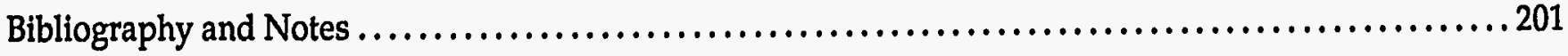




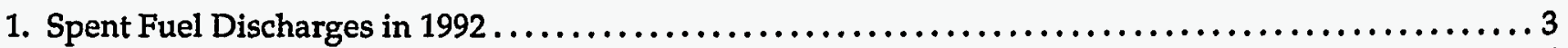

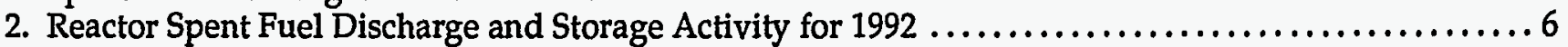

3. Temporarily Discharged Assemblies. ............................................... 11

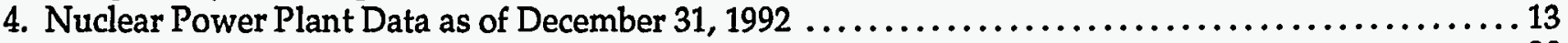

5. Annual Spent Fuel Discharges and Burnup, 1968-1992 .............................. 20

6. Annual Spent Fuel Burnup (in Assemblies), 1968-1992 . . . . . . . . . . . . . . . . . . . . . . 24

7. Metric Tons of Uranium in Annual Spent Fuel Burnup, 1968-1992 . . . . . . . . . . . . . . . . . . 25

8. U.S. Burnup Distribution for All Discharged Assemblies . . . . . . . . . . . . . . . . . . . . . . . . . . 26

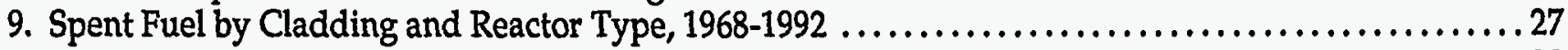

10. Reactors Discharging Stainless Steel Clad Assemblies . . . . . . . . . . . . . . . . . . . . . . . . . . 28

11. Burnup Versus En richment by Cladding and Reactor Type (in Assemblies) $\ldots \ldots \ldots \ldots \ldots \ldots \ldots \ldots, \ldots \ldots$

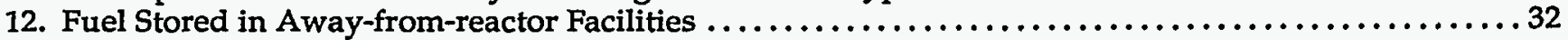

13. Site Capacities and Inventories at Nuclear Power Plants as of December 31, 1992..............34

14. Site Capacities and Inventories at Away-from-reactor Storage Facilities as of December 31, $1992 \ldots . \ldots 4$

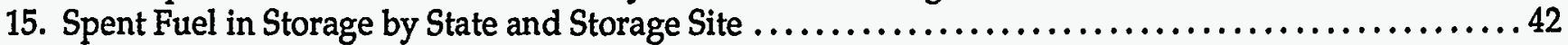

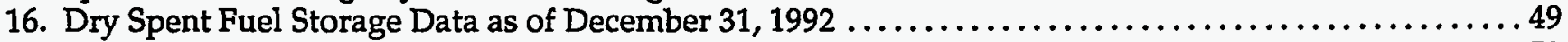

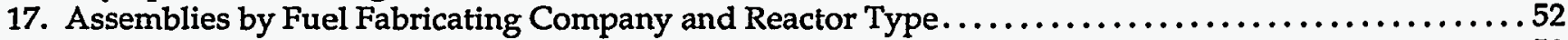

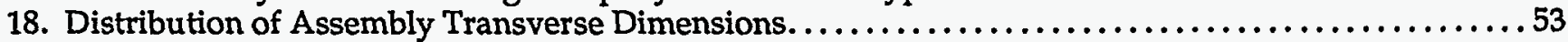

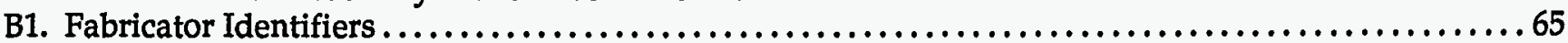

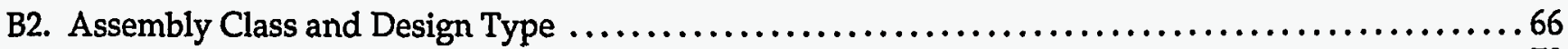

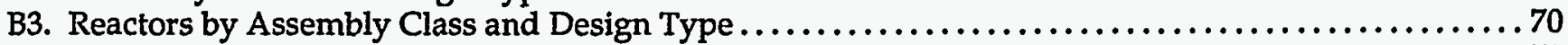

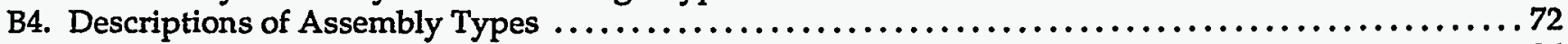

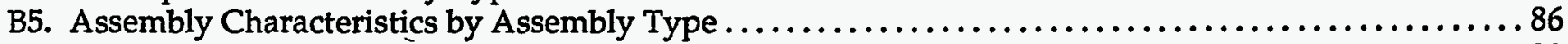

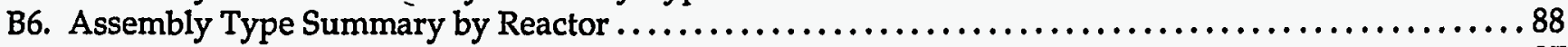

B7. Assembly Type Summary by Assembly Type. . . . . . . . . . . . . . . . . . . . . . . . . . . . . . . . 97

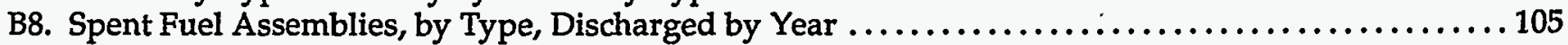

B9. Initial Uranium Content of Spent Fuel Assemblies, by Type, Discharged by Year ............. 107

B10. Burnup of Spent Fuel Assemblies, by Type, Discharged by Year . . . . . . . . . . . . . . . . . . . . . 109

\section{Figures}

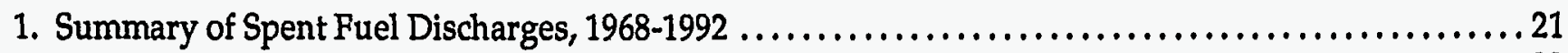

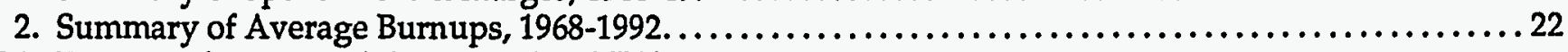

D1. Energy Information Administration (EIA)

Quality Assurance (QA) Report. 


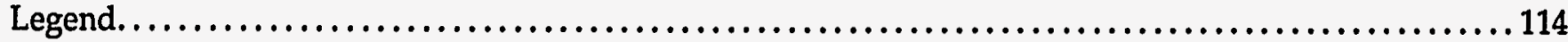

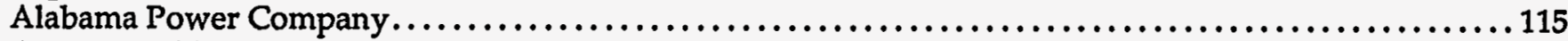

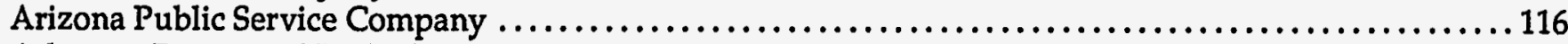

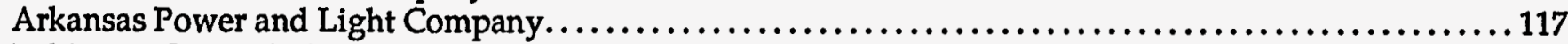

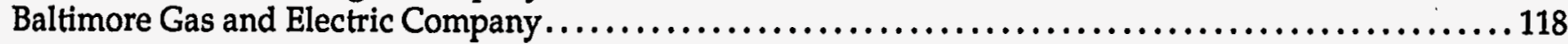

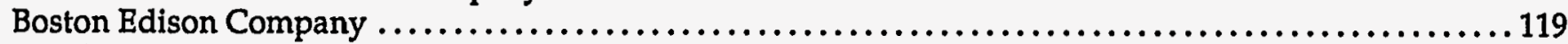

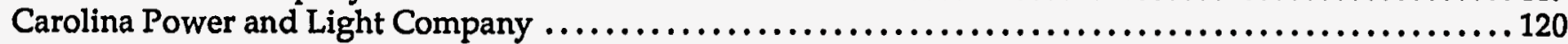

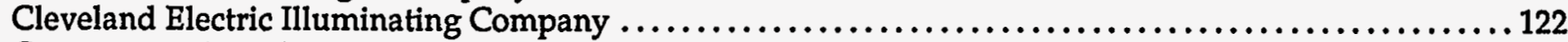

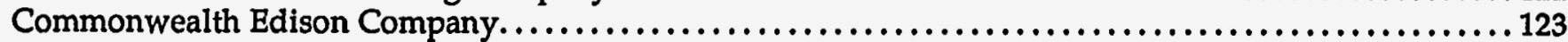

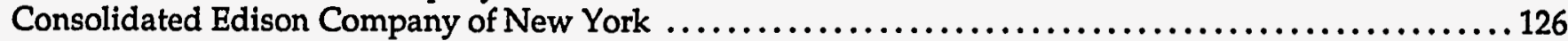

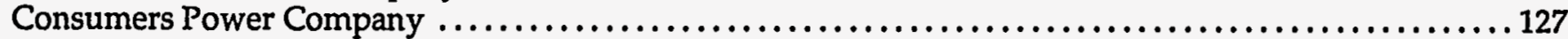

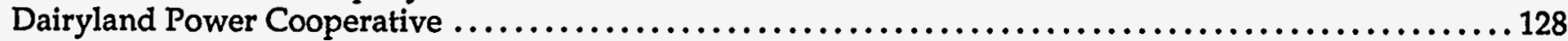

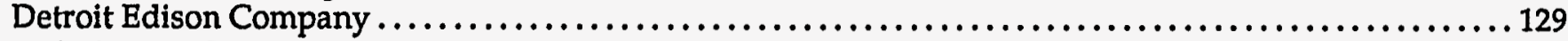

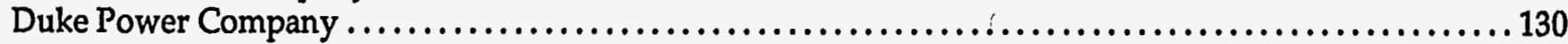

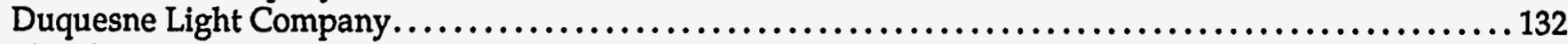

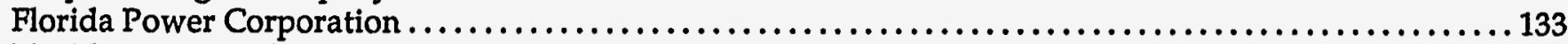

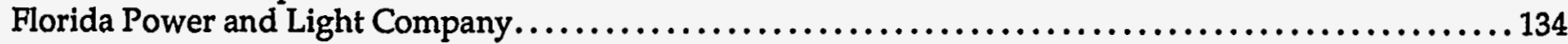

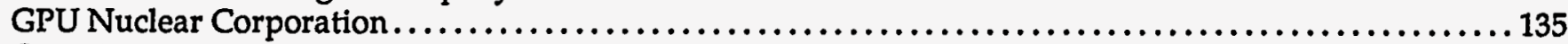

Georgia Power Company................................................................. 136

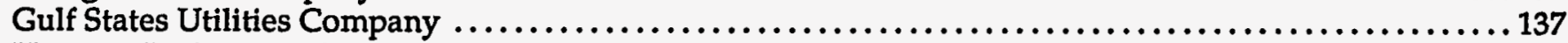

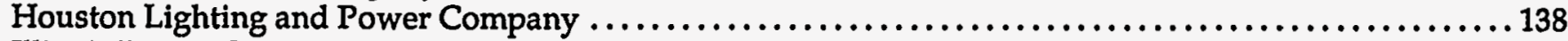

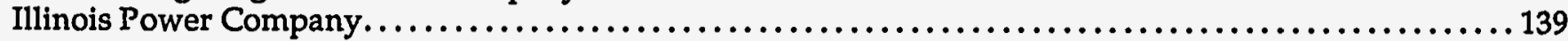

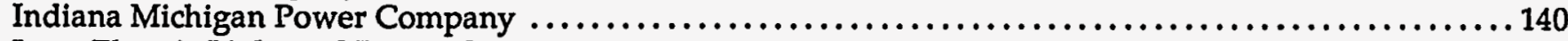

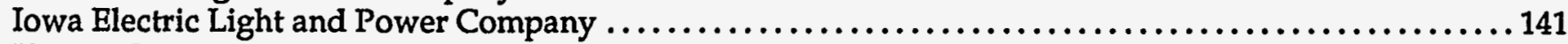

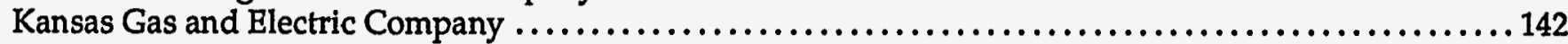

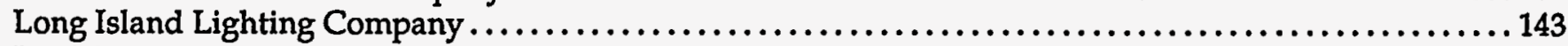

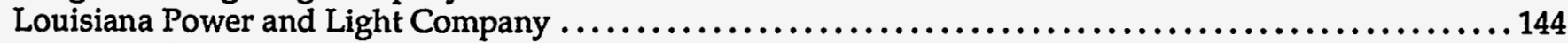

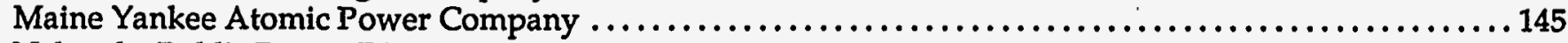

Nebraska Public Power District ...................................................... 146

New York Power Authority (a.k.a., Power Authority of the State of New York) $\ldots \ldots \ldots \ldots \ldots \ldots \ldots \ldots \ldots \ldots 147$

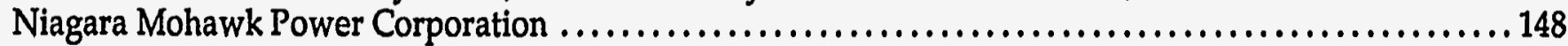

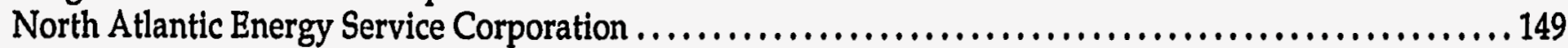

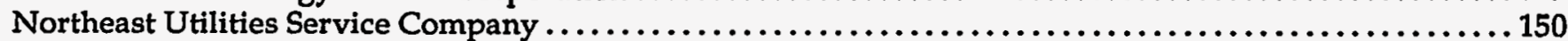

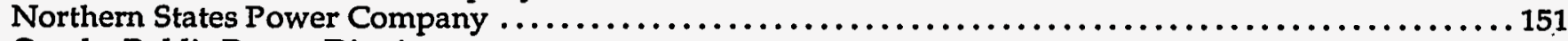

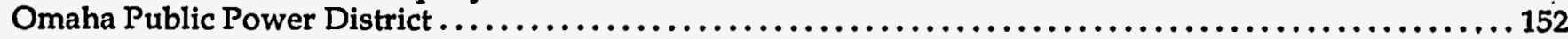

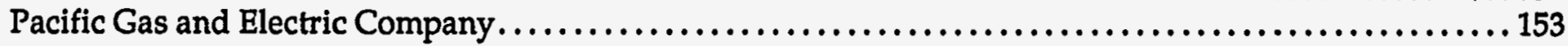

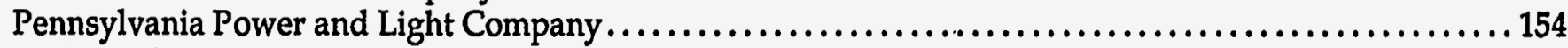

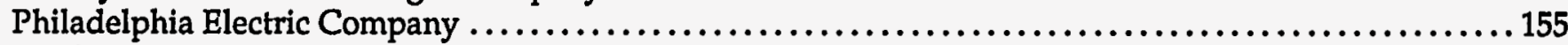

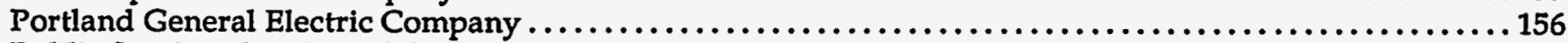

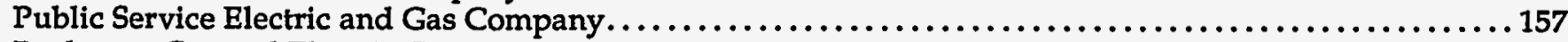

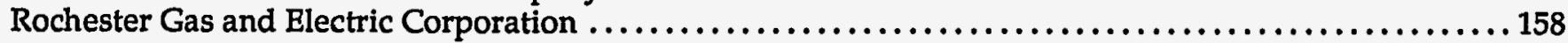

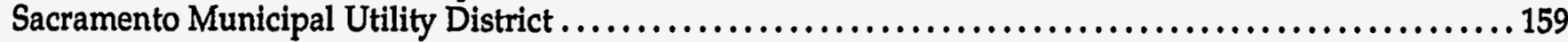

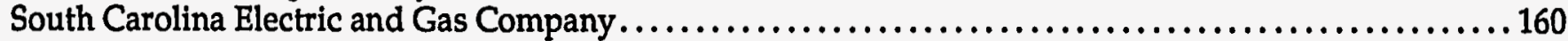

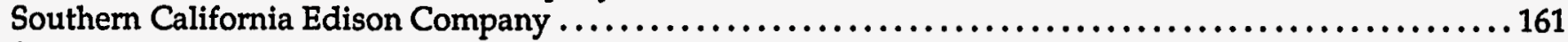

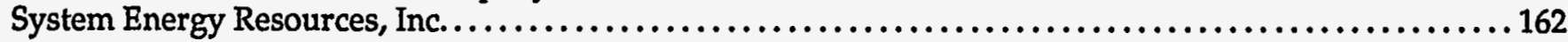

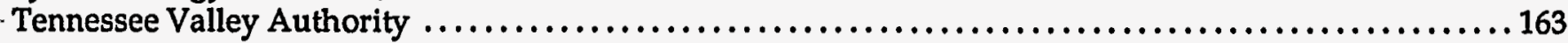

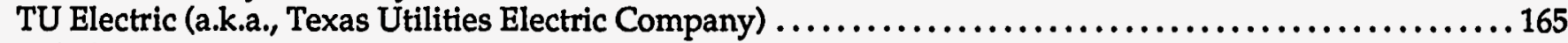

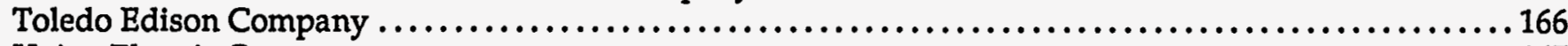

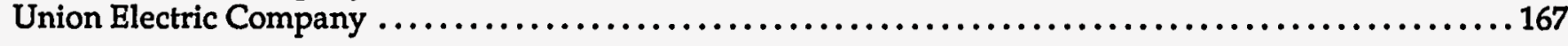


Vermont Yankee Nuclear Power Corporation . . . . . . . . . . . . . . . . . . . . . . . . . . . . . . . . . . . . . 168

Virginia Power (a.k.a., Virginia Electric and Power Company) $\ldots \ldots \ldots \ldots \ldots \ldots \ldots \ldots \ldots \ldots \ldots \ldots \ldots \ldots$

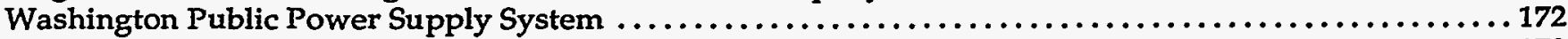

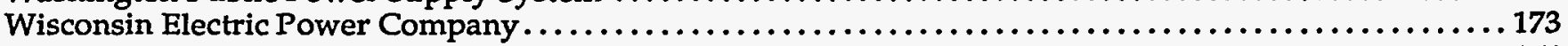

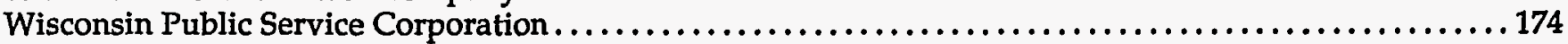

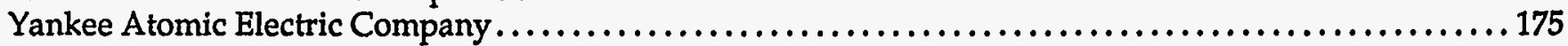

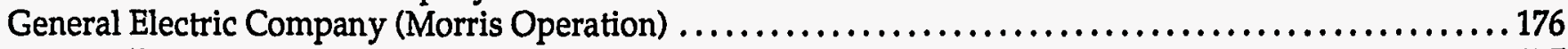

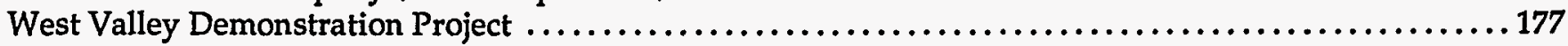

\section{Pictograms by Reactor}

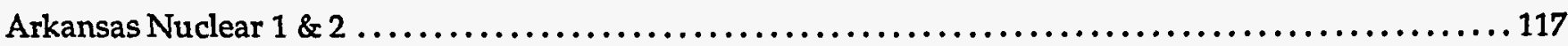

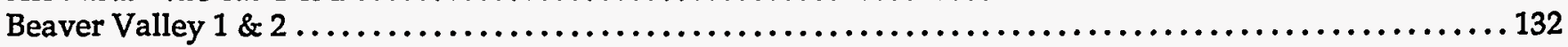

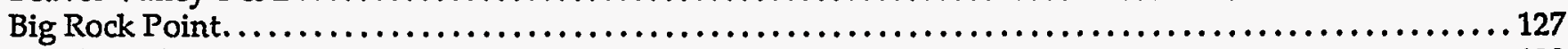

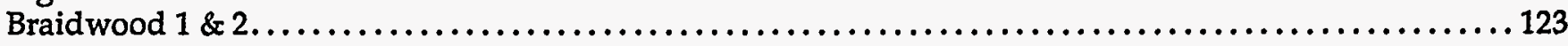

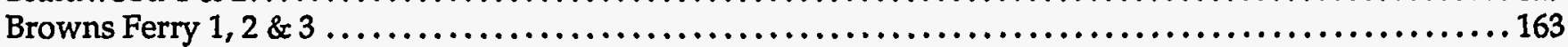

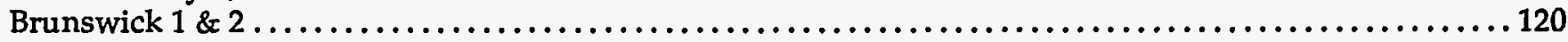

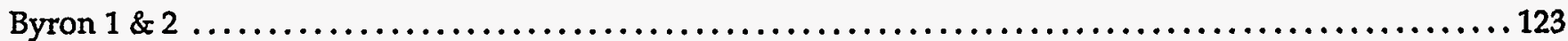

Callaway........................................................................ 167

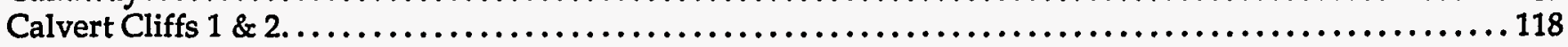

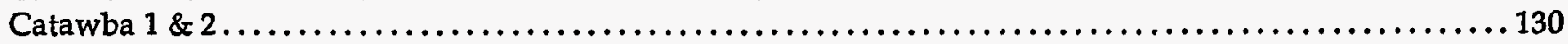

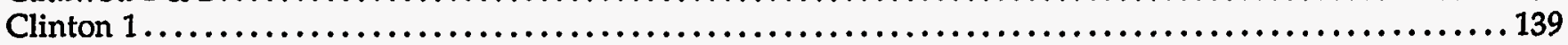

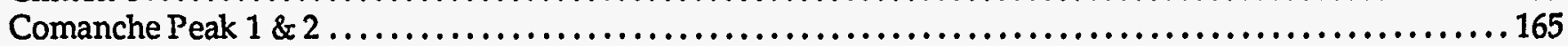

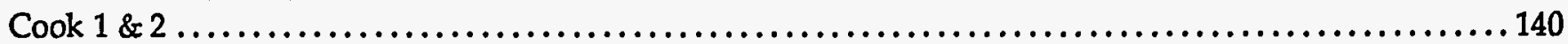

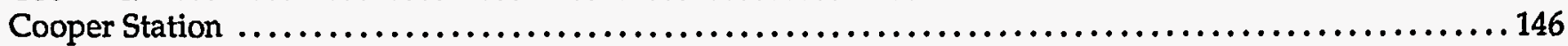

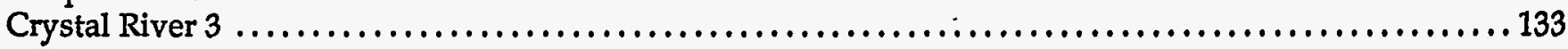

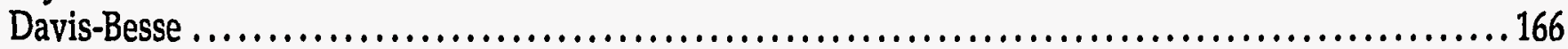

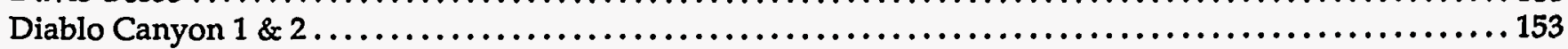

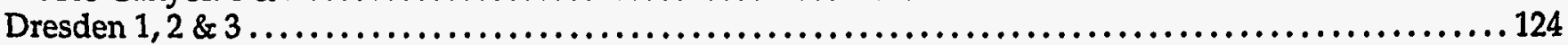

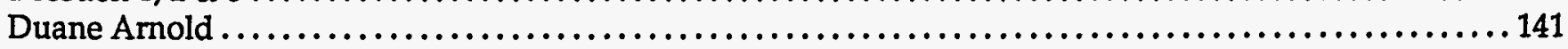

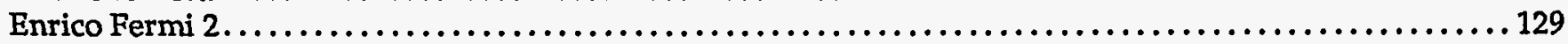

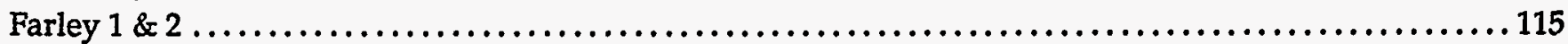

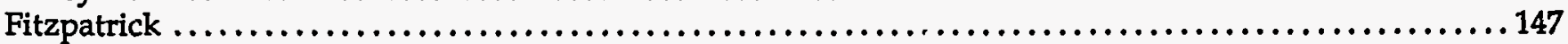

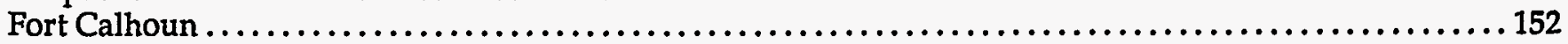

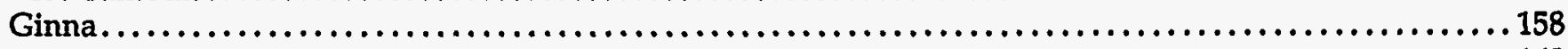

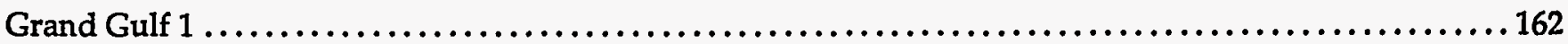

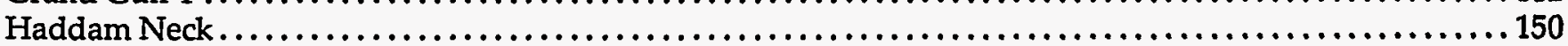

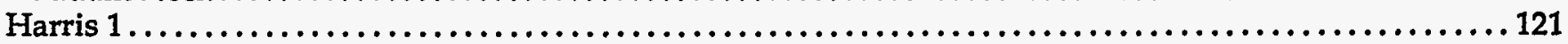

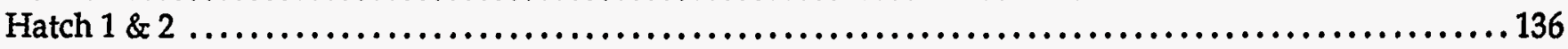

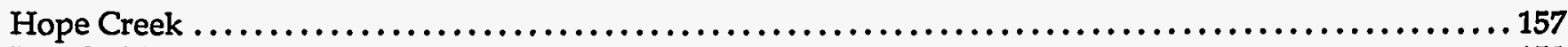

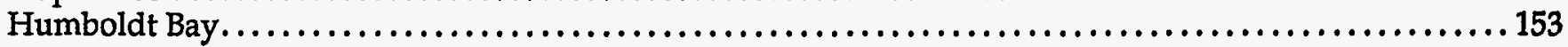

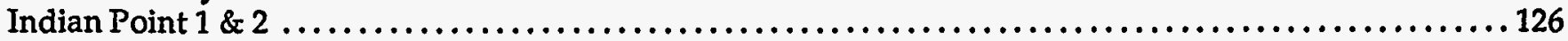

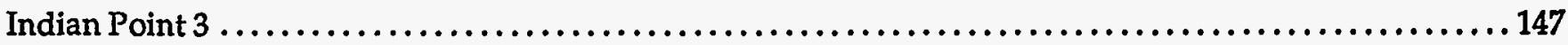

Kewaunee..................................................................... 174

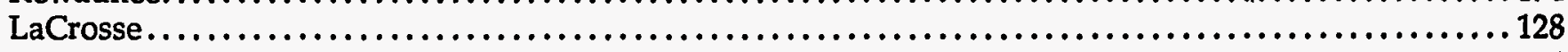

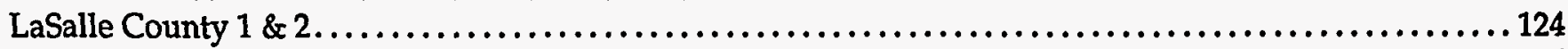

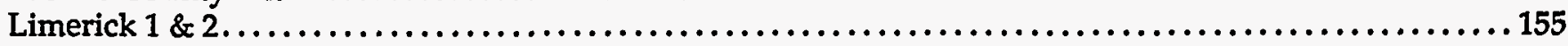

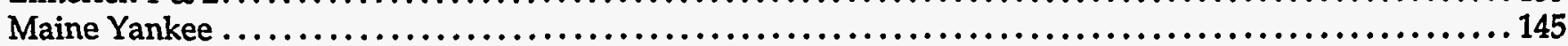

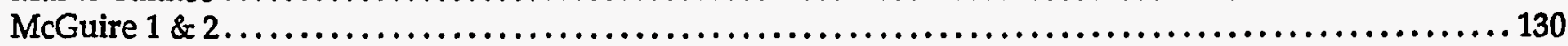

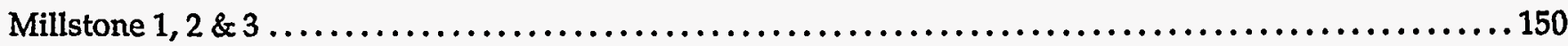




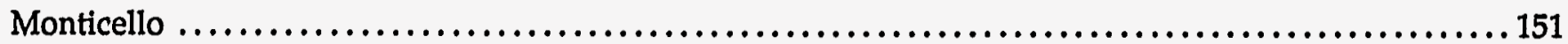

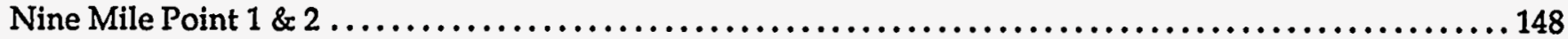

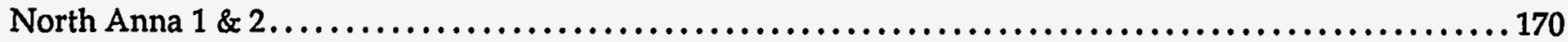

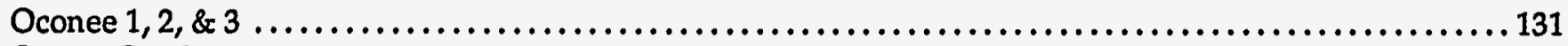

Oyster Creek ..................................................................... 135

Palisades.................................................................... 127

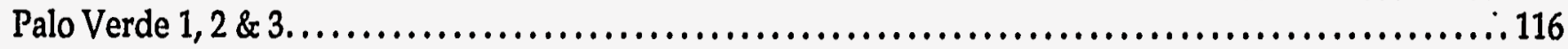

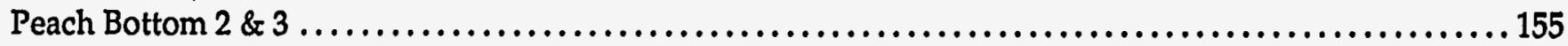

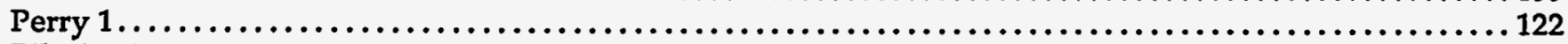

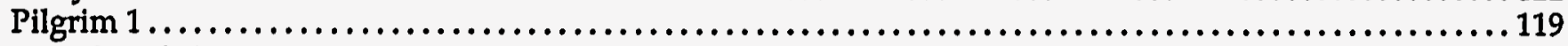

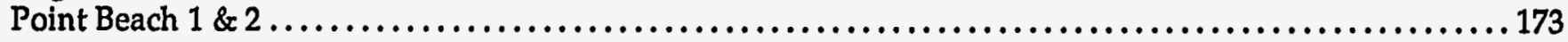

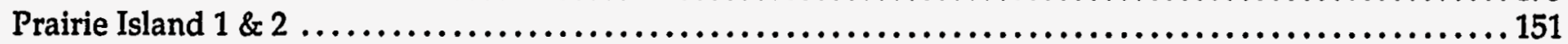

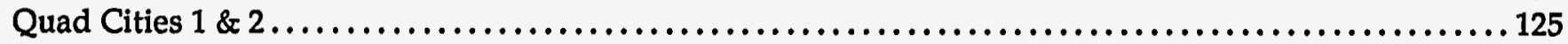

Rancho Seco..................................................................... 159

River Bend 1 .................................................................. 137

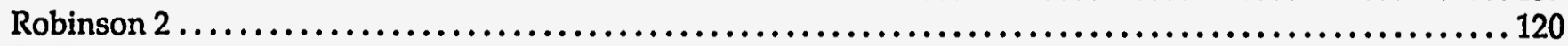

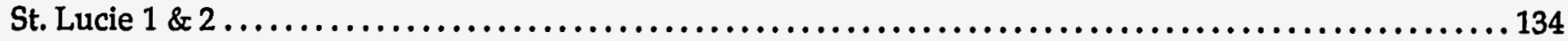

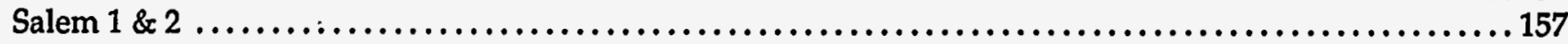

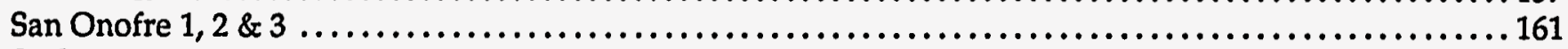

Seabrook....................................................................... 149

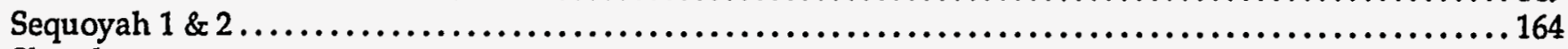

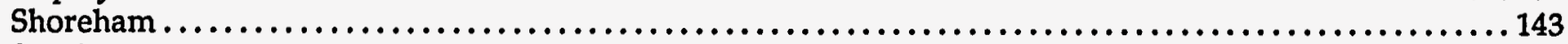

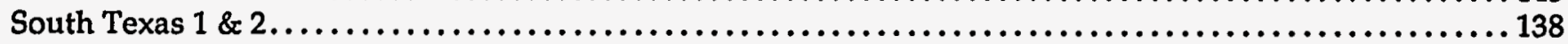

Summer..........................................................................160

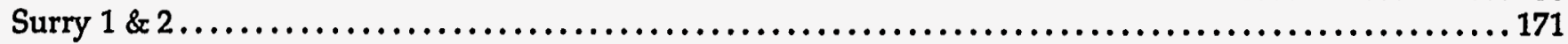

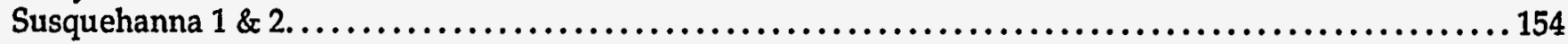

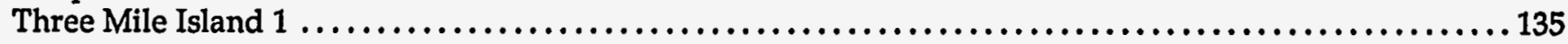

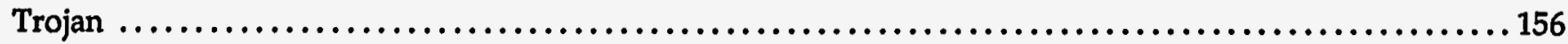

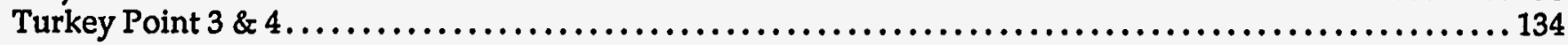

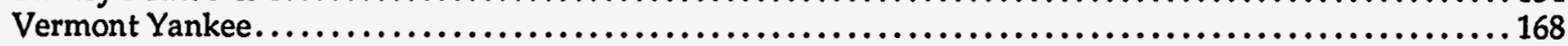

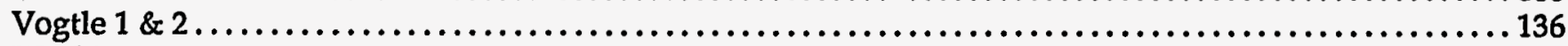

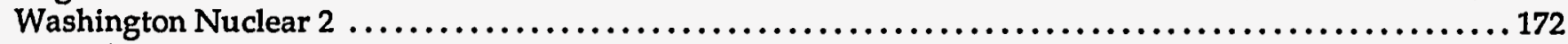

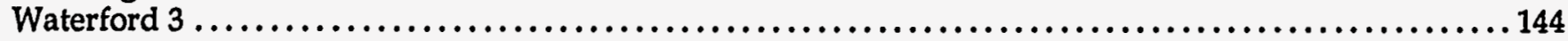

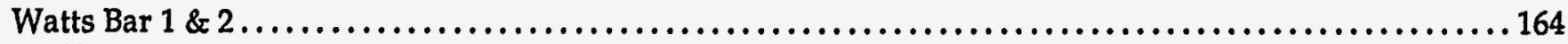

Wolf Creek 1 . ................................................................ 142

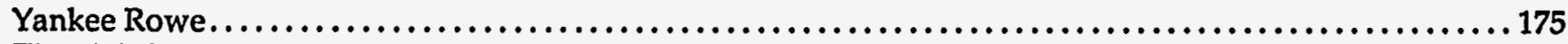

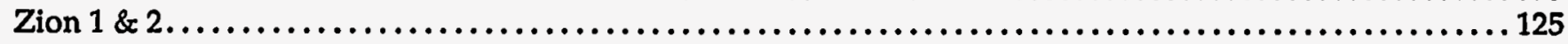




\title{
Canisters and Nonfuel Components at Commercial Nuclear Reactors
}

\author{
by \\ Kathy Gibbard \\ James Disbrow
}

\section{Introduction}

The storage of nonfuel assembly (NFA) hardware and its subsequent impact on a Federal Waste Management System has been the subject of much discussion because of the effect on waste acceptance procedures and transportation and disposal requirements. NFA hardware, if stored or disposed outside of fuel assemblies and in an integral form, would represent a significant addition to the volume of waste required to be handled. NFA hardware, if integral to a fuel assembly, could represent a significant addition to the total weight of each shipment.

The Form RW-859 "Nuclear Fuel Data" survey was revised to collect approximately 30 data elements on canisters and their contents and on nonfuel components (NFC) for each storage pool site. For the past 2 years, the Energy Information Administration (EIA) has collected these data as part of this survey from the operators of 123 commercial nuclear reactors. The purpose of this article is to present the most current data on canisters and NFC stored at these facilities as of December 31, 1992.

\section{Highlights}

A total of $\mathbf{2 8 6}$ canisters have been reported as being in storage pools as of December 31, 1992. Because all but one Form RW-859 respondent reported the number of canisters on either the 1991 or 1992 survey, and the one that did not report had no canisters in earlier years, these 286 canisters should represent the total in pools as of the above date. Detailed inventories were not reported, however, for 51 of these canisters, representing 17.8 percent of the total. Additionally, 264 open baskets were also reported to contain miscellaneous spent nuclear fuel (SNF) and nonfuel materials. All of the 286 canisters meet the dimensional envelope requirements specified for disposal of "standard fuel" under the
Standard Contract for Disposal of Spent Nuclear Fuel and/or High-Level Radioactive Waste (10 CFR 961); most of the baskets do not.

A total of 40,194 pieces of NFA hardware have been reported as being in storage at reactor sites. This total represents only the NFA hardware reported by the owners of 95 of the 109 reactors in generic assembly classes (e.g., Babcock \& Wilcox (B\&W) 15X15, Westinghouse (WE) 17X17, General Electric (GE) 4, 5, 6, Combustion Engineering (CE) System 80, etc.). The remaining 14 reactors either did not report nonfuel components data or reported no NFA hardware components. This article does not address the nonfuel components (including NFA hardware) reported by owners of reactors in single-reactor assembly classes (e.g., Dresden 1, Yankee Rowe, Palisades, LaCrosse, etc.).

\section{Canisters}

A canister is a container made of stainless steel or an inert alloy which holds SNF, high-level radioactive waste or nonfuel components. A canister may contain an intact spent fuel assembly, consolidated spent fuel assemblies, spent fuel rods or pieces, NFA hardware, nonfuel disassembly hardware, or other fuel and nonfuel material. These canisters are temporarily stored in spent fuel pools at reactor sites and occupy one or more slots in the pool. When full, a container is typically sealed with a lid and welded shut. Waste may also be placed in rodlet or garbage and debris (GAD) baskets, which are also stored in the spent fuel pools, but not necessarily in the pool racks.

Because many utilities face spent fuel storage constraints, the number of canisters stored at these facilities and the contents of these canisters are important concerns. A few utilities have used fuel consolidation campaigns as a means of preserving fuel assemblies and placing them in a canister in a grid storage pool space. 
Fuel is consolidated by removing rods with spacing closer than that of an intact assembly. In addition to maximizing density and lowering criticality, heat transfer characteristics are also improved. The empty assembly skeletons from consolidation may be compressed and either stored or disposed as low-level radioactive waste.

Table FE1 presents a summary of data on canisters, baskets, and their contents as reported on the Form RW-859 survey. The data in the table are compiled by storage pool site. Due to different reporting schedules, not all respondents were required to report on the 1992 Form RW-859 survey, so data for 13 storage pool sites have been carried over from 1991. In addition, one site did not report canister data for either survey year. Of the 286 canisters reported, 151 contain spent fuel (intact assemblies, consolidated assemblies, or fuel rods/pieces) and 149 contain nonfuel components (14 contain both fuel and nonfuel). The specific contents of 51 canisters were not reported so these tables should not be considered representative of the complete enumeration of these data. A total of 264 open baskets were also reported, containing miscellaneous fuel and nonfuel materials, garbage and debris.

Data on the contents of canisters containing spent nuclear fuel (intact assemblies, rods and/or pieces, or consolidated assemblies) are summarized in Table FE2. The 151 canisters containing SNF can be further subdivided by whether the fuel was irradiated in a boilingwater reactor (BWR) or pressurized-water reactor (PWR). Of the 42 canisters containing BWR SNF, 21 contain intact spent fuel assemblies, 17 contain fuel rods, and the contents of 4 canisters at one BWR are unknown. No fuel was consolidated at any BWRs. Of the 109 canisters containing PWR SNF, 16 contain intact assemblies, 52 contain fuel rods, and 36 canisters contain a total of 74 consolidated assemblies. These consolidation campaigns took place at Palisades, Oconee $1 \& 2$, Millstone 2, Prairie Island $1 \& 2$, and Ginna. The contents of 5 canisters at PWRs were not reported.

Data on the contents of canisters containing nonfuel components are summarized in Table FE3. Of the 149 nonfuel canisters, the majority contained either nonfuel components (67 canisters) and/or disassembly hardware (35 canisters). The contents of 42 canisters were not reported so this table should not be considered representative of the complete enumeration of these data. Nonfuel canisters may include more than one class of components and a few are currently empty.
Table FE4 presents a summary of the dimensions of canisters, in inches, reported on the Form RW-859 survey. As the table shows, the most prevalent sizes of canisters are $157 \times 8 \times 8$ and $161 \times 9 \times 9$. No canisters were reported as having a length greater than the longest PWR assembly, which is 199 inches. The minimum canister length reported is 84 inches, the size of the shortest BWR assembly. Containers with a reported length of less than 84 inches are classified as baskets. The total column of the table presents the distribution of canister lengths; the rows present the cross-sectional dimensions (width and depth) of canisters reported. Some respondents submitted data on the contents of a particular canister, but did not submit the canister's dimensions. These canisters are represented as having "Missing Dimensions." Other respondents did not file canister-specific data (including dimensions) and fall under the "Data Not Reported" category.

\section{Nonfuel Components}

The term "nonfuel components" may be defined broadly to cover all non-fuel-bearing components which receive radiation during power generation. Generally included are:

- The components used to initiate, control, and monitor the chain reaction in the core, often called NFA hardware (neutron sources, control elements, burnable absorbers, incore instrumentation, etc.)

- The nonfuel portion of a fuel assembly, often called disassembly hardware (guide tubes, water rods, grids, nozzles, etc.); and

- Miscellaneous hardware used in the reactor core which is not a part of fuel assemblies (dummy assemblies, coupon trees, etc.).

The Standard Contract has a more limited definition of nonfuel components which includes most NFA hardware. The contract does not explicitly identify BWR control assemblies, which are located between, rather than within, the SNF assemblies. Nor does it mention disassembly hardware and miscellaneous hardware.

In terms of location of each piece of hardware, a noncanistered nonfuel component also can be distinguished by whether it is integral or nonintegral with an assembly. Nonfuel components may be included as an integral part of the SNF assemblies delivered for disposal. Nonfuel components are generally used within or between assemblies but are not necessarily permanently attached to assemblies. These nonfuel 
components are usually retired from service on a schedule that is different from that of the fuel assemblies.

Because all of the nonfuel components listed in the Standard Contract fall into the NFA hardware grouping, NFA hardware has been the focus of the EIA data collection efforts on nonfuel components. Some utilities have reported nonfuel components which are either disassembly hardware or miscellaneous hardware. However, the reporting and analysis which follow deal primarily with NFA hardware.

\section{Nonfuel Components - NFA Categories by Vendor}

Tables FE5, FE6, FE7, and FE8 summarize the responses of the utilities to the Form RW-859 survey section on discharged NFA hardware. These tables do not include either the other nonfuel components reported by these utilities or the nonfuel components (including NFA hardware) reported by owners of reactors in singlereactor assembly classes (e.g., Dresden 1, Yankee Rowe, Palisades, LaCrosse, etc.). Of the 109 generic assembly class reactors, 14 reactors either did not report nonfuel components data or reported no NFA hardware components. The components of NFA hardware which are used at various reactors vary significantly with vendors of the Nuclear Steam Supply System (NSSS), so each of the tables presents the NFA hardware reported for one of the major NSSS vendors (Babcock \& Wilcox, Combustion Engineering, General Electric, and Westinghouse).

\section{Storage of Integral NFA hardware}

The Form RW-859 survey also requested the quantities of NFA hardware which are stored in or attached to assemblies and the dimensions of those NFA hardware components. NFA hardware may have been integral with an assembly, noncanistered in baskets, destined for disposal as low-level radioactive waste, or stored in canisters. Approximately 85 percent of the 40,194 pieces of NFA hardware reported were integral with an assembly. Reactor-by-reactor percentages of NFA hardware stored in or attached to assemblies are given in Tables FE5, FE6, FE7, and FE8.

\section{NFA Hardware Discharge Rates}

The following factors may affect NFA hardware discharge rates:

- Changes in fuel management strategies

- Changeout of infrequently replaced items (control rods, etc)
- Early changeout of hafnium control rod assemblies

- Low-level radioactive waste

\section{Changes in Fuel Management Strategies}

Changes in fuel management strategies have impacted discharge rates of NFA hardware, particularly burnable absorber assemblies. Reactors built by Westinghouse and Babcock \& Wilcox used one set of burnable absorber assemblies with the initial reactor fuel. The use of burnable absorber assemblies was resumed in later cycles to control the excess reactivity associated with higher initial fuel enrichments. The effect on quantities of NFA hardware was that reactors built by Westinghouse and Babcock \& Wilcox typically began to use significantly larger quantities of NFA hardware each cycle; preliminary results indicate nearly a one-to-one ratio of burnable absorber assemblies with fresh fuel assemblies for both vendors.

Newer features, such as the use of integral fuel burnable absorbers by Westinghouse, are intended to once again reduce quantities of NFA hardware discharged by reactors.

\section{Changeout of Infrequently Replaced Items}

Many estimates of NFA hardware discharges are related to discharged fuel assemblies because the number of discharged fuel assemblies gives a simple and quantitative measure of a reactor's use. However, NFA hardware is discharged on one schedule, fuel assemblies on another. This may distort estimates of NFA hardware discharges, especially when dealing with infrequently replaced components, such as control element assemblies. For example, operators of reactors in the CE $14 \mathrm{X}$ 14 assembly class report having discharged nearly 500 control element assemblies and slightly over 4,000 fuel assemblies. Operators of reactors in the CE $16 \times 16$ assembly class report having discharged only 3 control element assemblies and nearly 2,000 fuel assemblies. For CE $14 \times 14$ reactors, the number of control element assemblies is approximately 12 percent of the number of discharged fuel assemblies; for CE $16 \times 16$ reactors, the number of control element assemblies is less than 0.2 percent of the discharged fuel, assemblies. The explanation is that the older reactors (CE $14 \times 14$ ) have completed a changeout of their control element assemblies, whereas the newer reactors (CE $16 \times 16)$ have only discharged defective or damaged control element assemblies. This effect can also be observed between the older and newer Westinghouse assembly classes (e.g., 
WE $14 \times 14$ and WE $15 \times 15$ versus WE $17 \times 17$ ). The effect in Westinghouse assembly classes is somewhat obscured by the early changeout of hafnium control rod assemblies from some WE $17 \times 17$ plants.

\section{Early Changeout of Hafnium Control Rod Assemblies}

Several of the WE $17 \times 17$ plants using hafnium control rod assemblies experienced unexpected hydrating and subsequent control rod swelling. As a result, these assemblies have been replaced at all or nearly all of the plants originally using them, sometimes after as little as a single cycle of use. This development means that the number of control rod assemblies at WE $17 \times 17$ plants will not be directly correlated to the number of discharged fuel assemblies.

\section{Low-Level Radioactive Waste}

When NFA hardware has reached the end of its useful life, it may exceed the Class $C$ limits for disposal established in $10 \mathrm{CFR}$, Part 61 for low-level radioactive wastes. Hardware which exceeds these limits is referred to as Greater-Than-Class-C wastes. NFA hardware which does not exceed these limits may be disposed as a low-level waste. Some utilities have disposed of NFA hardware in this manner, but the EIA does not collect this data on the Form RW-859 survey.

\section{Conclusion}

This information represents the publicly available data on NFA hardware components. The results obtained thus far in the area of canisters and nonfuel components present a framework from which further data may be collected. Because the nonfuel components section of the Form RW-859 is a recent addition to the survey, respondent answers were not always the same even though the reactors are similar. This is particularly apparent with respect to the number of fuel channels reported at BWR reactors. While some reactors report a number of fuel channels which are roughly equivalent to the number of discharged fuel assemblies, other reactors report quantities which are significantly lower and appear to represent the number of fuel channels which are not attached to assemblies. These differences in reporting must be understood in order to accurately estimate quantities which might be designated for disposal. The Form RW-859 survey is currently being redesigned to collect more thorough data in these areas. In particular, data will be collected on the contents of all containers, to include all storage structures into which fuel and nonfuel components may be placed. The need for further information will depend on the level of detail required by designers and systems engineers to plan for the handling and disposition of these components. 
Table FE1. Container Data as of December 31, 1992

\begin{tabular}{|c|c|c|c|c|c|c|c|}
\hline Electric Utility Name & Reactor Names & $\begin{array}{l}\text { Reactor } \\
\text { Type }\end{array}$ & $\begin{array}{l}\text { Pool } \\
\text { Site } \\
\text { ID }\end{array}$ & $\begin{array}{l}\text { Total } \\
\text { Canisters }\end{array}$ & $\begin{array}{c}\text { Canisters } \\
\text { Fuel }\end{array}$ & $\begin{array}{l}\text { Canisters } \\
\text { Nonfuel }\end{array}$ & Baskets $^{a}$ \\
\hline Alabama Power Company $\ldots \ldots \ldots \ldots \ldots$ & $\begin{array}{l}\text { Farley } 1 \\
\text { Farley } 2\end{array}$ & $\begin{array}{l}\text { PWR } \\
\text { PWR }\end{array}$ & $\begin{array}{l}0101 \\
0102\end{array}$ & $\begin{array}{l}1 \\
0\end{array}$ & $\begin{array}{l}1 \\
0\end{array}$ & $\begin{array}{l}0 \\
0\end{array}$ & $\begin{array}{l}0 \\
0\end{array}$ \\
\hline Arizona Public Service Company . . . . . . . . & $\begin{array}{l}\text { Palo Verde } 1 \\
\text { Palo Verde } 2 \\
\text { Palo Verde } 3\end{array}$ & $\begin{array}{l}\text { PWR } \\
\text { PWR } \\
\text { PWR }\end{array}$ & $\begin{array}{l}0301 \\
0302 \\
0303\end{array}$ & $\begin{array}{l}8 \\
5 \\
5\end{array}$ & $\begin{array}{l}2 \\
1 \\
1\end{array}$ & $\begin{array}{l}6 \\
4 \\
4\end{array}$ & $\begin{array}{l}0 \\
0 \\
0\end{array}$ \\
\hline 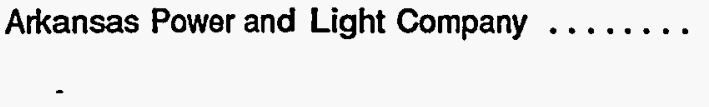 & $\begin{array}{l}\text { Arkansas Nuclear } 1 \\
\text { Arkansas Nuclear } 2\end{array}$ & $\begin{array}{l}\text { PWR } \\
\text { PWR }\end{array}$ & $\begin{array}{l}0401 \\
0402\end{array}$ & $\begin{array}{l}17 \\
14\end{array}$ & $\begin{array}{l}1 \\
1\end{array}$ & $\begin{array}{l}16 \\
13\end{array}$ & $\begin{array}{l}1 \\
1\end{array}$ \\
\hline Baltimore Gas and Electric Company ....... & Calvert Cliffs $1 \& 2$ & PWR & 0501 & 21 & 0 & 21 & 2 \\
\hline Boston Edison Company . . . . . . . . . . . & Pilgrim 1 & BWR & 0601 & 1 & 1 & 0 & 0 \\
\hline Carolina Power and Light Company . . . . . . . . & $\begin{array}{l}\text { Brunswick } 1 \\
\text { Brunswick } 2 \\
\text { Harris } 1 \\
\text { Robinson } 2\end{array}$ & $\begin{array}{l}\text { BWR } \\
\text { BWR } \\
\text { PWR } \\
\text { PWR }\end{array}$ & $\begin{array}{l}0701 \\
0702 \\
0703 \\
0705\end{array}$ & $\begin{array}{l}0 \\
1 \\
1 \\
3\end{array}$ & $\begin{array}{l}0 \\
1 \\
1 \\
1\end{array}$ & $\begin{array}{l}0 \\
0 \\
0 \\
2\end{array}$ & $\begin{array}{l}0 \\
1 \\
1 \\
0\end{array}$ \\
\hline $\begin{array}{l}\text { Cleveland Electric Illuminating } \\
\text { Company } \ldots \ldots \ldots \ldots \ldots \ldots \ldots \ldots \ldots \ldots\end{array}$ & Perry 1 & BWR & 0901 & 4 & 4 & $\mathbf{0}$ & 0 \\
\hline Commonwealth Edison Company .......... & $\begin{array}{l}\text { Braidwood } 1 \text { \& } 2 \\
\text { Byron } 1 \text { \& } 2 \\
\text { Dresden } 1 \\
\text { Dresden } 2 \\
\text { Dresden } 3 \\
\text { LaSalle County } 1 \text { \& } 2 \\
\text { Quad Cities } 1 \text { \& } 2 \\
\text { Zion } 1 \text { \& } 2\end{array}$ & $\begin{array}{l}\text { PWR } \\
\text { PWR } \\
\text { BWR } \\
\text { BWR } \\
\text { BWR } \\
\text { BWR } \\
\text { BWR } \\
\text { PWR }\end{array}$ & $\begin{array}{l}1001 \\
1003 \\
1005 \\
1006 \\
1007 \\
1008 \\
1010 \\
1012\end{array}$ & $\begin{array}{l}3 \\
2 \\
4 \\
2 \\
2 \\
1 \\
4 \\
3\end{array}$ & $\begin{array}{l}1 \\
2 \\
1 \\
2 \\
2 \\
1 \\
4 \\
1\end{array}$ & $\begin{array}{l}2 \\
0 \\
3 \\
0 \\
0 \\
0 \\
0 \\
2\end{array}$ & $\begin{array}{l}1 \\
1 \\
4 \\
2 \\
2 \\
4 \\
0 \\
0\end{array}$ \\
\hline $\begin{array}{l}\text { Consolidated Edison Company } \\
\text { of New York } \ldots \ldots \ldots \ldots \ldots \ldots \ldots \ldots\end{array}$ & $\begin{array}{l}\text { Indian Point } 1 \\
\text { Indian Point } 2\end{array}$ & $\begin{array}{l}\text { PWR } \\
\text { PWR }\end{array}$ & $\begin{array}{l}1101 \\
1102\end{array}$ & $\begin{array}{l}0 \\
0\end{array}$ & $\begin{array}{l}0 \\
0\end{array}$ & $\begin{array}{l}0 \\
0\end{array}$ & $\begin{array}{l}0 \\
1\end{array}$ \\
\hline Consumers Power Company ............ & $\begin{array}{l}\text { Big Rock Point } \\
\text { Palisades }\end{array}$ & $\begin{array}{l}\text { BWR } \\
\text { PWR }\end{array}$ & $\begin{array}{l}1201 \\
1204\end{array}$ & $\begin{array}{l}2 \\
6\end{array}$ & $\begin{array}{l}0 \\
6\end{array}$ & $\begin{array}{l}2 \\
0\end{array}$ & $\begin{array}{r}11 \\
0\end{array}$ \\
\hline Dairyland Power Cooperative . . . . . . . . . . & LaCrosse & BWR & 1301 & 0 & 0 & 0 & 1 \\
\hline
\end{tabular}

See footnotes at end of table. 
Table FE1. Container Data as of December 31, 1992 (Continued)

\begin{tabular}{|c|c|c|c|c|c|c|c|}
\hline Electric Utility Name & Reactor Names & $\begin{array}{c}\text { Reactor } \\
\text { Type }\end{array}$ & $\begin{array}{l}\text { Pool } \\
\text { Site } \\
\text { ID }\end{array}$ & $\begin{array}{c}\text { Total } \\
\text { Canisters }\end{array}$ & $\begin{array}{c}\text { Canisters } \\
\text { Fuel }\end{array}$ & $\begin{array}{l}\text { Canisters } \\
\text { Nonfuel }\end{array}$ & Baskets $^{a}$ \\
\hline Detroit Edison Company . . . . . . . . . . . . & Enrico Fermi 2 & BWR & 1402 & 0 & 0 & 0 & 0 \\
\hline Duke Power Company . . . . . . . . . . . & $\begin{array}{l}\text { Catawba } 1 \\
\text { Catawba } 2 \\
\text { McGuire } 1 \\
\text { McGuire } 2 \\
\text { Oconee } 1 \text { \& } 2 \\
\text { Oconee } 3\end{array}$ & $\begin{array}{l}\text { PWR } \\
\text { PWR } \\
\text { PWR } \\
\text { PWR } \\
\text { PWR } \\
\text { PWR }\end{array}$ & $\begin{array}{l}1501 \\
1502 \\
1504 \\
1505 \\
1506 \\
1508\end{array}$ & $\begin{array}{l}0 \\
0 \\
2 \\
1 \\
4 \\
1\end{array}$ & $\begin{array}{l}0 \\
0 \\
2 \\
1 \\
4 \\
1\end{array}$ & $\begin{array}{l}0 \\
0 \\
0 \\
0 \\
0 \\
0\end{array}$ & $\begin{array}{l}0 \\
0 \\
0 \\
0 \\
0 \\
0\end{array}$ \\
\hline Duquesne Light Company . . . . . . . . . . . & $\begin{array}{l}\text { Beaver Valley } 1 \\
\text { Beaver Valley } 2\end{array}$ & $\begin{array}{l}\text { PWR } \\
\text { PWR }\end{array}$ & $\begin{array}{l}1601 \\
1602\end{array}$ & $\begin{array}{r}11 \\
1\end{array}$ & $\begin{array}{l}3 \\
1\end{array}$ & $\begin{array}{l}8 \\
0\end{array}$ & $\begin{array}{l}3 \\
1\end{array}$ \\
\hline Florida Power Corporation . . . . . . . . . & Crystal River 3 & PWR & 1701 & 2 & 1 & 1 & 2 \\
\hline Florida Power and Light Company . . . . . . . . & $\begin{array}{l}\text { St. Lucie } 1 \\
\text { St. Lucie } 2 \\
\text { Turkey Point } 3 \\
\text { Turkey Point } 4\end{array}$ & $\begin{array}{l}\text { PWR } \\
\text { PWR } \\
\text { PWR } \\
\text { PWR }\end{array}$ & $\begin{array}{l}1801 \\
1802 \\
1803 \\
1804\end{array}$ & $\begin{array}{l}5 \\
2 \\
1 \\
0\end{array}$ & $\begin{array}{l}1 \\
1 \\
1 \\
0\end{array}$ & $\begin{array}{l}4 \\
1 \\
0 \\
0\end{array}$ & $\begin{array}{l}4 \\
2 \\
3 \\
1\end{array}$ \\
\hline GPU Nuclear Corporation $\ldots \ldots \ldots \ldots \ldots \ldots$ & $\begin{array}{l}\text { Three Mile Island } 1 \\
\text { Oyster Creek }\end{array}$ & $\begin{array}{l}\text { PWR } \\
\text { BWR }\end{array}$ & $\begin{array}{l}1901 \\
1903\end{array}$ & $\begin{array}{r}0 \\
18\end{array}$ & $\begin{array}{r}0 \\
18\end{array}$ & $\begin{array}{l}0 \\
0\end{array}$ & $\begin{array}{l}1 \\
0\end{array}$ \\
\hline Georgia Power Company ............. & $\begin{array}{l}\text { Hatch } 1 \& 2 \\
\text { Vogtle } 1 \& 2\end{array}$ & $\begin{array}{l}\text { BWR } \\
\text { PWR }\end{array}$ & $\begin{array}{l}2001 \\
2003\end{array}$ & $\begin{array}{l}0 \\
0\end{array}$ & $\begin{array}{l}0 \\
0\end{array}$ & $\begin{array}{l}0 \\
0\end{array}$ & $\begin{array}{l}5 \\
0\end{array}$ \\
\hline Gulf States Utilities Company . . . . . . . . & River Bend 1 & BWR & 2101 & 0 & 0 & 0 & 0 \\
\hline $\begin{array}{l}\text { Houston Lighting and Power } \\
\text { Company } \ldots \ldots \ldots \ldots \ldots \ldots \ldots \ldots\end{array}$ & $\begin{array}{l}\text { South Texas } 1 \\
\text { South Texas } 2\end{array}$ & $\begin{array}{l}\text { PWR } \\
\text { PWR }\end{array}$ & $\begin{array}{l}2201 \\
2202\end{array}$ & $\frac{2}{2}$ & $\begin{array}{l}0 \\
0\end{array}$ & $\begin{array}{l}2 \\
2\end{array}$ & $\begin{array}{l}1 \\
2\end{array}$ \\
\hline Illinois Power Company . . . . . . . . . . . . . . & Clinton 1 & BWR & 2301 & 0 & 0 & 0 & 1 \\
\hline $\begin{array}{l}\text { Indiana Michigan Power Company ......... } \\
\text { lowa Electric Light and }\end{array}$ & $\begin{array}{l}\text { Cook } 1 \\
\text { Cook } 2\end{array}$ & $\begin{array}{l}\text { PWR } \\
\text { PWR }\end{array}$ & $\begin{array}{l}5801 \\
5801\end{array}$ & $\begin{array}{l}3 \\
3\end{array}$ & $\begin{array}{l}1 \\
0\end{array}$ & $\begin{array}{l}2 \\
3\end{array}$ & $\begin{array}{l}0 \\
0\end{array}$ \\
\hline Power Company $\ldots \ldots \ldots \ldots \ldots \ldots$ & Duane Arnold & BWR & 2401 & $\mathbf{0}$ & 0 & 0 & 0 \\
\hline
\end{tabular}

See footnotes at end of table. 
Table FE1. Contalner Data as of December 31, 1992 (Continued)

\begin{tabular}{|c|c|c|c|c|c|c|c|}
\hline Electric Utility Name & Reactor Names & $\begin{array}{l}\text { Reactor } \\
\text { Type }\end{array}$ & $\begin{array}{l}\text { Pool } \\
\text { Site } \\
\text { ID }\end{array}$ & $\begin{array}{c}\text { Total } \\
\text { Canisters }\end{array}$ & $\begin{array}{c}\text { Canisters } \\
\text { Fuel }\end{array}$ & $\begin{array}{l}\text { Canisters } \\
\text { Nonfuel }\end{array}$ & Baskets $^{\mathrm{a}}$ \\
\hline \multicolumn{8}{|l|}{ Kansas Gas and Electric } \\
\hline Company $\ldots \ldots \ldots \ldots \ldots \ldots \ldots$ & Wolf Creek 1 & PWR & 2501 & 0 & 0 & 0 & 3 \\
\hline Long Island Lighting Company . . . . . . . . . . . & Shoreham & BWR & 2601 & 0 & 0 & 0 & 0 \\
\hline \multicolumn{8}{|l|}{ Louisiana Power and Light } \\
\hline Company $\ldots \ldots \ldots \ldots \ldots \ldots \ldots$ & Waterford 3 & PWR & 2701 & 4 & 2 & 4 & 0 \\
\hline \multicolumn{8}{|l|}{ Maine Yankee Atomic Power } \\
\hline Company $\ldots \ldots \ldots \ldots \ldots \ldots \ldots$ & Maine Yankee & PWR & 2801 & 0 & 0 & 0 & 26 \\
\hline Nebraska Public Power District $\ldots . \ldots \ldots \ldots$ & Cooper Station & BWR & 3001 & 0 & 0 & 0 & 0 \\
\hline New York Power Authority . . . . . . . . . . . . . & $\begin{array}{l}\text { Fitzpatrick } \\
\text { Indian Point } 3\end{array}$ & $\begin{array}{l}\text { BWR } \\
\text { PWR }\end{array}$ & $\begin{array}{l}3901 \\
3902\end{array}$ & $\begin{array}{l}0 \\
0\end{array}$ & $\begin{array}{l}0 \\
0\end{array}$ & $\begin{array}{l}0 \\
0\end{array}$ & $\begin{array}{l}1 \\
1\end{array}$ \\
\hline Niagara Mohawk Power Corporation ....... & $\begin{array}{l}\text { Nine Mile Point } 1 \\
\text { Nine Mile Point } 2\end{array}$ & $\begin{array}{l}\text { BWR } \\
\text { BWR }\end{array}$ & $\begin{array}{l}3101 \\
3102\end{array}$ & $\begin{array}{l}0 \\
0\end{array}$ & $\begin{array}{l}0 \\
0\end{array}$ & $\begin{array}{l}0 \\
0\end{array}$ & $\begin{array}{l}0 \\
0\end{array}$ \\
\hline North Atlantic Energy Service Corporation .... & Seabrook & PWR & 5901 & 0 & 0 & 0 & $\mathbf{0}$ \\
\hline Northeast Utilities Service Company . . . . . . . . & $\begin{array}{l}\text { Millstone } 1 \\
\text { Millstone } 2 \\
\text { Millstone } 3 \\
\text { Haddam Neck }\end{array}$ & $\begin{array}{l}\text { BWR } \\
\text { PWR } \\
\text { PWR } \\
\text { PWR }\end{array}$ & $\begin{array}{l}3201 \\
3202 \\
3203 \\
5701\end{array}$ & $\begin{array}{r}1 \\
10 \\
0 \\
9\end{array}$ & $\begin{array}{l}1 \\
6 \\
0 \\
5\end{array}$ & $\begin{array}{l}0 \\
4 \\
0 \\
4\end{array}$ & $\begin{array}{r}0 \\
13 \\
1 \\
5\end{array}$ \\
\hline Northern States Power Company . . . . . . . . . & $\begin{array}{l}\text { Monticello } \\
\text { Prairie Island } 1 \text { \& } 2\end{array}$ & $\begin{array}{l}\text { BWR } \\
\text { PWR }\end{array}$ & $\begin{array}{l}3301 \\
3302\end{array}$ & $\begin{array}{r}0 \\
22\end{array}$ & $\begin{array}{r}0 \\
20\end{array}$ & $\begin{array}{l}0 \\
2\end{array}$ & $\begin{array}{l}0 \\
0\end{array}$ \\
\hline Omaha Public Power District . . . . . . . . . . & Fort Calhoun & PWR & 3401 & 0 & 0 & 0 & $\mathbf{0}$ \\
\hline Pacific Gas and Electric Company ......... & $\begin{array}{l}\text { Diablo Canyon } 1 \\
\text { Diablo Canyon } 2 \\
\text { Humboldt Bay }\end{array}$ & $\begin{array}{l}\text { PWR } \\
\text { PWR } \\
\text { BWR }\end{array}$ & $\begin{array}{l}3501 \\
3502 \\
3503\end{array}$ & $\begin{array}{l}0 \\
1 \\
1\end{array}$ & $\begin{array}{l}0 \\
1 \\
1\end{array}$ & $\begin{array}{l}0 \\
0 \\
0\end{array}$ & $\begin{array}{l}0 \\
1 \\
7\end{array}$ \\
\hline $\begin{array}{l}\text { Pennsylvania Power and Light } \\
\text { Company } \ldots \ldots \ldots \ldots \ldots \ldots \ldots \ldots\end{array}$ & Susquehanna $1 \& 2$ & BWR & 3601 & 1 & 1 & 0 & $\mathbf{0}$ \\
\hline
\end{tabular}


Table FE1. Container Data as of December 31, 1992 (Continued)

\begin{tabular}{|c|c|c|c|c|c|c|c|}
\hline Electric Utllity Name & Reactor Names & $\begin{array}{c}\text { Reactor } \\
\text { Typo }\end{array}$ & $\begin{array}{c}\text { Pool } \\
\text { Site } \\
\text { ID }\end{array}$ & $\begin{array}{c}\text { Total } \\
\text { Canisters } \\
\end{array}$ & $\begin{array}{c}\text { Canisters } \\
\text { Fuel }\end{array}$ & $\begin{array}{l}\text { Canisters } \\
\text { Nonfuel }\end{array}$ & Baskets $^{a}$ \\
\hline Philadelphia Electric Company . . . . . . . . . & $\begin{array}{l}\text { Limerick } 1 \text { \& } 2 \\
\text { Peach Bottom } 2 \\
\text { Peach Bottom } 3\end{array}$ & $\begin{array}{l}\text { BWR } \\
\text { BWR } \\
\text { BWR }\end{array}$ & $\begin{array}{l}3701 \\
3704 \\
3705\end{array}$ & $\begin{array}{l}5 \\
2 \\
1\end{array}$ & $\begin{array}{l}0 \\
2 \\
1\end{array}$ & $\begin{array}{l}5 \\
0 \\
0\end{array}$ & $\begin{array}{r}8 \\
4 \\
11\end{array}$ \\
\hline Portland General Electric Company ........ & Trojan & PWR & 3801 & 22 & 12 & 21 & 0 \\
\hline Public Service Company of Colorado . . . . . . . & Fort St. Vrain & HTGR & 4101 & 0 & 0 & 0 & 0 \\
\hline Public Service Electric and Gas Company .... & $\begin{array}{l}\text { Hope Creek } \\
\text { Salem } 1 \\
\text { Salem } 2\end{array}$ & $\begin{array}{l}\text { BWR } \\
\text { PWR } \\
\text { PWR }\end{array}$ & $\begin{array}{l}4201 \\
4402 \\
4203\end{array}$ & $\begin{array}{l}0 \\
0 \\
0\end{array}$ & $\begin{array}{l}0 \\
0 \\
0\end{array}$ & $\begin{array}{l}0 \\
0 \\
0\end{array}$ & $\begin{array}{r}0 \\
18 \\
7\end{array}$ \\
\hline Rochester Gas and Electric Corporation ...... & Ginna & PWR & 4401 & 10 & 8 & 2 & 2 \\
\hline Sacramento Municipal Utility District . . . . . . . & Rancho Seco & PWR & 4501 & 0 & 0 & 0 & 0 \\
\hline South Carolina Electric and Gas Company .... & Summer & PWR & 4601 & 1 & 1 & 0 & 1 \\
\hline Southern California Edison Company ....... & $\begin{array}{l}\text { San Onofre } 1 \\
\text { San Onofre } 2 \\
\text { San Onofre } 3\end{array}$ & $\begin{array}{l}\text { PWR } \\
\text { PWR } \\
\text { PWR }\end{array}$ & $\begin{array}{l}4701 \\
4702 \\
4703\end{array}$ & $\begin{array}{l}0 \\
0 \\
0\end{array}$ & $\begin{array}{l}0 \\
0 \\
0\end{array}$ & $\begin{array}{l}0 \\
0 \\
0\end{array}$ & $\begin{array}{l}4 \\
8 \\
9\end{array}$ \\
\hline System Energy Resources, Inc. . . . . . . . . & Grand Gulf 1 & BWR & 2901 & 1 & 1 & 0 & 18 \\
\hline Tennessee Valley Authority . . . . . . . . . . . & $\begin{array}{l}\text { Bellefonte } 1 \\
\text { Bellefonte } 2 \\
\text { Browns Ferry } 1 \text { \& } 2 \\
\text { Browns Ferry } 3 \\
\text { Sequoyah } 1 \text { \& } 2 \\
\text { Watts Bar } 1 \text { \& } 2\end{array}$ & $\begin{array}{l}\text { PWR } \\
\text { PWR } \\
\text { BWR } \\
\text { BWR } \\
\text { PWR } \\
\text { PWR }\end{array}$ & $\begin{array}{l}4801 \\
4802 \\
4803 \\
4805 \\
4808 \\
4810\end{array}$ & $\begin{array}{l}0 \\
0 \\
1 \\
0 \\
0 \\
0\end{array}$ & $\begin{array}{l}0 \\
0 \\
1 \\
0 \\
0 \\
0\end{array}$ & $\begin{array}{l}0 \\
0 \\
0 \\
0 \\
0 \\
0\end{array}$ & $\begin{array}{l}0 \\
0 \\
0 \\
0 \\
0 \\
0\end{array}$ \\
\hline TU Electric $\ldots \ldots \ldots \ldots \ldots \ldots \ldots$ & Comanche Peak $1 \& 2$ & PWR & 4901 & 0 & 0 & 0 & 37 \\
\hline Toledo Edison Company . . . . . . . . . . . . . & Davis-Besse & PWR & 5001 & 3 & 1 & 2 & 3 \\
\hline Union Electric Company $\ldots \ldots \ldots \ldots \ldots$ & Callaway & PWR & 5101 & 1 & 1 & 0 & 1 \\
\hline
\end{tabular}

See footnotes at end of table. 
Table FE1. Container Data as of December 31, 1992 (Continued)

\begin{tabular}{|c|c|c|c|c|c|c|c|}
\hline Electric Utility Name & Reactor Names & $\begin{array}{l}\text { Reactor } \\
\text { Type }\end{array}$ & $\begin{array}{l}\text { Pool } \\
\text { Site } \\
\text { ID }\end{array}$ & $\begin{array}{c}\text { Total } \\
\text { Canisters }\end{array}$ & $\begin{array}{c}\text { Canisters } \\
\text { Fuel }\end{array}$ & $\begin{array}{l}\text { Canisters } \\
\text { Nonfuel }\end{array}$ & Baskets $^{a}$ \\
\hline \multicolumn{8}{|l|}{ Vermont Yankee Nuclear Power } \\
\hline Corporation $\ldots \ldots \ldots \ldots \ldots \ldots \ldots \ldots$ & Vermont Yankee & BWR & 6001 & 0 & 0 & 0 & 0 \\
\hline Virginia Power $\ldots \ldots \ldots \ldots \ldots \ldots \ldots$ & $\begin{array}{l}\text { North Anna } 1 \& 2 \\
\text { Surry } 1 \& 2\end{array}$ & $\begin{array}{l}\text { PWR } \\
\text { PWR }\end{array}$ & $\begin{array}{l}5201 \\
5203\end{array}$ & $\begin{array}{l}3 \\
3\end{array}$ & $\begin{array}{l}1 \\
2\end{array}$ & $\begin{array}{l}2 \\
2\end{array}$ & $\begin{array}{l}2 \\
8\end{array}$ \\
\hline \multicolumn{8}{|l|}{ Washington Public Power } \\
\hline Supply System $\ldots \ldots \ldots \ldots \ldots \ldots$ & Washington Nuclear 2 & BWR & 5302 & 0 & 0 & 0 & 1 \\
\hline Wisconsin Electric Power Company . . . . . . & Point Beach 1 \& 2 & PWR & 5401 & 16 & 13 & 3 & 0 \\
\hline Wisconsin Public Service Corporation $\ldots . .$. & Kewaunee & PWR & 5701 & 0 & 0 & 0 & 0 \\
\hline Yankee Atomic Electric Company $\ldots . . . \ldots$ & Yankee Rowe & PWR & 5601 & 0 & 0 & 0 & 0 \\
\hline$\ldots \ldots \ldots \ldots \ldots$ & 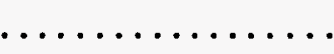 & & & $b_{286}$ & 151 & 149 & 264 \\
\hline
\end{tabular}

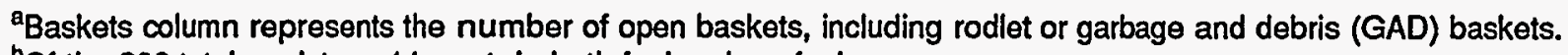

b $O$ the 286 total canisters, 14 contain both fuel and nonfuel.

$P W R=$ Pressurized-water reactor; BWR $=$ Boiling-water reactor; $H T G R=$ High-temperature, gas-cooled reactor

Notes: Data for the following pool sites are as of December 31, 1991 - Pilgrim 1, Indian Point 1 \& 2, Big Rock Point, Beaver Valley 1, Three Mile Island 1,

Wolf Crieek 1, Cooper Station, Diablo Canyon 2, Salem 2, Summer, Davis-Besse, and Yankee Rowe. Shoreham did not report for either 1991 or 1992.

Source: Energy Information Administration, Form RW-859, "Nuclear Fuel Data" (1992). 
Table FE2. Canisters Containing Spent Fuel as of December 31, 1992

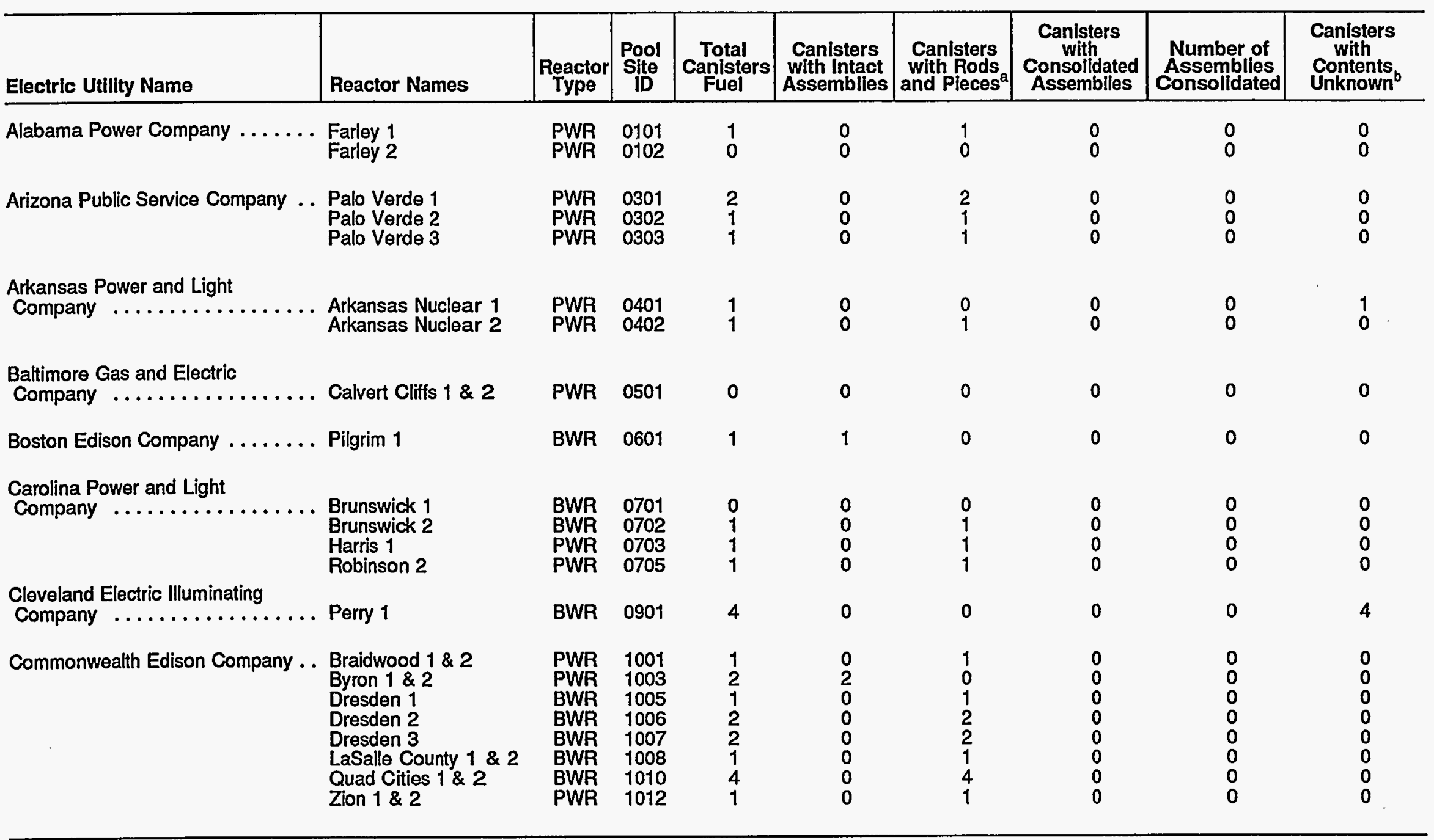

See footnotes at end of table. 
Table FE2. Canisters Containing Spent Fuel as of December 31, 1992 (Continued)

\begin{tabular}{|c|c|c|c|c|c|c|c|c|c|}
\hline Electric Utility Name & Reactor Names & $\mid \begin{array}{c}\text { Reactor } \\
\text { Type }\end{array}$ & $\begin{array}{c}\text { Pool } \\
\text { Site } \\
\text { ID }\end{array}$ & $\begin{array}{c}\text { Total } \\
\text { Canisters } \\
\text { Fuel }\end{array}$ & $\begin{array}{c}\text { Canisters } \\
\text { with Intact } \\
\text { Assemblies }\end{array}$ & $\begin{array}{c}\text { Canisters } \\
\text { with Rods } \\
\text { and Pieces }\end{array}$ & $\begin{array}{c}\text { Canisters } \\
\text { with } \\
\text { Consolidated } \\
\text { Assemblies }\end{array}$ & $\begin{array}{c}\text { Number of } \\
\text { Assemblies } \\
\text { Consolidated }\end{array}$ & $\begin{array}{l}\text { Canisters } \\
\text { with } \\
\text { Contents } \\
\text { Unknown } \\
\end{array}$ \\
\hline $\begin{array}{l}\text { Consolidated Edison Company } \\
\text { of New York } \ldots \ldots \ldots \ldots \ldots \ldots\end{array}$ & $\begin{array}{l}\text { Indian Point } 1 \\
\text { Indian Point } 2\end{array}$ & $\begin{array}{l}\text { PWR } \\
\text { PWR }\end{array}$ & $\begin{array}{l}1101 \\
1102\end{array}$ & $\begin{array}{l}0 \\
0\end{array}$ & $\begin{array}{l}0 \\
0\end{array}$ & $\begin{array}{l}0 \\
0\end{array}$ & $\begin{array}{l}0 \\
0\end{array}$ & $\begin{array}{l}0 \\
0\end{array}$ & $\begin{array}{l}0 \\
0\end{array}$ \\
\hline Consumers Power Company . . . . & $\begin{array}{l}\text { Big Rock Point } \\
\text { Palisades }\end{array}$ & $\begin{array}{l}\text { BWR } \\
\text { PWR }\end{array}$ & $\begin{array}{l}1201 \\
1204\end{array}$ & $\begin{array}{l}0 \\
6\end{array}$ & $\begin{array}{l}0 \\
0\end{array}$ & $\begin{array}{l}0 \\
1\end{array}$ & $\begin{array}{l}0 \\
5\end{array}$ & $\stackrel{0}{17}$ & $\begin{array}{l}0 \\
0\end{array}$ \\
\hline Dairyland Power Cooperative ..... & LaCrosse & BWR & 1301 & 0 & 0 & 0 & 0 & 0 & 0 \\
\hline Detroit Edison Company . . . . . . . & Enrico Fermi 2 & BWR & 1402 & 0 & 0 & 0 & 0 & 0 & 0 \\
\hline Duke Power Company $\ldots . . .$. & $\begin{array}{l}\text { Catawba } 1 \\
\text { Catawba } 2 \\
\text { McGuire } 1 \\
\text { McGuire } 2 \\
\text { Oconee } 1 \text { \& } 2 \\
\text { Ocones } 3\end{array}$ & $\begin{array}{l}\text { PWR } \\
\text { PWR } \\
\text { PWR } \\
\text { PWR } \\
\text { PWR } \\
\text { PWR }\end{array}$ & $\begin{array}{l}1501 \\
1502 \\
1504 \\
1505 \\
1506 \\
1508\end{array}$ & $\begin{array}{l}0 \\
0 \\
2 \\
1 \\
4 \\
1\end{array}$ & $\begin{array}{l}0 \\
0 \\
0 \\
0 \\
0 \\
0\end{array}$ & $\begin{array}{l}0 \\
0 \\
2 \\
1 \\
2 \\
1\end{array}$ & $\begin{array}{l}0 \\
0 \\
0 \\
0 \\
2 \\
0\end{array}$ & $\begin{array}{l}0 \\
0 \\
0 \\
0 \\
4 \\
0\end{array}$ & $\begin{array}{l}0 \\
0 \\
0 \\
0 \\
0 \\
0\end{array}$ \\
\hline Duquesne Light Company . . . . . . & $\begin{array}{l}\text { Beaver Valley } 1 \\
\text { Beaver Valiey } 2\end{array}$ & $\begin{array}{l}\text { PWR } \\
\text { PWR }\end{array}$ & $\begin{array}{l}1601 \\
1602\end{array}$ & $\begin{array}{l}3 \\
1\end{array}$ & $\begin{array}{l}2 \\
0\end{array}$ & $\begin{array}{l}1 \\
1\end{array}$ & $\begin{array}{l}0 \\
0\end{array}$ & $\begin{array}{l}0 \\
0\end{array}$ & $\begin{array}{l}0 \\
0\end{array}$ \\
\hline Florida Power Corporation $\ldots \ldots$ & Crystal River 3 & PWR & 1701 & 1 & 0 & 1 & 0 & 0 & 0 \\
\hline Florida Power and Light Company . & $\begin{array}{l}\text { St. Lucie } 1 \\
\text { St. Lucie } 2 \\
\text { Turkey Point } 3 \\
\text { Turkey Point } 4\end{array}$ & $\begin{array}{l}\text { PWR } \\
\text { PWR } \\
\text { PWR } \\
\text { PWR }\end{array}$ & $\begin{array}{l}1801 \\
1802 \\
1803 \\
1804\end{array}$ & $\begin{array}{l}1 \\
1 \\
1 \\
0\end{array}$ & $\begin{array}{l}0 \\
0 \\
0 \\
0\end{array}$ & $\begin{array}{l}0 \\
0 \\
1 \\
0\end{array}$ & $\begin{array}{l}0 \\
0 \\
0 \\
0\end{array}$ & $\begin{array}{l}0 \\
0 \\
0 \\
0\end{array}$ & $\begin{array}{l}1 \\
1 \\
0 \\
0\end{array}$ \\
\hline GPU Nuclear Corporation . . . . . . & $\begin{array}{l}\text { Three Mile Island } 1 \\
\text { Oyster Creek }\end{array}$ & $\begin{array}{l}\text { PWR } \\
\text { BWR }\end{array}$ & $\begin{array}{l}1901 \\
1903\end{array}$ & $\begin{array}{r}0 \\
18\end{array}$ & $\begin{array}{r}0 \\
18\end{array}$ & $\begin{array}{l}0 \\
0\end{array}$ & $\begin{array}{l}0 \\
0\end{array}$ & $\begin{array}{l}0 \\
0\end{array}$ & $\begin{array}{l}0 \\
0\end{array}$ \\
\hline Georgia Power Company ...... & $\begin{array}{l}\text { Hatch } 1 \& 2 \\
\text { Vogtle } 1 \& 2\end{array}$ & $\begin{array}{l}\text { BWR } \\
\text { PWR }\end{array}$ & $\begin{array}{l}2001 \\
2003\end{array}$ & $\begin{array}{l}0 \\
0\end{array}$ & $\begin{array}{l}0 \\
0\end{array}$ & $\begin{array}{l}0 \\
0\end{array}$ & $\begin{array}{l}0 \\
0\end{array}$ & $\begin{array}{l}0 \\
0\end{array}$ & $\begin{array}{l}0 \\
0\end{array}$ \\
\hline
\end{tabular}

See footnotes at end of table. 
Table FE2. Canisters Containing Spent Fuel as of December 31, 1992 (Continued)

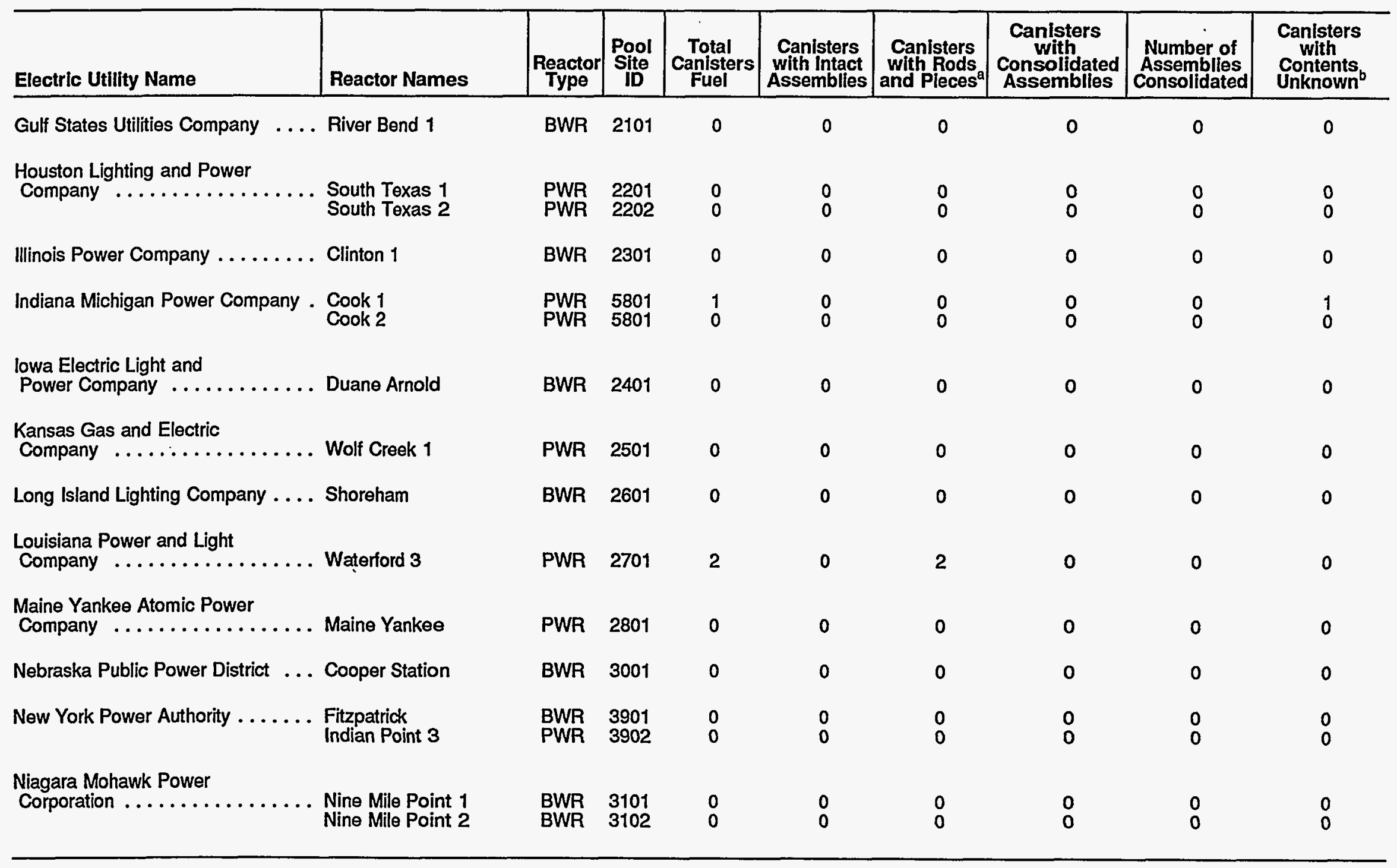

See footnotes at end of table. 
Table FE2. Canisters Containing Spent Fuel as of December 31, 1992 (Continued)

\begin{tabular}{|c|c|c|c|c|c|c|c|c|c|}
\hline Electric Utility Name & Reactor Names & Reactor & $\begin{array}{l}\text { Pool } \\
\text { Site } \\
\text { ID }\end{array}$ & $\begin{array}{c}\text { Total } \\
\text { Canisters } \\
\text { Fuel }\end{array}$ & $\begin{array}{l}\text { Canisters } \\
\text { with Intact } \\
\text { Assemblies }\end{array}$ & $\begin{array}{c}\text { Canisters } \\
\text { with Rods } \\
\text { and Pieces }\end{array}$ & $\begin{array}{l}\text { Canisters } \\
\text { with } \\
\text { Consolidated } \\
\text { Assemblies }\end{array}$ & $\begin{array}{c}\text { Number of } \\
\text { Assemblies } \\
\text { Consolidated }\end{array}$ & $\begin{array}{c}\text { Canisters } \\
\text { with } \\
\text { Contents } \\
\text { Unknown }^{b}\end{array}$ \\
\hline $\begin{array}{l}\text { North Atlantic Energy Service } \\
\text { Corporation . . . . . . . . . . . }\end{array}$ & Seabrook & PWR & 5901 & 0 & 0 & 0 & 0 & 0 & 0 \\
\hline $\begin{array}{l}\text { Northeast Utilities Service } \\
\text { Company } \ldots \ldots \ldots \ldots \ldots \ldots\end{array}$ & $\begin{array}{l}\text { Millstone } 1 \\
\text { Millstone } 2 \\
\text { Millstone } 3 \\
\text { Haddam Neck }\end{array}$ & $\begin{array}{l}\text { BWR } \\
\text { PWR } \\
\text { PWR } \\
\text { PWR }\end{array}$ & $\begin{array}{l}3201 \\
3202 \\
3203 \\
5701\end{array}$ & $\begin{array}{l}1 \\
6 \\
0 \\
5\end{array}$ & $\begin{array}{l}0 \\
0 \\
0 \\
0\end{array}$ & $\begin{array}{l}1 \\
3 \\
0 \\
5\end{array}$ & $\begin{array}{l}0 \\
3 \\
0 \\
0\end{array}$ & $\begin{array}{l}0 \\
6 \\
0 \\
0\end{array}$ & $\begin{array}{l}0 \\
0 \\
0 \\
0\end{array}$ \\
\hline Northern States Power Company .. & $\begin{array}{l}\text { Monticello } \\
\text { Prairie Island } 1 \text { \& } 2\end{array}$ & $\begin{array}{l}\text { BWR } \\
\text { PWR }\end{array}$ & $\begin{array}{l}3301 \\
3302\end{array}$ & $\begin{array}{r}0 \\
20\end{array}$ & $\begin{array}{l}0 \\
0\end{array}$ & $\begin{array}{l}0 \\
2\end{array}$ & $\begin{array}{r}0 \\
18\end{array}$ & $\begin{array}{r}0 \\
36\end{array}$ & $\begin{array}{l}0 \\
0\end{array}$ \\
\hline Omaha Public Power District ..... & Fort Calhoun & PWR & 3401 & 0 & 0 & 0 & 0 & 0 & 0 \\
\hline Pacific Gas and Electric Company . & $\begin{array}{l}\text { Diablo Canyon } 1 \\
\text { Diablo Canyon } 2 \\
\text { Humboldt Bay }\end{array}$ & $\begin{array}{l}\text { PWR } \\
\text { PWR } \\
\text { BWR }\end{array}$ & $\begin{array}{l}3501 \\
3502 \\
3503\end{array}$ & $\begin{array}{l}0 \\
1 \\
1\end{array}$ & $\begin{array}{l}0 \\
0 \\
1\end{array}$ & $\begin{array}{l}0 \\
1 \\
0\end{array}$ & $\begin{array}{l}0 \\
0 \\
0\end{array}$ & $\begin{array}{l}0 \\
0 \\
0\end{array}$ & $\begin{array}{l}0 \\
0 \\
0\end{array}$ \\
\hline $\begin{array}{l}\text { Pennsylvania Power and Light } \\
\text { Company } \ldots \ldots \ldots \ldots \ldots \ldots\end{array}$ & Susquehanna $1 \& 2$ & BWR & 3601 & 1 & 0 & 1 & 0 & 0 & 0 \\
\hline Philadelphia Electric Company .... & $\begin{array}{l}\text { Limerick } 1 \text { \& } 2 \\
\text { Peach Bottom } 2 \\
\text { Peach Bottom } 3\end{array}$ & $\begin{array}{l}\text { BWR } \\
\text { BWR } \\
\text { BWR }\end{array}$ & $\begin{array}{l}3701 \\
3704 \\
3705\end{array}$ & $\begin{array}{l}0 \\
2 \\
1\end{array}$ & $\begin{array}{l}0 \\
0 \\
0\end{array}$ & $\begin{array}{l}0 \\
2 \\
1\end{array}$ & $\begin{array}{l}0 \\
0 \\
0\end{array}$ & $\begin{array}{l}0 \\
0 \\
0\end{array}$ & $\begin{array}{l}0 \\
0 \\
0\end{array}$ \\
\hline $\begin{array}{l}\text { Portland General Electric } \\
\text { Company } \ldots \ldots \ldots \ldots \ldots\end{array}$ & Trojan & PWR & 3801 & 12 & 0 & 12 & 0 & 0 & 0 \\
\hline $\begin{array}{l}\text { Public Service Company of } \\
\text { Colorado } \ldots \ldots \ldots \ldots \ldots \ldots\end{array}$ & Fort St. Vrain & HTGR & 4101 & 0 & 0 & 0 & 0 & 0 & 0 \\
\hline $\begin{array}{l}\text { Public Service Electric and Gas } \\
\text { Company } \ldots \ldots \ldots \ldots \ldots \ldots \ldots\end{array}$ & $\begin{array}{l}\text { Hope Creek } \\
\text { Salem } 1 \\
\text { Salem } 2\end{array}$ & $\begin{array}{l}\text { BWR } \\
\text { PWR } \\
\text { PWR }\end{array}$ & $\begin{array}{l}4201 \\
4202 \\
4203\end{array}$ & $\begin{array}{l}0 \\
0 \\
0\end{array}$ & $\begin{array}{l}0 \\
0 \\
0\end{array}$ & $\begin{array}{l}0 \\
0 \\
0\end{array}$ & $\begin{array}{l}0 \\
0 \\
0\end{array}$ & $\begin{array}{l}0 \\
0 \\
0\end{array}$ & $\begin{array}{l}0 \\
0 \\
0\end{array}$ \\
\hline
\end{tabular}

See footnotes at end of table. 
Table FE2. Canisters Containing Spent Fuel as of December 31, 1992 (Continued)

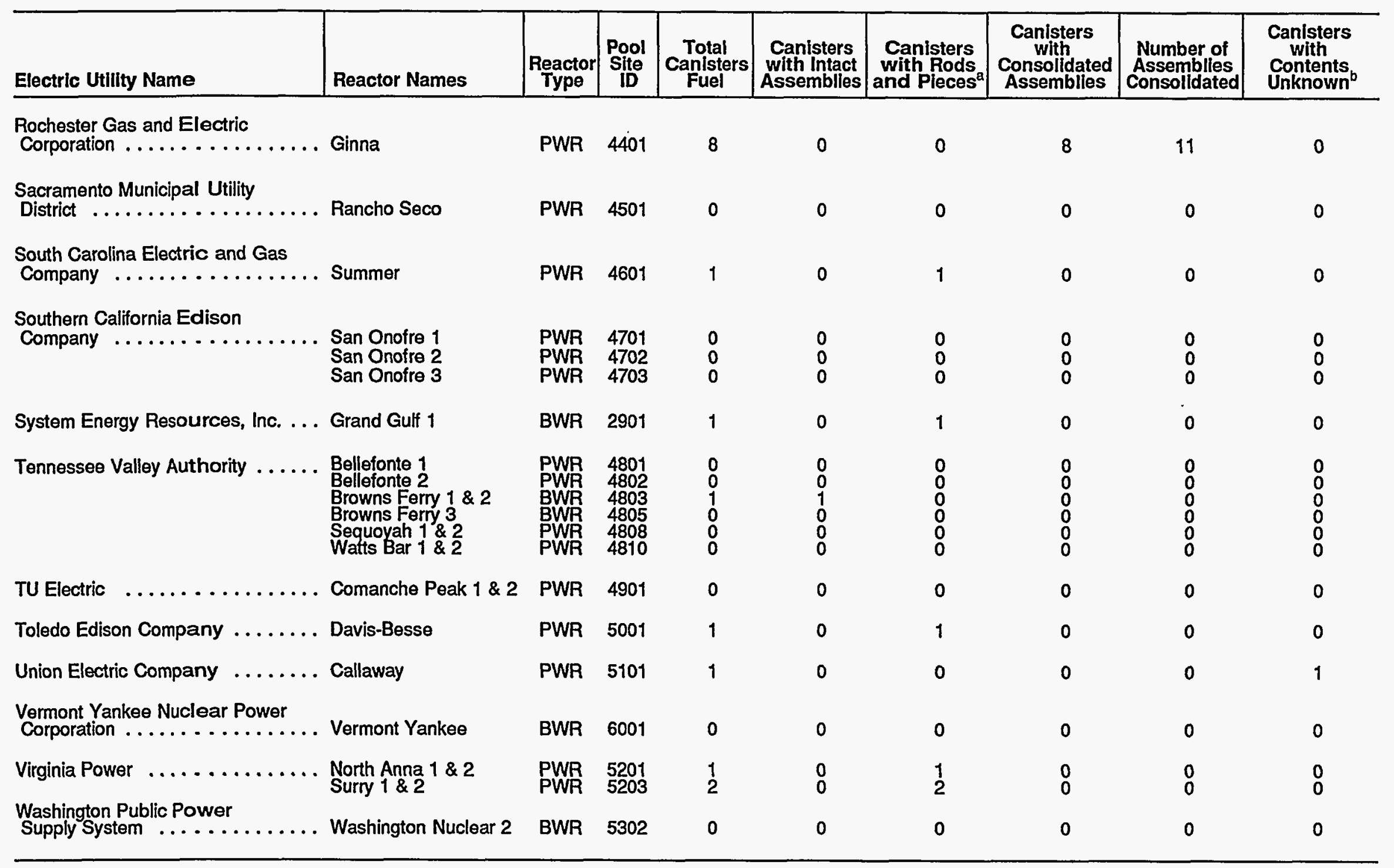

See footriotes at end of table. 
Table FE2. Canisters Containing Spent Fuel as of December 31, 1992 (Continued)

\begin{tabular}{|c|c|c|c|c|c|c|c|c|c|}
\hline Electric Utility Name & Reactor Names & $\begin{array}{c}\text { Reactor } \\
\text { Type } \\
\end{array}$ & $\begin{array}{c}\text { Pool } \\
\text { Site } \\
\text { ID }\end{array}$ & $\begin{array}{c}\text { Total } \\
\text { Canisters } \\
\text { Fuel }\end{array}$ & $\begin{array}{c}\text { Canisters } \\
\text { with Intact } \\
\text { Assemblies }\end{array}$ & $\begin{array}{c}\text { Canisters } \\
\text { with Rods } \\
\text { and Pleces } \\
\end{array}$ & $\begin{array}{c}\text { Canisters } \\
\text { with } \\
\text { Consolidated } \\
\text { Assemblies }\end{array}$ & $\begin{array}{c}\text { Number of } \\
\text { Assemblies } \\
\text { Consolidated }\end{array}$ & $\begin{array}{c}\text { Canisters } \\
\text { with } \\
\text { Contents } \\
\text { Unknown }\end{array}$ \\
\hline $\begin{array}{l}\text { Wisconsin Electric Power } \\
\text { Company } \ldots \ldots \ldots \ldots \ldots\end{array}$ & Point Beach $1 \& 2$ & PWR & 5401 & 13 & 12 & 1 & 0 & 0 & $\mathbf{0}$ \\
\hline $\begin{array}{l}\text { Wisconsin Public Service } \\
\text { Corporation } \ldots \ldots \ldots \ldots \ldots\end{array}$ & Kewaunee & PWR & 5701 & 0 & 0 & 0 & 0 & 0 & $\mathbf{0}$ \\
\hline Yankee Atomic Electric Company & Yankee Rowe & PWR & 5601 & 0 & 0 & 0 & 0 & 0 & 0 \\
\hline Total BWR . . . . . . . . . . . & $\ldots \ldots \ldots \ldots$ & .... & $\ldots$. & 42 & 21 & 17 & $\mathbf{0}$ & 0 & 4 \\
\hline Total PWR $\ldots \ldots \ldots \ldots \ldots$ & $\cdots \ldots \ldots \ldots$ & $\cdots$ & $\cdots \cdots$ & 109 & 16 & 52 & 36 & 74 & 5 \\
\hline TOTAL .............. & $\ldots \ldots \ldots \ldots$ & $\cdots$ & $\cdots \cdots$ & 151 & 37 & 69 & 36 & 74 & 9 \\
\hline
\end{tabular}

${ }^{a}$ Canisters with Rods and Pieces column represents the number of canisters containing intact fuel rods from consolidation, reconstitution, or reconstruction.

${ }^{b}$ Canisters with Contents Unknown column represents the number of canisters containing fuel for which specific data on the contents of the canister were not reported on the Form RW-859 survey.

PWR $=$ Pressurized-water reactor; BWR = Boiling-water reactor; HTGR $=$ High-temperature, gas-cooled reactor.

Notes: Data for the following pool sites are as of December 31, 1991 - Pilgrim 1, Indian Point 1 \& 2, Big Rock Point, Beaver Valley 1, Three Mile Island 1, Wolf

Creek 1, Cooper Station, Diablo Canyon 2, Salem 2, Summer, Davis-Besse, and Yankee Rowe. Shoreham did not report for either 1991 or 1992.

Source: Energy Information Administration, Form RW-859, "Nuclear Fuel Data" (1992). 


\begin{tabular}{|c|c|c|c|c|c|c|c|c|c|}
\hline Electric Utility Name & Reactor Names & $\begin{array}{c}\text { Reactor } \\
\text { Type }\end{array}$ & $\begin{array}{l}\text { Pool } \\
\text { Site } \\
\text { ID }\end{array}$ & \begin{tabular}{c|} 
Total \\
Canisters \\
Nonfuel $^{\mathrm{a}}$ \\
\end{tabular} & Debris $^{b}$ & $\begin{array}{c}\text { Nonfuel } \\
\text { Disassembly } \\
\text { Hardware }\end{array}$ & $\begin{array}{c}\text { Nonfuel } \\
\text { Components }\end{array}$ & Other ${ }^{d}$ & $\begin{array}{l}\text { Canisters } \\
\text { with } \\
\text { Contents } \\
\text { Unknown } \\
\end{array}$ \\
\hline Alabama Power Company .......... & $\begin{array}{l}\text { Farley } 1 \\
\text { Farley } 2\end{array}$ & $\begin{array}{l}\text { PWR } \\
\text { PWR }\end{array}$ & $\begin{array}{l}0101 \\
0102\end{array}$ & $\begin{array}{l}0 \\
0\end{array}$ & $\begin{array}{l}0 \\
0\end{array}$ & $\begin{array}{l}0 \\
0\end{array}$ & $\begin{array}{l}0 \\
0\end{array}$ & $\begin{array}{l}0 \\
0\end{array}$ & $\begin{array}{l}0 \\
0\end{array}$ \\
\hline Arizona Public Service Company . . . . . . . & $\begin{array}{l}\text { Palo Verde } 1 \\
\text { Palo Verde } 2 \\
\text { Palo Verde } 3\end{array}$ & $\begin{array}{l}\text { PWR } \\
\text { PWR } \\
\text { PWR }\end{array}$ & $\begin{array}{l}0301 \\
0302 \\
0303\end{array}$ & $\begin{array}{l}6 \\
4 \\
4\end{array}$ & $\begin{array}{l}0 \\
0 \\
0\end{array}$ & $\begin{array}{l}5 \\
4 \\
4\end{array}$ & $\begin{array}{l}5 \\
4 \\
4\end{array}$ & $\begin{array}{l}0 \\
0 \\
0\end{array}$ & $\begin{array}{l}0 \\
0 \\
0\end{array}$ \\
\hline Arkansas Power and Light Company .... & $\begin{array}{l}\text { Arkansas Nuclear } 1 \\
\text { Arkansas Nuclear } 2\end{array}$ & $\begin{array}{l}\text { PWR } \\
\text { PWR }\end{array}$ & $\begin{array}{l}0401 \\
0402\end{array}$ & $\begin{array}{l}16 \\
13\end{array}$ & $\begin{array}{l}0 \\
0\end{array}$ & $\begin{array}{l}0 \\
0\end{array}$ & $\begin{array}{l}0 \\
0\end{array}$ & $\begin{array}{l}0 \\
0\end{array}$ & $\begin{array}{r}16 \\
13\end{array}$ \\
\hline Baltimore Gas and Electric Company .... & Calvert Cliffs $1 \& 2$ & PWR & 0501 & 21 & 0 & 4 & 7 & 10 & 0 \\
\hline Boston Edison Company . . . . . . . . . . & Pilgrim 1 & BWR & 0601 & 0 & 0 & 0 & 0 & 0 & 0 \\
\hline Carolina Power and Light Company ..... & $\begin{array}{l}\text { Brunswick } 1 \\
\text { Brunswick } 2 \\
\text { Harris } 1 \\
\text { Robinson } 2\end{array}$ & $\begin{array}{l}\text { BWR } \\
\text { BWR } \\
\text { PWR } \\
\text { PWR }\end{array}$ & $\begin{array}{l}0701 \\
0702 \\
0703 \\
0705\end{array}$ & $\begin{array}{l}0 \\
0 \\
0 \\
2\end{array}$ & $\begin{array}{l}0 \\
0 \\
0 \\
0\end{array}$ & $\begin{array}{l}0 \\
0 \\
0 \\
0\end{array}$ & $\begin{array}{l}0 \\
0 \\
0 \\
0\end{array}$ & $\begin{array}{l}0 \\
0 \\
0 \\
2\end{array}$ & $\begin{array}{l}0 \\
0 \\
0 \\
0\end{array}$ \\
\hline $\begin{array}{l}\text { Cleveland Electric Illuminating } \\
\text { Company } \ldots \ldots \ldots \ldots \ldots \ldots \ldots \ldots \ldots\end{array}$ & Perry 1 & BWR & 0901 & 0 & 0 & 0 & $\mathbf{0}$ & 0 & 0 \\
\hline Commonwealth Edison Company ... & $\begin{array}{l}\text { Braidwood } 1 \text { \& } 2 \\
\text { Byron } 1 \text { \& } 2 \\
\text { Dresden } 1 \\
\text { Dresden } 2 \\
\text { Dresden } 3 \\
\text { LaSalle County } 1 \text { \& } 2 \\
\text { Quad Cities } 1 \text { \& } 2 \\
\text { Zion } 1 \text { \& } 2\end{array}$ & $\begin{array}{l}\text { PWR } \\
\text { PWR } \\
\text { BWR } \\
\text { BWR } \\
\text { BWR } \\
\text { BWR } \\
\text { BWR } \\
\text { PWR }\end{array}$ & $\begin{array}{l}1001 \\
1003 \\
1005 \\
1006 \\
1007 \\
1008 \\
1010 \\
1012\end{array}$ & $\begin{array}{l}2 \\
0 \\
3 \\
0 \\
0 \\
0 \\
0 \\
2\end{array}$ & $\begin{array}{l}0 \\
0 \\
0 \\
0 \\
0 \\
0 \\
0 \\
0\end{array}$ & $\begin{array}{l}0 \\
0 \\
0 \\
0 \\
0 \\
0 \\
0 \\
0\end{array}$ & $\begin{array}{l}1 \\
0 \\
3 \\
0 \\
0 \\
0 \\
0 \\
0\end{array}$ & $\begin{array}{l}2 \\
0 \\
0 \\
0 \\
0 \\
0 \\
0 \\
2\end{array}$ & $\begin{array}{l}0 \\
0 \\
0 \\
0 \\
0 \\
0 \\
0 \\
0\end{array}$ \\
\hline $\begin{array}{l}\text { Consolidated Edison Company } \\
\text { of New York } \ldots \ldots \ldots \ldots \ldots \ldots \ldots \ldots\end{array}$ & $\begin{array}{l}\text { Indian Point } 1 \\
\text { Indian Point } 2\end{array}$ & $\begin{array}{l}\text { PWR } \\
\text { PWR }\end{array}$ & $\begin{array}{l}1101 \\
1102\end{array}$ & $\begin{array}{l}0 \\
0\end{array}$ & $\begin{array}{l}0 \\
0\end{array}$ & $\begin{array}{l}0 \\
0\end{array}$ & $\begin{array}{l}0 \\
0\end{array}$ & $\begin{array}{l}0 \\
0\end{array}$ & $\begin{array}{l}0 \\
0\end{array}$ \\
\hline Consumers Power Company . . . . . . . . & $\begin{array}{l}\text { Big Rock Point } \\
\text { Palisades }\end{array}$ & $\begin{array}{l}\text { BWR } \\
\text { PWR }\end{array}$ & $\begin{array}{l}1201 \\
1204\end{array}$ & $\begin{array}{l}2 \\
0\end{array}$ & $\begin{array}{l}0 \\
0\end{array}$ & $\begin{array}{l}0 \\
0\end{array}$ & $\begin{array}{l}0 \\
0\end{array}$ & $\begin{array}{l}0 \\
0\end{array}$ & $\begin{array}{l}2 \\
0\end{array}$ \\
\hline Dairyland Power Cooperative ......... & LaCrosse & BWR & 1301 & 0 & 0 & 0 & 0 & 0 & 0 \\
\hline
\end{tabular}


Table FE3. Canisters Containing Nonfuel Components as of December 31, 1992 (Continued)

\begin{tabular}{|c|c|c|c|c|c|c|c|c|c|}
\hline Electric Utility Name & Reactor Names & $\begin{array}{l}\text { Reactor } \\
\text { Type }\end{array}$ & $\begin{array}{c}\text { Pool } \\
\text { Site } \\
\text { ID }\end{array}$ & $\begin{array}{c}\text { Total } \\
\text { Canisters } \\
\text { Nonfuel }^{\mathrm{a}}\end{array}$ & Debris $^{b}$ & $\begin{array}{c}\text { Nonfuel } \\
\text { Disassembly } \\
\text { Hardware }\end{array}$ & $\begin{array}{c}\text { Nonfuel } \\
\text { Components }^{c}\end{array}$ & Other $^{d}$ & \begin{tabular}{|c|} 
Canisters \\
with \\
Contents \\
Unknown \\
\end{tabular} \\
\hline Detroit Edison Company ............ & Enrico Fermi 2 & BWR & 1402 & 0 & 0 & 0 & 0 & 0 & 0 \\
\hline Duke Power Company $\ldots \ldots \ldots \ldots$ & $\begin{array}{l}\text { Catawba } 1 \\
\text { Catawba } 2 \\
\text { McGuire } 1 \\
\text { McGuire } 2 \\
\text { Oconee } 1 \text { \& } 2 \\
\text { Oconee } 3\end{array}$ & $\begin{array}{l}\text { PWR } \\
\text { PWR } \\
\text { PWR } \\
\text { PWR } \\
\text { PWR } \\
\text { PWR }\end{array}$ & $\begin{array}{l}1501 \\
1502 \\
1504 \\
1505 \\
1506 \\
1508\end{array}$ & $\begin{array}{l}0 \\
0 \\
0 \\
0 \\
0 \\
0\end{array}$ & $\begin{array}{l}0 \\
0 \\
0 \\
0 \\
0 \\
0\end{array}$ & $\begin{array}{l}0 \\
0 \\
0 \\
0 \\
0 \\
0\end{array}$ & $\begin{array}{l}0 \\
0 \\
0 \\
0 \\
0 \\
0\end{array}$ & $\begin{array}{l}0 \\
0 \\
0 \\
0 \\
0 \\
0\end{array}$ & $\begin{array}{l}0 \\
0 \\
0 \\
0 \\
0 \\
0\end{array}$ \\
\hline Duquesne Light Company . . . . . . . . . & $\begin{array}{l}\text { Beaver Valley } 1 \\
\text { Beaver Valley } 2\end{array}$ & $\begin{array}{l}\text { PWR } \\
\text { PWR }\end{array}$ & $\begin{array}{l}1601 \\
1602\end{array}$ & $\begin{array}{l}8 \\
0\end{array}$ & $\begin{array}{l}0 \\
0\end{array}$ & $\begin{array}{l}2 \\
0\end{array}$ & $\begin{array}{l}8 \\
0\end{array}$ & $\begin{array}{l}8 \\
0\end{array}$ & $\begin{array}{l}0 \\
0\end{array}$ \\
\hline Florida Power Corporation $\quad \ldots \ldots \ldots \ldots$ & Crystal River 3 & PWR & 1701 & 1 & 0 & 1 & 1 & 1 & 0 \\
\hline Florida Power and Light Company ..... . & $\begin{array}{l}\text { St. Lucie } 1 \\
\text { St. Lucie } 2 \\
\text { Turkey Point } 3 \\
\text { Turkey Point } 4\end{array}$ & $\begin{array}{l}\text { PWR } \\
\text { PWR } \\
\text { PWR } \\
\text { PWA }\end{array}$ & $\begin{array}{l}1801 \\
1802 \\
1803 \\
1804\end{array}$ & $\begin{array}{l}4 \\
1 \\
0 \\
0\end{array}$ & $\begin{array}{l}0 \\
0 \\
0 \\
0\end{array}$ & $\begin{array}{l}0 \\
0 \\
0 \\
0\end{array}$ & $\begin{array}{l}0 \\
0 \\
0 \\
0\end{array}$ & $\begin{array}{l}0 \\
0 \\
0 \\
0\end{array}$ & $\begin{array}{l}4 \\
1 \\
0 \\
0\end{array}$ \\
\hline GPU Nuclear Corporation . . . . . . . . . & $\begin{array}{l}\text { Three Mile Island } 1 \\
\text { Oyster Creek }\end{array}$ & $\begin{array}{l}\text { PWR } \\
\text { BWR }\end{array}$ & $\begin{array}{l}1901 \\
1903\end{array}$ & $\begin{array}{l}0 \\
0\end{array}$ & $\begin{array}{l}0 \\
0\end{array}$ & $\begin{array}{l}0 \\
0\end{array}$ & $\begin{array}{l}0 \\
0\end{array}$ & $\begin{array}{l}0 \\
0\end{array}$ & $\begin{array}{l}0 \\
0\end{array}$ \\
\hline \multicolumn{10}{|l|}{-} \\
\hline Georgia Power Company ......... & $\begin{array}{l}\text { Hatch } 1 \text { \& } 2 \\
\text { Vogtle } 1 \text { \& } 2\end{array}$ & $\begin{array}{l}\text { BWR } \\
\text { PWR }\end{array}$ & $\begin{array}{l}2001 \\
2003\end{array}$ & $\begin{array}{l}0 \\
0\end{array}$ & $\begin{array}{l}0 \\
0\end{array}$ & $\begin{array}{l}0 \\
0\end{array}$ & $\begin{array}{l}0 \\
0\end{array}$ & $\begin{array}{l}0 \\
0\end{array}$ & $\begin{array}{l}0 \\
0\end{array}$ \\
\hline Gulf States Utilities Company $\ldots \ldots \ldots$ & River Bend 1 & BWR & 2101 & 0 & 0 & 0 & 0 & 0 & 0 \\
\hline $\begin{array}{l}\text { Houston Lighting and Power } \\
\text { Company } \ldots \ldots \ldots \ldots \ldots \ldots \ldots \ldots\end{array}$ & $\begin{array}{l}\text { South Texas } 1 \\
\text { South Texas } 2\end{array}$ & $\begin{array}{l}\text { PWR } \\
\text { PWR }\end{array}$ & $\begin{array}{l}2201 \\
2202\end{array}$ & $\begin{array}{l}2 \\
2\end{array}$ & $\begin{array}{l}0 \\
0\end{array}$ & $\begin{array}{l}0 \\
0\end{array}$ & $\begin{array}{l}1 \\
0\end{array}$ & $\begin{array}{l}0 \\
0\end{array}$ & $\begin{array}{l}0 \\
0\end{array}$ \\
\hline Illinois Power Company . . . . . . . . . . & Clinton 1 & BWR & 2301 & 0 & 0 & 0 & 0 & 0 & 0 \\
\hline Indiana Michigan Power Company . & $\begin{array}{l}\text { Cook } 1 \\
\text { Cook } 2\end{array}$ & $\begin{array}{l}\text { PWR } \\
\text { PWR }\end{array}$ & $\begin{array}{l}5801 \\
5801\end{array}$ & $\begin{array}{l}2 \\
3\end{array}$ & $\begin{array}{l}0 \\
0\end{array}$ & $\begin{array}{l}0 \\
0\end{array}$ & $\begin{array}{l}0 \\
0\end{array}$ & $\begin{array}{l}2 \\
3\end{array}$ & $\begin{array}{l}0 \\
0\end{array}$ \\
\hline
\end{tabular}


Table FE3. Canisters Containing Nonfuel Components as of December 31, 1992 (Continued)

\begin{tabular}{|c|c|c|c|c|c|c|c|c|c|}
\hline Electric Utility Name & Reactor Names & $\begin{array}{c}\text { Reactor } \\
\text { Type }\end{array}$ & $\begin{array}{l}\text { Pool } \\
\text { Site } \\
\text { ID }\end{array}$ & $\begin{array}{c}\text { Total } \\
\text { Canisters } \\
\text { Nonfuel }^{\mathrm{a}}\end{array}$ & Debris $^{b}$ & $\begin{array}{c}\text { Nonfuel } \\
\text { Disassembly } \\
\text { Hardware }\end{array}$ & $\begin{array}{c}\text { Nonfuel } \\
\text { Components }^{c}\end{array}$ & Other ${ }^{d}$ & $\begin{array}{l}\text { Canisters } \\
\text { with } \\
\text { Contents } \\
\text { Unknown }\end{array}$ \\
\hline $\begin{array}{l}\text { lowa Electric Light and } \\
\text { Power Company } \ldots \ldots \ldots \ldots \ldots \ldots\end{array}$ & Duane Arnold & BWR & 2401 & 0 & 0 & 0 & 0 & 0 & 0 \\
\hline $\begin{array}{l}\text { Kansas Gas and Electric } \\
\text { Company } \ldots \ldots \ldots \ldots \ldots \ldots \ldots\end{array}$ & Wolf Creek 1 & PWR & 2501 & 0 & 0 & 0 & 0 & 0 & 0 \\
\hline Long Island Lighting Company . . . . . . . . & Shoreham & BWR & 2601 & 0 & 0 & 0 & 0 & 0 & 0 \\
\hline $\begin{array}{l}\text { Louisiana Power and Light } \\
\text { Company } \ldots \ldots \ldots \ldots \ldots \ldots \ldots\end{array}$ & Waterford 3 & PWR & 2701 & 4 & 0 & 4 & 4 & 0 & 0 \\
\hline $\begin{array}{l}\text { Maine Yankee Atomic Power } \\
\text { Company } \ldots \ldots \ldots \ldots \ldots \ldots \ldots\end{array}$ & Maine Yankee & PWR & 2801 & 0 & 0 & 0 & 0 & 0 & 0 \\
\hline Nebraska Public Power District ... . . . . & Cooper Station & BWR & 3001 & 0 & 0 & 0 & 0 & 0 & 0 \\
\hline New York Power Authority . . . . . . . . . . . & $\begin{array}{l}\text { Fitzpatrick } \\
\text { Indian Point } 3\end{array}$ & $\begin{array}{l}\text { BWR } \\
\text { PWR }\end{array}$ & $\begin{array}{l}3901 \\
3902\end{array}$ & $\begin{array}{l}0 \\
0\end{array}$ & $\begin{array}{l}0 \\
0\end{array}$ & $\begin{array}{l}0 \\
0\end{array}$ & $\begin{array}{l}0 \\
0\end{array}$ & $\begin{array}{l}0 \\
0\end{array}$ & $\begin{array}{l}0 \\
0\end{array}$ \\
\hline Niagara Mohawk Power Corporation ... & $\begin{array}{l}\text { Nine Mile Point } 1 \\
\text { Nine Mile Point } 2\end{array}$ & $\begin{array}{l}\text { BWR } \\
\text { BWR }\end{array}$ & $\begin{array}{l}3101 \\
3102\end{array}$ & $\begin{array}{l}0 \\
0\end{array}$ & $\begin{array}{l}0 \\
0\end{array}$ & $\begin{array}{l}0 \\
0\end{array}$ & $\begin{array}{l}0 \\
0\end{array}$ & $\begin{array}{l}0 \\
0\end{array}$ & $\begin{array}{l}0 \\
0\end{array}$ \\
\hline North Atlantic Energy Service Corporation & Seabrook & PWR & 5901 & 0 & 0 & 0 & 0 & 0 & 0 \\
\hline Northeast Utilities Service Company . . . . . & $\begin{array}{l}\text { Millstone } 1 \\
\text { Millstone } 2 \\
\text { Millstone } 3 \\
\text { Haddam Neck }\end{array}$ & $\begin{array}{l}\text { BWR } \\
\text { PWR } \\
\text { PWR } \\
\text { PWR }\end{array}$ & $\begin{array}{l}3201 \\
3202 \\
3203 \\
5701\end{array}$ & $\begin{array}{l}0 \\
4 \\
0 \\
4\end{array}$ & $\begin{array}{l}0 \\
1 \\
0 \\
0\end{array}$ & $\begin{array}{l}0 \\
3 \\
0 \\
0\end{array}$ & $\begin{array}{l}0 \\
0 \\
0 \\
1\end{array}$ & $\begin{array}{l}0 \\
0 \\
0 \\
3\end{array}$ & $\begin{array}{l}0 \\
0 \\
0 \\
0\end{array}$ \\
\hline Northern States Power Company . . . . . . . & $\begin{array}{l}\text { Monticello } \\
\text { Prairie Island } 1 \text { \& } 2\end{array}$ & $\begin{array}{l}\text { BWR } \\
\text { PWR }\end{array}$ & $\begin{array}{l}3301 \\
3302\end{array}$ & $\begin{array}{l}0 \\
2\end{array}$ & $\begin{array}{l}0 \\
0\end{array}$ & $\begin{array}{l}0 \\
0\end{array}$ & $\begin{array}{l}0 \\
2\end{array}$ & $\begin{array}{l}0 \\
0\end{array}$ & $\begin{array}{l}0 \\
0\end{array}$ \\
\hline Omaha Public Power District . . . . . . . . . & Fort Calhoun & PWR & 3401 & 0 & 0 & 0 & 0 & 0 & 0 \\
\hline Pacilic Gas and Electric Company ... . . . & $\begin{array}{l}\text { Diablo Canyon } 1 \\
\text { Diablo Canyon } 2 \\
\text { Humboldt Bay }\end{array}$ & $\begin{array}{l}\text { PWR } \\
\text { PWR } \\
\text { BWR }\end{array}$ & $\begin{array}{l}3501 \\
3502 \\
3503\end{array}$ & $\begin{array}{l}0 \\
0 \\
0\end{array}$ & $\begin{array}{l}0 \\
0 \\
0\end{array}$ & $\begin{array}{l}0 \\
0 \\
0\end{array}$ & $\begin{array}{l}0 \\
0 \\
0\end{array}$ & $\begin{array}{l}0 \\
0 \\
0\end{array}$ & $\begin{array}{l}0 \\
0 \\
0\end{array}$ \\
\hline
\end{tabular}

See footnotes at end of table. 
Tablē FE3. Canisters Containing Nonfuel Components as of December 31, 1992 (Continued)

\begin{tabular}{|c|c|c|c|c|c|c|c|c|c|}
\hline Electric Utility Name & Reactor Names & $\begin{array}{l}\text { Reactor } \\
\text { Type }\end{array}$ & $\begin{array}{l}\text { Pool } \\
\text { Site } \\
\text { ID }\end{array}$ & $\begin{array}{c}\text { Total } \\
\text { Canisters } \\
\text { Nonfuel }^{a}\end{array}$ & Debris $^{b}$ & $\begin{array}{c}\text { Nonfuel } \\
\text { Disassembly } \\
\text { Hardware }\end{array}$ & $\begin{array}{c}\text { Nonfuel } \\
\text { Components }^{c}\end{array}$ & Other ${ }^{d}$ & $\begin{array}{l}\text { Canisters } \\
\text { with } \\
\text { Contents } \\
\text { Unknown }\end{array}$ \\
\hline $\begin{array}{l}\text { Pennsylvania Power and Light } \\
\text { Company } \ldots \ldots \ldots \ldots \ldots \ldots \ldots \ldots\end{array}$ & Susquehanna $1 \& 2$ & BWR & 3601 & 0 & 0 & 0 & 0 & 0 & 0 \\
\hline Philadelphia Electric Company . . . . . . . & $\begin{array}{l}\text { Limerick } 1 \text { \& } 2 \\
\text { Peach Bottom } 2 \\
\text { Peach Bottom } 3\end{array}$ & $\begin{array}{l}\text { BWR } \\
\text { BWR } \\
\text { BWR }\end{array}$ & $\begin{array}{l}3701 \\
3704 \\
3705\end{array}$ & $\begin{array}{l}5 \\
0 \\
0\end{array}$ & $\begin{array}{l}0 \\
0 \\
0\end{array}$ & $\begin{array}{l}0 \\
0 \\
0\end{array}$ & $\begin{array}{l}0 \\
0 \\
0\end{array}$ & $\begin{array}{l}0 \\
0 \\
0\end{array}$ & $\begin{array}{l}5 \\
0 \\
0\end{array}$ \\
\hline Portland General Electric Company ..... & Trojan & PWR & 3801 & 21 & 5 & 6 & 20 & 0 & 0 \\
\hline Public Service Company of Colorado .... & Fort St. Vrain & HTGR & 4101 & 0 & 0 & 0 & 0 & 0 & 0 \\
\hline Public Service Electric and Gas Company & $\begin{array}{l}\text { Hope Creek } \\
\text { Salem } 1 \\
\text { Salem } 2\end{array}$ & $\begin{array}{l}\text { BWR } \\
\text { PWR } \\
\text { PWR }\end{array}$ & $\begin{array}{l}4201 \\
4402 \\
4203\end{array}$ & $\begin{array}{l}0 \\
0 \\
0\end{array}$ & $\begin{array}{l}0 \\
0 \\
0\end{array}$ & $\begin{array}{l}0 \\
0 \\
0\end{array}$ & $\begin{array}{l}0 \\
0 \\
0\end{array}$ & $\begin{array}{l}0 \\
0 \\
0\end{array}$ & $\begin{array}{l}0 \\
0 \\
0\end{array}$ \\
\hline Rochester Gas and Electric Corporation .. & Ginna & PWR & 4401 & 2 & 0 & 0 & 2 & 0 & 0 \\
\hline Sacramento Municipal Utility District . . . . . & Rancho Seco & PWR & 4501 & 0 & 0 & 0 & 0 & 0 & 0 \\
\hline South Carolina Electric and Gas Company & Summer & PWR & 4601 & 0 & 0 & 0 & 0 & 0 & 0 \\
\hline Southern California Edison Company .... & $\begin{array}{l}\text { San Onofre } 1 \\
\text { San Onofre 2 } \\
\text { San Onofre } 3\end{array}$ & $\begin{array}{l}\text { PWR } \\
\text { PWR } \\
\text { PWR }\end{array}$ & $\begin{array}{l}4701 \\
4702 \\
4703\end{array}$ & $\begin{array}{l}0 \\
0 \\
0\end{array}$ & $\begin{array}{l}0 \\
0 \\
0\end{array}$ & $\begin{array}{l}0 \\
0 \\
0\end{array}$ & $\begin{array}{l}0 \\
0 \\
0\end{array}$ & $\begin{array}{l}0 \\
0 \\
0\end{array}$ & $\begin{array}{l}0 \\
0 \\
0\end{array}$ \\
\hline System Energy Resources, Inc. . . . . . . . & Grand Gulf 1 & BWR & 2901 & 0 & 0 & 0 & 0 & 0 & 0 \\
\hline Tennessee Valley Authority . . . . . . . . . & $\begin{array}{l}\text { Bellefonte } 1 \\
\text { Bellefonte } 2 \\
\text { Browns Ferry } 1 \text { \& } 2 \\
\text { Browns Ferry } 3 \\
\text { Sequoyah } 1 \text { \& } 2 \\
\text { Watts Bar } 1 \text { \& } 2\end{array}$ & $\begin{array}{l}\text { PWR } \\
\text { PWR } \\
\text { BWR } \\
\text { BWR } \\
\text { PWR } \\
\text { PWR }\end{array}$ & $\begin{array}{l}4801 \\
4802 \\
4803 \\
4805 \\
4808 \\
4810\end{array}$ & $\begin{array}{l}0 \\
0 \\
0 \\
0 \\
0 \\
0\end{array}$ & $\begin{array}{l}0 \\
0 \\
0 \\
0 \\
0 \\
0\end{array}$ & $\begin{array}{l}0 \\
0 \\
0 \\
0 \\
0 \\
0\end{array}$ & $\begin{array}{l}0 \\
0 \\
0 \\
0 \\
0 \\
0\end{array}$ & $\begin{array}{l}0 \\
0 \\
0 \\
0 \\
0 \\
0\end{array}$ & $\begin{array}{l}0 \\
0 \\
0 \\
0 \\
0 \\
0\end{array}$ \\
\hline TU Electric $\ldots \ldots \ldots \ldots \ldots$ & Comanche Peak $1 \& 2$ & PWR & 4901 & 0 & 0 & 0 & 0 & 0 & 0 \\
\hline Toledo Edison Company . . . . . . . . . . . & Davis-Besse & PWR & 5001 & 2 & 0 & 2 & 0 & 0 & 0 \\
\hline
\end{tabular}


Table FE3. Canisters Containing Nonfuel Components as of December 31, 1992 (Continued)

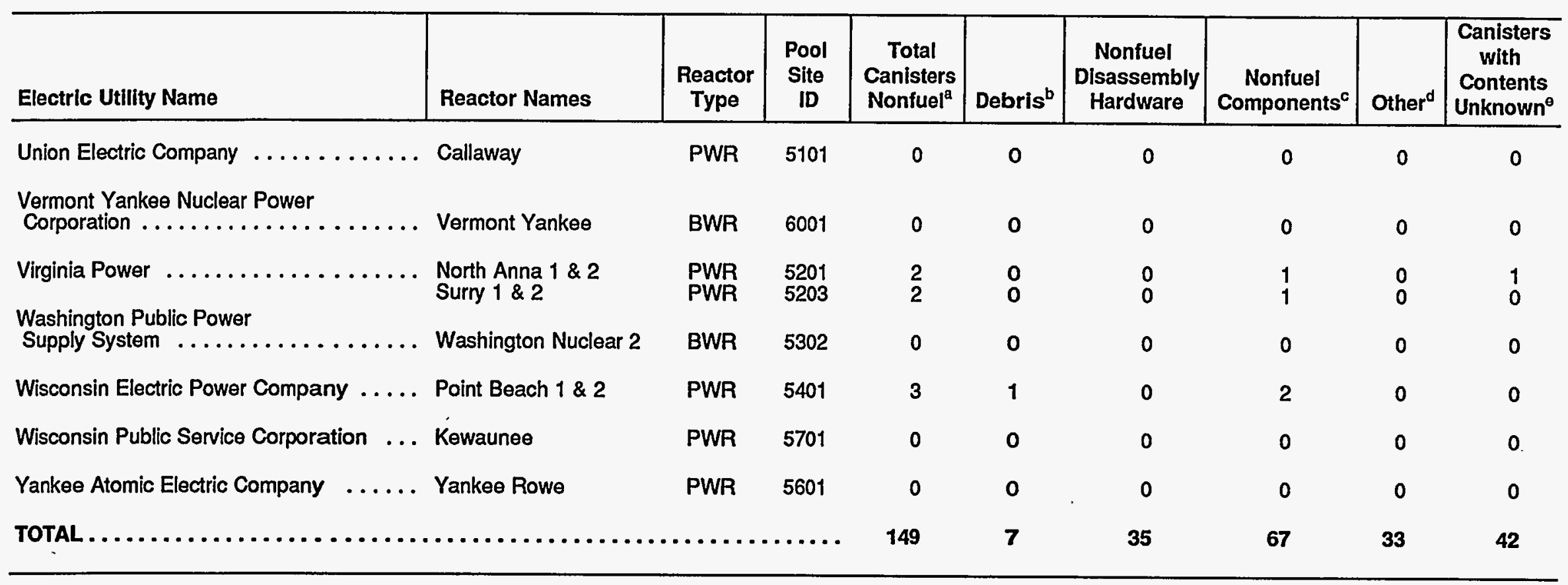

a Total Canisters Nonfuel column may not equal sum of individual canister contents columns since canisters may contain more than one category or be empty. bebris column represents canisters containing fuel rod pieces, fuel pellets, etc.

cNonfuel Components column represents canisters containing nonfuel assembly hardware.

dOther column represents canisters containing other nonfuel material.

${ }^{\circ}$ Canisters with Contents Unknown column represents the number of canisters containing nonfuel components for which specific data on the contents of the canister were not reported on the Form RW-859 survey.

PWR $=$ Pressurized-water reactor; BWR = Boiling-water reactor; $H T G R=$ High-temperature, gas-cooled reactor.

Notes: Data for the following pool sites are as of December 31, 1991 - Pilgrim 1, Indian Point 1 \& 2, Big Rock Point, Beaver Valley 1, Three Mile Island 1, Wolf

Creek 1, Cooper Station, Diablo Canyon 2, Salem 2, Summer, Davis-Besse, and Yankee Rowe. Shoreham did not report for either 1991 or 1992.

Source: Energy Information Administration, Form RW-859, "Nuclear Fuel Data" (1992). 
Table FE4. Canister Dimensions

\begin{tabular}{|c|c|c|c|c|c|c|c|c|c|c|c|c|c|c|}
\hline \multirow[b]{2}{*}{$\begin{array}{l}\text { Length } \\
\text { (inches) }\end{array}$} & \multicolumn{11}{|c|}{ Width $\times$ Depth (inches) } & \multirow[b]{2}{*}{$\begin{array}{c}\text { Missing } \\
\text { Dimensions }^{\mathrm{a}}\end{array}$} & \multirow[b]{2}{*}{$\begin{array}{c}\text { Data } \\
\text { Not } \\
\text { Reported }^{b}\end{array}$} & \multirow[b]{2}{*}{ Total } \\
\hline & $3 \times 3$ & $5 \times 5$ & $6 \times 6$ & $7 \times 7$ & $8 \times 8$ & $9 \times 9$ & $10 \times 10$ & $12 \times 12$ & $14 \times 14$ & $16 \times 16$ & $19 \times 19$ & & & \\
\hline 84 & - & - & - & - & - & - & - & 1 & - & - & - & - & - & 1 \\
\hline 100 & - & - & 1 & - & - & - & - & - & - & - & - & - & - & 1 \\
\hline 138 & - & - & - & - & 9 & - & - & - & - & - & - & - & - & 9 \\
\hline 144 & - & - & 1 & -- & - & 6 & - & - & - & - & - & - & - & 7 \\
\hline 152 & - & - & 1 & -- & - & - & - & - & - & - & - & - & - & 1 \\
\hline 153 & - & - & - & - & 1 & - & - & - & - & - & - & - & -- & 1 \\
\hline 155 & - & - & - & - & 4 & - & - & - & - & - & - & - & - & 4 \\
\hline 156 & - & - & - & - & 1 & 8 & - & - & - & - & - & - & - & 9 \\
\hline 157 & - & - & - & - & 24 & - & - & - & - & - & - & - & - & 24 \\
\hline 158 & - & - & - & - & - & 4 & - & - & - & - & - & - & - & 4 \\
\hline 159 & - & 2 & - & - & 6 & 1 & - & - & - & - & - & - & - & 9 \\
\hline 160 & - & - & - & 5 & 5 & 3 & - & - & - & - & - & - & -- & 13 \\
\hline 161 & - & - & - & - & 19 & 22 & - & - & - & - & - & - & - & 41 \\
\hline 163 & - & - & 1 & - & - & 2 & - & - & - & -- & - & - & - & 3 \\
\hline 166 & - & - & - & - & 1 & - & - & - & - & - & - & - & - & 1 \\
\hline 168 & - & - & - & - & 10 & - & - & - & - & - & - & - & - & 10 \\
\hline 170 & - & - & - & - & - & - & 5 & - & 2 & - & 2 & - & - & 9 \\
\hline 171 & -2 & - & - & - & 2 & - & - & - & - & - & - & - & - & 4 \\
\hline 174 &. & 1 & - & - & - & - & - & - & - & - & - & - & - & 1 \\
\hline 176 & - & - & 4 & - & - & - & - & - & - & 1 & - & - & - & 5 \\
\hline 180 & - & - & - & - & 16 & 18 & 1 & - & - & - & - & - & - & 35 \\
\hline 186 & - & - & - & - & 1 & - & - & - & - & - & - & - & - & 1 \\
\hline 189 & - & - & - & - & 3 & - & - & - & - & - & - & - & - & 3 \\
\hline 192 & - & - & -- & - & - & 2 & - & - & - & - & - & - & - & 2 \\
\hline $\begin{array}{c}\text { Missing } \\
\text { Dimensions }^{\mathrm{a}}\end{array}$ & - & - & - & - & - & - & - & - & - & - & - & 37 & - & 37 \\
\hline $\begin{array}{l}\text { Data Not } \\
\text { Reported }^{b}\end{array}$ & - & - & - & - & - & - & - & - & - & - & - & - & 51 & 51 \\
\hline TOTAL & 2 & 3 & 8 & 5 & 102 & 66 & 6 & 1 & 2 & 1 & 2 & 37 & 51 & 286 \\
\hline
\end{tabular}

anMissing Dimensions" represents the canisters for which contents were reported, but dimensions were not.

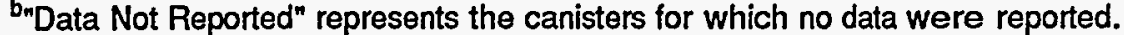

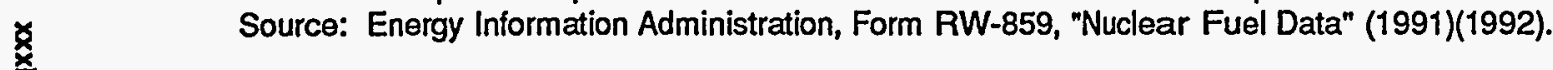


Table FE5. Nonfuel Assembly (NFA) Hardware from Babcock \& Wilcox Reactors

\begin{tabular}{|c|c|c|c|c|c|c|c|c|c|}
\hline \multirow[b]{2}{*}{ Reactor Names ${ }^{a}$} & \multirow[b]{2}{*}{$\begin{array}{c}\text { Permanently } \\
\text { Dlscharged } \\
\text { Fuel } \\
\text { Assemblies }\end{array}$} & \multicolumn{5}{|c|}{ NFA Hardware } & \multirow[b]{2}{*}{$\begin{array}{c}\text { Total } \\
\text { Reported } \\
\text { NFA } \\
\text { Hardware } \\
\text { Items }\end{array}$} & \multirow[b]{2}{*}{$\begin{array}{c}\text { NFA } \\
\text { Hardware } \\
\text { Items } \\
\text { Reported } \\
\text { In/Attached } \\
\text { to Fuel } \\
\text { Assemblies }\end{array}$} & \multirow[b]{2}{*}{$\begin{array}{l}\text { Percentage } \\
\text { of NFA } \\
\text { Hardware } \\
\text { Items } \\
\text { In/Attached } \\
\text { to Fuel } \\
\text { Assemblies }\end{array}$} \\
\hline & & $\begin{array}{c}\text { Burnable } \\
\text { Polson Rod } \\
\text { Assemblies }\end{array}$ & $\begin{array}{c}\text { Axial Power } \\
\text { Shaping } \\
\text { Assemblies }\end{array}$ & $\begin{array}{l}\text { Control Rod } \\
\text { Assemblies }\end{array}$ & $\begin{array}{l}\text { Neutron } \\
\text { Sources }\end{array}$ & $\begin{array}{c}\text { Orifice } \\
\text { Rod } \\
\text { Assemblies }\end{array}$ & & & \\
\hline Arkansas Nuclear $1 \ldots \ldots \ldots$ & 624 & 0 & 0 & 0 & 2 & ${ }^{b} 66$ & 68 & 66 & $97 \%$ \\
\hline Crystal River $3 \ldots \ldots \ldots \ldots$ & 531 & 232 & 8 & 61 & 2 & 2 & 305 & 305 & $100 \%$ \\
\hline Davis-Besse ............. & 386 & 180 & 8 & 0 & 4 & 2 & 194 & 194 & $100 \%$ \\
\hline Oconee $1 \& 2 \ldots \ldots \ldots \ldots$ & $c_{1,562}$ & d891 & ${ }^{d} 35$ & $d_{178}$ & $d_{6}$ & $d_{15}$ & 1,125 & 1,119 & $99 \%$ \\
\hline Oconee $3 . . . \ldots \ldots \ldots . . .$. & 748 & 347 & 13 & 4 & 2 & 7 & 373 & 372 & $100 \%$ \\
\hline Rancho Seco $\ldots \ldots \ldots \ldots$ & 493 & 272 & ${ }^{\theta} 16$ & 61 & 4 & 62 & 415 & 0 & $0 \%$ \\
\hline Three Mile Island $1 \ldots$ & 513 & 268 & 8 & 13 & 4 & ${ }^{\mathrm{b}} 77$ & 370 & 366 & $99 \%$ \\
\hline Total From Reporting Reactors & 4,857 & 2,190 & 88 & 317 & 24 & 231 & 2,850 & 2,422 & $85 \%$ \\
\hline $\begin{array}{l}\text { Percentage of Permanently Dis } \\
\text { Assemblies ............ }\end{array}$ & $\begin{array}{l}\text { arged Fuel } \\
\ldots \ldots \ldots \ldots\end{array}$ & $45.09 \%$ & $1.81 \%$ & $6.53 \%$ & $0.49 \%$ & $4.76 \%$ & & & \\
\hline
\end{tabular}

a Includes all Babcock \& Wilcox reactors.

b Quantity is the number reported in the pool plus the number reported in assemblies.

${ }^{c}$ Number of fuel assemblies in pool represents fuel discharged from two reactors into one pool.

d Number of nonfuel components in pool represents components discharged from two reactors into one pool.

- Includes 8 axial power shaping assemblies and 8 gray axial power shaping assemblies.

Note: Data for the following pool sites are as of December 31, 1991 - Three Mile Island 1 and Davis-Besse.

Source: Energy Information Administration, Form RW-859, "Nuclear Fuel Data" (1992). 


\section{Table FE6. Nonfuel Assembly (NFA) Hardware from Combustion Engineering Reactors}

\begin{tabular}{|c|c|c|c|c|c|c|c|c|}
\hline & \multirow[b]{2}{*}{ Reactor Names $^{a}$} & \multirow[b]{2}{*}{$\begin{array}{c}\text { Permanently } \\
\text { Discharged } \\
\text { Fuel } \\
\text { Assemblies }\end{array}$} & \multicolumn{3}{|c|}{ NFA Hardware } & \multirow[b]{2}{*}{$\begin{array}{c}\text { Total } \\
\text { Reported } \\
\text { NFA } \\
\text { Hardware } \\
\text { Items }\end{array}$} & \multirow[b]{2}{*}{$\begin{array}{c}\text { NFA } \\
\text { Hardware } \\
\text { Items } \\
\text { Reported } \\
\text { In/Attached } \\
\text { to Fuel } \\
\text { Assemblies }\end{array}$} & \multirow[b]{2}{*}{$\begin{array}{c}\text { Percentage } \\
\text { of NFA } \\
\text { Hardware } \\
\text { Items } \\
\text { In/Attached } \\
\text { to Fuel } \\
\text { Assemblies }\end{array}$} \\
\hline & & & $\begin{array}{c}\text { Control } \\
\text { Element } \\
\text { Assemblies }\end{array}$ & $\begin{array}{l}\text { Neutron } \\
\text { Sources }\end{array}$ & $\begin{array}{l}\text { Control } \\
\text { Element } \\
\text { Assembly } \\
\text { Flow Plugs }\end{array}$ & & & \\
\hline \multirow{9}{*}{ 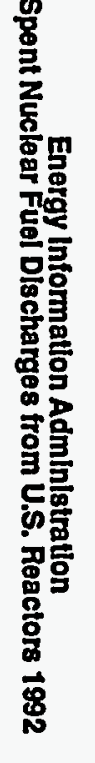 } & Arkansas Nuclear $2 \ldots \ldots \ldots \ldots \ldots$ & 564 & 0 & 2 & 0 & 2 & 2 & $100 \%$ \\
\hline & Calvert Cliffs $1 \& 2 \ldots \ldots \ldots \ldots$ & $b_{1,412}$ & cad 177 & $\mathrm{c}, \mathrm{d}_{2}$ & 0 & 179 & 177 & $99 \%$ \\
\hline & Maine Yankee $\ldots \ldots \ldots \ldots \ldots$ & 1,054 & $c_{93}$ & $c_{1}$ & 0 & 94 & 94 & $100 \%$ \\
\hline & Millstone $2 \ldots \ldots \ldots \ldots \ldots \ldots$ & 784 & 82 & 4 & 8 & 94 & 91 & $97 \%$ \\
\hline & San Onotre $2 \ldots \ldots \ldots \ldots \ldots$ & 484 & $c_{1}$ & 0 & 0 & 1 & 1 & $100 \%$ \\
\hline & San Onofre $3 \ldots \ldots \ldots \ldots \ldots$ & 484 & $c_{1}$ & 0 & 0 & 1 & 1 & $100 \%$ \\
\hline & Waterford $3 \ldots \ldots \ldots \ldots$ & 428 & 1 & 0 & 0 & 1 & 1 & $100 \%$ \\
\hline & Total From Reporting Reactors . . . . & 6,006 & 494 & 11 & 8 & 513 & 440 & $86 \%$ \\
\hline & $\begin{array}{l}\text { Percentage of Permanently Discharge } \\
\text { Assembles } \ldots \ldots \ldots \ldots \ldots \ldots \ldots\end{array}$ & Fuel & $8.23 \%$ & $0.18 \%$ & $0.13 \%$ & & & \\
\hline
\end{tabular}

a Includes all Combustion Engineering reactors except Palo Verde 1, Palo Verde 2, and Palo Verde 3 which did not report these data.

b Number of fuel assemblies in pool represents fuel discharged from two reactors into separate pools joined by a transier canal.

c Quantity is the number reported in the pool plus the number reported in assemblies.

d Number of nonfuel components in pool represents components stored in fuel discharged from two reactors into separate pools joined by a transfer canal.

Source: Energy Information Administration, Form RW-859, "Nuclear Fuel Data" (1992). 


\begin{tabular}{|c|c|c|c|c|c|c|c|c|c|}
\hline \multirow[b]{2}{*}{ Reactor Names ${ }^{a}$} & \multirow[b]{2}{*}{$\begin{array}{c}\text { Permanently } \\
\text { Discharged } \\
\text { Fuel } \\
\text { Assemblies }\end{array}$} & \multicolumn{5}{|c|}{ NFA Hardware } & \multirow[b]{2}{*}{$\begin{array}{c}\text { Total } \\
\text { Reported } \\
\text { NFA } \\
\text { Hardware } \\
\text { Items }\end{array}$} & \multirow[b]{2}{*}{$\begin{array}{c}\text { NFA } \\
\text { Hardware } \\
\text { Items } \\
\text { Reported } \\
\text { In/Attached } \\
\text { to Fuel } \\
\text { Assemblles }\end{array}$} & \multirow[b]{2}{*}{$\begin{array}{l}\text { Percentage } \\
\text { of NFA } \\
\text { Hardware } \\
\text { Items } \\
\text { In/Attached } \\
\text { to Fuel } \\
\text { Assemblies }\end{array}$} \\
\hline & & $\begin{array}{c}\text { Fuel } \\
\text { Channels }\end{array}$ & $\begin{array}{l}\text { Control } \\
\text { Blades }\end{array}$ & $\begin{array}{l}\text { Neutron } \\
\text { Sources }\end{array}$ & $\begin{array}{l}\text { Local } \\
\text { Power } \\
\text { Range } \\
\text { Monitors }\end{array}$ & $\begin{array}{c}\text { Other } \\
\text { Instrumentation }\end{array}$ & & & \\
\hline Browns Ferry $1, \ldots \ldots \ldots \ldots$ & 1,488 & 1,467 & 1 & 0 & 0 & 0 & 1,468 & 1,464 & $100 \%$ \\
\hline Browns Ferry $2 \ldots \ldots \ldots \ldots$ & 1,440 & 1,409 & 3 & 0 & 0 & 1 & 1,413 & 1,409 & $100 \%$ \\
\hline Browns Ferry $3 . \ldots \ldots \ldots \ldots$ & 1,029 & 1,018 & 35 & 0 & 0 & 0 & 1,053 & 1,004 & $95 \%$ \\
\hline Brunswick $1 \ldots \ldots \ldots \ldots$ & 1,215 & 11 & 0 & 0 & 0 & 0 & 11 & 0 & $0 \%$ \\
\hline Brunswick $2 \ldots \ldots \ldots \ldots$ & 1,400 & 6 & 0 & 0 & 0 & 0 & 6 & 0 & $0 \%$ \\
\hline 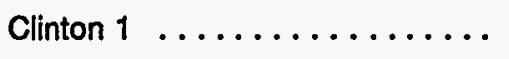 & 568 & 0 & 0 & 5 & 17 & 11 & 33 & 0 & $0 \%$ \\
\hline Cooper Station ............. & 1,676 & 93 & 19 & 0 & 34 & 0 & 146 & 0 & $0 \%$ \\
\hline Dresden $2 \ldots \ldots \ldots \ldots \ldots$ & 2,605 & 23 & 15 & 0 & 0 & 0 & 38 & 0 & $0 \%$ \\
\hline Dresden $3 \ldots \ldots \ldots \ldots$ & 1,864 & 70 & 56 & 0 & 5 & 0 & 131 & 0 & $0 \%$ \\
\hline Enrico Fermi $2 \ldots \ldots \ldots \ldots$ & 672 & 2 & 165 & 0 & 6 & 0 & 173 & 0 & $0 \%$ \\
\hline Fitzpatrick................ & 1,684 & 0 & 51 & 0 & 15 & 0 & 66 & 0 & $0 \%$ \\
\hline Grand Gulf $1 \ldots \ldots \ldots \ldots$ & 1,384 & 0 & 0 & 7 & 31 & 13 & 51 & 0 & $0 \%$ \\
\hline Hatch $1 \& 2 \ldots \ldots \ldots \ldots \ldots$ & $b_{3,376}$ & $c_{3,381}$ & 0 & 0 & 0 & 0 & 3,381 & 3,381 & $d_{100 \%}$ \\
\hline Hope Creek.........$\ldots$. & 1,008 & 1,008 & 27 & 1 & 8 & 0 & 1,044 & 1,008 & $97 \%$ \\
\hline LaSalle County $1 \& 2 \ldots \ldots \ldots$ & $b_{1,920}$ & ${ }^{\theta_{1,512}}$ & 9 & 14 & 88 & 33 & 1,656 & 1,472 & $89 \%$ \\
\hline Millstone $1, \ldots \ldots \ldots \ldots \ldots$ & 2,116 & ${ }^{\theta} 1,800$ & 0 & 0 & 0 & 0 & 1,800 & 1,800 & $100 \%$ \\
\hline Monticello............... & 1,640 & 559 & 21 & $\mathbf{0}$ & 13 & 0 & 593 & 492 & $83 \%$ \\
\hline Nine Mile Point 1 .......... & 1,620 & 1,620 & 1 & 0 & 2 & 0 & 1,623 & 0 & $0 \%$ \\
\hline Nine Mile Point $2 \ldots \ldots \ldots$ & 444 & 444 & 0 & 4 & 0 & 0 & 448 & 0 & $0 \%$ \\
\hline Oyster Creek .............. & 1,876 & 1,389 & 13 & 2 & 0 & 0 & 1,404 & 1,389 & $99 \%$ \\
\hline Peach Bottom $2 \ldots \ldots \ldots \ldots$ & 2,164 & 5 & 0 & 0 & 0 & 0 & 5 & 0 & $0 \%$ \\
\hline Peach Bottom $3 \ldots \ldots . .$. & 1,944 & 0 & 41 & 1 & 0 & 0 & 42 & 0 & $0 \%$ \\
\hline
\end{tabular}

See footnotes at end of table. 
Table FE7. Nonfuel Assembly (NFA) Hardware from General Electric Reactors (Continued)

\begin{tabular}{|c|c|c|c|c|c|c|c|c|c|}
\hline \multirow[b]{2}{*}{ Reactor Names ${ }^{a}$} & \multirow[b]{2}{*}{$\begin{array}{c}\text { Permanently } \\
\text { Discharged } \\
\text { Fuel } \\
\text { Assemblies }\end{array}$} & \multicolumn{5}{|c|}{ NFA Hardware } & \multirow[b]{2}{*}{$\begin{array}{c}\text { Total } \\
\text { Reported } \\
\text { NFA } \\
\text { Hardware } \\
\text { Items }\end{array}$} & \multirow[b]{2}{*}{$\begin{array}{c}\text { NFA } \\
\text { Hardware } \\
\text { Items } \\
\text { Reported } \\
\text { In/Attached } \\
\text { to Fuel } \\
\text { Assemblies }\end{array}$} & \multirow[b]{2}{*}{$\begin{array}{l}\text { Percentage } \\
\text { of NFA } \\
\text { Hardware } \\
\text { Items } \\
\text { In/Attached } \\
\text { to Fuel } \\
\text { Assemblies }\end{array}$} \\
\hline & & $\begin{array}{c}\text { Fuel } \\
\text { Channels }\end{array}$ & $\begin{array}{l}\text { Control } \\
\text { Blades }\end{array}$ & $\begin{array}{l}\text { Neutron } \\
\text { Sources }\end{array}$ & $\begin{array}{l}\text { Local } \\
\text { Power } \\
\text { Range } \\
\text { Monitors }\end{array}$ & $\begin{array}{c}\text { Other } \\
\text { Instrumentation }\end{array}$ & & & \\
\hline Perry $1 \ldots \ldots \ldots \ldots \ldots$ & 744 & 0 & 24 & 11 & 43 & 0 & 78 & 0 & $0 \%$ \\
\hline Pilgrim $1 \ldots \ldots \ldots \ldots \ldots$ & 1,488 & ${ }^{\theta_{1}, 489}$ & 55 & 1 & 102 & 42 & 1,689 & 1,514 & $90 \%$ \\
\hline Quad Cities 1 \& $2 \ldots \ldots \ldots \ldots$. & $b_{2,053}$ & $c, \theta_{370}$ & ${ }^{6} 6$ & 0 & $c_{5}$ & $c_{8}$ & 389 & 369 & $95 \%$ \\
\hline River Bend $1 \ldots \ldots \ldots \ldots$ & 764 & 0 & 24 & 5 & 18 & 6 & 53 & 0 & $0 \%$ \\
\hline Vermont Yankee ............ & 1,850 & 1,366 & 0 & 0 & 0 & 0 & 1,366 & 1,366 & $100 \%$ \\
\hline Total From Reporting Reactors. & 42,032 & 19,042 & 566 & 51 & 387 & 114 & 20,160 & 16,668 & $83 \%$ \\
\hline $\begin{array}{l}\text { Percentage of Permanently Disch } \\
\text { Assemblies . . . . . . . . . }\end{array}$ & ged Fuel & $45.30 \%$ & $1.36 \%$ & $0.11 \%$ & $0.92 \%$ & $0.25 \%$ & & & \\
\hline
\end{tabular}

a Includes all General Electric reactors except Duane Arnold, Limerick 1 \& 2, Shoreham, Susquehanna 1 \& 2, and Washington Nuclear 2 which did not report these data.

${ }^{b}$ Number of fuel assemblies in pool represents fuel discharged from two reactors into separate pools joined by a transfer canal.

c Number of nonfuel components in pool represents components discharged from two reactors into separate pools joined by a transfer canal.

Although all nonfuel components are reported as being in fuel assemblies, more nonfuel components were reported than discharged fuel assemblies.

- Quantity is the number reported in the pool plus the number reported in assemblies.

Note: Data for the following pool sites are as of December 31, 1991 - Pilgrim 1 and Cooper Station.

Source: Energy Information Administration, Form RW-859, "Nuclear Fuel Data" (1992). 


\begin{tabular}{|c|c|c|c|c|c|c|c|c|c|}
\hline \multirow[b]{2}{*}{ Reactor Names ${ }^{a}$} & \multirow[b]{2}{*}{$\begin{array}{c}\text { Permanently } \\
\text { Discharged } \\
\text { Fuel } \\
\text { Assemblles }\end{array}$} & \multicolumn{5}{|c|}{ NFA Hardware } & \multirow[b]{2}{*}{$\begin{array}{c}\text { Total } \\
\text { Reported } \\
\text { NFA } \\
\text { Hardware } \\
\text { Items }\end{array}$} & \multirow[b]{2}{*}{$\begin{array}{c}\text { NFA } \\
\text { Hardware } \\
\text { Items } \\
\text { Reported } \\
\text { In/Attached } \\
\text { to Fuel } \\
\text { Assemblies }\end{array}$} & \multirow[b]{2}{*}{$\begin{array}{c}\text { Percentage } \\
\text { of NFA } \\
\text { Hardware } \\
\text { Items } \\
\text { In/Attached } \\
\text { to Fuel } \\
\text { Assemblles }\end{array}$} \\
\hline & & $\begin{array}{c}\text { Burnable } \\
\text { Poison } \\
\text { Rod } \\
\text { Assemblies }\end{array}$ & $\begin{array}{l}\text { Thimble } \\
\text { Plug } \\
\text { Assemblies }\end{array}$ & $\begin{array}{c}\text { Rod } \\
\text { Cluster } \\
\text { Control } \\
\text { Assemblies }\end{array}$ & $\begin{array}{l}\text { Neutron } \\
\text { Sources }\end{array}$ & $\begin{array}{c}\text { Incore } \\
\text { Instrumentation }\end{array}$ & & & \\
\hline Beaver Valley 1 . . . . . . . & 498 & 239 & 106 & 6 & 4 & 0 & 355 & 353 & $99 \%$ \\
\hline Beaver Valley $2 \ldots \ldots \ldots$ & 187 & 68 & ${ }^{b} 140$ & 0 & 2 & 0 & 210 & 183 & $87 \%$ \\
\hline Braidwood $1 \& 2 \ldots \ldots \ldots \ldots$ & $c_{404}$ & 336 & 23 & 59 & 4 & 3 & 425 & 276 & $65 \%$ \\
\hline 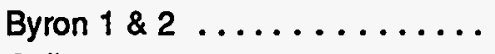 & $c_{596}$ & 452 & 17 & 107 & 4 & 0 & 580 & 384 & $66 \%$ \\
\hline Callaway $\ldots \ldots \ldots \ldots \ldots$ & 452 & 230 & 145 & 55 & 2 & 17 & 449 & 383 & $85 \%$ \\
\hline Catawba $1 \ldots \ldots \ldots \ldots$ & 408 & 322 & 27 & 8 & 2 & 0 & 359 & 334 & $93 \%$ \\
\hline Catawba $2 \ldots \ldots \ldots \ldots$ & 280 & 221 & 30 & 5 & 2 & 0 & 258 & 224 & $87 \%$ \\
\hline Comanche Peak 1 \& $2 \ldots \ldots$ & 117 & 152 & 21 & 0 & 4 & 3 & 180 & 117 & $65 \%$ \\
\hline Cook $1 \ldots \ldots \ldots \ldots$ & 866 & 415 & 143 & 20 & 4 & 0 & 582 & 582 & $100 \%$ \\
\hline Cook $2 \ldots \ldots \ldots \ldots \ldots$ & 657 & 461 & 6 & 2 & 4 & 0 & 473 & 463 & $98 \%$ \\
\hline Diablo Canyon 1 . . . . . . . & 376 & 188 & 94 & 0 & 3 & 5 & 290 & 261 & $90 \%$ \\
\hline Diablo Canyon $2 \ldots . . . . .$. & 308 & 136 & 0 & 0 & 3 & 6 & 145 & 122 & $84 \%$ \\
\hline Farley $1 \ldots \ldots \ldots \ldots$ & 655 & 431 & 177 & 5 & 4 & 83 & 700 & 617 & $88 \%$ \\
\hline Farley $2 \ldots \ldots \ldots \ldots \ldots$ & 498 & 398 & 106 & 0 & 2 & 0 & 506 & 498 & $98 \%$ \\
\hline Ginna ............... & 701 & 47 & 121 & 36 & 10 & 0 & 214 & 213 & $99 \%$ \\
\hline Harris $1 \ldots \ldots \ldots \ldots$ & 224 & 110 & 7 & 57 & 3 & 14 & 191 & 177 & $93 \%$ \\
\hline Indian Point $2 \ldots \ldots \ldots \ldots$ & 660 & 414 & 175 & 1 & 10 & 0 & 600 & 600 & $100 \%$ \\
\hline Indian Point $3 \ldots \ldots \ldots \ldots$ & 582 & 0 & 1 & 0 & 0 & 0 & 1 & 0 & $0 \%$ \\
\hline Kewaunee ............... & 604 & 270 & 102 & 68 & 7 & 72 & 519 & 447 & $86 \%$ \\
\hline McGuire $1 \ldots \ldots \ldots \ldots$ & 484 & 423 & 61 & 8 & 3 & 0 & 495 & 448 & $91 \%$ \\
\hline McGuire $2 \ldots \ldots \ldots \ldots$ & 476 & 668 & 61 & 10 & 2 & 0 & 741 & 735 & $99 \%$ \\
\hline Millstone $3 \ldots \ldots \ldots$ & 248 & ${ }^{d} 140$ & ${ }^{d} 91$ & ${ }^{d} 40$ & $d_{2}$ & 2 & 275 & 236 & $86 \%$ \\
\hline North Anna $1 \& 2 \ldots \ldots \ldots$ & $c_{959}$ & ${ }^{8} 841$ & ${ }^{0} 146$ & ${ }^{\theta} 6$ & ${ }^{0} 12$ & ${ }^{\theta} 0$ & 1,005 & 1,005 & $t_{100 \%}$ \\
\hline Point Beach $1 \& 2 \ldots \ldots \ldots \ldots$ & $c_{1,202}$ & $\mathrm{~d}_{361}$ & $d_{320}$ & ${ }^{d} 66$ & $d_{14}$ & 0 & 761 & 755 & $99 \%$ \\
\hline Robinson $2 \ldots \ldots \ldots \ldots$ & 768 & 4 & 2 & 6 & 5 & 33 & 50 & 11 & $22 \%$ \\
\hline Salem $1 \ldots \ldots \ldots \ldots$ & 631 & ${ }^{d} 505$ & $d_{135}$ & 22 & $d_{5}$ & 0 & 667 & 594 & $89 \%$ \\
\hline
\end{tabular}

See footnotes at end of table. 
Table FE8. Nonfuel Assembly (NFA) Hardware from Westinghouse Reactors (Continued)

\begin{tabular}{|c|c|c|c|c|c|c|c|c|c|}
\hline \multirow[b]{2}{*}{ Reactor Names ${ }^{a}$} & \multirow[b]{2}{*}{$\begin{array}{c}\text { Permanently } \\
\text { Discharged } \\
\text { Fuel } \\
\text { Assemblies }\end{array}$} & \multicolumn{5}{|c|}{ NFA Hardware } & \multirow[b]{2}{*}{$\begin{array}{c}\text { Total } \\
\text { Reported } \\
\text { NFA } \\
\text { Hardware } \\
\text { Items }\end{array}$} & \multirow[b]{2}{*}{$\begin{array}{c}\text { NFA } \\
\text { Hardware } \\
\text { Items } \\
\text { Reported } \\
\text { In/Attached } \\
\text { to Fuel } \\
\text { Assemblies }\end{array}$} & \multirow[b]{2}{*}{$\begin{array}{c}\text { Percentage } \\
\text { of NFA } \\
\text { Hardware } \\
\text { Items } \\
\text { In/Attached } \\
\text { to Fuel } \\
\text { Assemblies }\end{array}$} \\
\hline & & $\begin{array}{c}\text { Burnable } \\
\text { Poison } \\
\text { Rod } \\
\text { Assemblles }\end{array}$ & $\begin{array}{l}\text { Thimble } \\
\text { Plug } \\
\text { Assemblles }\end{array}$ & $\begin{array}{c}\text { Rod } \\
\text { Cluster } \\
\text { Control } \\
\text { Assemblies }\end{array}$ & $\begin{array}{l}\text { Neutron } \\
\text { Sources }\end{array}$ & $\begin{array}{c}\text { Incore } \\
\text { Instrumentation }\end{array}$ & & & \\
\hline Salem $2 \ldots \ldots \ldots \ldots \ldots$ & 432 & $d_{236}$ & $d_{61}$ & 0 & $d_{3}$ & 0 & 300 & 300 & $100 \%$ \\
\hline Seabrook ............... & 136 & 90 & 2 & 7 & 2 & 0 & 101 & 60 & $59 \%$ \\
\hline Sequoyah $1 \ldots \ldots \ldots \ldots$ & 350 & 260 & 2 & 8 & 4 & 0 & 274 & 274 & $100 \%$ \\
\hline Sequoyah $2 \ldots \ldots \ldots \ldots$ & 387 & 236 & 1 & 2 & 2 & 0 & 241 & 241 & $100 \%$ \\
\hline South Texas $1 \ldots \ldots \ldots$. & 229 & 189 & 3 & 57 & 2 & 0 & 251 & 251 & ${ }^{1} 100 \%$ \\
\hline South Texas $2 \ldots \ldots \ldots \ldots$ & 116 & 98 & 0 & 57 & 2 & 0 & 157 & 157 & $100 \%$ \\
\hline Summer ................ & 372 & 222 & 76 & 0 & 4 & 0 & 302 & 275 & $91 \%$ \\
\hline Surry $1 \& 2 \ldots \ldots \ldots \ldots \ldots$ & $c_{1,266}$ & ${ }^{8} 506$ & ${ }^{8} 77$ & ${ }^{0} 109$ & ${ }^{9} 7$ & ${ }^{\circ}$ & 699 & 699 & $100 \%$ \\
\hline Trojan............. & 780 & 92 & 140 & 8 & 4 & 0 & 244 & 244 & $100 \%$ \\
\hline Turkey Point $3 \ldots \ldots \ldots$ & 605 & 246 & 191 & 10 & 2 & 0 & 449 & 448 & $100 \%$ \\
\hline Turkey Point $4 \ldots \ldots \ldots \ldots$ & 547 & 296 & 115. & 9 & 2 & 0 & 422 & 422 & $100 \%$ \\
\hline Vogtle $1 \& 2 \ldots \ldots \ldots \ldots$ & ${ }^{8} 400$ & ${ }^{8} 430$ & $e_{1}$ & ${ }^{\theta_{106}}$ & $\theta_{2}$ & $e_{19}$ & 558 & 400 & $72 \%$ \\
\hline Wolf Creak $1 \ldots \ldots \ldots \ldots$ & 340 & 274 & 69 & 55 & 2 & 11 & 411 & 319 & $78 \%$ \\
\hline Zion $1 \& 2 \ldots \ldots \ldots \ldots$ & $c_{1,608}$ & $d 826$ & $d_{340}$ & $d_{55}$ & d8 & 2 & 1,231 & 1,229 & $100 \%$ \\
\hline Total From Reporting Reactors & 21,409 & 11,833 & 3,335 & 1,070 & 163 & 270 & 16,671 & 15,337 & $92 \%$ \\
\hline $\begin{array}{l}\text { Percentage of Permanently Disc } \\
\text { Assemblles } \ldots \ldots \ldots \ldots \ldots \ldots \text {. }\end{array}$ & $\begin{array}{l}\text { harged Fuel } \\
\text {........... }\end{array}$ & $55.27 \%$ & $15.58 \%$ & $5.00 \%$ & $0.76 \%$ & $1.26 \%$ & & & \\
\hline
\end{tabular}

ancludes all Westinghouse reactors except Prairie Island 1 \& 2 and Watts Bar 1 \& 2 which did not report these data.

${ }^{6}$ Quantity includes 32 water displacement assemblies.

c Number of nonfuel components in pool represents components discharged from two reactors into one pool.

"Quantity is the number reported in the pool plus the number reported in assemblies.

- Quantities reported for nonfuel components were identical and were assumed to be duplicate records.

Although all nonfuel components are reported as being in fuel assemblies, more nonfuel components were reported than discharged fuel assemblies.

g Number of fuel assemblies in pool represents fuel discharged from two reactors into separate pools joined by a transfer canal.

Note: Data for the following pool sites are as of December 31, 1991 - Indian Point 2, Beaver Valley 1, Wolf Creek 1, Diablo Canyon 2, Salem 2, and Summer. Source: Energy Information Administration, Form RW-859, "Nuclear Fuel Data" (1992). 


\section{Executive Summary}

The Energy Information Administration (EIA) of the U.S. Department of Energy (DOE) administers the Nuclear Fuel Data survey, Form RW-859. This form is used to collect data on every fuel assembly irradiated in commercial nuclear reactors operating in the United States, and on the current inventories and storage capacities of those reactors. These data are important to the design and operation of the equipment and facilities that DOE will use for the future acceptance, transport, and disposal of all spent fuel. The information presented in this report summarizes the detailed data collected on Form RW-859 and identifies evident trends in burnup and enrichment in these data. This report focuses on commercial light-water reactor (LWR) spent nuclear fuel reported as discharged as of December 31, 1992.

\section{Spent Nuclear Fuel Discharges}

A total of 91,039 assemblies, with an initial loading weight of uranium of 25,958 metric tons (MTU) (Table ES1), have been discharged from 117 commercial LWRs from 1968 through 1992. Electric utilities also reported their plans to reinsert 163 temporarily discharged assemblies into pressurized-water reactors (PWRs) and 5 temporarily discharged assemblies into boiling-water reactors (BWRs). Approximately 37 percent $(9,547.6 \mathrm{MTU})$ of the total discharges is from BWRs; 63 percent (16,410.1 MTU) is from PWRs. Reprocessed spent fuel and discharges from the damaged Three Mile Island Unit 2 reactor and from Fort St. Vrain, the only commercial high-temperature, gas-cooled reactor (HTGR), are not in the scope of this report.

The characteristics of the permanently discharged spent fuel have changed over time. Most spent commercial nuclear fuel disharged before 1972 was reprocessed. Starting in 1970, the annual average burnup for discharged BWR assemblies has shown a fairly steady increase to almost 29 gigawattdays thermal per metric ton of uranium (GWDt/MTU) in 1992. The average burnup has also continued to increase for PWRs, reaching a new high of 36 GWDt/MTU in 1992, the highest annual average yet attained.

Through 1992, a total of 3,448 of the assemblies covered by this report were shipped from electric utilities to 6 away-from-reactor storage facilities. The 3,448 assemblies do not include shipments of fuel between reactors. Typically, these assemblies were moved either to free up space in the discharging reactor's storage site or for use in a research program.

\section{Site Capacities and Inventories, 1992}

The total inventory of discharged LWR spent nuclear fuel in storage in the United States as of December 31, 1992, was 91,039 assemblies. The majority of spent nuclear fuel is stored in water filled pools, but 903 assemblies are in dry storage at Independent Spent Fuel Storage Installations (ISFSIs) at Virginia Power's Surry plant, Carolina Power and Light Company's Robinson 2 plant, and Duke Power Company's Oconee plant. This section includes as a fold-out a map showing the location of the commercial nuclear reactors and planned and existing ISFSIs. The current licensed storage capacity is 205,731 assemblies. The total maximum storage capacity of all storage sites, as reported by electric utilities, and off-site storage is 215,989 assemblies. However, the excess of total maximum capacity over current total inventory does not reflect the shortage of pool storage in many individual cases. Of the 111 reactors expected to be in operation in the year 2000, 16 reactors appear to require expansion above current pool maximums before 2000 .

The quantities of spent nuclear fuel in storage at nuclear power plants and away-from-reactor facilities aggregated to the State level account for all permanently and temporarily discharged assemblies (Figures ES1 and ES2). 
Table ES1. Total U.S. Commercial Spent Nuclear Fuel Discharges, 1968-1992

\begin{tabular}{|c|c|c|c|}
\hline $\begin{array}{c}\text { Reactor } \\
\text { Typo }\end{array}$ & $\begin{array}{l}\text { Stored at } \\
\text { Reactor } \\
\text { Sites }\end{array}$ & $\begin{array}{c}\text { Stored at } \\
\text { Away-from-reactor } \\
\text { Facilities }\end{array}$ & Total \\
\hline Boiling-Water Reactor ....... & 49,645 & 2,957 & 52,602 \\
\hline \multirow[t]{2}{*}{ TOTAL ........ } & 87,591 & 3,448 & 91,039 \\
\hline & \multicolumn{3}{|c|}{ Metrle Tons of Uranium } \\
\hline Boiling-Water Reactor ....... & $8,993.7$ & 553.9 & $9,547.6$ \\
\hline TOTAL ......... & $25,211.3$ & 746.4 & $25,957.7$ \\
\hline
\end{tabular}

Notes: A number of assemblies discharged prior to 1972 were reprocessed and are not included in this table. See Table 12 and Technical Note 7 in Appendix E for information on reprocessed assemblies. A total of 2,208 high-temperature, gas-cooled reactor (HTGR) fuel elements, with initial uranium content equal to 25.0 metric tons of uranium (MTU), were discharged. These HTGR fuel elements are not included in the above table. See Technical Note 6 in Appendix $E$.

Source: Energy Information Administration, Form RW-859, "Nuclear Fuel Data" (1992). 


\section{Figure ES1. Number of Assemblies of Spent Nuclear Fuel in Storage at U.S. Nuclear Power Plants and Away-from-reactor Facilities, by State}

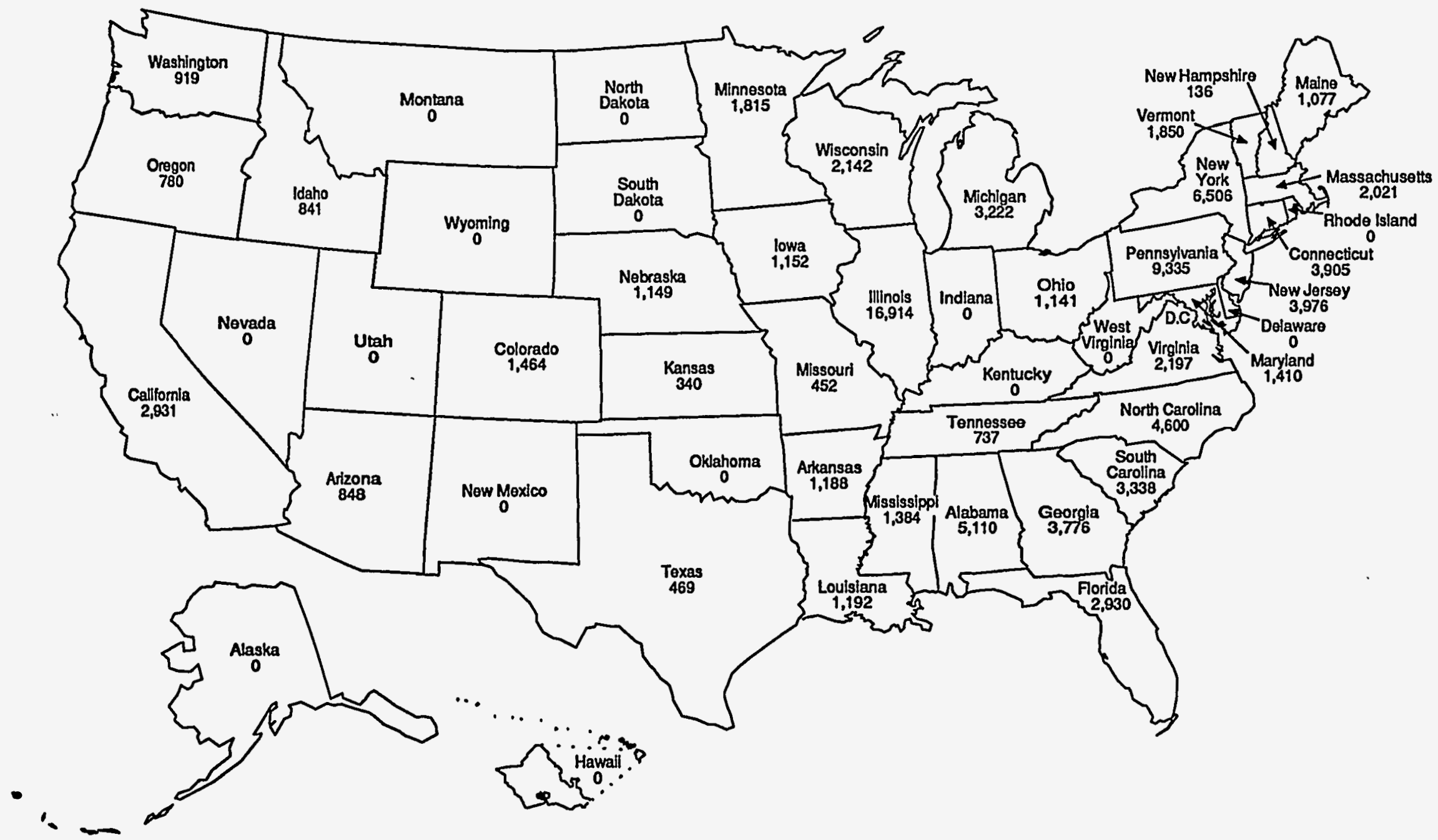

Notes: A total of 2,208 high-temperature, gas-cooled reactor (HTGR) fuel elements are in storage (744 stored in ldaho and 1,464 in Colorado). These HTGR fuel elements are reflected on this map but are not included in Table ES1. Numbers in the above map represent assemblies stored at nuclear power plant sites and away-from-reactor facilities, and include both permanently and temporarily discharged assemblies.

Source: Energy Information Administration, Form RW-859, "Nuclear Fuel Data" (1992) 
Figure ES2. Initial Uranium Content of Spent Nuclear Fuel in Storage at U.S. Nuclear Power Plants and Away-from-reactor Facilities, by State

(Metric tons of uranium)

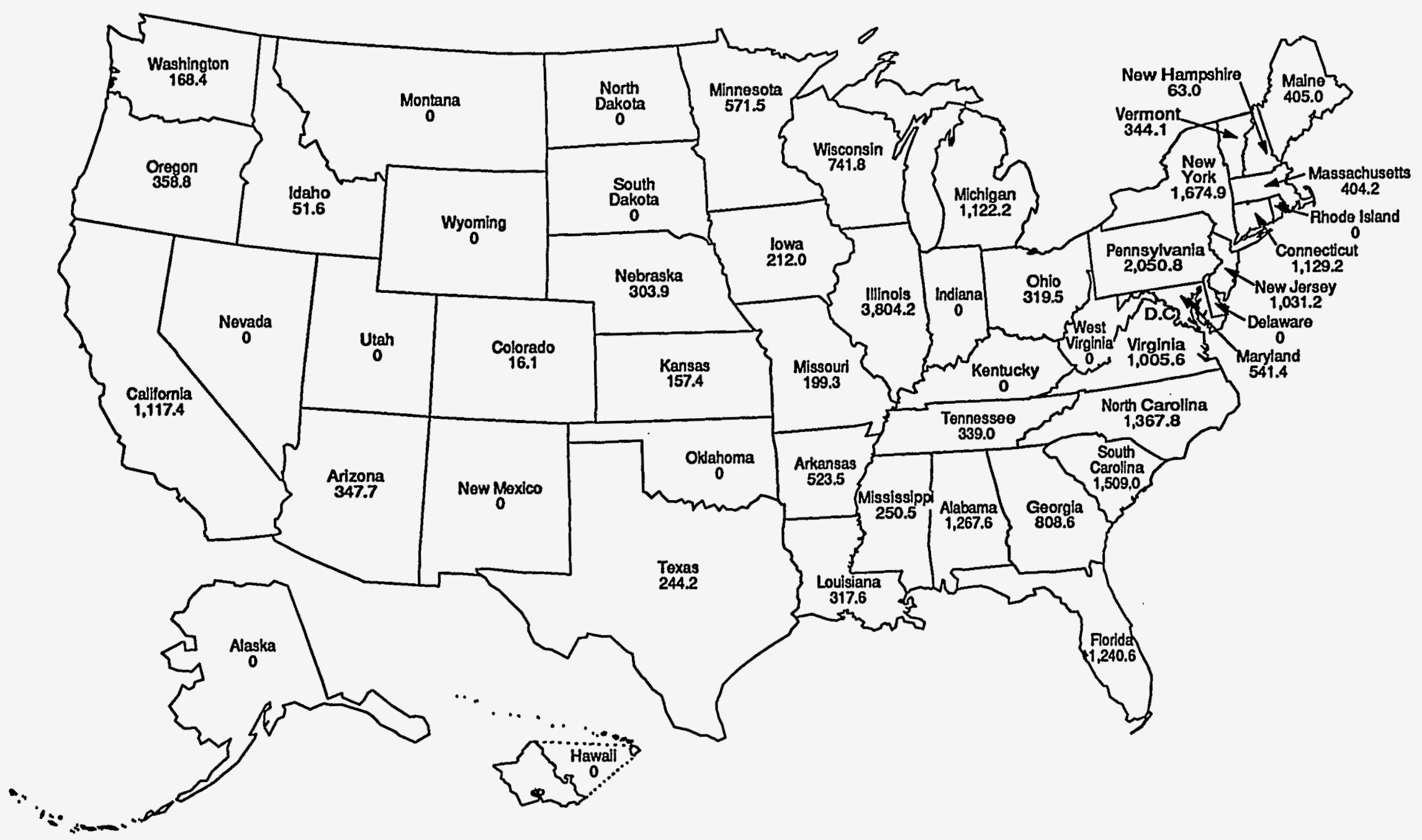

Notes: A total of 2,208 hlgh-temperature, gas-cooled reactor (HTGR) fuel elements are in storage (744 with inlial uranium content equal to 8.9 metric tons of uranium (MTU) stored in (daho and 1,464 with initial uranium content equal to 16.1 MTU stored in Colorado). These HTGR fuel elements are reflected on this map but are not included in Table ES1. Numbers in the above map represent the initial uranium content of assemblies stored at nuclear power plant sites and away-from-reactor faclities, and inciude both permanently and temporarily discharged assemblies.

Source: Energy Information Administration, Form RW-859,"Nuciear Fuel Data" (1992). 


\section{Assembly Type Characteristics}

One hundred twenty-four reactor fuel assembly types have been identified for use in domestic commercial LWRs. These can be broadly grouped by the two LWR types, BWR and PWR. Each assembly type has a unique combination of distinguishing characteristics such as envelope dimensions, rods per assembly, cladding type, and structural materials. Some types may fit in several different reactors. In some cases, a single reactor may use more than one type. Initial enrichment and discharge burnup can vary significantly within a single assembly type.
Forty-five BWR fuel assembly types have been discharged from 41 BWRs through 1992. However, five types make up 58 percent of the BWR assemblies discharged. General Electric fabricated about 91 percent of the discharged BWR assemblies. As fabricated, most are 5.44 inches wide, 176.2 inches long, and contain 183 to 195 kilograms $(\mathrm{kg})$ of uranium. Seventy-six. PWRs have discharged 47 PWR fuel assembly types. Five types make up 56 percent of the PWR assemblies discharged. Westinghouse fabricated about 58 percent of the discharged PWR assemblies. As built, most are 8.44 inches wide, 159.8 inches long, and contain 425 to $460 \mathrm{~kg}$ of uranium. 


\section{Spent Fuel Discharges}

\section{Reactor Spent Fuel Discharge and Storage Activity for 1992}

A total of 117 U.S. commercial light-water reactors (LWRs) have discharged spent nuclear fuel as of December 31, 1992. No reactors discharged spent fuel for the first time in 1992. Two reactors, Portland General Electric Company's Trojan and Southern California Edison Company's San Onofre 1 were permanently shut down in 1992, leaving 108 reactors in operation.

A total of 69 reactors discharged 7,782 spent nuclear fuel assemblies in 1992 (Table 1). Of these, 7,737 were permanently discharged assemblies and 45 were classified as temporarily discharged assemblies. A total of 4,024 boiling-water reactor (BWR) assemblies, containing 728.7 metric tons of uranium (MTU) were permanently discharged from 20 BWRs. A total of 3,713 pressurized-water reactor (PWR) assemblies, containing 1,601.0 MTU were permanently discharged from 49 PWRs. There are 4 BWR and 41 PWR temporarily discharged assemblies.

The total inventory of LWR spent nuclear fuel in storage in the United States as of December 31, 1992, is 91,039 assemblies, containing 25,957.7 MTU. These totals represent an increase from 1991 of 7,439 assemblies containing 2,226.3 MTU. Table 2 summarizes the changes in inventories at nuclear power plants for 1992. As shown in the table, the number of assemblies discharged in 1992 does not match the increase in the number of assemblies stored, because of reinsertion of previously discharged assemblies, transfer of assemblies among storage pools, and revisions to historical survey data from Form RW-859.

Temporarily discharged assemblies are assemblies that utilities have identified for future reinsertion. The cumulative number of discharged assemblies classified as temporarily discharged decreased from 213 assemblies at the end of 1991 to 168 assemblies at the end of 1992 (Table 3).

\section{Nuclear Power Plant Data}

All U.S. commercial LWRs which have discharged and/or are storing nuclear fuel assemblies as of December 31, 1992, are reported on Form RW-859. A total of 117 nuclear plants fall into this category: 41 BWRs and 76 PWRs. Three additional PWRs are expected to discharge spent fuel in the future. Table 4 lists data for U.S. commercial nuclear power reactors as of December 31, 1992. The list includes:

- Electric utility name

- Reactor name

- State in which the reactor is located

- Reactor type

- Reactor vendor

- Summer capability rating (net) in megawatts electric (MWe)

- Number of assemblies in the reactor core during its operation (core size)

- Startup date (that is, the year in which electricity was first generated from the reactor)

- License expiration date

- Year projected by the utility for loss of the ability to operate absent pickup of spent fuel by the DOE

- Actual or projected retirement date.

The license expiration date and the electric utility's projected retirement date are generally the same for each reactor. However, some utilities are expected to apply for reactor license renewal to prolong the productive lives of their reactors. All reactors that have shut down to date have done so before their license expiration dates. Some have shut down permanently and relinquished the operating licenses granted by the Nuclear Regulatory Commission. These are referred to as "retired" reactors. Others have shut down but have not surrendered their licenses. These reactors are in a "shutdown mode." 
Additional storage capacity is projected to be needed when a current storage facility is filled to capacity, based on an understanding of current storage conditions. Form RW-859 defines being filled to capacity as the loss of ability to discharge spent fuel. The EIA collects data based on the current plans for each commercial reactor for spent fuel storage. It also collects the estimated date on which the utility would discontinue reactor operation because storage space is lacking for discharged fuel, unless DOE picks up spent fuel. This date may change in time as utilities implement plans to rerack existing spent fuel storage pools, expand their storage capacities through licensed dry storage facilities, ship spent fuel, and modify fuel cycle management programs. 


\begin{tabular}{|c|c|c|c|c|c|c|c|}
\hline Electrlo UtIIIty Name & Reactor Name & $\begin{array}{c}\text { Reactor } \\
\text { Typo }\end{array}$ & $\begin{array}{c}\text { Regctor } \\
\text { ID }\end{array}$ & $\begin{array}{l}\text { Number of } \\
\text { Discherged } \\
\text { Assembiles }\end{array}$ & $\begin{array}{c}\text { Inlilal Uranium } \\
\text { Content of } \\
\text { Discharged } \\
\text { Assemblies } \\
\text { (MTU) }\end{array}$ & $\begin{array}{l}\text { Number of } \\
\text { Temporarlly } \\
\text { Discharged } \\
\text { Assembles }\end{array}$ & $\begin{array}{c}1992 \\
\text { Reactor } \\
\text { Cyclo } \\
\text { Shutdown } \\
\text { Date }\end{array}$ \\
\hline Alabama Power Company ... & $\begin{array}{l}\text { Farley } 1 \\
\text { Farley } 2\end{array}$ & $\begin{array}{l}\text { PWR } \\
\text { PWR }\end{array}$ & $\begin{array}{l}0101 \\
0102\end{array}$ & $\begin{array}{l}54 \\
61\end{array}$ & $\begin{array}{l}25.0 \\
28.3\end{array}$ & $\begin{array}{l}0 \\
0\end{array}$ & $\begin{array}{l}\text { Sep. } 25 \\
\text { Mar. } 06\end{array}$ \\
\hline $\begin{array}{l}\text { Arizona Publle Servlce } \\
\text { Company } \ldots \ldots \ldots \ldots \ldots \ldots\end{array}$ & $\begin{array}{l}\text { Palo Verde } 1 \\
\text { Palo Verde } 3\end{array}$ & $\begin{array}{l}\text { PWR } \\
\text { PWR }\end{array}$ & $\begin{array}{l}0301 \\
0303\end{array}$ & $\begin{array}{r}97 \\
117\end{array}$ & $\begin{array}{l}40.4 \\
47.5\end{array}$ & $\begin{array}{l}0 \\
0\end{array}$ & $\begin{array}{l}\text { Feb. } 15 \\
\text { Sep. } 19\end{array}$ \\
\hline $\begin{array}{l}\text { Arkansas Power and } \\
\text { Llght Company } . \ldots \ldots \ldots \ldots\end{array}$ & $\begin{array}{l}\text { Arkansas Nuclear } 1 \\
\text { Arkansas Nuclear } 2\end{array}$ & $\begin{array}{l}\text { PWR } \\
\text { PWR }\end{array}$ & $\begin{array}{l}0401 \\
0402\end{array}$ & $\begin{array}{l}61 \\
76\end{array}$ & $\begin{array}{l}28.3 \\
30.9\end{array}$ & $\begin{array}{l}0 \\
0\end{array}$ & $\begin{array}{l}\text { Feb. } 28 \\
\text { Sep. } 04\end{array}$ \\
\hline $\begin{array}{l}\text { Baltimore Gas and } \\
\text { Electrlc Company ......... }\end{array}$ & Calvert Cliffs 1 & PWR & 0501 & 85 & 33.0 & 0 & Mar. 19 \\
\hline $\begin{array}{l}\text { Carolina Power and Light } \\
\text { Company } \ldots \ldots \ldots \ldots \ldots \ldots\end{array}$ & $\begin{array}{l}\text { Harris } 1 \\
\text { Robinson } 2\end{array}$ & $\begin{array}{l}\text { PWR } \\
\text { PWR }\end{array}$ & $\begin{array}{l}0703 \\
0705\end{array}$ & $\begin{array}{l}64 \\
49\end{array}$ & $\begin{array}{l}29.0 \\
20.8\end{array}$ & $\begin{array}{l}0 \\
0\end{array}$ & $\begin{array}{l}\text { Sep. } 12 \\
\text { Mar. } 27\end{array}$ \\
\hline $\begin{array}{l}\text { Cleveland Electrle } \\
\text { Illuminating Company...... }\end{array}$ & Perry 1 & BWR & 0901 & 204 & 36.9 & 4 & Mar. 21 \\
\hline $\begin{array}{l}\text { Commonwealth Edison } \\
\text { Company } \ldots \ldots \ldots \ldots \ldots \ldots\end{array}$ & $\begin{array}{l}\text { Braldwood } 1 \\
\text { Byron } 2 \\
\text { LaSalle County } 1 \\
\text { LaSalle County } 2 \\
\text { Quad Cities } 1 \\
\text { Quad Citles } 2 \\
\text { Zlon } 1 \\
\text { Zion } 2\end{array}$ & $\begin{array}{l}\text { PWR } \\
\text { PWR } \\
\text { BWR } \\
\text { BWR } \\
\text { BWR } \\
\text { BWR } \\
\text { PWR } \\
\text { PWR }\end{array}$ & $\begin{array}{l}1001 \\
1004 \\
1008 \\
1009 \\
1010 \\
1011 \\
1012 \\
1013\end{array}$ & $\begin{array}{r}84 \\
89 \\
200 \\
224 \\
152 \\
152 \\
76 \\
84\end{array}$ & $\begin{array}{l}35.7 \\
37.7 \\
37.0 \\
41.3 \\
26.8 \\
26.8 \\
35.0 \\
38.6\end{array}$ & $\begin{array}{l}0 \\
0 \\
0 \\
0 \\
0 \\
0 \\
0 \\
0\end{array}$ & $\begin{array}{l}\text { Sep. } 05 \\
\text { Feb. } 28 \\
\text { Oct. } 03 \\
\text { Jan. } 04 \\
\text { Sep. } 20 \\
\text { Jan. } 01 \\
\text { Nov. } 12 \\
\text { Nov. } 12\end{array}$ \\
\hline $\begin{array}{l}\text { Consumers Power } \\
\text { Company } \ldots \ldots \ldots \ldots \ldots\end{array}$ & Palisades & PWR & 1204 & 76 & 29.7 & 0 & Fob. 06 \\
\hline Detrolt Edison Company .... & Enrico Fermi 2 & BWR & 1402 & 316 & 56.9 & 0 & Sep. 11 \\
\hline Duke Power Company ...... & 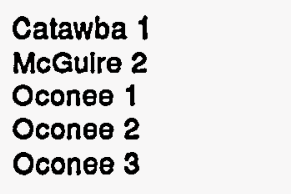 & $\begin{array}{l}\text { PWR } \\
\text { PWR } \\
\text { PWR } \\
\text { PWR } \\
\text { PWR }\end{array}$ & $\begin{array}{l}1501 \\
1505 \\
1506 \\
1507 \\
1508\end{array}$ & $\begin{array}{l}80 \\
76 \\
56 \\
73 \\
56\end{array}$ & $\begin{array}{l}34.0 \\
32.3 \\
25.9 \\
33.8 \\
25.9\end{array}$ & $\begin{array}{l}0 \\
0 \\
0 \\
0 \\
0\end{array}$ & $\begin{array}{l}\text { Jul. } 10 \\
\text { Jan. } 09 \\
\text { Dec. } 03 \\
\text { Jan. } 09 \\
\text { Jul. } 21\end{array}$ \\
\hline Duquesne Light Company ... . & Beaver Valley 2 & PWR & 1602 & 69 & 29.9 & 4 & Mar. 13 \\
\hline Florida Power Corporation... & Crystal River 3 & PWR & 1701 & 76 & 35.2 & 0 & May 30 \\
\hline $\begin{array}{l}\text { Florida Power and Light } \\
\text { Company ............... }\end{array}$ & $\begin{array}{l}\text { St. Lucle } 2 \\
\text { Turkey Polnt } 3\end{array}$ & $\begin{array}{l}\text { PWR } \\
\text { PWR }\end{array}$ & $\begin{array}{l}1802 \\
1803\end{array}$ & $\begin{array}{l}68 \\
57\end{array}$ & $\begin{array}{l}25.8 \\
26.2\end{array}$ & $\begin{array}{l}0 \\
0\end{array}$ & $\begin{array}{l}\text { Apr. } 20 \\
\text { Aug. } 24\end{array}$ \\
\hline GPU Nuclear Corporation ... & Oyster Creek & BWR & 1903 & 168 & 29.6 & 0 & Nov. 28 \\
\hline Goorgla Power Company.... & $\begin{array}{l}\text { Hatch } 2 \\
\text { Vogtle } 2\end{array}$ & $\begin{array}{l}\text { BWR } \\
\text { PWR }\end{array}$ & $\begin{array}{l}2002 \\
2004\end{array}$ & $\begin{array}{r}176 \\
93\end{array}$ & $\begin{array}{l}32.6 \\
43.1\end{array}$ & $\begin{array}{l}0 \\
0\end{array}$ & $\begin{array}{l}\text { Sep. } 15 \\
\text { Mar. } 11\end{array}$ \\
\hline
\end{tabular}

See footnotes at end of table. 
Table 1. Spent Fuel Discharges in 1992 (Continued)

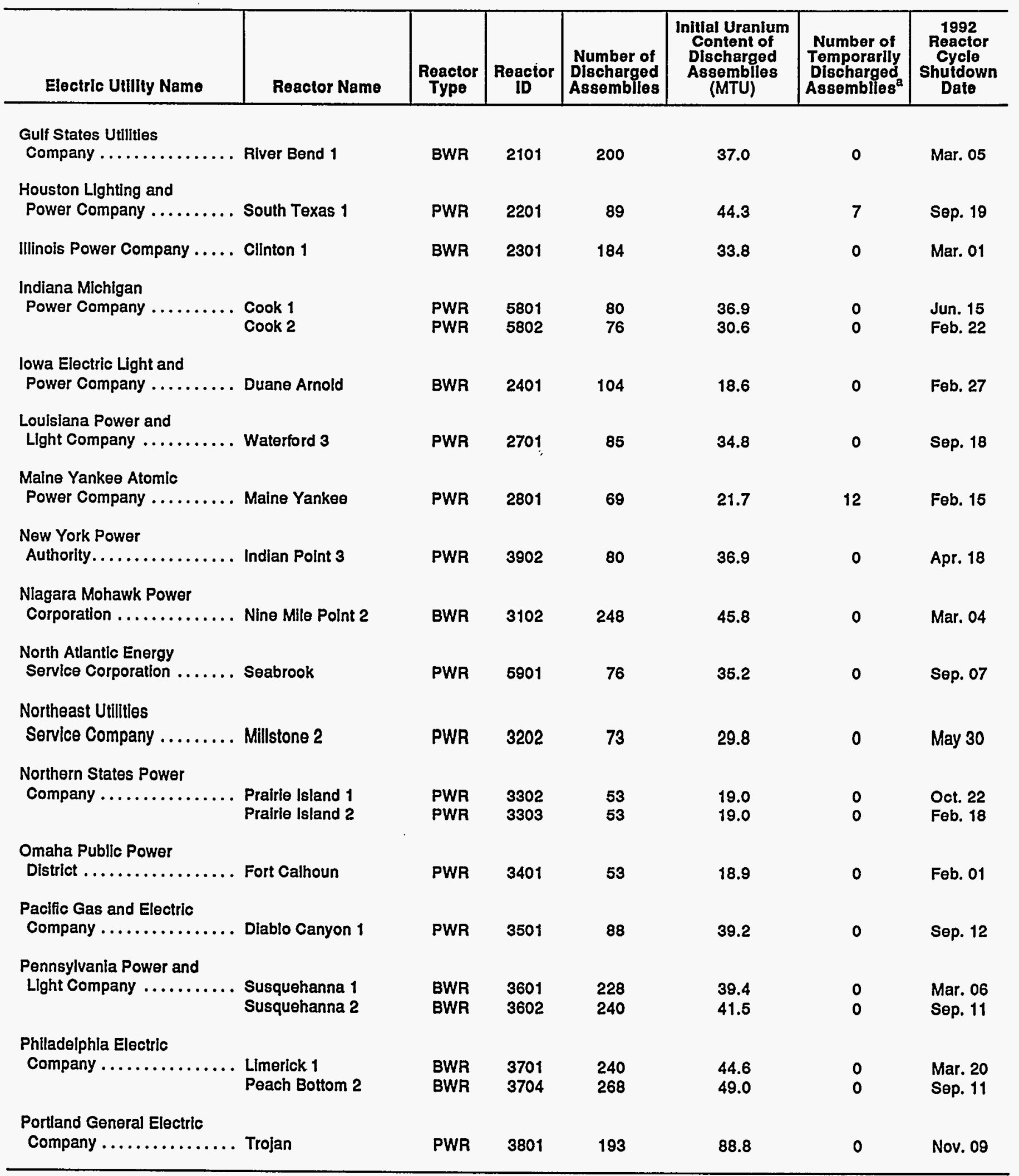

See footnotes at end of table. 
Table 1. Spent Fuel Discharges In 1992 (Continued)

\begin{tabular}{|c|c|c|c|c|c|c|c|}
\hline Electrlc Utillity Name & Reactor Name & $\begin{array}{c}\text { Reactor } \\
\text { Type }\end{array}$ & $\underset{\text { ID }}{\text { Reactor }}$ & $\begin{array}{l}\text { Number of } \\
\text { Discharged } \\
\text { Assemblies }\end{array}$ & $\begin{array}{c}\text { Initlal Uranium } \\
\text { Content of } \\
\text { Dlscharged } \\
\text { Assemblies } \\
\text { (MTU) }\end{array}$ & $\begin{array}{c}\text { Number of } \\
\text { Tomporarily } \\
\text { Dlscharged } \\
\text { Assemblies }\end{array}$ & $\begin{array}{c}1992 \\
\text { Reactor } \\
\text { Cyclo } \\
\text { Shutdown } \\
\text { Date }\end{array}$ \\
\hline $\begin{array}{l}\text { Publlc Service Electric } \\
\text { and Gas Company .... }\end{array}$ & $\begin{array}{l}\text { Hope Creek } \\
\text { Salem } 1\end{array}$ & $\begin{array}{l}\text { BWR } \\
\text { PWR }\end{array}$ & $\begin{array}{l}4201 \\
4202\end{array}$ & $\begin{array}{r}248 \\
93\end{array}$ & $\begin{array}{l}46.0 \\
40.6\end{array}$ & $\begin{array}{l}0 \\
5\end{array}$ & $\begin{array}{l}\text { Sep. } 12 \\
\text { Apr. } 04\end{array}$ \\
\hline $\begin{array}{l}\text { Rochester Gas and } \\
\text { Electrlc Corporation ..... }\end{array}$ & Ginna & PWR & 4401 & 37 & 13.0 & 0 & Mar. 27 \\
\hline $\begin{array}{l}\text { Southern Californla } \\
\text { Edlson Company....... }\end{array}$ & $\begin{array}{l}\text { San Onofre } 1 \\
\text { San Onofre } 3\end{array}$ & $\begin{array}{l}\text { PWR } \\
\text { PWR }\end{array}$ & $\begin{array}{l}4701 \\
4703\end{array}$ & $\begin{array}{l}157 \\
109\end{array}$ & $\begin{array}{l}58.1 \\
44.2\end{array}$ & $\begin{array}{l}0 \\
0\end{array}$ & $\begin{array}{l}\text { Nov.30 } \\
\text { Jan. } 24\end{array}$ \\
\hline 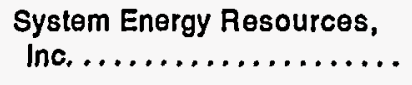 & Grand Gulf 1 & BWR & 2901 & 272 & 47.9 & 0 & Apr. 17 \\
\hline $\begin{array}{l}\text { Tennessee Valley } \\
\text { Authorlty......... }\end{array}$ & Sequoyah 2 & PWR & 4809 & 93 & 43.1 & 0 & Mar. 13 \\
\hline TU Electric.............. & Comanche Peak 1 & PWR & 4901 & 64 & 29.4 & 0 & Oct. 22 \\
\hline Unlon Electric Company.... & Callaway & PWR & 5101 & 101 & 42.9 & 0 & Mar. 20 \\
\hline $\begin{array}{c}\text { Vermont Yankee Nuclear } \\
\text { Power Corporation ..... }\end{array}$ & Vermont Yankee & BWR & 6001 & 128 & 23.5 & 0 & Fob. 29 \\
\hline VIriginia Power ... & $\begin{array}{l}\text { North Anna } 2 \\
\text { Surry } 1\end{array}$ & $\begin{array}{l}\text { PWR } \\
\text { PWR }\end{array}$ & $\begin{array}{l}5202 \\
5203\end{array}$ & $\begin{array}{l}75 \\
65\end{array}$ & $\begin{array}{l}34.2 \\
29.9\end{array}$ & $\begin{array}{l}1 \\
0\end{array}$ & $\begin{array}{l}\text { Feb. } 26 \\
\text { Feb. } 29\end{array}$ \\
\hline $\begin{array}{l}\text { Washington Publle Power } \\
\text { Supply System .......... }\end{array}$ & Washington Nuclear 2 & BWR & 5302 & 76 & 13.8 & 0 & Apr. 18 \\
\hline $\begin{array}{c}\text { Wisconsin Electric Power } \\
\text { Company.............. }\end{array}$ & $\begin{array}{l}\text { Point Beach } 1 \\
\text { Point Beach } 2\end{array}$ & $\begin{array}{l}\text { PWR } \\
\text { PWR }\end{array}$ & $\begin{array}{l}5401 \\
5402\end{array}$ & $\begin{array}{l}29 \\
29\end{array}$ & $\begin{array}{l}10.5 \\
10.5\end{array}$ & $\begin{array}{l}0 \\
0\end{array}$ & $\begin{array}{l}\text { Apr. } 10 \\
\text { Sep. } 25\end{array}$ \\
\hline $\begin{array}{l}\text { Wlsconsin Public Service } \\
\text { Corporation ............ }\end{array}$ & Kewaunee & PWR & 5501 & 57 & 17.0 & 12 & Mar. 06 \\
\hline Total BWR.............. & & & & 4,028 & 728.7 & 4 & \\
\hline Total PWR.............. & & & & 3,754 & $1,601.0$ & 41 & \\
\hline TOTAL $\ldots \ldots \ldots \ldots \ldots \ldots$ & & & & 7,782 & $2,329.8$ & 45 & \\
\hline
\end{tabular}

aThese numbers are included in the number of discharged assemblles column.

MTU = Metrlc tons of uranium; PWR = Pressurized-water reactor; BWR = Boiling-water reactor.

Note: Totals may not equal sum of components because of independent rounding. See Technical Note 11 in Appendix E.

Source: Energy Information Administration, Form RW-859, "Nuclear Fuel Data" (1992). 
Table 2. Reactor Spent Fuel Discharge and Storage Activity for 1992

\begin{tabular}{|c|c|c|c|c|c|c|c|c|}
\hline Electrlc Utility Name & Reactor Name & $\begin{array}{c}\text { Reactor } \\
\text { Type }\end{array}$ & $\begin{array}{c}\text { Pool Site } \\
\text { ID }\end{array}$ & $\begin{array}{l}1991 \text { Assembly } \\
\text { Total }\end{array}$ & $\begin{array}{c}1992 \text { Assembly } \\
\text { Discharges }\end{array}$ & $\begin{array}{c}1992 \text { Assembly } \\
\text { Reinsertions }\end{array}$ & $\begin{array}{l}\text { Other (see } \\
\text { footnotes) }\end{array}$ & $\begin{array}{l}1992 \text { Assembly } \\
\text { Total }\end{array}$ \\
\hline Alabama Power Company .... & $\begin{array}{l}\text { Farley } 1 \\
\text { Farley } 2\end{array}$ & $\begin{array}{l}\text { PWR } \\
\text { PWR }\end{array}$ & $\begin{array}{l}0101 \\
0102\end{array}$ & $\begin{array}{l}603 \\
441\end{array}$ & $\begin{array}{l}54 \\
61\end{array}$ & $\begin{array}{l}-2 \\
-4\end{array}$ & & $\begin{array}{l}655 \\
498\end{array}$ \\
\hline $\begin{array}{l}\text { Arizona Public Service } \\
\text { Company } . . \ldots \ldots \ldots \ldots \ldots \ldots\end{array}$ & $\begin{array}{l}\text { Palo Verde } 1 \\
\text { Palo Verde } 2 \\
\text { Palo Verde } 3\end{array}$ & $\begin{array}{l}\text { PWR } \\
\text { PWR } \\
\text { PWR }\end{array}$ & $\begin{array}{l}0301 \\
0302 \\
0303\end{array}$ & $\begin{array}{l}188 \\
288 \\
192\end{array}$ & $\begin{array}{r}97 \\
117\end{array}$ & $\begin{array}{r}-9 \\
-25\end{array}$ & & $\begin{array}{l}276 \\
288 \\
284\end{array}$ \\
\hline $\begin{array}{l}\text { Arkansas Power and } \\
\text { Light Company............ }\end{array}$ & $\begin{array}{l}\text { Arkansas Nuclear } 1 \\
\text { Arkansas Nuclear } 2\end{array}$ & $\begin{array}{l}\text { PWR } \\
\text { PWR }\end{array}$ & $\begin{array}{l}0401 \\
0402\end{array}$ & $\begin{array}{l}564 \\
488\end{array}$ & $\begin{array}{l}61 \\
76\end{array}$ & -1 & & $\begin{array}{l}624 \\
564\end{array}$ \\
\hline $\begin{array}{l}\text { Baltimore Gas and } \\
\text { Electric Company .......... }\end{array}$ & $\begin{array}{l}\text { Calvert Cliffs } 1 \\
\text { Calvert Cliffs } 2\end{array}$ & $\begin{array}{l}\text { PWR } \\
\text { PWR }\end{array}$ & 0501 & $\begin{array}{l}714 \\
612\end{array}$ & 85 & -1 & & $\begin{array}{l}798 \\
612\end{array}$ \\
\hline Boston Edison Company ..... & Pilgrim 1 & BWR & 0601 & 1,489 & & & $a_{-1}$ & 1,488 \\
\hline $\begin{array}{l}\text { Carolina Power and Light } \\
\text { Company } \ldots \ldots \ldots \ldots \ldots \ldots\end{array}$ & $\begin{array}{l}\text { Brunswick } 1 \\
\text { Brunswick } 1 \\
\text { Brunswick } 2 \\
\text { Brunswick } 2 \\
\text { Harris } 1 \\
\text { Harris } 1 \\
\text { Robinson } 2 \\
\text { Dry Storage }\end{array}$ & $\begin{array}{l}\text { BWR } \\
\text { PWR } \\
\text { BWR } \\
\text { PWR } \\
\text { PWR } \\
\text { BWR } \\
\text { PWR } \\
\text { PWR }\end{array}$ & $\begin{array}{l}0701 \\
0701 \\
0702 \\
0702 \\
0703 \\
0703 \\
0705 \\
07050\end{array}$ & $\begin{array}{r}1,090 \\
160 \\
1,113 \\
144 \\
276 \\
413 \\
247 \\
56\end{array}$ & $\begin{array}{l}64 \\
49\end{array}$ & -4 & $\begin{array}{l}{ }^{b} 84 \\
-84\end{array}$ & $\begin{array}{r}1,090 \\
160 \\
1,113 \\
144 \\
420 \\
413 \\
212 \\
56\end{array}$ \\
\hline $\begin{array}{l}\text { Cleveland Electric } \\
\text { Illuminating Company ........ }\end{array}$ & Perry 1 & $\begin{array}{l}\text { BWR } \\
\text { BWR }\end{array}$ & $\begin{array}{l}0901 \\
0902\end{array}$ & 544 & 204 & & & 748 \\
\hline $\begin{array}{l}\text { Commonwealth Edison } \\
\text { Company .............. }\end{array}$ & $\begin{array}{l}\text { Braidwood } 1 \\
\text { Braldwood } 2 \\
\text { Byron } 1 \\
\text { Byron } 2 \\
\text { Dresden } 1 \\
\text { Dresden } 2 \\
\text { Dresden } 3 \\
\text { LaSalle County } 1 \\
\text { LaSalle County } 2 \\
\text { Quad Cities } 1 \\
\text { Quad Cities } 2 \\
\text { Zion } 1 \\
\text { Zion } 2\end{array}$ & $\begin{array}{l}\text { PWR } \\
\text { PWR } \\
\text { PWR } \\
\text { PWR } \\
\text { BWR } \\
\text { BWR } \\
\text { BWR } \\
\text { BWR } \\
\text { BWR } \\
\text { BWR } \\
\text { BWR } \\
\text { PWR } \\
\text { PWR }\end{array}$ & $\begin{array}{l}1001 \\
1003 \\
1005 \\
1006 \\
1007 \\
1008 \\
1010 \\
1012\end{array}$ & $\begin{array}{r}320 \\
512 \\
\\
683 \\
1,954 \\
1,968 \\
1,496 \\
0 \\
3,692 \\
0 \\
1,448\end{array}$ & $\begin{array}{r}200 \\
224 \\
152 \\
152 \\
76 \\
84\end{array}$ & -5 & & $\begin{array}{r}404 \\
596 \\
683 \\
1,954 \\
1,968 \\
1,920 \\
3,996 \\
1,608\end{array}$ \\
\hline
\end{tabular}

See footnotes at end of table. 
Table 2. Reactor Spent Fuel Discharge and Storage Activity for 1992 (Continued)

\begin{tabular}{|c|c|c|c|c|c|c|c|c|}
\hline Electric Utllity Name & Reactor Name & $\begin{array}{l}\text { Reactor } \\
\text { Type }\end{array}$ & $\underset{\text { ID }}{\text { Pool Site }}$ & $\begin{array}{l}1991 \text { Assembly } \\
\text { Total }\end{array}$ & $\begin{array}{l}1992 \text { Assembly } \\
\text { Discharges }\end{array}$ & $\begin{array}{l}1992 \text { Assembly } \\
\text { Reinsertions }\end{array}$ & $\begin{array}{l}\text { Other (see } \\
\text { footnotes) }\end{array}$ & $\begin{array}{l}1992 \text { Assembly } \\
\text { Total }\end{array}$ \\
\hline $\begin{array}{l}\text { Consolidated Edison } \\
\text { Company of New York ...... }\end{array}$ & $\begin{array}{l}\text { Indian Point } 1 \\
\text { Indian Point } 2\end{array}$ & $\begin{array}{l}\text { PWR } \\
\text { PWR }\end{array}$ & $\begin{array}{l}1101 \\
1102\end{array}$ & $\begin{array}{l}160 \\
668\end{array}$ & & & & $\begin{array}{l}160 \\
668\end{array}$ \\
\hline $\begin{array}{l}\text { Consumers Power } \\
\text { Company } \ldots \ldots \ldots \ldots \ldots \ldots\end{array}$ & $\begin{array}{l}\text { Big Rock Point } \\
\text { Palisades }\end{array}$ & $\begin{array}{l}\text { BWR } \\
\text { PWR }\end{array}$ & $\begin{array}{l}1201 \\
1204\end{array}$ & $\begin{array}{l}294 \\
657\end{array}$ & 76 & & & $\begin{array}{l}294 \\
733\end{array}$ \\
\hline $\begin{array}{l}\text { Dairyland Power } \\
\text { Cooperative } \ldots \ldots \ldots \ldots \ldots\end{array}$ & LaCrosse & BWR & 1301 & 333 & & & & 333 \\
\hline Detroit Edison Company...... & Enrico Fermi 2 & BWR & 1402 & 448 & 316 & -92 & & 672 \\
\hline Duke Power Company ........ & $\begin{array}{l}\text { Catawba } 1 \\
\text { Catawba } 2 \\
\text { McGuire } 1 \\
\text { McGuire } 2 \\
\text { Oconee } 1 \\
\text { Oconee } 2 \\
\text { Oconee } 3 \\
\text { Dry Storage }\end{array}$ & $\begin{array}{l}\text { PWR } \\
\text { PWR } \\
\text { PWR } \\
\text { PWR } \\
\text { PWR } \\
\text { PWR } \\
\text { PWR } \\
\text { PWR }\end{array}$ & $\begin{array}{l}1501 \\
1502 \\
1504 \\
1505 \\
1506 \\
1508 \\
1506 D\end{array}$ & $\begin{array}{l}336 \\
280 \\
519 \\
665 \\
954 \\
\\
580 \\
312\end{array}$ & $\begin{array}{l}80 \\
\\
76 \\
56 \\
73 \\
56\end{array}$ & $\begin{array}{l}-8 \\
-21\end{array}$ & $\begin{array}{r}c-48 \\
c-120 \\
168\end{array}$ & $\begin{array}{r}408 \\
280 \\
519 \\
741 \\
1,014 \\
\\
516 \\
480\end{array}$ \\
\hline Duquesne Light Company .... & $\begin{array}{l}\text { Beaver Valley } 1 \\
\text { Beaver Valley } 2\end{array}$ & $\begin{array}{l}\text { PWR } \\
\text { PWR }\end{array}$ & $\begin{array}{l}1601 \\
1602\end{array}$ & $\begin{array}{l}512 \\
124\end{array}$ & 69 & -1 & & $\begin{array}{l}512 \\
192\end{array}$ \\
\hline Florida Power Corporation .... & Crystal River 3 & PWR & 1701 & 472 & 76 & -12 & & 536 \\
\hline $\begin{array}{l}\text { Florida Power and Light } \\
\text { Company } \ldots \ldots \ldots \ldots \ldots \ldots \ldots\end{array}$ & $\begin{array}{l}\text { St. Lucie } 1 \\
\text { St. Lucle } 2 \\
\text { Turkey Point } 3 \\
\text { Turkey Point } 4\end{array}$ & $\begin{array}{l}\text { PWR } \\
\text { PWR } \\
\text { PWR } \\
\text { PWR }\end{array}$ & $\begin{array}{l}1801 \\
1802 \\
1803 \\
1804\end{array}$ & $\begin{array}{l}796 \\
396 \\
532 \\
546\end{array}$ & $\begin{array}{l}68 \\
57\end{array}$ & -1 & & $\begin{array}{l}796 \\
464 \\
588 \\
546\end{array}$ \\
\hline GPU Nuclear Corporation..... & $\begin{array}{l}\text { Three Mile Island } 1 \\
\text { Oyster Creek }\end{array}$ & $\begin{array}{l}\text { PWR } \\
\text { BWR }\end{array}$ & $\begin{array}{l}1901 \\
1903\end{array}$ & $\begin{array}{r}521 \\
1,708\end{array}$ & 168 & & & $\begin{array}{r}521 \\
1,876\end{array}$ \\
\hline Georgia Power Company ..... & $\begin{array}{l}\text { Hatch } 1 \\
\text { Hatch } 2 \\
\text { Vogtle } 1 \\
\text { Vogtle } 2\end{array}$ & $\begin{array}{l}\text { BWR } \\
\text { BWR } \\
\text { PWR } \\
\text { PWR }\end{array}$ & $\begin{array}{l}2001 \\
2003\end{array}$ & $\begin{array}{r}1,800 \\
1,400 \\
240 \\
84\end{array}$ & $\begin{array}{r}176 \\
93\end{array}$ & & $d_{-17}$ & $\begin{array}{r}1,800 \\
1,576 \\
223 \\
177\end{array}$ \\
\hline $\begin{array}{l}\text { Gulf States Utilities } \\
\text { Company } \ldots \ldots \ldots \ldots \ldots \ldots\end{array}$ & River Bend 1 & BWR & 2101 & 564 & 200 & & & 764 \\
\hline $\begin{array}{l}\text { Houston Lighting and } \\
\text { Power Company........... }\end{array}$ & $\begin{array}{l}\text { South Texas } 1 \\
\text { South Texas } 2\end{array}$ & $\begin{array}{l}\text { PWR } \\
\text { PWR }\end{array}$ & $\begin{array}{l}2201 \\
2202\end{array}$ & $\begin{array}{l}160 \\
116\end{array}$ & 89 & -13 & & $\begin{array}{l}236 \\
116\end{array}$ \\
\hline
\end{tabular}

See footnotes at end of table. 
Table 2. Reactor Spent Fuel Discharge and Storage Activity for 1992 (Continued)

\begin{tabular}{|c|c|c|c|c|c|c|c|c|}
\hline Electrlc Utillity Name & Reactor Name & $\begin{array}{c}\text { Reactor } \\
\text { Type }\end{array}$ & $\begin{array}{c}\text { Pool Site } \\
\text { ID }\end{array}$ & $\begin{array}{c}1991 \text { Assembly } \\
\text { Total }\end{array}$ & $\begin{array}{c}1992 \text { Assembly } \\
\text { Discharges }\end{array}$ & $\begin{array}{c}1992 \text { Assembly } \\
\text { Reinsertions }\end{array}$ & $\begin{array}{l}\text { Other (see } \\
\text { footnotes) }\end{array}$ & $\begin{array}{c}1992 \text { Assembly } \\
\text { Total }\end{array}$ \\
\hline Illinois Power Company ...... & Clinton 1 & BWR & 2301 & 384 & 184 & & & 568 \\
\hline $\begin{array}{l}\text { Indiana Michigan } \\
\text { Power Company } \ldots \ldots \ldots \ldots\end{array}$ & $\begin{array}{l}\text { Cook } 1 \\
\text { Cook } 2\end{array}$ & $\begin{array}{l}\text { PWR } \\
\text { PWR }\end{array}$ & 5801 & 1,367 & $\begin{array}{l}80 \\
76\end{array}$ & & & 1,523 \\
\hline $\begin{array}{l}\text { lowa Electric Light and } \\
\text { Power Company } \ldots \ldots \ldots \ldots\end{array}$ & Duane Arnold & BWA & 2401 & 1,048 & 104 & & & 1,152 \\
\hline $\begin{array}{l}\text { Kansas Gas and Electric } \\
\text { Company } \ldots \ldots \ldots \ldots \ldots \ldots\end{array}$ & Wolf Creek 1 & PWR & 2501 & 340 & & & & 340 \\
\hline $\begin{array}{l}\text { Long Island Lighting } \\
\text { Company } \ldots \ldots \ldots \ldots \ldots \ldots\end{array}$ & Shoreham & BWR & 2601 & 560 & & & & 560 \\
\hline $\begin{array}{l}\text { Louisiana Power and } \\
\text { Light Company............ }\end{array}$ & Waterford 3 & PWR & 2701 & 344 & 85 & -1 & & 428 \\
\hline $\begin{array}{l}\text { Maine Yankee Atomic } \\
\text { Power Company ........... }\end{array}$ & Maine Yankee & PWR & 2801 & 1,009 & 69 & -1 & & 1,077 \\
\hline $\begin{array}{l}\text { Nebraska Public Power } \\
\text { District. ................. }\end{array}$ & Cooper Station & BWR & 3001 & 620 & & & & 620 \\
\hline $\begin{array}{l}\text { New York Power } \\
\text { Authority ................. }\end{array}$ & $\begin{array}{l}\text { Fitzpatrick } \\
\text { Indian Point } 3\end{array}$ & $\begin{array}{l}\text { BWR } \\
\text { PWR }\end{array}$ & $\begin{array}{l}3901 \\
3902\end{array}$ & $\begin{array}{r}1,684 \\
504\end{array}$ & 80 & & & $\begin{array}{r}1,684 \\
584\end{array}$ \\
\hline $\begin{array}{l}\text { Niagara Mohawk Power } \\
\text { Corporation } . . \ldots \ldots \ldots \ldots \ldots\end{array}$ & $\begin{array}{l}\text { Nine Mile Point } 1 \\
\text { Nine Mile Point } 2\end{array}$ & $\begin{array}{l}\text { BWR } \\
\text { BWR }\end{array}$ & $\begin{array}{l}3101 \\
3102\end{array}$ & $\begin{array}{r}1,620 \\
196\end{array}$ & 248 & & & $\begin{array}{r}1,620 \\
444\end{array}$ \\
\hline $\begin{array}{l}\text { North Atlantic Energy } \\
\text { Service Corporation. ......... }\end{array}$ & Seabrook & PWR & 5901 & 60 & 76 & & & 136 \\
\hline $\begin{array}{l}\text { Northeast Utilities } \\
\text { Service Company ... }\end{array}$ & $\begin{array}{l}\text { Millstone } 1 \\
\text { Millstone } 2 \\
\text { Millstone } 3 \\
\text { Haddam Neck }\end{array}$ & $\begin{array}{l}\text { BWR } \\
\text { PWR } \\
\text { PWR } \\
\text { PWR }\end{array}$ & $\begin{array}{l}3201 \\
3202 \\
3203 \\
5701\end{array}$ & $\begin{array}{r}2,116 \\
712 \\
248 \\
757\end{array}$ & 73 & -1 & & $\begin{array}{r}2,116 \\
784 \\
248 \\
757\end{array}$ \\
\hline $\begin{array}{l}\text { Northern States Power } \\
\text { Company } . . \ldots \ldots \ldots\end{array}$ & $\begin{array}{l}\text { Monticello } \\
\text { Prairie Island } 1 \\
\text { Prairie Island } 2\end{array}$ & $\begin{array}{l}\text { BWR } \\
\text { PWR } \\
\text { PWR }\end{array}$ & $\begin{array}{l}3301 \\
3302\end{array}$ & $\begin{array}{r}582 \\
1,133\end{array}$ & $\begin{array}{l}53 \\
53\end{array}$ & $\begin{array}{l}-5 \\
-1\end{array}$ & & $\begin{array}{r}582 \\
1,233\end{array}$ \\
\hline $\begin{array}{l}\text { Omaha Public Power } \\
\text { District. ................. }\end{array}$ & Fort Calhoun & PWR & 3401 & 477 & 53 & -1 & & 529 \\
\hline
\end{tabular}

See footnotes at end of table. 
Table 2. Reactor Spent Fuel Discharge and Storage Activity for 1992 (Continued)

\begin{tabular}{|c|c|c|c|c|c|c|c|c|}
\hline Electric Utility Name & Reactor Name & $\begin{array}{l}\text { Reactor } \\
\text { Type }\end{array}$ & $\begin{array}{c}\text { Pool Site } \\
\text { ID }\end{array}$ & $\begin{array}{l}1991 \text { Assembly } \\
\text { Total }\end{array}$ & $\begin{array}{c}1992 \text { Assembly } \\
\text { Discharges }\end{array}$ & $\begin{array}{c}1992 \text { Assembly } \\
\text { Reinsertions }\end{array}$ & $\begin{array}{l}\text { Other (see } \\
\text { footnotes) }\end{array}$ & $\begin{array}{l}1992 \text { Assembly } \\
\text { Total }\end{array}$ \\
\hline $\begin{array}{l}\text { Pacific Gas and Electric } \\
\text { Company } \ldots \ldots \ldots \ldots \ldots \ldots\end{array}$ & $\begin{array}{l}\text { Diablo Canyon } 1 \\
\text { Diablo Canyon } 2 \\
\text { Humboldt Bay }\end{array}$ & $\begin{array}{l}\text { PWR } \\
\text { PWR } \\
\text { BWR }\end{array}$ & $\begin{array}{l}3501 \\
3502 \\
3503\end{array}$ & $\begin{array}{l}288 \\
308 \\
390\end{array}$ & 88 & & & $\begin{array}{l}376 \\
308 \\
390\end{array}$ \\
\hline $\begin{array}{l}\text { Pennsylvania Power and } \\
\text { Light Company............. }\end{array}$ & $\begin{array}{l}\text { Susquehanna } 1 \\
\text { Susquehanna } 2\end{array}$ & $\begin{array}{l}\text { BWR } \\
\text { BWR }\end{array}$ & 3601 & $\begin{array}{l}1,176 \\
1,000\end{array}$ & $\begin{array}{l}228 \\
240\end{array}$ & -4 & & $\begin{array}{l}1,404 \\
1,236\end{array}$ \\
\hline $\begin{array}{l}\text { Philadelphia Electric } \\
\text { Company } \ldots \ldots \ldots \ldots\end{array}$ & $\begin{array}{l}\text { Limerick } 1 \\
\text { Limerick } 2 \\
\text { Peach Bottom } 2 \\
\text { Peach Bottom } 3\end{array}$ & $\begin{array}{l}\text { BWR } \\
\text { BWR } \\
\text { BWR } \\
\text { BWR }\end{array}$ & $\begin{array}{l}3701 \\
3704 \\
3705\end{array}$ & $\begin{array}{r}900 \\
224 \\
1,894 \\
1,944\end{array}$ & $\begin{array}{l}240 \\
268\end{array}$ & & & $\begin{array}{r}1,140 \\
224 \\
2,162 \\
1,944\end{array}$ \\
\hline $\begin{array}{l}\text { Portland General Electric } \\
\text { Company } \ldots \ldots \ldots \ldots \ldots \ldots\end{array}$ & Trojan & PWR & 3801 & 587 & 193 & & & 780 \\
\hline $\begin{array}{l}\text { Public Service Electric } \\
\text { and Gas Company.......... }\end{array}$ & $\begin{array}{l}\text { Hope Creek } \\
\text { Salem } 1 \\
\text { Salem } 2\end{array}$ & $\begin{array}{l}\text { BWR } \\
\text { PWR } \\
\text { PWR }\end{array}$ & $\begin{array}{l}4201 \\
4202 \\
4203\end{array}$ & $\begin{array}{l}760 \\
588 \\
436\end{array}$ & $\begin{array}{r}248 \\
93\end{array}$ & -25 & & $\begin{array}{r}1,008 \\
656 \\
436\end{array}$ \\
\hline $\begin{array}{l}\text { Rochester Gas and } \\
\text { Electric Corporation......... }\end{array}$ & Ginna & PWR & 4401 & 633 & 37 & -9 & & 661 \\
\hline $\begin{array}{l}\text { Sacramento Municipal } \\
\text { Utility District ............. }\end{array}$ & Rancho Seco & PWR & 4501 & 493 & & & & 493 \\
\hline $\begin{array}{l}\text { South Carolina Electric } \\
\text { and Gas Company.......... }\end{array}$ & Summer & PWR & 4601 & 372 & & & & 372 \\
\hline $\begin{array}{l}\text { Southern California } \\
\text { Edison Company ........... }\end{array}$ & $\begin{array}{l}\text { San Onofre } 1 \\
\text { San Onofre } 2 \\
\text { San Onofre } 3\end{array}$ & $\begin{array}{l}\text { PWR } \\
\text { PWR } \\
\text { PWR }\end{array}$ & $\begin{array}{l}4701 \\
4702 \\
4703\end{array}$ & $\begin{array}{r}99 \\
554 \\
445\end{array}$ & $\begin{array}{l}157 \\
109\end{array}$ & -1 & $\begin{array}{r}-49 \\
49\end{array}$ & $\begin{array}{l}207 \\
554 \\
602\end{array}$ \\
\hline $\begin{array}{l}\text { System Energy Resources, } \\
\text { Inc. } \ldots \ldots \ldots \ldots \ldots \ldots \ldots \ldots \ldots\end{array}$ & Grand Gulf 1 & BWR & 2901 & 1,112 & 272 & & & 1,384 \\
\hline $\begin{array}{l}\text { Tennessee Valley } \\
\text { Authority.......... }\end{array}$ & $\begin{array}{l}\text { Browns Ferry } 1 \\
\text { Browns Ferry } 2 \\
\text { Browns Ferry } 3 \\
\text { Sequoyah } 1 \\
\text { Sequoyah } 2 \\
\text { Watts Bar } 1 \\
\text { Watts Bar } 2\end{array}$ & $\begin{array}{l}\text { BWR } \\
\text { BWR } \\
\text { BWR } \\
\text { PWR } \\
\text { PWR } \\
\text { PWR } \\
\text { PWR }\end{array}$ & $\begin{array}{l}4803 \\
4805 \\
4808 \\
4810\end{array}$ & $\begin{array}{r}1,488 \\
1,440 \\
1,029 \\
661 \\
0\end{array}$ & 93 & -1 & $f^{f}-16$ & $\begin{array}{r}1,488 \\
1,440 \\
1,029 \\
737 \\
0\end{array}$ \\
\hline
\end{tabular}

\footnotetext{
See footnotes at end of table.
} 
Table 2. Reactor Spent Fuel Discharge and Storage Activity for 1992 (Continued)

\begin{tabular}{|c|c|c|c|c|c|c|c|c|}
\hline Electric Utility Name & Reactor Name & $\begin{array}{c}\text { Reactor } \\
\text { Type }\end{array}$ & $\begin{array}{c}\text { Pool Site } \\
\text { ID }\end{array}$ & $\begin{array}{c}1991 \text { Assembly } \\
\text { Total }\end{array}$ & $\begin{array}{c}1992 \text { Assembly } \\
\text { Discharges }\end{array}$ & $\begin{array}{l}1992 \text { Assembly } \\
\text { Reinsertions }\end{array}$ & $\begin{array}{l}\text { Other (see } \\
\text { footnotes) }\end{array}$ & $\begin{array}{l}1992 \text { Assembly } \\
\text { Total }\end{array}$ \\
\hline TU Electric............. & $\begin{array}{l}\text { Comanche Peak } 1 \\
\text { Comanche Peak } 2\end{array}$ & $\begin{array}{l}\text { PWR } \\
\text { PWR }\end{array}$ & 4901 & $\begin{array}{r}56 \\
0\end{array}$ & 64 & -3 & & $\begin{array}{r}117 \\
0\end{array}$ \\
\hline Toledo Edison Company ..... & Davis-Besse & PWR & 5001 & 392 & & & & 392 \\
\hline Union Electric Company...... & Callaway & PWR & 5101 & 364 & 101 & -13 & & 452 \\
\hline $\begin{array}{l}\text { Vermont Yankee Nuclear } \\
\text { Power Corporation ......... }\end{array}$ & Vermont Yankee & BWR & 6001 & 1,722 & 128 & & & 1,850 \\
\hline Virginia Power. ............ & $\begin{array}{l}\text { North Anna } 1 \\
\text { North Anna } 2 \\
\text { Surry } 1 \\
\text { Surry } 2 \\
\text { Dry Storage }\end{array}$ & $\begin{array}{l}\text { PWR } \\
\text { PWR } \\
\text { PWR } \\
\text { PWR } \\
\text { PWR }\end{array}$ & $\begin{array}{l}5201 \\
5203 \\
52030\end{array}$ & $\begin{array}{l}933 \\
837 \\
304\end{array}$ & $\begin{array}{l}75 \\
65\end{array}$ & $\begin{array}{l}-9 \\
-2\end{array}$ & $\begin{array}{r}9.6 \\
\text { h- } 63 \\
63\end{array}$ & $\begin{array}{l}993 \\
837 \\
367\end{array}$ \\
\hline $\begin{array}{l}\text { Washington Public Power } \\
\text { Supply System .......... }\end{array}$ & Washington Nuclear 2 & BWR & 5302 & 836 & 76 & & & 912 \\
\hline $\begin{array}{l}\text { Wisconsin Electric Power } \\
\text { Company } \ldots \ldots \ldots \ldots \ldots \ldots\end{array}$ & $\begin{array}{l}\text { Point Beach } 1 \\
\text { Point Beach } 2\end{array}$ & $\begin{array}{l}\text { PWR } \\
\text { PWR }\end{array}$ & 5401 & 1,137 & $\begin{array}{l}29 \\
29\end{array}$ & $\begin{array}{l}-1 \\
-1\end{array}$ & & 1,193 \\
\hline $\begin{array}{l}\text { Wisconsin Public Service } \\
\text { Corporation } \ldots \ldots \ldots \ldots \ldots\end{array}$ & Kewaunee & PWR & 5501 & 584 & 57 & -25 & & 616 \\
\hline $\begin{array}{l}\text { Yankee Atomic Electric } \\
\text { Company .............. }\end{array}$ & Yankee Rowe & PWR & 5601 & 533 & & & & 533 \\
\hline $\begin{array}{l}\text { Total BWR. . . . } \ldots \ldots \ldots \ldots \\
\text { Total PWR. . . . . } \ldots \ldots \ldots \ldots\end{array}$ & $\ldots \ldots \ldots \ldots \ldots \ldots \ldots \ldots \ldots$ & $\cdots \cdots$ & $\cdots \cdots$ & $\begin{array}{l}45,714 \\
34,438\end{array}$ & $\begin{array}{l}4,028 \\
3,754\end{array}$ & $\begin{array}{r}-96 \\
-224\end{array}$ & $\begin{array}{r}-1 \\
-22\end{array}$ & $\begin{array}{l}49,645 \\
37,946\end{array}$ \\
\hline TOTAL (Nuclear Power Plant & Sites Only)......... & $\cdots \cdots$ & $\cdots \cdots$ & 80,152 & 7,782 & -320 & -23 & 87,591 \\
\hline Storage Facilities (From Tab) & (e 14)............. & $\cdots$ & $\cdots \cdots$ & 3,448 & - & - & $\cdots$ & 3,448 \\
\hline TOTAL (Including Storage Fa & cilittes)...$\ldots \ldots \ldots$ & $\cdots$ & - $\ldots \cdot$ & 83,600 & 7,782 & -320 & -23 & 91,039 \\
\hline
\end{tabular}

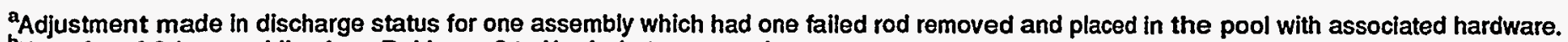

Transfer of $\mathbf{8 4}$ assemblies from Robinson 2 to Harris 1 storage pools.

TTransfer of 168 assemblies from Oconee storage pools to Oconee dry storage.

Total of 17 assemblies from Vogtle 1 reinserted incore at Vogtle 2.

-Transfer of 49 assemblies from San Onofre 1 to San Onofre 3 storage pool.

Total of 16 assemblies from Sequoyah 1 reinserted incore at Sequoyah 2.

9Total of 6 assemblies from North Anna 1 reinserted incore at North Anna 2.

hTransfer of 63 assemblles from Surry 1 to Surry dry storage.

PWR = Pressurized-water reactor; BWR = Boiling-water reactor.

$\overline{-}=$ Not applicable.

Source: Energy Information Administration, Form RW-859, "Nuclear Fuel Data" (1992). 
Table 3. Temporarlly Discharged Assemblies

\begin{tabular}{|c|c|c|c|c|c|c|}
\hline Electric UtIllity Name & Reactor Name & $\begin{array}{c}\text { Reactor } \\
\text { Type }\end{array}$ & $\begin{array}{l}\text { Pool } \\
\text { Slite } \\
\text { ID }\end{array}$ & $\begin{array}{c}\text { Temporarily } \\
\text { Dlscharged } \\
\text { Assemblles } \\
\text { Through } \\
1992\end{array}$ & $\begin{array}{l}\text { Tomporarlly } \\
\text { Discharged } \\
\text { Assemblles } \\
\text { Through } \\
1991\end{array}$ & $\begin{array}{l}\text { Increase or } \\
\text { Reduotlon In } \\
\text { Temporarlly } \\
\text { Dlscharged } \\
\text { Assemblies }\end{array}$ \\
\hline $\begin{array}{l}\text { Arizona Publle Service } \\
\text { Company ........... }\end{array}$ & $\begin{array}{l}\text { Palo Verde } 1 \\
\text { Palo Verde } 3\end{array}$ & $\begin{array}{l}\text { PWR } \\
\text { PWR }\end{array}$ & $\begin{array}{l}0301 \\
0303\end{array}$ & $\begin{array}{l}0 \\
1\end{array}$ & $\begin{array}{r}9 \\
25\end{array}$ & $\begin{array}{r}-8 \\
-24\end{array}$ \\
\hline $\begin{array}{l}\text { Carollna Power and Light } \\
\text { Company .............. }\end{array}$ & Brunswick 1 & BWR & 0701 & 1 & 1 & 0 \\
\hline $\begin{array}{l}\text { Cleveland Electric } \\
\text { Illuminating Company.......... }\end{array}$ & Perry 1 & BWR & 0901 & 4 & 0 & 4 \\
\hline $\begin{array}{l}\text { Consolldated Edison } \\
\text { Company of New York ......... }\end{array}$ & Indlan Point 2 & PWR & 1102 & 8 & 8 & 0 \\
\hline $\begin{array}{c}\text { Consumers Power } \\
\text { Company ......... }\end{array}$ & Pallsades & PWR & 1204 & 1 & 1 & 0 \\
\hline Duquesne Light Company ....... . & $\begin{array}{l}\text { Beaver Valley } 1 \\
\text { Beaver Valley } 2\end{array}$ & $\begin{array}{l}\text { PWR } \\
\text { PWR }\end{array}$ & $\begin{array}{l}1601 \\
1602\end{array}$ & $\begin{array}{r}14 \\
5\end{array}$ & $\begin{array}{r}14 \\
2\end{array}$ & $\begin{array}{l}0 \\
3\end{array}$ \\
\hline Florida Power Corporation....... & Crystal River 3 & PWR & 1701 & 5 & 17 & -12 \\
\hline GPU Nuclear Corporation ........ & Three Mile Island 1 & PWR & 1901 & 8 & 8 & 0 \\
\hline $\begin{array}{l}\text { Houston Lighting and } \\
\text { Power Company .... }\end{array}$ & South Texas 1 & PWR & 2201 & 7 & 0 & 7 \\
\hline $\begin{array}{l}\text { Maine Yankee Atomic } \\
\text { Power Company ............. }\end{array}$ & Maine Yankee & PWR & 2801 & 23 & 12 & 11 \\
\hline $\begin{array}{l}\text { New York Power } \\
\text { Authority................ }\end{array}$ & Indlan Point 3 & PWR & 3902 & 2 & 2 & 0 \\
\hline $\begin{array}{l}\text { Northeast Utillties } \\
\text { Service Company ........... }\end{array}$ & $\begin{array}{l}\text { Millstone } 2 \\
\text { Haddam Neck }\end{array}$ & $\begin{array}{l}\text { PWR } \\
\text { PWR }\end{array}$ & $\begin{array}{l}3202 \\
5701\end{array}$ & $\begin{array}{l}0 \\
1\end{array}$ & $\begin{array}{l}1 \\
1\end{array}$ & $\begin{array}{r}-1 \\
0\end{array}$ \\
\hline $\begin{array}{l}\text { Northern States Power } \\
\text { Company .................. }\end{array}$ & Prairle Island 1 & PWR & 3302 & 0 & 6 & -6 \\
\hline $\begin{array}{l}\text { Publle Service Electric } \\
\text { and Gas Company .... }\end{array}$ & $\begin{array}{l}\text { Salem } 1 \\
\text { Salem } 2\end{array}$ & $\begin{array}{l}\text { PWR } \\
\text { PWR }\end{array}$ & $\begin{array}{l}4202 \\
4203\end{array}$ & $\begin{array}{r}25 \\
4\end{array}$ & $\begin{array}{r}25 \\
0\end{array}$ & $\begin{array}{l}0 \\
4\end{array}$ \\
\hline TU Electric................... & Comanche Peak 1 & PWR & 4901 & 0 & 3 & -3 \\
\hline Toledo Edison Company ......... & Davis-Besse & PWR & 5001 & 6 & 6 & 0 \\
\hline
\end{tabular}

See footnotes at end of table. 
Table 3. Temporarily Discharged Assemblles (Continued)

\begin{tabular}{|c|c|c|c|c|c|c|}
\hline Electrlc Utility Name & Reactor Name & $\begin{array}{c}\text { Reactor } \\
\text { Type }\end{array}$ & $\begin{array}{l}\text { Pool } \\
\text { Site } \\
\text { ID }\end{array}$ & $\begin{array}{c}\text { Temporarlly } \\
\text { Dlscharged } \\
\text { Assemblles } \\
\text { Through } \\
1992\end{array}$ & $\begin{array}{c}\text { Temporarlly } \\
\text { Discharged } \\
\text { Assemblles } \\
\text { Through } \\
1991\end{array}$ & $\begin{array}{l}\text { Increase or } \\
\text { Reductlon in } \\
\text { Temporarlly } \\
\text { Discharged } \\
\text { Assemblles }\end{array}$ \\
\hline Virginia Power ............. & $\begin{array}{l}\text { North Anna } 1 \\
\text { Surry } 1\end{array}$ & $\begin{array}{l}\text { PWR } \\
\text { PWR }\end{array}$ & $\begin{array}{l}5201 \\
5203\end{array}$ & $\begin{array}{r}34 \\
7\end{array}$ & $\begin{array}{l}35 \\
21\end{array}$ & $\begin{array}{r}-1 \\
-14\end{array}$ \\
\hline $\begin{array}{l}\text { Wisconsin Public Service } \\
\text { Corporation ............... }\end{array}$ & Kewaunee & PWR & 5501 & 12 & 16 & -4 \\
\hline TOTAL .................. & & & & 168 & 213 & -45 \\
\hline
\end{tabular}

PWR = Pressurized-water reactor; BWR = Boiling-water reactor.

Note: Changes in number of temporarily discharged assemblies are due to discharge of additional temporarily discharged assemblies, reinsertion of previously discharged assemblies, and/or change in status of previously discharged assemblies.

Source: Energy Information Administration, Form RW-859, "Nuclear Fuel Data" (1992). 
Table 4. Nuclear Power Plant Data as of December 31, 1992

\begin{tabular}{|c|c|c|c|c|c|c|c|c|c|c|}
\hline Electric Utility Name & Reactor Name & State & $\begin{array}{l}\text { Reactor } \\
\text { Type }\end{array}$ & Vendor $^{\mathbf{a}}$ & $\begin{array}{c}\text { Net } \\
\text { Summer } \\
\text { Capabillty } \\
\text { (net MWe) }\end{array}$ & $\begin{array}{l}\text { Core Size } \\
\text { (number of } \\
\text { assemblies) }\end{array}$ & $\begin{array}{l}\text { Startup } \\
\text { (year) }\end{array}$ & $\begin{array}{c}\text { License } \\
\text { Expiration } \\
\text { (year) }^{d}\end{array}$ & $\begin{array}{c}\text { Loss of } \\
\text { Ability } \\
\text { to Operate } \\
\text { (year) }^{\circ}\end{array}$ & $\begin{array}{c}\text { Actual or } \\
\text { Projected } \\
\text { Retiremen } \\
\text { (year) }\end{array}$ \\
\hline Alabama Power Company .. & $\begin{array}{l}\text { Farley } 1 \\
\text { Farley } 2\end{array}$ & $\begin{array}{l}A L \\
A L\end{array}$ & $\begin{array}{l}\text { PWR } \\
\text { PWR }\end{array}$ & $\begin{array}{l}\text { WE } \\
W E\end{array}$ & $\begin{array}{l}815 \\
826\end{array}$ & $\begin{array}{l}157 \\
157\end{array}$ & $\begin{array}{l}1977 \\
1981\end{array}$ & $\begin{array}{l}2017 \\
2021\end{array}$ & $\begin{array}{l}2012 \\
2014\end{array}$ & $\begin{array}{l}2017 \\
2021\end{array}$ \\
\hline $\begin{array}{l}\text { Arizona Public Service } \\
\text { Company } . . \ldots \ldots \ldots \ldots \ldots\end{array}$ & $\begin{array}{l}\text { Palo Verde } 1 \\
\text { Palo Verde } 2 \\
\text { Palo Verde } 3\end{array}$ & $\begin{array}{l}A Z \\
A Z \\
A Z\end{array}$ & $\begin{array}{l}\text { PWR } \\
\text { PWR } \\
\text { PWR }\end{array}$ & $\begin{array}{l}C E \\
C E \\
C E\end{array}$ & $\begin{array}{l}1,270 \\
1,270 \\
1,270\end{array}$ & $\begin{array}{l}241 \\
241 \\
241\end{array}$ & $\begin{array}{l}1985 \\
1986 \\
1987\end{array}$ & $\begin{array}{l}2024 \\
2025 \\
2027\end{array}$ & $\begin{array}{l}2005 \\
2005 \\
2006\end{array}$ & $\begin{array}{l}2024 \\
2025 \\
2027\end{array}$ \\
\hline $\begin{array}{l}\text { Arkansas Power and } \\
\text { Light Company.......... }\end{array}$ & $\begin{array}{l}\text { Arkansas Nuclear } 1 \\
\text { Arkansas Nuclear } 2\end{array}$ & $\begin{array}{l}A R \\
A R\end{array}$ & $\begin{array}{l}\text { PWR } \\
\text { PWR }\end{array}$ & $\begin{array}{c}B \& W \\
C E\end{array}$ & $\begin{array}{l}836 \\
858\end{array}$ & $\begin{array}{l}177 \\
177\end{array}$ & $\begin{array}{l}1974 \\
1978\end{array}$ & $\begin{array}{l}2014 \\
2018\end{array}$ & $\begin{array}{l}1996 \\
1997\end{array}$ & $\begin{array}{l}2014 \\
2018\end{array}$ \\
\hline $\begin{array}{l}\text { Baltimore Gas and } \\
\text { Electric Company........ }\end{array}$ & $\begin{array}{l}\text { Caivert Cliffs } 1 \\
\text { Calvert Cliffs } 2\end{array}$ & $\begin{array}{l}\text { MD } \\
\text { MD }\end{array}$ & $\begin{array}{l}\text { PWR } \\
\text { PWR }\end{array}$ & $\begin{array}{l}C E \\
C E\end{array}$ & $\begin{array}{l}825 \\
825\end{array}$ & $\begin{array}{l}217 \\
217\end{array}$ & $\begin{array}{l}1975 \\
1976\end{array}$ & $\begin{array}{l}2014 \\
2016\end{array}$ & $\begin{array}{l}1994 \\
1993\end{array}$ & $\begin{array}{l}2016 \\
2016\end{array}$ \\
\hline Boston Edison Company ... & Pilgrim 1 & MA & BWR & GE & 665 & 580 & 1972 & 2012 & 2003 & 2012 \\
\hline $\begin{array}{l}\text { Carolina Power and Light } \\
\text { Company .............. }\end{array}$ & $\begin{array}{l}\text { Brunswick } 1 \\
\text { Brunswick } 2 \\
\text { Harris } 1 \\
\text { Robinson } 2\end{array}$ & $\begin{array}{l}\text { NC } \\
\text { NC } \\
\text { NC } \\
\text { SC }\end{array}$ & $\begin{array}{l}\text { BWR } \\
\text { BWR } \\
\text { PWR } \\
\text { PWR }\end{array}$ & $\begin{array}{l}\text { GE } \\
\text { GE } \\
\text { WE } \\
\text { WE }\end{array}$ & $\begin{array}{l}767 \\
754 \\
860 \\
683\end{array}$ & $\begin{array}{l}560 \\
560 \\
157 \\
157\end{array}$ & $\begin{array}{l}1976 \\
1975 \\
1987 \\
1970\end{array}$ & $\begin{array}{l}2016 \\
2014 \\
2026 \\
2010\end{array}$ & $\begin{array}{l}2002 \\
2001 \\
2018 \\
2002\end{array}$ & $\begin{array}{l}2016 \\
2014 \\
2026 \\
2010\end{array}$ \\
\hline $\begin{array}{l}\text { Cleveland Electric } \\
\text { Illuminating Company ..... }\end{array}$ & Perry 1 & $\mathrm{OH}$ & BWR & GE & 1,169 & 748 & 1986 & 2026 & 2009 & 2026 \\
\hline $\begin{array}{l}\text { Commonwealth Edison } \\
\text { Company } \ldots \ldots \ldots \ldots \ldots \text {. }\end{array}$ & $\begin{array}{l}\text { Braidwood 1 } \\
\text { Braldwood } 2 \\
\text { Byron 1 } \\
\text { Byron 2 } \\
\text { Dresden 1 } \\
\text { Dresden } 2 \\
\text { Dresden } 3 \\
\text { LaSalle County } 1 \\
\text { LaSalle County } 2 \\
\text { Quad Cities } 1 \\
\text { Quad Cities } 2 \\
\text { Zion 1 } \\
\text { Zion } 2\end{array}$ & $\begin{array}{l}\text { IL } \\
\text { IL } \\
\text { IL } \\
\text { IL } \\
\text { IL } \\
\text { IL } \\
\text { IL } \\
\text { IL } \\
\text { IL } \\
\text { IL } \\
\text { IL }\end{array}$ & $\begin{array}{l}\text { PWR } \\
\text { PWR } \\
\text { PWR } \\
\text { PWR } \\
\text { BWR } \\
\text { BWR } \\
\text { BWR } \\
\text { BWR } \\
\text { BWR } \\
\text { BWR } \\
\text { BWR } \\
\text { PWR } \\
\text { PWR }\end{array}$ & $\begin{array}{l}\text { WE } \\
\text { WE } \\
\text { WE } \\
\text { WE } \\
\text { GE } \\
\text { GE } \\
\text { GE } \\
\text { GE } \\
\text { GE } \\
\text { GE } \\
\text { GE } \\
\text { WE } \\
\text { WE }\end{array}$ & $\begin{array}{r}1,090 \\
1,090 \\
1,120 \\
1,120 \\
200 \\
772 \\
773 \\
1,048 \\
1,048 \\
769 \\
769 \\
1,040 \\
1,040\end{array}$ & $\begin{array}{l}193 \\
193 \\
193 \\
193 \\
464 \\
724 \\
724 \\
764 \\
764 \\
724 \\
724 \\
193 \\
193\end{array}$ & $\begin{array}{l}1987 \\
1988 \\
1985 \\
1987 \\
1960 \\
1970 \\
1971 \\
1982 \\
1984 \\
1972 \\
1972 \\
1973 \\
1973\end{array}$ & $\begin{array}{l}2026 \\
2027 \\
2024 \\
2026 \\
1996 \\
2006 \\
2011 \\
2022 \\
2023 \\
2012 \\
2012 \\
2013 \\
2013\end{array}$ & $\begin{array}{r}2012 \\
2013 \\
2011 \\
2011 \\
5 D \\
2000 \\
2001 \\
2013 \\
2015 \\
2007 \\
2006 \\
2006 \\
2006\end{array}$ & $\begin{array}{l}2028 \\
2028 \\
2025 \\
2027 \\
1984 \\
2010 \\
2013 \\
2024 \\
2024 \\
2013 \\
2013 \\
2013 \\
2014\end{array}$ \\
\hline
\end{tabular}

See footnotes at end of table. 
Table 4. Nuclear Power Plant Data as of December 31, 1992 (Continued)

\begin{tabular}{|c|c|c|c|c|c|c|c|c|c|c|}
\hline Electric Utility Name & Reactor Name & State & $\begin{array}{l}\text { Reactor } \\
\text { Type }\end{array}$ & Vendor $^{\mathrm{a}}$ & $\begin{array}{c}\text { Net } \\
\text { Summer } \\
\text { Capability } \\
\text { (net MWe) }\end{array}$ & $\begin{array}{l}\text { Core Size } \\
\text { (number of } \\
\text { assemblies) }\end{array}$ & $\underset{\text { (year) }}{\text { Startup }}$ & $\begin{array}{l}\text { Licenso } \\
\text { Expiration } \\
\text { (year) }^{d}\end{array}$ & $\begin{array}{c}\text { Loss of } \\
\text { Ability } \\
\text { to Operate } \\
\text { (year) }^{\circ}\end{array}$ & $\begin{array}{c}\text { Actual or } \\
\text { Projected } \\
\text { Retirement } \\
\text { (year) }\end{array}$ \\
\hline $\begin{array}{l}\text { Consolidated Edison } \\
\text { Company of New York .... }\end{array}$ & $\begin{array}{l}\text { Indian Point } 1 \\
\text { Indian Point } 2\end{array}$ & $\begin{array}{l}\text { NY } \\
\text { NY }\end{array}$ & $\begin{array}{l}\text { PWR } \\
\text { PWR }\end{array}$ & $\begin{array}{l}\text { B\&W } \\
\text { WE }\end{array}$ & $\begin{array}{l}265 \\
931\end{array}$ & $\begin{array}{l}120 \\
193\end{array}$ & $\begin{array}{l}1962 \\
1973\end{array}$ & $\begin{array}{l}1980 \\
2013\end{array}$ & $\begin{array}{r}R D \\
2003\end{array}$ & $\begin{array}{l}1980 \\
2013\end{array}$ \\
\hline $\begin{array}{l}\text { Consumers Power } \\
\text { Company } \ldots \ldots \ldots \ldots \ldots\end{array}$ & $\begin{array}{l}\text { Big Rock Point } \\
\text { Palisades }\end{array}$ & $\begin{array}{l}\mathrm{MI} \\
\mathrm{MI}\end{array}$ & $\begin{array}{l}\text { BWR } \\
\text { PWR }\end{array}$ & $\begin{array}{l}\mathrm{GE} \\
\mathrm{CE}\end{array}$ & $\begin{array}{r}67 \\
755\end{array}$ & $\begin{array}{r}84 \\
204\end{array}$ & $\begin{array}{l}1962 \\
1971\end{array}$ & $\begin{array}{l}2000 \\
2007\end{array}$ & $\begin{array}{l}1999 \\
2007\end{array}$ & $\begin{array}{l}2000 \\
2011\end{array}$ \\
\hline $\begin{array}{l}\text { Dairyland Power } \\
\text { Cooperative } \ldots \ldots \ldots \ldots\end{array}$ & LaCrosse & WI & BWR & $A C$ & 50 & 72 & 1968 & 2031 & RD & 1987 \\
\hline Detroit Edison Company.... & Enrico Fermi 2 & Mi & BWR & GE & 1,060 & 764 & 1985 & 2025 & 2006 & 2025 \\
\hline Duke Power Company ..... & $\begin{array}{l}\text { Catawba } 1 \\
\text { Catawba } 2 \\
\text { McGuire } 1 \\
\text { McGuire } 2 \\
\text { Oconee } 1 \\
\text { Oconee } 2 \\
\text { Oconee } 3\end{array}$ & $\begin{array}{l}\text { SC } \\
\text { SC } \\
\text { NC } \\
\text { NC } \\
\text { SC } \\
\text { SC } \\
\text { SC }\end{array}$ & $\begin{array}{l}\text { PWR } \\
\text { PWR } \\
\text { PWR } \\
\text { PWR } \\
\text { PWR } \\
\text { PWR } \\
\text { PWR }\end{array}$ & $\begin{array}{l}\text { WE } \\
\text { WE } \\
\text { WE } \\
\text { WE } \\
\text { B\&W } \\
\text { B\&W } \\
\text { B\&W }\end{array}$ & $\begin{array}{r}1,129 \\
1,129 \\
1,129 \\
1,129 \\
846 \\
846 \\
846\end{array}$ & $\begin{array}{l}193 \\
193 \\
193 \\
193 \\
177 \\
177 \\
177\end{array}$ & $\begin{array}{l}1985 \\
1986 \\
1981 \\
1983 \\
1973 \\
1973 \\
1974\end{array}$ & $\begin{array}{l}2024 \\
2026 \\
2021 \\
2023 \\
2013 \\
2013 \\
2014\end{array}$ & $\begin{array}{l}2011 \\
2011 \\
2007 \\
2006 \\
2010 \\
2010 \\
2011\end{array}$ & $\begin{array}{l}2025 \\
2026 \\
2021 \\
2023 \\
2013 \\
2013 \\
2014\end{array}$ \\
\hline Duquesne Light Company .. & $\begin{array}{l}\text { Beaver Valley } 1 \\
\text { Beaver Valley } 2\end{array}$ & $\begin{array}{l}\text { PA } \\
\text { PA }\end{array}$ & $\begin{array}{l}\text { PWR } \\
\text { PWR }\end{array}$ & $\begin{array}{l}\text { WE } \\
\text { WE }\end{array}$ & $\begin{array}{l}810 \\
833\end{array}$ & $\begin{array}{l}157 \\
157\end{array}$ & $\begin{array}{l}1976 \\
1987\end{array}$ & $\begin{array}{l}2016 \\
2027\end{array}$ & $\begin{array}{l}2013 \\
2008\end{array}$ & $\begin{array}{l}2016 \\
2026\end{array}$ \\
\hline Florida Power Corporation. . & Crystal River 3 & FL & PWR & B\&W & 820 & 177 & 1977 & 2016 & 2010 & 2016 \\
\hline $\begin{array}{l}\text { Florida Power and Light } \\
\text { Company ............. }\end{array}$ & $\begin{array}{l}\text { St. Lucie } 1 \\
\text { St. Lucie } 2 \\
\text { Turkey Point } 3 \\
\text { Turkey Point } 4\end{array}$ & $\begin{array}{l}\text { FL } \\
F L \\
F L \\
F L\end{array}$ & $\begin{array}{l}\text { PWR } \\
\text { PWR } \\
\text { PWR } \\
\text { PWR }\end{array}$ & $\begin{array}{l}\text { CE } \\
\text { CE } \\
\text { WE } \\
\text { WE }\end{array}$ & $\begin{array}{l}839 \\
839 \\
666 \\
666\end{array}$ & $\begin{array}{l}217 \\
217 \\
157 \\
157\end{array}$ & $\begin{array}{l}1976 \\
1983 \\
1972 \\
1973\end{array}$ & $\begin{array}{l}2016 \\
2023 \\
2007 \\
2007\end{array}$ & $\begin{array}{l}2016 \\
2023 \\
2007 \\
2007\end{array}$ & $\begin{array}{l}2016 \\
2023 \\
2007 \\
2007\end{array}$ \\
\hline GPU Nuclear Corporation .. & $\begin{array}{l}\text { Three Mile island } 1 \\
\text { Oyster Creek }\end{array}$ & $\begin{array}{l}\text { PA } \\
\text { NJ }\end{array}$ & $\begin{array}{l}\text { PWR } \\
\text { BWR }\end{array}$ & $\begin{array}{l}\text { B\&W } \\
\text { GE }\end{array}$ & $\begin{array}{l}808 \\
610\end{array}$ & $\begin{array}{l}177 \\
560\end{array}$ & $\begin{array}{l}1974 \\
1969\end{array}$ & $\begin{array}{l}2014 \\
2009\end{array}$ & $\begin{array}{l}2014 \\
2000\end{array}$ & $\begin{array}{l}2014 \\
2009\end{array}$ \\
\hline Georgia Power Company... & $\begin{array}{l}\text { Hatch } 1 \\
\text { Hatch } 2 \\
\text { Vogtle } 1 \\
\text { Vogtle } 2\end{array}$ & $\begin{array}{l}\text { GA } \\
\text { GA } \\
\text { GA } \\
\text { GA }\end{array}$ & $\begin{array}{l}\text { BWR } \\
\text { BWR } \\
\text { PWR } \\
\text { PWR }\end{array}$ & $\begin{array}{l}\text { GE } \\
\text { GE } \\
\text { WE } \\
\text { WE }\end{array}$ & $\begin{array}{r}748 \\
770 \\
1,110 \\
1,114\end{array}$ & $\begin{array}{l}560 \\
560 \\
193 \\
193\end{array}$ & $\begin{array}{l}1974 \\
1978 \\
1987 \\
1989\end{array}$ & $\begin{array}{l}2014 \\
2018 \\
2027 \\
2029\end{array}$ & $\begin{array}{l}2003 \\
2004 \\
2011 \\
2010\end{array}$ & $\begin{array}{l}2014 \\
2018 \\
2027 \\
2029\end{array}$ \\
\hline $\begin{array}{l}\text { Gulf States Utilities } \\
\text { Company } \ldots \ldots \ldots \ldots \ldots \ldots\end{array}$ & River Bend 1 & LA & BWR & GE & 931 & 624 & 1985 & 2025 & 2003 & 2025 \\
\hline
\end{tabular}

See footnotes at end of table. 
Table 4. Nuclear Power Plant Data as of December 31, 1992 (Continued)

\begin{tabular}{|c|c|c|c|c|c|c|c|c|c|c|}
\hline Electric Utility Name & Reactor Name & State & $\begin{array}{l}\text { Reactor } \\
\text { Type }\end{array}$ & Vendor $^{a}$ & $\begin{array}{c}\text { Net } \\
\text { Summer } \\
\text { Capability } \\
\text { (net MWe) }\end{array}$ & $\begin{array}{l}\text { Core Size } \\
\text { (number of } \\
\text { assemblies) }\end{array}$ & $\underset{\text { (year) }^{\text {Startup }}}{ }$ & $\begin{array}{l}\text { License } \\
\text { Expiration } \\
\text { (year) }^{\text {(yea }}\end{array}$ & $\begin{array}{l}\text { Loss of } \\
\text { Ability } \\
\text { to Operate } \\
\text { (year) }^{\circ}\end{array}$ & $\begin{array}{l}\text { Actual or } \\
\text { Projected } \\
\text { Retirement } \\
\text { (year) }\end{array}$ \\
\hline $\begin{array}{l}\text { Houston Lighting and } \\
\text { Power Company.......... }\end{array}$ & $\begin{array}{l}\text { South Texas } 1 \\
\text { South Texas } 2\end{array}$ & $\begin{array}{l}T X \\
T X\end{array}$ & $\begin{array}{l}\text { PWR } \\
\text { PWR }\end{array}$ & $\begin{array}{l}\text { WE } \\
\text { WE }\end{array}$ & $\begin{array}{l}1,241 \\
1,241\end{array}$ & $\begin{array}{l}193 \\
193\end{array}$ & $\begin{array}{l}1988 \\
1989\end{array}$ & $\begin{array}{l}2027 \\
2028\end{array}$ & $\begin{array}{l}2027 \\
2028\end{array}$ & $\begin{array}{l}2027 \\
2028\end{array}$ \\
\hline Illinois Power Company .... & Clinton 1 & IL & BWR & GE & 930 & 624 & 1987 & 2026 & 2008 & 2026 \\
\hline $\begin{array}{l}\text { Indiana Michigan } \\
\text { Power Company......... }\end{array}$ & $\begin{array}{l}\text { Cook } 1 \\
\text { Cook } 2\end{array}$ & $\begin{array}{l}\text { MI } \\
\text { MI }\end{array}$ & $\begin{array}{l}\text { PWR } \\
\text { PWR }\end{array}$ & $\begin{array}{l}\text { WE } \\
\text { WE }\end{array}$ & $\begin{array}{l}1,000 \\
1,060\end{array}$ & $\begin{array}{l}193 \\
193\end{array}$ & $\begin{array}{l}1975 \\
1978\end{array}$ & $\begin{array}{l}2014 \\
2017\end{array}$ & $\begin{array}{l}2011 \\
2011\end{array}$ & $\begin{array}{l}2014 \\
2017\end{array}$ \\
\hline $\begin{array}{l}\text { lowa Electric Light and } \\
\text { Power Company.......... }\end{array}$ & Duane Arnold & IA & BWR & GE & 515 & 368 & 1974 & 2014 & 1999 & 2014 \\
\hline $\begin{array}{l}\text { Kansas Gas and Electric } \\
\text { Company } \ldots \ldots \ldots \ldots \ldots \ldots\end{array}$ & Wolf Creek 1 & KS & PWR & WE & 1,159 & 193 & 1985 & 2025 & 2011 & 2025 \\
\hline $\begin{array}{l}\text { Long island Lighting } \\
\text { Company } \ldots \ldots \ldots \ldots \ldots\end{array}$ & Shoreham & NY & BWR & GE & 849 & 560 & 1986 & 2013 & SD & 1987 \\
\hline $\begin{array}{l}\text { Louisiana Power and } \\
\text { Light Company........... }\end{array}$ & Waterford 3 & LA & PWR & CE & 1,075 & 217 & 1985 & 2024 & 2000 & 2024 \\
\hline $\begin{array}{l}\text { Maine Yankee Atomic } \\
\text { Power Company......... }\end{array}$ & Maine Yankee & ME & PWR & CE & 870 & 217 & 1972 & 2008 & 1999 & 2008 \\
\hline $\begin{array}{l}\text { Nebraska Public Power } \\
\text { District................. }\end{array}$ & Cooper Station & NE & BWR & GE & 778 & 548 & 1974 & 2014 & 2006 & 2014 \\
\hline $\begin{array}{l}\text { New York Power } \\
\text { Authority............... }\end{array}$ & $\begin{array}{l}\text { Fitzpatrick } \\
\text { Indian Point } 3\end{array}$ & $\begin{array}{l}\text { NY } \\
\text { NY }\end{array}$ & $\begin{array}{l}\text { BWR } \\
\text { PWR }\end{array}$ & $\begin{array}{l}\text { GE } \\
\text { WE }\end{array}$ & $\begin{array}{l}800 \\
980\end{array}$ & $\begin{array}{l}560 \\
193\end{array}$ & $\begin{array}{l}1975 \\
1976\end{array}$ & $\begin{array}{l}2014 \\
2015\end{array}$ & $\begin{array}{l}2003 \\
2006\end{array}$ & $\begin{array}{l}2014 \\
2015\end{array}$ \\
\hline $\begin{array}{l}\text { Niagara Mohawk Power } \\
\text { Corporation.............. }\end{array}$ & $\begin{array}{l}\text { Nine Mile Point } 1 \\
\text { Nine Mile Point } 2\end{array}$ & $\begin{array}{l}\text { NY } \\
\text { NY }\end{array}$ & $\begin{array}{l}\text { BWR } \\
\text { BWR }\end{array}$ & $\begin{array}{l}\mathrm{GE} \\
\mathrm{GE}\end{array}$ & $\begin{array}{r}605 \\
1,045\end{array}$ & $\begin{array}{l}532 \\
764\end{array}$ & $\begin{array}{l}1969 \\
1987\end{array}$ & $\begin{array}{l}2009 \\
2026\end{array}$ & $\begin{array}{l}2005 \\
2017\end{array}$ & $\begin{array}{l}2008 \\
2026\end{array}$ \\
\hline $\begin{array}{l}\text { North Atlantic Energy } \\
\text { Service Corporation....... }\end{array}$ & Seabrook & $\mathrm{NH}$ & PWR & WE & 1,150 & 193 & 1990 & 2026 & 2012 & 2030 \\
\hline $\begin{array}{l}\text { Northeast Utilities } \\
\text { Service Company ........ }\end{array}$ & $\begin{array}{l}\text { Millstone } 1 \\
\text { Millstone } 2 \\
\text { Millstone } 3 \\
\text { Haddam Neck }\end{array}$ & $\begin{array}{l}\text { CT } \\
\text { CT } \\
\text { CT } \\
\text { CT }\end{array}$ & $\begin{array}{l}\text { BWR } \\
\text { PWR } \\
\text { PWR } \\
\text { PWR }\end{array}$ & $\begin{array}{l}\text { GE } \\
\text { CE } \\
\text { WE } \\
\text { WE }\end{array}$ & $\begin{array}{r}652 \\
863 \\
1,137 \\
560\end{array}$ & $\begin{array}{l}580 \\
217 \\
193 \\
157\end{array}$ & $\begin{array}{l}1970 \\
1975 \\
1986 \\
1967\end{array}$ & $\begin{array}{l}2010 \\
2015 \\
2025 \\
2007\end{array}$ & $\begin{array}{l}2004 \\
2000 \\
2002 \\
2002\end{array}$ & $\begin{array}{l}2010 \\
2015 \\
2025 \\
2007\end{array}$ \\
\hline
\end{tabular}

See footnotes at end of table. 
Table 4. Nuclear Power Plant Data as of December 31, 1992 (Continued)

\begin{tabular}{|c|c|c|c|c|c|c|c|c|c|c|}
\hline Electric Utility Name & Reactor Name & State & $\begin{array}{c}\text { Reactor } \\
\text { Type }\end{array}$ & Vendor ${ }^{2}$ & $\begin{array}{c}\text { Net } \\
\text { Summer } \\
\text { Capability } \\
\text { (net MWe) }\end{array}$ & $\begin{array}{c}\text { Core Size } \\
\text { (number of } \\
\text { assemblies) }\end{array}$ & $\begin{array}{c}\text { Startup } \\
\text { (year) }^{E}\end{array}$ & $\begin{array}{c}\text { License } \\
\text { Expiration } \\
\text { (year) }^{\mathrm{g}}\end{array}$ & $\begin{array}{c}\text { Loss of } \\
\text { Ability } \\
\text { to Operate } \\
\text { (year) }^{\circ}\end{array}$ & $\begin{array}{c}\text { Actual or } \\
\text { Projected } \\
\text { Retirement } \\
\text { (year) }\end{array}$ \\
\hline $\begin{array}{l}\text { Northern States Power } \\
\text { Company } \ldots \ldots \ldots \ldots \ldots \ldots\end{array}$ & $\begin{array}{l}\text { Monticello } \\
\text { Prairie Island } 1 \\
\text { Prairie Island } 2\end{array}$ & $\begin{array}{l}M N \\
M N \\
M N\end{array}$ & $\begin{array}{l}\text { BWR } \\
\text { PWR } \\
\text { PWR }\end{array}$ & $\begin{array}{l}\text { GE } \\
\text { WE } \\
\text { WE }\end{array}$ & $\begin{array}{l}532 \\
510 \\
505\end{array}$ & $\begin{array}{l}484 \\
121 \\
121\end{array}$ & $\begin{array}{l}1971 \\
1973 \\
1974\end{array}$ & $\begin{array}{l}2010 \\
2013 \\
2014\end{array}$ & $\begin{array}{l}2004 \\
1995 \\
1995\end{array}$ & $\begin{array}{l}2010 \\
2013 \\
2014\end{array}$ \\
\hline $\begin{array}{l}\text { Omaha Public Power } \\
\text { District ................ }\end{array}$ & Fort Calhoun & NE & PWR & $\mathrm{CE}$ & 476 & 133 & 1973 & 2008 & 2002 & 2008 \\
\hline $\begin{array}{l}\text { Pacific Gas and Electric } \\
\text { Company } \ldots \ldots \ldots \ldots \ldots\end{array}$ & $\begin{array}{l}\text { Diablo Canyon } 1 \\
\text { Diablo Canyon } 2 \\
\text { Humboldt Bay }\end{array}$ & $\begin{array}{l}\text { CA } \\
\text { CA } \\
\text { CA }\end{array}$ & $\begin{array}{l}\text { PWR } \\
\text { PWR } \\
\text { BWR }\end{array}$ & $\begin{array}{l}\text { WE } \\
\text { WE } \\
\text { GE }\end{array}$ & $\begin{array}{r}1,073 \\
1,087 \\
65\end{array}$ & $\begin{array}{l}193 \\
193 \\
184\end{array}$ & $\begin{array}{l}1984 \\
1985 \\
1963\end{array}$ & $\begin{array}{l}2008 \\
2010 \\
2015\end{array}$ & $\begin{array}{r}2004 \\
2011 \\
\text { SD }\end{array}$ & $\begin{array}{l}2021 \\
2026 \\
1976\end{array}$ \\
\hline $\begin{array}{l}\text { Pennsylvania Power and } \\
\text { Light Company........... }\end{array}$ & $\begin{array}{l}\text { Susquehanna } 1 \\
\text { Susquehanna } 2\end{array}$ & $\begin{array}{l}\text { PA } \\
\text { PA }\end{array}$ & $\begin{array}{l}\text { BWR } \\
\text { BWR }\end{array}$ & $\begin{array}{l}\mathrm{GE} \\
\mathrm{GE}\end{array}$ & $\begin{array}{l}1,040 \\
1,044\end{array}$ & $\begin{array}{l}764 \\
764\end{array}$ & $\begin{array}{l}1982 \\
1984\end{array}$ & $\begin{array}{l}2022 \\
2024\end{array}$ & $\begin{array}{l}2001 \\
2001\end{array}$ & $\begin{array}{l}2022 \\
2024\end{array}$ \\
\hline $\begin{array}{l}\text { Philadelphia Electric } \\
\text { Company } \ldots \ldots \ldots \ldots \ldots\end{array}$ & $\begin{array}{l}\text { Limerick } 1 \\
\text { Limerick } 2 \\
\text { Peach Bottom } 2 \\
\text { Peach Bottom } 3\end{array}$ & $\begin{array}{l}\text { PA } \\
\text { PA } \\
\text { PA } \\
\text { PA }\end{array}$ & $\begin{array}{l}\text { BWR } \\
\text { BWR } \\
\text { BWR } \\
\text { BWR }\end{array}$ & $\begin{array}{l}\mathrm{GE} \\
\mathrm{GE} \\
\mathrm{GE} \\
\mathrm{GE}\end{array}$ & $\begin{array}{l}1,055 \\
1,055 \\
1,051 \\
1,035\end{array}$ & $\begin{array}{l}764 \\
764 \\
764 \\
764\end{array}$ & $\begin{array}{l}1985 \\
1989 \\
1974 \\
1974\end{array}$ & $\begin{array}{l}2024 \\
2029 \\
2008 \\
2008\end{array}$ & $\begin{array}{l}2000 \\
1996 \\
1998 \\
1999\end{array}$ & $\begin{array}{l}2024 \\
2029 \\
2008 \\
2008\end{array}$ \\
\hline $\begin{array}{l}\text { Portland General Electric } \\
\text { Company } \ldots \ldots \ldots \ldots \ldots \ldots\end{array}$ & Trojan & OR & PWR & WE & 1,104 & 193 & 1975 & 2011 & SD & 1992 \\
\hline $\begin{array}{l}\text { Public Service Electric } \\
\text { and Gas Company........ }\end{array}$ & $\begin{array}{l}\text { Hope Creek } \\
\text { Salem } 1 \\
\text { Salem } 2\end{array}$ & $\begin{array}{l}\text { NJ } \\
\text { NJ } \\
\text { NJ }\end{array}$ & $\begin{array}{l}\text { BWR } \\
\text { PWR } \\
\text { PWR }\end{array}$ & $\begin{array}{l}\text { GE } \\
W E \\
W E\end{array}$ & $\begin{array}{l}1,031 \\
1,106 \\
1,106\end{array}$ & $\begin{array}{l}764 \\
193 \\
193\end{array}$ & $\begin{array}{l}1986 \\
1976 \\
1981\end{array}$ & $\begin{array}{l}2026 \\
2016 \\
2020\end{array}$ & $\begin{array}{l}2010 \\
2002 \\
2006\end{array}$ & $\begin{array}{l}2026 \\
2016 \\
2020\end{array}$ \\
\hline $\begin{array}{l}\text { Rochester Gas and } \\
\text { Electric Corporation....... }\end{array}$ & Ginna & NY & PWR & WE & 470 & 121 & 1969 & 2009 & 1999 & 2009 \\
\hline $\begin{array}{l}\text { Sacramento Municipal } \\
\text { Utility District ............ }\end{array}$ & Rancho Seco & CA & PWR & $B \& W$ & 918 & 177 & 1974 & 2008 & SD & 1989 \\
\hline $\begin{array}{l}\text { South Carolina Electric } \\
\text { and Gas Company........ }\end{array}$ & Summer & sc & PWR & WE & 885 & 157 & 1982 & 2022 & 2008 & 2035 \\
\hline $\begin{array}{l}\text { Southern California } \\
\text { Edison Company... }\end{array}$ & $\begin{array}{l}\text { San Onofre } 1 \\
\text { San Onofre } 2 \\
\text { San Onofre } 3\end{array}$ & $\begin{array}{l}\text { CA } \\
\text { CA } \\
\text { CA }\end{array}$ & $\begin{array}{l}\text { PWR } \\
\text { PWR } \\
\text { PWR }\end{array}$ & $\begin{array}{l}\text { WE } \\
\text { CE } \\
\text { CE }\end{array}$ & $\begin{array}{r}436 \\
1,070 \\
1,080\end{array}$ & $\begin{array}{l}157 \\
217 \\
217\end{array}$ & $\begin{array}{l}1967 \\
1982 \\
1983\end{array}$ & $\begin{array}{l}2004 \\
2013 \\
2013\end{array}$ & $\begin{array}{r}S D \\
2005 \\
2005\end{array}$ & $\begin{array}{l}1992 \\
2013 \\
2013\end{array}$ \\
\hline
\end{tabular}

See footnotes at end of table. 
Table 4. Nuclear Power Plant Data as of December 31, 1992 (Continued)

\begin{tabular}{|c|c|c|c|c|c|c|c|c|c|c|}
\hline Electric Utility Name & Reactor Name & State & $\begin{array}{l}\text { Reactor } \\
\text { Type }\end{array}$ & Vendor $^{a}$ & $\begin{array}{c}\text { Net } \\
\text { Summer } \\
\text { Capability } \\
\text { (net MWe) }\end{array}$ & $\begin{array}{l}\text { Core Size } \\
\text { (number of } \\
\text { assemblies) }\end{array}$ & $\begin{array}{l}\text { Startup } \\
\text { (year) }^{c}\end{array}$ & $\begin{array}{l}\text { License } \\
\text { Expiratign } \\
\text { (year) }^{d}\end{array}$ & $\begin{array}{c}\text { Loss of } \\
\text { Abillty } \\
\text { to Operate } \\
\text { (year) }^{\theta}\end{array}$ & $\begin{array}{c}\text { Actual or } \\
\text { Projected } \\
\text { Retirement } \\
\text { (year) }\end{array}$ \\
\hline $\begin{array}{l}\text { System Energy Resources, } \\
\text { Inc. } . . . \ldots \ldots \ldots \ldots \ldots \ldots\end{array}$ & Grand Gulf 1 & MS & BWR & GE & 1.143 & 800 & 1984 & 2022 & 2005 & 2022 \\
\hline $\begin{array}{l}\text { Tennessee Valley } \\
\text { Authority .............. }\end{array}$ & $\begin{array}{l}\text { Browns Ferry } 1 \\
\text { Browns Ferry } 2 \\
\text { Browns Ferry } 3 \\
\text { Sequoyah } 1 \\
\text { Sequoyah } 2 \\
\text { Watts Bar } 1 \\
\text { Watts Bar } 2\end{array}$ & $\begin{array}{l}A L \\
A L \\
A L \\
T N \\
T N \\
T N \\
T N\end{array}$ & $\begin{array}{l}\text { BWR } \\
\text { BWR } \\
\text { BWR } \\
\text { PWR } \\
\text { PWR } \\
\text { PWR } \\
\text { PWR }\end{array}$ & $\begin{array}{l}\text { GE } \\
\text { GE } \\
\text { GE } \\
\text { WE } \\
\text { WE } \\
\text { WE } \\
\text { WE }\end{array}$ & $\begin{array}{l}1,065 \\
1,065 \\
1,065 \\
1,148 \\
1,148 \\
1,170 \\
1,170\end{array}$ & $\begin{array}{l}764 \\
764 \\
764 \\
193 \\
193 \\
193 \\
193\end{array}$ & $\begin{array}{l}1973 \\
1974 \\
1976 \\
1980 \\
1981 \\
1994 \\
1996\end{array}$ & $\begin{array}{r}2013 \\
2014 \\
2016 \\
2020 \\
2021 \\
C \\
C\end{array}$ & $\begin{array}{l}2006 \\
2006 \\
2006 \\
2002 \\
2002 \\
2007 \\
2007\end{array}$ & $\begin{array}{l}2013 \\
2014 \\
2016 \\
2020 \\
2021 \\
2030 \\
2032\end{array}$ \\
\hline TU Electric ............. & $\begin{array}{l}\text { Comanche Peak } 1 \\
\text { Comanche Peak } 2\end{array}$ & $\begin{array}{l}T X \\
T X\end{array}$ & $\begin{array}{l}\text { PWR } \\
\text { PWR }\end{array}$ & $\begin{array}{l}\text { WE } \\
\text { WE }\end{array}$ & $\begin{array}{l}1,150 \\
1,150\end{array}$ & $\begin{array}{l}193 \\
193\end{array}$ & $\begin{array}{l}1990 \\
1993\end{array}$ & $\begin{array}{r}2030 \\
C\end{array}$ & $\begin{array}{l}2020 \\
2021\end{array}$ & $\begin{array}{l}2030 \\
2030\end{array}$ \\
\hline Toledo Edison Company ... & Davis-Besse & $\mathrm{OH}$ & PWR & B\&W & 877 & 177 & 1977 & 2017 & NA & 2037 \\
\hline Union Electric Company.... & Callaway & MO & PWR & WE & 1,125 & 193 & 1984 & 2024 & 2007 & 2024 \\
\hline $\begin{array}{l}\text { Vermont Yankee Nuclear } \\
\text { Power Corporation........ }\end{array}$ & Vermont Yankee & VT & BWR & GE & 496 & 368 & 1972 & 2012 & 2004 & 2012 \\
\hline Virginia Power .......... & $\begin{array}{l}\text { North Anna } 1 \\
\text { North Anna } 2 \\
\text { Surry } 1 \\
\text { Surry } 2\end{array}$ & $\begin{array}{l}\text { VA } \\
\text { VA } \\
\text { VA } \\
\text { VA }\end{array}$ & $\begin{array}{l}\text { PWR } \\
\text { PWR } \\
\text { PWR } \\
\text { PWR }\end{array}$ & $\begin{array}{l}\text { WE } \\
\text { WE } \\
\text { WE } \\
\text { WE }\end{array}$ & $\begin{array}{l}848 \\
909 \\
781 \\
781\end{array}$ & $\begin{array}{l}157 \\
157 \\
157 \\
157\end{array}$ & $\begin{array}{l}1978 \\
1980 \\
1972 \\
1973\end{array}$ & $\begin{array}{l}2018 \\
2020 \\
2012 \\
2013\end{array}$ & $\begin{array}{l}2000 \\
2000 \\
2012 \\
2013\end{array}$ & $\begin{array}{l}2018 \\
2020 \\
2012 \\
2013\end{array}$ \\
\hline $\begin{array}{l}\text { Washington Public Power } \\
\text { Supply System.......... }\end{array}$ & Washington Nuclear 2 & WA & BWR & GE & 1,100 & 764 & 1984 & 2023 & 1999 & 2023 \\
\hline $\begin{array}{l}\text { Wisconsin Electric Power } \\
\text { Company } . . \ldots \ldots \ldots \ldots \ldots\end{array}$ & $\begin{array}{l}\text { Point Beach } 1 \\
\text { Point Beach } 2\end{array}$ & $\begin{array}{l}W I \\
W I\end{array}$ & $\begin{array}{l}\text { PWR } \\
\text { PWR }\end{array}$ & $\begin{array}{l}\text { WE } \\
\text { WE }\end{array}$ & $\begin{array}{l}492 \\
492\end{array}$ & $\begin{array}{l}121 \\
121\end{array}$ & $\begin{array}{l}1970 \\
1972\end{array}$ & $\begin{array}{l}2010 \\
2013\end{array}$ & $\begin{array}{l}1996 \\
1996\end{array}$ & $\begin{array}{l}2010 \\
2013\end{array}$ \\
\hline
\end{tabular}

See footnotes at end of table. 


\begin{tabular}{|c|c|c|c|c|c|c|c|c|c|c|}
\hline Electric Utility Name & Reactor Name & State & $\begin{array}{l}\text { Reactor } \\
\text { Type }\end{array}$ & Vendor $^{\mathfrak{a}}$ & $\begin{array}{c}\text { Net } \\
\text { Summer } \\
\text { Capability } \\
\text { (net MWe) }\end{array}$ & $\begin{array}{c}\text { Core Size } \\
\text { (number of } \\
\text { assemblies) }\end{array}$ & $\begin{array}{l}\text { Startup } \\
(\text { year) }\end{array}$ & $\begin{array}{l}\text { License } \\
\text { Expiratlon } \\
\text { (year) }\end{array}$ & $\begin{array}{c}\text { Loss of } \\
\text { Abillty } \\
\text { to Operate } \\
\text { (year) }^{\text {(yea }}\end{array}$ & $\begin{array}{c}\text { Actual or } \\
\text { Projected } \\
\text { Retirement } \\
\text { (year) }\end{array}$ \\
\hline $\begin{array}{l}\text { Wisconsin Public Service } \\
\text { Corporation ............ }\end{array}$ & Kowaunee & WI & PWR & WE & 522 & 121 & 1974 & 2013 & 2002 & 2014 \\
\hline $\begin{array}{l}\text { Yankee Atomic Electric } \\
\text { Company ............. }\end{array}$ & Yankee Rowe & MA & PWR & WE & 175 & 76 & 1960 & 2000 & SD & 1992 \\
\hline
\end{tabular}

${ }^{a}$ Vendor codes are as follows: $A C=$ Allis Chalmers; $B \& W=$ Babcock \& Wilcox Company; $C E=A B B$ Combustion Engineering; GE = GE Nuclear Energy; WE = Westinghouse Electric.

Net Summer Capability (net MWe) data are not available on Form RW-859 data base. Data are from Energy Information Administration (EIA) publication World Nuclear Capacity and Fuel Cycle Requirements 1993, Table D1.

'Startup Year not available for all reactors on Form RW-859 data base. Startup Years for Watts Bar 1 \& 2 and Comanche Peak 2 are from ElA, World Nuclear Capacity and Fuel Cycle Requirements 1993, Table E1, Estimated Operable Date column.

License Expiration Year not available for all reactors on Form RW-859 data base. License Expiration Years for Watts Bar 1 \& 2 and Comanche Peak 2 are from ElA, World Nuclear Capacity and Fuel Cycle Requirements 1993, Table E1, Status column. License status codes: C = construction permit.

'These data are compiled directly from question 2.3 on Form RW-859. It reads as follows: "What is the estimated date on which you would not continue reactor operation, because of a lack of storage space for discharged fuel absent spent fuel pickup by DOE?"

PWR $=$ Pressurized-water reactor; $B W R=$ Boiling-Water reactor; $M W e=$ Megawatts electric.

$N A=$ Not available (no Loss of Ability to Operate Year date has been estimated by the utility). $S D=S h u t d o w n$ reactor. RD $=$ Retired reactor.

Source: Energy Information Administration, Form RW-859, "Nuclear Fuel Data" (1992). 


\section{Annual Discharges and Burnup}

A total of 91,039 spent nuclear fuel assemblies discharged through 1992 contained 25,958 MTU. By weight, approximately 37 percent of the spent nuclear fuel came from BWRs and 63 percent came from PWRs. In 1992, the number of PWR assemblies permanently discharged $(3,713)$ represents an increase from the previous high of 3,550 in 1990. The number of BWR assemblies permanently discharged $(4,024)$ represents an increase from the previous high of 3,924 reported in 1989.

Annual spent fuel discharges and burnup values are summarized in Table 5. Permanently discharged spent fuel for BWRs and PWRs is shown by number of assemblies and metric tons of uranium in Figure 1. Both show the number of assemblies and metric tons of uranium discharged in each year for BWRs and PWRs. The table also shows the annual average burnup in gigawattdays thermal per initial loading weight of uranium, in metric tons (GWDt/MTU), including assemblies discharged from nonequilibrium cycles.

Some electric utilities report plans to continue increasing burnup in the future. Increasing the concentration of U-235 in the enriched uranium product and changing the design specifications of the fuel rods to allow for longer in-core exposures achieves increases in burnup.

Electric utilities use higher fuel burnup to increase cycle lengths and/or to reduce spent fuel generation. Increasing cycle length allows the potential for reducing the relative outage time: for example, an annual cycle requires three refuelings in a 3-year period, while an 18-month cycle requires only two refuelings in the same time period. Thus, increased capacity factors can result from increased cycle length; however, increased lengths of fuel outages can offset this increase in capacity factors.
Starting in 1970, the annual average burnup for all discharged BWR assemblies have shown an increase to a high of 28.8 GWDt/MTU in 1992. Average burnups have fluctuated between 1984 and 1992 because of the number of reactors discharging first and second cycle assemblies. The average burnup for all PWR assemblies increased to 36.4 GWDt/MTU in 1992, the highest annual average yet attained. It had been fairly constant from 1980 through 1987 at roughly $30 \mathrm{GWDt} / \mathrm{MTU}$.

The last two columns in Table 5 show the burnup of equilibrium cycle discharges for BWRs and PWRs. Equilibrium cycle is an analytical term that refers to fuel cycles occurring after the initial two cycles of a reactor's operation and prior to its final cycle. It is an assumed condition in which the spent nuclear fuel from a facility has a relatively constant composition from cycle to cycle. In a reactor, this condition typically begins with the third or fourth fuel loading, depending on the portion of the core being replaced. (For the purpose of this report, it is assumed to begin with the third fuel loading.) For example, the first fuel loading may have three sectors; one has an enrichment of 1.7 percent, the second an enrichment of 2.7 percent, and the third an enrichment of 3.7 percent. The first sector would be discharged at the end of the first cycle, with an average burnup of 10 GWDt/MTU; the second sector would be discharged at the end of the second cycle, with a burnup of 20 GWDt/MTU; the third sector would be the first equilibrium cycle discharge and would be discharged at the end of the third cycle.

For BWRs, the average equilibrium spent fuel discharge burnup in 1992 was 29.4 GWDt/MTU. For PWRs, the average equilibrium spent fuel discharge burnup in 1992 was $38.7 \mathrm{GWDt} / \mathrm{MTU}$. The trends in achieved burnup levels for BWR and PWR spent fuel discharged to date are shown in Figure 2. The graph shows that PWR spent fuel has achieved increasingly higher burnup levels than BWR spent fuel. 
Table 5. Annual Spent Fuel Discharges and Burnup, 1968-1992

\begin{tabular}{|c|c|c|c|c|c|c|c|c|c|c|}
\hline \multirow[b]{3}{*}{ Year } & \multirow{2}{*}{\multicolumn{3}{|c|}{ Number of Assemblies }} & \multirow{2}{*}{\multicolumn{3}{|c|}{ Metrlc Tons of Uranium }} & \multicolumn{4}{|c|}{ Average Burnup (GWDt/MTU) } \\
\hline & & & & & & & \multicolumn{2}{|c|}{ All Discharged Assemblles } & \multicolumn{2}{|c|}{ Equillbrium Cycle Discharges } \\
\hline & BWR & PWR & Total & BWR & PWR & Total & BWR & PWR & BWR & PWR \\
\hline $1968 \ldots \ldots \ldots \ldots$ & 5 & 0 & 5 & 0.6 & 0.0 & 0.6 & 1.6 & 0.0 & 1.6 & 0.0 \\
\hline $1969 \ldots \ldots \ldots \ldots$ & 96 & 0 & 96 & 9.8 & 0.0 & 9.8 & 15.2 & 0.0 & 15.2 & 0.0 \\
\hline $1970 \ldots \ldots \ldots \ldots$ & 29 & 99 & 128 & 5.6 & 39.0 & 44.6 & 0.3 & 18.4 & 0.0 & 0.0 \\
\hline $1971 \ldots \ldots \ldots \ldots$ & 408 & 113 & 521 & 64.0 & 44.5 & 108.5 & 5.8 & 23.9 & 16.6 & 0.0 \\
\hline $1972 \ldots \ldots \ldots \ldots$ & 771 & 282 & 1,053 & 141.5 & 99.9 & 241.4 & 6.4 & 21.9 & 16.3 & 28.0 \\
\hline $1973 \ldots \ldots \ldots \ldots$ & 576 & 165 & 741 & 95.1 & 67.1 & 162.2 & $12.4^{\circ}$ & 23.7 & 15.9 & 28.1 \\
\hline $1974 \ldots \ldots \ldots \ldots$ & 1,313 & 575 & 1,888 & 244.6 & 207.7 & 452.3 & 12.7 & 18.9 & 14.5 & 25.7 \\
\hline $1975 \ldots \ldots \ldots \ldots$ & 1,223 & 797 & 2,020 & 225.6 & 321.8 & 547.4 & 16.9 & 18.1 & 17.3 & 27.3 \\
\hline $1976 \ldots \ldots \ldots \ldots$ & 1,666 & 931 & 2,597 & 297.4 & 401.0 & 698.3 & 13.4 & 22.2 & 16.7 & 25.8 \\
\hline $1977 \ldots \ldots \ldots \ldots$ & 2,047 & 1,107 & 3,154 & 382.9 & 466.9 & 849.8 & 16.6 & 25.1 & 19.0 & 27.8 \\
\hline $1978 \ldots \ldots \ldots \ldots$ & 2,239 & 1,666 & 3,905 & 383.2 & 699.0 & $1,082.3$ & 19.8 & 26.4 & 22.1 & 28.7 \\
\hline $1979 \ldots \ldots \ldots \ldots$ & 2,131 & 1,662 & 3,793 & 399.8 & 721.2 & $1,121.1$ & 22.4 & 27.0 & 23.7 & 30.2 \\
\hline $1980 \ldots \ldots \ldots \ldots$ & 3,330 & 1,456 & 4,786 & 619.9 & 618.1 & $1,237.9$ & 22.4 & 29.7 & 23.3 & 30.7 \\
\hline $1981 \ldots \ldots \ldots \ldots$ & 2,467 & 1,585 & 4,052 & 458.7 & 675.9 & $1,134.6$ & 23.9 & 30.2 & 23.9 & 30.9 \\
\hline $1982 \ldots \ldots \ldots \ldots$ & 1,951 & 1,491 & 3,442 & 357.2 & 640.4 & 997.6 & 24.7 & 29.7 & 24.9 & 32.3 \\
\hline $1983 \ldots \ldots \ldots \ldots$ & 2,698 & 1,778 & 4,476 & 491.3 & 772.2 & $1,263.5$ & 26.7 & 30.0 & 26.7 & 31.6 \\
\hline $1984 \ldots \ldots \ldots \ldots$ & 2,736 & 1,938 & 4,674 & 498.0 & 841.7 & $1,339.8$ & 25.4 & 29.5 & 25.4 & 32.1 \\
\hline $1985 \ldots \ldots \ldots \ldots$ & 2,835 & 2,037 & 4,872 & 514.6 & 861.3 & $1,375.9$ & 23.4 & 31.8 & 25.9 & 33.2 \\
\hline $1986 \ldots \ldots \ldots \ldots$ & 2,551 & 2,301 & 4,852 & 458.2 & $1,000.9$ & $1,459.0$ & 21.0 & 30.4 & 27.3 & 33.9 \\
\hline $1987 \ldots \ldots \ldots \ldots$ & 3,876 & 2,604 & 6,480 & 699.4 & $1,113.8$ & $1,813.2$ & 19.2 & 31.3 & 27.0 & 34.4 \\
\hline $1988 \ldots \ldots \ldots \ldots$ & 2,956 & 2,644 & 5,600 & 535.6 & $1,125.2$ & $1,660.8$ & 24.1 & 33.3 & 27.0 & 35.4 \\
\hline $1989 \ldots \ldots \ldots \ldots$ & 3,924 & 2,810 & 6,734 & 714.9 & $1,226.7$ & $1,941.7$ & 22.0 & 32.5 & 27.3 & 36.8 \\
\hline $1990 \ldots \ldots \ldots \ldots$ & 3,485 & 3,550 & 7,035 & 632.8 & $1,531.9$ & $2,164.7$ & 25.0 & 34.0 & 27.8 & 36.0 \\
\hline $1991 \ldots \ldots \ldots \ldots$ & 3,260 & 2,970 & 6,230 & 588.0 & $1,297.7$ & $1,885.6$ & 28.2 & 35.2 & 30.4 & 37.3 \\
\hline $1992^{a} \ldots \ldots \ldots \ldots$ & 4,024 & 3,713 & 7,737 & 728.7 & $1,601.0$ & $2,329.8$ & 28.8 & 36.4 & 29.4 & 38.7 \\
\hline Temps ${ }^{b} \ldots \ldots \ldots \ldots$ & 5 & 163 & 168 & 0.2 & 35.1 & 35.3 & 23.0 & 24.2 & 23.0 & 26.2 \\
\hline TOTAL......... & 52,602 & $\mathbf{3 8 , 4 3 7}$ & 91,039 & $9,547.6$ & $16,410.1$ & $25,957.7$ & 22.3 & 30.9 & 24.8 & 33.3 \\
\hline
\end{tabular}

a Some data for previous years have been revised. Current-year data may be revised in future publications. When utilities reinsert assemblies discharged in previous years, historical totals change. See Technical Note 12 in Appendix E.

Temps are temporarily discharged assemblies, as of December 31, 1992, as reported on Form RW-859.

PWR = Pressurized-water reactor; BWR = Boiling-Water reactor; GWDt/MTU = Gigawattdays thermal per metric ton of uranium.

Notes: A total of 2,208 high-temperature, gas-cooled reactor (HTGR) fuel elements, with initial uranium content equal to $25.0 \mathrm{MTU}$, were discharged. These HTGR fuel elements are not included in the above table. See Technical Note 6 in Appendix E. Totals may not equal sum of components because of independent rounding. See Technical Note 11 in Appendix $E$.

Source: Energy Information Administration, Form RW-859, "Nuclear Fuel Data* (1992). 
Flgure 1. Summary of Spent Fuel Discharges, 1968-1992
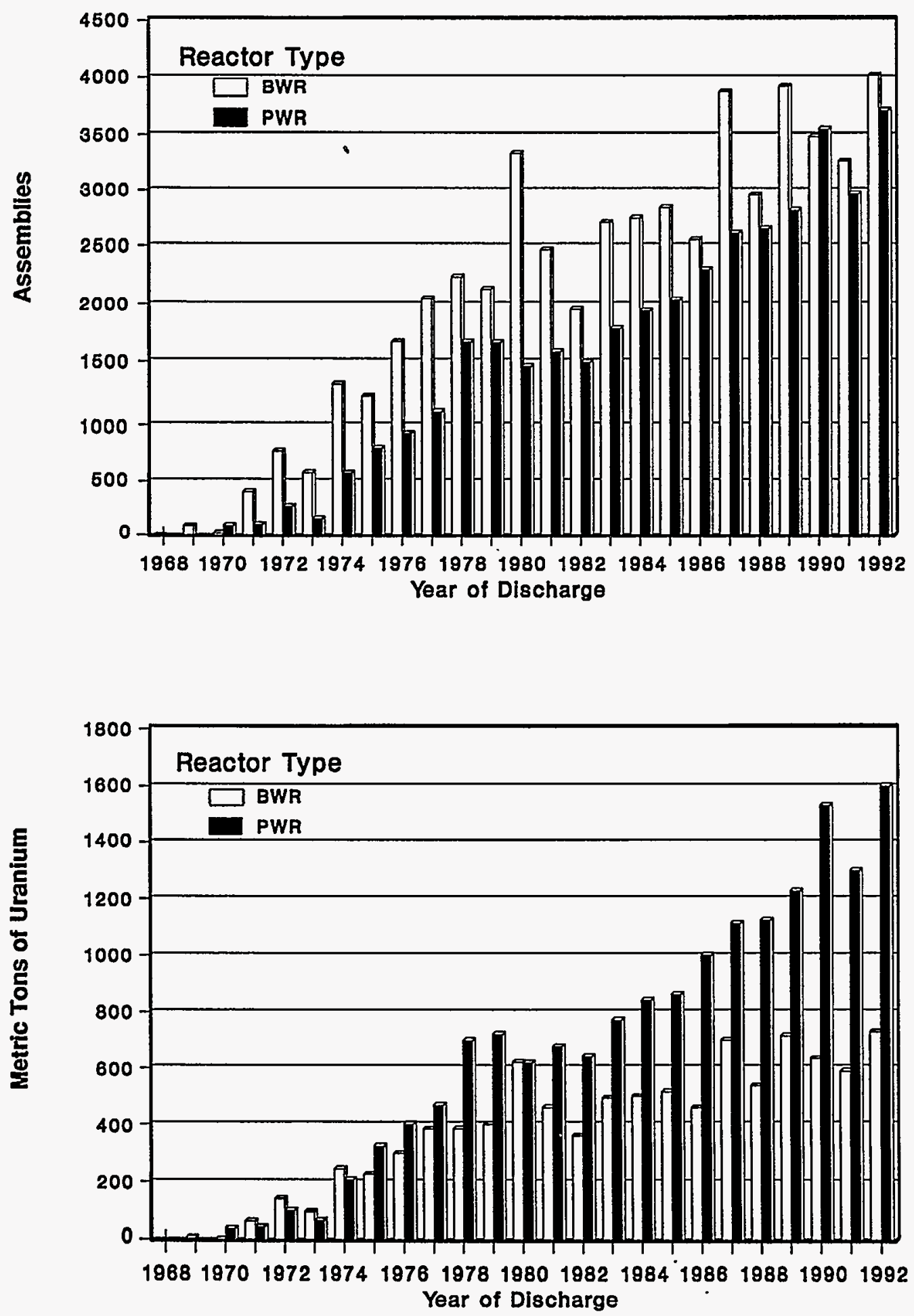

BWR = Boiling-water reactor; PWR = Pressurized-water reactor.

Notes: A number of assemblies discharged prior to 1972 were reprocessed and are not included in this figure. See Table 12 and Technical Note 7 In Appendix E for information on reprocessed assemblies. Graphs do not include 2,208 high-temperature, gas-cooled reactor (HTGR) fuel elements and 168 temporarily discharged assemblles.

Source: Energy Information Administration, Form RW-859, "Nuclear Fuel Data" (1992). 
Figure 2. Summary of Average Burnups, 1968-1992
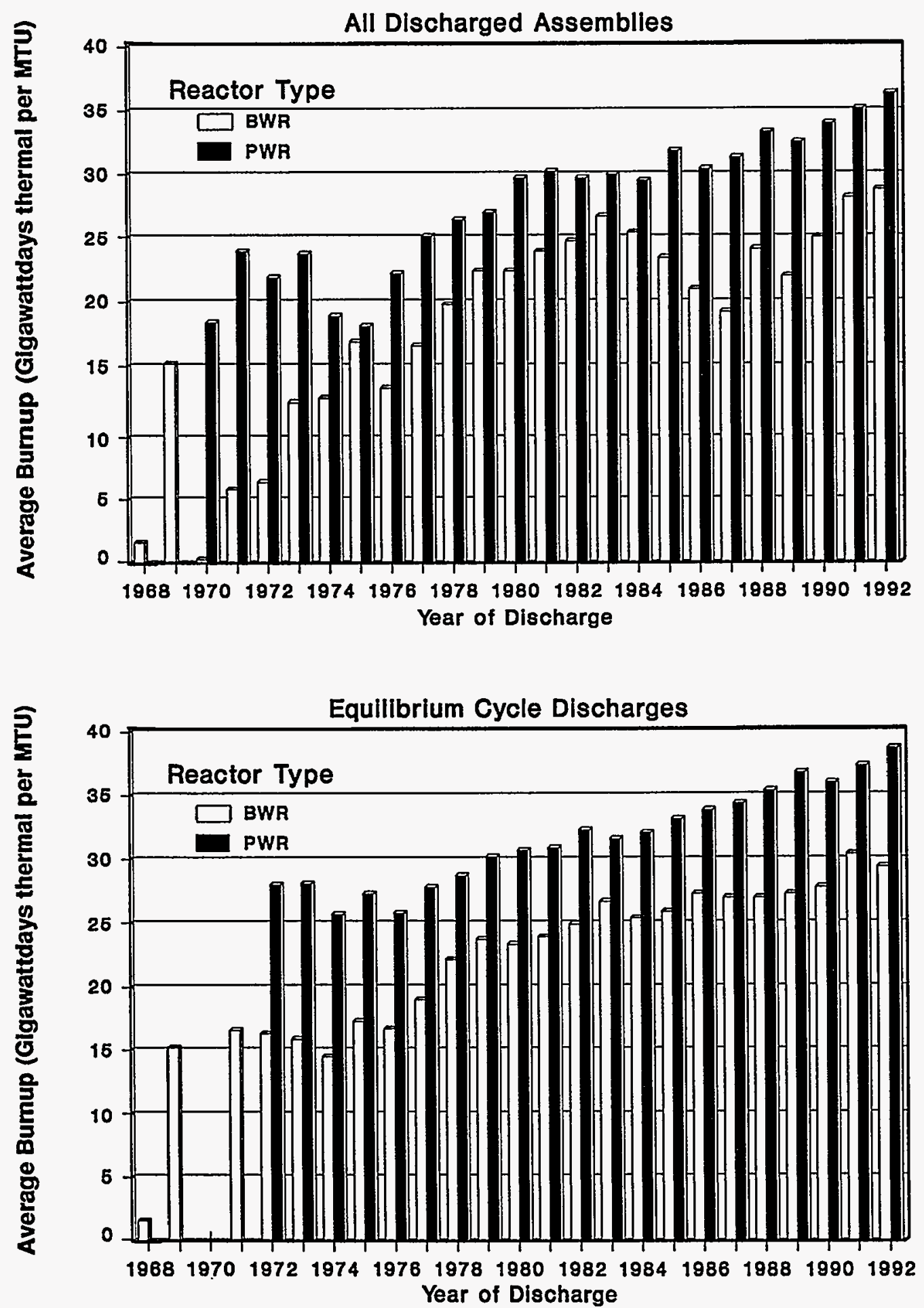

BWR = Boiling-water reactor; PWR $=$ Pressurized-water reactor; MTU $=$ Metric tons of uranium.

Notes: A number of assemblies discharged prior to 1972 were reprocessed and are not included in this figure. See Table 12 and Technical Note 7 in Appendix E for information on reprocessed assemblies. Graphs do not included 2,208 high-temperature, gas-cooled reactor (HTGR) fuel elements and 168 temporarily discharged assemblies. Equilibrium Cycle Discharges exclude discharge data for cycles 1 and 2 of each reactor.

Source: Energy Information Administration, Form RW-859, "Nuclear Fuel Data" (1992). 
The annual spent fuel burnup distributions in number of BWR and PWR assemblies are presented in Table 6. Data are presented by year of discharge and burnup range, in intervals of 5 GWDt/MTU. The spent fuel burnup of assemblies has increased over time, as indicated by the progression of larger concentrations of assemblies in higher burnup categories in most recent years. For example, 1,996 BWR assemblies discharged in 1992 (49.6 percent) fall in the range of 30 to $35 \mathrm{GWDt} / \mathrm{MTU}$, the highest percentage for that range for any year. A total of 1,499 PWR assemblies (40.4 percent) had burnups greater than $40 \mathrm{GWDt} / \mathrm{MTU}$ in 1992. This total increased dramatically from the 756 PWR assemblies ( 25.5 percent) in 1991, thus continuing the pattern for larger concentrations of assemblies in higher burnup categories.

On the high end of the discharge burnups, only 7 BWR assemblies have exceded $40 \mathrm{GWDt} / \mathrm{MTU}$, while 58 PWR assemblies have exceded $50 \mathrm{GWDt} / \mathrm{MTU}$. The annual spent fuel burnup distribution by weight for BWR and PWR assemblies, in categories identical to those in Table 6, are shown in Table 7. As expected, the results parallel those shown in Table 6 . These increases show the effect of the improved fuel management practices being used to provide higher burnups.

Assembly weights, burnups, and enrichment data by burnup range for BWRs and PWRs are compared in Table 8. Examination of the data shows that BWRs have discharged over 57 percent of the assemblies, yet PWRs account for 63 percent of the uranium (by weight) that has been discharged; PWR assemblies contain substantially more uranium than BWR assemblies ( $450 \mathrm{~kg}$ and $200 \mathrm{~kg}$, respectively). Of the discharged BWR assemblies, 69 percent (by initial loading weight of uranium) is concentrated in the 15 to $30 \mathrm{GWDt} / \mathrm{MTU}$ range, and of the discharged PWR assemblies, 71 percent is concentrated in the 25 to 40 GWDt/MTU range (Table 8).

Data for reprocessed fuel assemblies have not been collected and, therefore, are not addressed in this report. Data for Fort St. Vrain have been omitted, since it is not an LWR. Three Mile Island Unit 2 ceased operation in 1979. The reactor core is now part of a DOE test and evaluation program; thus, because of this unique situation, the fuel from that reactor is not included in this report.

\section{Cladding}

Early reactors used stainless steel cladding for their fuel assemblies. This was less than perfect because stainless steel is a neutron absorber or poison to the nuclear reaction. As a result, Zircaloy-2 (for BWRs) and Zircaloy-4 (for PWRs) have been used as the cladding material of choice since the early 1970s. For simplicity of data presentation, the designation zircaloy is used for both cladding types. Current LWRs use zircaloy cladding almost exclusively. Zircaloy cladding provides better neutron utilization, and is also more resistant to corrosion under the harsh physical and chemical conditions present in the reactor core. Only one reactor still has some stainless steel clad fuel rods in core.

Advances in the fabrication and treatment of zircaloy clad fuel have reduced fuel failures significantly. This has resulted in higher burnups and, to some extent, improved fuel economy. This trend is expected to continue as assemblies with the improved cladding are loaded with higher enrichments (over 4 percent enrichment), irradiated, and discharged. Zircaloy cladding is also usually much less radioactive after being discharged from the core than is stainless steel.

The number of spent fuel assemblies and MTU of spent fuel discharged by year and by fuel rod cladding type are presented in Table 9. The table shows that most discharged spent fuel is zircaloy clad. Based on MTU, 99 percent of BWR fuel is zircaloy clad, and 96 percent of PWR fuel is zircaloy clad. Overall, only 3 percent of discharged fuel is stainless steel clad.

Only five reactors have discharged fuel rods with stainless steel cladding (Table 10). Of these, four reactors (Indian Point 1, LaCrosse, San Onofre 1, and Yankee Rowe) are permanently shutdown. Yankee Rowe switched to zircaloy clad fuel in the mid-1970's. Haddam Neck is in the process of switching to zircaloy clad fuel. After Haddam Neck has completed its switch to zircaloy clad fuel, no U.S. commercial reactor will be using stainless steel clad fuel. 
Table 6. Annual Spent Fuel Burnup (in Assemblies), 1968-1992

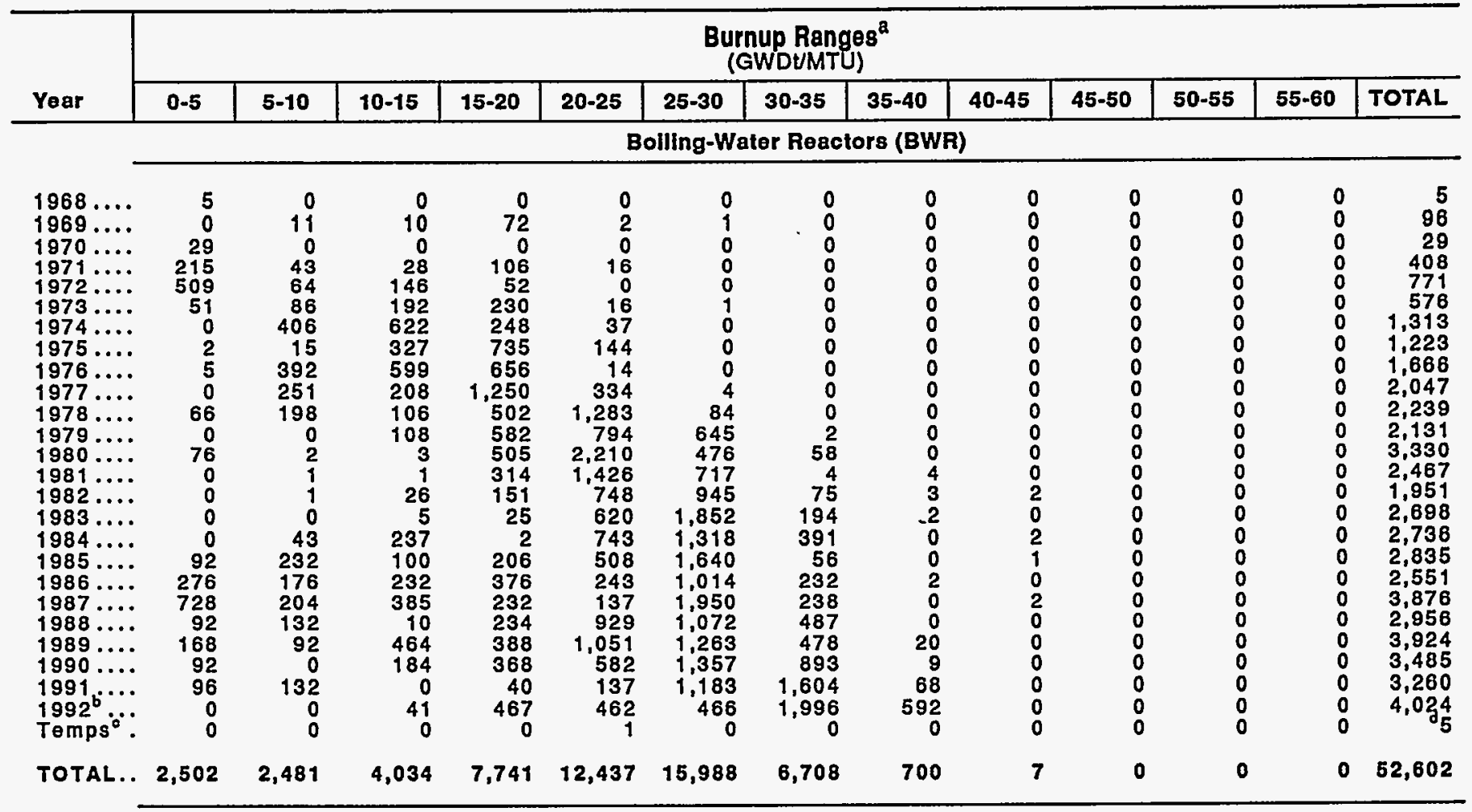

\section{Pressurlzed-Water Reactors (PWR)}

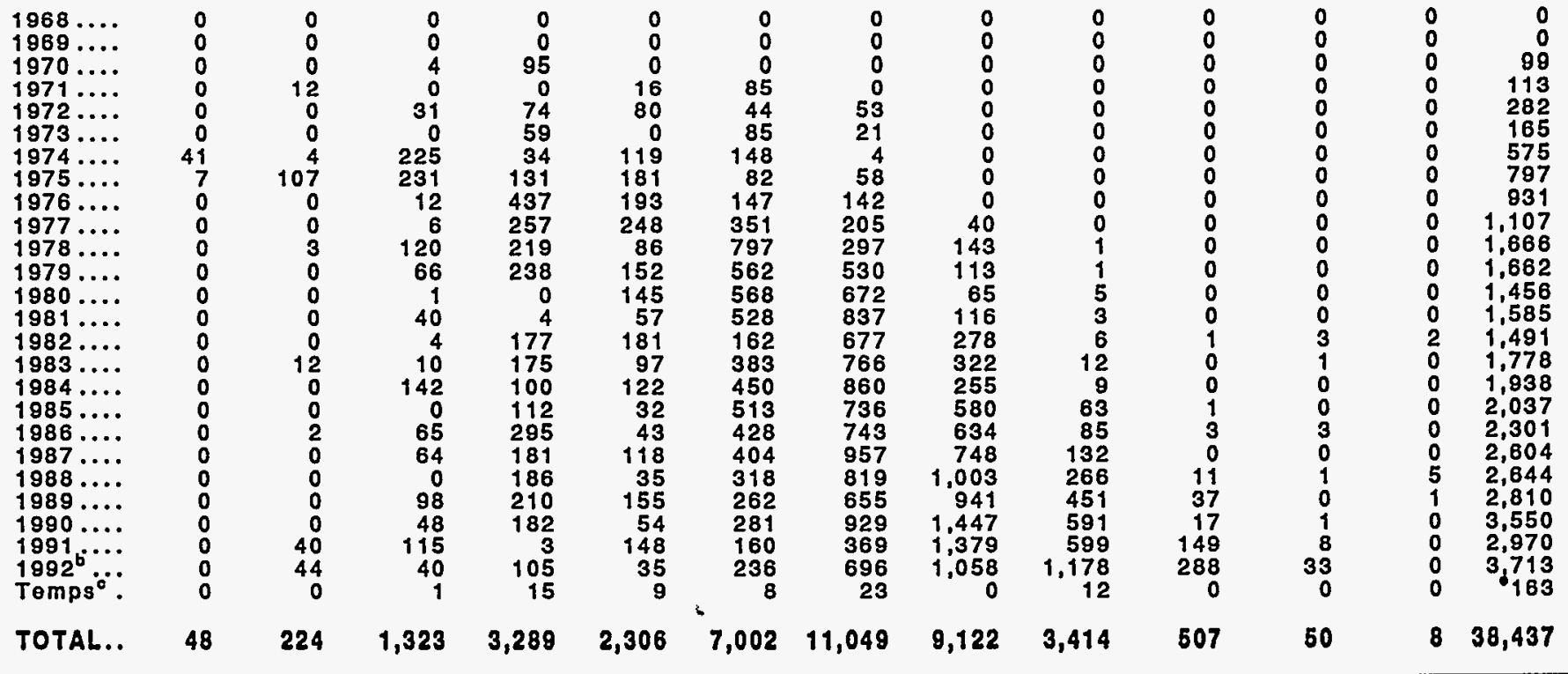

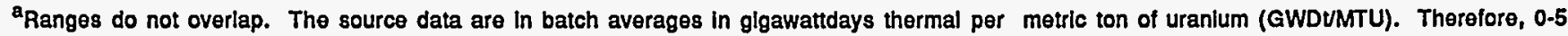
GWDUMTU means 0 through 4.999 GWDUMTU; 5-10 GWDUMTU means 5.000-9.999 GWDUMTU; etc. See Technical Note 9 in Appendlx E.

${ }^{b}$ Some data for prevlous years have been revised. Current-y ear data may be revised in future publications. When utllities reinsert assemblles discharged In previous years, hlstorical totals change. See Technical Note 12 in Appendlx E.

CTemps are temporarily discharged assemblles, as of December 31, 1992, as reported on Form RW-859.

Includes 4 temporarlly discharged BWR assemblles with missing burnup data. See Technical Note 10 in Appendlx E.

Inciudes 95 temporarlly dlscharged PWR assemblles with mlssing burnup data. See Technical Note 10 in Appendix E.

Note: A total of 2,208 hlgh-temperature, gas-cooled reactor (HTGR) fuel elements, with inital uranlum content equal to $25.0 \mathrm{MTU}$, were dlscharged. These HTGR fuel elements are not Included in the above table. See Technlcal Note 6 In Appendlx E.

Source: Energy Information Administration, Form RW-859, "Nuclear Fuel Data" (1992). 
Table 7. Metric Tons of Uranium in Annual Spent Fuel Burnup, 1968-1992

\begin{tabular}{|c|c|c|c|c|c|c|c|c|c|c|c|c|c|}
\hline \multirow[b]{2}{*}{ Year } & \multicolumn{13}{|c|}{$\begin{array}{c}\text { Burnup Ranges }{ }^{a} \\
\text { (GWDt/MTU) }\end{array}$} \\
\hline & 0.5 & $5-10$ & $10-15$ & $15-20$ & $20-25$ & $25-30$ & $30-35$ & 35.40 & 40.45 & $45-50$ & $50-55$ & $55 \cdot 60$ & TOTAL \\
\hline & \multicolumn{13}{|c|}{ Bolling-Water Reactors (BWR) } \\
\hline 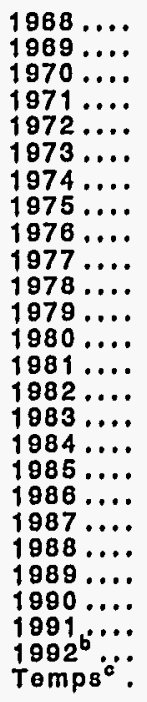 & $\begin{array}{r}0.6 \\
0.0 \\
5.6 \\
41.5 \\
97.9 \\
9.7 \\
0.0 \\
0.3 \\
0.8 \\
0.0 \\
6.3 \\
0.0 \\
14.0 \\
0.0 \\
0.0 \\
0.0 \\
0.0 \\
18.9 \\
50.8 \\
133.5 \\
17.0 \\
30.9 \\
17.0 \\
17.8 \\
0.0 \\
0.0\end{array}$ & $\begin{array}{r}0.0 \\
1.2 \\
0.0 \\
8.1 \\
12.1 \\
16.5 \\
78.4 \\
1.7 \\
67.1 \\
48.0 \\
32.4 \\
0.0 \\
0.4 \\
0.2 \\
0.2 \\
0.0 \\
7.9 \\
42.5 \\
32.4 \\
36.1 \\
24.5 \\
16.9 \\
0.0 \\
24.6 \\
0.0 \\
0.0\end{array}$ & $\begin{array}{r}0.0 \\
1.0 \\
0.0 \\
2.8 \\
27.6 \\
30.9 \\
117.7 \\
62.0 \\
108.7 \\
40.3 \\
13.1 \\
18.6 \\
0.6 \\
0.2 \\
4.6 \\
0.8 \\
43.0 \\
18.3 \\
42.5 \\
68.8 \\
1.8 \\
85.3 \\
34.0 \\
0.0 \\
7.6 \\
0.0\end{array}$ & $\begin{array}{r}0.0 \\
7.3 \\
0.0 \\
10.0 \\
4.0 \\
36.4 \\
44.7 \\
136.4 \\
118.4 \\
235.0 \\
84.2 \\
108.7 \\
93.3 \\
58.1 \\
25.6 \\
2.9 \\
0.3 \\
35.8 \\
66.6 \\
40.8 \\
42.9 \\
71.8 \\
67.6 \\
7.2 \\
88.1 \\
0.0\end{array}$ & $\begin{array}{r}0.0 \\
0.2 \\
0.0 \\
1.6 \\
0.0 \\
1.5 \\
3.8 \\
25.3 \\
2.3 \\
58.9 \\
232.0 \\
149.2 \\
413.3 \\
265.4 \\
138.5 \\
113.5 \\
136.2 \\
93.2 \\
43.1 \\
24.7 \\
168.3 \\
193.2 \\
108.2 \\
24.0 \\
85.1 \\
0.2\end{array}$ & $\begin{array}{r}0.0 \\
0.1 \\
0.0 \\
0.0 \\
0.0 \\
0.1 \\
0.0 \\
0.0 \\
0.0 \\
0.7 \\
15.2 \\
123.1 \\
87.6 \\
133.3 \\
173.6 \\
337.8 \\
239.5 \\
297.4 \\
180.7 \\
352.4 \\
192.4 \\
227.7 \\
247.5 \\
215.0 \\
83.9 \\
0.0\end{array}$ & $\begin{array}{r}0.0 \\
0.0 \\
0.0 \\
0.0 \\
0.0 \\
0.0 \\
0.0 \\
0.0 \\
0.0 \\
0.0 \\
0.0 \\
0.3 \\
10.7 \\
0.7 \\
13.8 \\
35.7 \\
70.8 \\
10.2 \\
41.7 \\
42.9 \\
88.7 \\
85.5 \\
158.9 \\
287.2 \\
362.7 \\
0.0\end{array}$ & $\begin{array}{r}0.0 \\
0.0 \\
0.0 \\
0.0 \\
0.0 \\
0.0 \\
0.0 \\
0.0 \\
0.0 \\
0.0 \\
0.0 \\
0.0 \\
0.0 \\
0.7 \\
0.6 \\
0.4 \\
0.0 \\
0.0 \\
0.4 \\
0.0 \\
0.0 \\
3.6 \\
1.6 \\
12.1 \\
103.4 \\
0.0\end{array}$ & $\begin{array}{l}0.0 \\
0.0 \\
0.0 \\
0.0 \\
0.0 \\
0.0 \\
0.0 \\
0.0 \\
0.0 \\
0.0 \\
0.0 \\
0.0 \\
0.0 \\
0.0 \\
0.4 \\
0.0 \\
0.4 \\
0.2 \\
0.0 \\
0.4 \\
0.0 \\
0.0 \\
0.0 \\
0.0 \\
0.0 \\
0.0\end{array}$ & $\begin{array}{l}0.0 \\
0.0 \\
0.0 \\
0.0 \\
0.0 \\
0.0 \\
0.0 \\
0.0 \\
0.0 \\
0.0 \\
0.0 \\
0.0 \\
0.0 \\
0.0 \\
0.0 \\
0.0 \\
0.0 \\
0.0 \\
0.0 \\
0.0 \\
0.0 \\
0.0 \\
0.0 \\
0.0 \\
0.0 \\
0.0\end{array}$ & $\begin{array}{l}0.0 \\
0.0 \\
0.0 \\
0.0 \\
0.0 \\
0.0 \\
0.0 \\
0.0 \\
0.0 \\
0.0 \\
0.0 \\
0.0 \\
0.0 \\
0.0 \\
0.0 \\
0.0 \\
0.0 \\
0.0 \\
0.0 \\
0.0 \\
0.0 \\
0.0 \\
0.0 \\
0.0 \\
0.0 \\
0.0\end{array}$ & $\begin{array}{l}0.0 \\
0.0 \\
0.0 \\
0.0 \\
0.0 \\
0.0 \\
0.0 \\
0.0 \\
0.0 \\
0.0 \\
0.0 \\
0.0 \\
0.0 \\
0.0 \\
0.0 \\
0.0 \\
0.0 \\
0.0 \\
0.0 \\
0.0 \\
0.0 \\
0.0 \\
0.0 \\
0.0 \\
0.0 \\
0.0\end{array}$ & $\begin{array}{r}0.6 \\
9.8 \\
5.6 \\
64.0 \\
141.5 \\
95.1 \\
244.6 \\
225.6 \\
297.4 \\
382.9 \\
383.2 \\
399.8 \\
619.9 \\
458.7 \\
357.2 \\
491.3 \\
498.0 \\
514.6 \\
458.2 \\
699.4 \\
535.6 \\
714.9 \\
632.8 \\
588.0 \\
728.7 \\
0.2\end{array}$ \\
\hline TOTAL.. & 460.7 & 451.0 & 730.4 & $1,384.0$ & $2,279.7$ & $2,908.1$ & $1,209.8$ & 122.6 & 1.3 & 0.0 & 0.0 & 0.0 & $9,547.6$ \\
\hline
\end{tabular}

Pressurized-Water Reactors (PWR)

\begin{tabular}{|c|c|c|c|c|c|c|c|c|c|c|c|c|c|}
\hline 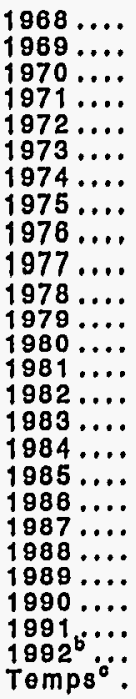 & $\begin{array}{l}0.0 \\
0.0 \\
0.0 \\
0.0 \\
0.0 \\
0.0 \\
7.4 \\
2.7 \\
0.0 \\
0.0 \\
0.0 \\
0.0 \\
0.0 \\
0.0 \\
0.0 \\
0.0 \\
0.0 \\
0.0 \\
0.0 \\
0.0 \\
0.0 \\
0.0 \\
0.0 \\
0.0 \\
0.0 \\
0.0\end{array}$ & $\begin{array}{r}0.0 \\
0.0 \\
0.0 \\
4.6 \\
0.0 \\
0.0 \\
1.5 \\
42.6 \\
0.0 \\
0.0 \\
1.4 \\
0.0 \\
0.0 \\
0.0 \\
0.0 \\
5.5 \\
0.0 \\
0.0 \\
0.8 \\
0.0 \\
0.0 \\
0.0 \\
0.0 \\
9.2 \\
19.8 \\
0.0\end{array}$ & $\begin{array}{r}0.0 \\
0.0 \\
1.7 \\
0.0 \\
11.9 \\
0.0 \\
86.4 \\
95.0 \\
5.6 \\
2.8 \\
47.9 \\
30.6 \\
0.4 \\
17.2 \\
1.8 \\
4.0 \\
58.0 \\
0.0 \\
27.6 \\
27.2 \\
0.0 \\
48.0 \\
24.0 \\
53.2 \\
14.8 \\
0.5\end{array}$ & $\begin{array}{r}0.0 \\
0.0 \\
37.3 \\
0.0 \\
29.3 \\
26.2 \\
13.6 \\
53.6 \\
194.2 \\
108.3 \\
89.8 \\
109.4 \\
0.0 \\
1.9 \\
81.1 \\
80.6 \\
45.2 \\
49.0 \\
132.0 \\
78.1 \\
83.9 \\
91.4 \\
85.2 \\
1.4 \\
43.7 \\
6.5\end{array}$ & $\begin{array}{r}0.0 \\
0.0 \\
0.0 \\
6.2 \\
27.8 \\
0.0 \\
40.5 \\
79.4 \\
82.4 \\
113.1 \\
39.1 \\
64.0 \\
66.8 \\
25.8 \\
80.4 \\
44.2 \\
56.3 \\
13.6 \\
19.3 \\
53.4 \\
15.0 \\
68.6 \\
24.0 \\
79.5 \\
15.0 \\
4.0\end{array}$ & $\begin{array}{r}0.0 \\
0.0 \\
0.0 \\
33.7 \\
8.9 \\
33.3 \\
57.3 \\
25.3 \\
63.3 \\
140.3 \\
336.9 \\
232.3 \\
241.8 \\
228.5 \\
61.4 \\
168.9 \\
198.4 \\
217.0 \\
180.2 \\
175.7 \\
139.2 \\
112.1 \\
127.5 \\
60.5 \\
111.8 \\
3.7\end{array}$ & $\begin{array}{r}0.0 \\
0.0 \\
0.0 \\
0.0 \\
22.1 \\
7.6 \\
1.1 \\
23.1 \\
55.4 \\
87.1 \\
123.1 \\
234.3 \\
280.6 \\
351.1 \\
292.0 \\
331.8 \\
374.8 \\
317.8 \\
335.4 \\
411.9 \\
349.6 \\
286.7 \\
398.0 \\
159.4 \\
304.1 \\
9.9\end{array}$ & $\begin{array}{r}0.0 \\
0.0 \\
0.0 \\
0.0 \\
0.0 \\
0.0 \\
0.0 \\
0.0 \\
0.0 \\
15.4 \\
60.4 \\
50.1 \\
26.3 \\
50.1 \\
118.3 \\
131.4 \\
104.8 \\
239.4 \\
268.0 \\
315.8 \\
427.4 \\
415.0 \\
616.4 \\
609.9 \\
453.0 \\
0.0\end{array}$ & $\begin{array}{r}0.0 \\
0.0 \\
0.0 \\
0.0 \\
0.0 \\
0.0 \\
0.0 \\
0.0 \\
0.0 \\
0.0 \\
0.4 \\
0.5 \\
2.0 \\
1.3 \\
2.7 \\
5.4 \\
4.1 \\
24.1 \\
35.0 \\
51.8 \\
103.1 \\
189.3 \\
249.4 \\
257.1 \\
505.0 \\
5.6\end{array}$ & $\begin{array}{r}0.0 \\
0.0 \\
0.0 \\
0.0 \\
0.0 \\
0.0 \\
0.0 \\
0.0 \\
0.0 \\
0.0 \\
0.0 \\
0.0 \\
0.0 \\
0.0 \\
0.4 \\
0.0 \\
0.0 \\
0.4 \\
1.3 \\
0.0 \\
4.6 \\
15.2 \\
7.0 \\
64.2 \\
119.0 \\
0.0\end{array}$ & $\begin{array}{r}0.0 \\
0.0 \\
0.0 \\
0.0 \\
0.0 \\
0.0 \\
0.0 \\
0.0 \\
0.0 \\
0.0 \\
0.0 \\
0.0 \\
0.0 \\
0.0 \\
1.3 \\
0.5 \\
0.0 \\
0.0 \\
1.3 \\
0.0 \\
0.4 \\
0.0 \\
0.3 \\
3.4 \\
14.8 \\
0.0\end{array}$ & $\begin{array}{l}0.0 \\
0.0 \\
0.0 \\
0.0 \\
0.0 \\
0.0 \\
0.0 \\
0.0 \\
0.0 \\
0.0 \\
0.0 \\
0.0 \\
0.0 \\
0.0 \\
0.9 \\
0.0 \\
0.0 \\
0.0 \\
0.0 \\
0.0 \\
2.0 \\
0.4 \\
0.0 \\
0.0 \\
0.0 \\
0.0\end{array}$ & $\begin{array}{r}0.0 \\
0.0 \\
39.0 \\
44.5 \\
99.9 \\
67.1 \\
207.7 \\
321.8 \\
401.0 \\
466.9 \\
699.0 \\
721.2 \\
618.1 \\
675.9 \\
640.4 \\
772.2 \\
841.7 \\
861.3 \\
1,000.9 \\
1,113.8 \\
1,125.2 \\
1,226.7 \\
1,531.9 \\
1,297.7 \\
1,601.0 \\
935.1\end{array}$ \\
\hline OTAL. & 10.2 & 85.4 & 558.6 & $1,441.7$ & $1,018.5$ & $2,958.1$ & $4,756.9$ & $3,901.7$ & $1,436.7$ & 212.1 & 21.9 & 3.3 & $6,410.1$ \\
\hline
\end{tabular}

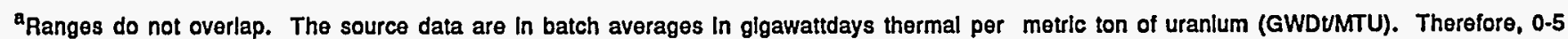
GWDU/MTU means 0 through 4.999 GWDUMTU; 5-10 GWDt/MTU means 5.000-9.999 GWDt/MTU; etc. See Technical Note 9 in Appendix E.

bsome data for prevlous years have been revised. Current-year data may be revised in future publlcatlons. When utllitles relnsert assemblles discharged In prevlous years, historical totals change. See Technical Note 12 in Appendlx E.

CTemps are temporarlly dlscharged assemblles, as of December 31, 1992, as reported on Form RW-859.

Includes 4.9 MTU for temporarily discharged PWR assemblies with missing burnup data. See Technical Note 10 in Appendlx E.

Notes: A total of 2,208 hlgh-temperature, gas-cooled reactor (HTGR) fuel elements, with Initlal uranlum content equal to $25.0 \mathrm{MTU}$, were discharged. These HTGR fuel elements are not Included in the above table. See Technical Note 6 in Appendlx E. Totals may not equal sum of components because of Independent rounding. See Technical Note 11 in Appendlx E.

Source: Energy Informatlon Adm!nlstration, Form RW-859, "Nuclear Fuel Data" (1992). 
Table 8. U.S. Burnup Distribution for All Discharged Assemblies

\begin{tabular}{|c|c|c|c|c|}
\hline $\begin{array}{c}\text { Burnup Ranges } \\
\text { (GWDU/MTU) }\end{array}$ & Assemblles & $\begin{array}{c}\text { Initial } \\
\text { Uranium Content } \\
\text { (MTU) }\end{array}$ & $\begin{array}{c}\text { Average } \\
\text { Burnup } \\
\text { (GWDt/MTU) }\end{array}$ & $\begin{array}{c}\text { Enrichment } \\
\text { (welght percent) }\end{array}$ \\
\hline $\begin{array}{r}0-5 \ldots \ldots \ldots \\
5-10 \ldots \ldots \\
10-15 \ldots \ldots \\
15-20 \ldots \ldots \\
20-25 \ldots \ldots \ldots \\
25-30 \ldots \ldots \\
30-35 \ldots \ldots \\
35-40 \ldots \ldots \\
40-45 \ldots \ldots \\
\end{array}$ & $\begin{array}{r}2,502 \\
2,481 \\
4,034 \\
7,741 \\
12,437 \\
15,988 \\
6,708 \\
700 \\
7\end{array}$ & $\begin{array}{r}460.7 \\
451.0 \\
730.4 \\
1,384.0 \\
2,279.7 \\
2,908.1 \\
1,209.8 \\
122.6 \\
1.3\end{array}$ & $\begin{array}{r}2.7 \\
8.4 \\
12.5 \\
17.8 \\
22.8 \\
27.3 \\
31.6 \\
36.0 \\
42.3\end{array}$ & $\begin{array}{l}1.46 \\
1.52 \\
2.06 \\
2.13 \\
2.39 \\
2.66 \\
2.88 \\
3.10 \\
2.62\end{array}$ \\
\hline TOTAL....... & $b_{52,602}$ & $9,547.6$ & 22.3 & 2.39 \\
\hline
\end{tabular}

\begin{tabular}{|c|c|c|c|c|}
\hline \multicolumn{5}{|c|}{ Pressurized-Water Reactors (PWR) } \\
\hline $\begin{array}{r}0-5 \ldots \ldots \ldots \\
5-10 \ldots \ldots \ldots \\
10-15 \ldots \ldots \\
15-20 \ldots \ldots \\
20-25 \ldots \ldots \ldots \\
25-30 \ldots \ldots \\
30-35 \ldots \ldots \\
35-40 \ldots \ldots \\
40-45 \ldots \ldots \\
45-50 \ldots \ldots \\
50-55 \ldots \ldots \\
55-60 \ldots \ldots \\
\end{array}$ & $\begin{array}{r}48 \\
224 \\
1,323 \\
3,289 \\
2,306 \\
7,002 \\
11,049 \\
9,122 \\
3,414 \\
507 \\
50 \\
8\end{array}$ & $\begin{array}{r}10.2 \\
85.4 \\
558.6 \\
1,441.7 \\
1,018.5 \\
2,958.1 \\
4,756.9 \\
3,901.7 \\
1,436.7 \\
212.1 \\
21.9 \\
3.3\end{array}$ & $\begin{array}{r}4.6 \\
8.7 \\
12.8 \\
17.5 \\
22.5 \\
27.6 \\
32.4 \\
37.0 \\
41.4 \\
46.4 \\
51.0 \\
56.2\end{array}$ & $\begin{array}{l}3.61 \\
3.08 \\
2.13 \\
2.18 \\
2.61 \\
2.86 \\
3.17 \\
3.39 \\
3.57 \\
3.68 \\
3.74 \\
3.54\end{array}$ \\
\hline TOTAL....... & ${ }^{c_{38,437}}$ & $d_{16,410.1}$ & 30.9 & 3.05 \\
\hline TOTAL........ & 91,039 & $25,957.7$ & 27.7 & 2.81 \\
\hline
\end{tabular}

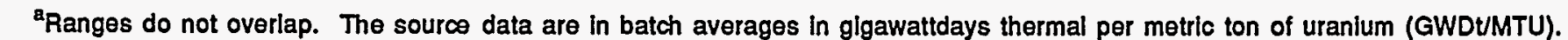
Therefore, 0-5 GWDt/MTU means 0 through 4.999 GWDt/MTU; 5-10 GWDt/MTU means 5.000-9.999 GWDt/MTU; etc. See Technical Note 9 In Appendlx E.

Wo assigned burnup range for 4 temporarily dlscharged BWR assemblies. These BWR assemblles are included in the Total columns. Seo Technical Note 10 in Appendix E.

'No assigned burnup range for 95 temporarily discharged PWR assemblies. These PWR assemblies are included in the Total columns. See Technical Note 10 in Appendix E.

IIncludes 4.9 MTU for temporarlly discharged PWR assemblles with no assigned burnup range. These welghts are included in the Total columns. See Technical Note 10 in Appendix E.

Notes: A total of 2,208 high-temperature, gas-cooled reactor (HTGR) fuel elements, with initial uranium content equal to 25.0 MTU, were discharged. These HTGR fuel elements are not included in the Total Reactors section of the table. See Technical Note 6 in Appendix E. Totals may not equal sum of components because of independent rounding. See Technical Note 11 in Appendlx E.

Source: Energy Information Administration, Form RW-859, "Nuclear Fuel Data" (1992). 
Table 9. Spent Fuel by Cladding and Reactor Type, 1968-1992

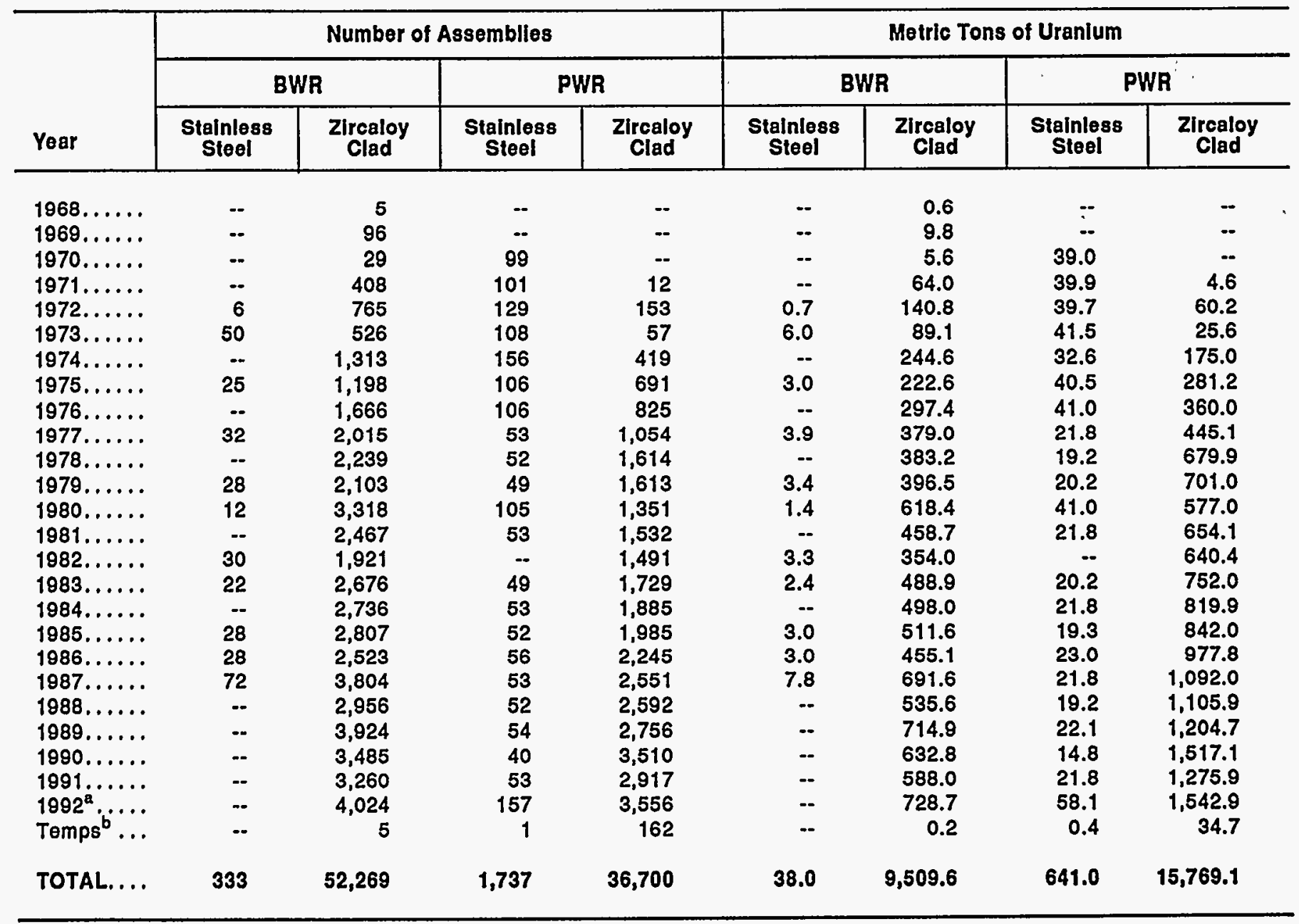

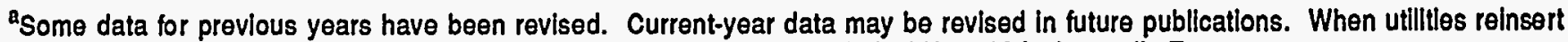
assemblies discharged In prevlous years, historical totals change. Seo Technical Note 12 in Appendix E.

${ }^{6}$ Temps are temporarily discharged assemblies, as of Decomber 31, 1992, as reported on Form RW-859.

BWA = Boilling-water reactor; PWR = Pressurized-water reactor.

-. = Not appllcable.

Notes: A total of 2,208 hlgh-temperature, gas-cooled reactor (HTGR) zircaloy clad fuel elements, with initial uranlum content equal to 25.0 metric tons of uranlum (MTU), were discharged. These HTGR fuel elements are not included in the above table. See Technical Note 6 In Appendix E. Totals may not equal sum of components because of independent rounding. See Technical Note 11 in Appendix $E$.

Source: Energy Information Administration, Form RW-859, "Nuclear Fuel Data" (1992). 
Table 10. Reactors Discharging Stainless Steel Clad Assemblies

\begin{tabular}{|c|c|c|c|c|c|c|c|c|}
\hline \multirow[b]{2}{*}{$\begin{array}{c}\text { Reactor } \\
\text { Name }\end{array}$} & \multicolumn{4}{|c|}{ Number of Assemblies } & \multicolumn{4}{|c|}{ Metrle Tons of Uranium } \\
\hline & $\begin{array}{c}\text { Stainless } \\
\text { Steel }\end{array}$ & $\begin{array}{c}\text { Zircaloy } \\
\text { Clad }\end{array}$ & $\begin{array}{c}\text { Stainless } \\
\text { Steol }\end{array}$ & $\begin{array}{c}\text { ZIrcaloy } \\
\text { Clad }\end{array}$ & $\begin{array}{l}\text { Stainless } \\
\text { Steel }\end{array}$ & $\begin{array}{c}\text { Zircaloy } \\
\text { Clad }\end{array}$ & $\begin{array}{l}\text { Stainless } \\
\text { Steel }\end{array}$ & $\begin{array}{c}\text { Zircaloy } \\
\text { Clad }\end{array}$ \\
\hline Haddam Neck .......... & -- & -- & 836 & 4 & -- & -- & 345.2 & 1.5 \\
\hline Indian Point $1 \ldots \ldots \ldots$ & -- & $\cdots$ & 160 & -- & - & - & 30.6 & -- \\
\hline Yankee Rowe ........... & -- & -- & 76 & 457 & -- & -- & 20.8 & 106.5 \\
\hline TOTAL $\ldots \ldots \ldots \ldots \ldots$ & 333 & $\ldots$ & 1,737 & 461 & 38.0 & -. & 641.0 & 107.9 \\
\hline
\end{tabular}

BWR = Boiling-water reactor; PWR = Pressurized-water reactor.

- = Not appllcable.

Note: Totals may not equal sum of components because of independent rounding. See Technical Note 11 in Appendix E.

Source: Energy Information Administration, Form RW-859, "Nuclear Fuel Data" (1992). 


\section{Enrichment and Burnup}

The distribution of spent fuel assemblies discharged through 1992 by enrichment (weight percent) and burnup ranges is shown in Table 11. Separate data are displayed for each combination of reactor type (BWR and PWR) and cladding (zircaloy and stainless steel). Enrichments (weight percents) for stainless steel clad assemblies are higher than those for zircaloy clad assemblies, because stainless steel is a neutron poison. (Four PWR mixed-oxide assemblies were discharged from San Onofre 1. These assemblies are shown in the PWR stainless steel category in the 0.6 to 1.0 range, as the plutonium content of these assemblies is not reflected in the table.)

\section{Utility Shipments to Away-from-reactor Storage Facilities}

Spent fuel shipments may be of two types, both of which are regulated by the U.S. Nuclear Regulatory Commission and are monitored by State and local governments. The first is the transfer of spent fuel assemblies within an electric utility. These transfers allow for the unused spent fuel storage capacity at one site to be used as additional storage for a reactor that may be running low on spent fuel storage capability. They also help utilities to use the available capacity efficiently and delay the need to build additional storage capabilities. These intrautility transfers are not covered in this report because they have no effect on a particular utility's storage capability. A second type of shipment is from a utility to an away-from-reactor storage facility (Table 12). There were no shipments of spent fuel from a utility to a storage facility in 1992. 
Table 11. Burnup Versus Enrichment by Cladding and Reactor Type (in Assemblies)

\begin{tabular}{|c|c|c|c|c|c|c|c|c|c|c|c|c|c|}
\hline \multirow{2}{*}{$\begin{array}{l}\text { Enrichment } \\
\text { (weight percent) }^{a}\end{array}$} & \multicolumn{13}{|c|}{ Burnup Ranges (GWDt/MTU) ${ }^{\mathfrak{a}}$} \\
\hline & $0-5$ & $5-10$ & $10-15$ & $15-20$ & $20-25$ & 25-30 & $30-35$ & $35-40$ & $40-45$ & $45-50$ & $50-55$ & $55-60$ & Total \\
\hline & \multicolumn{13}{|c|}{ Boiling-Water Reactor: Stainless Steel } \\
\hline $0.0-0.6 \ldots \ldots \ldots$ & $-\infty$ & -- & - & - & -- & -- & - & - & -- & - & -- & - & - \\
\hline $0.6-1.0 \ldots \ldots \ldots$ & -- & $\cdots$ & - & -- & -- & -- & - & - & - & - & -- & -- & - \\
\hline $1.0-1.4 \ldots \ldots \ldots$ & - & - & $\cdots$ & - & -- & -- & -- & - & - & - & -- & - & - \\
\hline $1.4-1.8 \ldots \ldots \ldots \ldots$ & -- & - & -- & - & - & -- & - & - & $-\infty$ & - & -- & -- & - \\
\hline $1.8-2.2 \ldots \ldots \ldots \ldots$ & $\cdots$ & -- & $\cdots$ & - & -- & -- & - & - & - & - & -- & -- & - \\
\hline $2.2-2.6 \ldots \ldots \ldots \ldots$ & $\cdots$ & -- & -- & - & - & -- & -- & -- & -- & -. & - & -- & -- \\
\hline $2.6-3.0 \ldots \ldots \ldots$ & -- & $-\cdots$ & - & - & - & -- & - & -- & -- & - & - & - & - \\
\hline $3.0-3.4 \ldots \ldots \ldots \ldots$ & -- & $\ddot{n}$ & $\ddot{n}$ & - & -- & -- & -- & -- & -- & -- & - & -- & - \\
\hline $3.4-3.8 \ldots \ldots \ldots$ & -- & 25 & 88 & 143 & 4 & -- & -- & -- & -- & - & - & - & 260 \\
\hline $3.8-4.2 \ldots \ldots \ldots \ldots$ & -- & 2 & 32 & 39 & -- & -- & - & - & - & - & - & - & 73 \\
\hline $4.2-4.6 \ldots \ldots \ldots \ldots$ & -- & - & - & - & - & -- & -- & -- & -- & $\cdots$ & -- & -- & $\cdots$ \\
\hline $4.6-5.0 \ldots \ldots \ldots$ & -- & -- & -- & - & -- & -- & - & -- & - & - & - & -- & - \\
\hline \multirow[t]{2}{*}{ TOTAL ......... } & - & 27 & 120 & 182 & 4 & - & - & - & - & - & - & - & 333 \\
\hline & \multicolumn{13}{|c|}{ Boiling-Water Reactor: Zircaloy Clad } \\
\hline $0.0-0.6 \ldots \ldots \ldots$ & - & - & -- & - & - & -- & - & - & - & -- & - & - & $b_{4}$ \\
\hline $0.6-1.0 \ldots \ldots \ldots \ldots$ & 1,060 & 536 & 140 & -- & -- & - & - & -- & - & -- & -- & -- & 1,736 \\
\hline $1.0-1.4 \ldots \ldots \ldots \ldots$ & $\cdots$ & 932 & 277 & 108 & -- & -- & -- & - & - & - & -- & - & 1,317 \\
\hline $1.4-1.8 \ldots \ldots \ldots$ & 4 & 172 & 188 & 388 & 37 & - & - & - & - & - & - & - & 789 \\
\hline $1.8-2.2 \ldots \ldots \ldots \ldots$ & 1,314 & 231 & 2,246 & 4,245 & 2,498 & 180 & - & 1 & - & - & -- & -- & 10,715 \\
\hline $2.2-2.6 \ldots \ldots \ldots \ldots$ & 118 & 526 & 525 & 2,508 & 7,301 & 3,773 & 75 & 56 & - & - & -- & -- & 14,882 \\
\hline $2.6-3.0 \ldots \ldots \ldots \ldots$ & - & 49 & 260 & 261 & 2,381 & 11,097 & 4,092 & 131 & 7 & - & - & - & 18,278 \\
\hline $3.0-3.4 \ldots \ldots \ldots \ldots$ & -- & - & 198 & 8 & 124 & 884 & 2,535 & 512 & - & - & -- & - & 4,261 \\
\hline $3.4-3.8 \ldots \ldots \ldots \ldots$ & 6 & 8 & 80 & 41 & 92 & 40 & 6 & -- & - & - & -- & - & 273 \\
\hline $3.8-4.2 \ldots \ldots \ldots \ldots$ & -- & -- & -- & -- & $\cdots$ & 14 & - & - & -- & -- & -- & - & 14 \\
\hline $4.2-4.6 \ldots \ldots \ldots \ldots$ & -- & -- & -- & -- & - & - & -- & - & - & - & - & - & - \\
\hline $4.6-5.0 \ldots \ldots \ldots$ & -- & -- & -- & $-\cdots$ & - & - & -- & -- & - & - & - & - & - \\
\hline TOTAL ......... & 2,502 & 2,454 & 3,914 & 7,559 & 12,433 & 15,988 & 6,708 & 700 & 7 & - & - & - & 52,269 \\
\hline
\end{tabular}

See footnotes at end of table. 
Table 11. Burnup Versus Enrichment by Cladding and Reactor Type (in Assemblies) (Continued)

\begin{tabular}{|c|c|c|c|c|c|c|c|c|c|c|c|c|c|}
\hline \multirow{2}{*}{$\begin{array}{l}\text { Enrichment } \\
\text { (weight percent) }^{\mathbf{a}}\end{array}$} & \multicolumn{13}{|c|}{ Burnup Ranges (GWDt/MTU) ${ }^{a}$} \\
\hline & $0-5$ & $5-10$ & $10-15$ & $15-20$ & $20-25$ & $25-30$ & $30-35$ & $35-40$ & $40-45$ & $45-50$ & $50-55$ & $55-60$ & Total \\
\hline & \multicolumn{13}{|c|}{ Pressurized-Water Reactor: Stainless Steel } \\
\hline $0.0-0.6 \ldots \ldots \ldots$ & - & -- & -- & - & - & - & - & - & - & - & - & - & - \\
\hline $0.6-1.0 \ldots \ldots \ldots$ & $\cdots$ & -- & -- & 4 & - & - & -- & - & - & -- & -- & - & 4 \\
\hline $1.0-1.4 \ldots \ldots \ldots$ & -- & -- & -- & - & - & - & - & - & - & - & - & - & - \\
\hline $1.4-1.8 \ldots \ldots \ldots \ldots$ & - & -- & -- & - & - & -- & - & - & -- & $\cdots$ & - & - & - \\
\hline $1.8-2.2 \ldots \ldots \ldots \ldots$ & - & -- & -- & - & - & - & - & - & -- & - & - & - & - \\
\hline $2.2-2.6 \ldots \ldots \ldots \ldots$ & -- & -- & -- & - & - & -- & - & - & -- & - & $\cdots$ & - & - \\
\hline $2.6-3.0 \ldots \ldots \ldots$ & $-\cdots$ & -- & -- & - & - & -- & - & - & -- & -- & $\cdots$ & - & - \\
\hline $3.0-3.4 \ldots \ldots \ldots \ldots$ & -- & -- & - & 95 & 8 & 46 & - & - & -- & -- & - & - & 149 \\
\hline $3.4-3.8 \ldots \ldots \ldots \ldots$ & -- & $\ddot{-}$ & 4 & $\bar{n}$ & 10 & 90 & 53 & 4 & -- & -- & -- & -- & 161 \\
\hline $3.8-4.2 \ldots \ldots \ldots \ldots$ & -- & 2 & 85 & 52 & 41 & 197 & 798 & 132 & -- & -- & -- & -- & 1,307 \\
\hline $4.2-4.6 \ldots \ldots \ldots \ldots$ & 40 & -- & $\cdots$ & - & - & $\overline{--}$ & - & -- & -- & -- & -- & -- & 40 \\
\hline $4.6-5.0 \ldots \ldots \ldots$ & -- & -- & - & - & 32 & 36 & 8 & - & -- & - & - & - & 76 \\
\hline \multirow[t]{2}{*}{ TOTAL ......... } & 40 & 2 & 89 & 151 & 91 & 369 & 859 & 136 & - & - & - & - & 1,737 \\
\hline & \multicolumn{13}{|c|}{ Pressurized-Water Reactor: Zircaloy Clad } \\
\hline $0.0-0.6 \ldots \ldots \ldots$ & -- & -- & $\cdots$ & - & - & - & -- & -- & - & - & - & - & ${ }^{c} 95$ \\
\hline $0.6-1.0 \ldots \ldots \ldots \ldots$ & -- & -- & -- & - & - & - & - & - & -- & - & $\cdots$ & - & -- \\
\hline $1.0-1.4 \ldots \ldots \ldots \ldots$ & -- & $\cdots$ & -- & - & $\overline{-}$ & - & - & -. & -- & - & -- & - & - \\
\hline $1.4-1.8 \ldots \ldots \ldots \ldots$ & - & 25 & 418 & 78 & 7 & - & - & -- & -- & - & -- & - & 528 \\
\hline $1.8-2.2 \ldots \ldots \ldots \ldots$ & 7 & 3 & 570 & 2,116 & 227 & 97 & - & -- & -- & - & -- & -- & 3,020 \\
\hline $2.2-2.6 \ldots \ldots \ldots \ldots$ & -- & -- & 69 & 796 & 948 & 993 & 5 & -- & 1 & $-\overline{-}$ & - & - & 2,812 \\
\hline $2.6-3.0 \ldots \ldots \ldots \ldots$ & -- & -- & 4 & 57 & 631 & 3,456 & 2,516 & 176 & 82 & 5 & -- & -- & 6,927 \\
\hline $3.0-3.4 \ldots \ldots \ldots \ldots$ & -- & 98 & 126 & 64 & 319 & 1,630 & 6,363 & 4,037 & 505 & 24 & 5 & 1 & 13,172 \\
\hline $3.4-3.8 \ldots \ldots \ldots \ldots$ & 1 & 56 & 36 & 18 & 66 & 346 & 1,218 & 4,105 & 1,921 & 236 & 11 & 6 & 8,020 \\
\hline $3.8-4.2 \ldots \ldots \ldots \ldots$ & $-\infty$ & 40 & 11 & 9 & 11 & 111 & 88 & 668 & 905 & 223 & 29 & 1 & 2,096 \\
\hline $4.2-4.6 \ldots \ldots \ldots \ldots$ & -- & -- & -- & - & 6 & - & - & -- & - & 19 & 5 & - & 30 \\
\hline $4.6-5.0 \ldots \ldots \ldots \ldots$ & $\cdots$ & -- & -- & - & - & - & - & $\cdots$ & $\cdots$ & $\cdots$ & $\cdots$ & $\cdots$ & - \\
\hline TOTAL ......... & 8 & 222 & 1,234 & 3,138 & 2,215 & 6,633 & 10,190 & 8,986 & 3,414 & 507 & 50 & 8 & 36,700 \\
\hline
\end{tabular}

a Ranges do not overlap. See Technical Note 9 in Appendix E

Wo enrichment (weight percent) is reported for 4 temporarily discharged BWR assemblies. These BWR assemblies are included in the Total Column. See Technical Note 10 in Appendix $E$.

No enrichment (weight percent) is reported for 95 temporarily discharged PWR assemblies. These PWR assemblies are included in the Total Column. See Technical Note 10 in Appendix E.

GWDUMTU = Gigawattdays thermal per metric ton of uranium

- - Not applicable.

Note: A total of 2,208 high-temperature, gas-cooled reactor (HTGR) fuel elements, with initial uranium content equal to $25.0 \mathrm{MTU}$, were discharged. These HTGR fuel elements are not included in the above table. See Technical Note 6 in Appendix E. 
Table 12. Fuel Stored in Away-from-reactor Facilities

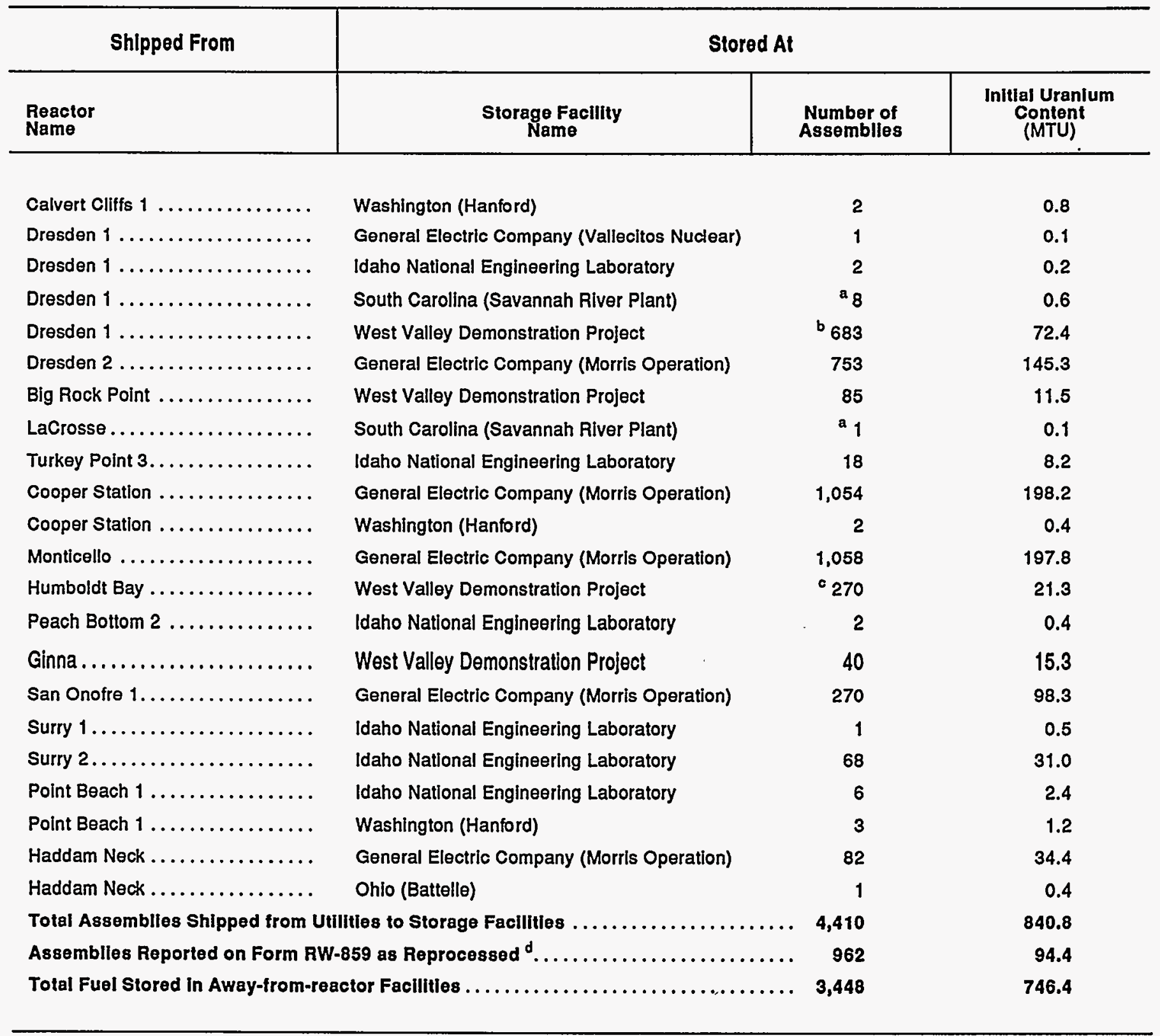

${ }^{a} A l l$ assemblies shipped to South Carolina (Savannah River Plant) have been reprocessed.

b Assemblies shipped from Dresden 1 to West Valley Demonstration Project have since been reprocessed.

${ }^{c}$ Assemblies shipped from Humboldt Bay to West Valley Demonstration Project have since been reprocessed.

Complete historical data are not available for reprocessed assemblies. See Technical Note 7 in Appendix E.

Notes: A total of 744 high-temperature, gas-cooled reactor (HTGR) fuel elements, with initial uranium content equal to 8.9 metric tons of uranium (MTU), were shipped from Fort St. Vrain to Idaho National Engineering Laboratory. These HTGR fuel elements are not included In the above table. See Technical Note 6 in Appendix E. Round-trip shipments are not Included in this table. Totals may not equal sum of components because of independent rounding. See Technical Note 11 in Appendix $E$.

Source: Energy Information Administration, Form RW-859, "Nuclear Fuel Data" (1992). 


\section{Site Capacities, Inventories, and Dry Storage at Utilities and Storage Facilities}

\section{Site Capacities and Inventories at Utilities}

The total licensed capacity for storage of spent nuclear fuel in the United States as of December 31, 1992, was 205,731 slots or cells; each slot is capable of holding one assembly (Table 13). The reported maximum capacity was 215,989 including 3,932 dry storage slots, 3,061 off-site slots, and 208,996 on-site slots in storage pools. These totals change slightly as the calculation assumptions change. Footnotes $h$ and $i$ of Table 13 explain the assumptions used in calculating this total.

The respondents were asked to report their maximum established spent fuel storage capacity for each site (in number of assemblies). The definition used by EIA was "the maximum number of intact assemblies that will be able to be stored at some point in the future (between the reporting date and the reactor's end of life) considering any established or current studies or engineering evaluations, at the time of submittal for licensing approval from the U.S. Nuclear Regulatory Commission (NRC)" (see Technical Note 13). The maximum storage capacity can change from year to year as reflected in Table 13 and the pictograms in Appendix $C$.

Many electric utilities are in the process of reracking their storage pools to achieve a higher maximum capacity; some have inaccessible slots that lower their maximum capacity. For example, the Duke Power Company's Oconee 3 power plant storage pool has four slots which are currently unusable because of equipment interference in addition to three slots which will never be used for spent nuclear fuel. The Northern States Power Company's Monticello power plant storage pool has a maximum established spent fuel storage capacity listed as 2,210 assemblies. This figure does not include seven slots which will never be used for spent nuclear fuel. An additional 20 slots are used for storing nonfuel bearing components (control blades) and are also unusable at this time.
Reworking of these 20 cells may produce a maximum established spent fuel storage capacity of 2,230 in the future.

The total initial uranium content of assemblies in storage is 25,958 metric tons of uranium (MTU). The total inventory of light-water reactor (LWR) spent nuclear fuel in storage in the United States as of December 31,1992 , is 91,039 assemblies (Table 13). Of these, 87,591 assemblies are in storage at 117 reactors that have been or are discharging and/or storing nuclear fuel assemblies, including 903 assemblies in the Independent Spent Fuel Storage Installations (ISFSIs) at Virginia Power's Surry plant, Carolina Power and Light Company's Robinson 2 plant, and Duke Power Company's Oconee plant. An additional 3,448 assemblies have been shipped to away-from-reactor storage facilities.

\section{Site Capacities and Inventories at Storage Facilities}

More than 99 percent of the assemblies at U.S. awayfrom-reactor storage facilities are located at General Electric Morris (Morris, Illinois), DOE's Idaho National Engineering Laboratory (Idaho Falls, Idaho), and the West Valley Demonstration Project (West Valley, New York) (Table 14). There are seven assemblies stored at Hanford, Washington, and one each at Columbus, Ohio, and Vallecitos, California. Table 15 corresponds to the maps which list all spent fuel in storage by State (Figures ES2 and ES3). LWR spent nuclear fuel is stored in 34 States (Figure ES2). Additionally, high-temperature, gas-cooled reactor (HTGR) spent fuel elements are currently being stored in Colorado and Idaho. The data in Table 15 and both Figures ES2 and ES3 include all permanently discharged assemblies and all temporarily discharged assemblies with uranium content that are scheduled to be reinserted at a later date. The away-from-reactor storage facilities are also included in the State totals. 
Table 13. Site Capacities and Inventories at Nuclear Power Plants as of December 31, 1992

\begin{tabular}{|c|c|c|c|c|c|c|c|c|}
\hline Electric UtIlity Name & $\begin{array}{l}\text { Reactor/Storage } \\
\text { Site Name }\end{array}$ & $\begin{array}{l}\text { Reactor } \\
\text { Type }\end{array}$ & $\begin{array}{l}\text { Pool } \\
\text { Site } \\
\text { ID }\end{array}$ & $\begin{array}{l}\text { Licensed } \\
\text { Storage } \\
\text { Capacitya }\end{array}$ & $\begin{array}{l}\text { Maximum } \\
\text { Establlshed } \\
\text { Storage } \\
\text { Capacity }\end{array}$ & $\begin{array}{c}\text { Current } \\
\text { Inventory }\end{array}$ & $\begin{array}{c}\text { Initlal Uranlum } \\
\text { Content of } \\
\text { Pool Inventory } \\
\text { (MTU) }\end{array}$ & $\begin{array}{l}\text { Number of } \\
\text { Temporarily } \\
\text { Discharged } \\
\text { Assemblies }^{d}\end{array}$ \\
\hline Alabama Power Company .. & $\begin{array}{l}\text { Farley } 1 \\
\text { Farley } 2\end{array}$ & $\begin{array}{l}\text { PWR } \\
\text { PWR }\end{array}$ & $\begin{array}{l}0101 \\
0102\end{array}$ & $\begin{array}{l}1,407 \\
1,407\end{array}$ & $\begin{array}{l}1,407 \\
1,407\end{array}$ & $\begin{array}{l}655 \\
498\end{array}$ & $\begin{array}{l}302.0 \\
229.6\end{array}$ & $\begin{array}{l}0 \\
0\end{array}$ \\
\hline $\begin{array}{l}\text { Arizona Public Service } \\
\text { Company } \ldots \ldots \ldots \ldots \ldots \ldots\end{array}$ & $\begin{array}{l}\text { Palo Verde } 1 \\
\text { Palo Verde } 2 \\
\text { Palo Verde } 3\end{array}$ & $\begin{array}{l}\text { PWR } \\
\text { PWR } \\
\text { PWR }\end{array}$ & $\begin{array}{l}0301 \\
0302 \\
0303\end{array}$ & $\begin{array}{l}665 \\
665 \\
665\end{array}$ & $\begin{array}{l}1,323 \\
1,323 \\
1,322\end{array}$ & $\begin{array}{l}276 \\
288 \\
284\end{array}$ & $\begin{array}{l}113.7 \\
118.1 \\
115.9\end{array}$ & $\begin{array}{l}0 \\
0 \\
1\end{array}$ \\
\hline $\begin{array}{l}\text { Arkansas Power and } \\
\text { Light Company.......... }\end{array}$ & $\begin{array}{l}\text { Arkansas Nuclear } 1 \\
\text { Arkansas Nuclear } 2\end{array}$ & $\begin{array}{l}\text { PWR } \\
\text { PWR }\end{array}$ & $\begin{array}{l}0401 \\
0402\end{array}$ & $\begin{array}{l}968 \\
988\end{array}$ & $\begin{array}{l}948 \\
933\end{array}$ & $\begin{array}{l}624 \\
564\end{array}$ & $\begin{array}{l}288.9 \\
234.6\end{array}$ & $\begin{array}{l}0 \\
0\end{array}$ \\
\hline $\begin{array}{l}\text { Baltimore Gas and } \\
\text { Electric Company......... }\end{array}$ & $\begin{array}{l}\text { Calvert Cliffs } 1 \\
\text { Calvert Cliffs } 2\end{array}$ & $\begin{array}{l}\text { PWR } \\
\text { PWR }\end{array}$ & 0501 & $\begin{array}{r}830 \\
1,000\end{array}$ & $\begin{array}{l}800 \\
988\end{array}$ & $\begin{array}{l}798 \\
612\end{array}$ & $\begin{array}{l}306.4 \\
235.0\end{array}$ & $\begin{array}{l}0 \\
0\end{array}$ \\
\hline Boston Edison Company ... & Pilgrim 1 & BWR & 0601 & 2,320 & 2,304 & 1,488 & 276.8 & 0 \\
\hline $\begin{array}{l}\text { Carolina Power and Light } \\
\text { Company } \ldots \ldots \ldots \ldots \ldots \ldots\end{array}$ & $\begin{array}{l}{ }^{\circ} \text { Brunswick } 1 \\
\text { Brunswick } 1 \\
{ }^{\circ} \text { Brunswick } 2 \\
\text { Brunswick } 2 \\
\text { 'Harris } 1 \\
{ }^{9} \text { Harris } 1 \\
\text { Robinson } 2 \\
\text { Dry Storage }\end{array}$ & $\begin{array}{l}\text { BWR } \\
\text { fPWR } \\
\text { BWR } \\
\text { fPWR } \\
\text { PWR } \\
\text { BWR } \\
\text { PWR } \\
\text { PWR }\end{array}$ & $\begin{array}{l}0701 \\
0701 \\
0702 \\
0702 \\
0703 \\
0703 \\
0705 \\
0705 D\end{array}$ & $\begin{array}{r}1,803 \\
160 \\
1,839 \\
144 \\
4,184 \\
5,808 \\
544 \\
56\end{array}$ & $\begin{array}{r}1,803 \\
160 \\
1,839 \\
144 \\
1,832 \\
2,541 \\
537 \\
56\end{array}$ & $\begin{array}{r}1,090 \\
160 \\
1,113 \\
144 \\
420 \\
413 \\
212 \\
56\end{array}$ & $\begin{array}{r}200.8 \\
71.4 \\
205.9 \\
65.5 \\
187.1 \\
76.8 \\
91.4 \\
24.1\end{array}$ & $\begin{array}{l}1 \\
0 \\
0 \\
0 \\
0 \\
0 \\
0 \\
0\end{array}$ \\
\hline $\begin{array}{l}\text { Cleveland Electric } \\
\text { Illuminating Company ...... }\end{array}$ & Perry 1 & $\begin{array}{l}\text { BWR } \\
\text { BWR }\end{array}$ & $\begin{array}{l}0901 \\
0902\end{array}$ & $\begin{array}{l}2,400 \\
1,620\end{array}$ & $\begin{array}{l}2,400 \\
1,620\end{array}$ & $\begin{array}{r}748 \\
0\end{array}$ & $\begin{array}{r}137.6 \\
0.0\end{array}$ & $\begin{array}{l}4 \\
0\end{array}$ \\
\hline
\end{tabular}

See footnotes at end of table. 
Table 13. Site Capacities and Inventories at Nuclear Power Plants as of December 31, 1992 (Continued)

\begin{tabular}{|c|c|c|c|c|c|c|c|c|}
\hline Electric UtIlity Name & $\begin{array}{l}\text { Reactor/Storage } \\
\text { Site Name }\end{array}$ & $\begin{array}{l}\text { Reactor } \\
\text { Type }\end{array}$ & $\begin{array}{l}\text { Pool } \\
\text { Site } \\
\text { ID }\end{array}$ & $\begin{array}{l}\text { Llcensed } \\
\text { Storage } \\
\text { Capacity }\end{array}$ & $\begin{array}{c}\text { Maximum } \\
\text { Established } \\
\text { Storage } \\
\text { Capacityb }\end{array}$ & $\begin{array}{c}\text { Current } \\
\text { Inventory }\end{array}$ & $\begin{array}{c}\text { Initial Uranium } \\
\text { Content of } \\
\text { Pool Inventory } \\
\text { (MTU) }\end{array}$ & $\begin{array}{l}\text { Number of } \\
\text { Temporarily } \\
\text { Discharged } \\
\text { Assemblies }\end{array}$ \\
\hline $\begin{array}{l}\text { Commonwealth Edison } \\
\text { Company ............. }\end{array}$ & $\begin{array}{l}\text { Braidwood } 1 \\
\text { Braidwood } 2 \\
\text { Byron } 1 \\
\text { Byron } 2 \\
\text { Dresden } 1 \\
\text { Dresden } 2 \\
\text { Dresden } 3 \\
\text { LaSalle County } 1 \\
\text { LaSalle County } 2 \\
\text { Quad Cities } 1 \\
\text { Quad Cities } 2 \\
\text { Zion } 1 \\
\text { Zion } 2\end{array}$ & $\begin{array}{l}\text { PWR } \\
\text { PWR } \\
\text { PWR } \\
\text { PWR } \\
\text { BWR } \\
\text { BWR } \\
\text { BWR } \\
\text { BWR } \\
\text { BWR } \\
\text { BWR } \\
\text { BWR } \\
\text { PWR } \\
\text { PWR }\end{array}$ & $\begin{array}{l}1001 \\
1003 \\
1005 \\
1006 \\
1007 \\
1008 \\
1010 \\
\\
1012\end{array}$ & $\begin{array}{r}2,870 \\
\\
2,870 \\
\\
720 \\
3,537 \\
3,537 \\
5,153 \\
0 \\
7,554 \\
0 \\
2,112\end{array}$ & $\begin{array}{r}2,834 \\
\\
2,824 \\
\\
720 \\
3,537 \\
3,537 \\
8,083 \\
0 \\
7,551 \\
0 \\
3,012\end{array}$ & $\begin{array}{r}404 \\
596 \\
\\
683 \\
1,954 \\
1,968 \\
1,920 \\
0 \\
3,996 \\
0 \\
1,608\end{array}$ & \begin{tabular}{r|}
171.6 \\
253.2 \\
\\
69.7 \\
353.0 \\
355.6 \\
351.4 \\
0.0 \\
737.2 \\
0.0 \\
734.0
\end{tabular} & $\begin{array}{l}0 \\
0 \\
0 \\
0 \\
0 \\
0 \\
0 \\
0 \\
0 \\
0\end{array}$ \\
\hline $\begin{array}{l}\text { Consolidated Edison } \\
\text { Company of New York .... }\end{array}$ & $\begin{array}{l}\text { Indian Point } 1 \\
\text { Indian Point } 2\end{array}$ & $\begin{array}{l}\text { PWR } \\
\text { PWR }\end{array}$ & $\begin{array}{l}1101 \\
1102\end{array}$ & $\begin{array}{r}756 \\
1,374\end{array}$ & $\begin{array}{r}756 \\
1,374\end{array}$ & $\begin{array}{l}160 \\
668\end{array}$ & $\begin{array}{r}30.6 \\
303.3\end{array}$ & $\begin{array}{l}0 \\
8\end{array}$ \\
\hline $\begin{array}{l}\text { Consumers Power } \\
\text { Company } . \ldots \ldots \ldots \ldots \ldots\end{array}$ & $\begin{array}{l}\text { Big Rock Point } \\
\text { Pallsades }\end{array}$ & $\begin{array}{l}\text { BWR } \\
\text { PWR }\end{array}$ & $\begin{array}{l}1201 \\
1204\end{array}$ & $\begin{array}{l}441 \\
892\end{array}$ & $\begin{array}{r}441 \\
1,075\end{array}$ & $\begin{array}{l}294 \\
733\end{array}$ & $\begin{array}{r}38.5 \\
292.5\end{array}$ & $\begin{array}{l}0 \\
1\end{array}$ \\
\hline $\begin{array}{l}\text { Dairyland Power } \\
\text { Cooperative } \ldots \ldots \ldots \ldots \text {. }\end{array}$ & LaCrosse & BWR & 1301 & 440 & 440 & 333 & 38.0 & 0 \\
\hline Detroit Edison Company.... & Enrico Fermi 2 & BWR & 1402 & 2,383 & 2,383 & 672 & 121.6 & 0 \\
\hline Duke Power Company ..... & $\begin{array}{l}\text { Catawba } 1 \\
\text { Catawba } 2 \\
\text { McGuire } 1 \\
\text { McGuire } 2 \\
\text { Oconee } 1 \\
\text { Oconee } 2 \\
\text { Oconee } 3 \\
\text { Dry Storage }\end{array}$ & $\begin{array}{l}\text { PWR } \\
\text { PWR } \\
\text { PWR } \\
\text { PWR } \\
\text { PWR } \\
\text { PWR } \\
\text { PWR } \\
\text { PWR }\end{array}$ & $\begin{array}{l}1501 \\
1502 \\
1504 \\
1505 \\
1506 \\
\\
1508 \\
15060\end{array}$ & $\begin{array}{r}1,419 \\
1,418 \\
1,463 \\
1,463 \\
1,312 \\
\\
825 \\
2,112\end{array}$ & $\begin{array}{r}2,615 \\
2,615 \\
1,387 \\
1,381 \\
1,311 \\
\\
818 \\
2,112\end{array}$ & $\begin{array}{r}408 \\
280 \\
519 \\
741 \\
1,014 \\
\\
\\
516 \\
480\end{array}$ & $\begin{array}{l}173.0 \\
119.0 \\
228.4 \\
331.9 \\
470.0 \\
\\
239.2 \\
222.7\end{array}$ & $\begin{array}{l}0 \\
0 \\
0 \\
0 \\
0 \\
0 \\
0\end{array}$ \\
\hline
\end{tabular}

See footnotes at end of table. 
Table 13. Site Capacities and Inventories at Nuclear Power Plants as of December 31, 1992 (Continued)

\begin{tabular}{|c|c|c|c|c|c|c|c|c|}
\hline Electric Utility Name & $\begin{array}{l}\text { Reactor/Storage } \\
\text { Site Name }\end{array}$ & $\begin{array}{l}\text { Reactor } \\
\text { Type }\end{array}$ & $\begin{array}{l}\text { Pool } \\
\text { Site } \\
\text { ID }\end{array}$ & $\begin{array}{l}\text { Licensed } \\
\text { Storage } \\
\text { Capacily }^{a}\end{array}$ & $\begin{array}{l}\text { Maximum } \\
\text { Established } \\
\text { Storage } \\
\text { Capacity }^{b}\end{array}$ & $\begin{array}{c}\text { Current } \\
\text { Inventory }\end{array}$ & $\begin{array}{c}\text { Initial Uranium } \\
\text { Content of } \\
\text { Pool Inventory } \\
\text { (MTU) }\end{array}$ & $\begin{array}{l}\text { Number of } \\
\text { Temporarily } \\
\text { Discharged } \\
\text { Assemblies }^{\mathrm{d}}\end{array}$ \\
\hline Duquesne Light Company .. & $\begin{array}{l}\text { Beaver Valley } 1 \\
\text { Beaver Valley } 2\end{array}$ & $\begin{array}{l}\text { PWR } \\
\text { PWR }\end{array}$ & $\begin{array}{l}1601 \\
1602\end{array}$ & $\begin{array}{r}833 \\
1,088\end{array}$ & $\begin{array}{l}1,621 \\
1,088\end{array}$ & $\begin{array}{l}512 \\
192\end{array}$ & $\begin{array}{r}236.0 \\
88.5\end{array}$ & $\begin{array}{r}14 \\
5\end{array}$ \\
\hline Florida Power Corporation... & Crystal River 3 & PWR & 1701 & 1,357 & 1,357 & 536 & 248.7 & 5 \\
\hline $\begin{array}{l}\text { Florida Power and Light } \\
\text { Company .............. }\end{array}$ & $\begin{array}{l}\text { St. Lucie } 1 \\
\text { St. Lucie } 2 \\
\text { Turkey Point } 3 \\
\text { Turkey Point } 4\end{array}$ & $\begin{array}{l}\text { PWR } \\
\text { PWR } \\
\text { PWR } \\
\text { PWR }\end{array}$ & $\begin{array}{l}1801 \\
1802 \\
1803 \\
1804\end{array}$ & $\begin{array}{l}1,706 \\
1,584 \\
1,404 \\
1,404\end{array}$ & $\begin{array}{l}1,705 \\
1,076 \\
1,367 \\
1,376\end{array}$ & $\begin{array}{l}796 \\
464 \\
588 \\
546\end{array}$ & $\begin{array}{l}300.1 \\
175.9 \\
267.5 \\
248.4\end{array}$ & $\begin{array}{l}0 \\
0 \\
0 \\
0\end{array}$ \\
\hline GPU Nuclear Corporation... & $\begin{array}{l}\text { Three Mile Island } 1 \\
\text { Oyster Creek }\end{array}$ & $\begin{array}{l}\text { PWR } \\
\text { BWR }\end{array}$ & $\begin{array}{l}1901 \\
1903\end{array}$ & $\begin{array}{r}752 \\
2,600\end{array}$ & $\begin{array}{l}1,284 \\
2,600\end{array}$ & $\begin{array}{r}521 \\
1,876\end{array}$ & $\begin{array}{l}238.1 \\
343.3\end{array}$ & $\begin{array}{l}8 \\
0\end{array}$ \\
\hline Georgia Power Company... & $\begin{array}{l}\text { Hatch } 1 \\
\text { Hatch } 2 \\
\text { Vogtle } 1 \\
\text { Vogtle } 2\end{array}$ & $\begin{array}{l}\text { BWR } \\
\text { BWR } \\
\text { PWR } \\
\text { PWR }\end{array}$ & $\begin{array}{l}2001 \\
2003\end{array}$ & $\begin{array}{r}3,181 \\
2,845 \\
288 \\
2,098\end{array}$ & $\begin{array}{r}3,101 \\
2,845 \\
284 \\
1,999\end{array}$ & $\begin{array}{r}1,800 \\
1,576 \\
223 \\
177\end{array}$ & $\begin{array}{r}333.0 \\
290.0 \\
103.7 \\
81.9\end{array}$ & $\begin{array}{l}0 \\
0 \\
0 \\
0\end{array}$ \\
\hline $\begin{array}{l}\text { Gulf States Utilities } \\
\text { Company } \ldots \ldots \ldots \ldots \ldots \ldots\end{array}$ & River Bend 1 & BWR & 2101 & 2,680 & 3,172 & 764 & 141.3 & 0 \\
\hline $\begin{array}{l}\text { Houston Lighting and } \\
\text { Power Company.......... }\end{array}$ & $\begin{array}{l}\text { South Texas } 1 \\
\text { South Texas } 2\end{array}$ & $\begin{array}{l}\text { PWR } \\
\text { PWR }\end{array}$ & $\begin{array}{l}2201 \\
2202\end{array}$ & $\begin{array}{l}1,969 \\
1,969\end{array}$ & $\begin{array}{l}1,959 \\
1,958\end{array}$ & $\begin{array}{l}236 \\
116\end{array}$ & $\begin{array}{r}127.9 \\
62.6\end{array}$ & $\begin{array}{l}7 \\
0\end{array}$ \\
\hline Illinois Power Company .... & Clinton 1 & BWR & 2301 & 2,512 & 2,512 & 568 & 104.5 & 0 \\
\hline $\begin{array}{l}\text { Indiana Michigan } \\
\text { Power Company......... }\end{array}$ & $\begin{array}{l}\text { Cook } 1 \\
\text { Cook } 2\end{array}$ & $\begin{array}{l}\text { PWR } \\
\text { PWR }\end{array}$ & 5801 & 2,050 & 3,613 & 1,523 & 669.6 & $\mathbf{0}$ \\
\hline $\begin{array}{l}\text { lowa Electric Light and } \\
\text { Power Company.......... }\end{array}$ & Duane Arnold & BWR & 2401 & 2,050 & 1,898 & 1,152 & 212.0 & 0 \\
\hline $\begin{array}{l}\text { Kansas Gas and Electric } \\
\text { Company } . . . \ldots \ldots \ldots \ldots\end{array}$ & Wolf Creek 1 & PWR & 2501 & 1,340 & 1,327 & 340 & 157.4 & 0 \\
\hline
\end{tabular}

See footnotes at end of table. 
Table 13. Site Capacities and Inventories at Nuclear Power Plants as of December 31, 1992 (Continued)

\begin{tabular}{|c|c|c|c|c|c|c|c|c|}
\hline Electric Utility Name & $\begin{array}{l}\text { Reactor/Storage } \\
\text { Site Name }\end{array}$ & $\begin{array}{l}\text { Reactor } \\
\text { Type }\end{array}$ & $\begin{array}{l}\text { Pool } \\
\text { Site } \\
\text { ID }\end{array}$ & $\begin{array}{l}\text { Licensed } \\
\text { Storage } \\
\text { Capacity }^{a}\end{array}$ & $\begin{array}{l}\text { Maximum } \\
\text { Established } \\
\text { Storage } \\
\text { Capacity }^{\mathrm{b}}\end{array}$ & $\begin{array}{c}\text { Current } \\
\text { Inventory }\end{array}$ & $\begin{array}{c}\text { Initial Uranium } \\
\text { Content of } \\
\text { Pool Inventory } \\
\text { (MTU) }\end{array}$ & $\begin{array}{l}\text { Number of } \\
\text { Temporarily } \\
\text { Discharged }^{\text {Assemblies }}\end{array}$ \\
\hline $\begin{array}{l}\text { Long Island Lighting } \\
\text { Company } . . \ldots \ldots \ldots \ldots \ldots\end{array}$ & Shoreham & BWR & 2601 & 2,436 & 2,685 & 560 & 102.5 & $\mathbf{0}$ \\
\hline $\begin{array}{l}\text { Louisana Power and } \\
\text { Light Company } \ldots \ldots \ldots \ldots\end{array}$ & Waterford 3 & PWR & 2701 & 1,088 & 1,070 & 428 & 176.3 & $\mathbf{0}$ \\
\hline $\begin{array}{l}\text { Maine Yankee Atomic } \\
\text { Power Company ......... }\end{array}$ & Maine Yankee & PWR & 2801 & 1,476 & 1,464 & 1,077 & 405.0 & 23 \\
\hline $\begin{array}{l}\text { Nebraska Public Power } \\
\text { District ............... }\end{array}$ & Cooper Station & BWR & 3001 & 2,366 & 2,366 & 620 & 113.5 & 0 \\
\hline $\begin{array}{l}\text { New York Power } \\
\text { Authority.............. }\end{array}$ & $\begin{array}{l}\text { Fitzpatrick } \\
\text { Indian Point } 3\end{array}$ & $\begin{array}{l}\text { BWR } \\
\text { PWR }\end{array}$ & $\begin{array}{l}3901 \\
3902\end{array}$ & $\begin{array}{l}2,797 \\
1,345\end{array}$ & $\begin{array}{l}2,797 \\
1,340\end{array}$ & $\begin{array}{r}1,684 \\
584\end{array}$ & $\begin{array}{l}311.5 \\
266.9\end{array}$ & $\begin{array}{l}0 \\
2\end{array}$ \\
\hline $\begin{array}{l}\text { Niagara Mohawk Power } \\
\text { Corporation } . . . \ldots \ldots \ldots \ldots\end{array}$ & $\begin{array}{l}\text { Nine Mile Point } 1 \\
\text { Nine Mile Point } 2\end{array}$ & $\begin{array}{l}\text { BWR } \\
\text { BWR }\end{array}$ & $\begin{array}{l}3101 \\
3102\end{array}$ & $\begin{array}{l}2,776 \\
4,049\end{array}$ & $\begin{array}{l}2,324 \\
2,528\end{array}$ & $\begin{array}{r}1,620 \\
444\end{array}$ & $\begin{array}{r}301.3 \\
82.1\end{array}$ & $\begin{array}{l}0 \\
0\end{array}$ \\
\hline $\begin{array}{l}\text { North Atlantic Energy } \\
\text { Servic Corporation ....... }\end{array}$ & Seabrook & PWR & 5901 & 1,236 & 1,236 & 136 & 63.0 & $\mathbf{0}$ \\
\hline $\begin{array}{l}\text { Northeast Utilities } \\
\text { Service Company ........ }\end{array}$ & $\begin{array}{l}\text { Millstone } 1 \\
\text { Millstone } 2 \\
\text { Millstone } 3 \\
\text { Haddam Neck }\end{array}$ & $\begin{array}{l}\text { BWR } \\
\text { PWR } \\
\text { PWR } \\
\text { PWR }\end{array}$ & $\begin{array}{l}3201 \\
3202 \\
3203 \\
5701\end{array}$ & $\begin{array}{r}3,229 \\
1,072 \\
756 \\
1,172\end{array}$ & $\begin{array}{r}3,229 \\
1,065 \\
756 \\
1,167\end{array}$ & $\begin{array}{r}2,116 \\
784 \\
248 \\
757\end{array}$ & $\begin{array}{l}391.5 \\
311.2 \\
114.6 \\
311.9\end{array}$ & $\begin{array}{l}0 \\
0 \\
0 \\
1\end{array}$ \\
\hline $\begin{array}{l}\text { Northern States Power } \\
\text { Company } \ldots \ldots \ldots \ldots \ldots \ldots\end{array}$ & $\begin{array}{l}\text { Monticello } \\
\text { Prairie Island } 1 \\
\text { Prairie Island } 2\end{array}$ & $\begin{array}{l}\text { BWR } \\
\text { PWR } \\
\text { PWR }\end{array}$ & $\begin{array}{l}3301 \\
3302\end{array}$ & $\begin{array}{l}2,237 \\
1,386\end{array}$ & $\begin{array}{l}2,210 \\
1,354\end{array}$ & $\begin{array}{r}582 \\
1,233\end{array}$ & $\begin{array}{l}104.8 \\
466.7\end{array}$ & $\begin{array}{l}0 \\
0\end{array}$ \\
\hline $\begin{array}{l}\text { Omaha Public Power } \\
\text { District ............... }\end{array}$ & Fort Calhoun & PWR & 3401 & 729 & 729 & 529 & 190.4 & 0 \\
\hline
\end{tabular}

See footnotes at end of table. 
Table 13. Site Capacities and Inventories at Nuclear Power Plants as of December 31, 1992 (Continued)

\begin{tabular}{|c|c|c|c|c|c|c|c|c|}
\hline Electrlc Utility Name & $\begin{array}{l}\text { Reactor/Storage } \\
\text { Site Name }\end{array}$ & $\begin{array}{c}\text { Reactor } \\
\text { Type }\end{array}$ & $\begin{array}{l}\text { Pool } \\
\text { Site } \\
\text { ID }\end{array}$ & $\begin{array}{l}\text { Ucensed } \\
\text { Storage } \\
\text { Capacily }\end{array}$ & $\begin{array}{l}\text { Maximum } \\
\text { Established } \\
\text { Storage } \\
\text { Capacity }\end{array}$ & $\begin{array}{l}\text { Current } \\
\text { Inventory }\end{array}$ & $\begin{array}{c}\text { Initial Uranium } \\
\text { Content of } \\
\text { Pool Inventory } \\
\text { (MTU) }\end{array}$ & $\begin{array}{l}\text { Number of } \\
\text { Temporarlly } \\
\text { Dlscharged } \\
\text { Assembiles }^{d}\end{array}$ \\
\hline $\begin{array}{l}\text { Pacific Gas and Electric } \\
\text { Company } \ldots \ldots \ldots \ldots \ldots \ldots\end{array}$ & $\begin{array}{l}\text { Diablo Canyon } 1 \\
\text { Diablo Canyon } 2 \\
\text { Humboldt Bay }\end{array}$ & $\begin{array}{l}\text { PWR } \\
\text { PWR } \\
\text { BWR }\end{array}$ & $\begin{array}{l}3501 \\
3502 \\
3503\end{array}$ & $\begin{array}{r}1,324 \\
1,324 \\
486\end{array}$ & $\begin{array}{r}1,324 \\
1,317 \\
485\end{array}$ & $\begin{array}{l}376 \\
308 \\
390\end{array}$ & $\begin{array}{r}171.8 \\
142.0 \\
28.9\end{array}$ & $\begin{array}{l}0 \\
0 \\
0\end{array}$ \\
\hline $\begin{array}{l}\text { Pennsylvania Power and } \\
\text { Light Company.......... }\end{array}$ & $\begin{array}{l}\text { Susquehanna } 1 \\
\text { Susquehanna } 2\end{array}$ & $\begin{array}{l}\text { BWR } \\
\text { BWR }\end{array}$ & 3601 & $\begin{array}{l}2,840 \\
2,840\end{array}$ & $\begin{array}{l}2,840 \\
2,840\end{array}$ & $\begin{array}{l}1,404 \\
1,236\end{array}$ & $\begin{array}{l}252.7 \\
221.3\end{array}$ & $\begin{array}{l}\mathbf{0} \\
\mathbf{0}\end{array}$ \\
\hline $\begin{array}{l}\text { Philadelphia Electric } \\
\text { Company } . \ldots \ldots \ldots \ldots \ldots\end{array}$ & $\begin{array}{l}\text { Limerick } 1 \\
\text { Limerick } 2 \\
\text { Peach Bottom } 2 \\
\text { Peach Bottom } 3\end{array}$ & $\begin{array}{l}\text { BWR } \\
\text { BWR } \\
\text { BWR } \\
\text { BWR }\end{array}$ & $\begin{array}{l}3701 \\
3704 \\
3705\end{array}$ & $\begin{array}{l}2,040 \\
2,040 \\
3,819 \\
3,819\end{array}$ & $\begin{array}{l}2,766 \\
2,030 \\
3,819 \\
3,814\end{array}$ & $\begin{array}{r}1,140 \\
224 \\
2,162 \\
1,944\end{array}$ & $\begin{array}{r}210.9 \\
41.7 \\
400.0 \\
361.6\end{array}$ & $\begin{array}{l}0 \\
0 \\
0 \\
0\end{array}$ \\
\hline $\begin{array}{l}\text { Portland General Electric } \\
\text { Company .............. }\end{array}$ & Trojan & PWR & 3801 & 1,408 & 1,395 & 780 & 358.8 & 0 \\
\hline $\begin{array}{l}\text { Public Service Electric } \\
\text { and Gas Company........ }\end{array}$ & $\begin{array}{l}\text { Hope Creek } \\
\text { Salem } 1 \\
\text { Salem } 2\end{array}$ & $\begin{array}{l}\text { BWR } \\
\text { PWR } \\
\text { PWR }\end{array}$ & $\begin{array}{l}4201 \\
4202 \\
4203\end{array}$ & $\begin{array}{l}4,006 \\
1,170 \\
1,170\end{array}$ & $\begin{array}{l}3,998 \\
1,117 \\
1,139\end{array}$ & $\begin{array}{r}1,008 \\
656 \\
436\end{array}$ & $\begin{array}{l}186.5 \\
300.4 \\
201.0\end{array}$ & $\begin{array}{r}0 \\
25 \\
4\end{array}$ \\
\hline $\begin{array}{l}\text { Rochester Gas and } \\
\text { Electric Corporation....... }\end{array}$ & Ginna & PWR & 4401 & 1,016 & 987 & 661 & 249.9 & 0 \\
\hline $\begin{array}{l}\text { Sacramento Municipal } \\
\text { Utillty District ........... }\end{array}$ & Rancho Seco & PWR & 4501 & 1,080 & 1,080 & 493 & 228.3 & $\mathbf{0}$ \\
\hline $\begin{array}{l}\text { South Carolina Electric } \\
\text { and Gas Company......... }\end{array}$ & Summer & PWR & 4601 & 1,276 & 1,276 & 372 & 169.6 & 0 \\
\hline $\begin{array}{l}\text { Southern California } \\
\text { Edison Company ......... }\end{array}$ & $\begin{array}{l}\text { San Onofre } 1 \\
\text { San Onofre } 2 \\
\text { San Onofre } 3\end{array}$ & $\begin{array}{l}\text { PWR } \\
\text { PWR } \\
\text { PWR }\end{array}$ & $\begin{array}{l}4701 \\
4702 \\
4703\end{array}$ & $\begin{array}{r}216 \\
1,542 \\
1,542\end{array}$ & $\begin{array}{r}216 \\
1,542 \\
1,542\end{array}$ & $\begin{array}{l}207 \\
554 \\
602\end{array}$ & $\begin{array}{r}76.2 \\
226.2 \\
243.9\end{array}$ & $\begin{array}{l}0 \\
0 \\
0\end{array}$ \\
\hline
\end{tabular}

See footnotes at end of table. 
Table 13. Site Capacities and Inventories at Nuclear Power Plants as of December 31, 1992 (Continued)

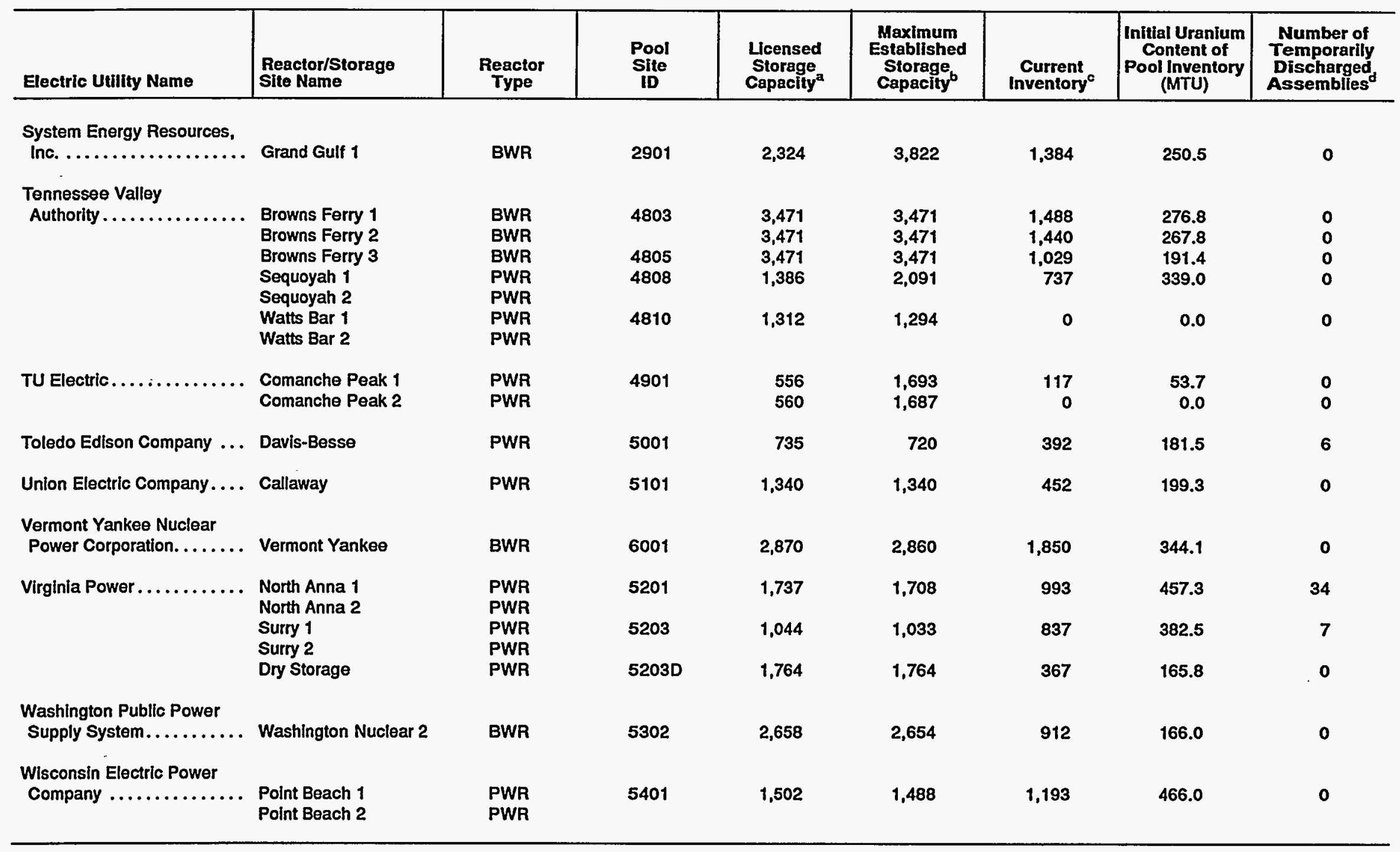

See footnotes at end of table. 
Table 13. Site Capacities and Inventories at Nuclear Power Plants as of December 31, 1992 (Continued)

\begin{tabular}{|c|c|c|c|c|c|c|c|c|}
\hline Electric Utillity Name & $\begin{array}{l}\text { Reactor/Storage } \\
\text { Site Name }\end{array}$ & $\begin{array}{l}\text { Reactor } \\
\text { Typo }\end{array}$ & $\begin{array}{c}\text { Pool } \\
\text { Site } \\
\text { ID }\end{array}$ & $\begin{array}{l}\text { Licensed } \\
\text { Storage } \\
\text { Capacity }^{\mathrm{a}}\end{array}$ & $\begin{array}{c}\text { Maximum } \\
\text { Established } \\
\text { Storage } \\
\text { Capacity }\end{array}$ & $\begin{array}{c}\text { Current } \\
\text { Inventory }\end{array}$ & $\begin{array}{c}\text { Initial Uranium } \\
\text { Content of } \\
\text { Pool Inventory } \\
\text { (MTU) }\end{array}$ & $\begin{array}{l}\text { Number of } \\
\text { Temporarily } \\
\text { Discharged } \\
\text { Assemblies }\end{array}$ \\
\hline $\begin{array}{l}\text { Wisconsin Public Service } \\
\text { Corporation. ............. }\end{array}$ & Kewaunee & PWR & 5501 & 990 & 990 & 616 & 237.8 & 12 \\
\hline $\begin{array}{l}\text { Yankee Atomic Electric } \\
\text { Company } . . . \ldots \ldots \ldots \ldots\end{array}$ & Yankee Rowe & PWR & 5601 & 721 & 721 & 533 & 127.4 & 0 \\
\hline TOTAL (Nuclear Power Plan & nt Sites Only) ... & ..... & $\cdots \cdots$ & $h_{202,670}$ & $h_{212,928}$ & 87,591 & $25,211.3$ & 168 \\
\hline Away-from-reactor Storage Fa & acilities (From Table & $\ldots \ldots$ & ........ & 3,061 & 3,061 & 3,448 & 746.4 & 0 \\
\hline TOTAL (Including Away-fro & m-reactor Storage & 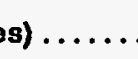 & & $i_{205,731}$ & $i_{215,989}$ & 91,039 & $25,957.7$ & 168 \\
\hline
\end{tabular}

Licensed Storage Capacity is the maximum number of spent nuclear fuel assemblies and canisters to be stored at a given site or spent nuclear fuel pool, as licensed by the Nuclear Regulatory Commission.

${ }^{6}$ Maximum Established Storage Capacity is the maximum established spent fuel capacity for the site defined by the DOE as the maximum number of intact assemblies that will be able to be stored at some point in the future (between the reporting date and the reactor's end of life) taking into account any established or current studies or engineering evaluations, at the time of submittal for licensing approval from the NRC.

c Current Inventory is the number of spent nuclear fuel assemblies stored at a given site or spent nuclear fuel pool, at a given point in time.

These numbers are included in the current inventory column.

Brunswick 1 and 2 are BWR reactors with the capability of storing both BWR and PWR fuel assemblies in their storage pools.

The current inventory of assemblies stored in the PWR portion of Brunswick pools came from the Robinson 2 reactor.

Garris 1 is a PWR reactor with the capability of storing both PWR and BWR fuel assemblies in its storage pools, based on future needs. The pools are licensed to hold up to 4,184 PWR or 5,808 BWR assemblies.

hLicensed storage capacity total and maximum established storage capacity total assume that Carolina Power and Light Company's Harris 1 site (capable of storing PWR and BWR fuel assemblies), will store BWR assemblies only. Totals assuming PWR assemblies only are 201,046 licensed storage capacity and 212,219 maximum established storage capacity.

Licensed storage capacity total and maximum established storage capacity total assume that Carolina Power and Light Company's Harris 1 site and General Electric Company's Morris Operation site (both capable of storing PWR and BWR fuel assemblies) will store BWR assemblies only. Totals assuming PWR assemblies only are 201,559 licensed storage capacity and 212,732 maximum established storage capacity.

MTU $=$ Metric tons of uranium; PWR = Pressurized-water reactor; BWR = Boiling-water reactor

Note: Totals may not equal sum of components because of independent rounding. See Technical Note 11 in Appendix E.

Source: Energy Information Administration, Form RW-859, "Nuclear Fuel Data" (1992). 
Table 14. Site Capacities and Inventories at Away-from-reactor Storage Facilities as of December 31,1992

\begin{tabular}{|c|c|c|c|c|c|c|c|c|}
\hline Storage Facility & $\begin{array}{l}\text { Contributing } \\
\text { Reactor }\end{array}$ & $\underset{\text { ID }}{\text { Reactor }}$ & $\begin{array}{c}\text { Reactor } \\
\text { Type }\end{array}$ & $\begin{array}{l}\text { Pool } \\
\text { Site } \\
\text { ID }\end{array}$ & $\begin{array}{l}\text { Licensed } \\
\text { Storage } \\
\text { Capacity a }\end{array}$ & $\begin{array}{l}\text { Maximum } \\
\text { Established } \\
\text { Storage } \\
\text { Capacity a }\end{array}$ & $\begin{array}{c}\text { Current } \\
\text { Inventory a }\end{array}$ & $\begin{array}{c}\text { Initial Uranium } \\
\text { Content of } \\
\text { Pool Inventory } \\
\text { (MTU) } \\
\end{array}$ \\
\hline $\begin{array}{l}\text { Babcock \& Wilcox } \\
\text { Company (Lynchburg)........... }\end{array}$ & & $\begin{array}{l}-- \\
--\end{array}$ & $\begin{array}{l}\text { PWR } \\
\text { BWR }\end{array}$ & 7101 & $\begin{array}{l}4 \\
4\end{array}$ & $\begin{array}{l}4 \\
4\end{array}$ & $\begin{array}{l}0 \\
0\end{array}$ & $\begin{array}{l}0.0 \\
0.0\end{array}$ \\
\hline 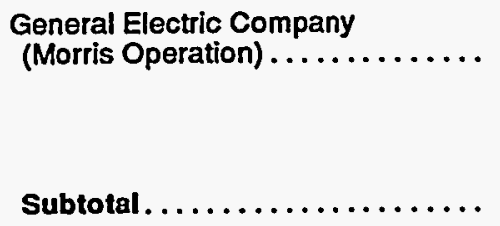 & $\begin{array}{l}\text { San Onofre 1 } \\
\text { Haddam Neck } \\
\text { Dresden } 2 \\
\text { Cooper Station } \\
\text { Monticello }\end{array}$ & $\begin{array}{l}4701 \\
5701 \\
1006 \\
3001 \\
3301\end{array}$ & $\begin{array}{l}\text { PWR } \\
\text { PWR } \\
\text { BWR } \\
\text { BWR } \\
\text { BWR }\end{array}$ & 6601 & $\begin{array}{r}b 380 \\
b_{2,928}\end{array}$ & $\begin{array}{r}380 \\
2,928\end{array}$ & $\begin{array}{r}270 \\
82 \\
753 \\
1,054 \\
1,058 \\
\mathbf{3 , 2 1 7}\end{array}$ & $\begin{array}{r}98.3 \\
34.4 \\
145.3 \\
198.2 \\
197.8 \\
674.0\end{array}$ \\
\hline $\begin{array}{l}\text { General Electric Company } \\
\text { (Vallecitos Nuclear)............ }\end{array}$ & Dresden 1 & 1005 & BWR & 6201 & & & 1 & 0.1 \\
\hline $\begin{array}{l}\text { Idaho National } \\
\text { Engineering Laboratory }{ }^{\circ} . \ldots \ldots \ldots\end{array}$ & $\begin{array}{l}\text { Turkey Point } 3 \\
\text { d Surry } 1 \\
\text { d Surry } 2 \\
\text { Point Beach } 1 \\
\text { Dresden } 1 \\
\text { Peach Bottom } 2\end{array}$ & $\begin{array}{l}1803 \\
5203 \\
5204 \\
5401 \\
1005 \\
3704\end{array}$ & $\begin{array}{l}\text { PWR } \\
\text { PWR } \\
\text { PWR } \\
\text { PWR } \\
\text { BWR } \\
\text { BWR }\end{array}$ & 7002 & $\begin{array}{l}-- \\
-- \\
-- \\
-- \\
--\end{array}$ & $\bar{m}+\overline{-}$ & $\begin{array}{r}18 \\
1 \\
68 \\
6 \\
2 \\
2 \\
97\end{array}$ & $\begin{array}{r}8.2 \\
0.5 \\
31.0 \\
2.4 \\
0.2 \\
0.4 \\
42.7\end{array}$ \\
\hline Ohio (Battelle) ${ }^{\circ} \ldots \ldots \ldots \ldots \ldots$ & Haddam Neck & 5701 & PWR & 7006 & - & - & 1 & 0.4 \\
\hline $\begin{array}{l}\text { Washington }\left(\text { Hanford) }{ }^{c} \ldots \ldots \ldots\right. \\
\text { Subtotal } \ldots \ldots \ldots \ldots \ldots \ldots \ldots\end{array}$ & $\begin{array}{l}\text { Calvert Clifts } 1 \\
\text { Point Beach } 1 \\
\text { Cooper Station }\end{array}$ & $\begin{array}{l}0501 \\
5401 \\
3001\end{array}$ & $\begin{array}{l}\text { PWR } \\
\text { PWR } \\
\text { BWR }\end{array}$ & 7007 & 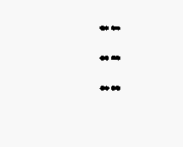 & $\begin{array}{l}- \\
-\end{array}$ & $\begin{array}{l}2 \\
3 \\
2 \\
7\end{array}$ & $\begin{array}{l}0.8 \\
1.2 \\
0.4 \\
2.4\end{array}$ \\
\hline $\begin{array}{l}\text { West Valley } \\
\text { Demonstration Project......... } \\
\text { Subtotal................ }\end{array}$ & $\begin{array}{l}\text { Ginna } \\
\text { Big Rock Point }\end{array}$ & $\begin{array}{l}4401 \\
1201\end{array}$ & $\begin{array}{l}\text { PWR } \\
\text { BWR }\end{array}$ & 7005 & $\begin{array}{l}0 \\
80 \\
85\end{array}$ & $\begin{array}{l}40 \\
85\end{array}$ & $\begin{array}{r}40 \\
85 \\
125\end{array}$ & $\begin{array}{l}15.3 \\
11.5 \\
26.8\end{array}$ \\
\hline TOTAL $\ldots \ldots \ldots \ldots \ldots \ldots \ldots$ & 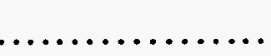 & . & $\ldots$ & & f 3,061 & $f^{\prime} \mathbf{3 , 0 6 1}$ & 3,448 & 746.4 \\
\hline
\end{tabular}

an number of assemblies (see Glossary for definition).

${ }^{b}$ General Electric Company (Morris Operation) is not licensed to receive any more assemblies.

These storage facilities are not surveyed by Form RW-859. The current inventory data for these facilities are shipments to these facilities which were reported by the individual utilities on Form RW-859. See Technical Note 2 in Appendix E. Licensed and maximum established storage capacity data are not applicable to these DOE facilities.

'Surry 1 and 2 spent fuel stored at ldaho National Engineering Laboratory is in dry storage.

owest Valley Demonstration Project is not licensed to receive any more assemblies.

'West Valley Demonstration Project is not licensed to receive any more assemblies. fuel assemblies), will store BWR assemblies only. Totals assuming PWR assemblies only are 513 licensed storage capacity and 513 maximum established storage capacity. MTU = Metric tons of uranium; PWR = Pressurized-water reactor; BWR = Boiling-water reactor.

MTU $=$ Metric tons

$=\quad$ Note: Totals may not equal sum of components because of independent rounding. See Technical Note 11 in Appendix E.

Source: Energy Information Administration, Form RW-859, "Nuclear Fuel Data" (1992). 
Table 15. Spent Fuel in Storage by State and Storage Site

\begin{tabular}{|c|c|c|c|c|c|c|c|}
\hline State & $\begin{array}{c}\text { Storage } \\
\text { Site }\end{array}$ & $\mid \begin{array}{c}\text { BWR } \\
\text { Assemblles }\end{array}$ & $\begin{array}{c}\text { BWR } \\
\begin{array}{c}\text { Initial Uranium } \\
\text { Content } \\
\text { (MTU) }\end{array}\end{array}$ & $\mid \begin{array}{c}\text { PWR } \\
\text { Assemblles }\end{array}$ & 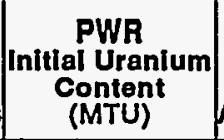 & $\begin{array}{c}\text { Total } \\
\text { Stored } \\
\text { Assemblles }\end{array}$ & $\begin{array}{c}\text { Tolal } \\
\text { Initial Uranium } \\
\text { Content } \\
\text { (MTU) }\end{array}$ \\
\hline $\begin{array}{l}\text { Alabama.... } \\
\text { Alabama .. }\end{array}$ & $\begin{array}{l}\text { Browns Ferry } 1 \\
\text { Browns Ferry } 3 \\
\text { Farley } 1 \\
\text { Farley } 2 \\
\text { Total }\end{array}$ & $\begin{array}{r}2,928 \\
1,029 \\
0 \\
0 \\
3,957\end{array}$ & $\begin{array}{r}544.6 \\
191.4 \\
0.0 \\
0.0 \\
736.0\end{array}$ & $\begin{array}{r}0 \\
0 \\
655 \\
498 \\
1,153\end{array}$ & $\begin{array}{r}0.0 \\
0.0 \\
302.0 \\
229.6 \\
531.6\end{array}$ & $\begin{array}{r}2,928 \\
1,029 \\
655 \\
498 \\
5,110\end{array}$ & $\begin{array}{r}544.6 \\
191.4 \\
302.0 \\
229.6 \\
1,267.6\end{array}$ \\
\hline $\begin{array}{l}\text { Arizona..... } \\
\text { Arizona ... }\end{array}$ & $\begin{array}{l}\text { Palo Verde } 1 \\
\text { Palo Verde } 2 \\
\text { Palo Verde } 3 \\
\text { Total }\end{array}$ & $\begin{array}{l}0 \\
0 \\
0 \\
0\end{array}$ & $\begin{array}{l}0.0 \\
0.0 \\
0.0 \\
0.0\end{array}$ & $\begin{array}{r}276 \\
288 \\
284 \\
848\end{array}$ & $\begin{array}{l}113.7 \\
118.1 \\
115.9 \\
347.7\end{array}$ & $\begin{array}{l}276 \\
288 \\
284 \\
848\end{array}$ & $\begin{array}{l}113.7 \\
118.1 \\
115.9 \\
347.7\end{array}$ \\
\hline $\begin{array}{c}\text { Arkansas ... } \\
\text { Arkansas.. }\end{array}$ & $\begin{array}{l}\text { Arkansas Nuclear } 1 \\
\text { Arkansas Nuclear } 2 \\
\text { Total }\end{array}$ & $\begin{array}{l}0 \\
0 \\
0\end{array}$ & $\begin{array}{l}0.0 \\
0.0 \\
0.0\end{array}$ & $\begin{array}{r}624 \\
564 \\
1,188\end{array}$ & $\begin{array}{l}288.9 \\
234.6 \\
523.5\end{array}$ & $\begin{array}{r}624 \\
564 \\
1,188\end{array}$ & $\begin{array}{l}288.9 \\
234.6 \\
523.5\end{array}$ \\
\hline $\begin{array}{l}\text { California... } \\
\text { Callfornia. }\end{array}$ & $\begin{array}{l}\text { Dlablo Canyon } 1 \\
\text { Diablo Canyon } 2 \\
\text { Humboldt Bay } \\
\text { Rancho Seco } \\
\text { San Onofre } 1 \\
\text { San Onofre } 2 \\
\text { San Onofre } 3 \\
\text { Vallecitos } \\
\text { Total }\end{array}$ & $\begin{array}{r}0 \\
0 \\
390 \\
0 \\
0 \\
0 \\
0 \\
1 \\
391\end{array}$ & $\begin{array}{r}0.0 \\
0.0 \\
28.9 \\
0.0 \\
0.0 \\
0.0 \\
0.0 \\
0.1 \\
29.0\end{array}$ & $\begin{array}{r}376 \\
308 \\
0 \\
493 \\
207 \\
554 \\
602 \\
0 \\
2,540\end{array}$ & \begin{tabular}{r|}
171.8 \\
142.0 \\
0.0 \\
228.3 \\
76.2 \\
226.2 \\
243.9 \\
0.0 \\
$1,088.4$
\end{tabular} & $\begin{array}{r}376 \\
308 \\
390 \\
493 \\
207 \\
554 \\
602 \\
1 \\
2,931\end{array}$ & $\begin{array}{r}171.8 \\
142.0 \\
28.9 \\
228.3 \\
76.2 \\
226.2 \\
243.9 \\
0.1 \\
1,117.4\end{array}$ \\
\hline $\begin{array}{r}\text { Colorado ... } \\
\text { Colorado.. }\end{array}$ & $\begin{array}{l}\text { Fort St. Vrain } \\
\text { Total }\end{array}$ & $\begin{array}{l}0 \\
0\end{array}$ & $\begin{array}{l}0.0 \\
0.0\end{array}$ & $\begin{array}{l}0 \\
0\end{array}$ & $\begin{array}{l}0.0 \\
0.0\end{array}$ & $\begin{array}{l}a_{1,464} \\
a_{1,464}\end{array}$ & $\begin{array}{l}a_{16.1} \\
a_{16.1}\end{array}$ \\
\hline $\begin{array}{l}\text { Connectlout. } \\
\text { Connectlou }\end{array}$ & $\begin{array}{l}\text { Haddam Neck } \\
\text { Millstone } 1 \\
\text { Millstone } 2 \\
\text { Millstone } 3 \\
\text { Total }\end{array}$ & $\begin{array}{r}0 \\
2,116 \\
0 \\
0 \\
2,116\end{array}$ & $\begin{array}{r}0.0 \\
391.5 \\
0.0 \\
0.0 \\
391.5\end{array}$ & $\begin{array}{r}757 \\
0 \\
784 \\
248 \\
1,789\end{array}$ & $\begin{array}{r}311.9 \\
0.0 \\
311.2 \\
114.6 \\
737.7\end{array}$ & $\begin{array}{r}757 \\
2,116 \\
784 \\
248 \\
3,905\end{array}$ & $\begin{array}{r}311.9 \\
391.5 \\
311.2 \\
114.6 \\
1,129.2\end{array}$ \\
\hline $\begin{array}{l}\text { Florida ...... } \\
\text { Florida.... }\end{array}$ & $\begin{array}{l}\text { Crystal River } 3 \\
\text { St. Lucle } 1 \\
\text { St. Lucie } 2 \\
\text { Turkey Point } 3 \\
\text { Turkey Point } 4 \\
\text { Total }\end{array}$ & $\begin{array}{l}0 \\
0 \\
0 \\
0 \\
0 \\
0\end{array}$ & $\begin{array}{l}0.0 \\
0.0 \\
0.0 \\
0.0 \\
0.0 \\
0.0\end{array}$ & $\begin{array}{r}536 \\
796 \\
464 \\
588 \\
546 \\
2,930\end{array}$ & $\begin{array}{r}248.7 \\
300.1 \\
175.9 \\
267.5 \\
248.4 \\
1,240.6\end{array}$ & $\begin{array}{r}536 \\
796 \\
464 \\
588 \\
546 \\
2,930\end{array}$ & $\begin{array}{r}248.7 \\
300.1 \\
175.9 \\
267.5 \\
248.4 \\
1,240.6\end{array}$ \\
\hline $\begin{array}{l}\text { Georgla .... } \\
\text { Georgla ... }\end{array}$ & $\begin{array}{l}\text { Hatch } 1 \\
\text { Vogtle } 1 \\
\text { Total }\end{array}$ & $\begin{array}{r}3,376 \\
0 \\
3,376\end{array}$ & $\begin{array}{r}623.0 \\
0.0 \\
623.0\end{array}$ & $\begin{array}{r}0 \\
400 \\
400\end{array}$ & $\begin{array}{r}0.0 \\
185.6 \\
185.6\end{array}$ & $\begin{array}{r}3,376 \\
400 \\
3,776\end{array}$ & $\begin{array}{l}623.0 \\
185.6 \\
808.6\end{array}$ \\
\hline $\begin{array}{l}\text { Idaho ..... } \\
\text { Idaho.... }\end{array}$ & $\begin{array}{l}\text { Idaho National } \\
\text { Engineering Laboratory } \\
\text { Total }\end{array}$ & 4 & 0.6 & 93 & 42.1 & $\begin{array}{l}b_{841} \\
b_{841}\end{array}$ & $\begin{array}{l}b_{51.6} \\
b_{51.6}\end{array}$ \\
\hline
\end{tabular}

See footnotes at end of table. 
Table 15. Spent Fuel In Storage by State and Storage Site (Continued)

\begin{tabular}{|c|c|c|c|c|c|c|}
\hline $\begin{array}{l}\text { Storege } \\
\text { Site }\end{array}$ & $\underset{\text { Assemblles }}{\text { BWR }}$ & $\begin{array}{c}\text { BWR } \\
\text { Initial Uranlum } \\
\text { Content } \\
\text { (MTU) }\end{array}$ & $\underset{\text { Posemblles }}{\text { PWR }}$ & $\begin{array}{c}\text { PWR } \\
\text { Inltial Uranlum } \\
\text { Content } \\
\text { (MTU) }\end{array}$ & $\begin{array}{c}\text { Total } \\
\text { Stored } \\
\text { Assemblles }\end{array}$ & $\begin{array}{l}\text { Total } \\
\text { Inltlal Uranium } \\
\text { Content } \\
\text { (MTU) }\end{array}$ \\
\hline $\begin{aligned} \text { Illinols............ } & \text { Braidwood 1 } \\
& \text { Byron 1 } \\
& \text { Clinton 1 } \\
& \text { Dresden 1 } \\
& \text { Dresden 2 } \\
& \text { Dresden } 3 \\
& \text { LaSalle County 1 } \\
& \text { Morrls } \\
& \text { Quad Clties 1 } \\
& \text { Zion 1 } \\
& \\
\text { IIIInols ......... } & \text { Total }\end{aligned}$ & $\begin{array}{r}0 \\
0 \\
568 \\
683 \\
1,954 \\
1,968 \\
1,920 \\
2,865 \\
3,996 \\
0 \\
13,954\end{array}$ & $\begin{array}{r}0.0 \\
0.0 \\
104.5 \\
69.7 \\
353.0 \\
355.6 \\
351.4 \\
541.3 \\
737.2 \\
0.0 \\
2,512.7\end{array}$ & $\begin{array}{r}404 \\
596 \\
0 \\
0 \\
0 \\
0 \\
0 \\
352 \\
0 \\
1,608 \\
2,960\end{array}$ & $\begin{array}{r}171.6 \\
253.2 \\
0.0 \\
0.0 \\
0.0 \\
0.0 \\
0.0 \\
132.7 \\
0.0 \\
734.0 \\
1,291.5\end{array}$ & $\begin{array}{r}404 \\
596 \\
568 \\
683 \\
1,954 \\
1,968 \\
1,920 \\
3,217 \\
3,996 \\
1,608 \\
16,914\end{array}$ & $\begin{array}{r}171.6 \\
253.2 \\
104.5 \\
69.7 \\
353.0 \\
355.6 \\
351.4 \\
674.0 \\
737.2 \\
734.0 \\
3,804.2\end{array}$ \\
\hline $\begin{array}{l}\text { Kansas........... Wolf Creok } 1 \\
\text { Kansas ....... Total }\end{array}$ & $\begin{array}{l}0 \\
0\end{array}$ & $\begin{array}{l}0.0 \\
0.0\end{array}$ & $\begin{array}{l}340 \\
340\end{array}$ & $\begin{array}{l}157.4 \\
157.4\end{array}$ & $\begin{array}{l}340 \\
340\end{array}$ & $\begin{array}{l}157.4 \\
157.4\end{array}$ \\
\hline $\begin{array}{c}\text { Loulsiana......... River Bend } 1 \\
\text { Loulslana ....... Total }\end{array}$ & $\begin{array}{r}764 \\
0 \\
764\end{array}$ & $\begin{array}{r}141.3 \\
0.0 \\
141.3\end{array}$ & $\begin{array}{r}0 \\
428 \\
428\end{array}$ & $\begin{array}{r}0.0 \\
176.3 \\
176.3\end{array}$ & $\begin{array}{r}764 \\
428 \\
1,192\end{array}$ & $\begin{array}{l}141.3 \\
176.3 \\
317.6\end{array}$ \\
\hline $\begin{array}{l}\text { Malne... . . . . . . . Maine Yankee } \\
\text { Malne..... . . . Total }\end{array}$ & $\begin{array}{l}0 \\
\mathbf{0}\end{array}$ & $\begin{array}{l}0.0 \\
0.0\end{array}$ & $\begin{array}{l}1,077 \\
1,077\end{array}$ & $\begin{array}{l}405.0 \\
405.0\end{array}$ & $\begin{array}{l}1,077 \\
1,077\end{array}$ & $\begin{array}{l}405.0 \\
405.0\end{array}$ \\
\hline $\begin{array}{l}\text { Maryland ......... Calvert Cliffs } 1 \\
\text { Maryland........ Total }\end{array}$ & $\begin{array}{l}0 \\
0\end{array}$ & $\begin{array}{l}0.0 \\
0.0\end{array}$ & $\begin{array}{l}1,410 \\
1,410\end{array}$ & $\begin{array}{l}541.4 \\
541.4\end{array}$ & $\begin{array}{l}1,410 \\
1,410\end{array}$ & $\begin{array}{l}541.4 \\
541.4\end{array}$ \\
\hline $\begin{array}{l}\text { Minnesota ........ Monticello } \\
\text { Prairie Island } 1 \\
\text { MInnesota....... Total }\end{array}$ & $\begin{array}{r}582 \\
0 \\
582\end{array}$ & $\begin{array}{r}104.8 \\
0.0 \\
104.8\end{array}$ & $\begin{array}{r}0 \\
1,233 \\
1,233\end{array}$ & $\begin{array}{r}0.0 \\
466.7 \\
466.7\end{array}$ & $\begin{array}{r}582 \\
1,233 \\
1,815\end{array}$ & $\begin{array}{l}104.8 \\
466.7 \\
571.5\end{array}$ \\
\hline $\begin{array}{l}\text { Mississippl. ...... G Grand Gulf } 1 \\
\text { MIssissippi . . . . Total }\end{array}$ & $\begin{array}{l}1,384 \\
1,384\end{array}$ & $\begin{array}{l}250.5 \\
250.5\end{array}$ & $\begin{array}{l}0 \\
0\end{array}$ & $\begin{array}{l}0.0 \\
0.0\end{array}$ & $\begin{array}{l}1,384 \\
1,384\end{array}$ & $\begin{array}{l}250.5 \\
250.5\end{array}$ \\
\hline $\begin{array}{l}\text { Mlssourl ......... Callaway } \\
\text { Mlssourl ....... Total }\end{array}$ & $\begin{array}{l}0 \\
0\end{array}$ & $\begin{array}{l}0.0 \\
0.0\end{array}$ & $\begin{array}{l}452 \\
452\end{array}$ & $\begin{array}{l}199.3 \\
199.3\end{array}$ & $\begin{array}{l}462 \\
452\end{array}$ & $\begin{array}{l}199.3 \\
199.3\end{array}$ \\
\hline $\begin{array}{c}\text { Nebraska......... Cooper Station } \\
\begin{array}{c}\text { Fort Calhoun } \\
\text { Nebraska........ Total }\end{array}\end{array}$ & $\begin{array}{r}620 \\
0 \\
620\end{array}$ & $\begin{array}{r}113.5 \\
0.0 \\
113.5\end{array}$ & $\begin{array}{r}0 \\
529 \\
529\end{array}$ & $\begin{array}{r}0.0 \\
190.4 \\
190.4\end{array}$ & $\begin{array}{r}620 \\
529 \\
1,149\end{array}$ & $\begin{array}{l}113.5 \\
190.4 \\
303.9\end{array}$ \\
\hline $\begin{array}{l}\text { New Hampshire. ... Seabrook } \\
\text { Now Hampshlre.. Total }\end{array}$ & $\begin{array}{l}0 \\
0\end{array}$ & $\begin{array}{l}0.0 \\
0.0\end{array}$ & $\begin{array}{l}136 \\
136\end{array}$ & $\begin{array}{l}63.0 \\
63.0\end{array}$ & $\begin{array}{l}136 \\
136\end{array}$ & $\begin{array}{l}63.0 \\
63.0\end{array}$ \\
\hline
\end{tabular}

See footnotes at end of table. 
Table 15. Spent Fuel in Storage by State and Storage S!te (Continued)

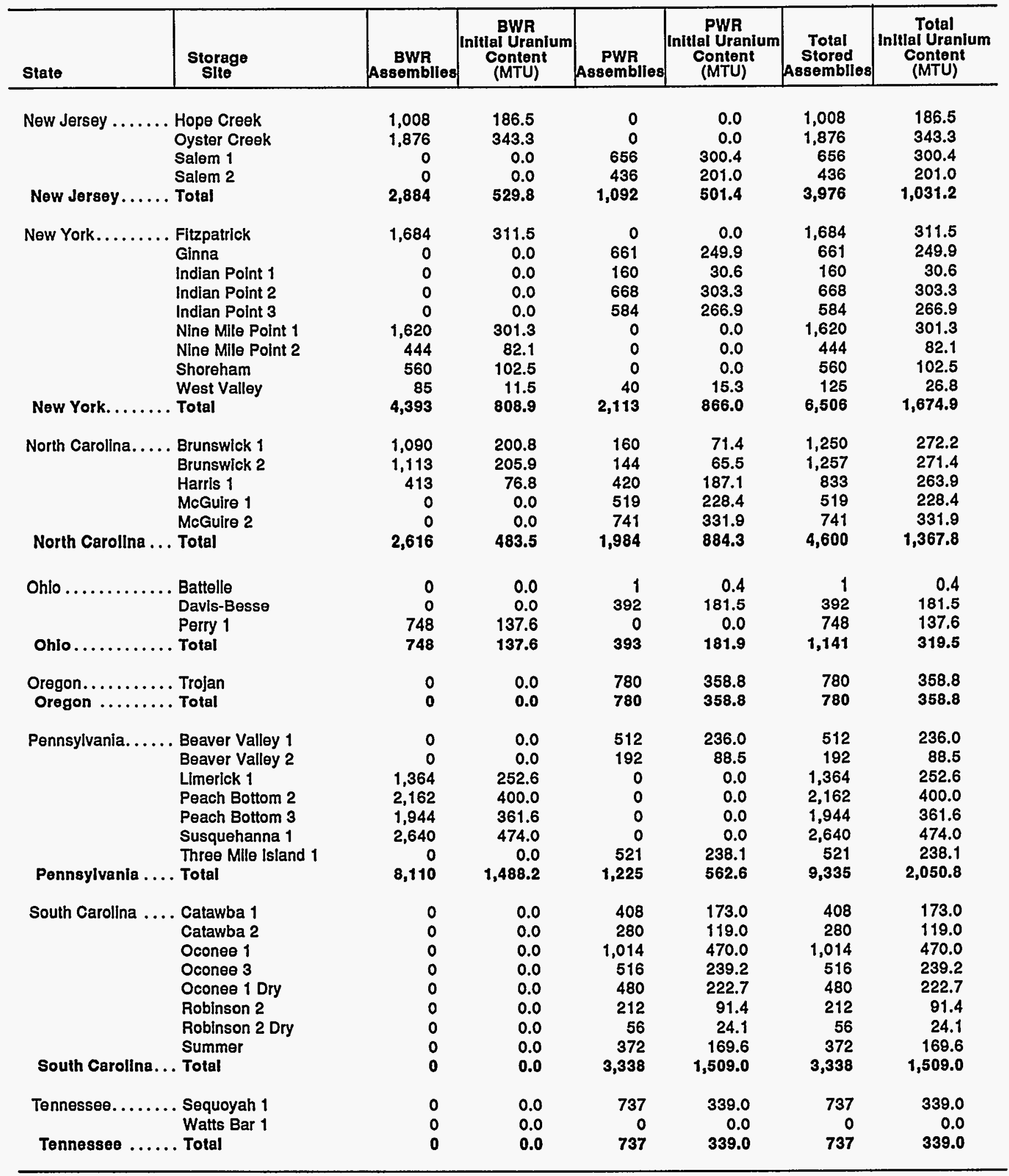

See footnotes at end of table. 
Table 15. Spent Fuel in Storage by State and Storage Site (Continued)

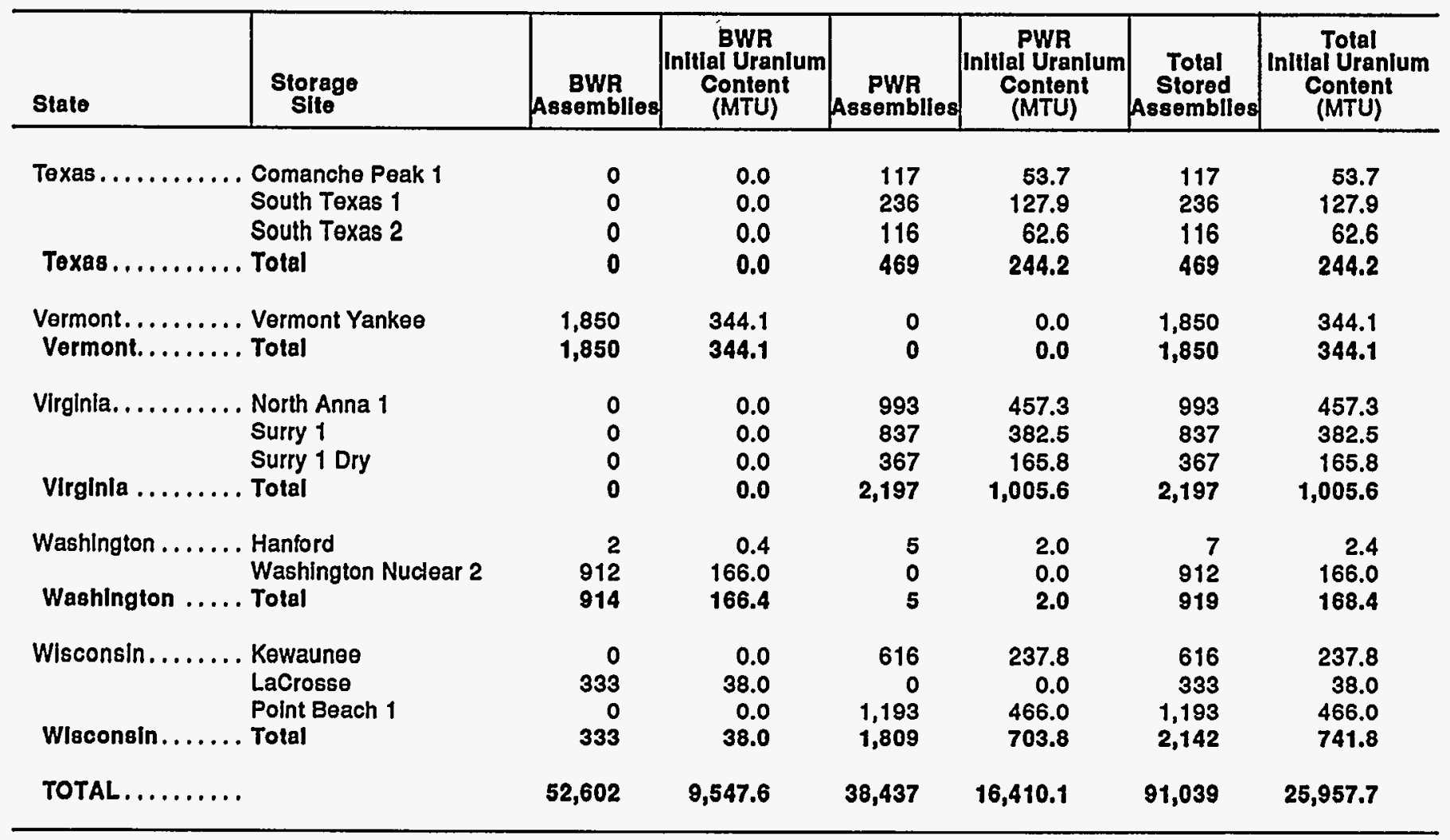

${ }^{a}$ These 1,464 high-temperature, gas-cooled reactor (HTGR) fuel elements are not Included in the table totals. Se日 Technical Note 6 In Appendix E.

${ }_{A}$ total of 744 HTGR fuel elements, with initial uranium content equal to 8.9 metric tons of uranium (MTU), were discharged. These HTGR fuel elements are not included in the above table totals. See Technical Note 6 in Appendix E.

BWR = Bolllng-Water reactor; PWR = Pressurized-water reactor; MTU = Metric tons of uranlum.

Note: Totals may not equal sum of components because of independent rounding. See Technlcal Note 11 in Appendix E.

Source: Energy Information Administration, Form RW-859, "Nuclear Fuel Data" (1992). 


\section{Dry Storage at Utilities}

In order to meet the demand for storage space, several utility companies have opted to increase their spent fuel storage capacity through the use of dry storage techniques. Many different dry storage techniques have been designed and are being, or will be, licensed and used. These include metal storage casks, concrete storage casks, metal canisters housed in concrete modules, and concrete storage vaults.

All U.S. commercial LWR's which are storing, or are planning to store nuclear fuel assemblies in an ISFSI as of December 31, 1992, are reported on Form RW-859. Several utilities have contributed supplementary dry storage information. Table 16 lists data for each of these utilities and affected reactors. Utilities are listed by the date the dry storage license was issued.

The three utilities that currently use the Nutec Horizontal Modular Storage (NUHOMS) Spent Fuel Storage System are Baltimore Gas and Electric Company, Carolina Power and Light Company, and Duke Power Company. Both GPU Nuclear Corporation and Sacramento Municipal Utility District plan to employ the NUHOMS system. The system consists of three major safety-related components: a dry shielded canister (DSC), which provides a high integrity containment boundary and a controlled storage environment for the fuel; a reinforced concrete horizontal storage module (HSM), which houses the stored DSC and provides radiation shielding, protection against natural phenomena, and an efficient means for decay heat removal; and a transfer cask, which provides for the safe shielded transfer of the DSC from the plant spent fuel pool to the storage module. The NUHOMS system is designed and licensed to the requirements of 10 CFR 72 and ANS/ANSI 57.9 for ISFSIs.

\section{Baltimore Gas and Electric Company}

The ISFSI at Baltimore Gas and Electric Company's Calvert Cliffs station is the NUHOMS-24P design. The Calvert Cliffs ISFSI has been designed as a life-ofplant storage facility. The ISFSI will have the capacity to store all spent fuel discharged from Calvert Cliffs Units 1 and 2, beyond the spent fuel pool capacity, up to the 40-year plant life, if necessary. The exact capacity needed is uncertain, and to limit capital investment until necessary, the ISFSI will be constructed in up to five phases. The ISFSI required the preparation of a 10 CFR 72 License Application, Safety Analysis Report, Environmental Report, and a Security Plan for NRC review and approval. The license material was prepared and submitted to the NRC in December 1989. Construction of the ISFSI west of the plant began in April 1991 after NRC approved the Environmental Report. The facility and its pre-operational testing were completed in October 1992. The ISFSI was licensed by the NRC on November 25, 1992. The license allows Baltimore Gas and Electric Company to place as many as 2,880 pool-cooled assemblies in casks that would then be placed in ISFSIs. Each cask can hold 24 assemblies, and there are currently 48 cask spaces in the storage module. The license has a 20 -year term, but the licensee may seek to extend the term before it expires.

\section{Carolina Power and Light Company}

The ISFSI for Carolina Power and Light Company's Robinson 2 plant is composed of eight HSMs, and is the NUHOMS-7P design. The HSM is a steelreinforced concrete structure which holds seven intact assemblies in each module. The ISFSI was licensed by the NRC in August 1986 to hold 56 assemblies. They are located inside the fenced area at the Robinson 2 plant site. The Carolina Power and Light Company also applied to the NRC for a license for an ISFSI to be built at its Brunswick plant. The ISFSI will be used only as a backup if shipping of spent nuclear fuel to the Harris plant is prohibited.

\section{Consumers Power Company}

In April 1993, the NRC approved the dry-cask storage of fuel that has already undergone pool cooling, and the use of the new VSC-24 cask by Sierra Nuclear Corporation at the Consumers Power Company's Palisades plant. The approval was challenged by the Michigan Attorney General and a citizen organization, the Lake Michigan Federation, on the grounds that the process should have entailed a full environmental impact statement, rather than the less elaborate environmental assessment. On May 10, 1993, the U.S. District Court for Western Michigan ruled that they did not have jurisdiction, and denied the request to prevent cask loading. The plaintiffs then took their request to the U.S. Court of Appeals for the Sixth Circuit, in Cincinnati. On May 17, 1993, a three-judge 
panel of that court declined to issue an emergency motion to stop cask loading; no decision had been rendered, at this writing, on the basic argument on the legality of the cask approval. The Palisades plant was the first to load spent fuel cask in its ISFSI under the NRC general licensing provisions. This ruling, under 10 CFR 72 subpart $K$, allows the use of certain cask/storage systems at licensed nuclear power plants without the need for further site-specific licensing. Plant personnel started loading casks on May 7, 1993, and by May 19, 1993, the job for the upcoming refueling was finished: two casks had each received 24 spent fuel assemblies, and had then been welded shut and placed on the storage pad. There are 6 more casks already on-site, and the license amendment allows for as many as 25 to be used at the plant-which would last Palisades through the end of its current licensed life, in 2007.

\section{Duke Power Company}

The Duke Power Company received its Oconee site license from the NRC in January 1990 to operate 88 modules of the NUHOMS-24P design. They are designed to store 24 pressurized-water reactor (PWR) assemblies per module; therefore, the maximum capacity is 2,112 assemblies. The first 20 modules were completed in 1990 and the second set of 20 were finished in 1992. Duke Power Company estimates that it will need 20 more modules by 1996 because of a lack of storage in their spent fuel pool. The schedule for loading the modules with fuel is as follows: in 1990, 4 modules were loaded; in 1991, 9 more modules were loaded; in 1992, 7 modules were loaded; in 1993, 4 modules will be loaded; in 1994, 5 modules will be loaded; in 1995, 5 modules will be loaded; in 1996, 5 modules will be loaded; in 1997, 5 modules will be loaded; and in 1998, 5 modules will be loaded.

\section{GPU Nuclear Corporation}

The GPU Nuclear Corporation has contracted Pacific Nuclear to engineer, license, and construct a spent fuel storage system for the Oyster Creek nuclear plant. The agreement includes the design and contruction of concrete modules and stainless steel containers for use in storing the plant's spent fuel on-site. The facility will employ Pacific Nuclear's NUHOMS technology. Pacific Nuclear will complete engineering and licensing work in 1994 and will begin delivering the fuel-storage equipment in 1995.

\section{Northern States Power Company}

The decision by the Minnesota Public Utilities Commission to allow 17 containers for aboveground spent fuel storage at Northern States Power Company's Prairie Island site was granted June 26, 1991. Although the storage capacity granted is less than the 48 casks the company asked for in its certificate of need submitted in April 1991, the 17 casks will enable it to continue operation of Prairie Island at least through the end of the century. The possibility of more cost effective measures, other than dry cask storage, being available at the end of the century prompted the Commission to limit the company to 17 casks. These measures include, but are not limited to, research and development in renewable energy resources, conservation, and load management. Without the additional storage, the two-unit plant might have been forced to cease operating in 1995. Northern States will be transferring the oldest spent fuel from its storage pool into the casks. The NRC requires that the fuel to be stored in aboveground casks be at least 10 years old; but the Commission is calling for it to be at least 15 years old. Site construction began in September 1992. The initial TN-40 cask order had been placed for seven casks. The first cask was scheduled for completion November 1993.

In August 1993, a Minnesota Supreme Court decision put a hold for at least 7 months on Northern States Power Company's plans for dry cask storage at Prairie Island. The State's highest court recently decided not to review an appeals court decision which overturned State regulators' approval of the on-site storage plan. The appeals court had found that plant licensee Northern States Power Company needs the Minnesota legislature's approval before using dry cask storage to supplement dwindling space in the plant's spent fuel pool.

A State law, enacted in the 1970s, requires the State legislature's approval for "permanent" spent fuel storage within Minnesota. The appeals court concluded, and the supreme court agreed, that Northern States' dry cask storage option constituted "permanent" storage, thus requiring State legislative approval.

The spent fuel pool will be full in 1994. The utility estimates that with no additional fuel storage space, the Prairie Island 2 reactor will shut down in May 1995 while the Prairie Island 1 reactor will shut down in January 1996. 
Before the supreme court ruling came down, Northern States had finished all the necessary preparatory site work for the spent fuel casks, including construction of the concrete pads on which the casks would sit. The utility also had contracted with Transnuclear, Inc., to purchase casks, and the first two were being fabricated. Cask fabrication has since been suspended pending approval from the State legislature.

Meanwhile, in October 1993 the NRC granted the utility a license for the Prairie Island site to store fuel in up to $48 \mathrm{TN}-40$ casks.

\section{Public Service Company of Colorado}

The NRC licensed the ISFSI at Public Service Company of Colorado's closed Fort St. Vrain plant on November 4, 1991. The ISFSI is a Modular Vault Dry Storage System, made up of six air-cooled concrete vaults. The ISFSI will hold the fuel in dry storage until such time as DOE, under its contract with the utility, takes possession of the material. The license authorizes storage in the ISFSI of up to 1,482 spent fuel elements, 37 reflector control rod elements, and 6 neutron source elements. In December 1991, the Public Service Company of Colorado transferred 18 elements to its ISFSI. In June 1992, 1,446 elements were transferred to the ISFSI, for a total of 1,464 which now reside in the ISFSI. The reflector elements and neutron source elements were disposed of offsite. The design lifetime of the facility is 40 years, although the current license is limited to 20 years. This facility is designed as a stand alone operation although it currently relies on a portion of the plant's security system.

\section{Sacramento Municipal Utility District}

The Sacramento Municipal Utility District (SMUD) first applied for a license with the NRC for an ISFSI at the Rancho Seco plant in October 1991. A revised application was submitted to the NRC in October 1993. The environmental assessment will be completed in early 1994, after which time construction of the ISFSI might commence. The design will be similar to the Oconee and Calvert Cliffs plants, the NUHOMS-24P storage model. The utility predicts that licensing of the ISFSI to store spent nuclear fuel will be issued by mid-1995. SMUD is also the first utility to apply for a license to the NRC for a transportable storage system under 10 CFR 71, which will license a cask and canisters so that they can be transported off-site to a repository.

\section{Toledo Edison Company}

Instead of reracking its wet pool, Toledo Edison Company chose to build an ISFSI at its Davis-Besse plant with an existing NRC-approved cask design. The utility plans to load the first fuel into the casks in 1995.

\section{VIrginla Power}

Virginia Power was the first U.S. utility to use dry storage for spent nuclear fuel. The Virginia Power ISFSI located at the Surry Power Station, Surry, Virginia, is a design using metal storage casks. It was licensed by the NRC in July 1986 for use of the CASTOR V/21. Use of the MC-10 and NAC-I28/ST has also been approved by the NRC. Each cask is 16 feet high, 8 feet in diameter, and weighs 110 to 120 tons when loaded with fuel. These three cask designs hold between 21 and 28 fuel assemblies. The casks sit on a pad 230 feet long, 32 feet wide, and 3 feet thick (reinforced concrete). Each pad will hold 28 casks, and the facility license provides for three pads. The facility and casks have been evaluated for extreme temperatures $\left(-20^{\circ} \mathrm{F}\right.$ to $\left.115^{\circ} \mathrm{F}\right)$, extreme wind (105 m.p.h.), snow and ice, loss of electrical power, loss of cask radiation shielding, tornados ( 300 m.p.h.), gas pipeline explosions, and cask seal leakage and drops. By the end of 1990, a total of 252 assemblies had been stored in 12 casks. By the end of 1991, 52 more assemblies were stored in 2 additional casks, one holding 28 assemblies, the other 24. In 1992, 3 more casks each holding 21 assemblies for a total of 63 were stored. This increased the total number of assemblies in dry storage at Surry to 367 by the end of 1992 . The following are projections for upcoming years: in 1993, 2 casks holding 49 assemblies; in 1994, 5 casks holding 117 assemblies; and in 1995, 4 casks holding 84 assemblies. The ISFSI has been licensed to hold up to 1,764 assemblies.

\section{Other UtIIItles}

The Wisconsin Electric Power Company's Point Beach plant, the Arkansas Power and Light Company's Arkansas Nuclear plant, and the New York Power Authority's Fitzpatrick plant do not have applications with the NRC but have contracts with vendors to provide on-site dry storage. 
Table 16. Dry Spent Fuel Storage Data as of December 31, 1992

\begin{tabular}{|c|c|c|c|c|c|c|c|c|c|c|c|}
\hline \multirow[b]{2}{*}{ Utility } & \multirow[b]{2}{*}{ Reactor } & \multirow{2}{*}{$\begin{array}{c}\text { Date } \\
\text { License } \\
\text { Issued/ } \\
\text { Submitted } \\
\text { Expected }^{\mathrm{a}} \text { ' }\end{array}$} & \multirow[b]{2}{*}{ Vendor } & \multirow[b]{2}{*}{$\begin{array}{l}\text { Storage } \\
\text { Type }\end{array}$} & \multirow[b]{2}{*}{ Model } & \multirow[b]{2}{*}{$\begin{array}{c}\text { Capacity } \\
\text { (assemblies) }\end{array}$} & \multicolumn{5}{|c|}{$\begin{array}{c}\text { Assemblies Stored } \\
\text { Through }\end{array}$} \\
\hline & & & & & & & 1989 & 1990 & 1991 & 1992 & Total \\
\hline $\begin{array}{l}\text { Baltimore Gas and } \\
\text { Electric Company....... }\end{array}$ & Calvert Cliffs $1 \& 2$ & $11 / 92$ & $\begin{array}{l}\text { Pacific Nuclear Fuel } \\
\text { Services, Inc. }\end{array}$ & $\begin{array}{l}\text { Concrete } \\
\text { Module }\end{array}$ & NUHOMS-24P & 24 PWR & - & - & - & - & - \\
\hline $\begin{array}{l}\text { Carolina Power and } \\
\text { Light Company......... }\end{array}$ & Robinson 2 & $08 / 86$ & $\begin{array}{l}\text { Pacific Nuclear Fuel } \\
\text { Services, Inc. }\end{array}$ & $\begin{array}{l}\text { Concrete } \\
\text { Module }\end{array}$ & NUHOMS-7P & 7 PWR & 56 & - & - & - & 56 \\
\hline $\begin{array}{l}\text { Carolina Power and } \\
\text { Light Company......... }\end{array}$ & Brunswick $1 \& 2$ & ${ }^{a} 05 / 89$ & $\begin{array}{l}\text { Pacific Nuclear Fuel } \\
\text { Services, Inc. }\end{array}$ & $\begin{array}{l}\text { Concrete } \\
\text { Module }\end{array}$ & NUHOMS-7P & 7 PWR & - & - & -- & -- & - \\
\hline $\begin{array}{l}\text { Consumers Power } \\
\text { Company } \ldots \ldots \ldots \ldots \ldots\end{array}$ & Palisades & ${ }^{a} 03 / 90$ & $\begin{array}{l}\text { Sierra Nuclear } \\
\text { Corporation }\end{array}$ & $\begin{array}{l}\text { Concrete } \\
\text { Cask }\end{array}$ & VSC-24 & 24 PWR & -- & - & - & - & - \\
\hline Duke Power Company .... & Oconee 1, 2, 3 & $01 / 90$ & $\begin{array}{l}\text { Pacific Nuclear Fuel } \\
\text { Services, Inc. }\end{array}$ & $\begin{array}{l}\text { Concrete } \\
\text { Module }\end{array}$ & NUHOMS-24P & 24 PWR & -- & 96 & 216 & 168 & 480 \\
\hline GPU Nuclear Corporation. . & Oyster Creek & ${ }^{b} 06 / 94$ & $\begin{array}{l}\text { Pacific Nuclear Fuel } \\
\text { Services, Inc. }\end{array}$ & $\begin{array}{l}\text { Concrete } \\
\text { Module }\end{array}$ & NUHOMS-52B & 52 BWR & -- & - & - & - & - \\
\hline $\begin{array}{l}\text { Northern States Power } \\
\text { Company } \ldots \ldots \ldots \ldots \ldots \ldots\end{array}$ & Prairie Island 1 \& 2 & $10 / 93$ & Transnuclear, Inc. & Metal Cask & TN-40 & 40 PWR & -- & - & - & - & -- \\
\hline $\begin{array}{l}\text { Public Service Company } \\
\text { of Colorado............ }\end{array}$ & Fort St. Vrain & $11 / 91$ & $\begin{array}{l}\text { Foster Wheeler Energy } \\
\text { Applications, Inc. }\end{array}$ & $\begin{array}{l}\text { Concrete } \\
\text { Vault }\end{array}$ & $\begin{array}{l}\text { Modular } \\
\text { Vault Dry } \\
\text { Storage }\end{array}$ & $\begin{array}{l}83 \text { PWR } \\
150 \text { BWR }\end{array}$ & -- & $\cdots$ & 18 & 1,446 & 1,464 \\
\hline $\begin{array}{l}\text { Sacramento Municipal } \\
\text { Utility District ........... }\end{array}$ & Rancho Seco & $a_{10 / 91}$ & $\begin{array}{l}\text { Pacific Nuclear Fuel } \\
\text { Services, Inc. }\end{array}$ & $\begin{array}{l}\text { Concrete } \\
\text { Module }\end{array}$ & NUHOMS-24P & 24 PWR & - & - & - & - & -- \\
\hline
\end{tabular}

See footnotes at end of table. 
Table 16. Dry Spent Fuel Storage Data as of December 31, 1992 (Continued)

\begin{tabular}{|c|c|c|c|c|c|c|c|c|c|c|c|}
\hline \multirow[b]{2}{*}{ Utility } & \multirow[b]{2}{*}{ Reactor } & \multirow{2}{*}{ 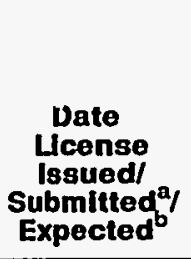 } & \multirow[b]{2}{*}{ Vendor } & \multirow[b]{2}{*}{$\begin{array}{c}\text { Storage } \\
\text { Type }\end{array}$} & \multirow[b]{2}{*}{ Model } & \multirow[b]{2}{*}{$\begin{array}{c}\text { Capacity } \\
\text { (assemblies) }\end{array}$} & \multicolumn{5}{|c|}{$\begin{array}{c}\text { Assemblies Stored } \\
\text { Through }\end{array}$} \\
\hline & & & & & & & 1989 & 1990 & 1991 & 1992 & Total \\
\hline \multirow[t]{3}{*}{ Virginia } & Surry $1 \& 2$ & $07 / 86$ & $\begin{array}{l}\text { General Nuclear } \\
\text { Systems, Inc. }\end{array}$ & & Castor V/21 & 21 PWR & 168 & 84 & -- & 63 & 315 \\
\hline & & & Westinghouse & Metal Cask & MC-10 & 24 PWR & - & -- & 24 & -- & 24 \\
\hline & & & $\begin{array}{l}\text { Nuclear Assurance } \\
\text { Corporation }\end{array}$ & Metal Cask & NAC-I28S $/ \mathrm{T}$ & 28 PWR & - & -- & 28 & -- & 28 \\
\hline \multicolumn{3}{|c|}{ Subtotal............. } & & & & & 168 & 84 & 52 & 63 & 367 \\
\hline \multicolumn{3}{|c|}{ TOTAL ............. } & & & & & 224 & 180 & ${ }^{c} 268$ & $c_{231}$ & c903 \\
\hline
\end{tabular}

a Date license application was submitted to the Nuclear Regulatory Commission.

bDate utility estimates license application to be approved by the Nuclear Regulatory Commission.

A total of 1,464 high-temperature, gas-cooled reactor (HTGR) fuel elements are in dry storage at the Fort St. Vrain reactor. These HTGR fuel elements are not included in the above table totals. See Technical Note 6 in Appendix E.

PWR = Pressurized-water reactor; BWR = Boiling-water reactor.

Source: Energy Information Administration, Form RW-859, "Nuclear Fuel Data" (1992). 


\section{Assembly Characteristics}

\section{Background}

A fuel rod, used in a nuclear power reactor, consists essentially of a stack of uranium dioxide $\left(\mathrm{UO}_{2}\right)$ pellets encapsulated within a metal tube that is sealed at both ends. Current light-water reactors (LWRs) use zircaloy cladding almost exclusively because of its lower neutron absorption relative to stainless steel. An LWR fuel assembly (or bundle) is a configuration of fuel rods assembled together for insertion into a reactor core.

Fuel assemblies are constructed from a number of individual fuel rods arranged together, generally in square arrays. Historically, these arrays have been of many different sizes. For example, one boiling-water reactor (BWR) design uses an array of 6 rods by 6 rods. BWR designs have had $6 \times 6,7 \times 7,8 \times 8,9 \times 9$, $10 \times 10$, and $11 \times 11$ fuel rod arrays. Pressurized-water reactor (PWR) designs have $13 \times 14,14 \times 14,15 \times 15$, $15 \times 16,16 \times 16,17 \times 17$, and $17 \times 18$ fuel rod arrays. Virtually all assemblies are stamped with a unique identifier.

In conjunction with Oak Ridge National Laboratory (ORNL), EIA has developed a categorization of assembly classes and assembly types within each class for spent fuel. This categorization, described in Appendix $B$, is based on the rod array configuration, fuel vendor, and other distinguishing characteristics, such as assembly length and width and type of fuel cladding. The eight-character assembly type codes listed in Appendix B are the codes used on Form RW-859. Neither enrichment nor discharge burnup affects the assembly type classification.

Some assembly types have had very limited use. For example, the Big Rock Point reactor has had 84-inchlong assemblies loaded with fuel rods in $7 \times 7,8 \times 8,9 \times 9$, and $11 \times 11$ array configurations. These assemblies had a range of initial loading weights of uranium and were fabricated by either General Electric, Nuclear Fuel Services, or Siemens Nuclear Fuel Company (formerly Advanced Nuclear Fuel Corporation, Exxon Nuclear Corporation, and Jersey Nuclear). These assemblies were manufactured exclusively for the Big Rock Point reactor.
Appendix B provides an excerpt from Form RW-859 instructions, titled "Assembly Types." This table defines the assembly type codes used in this report, and was the basis for developing Tables 17 and 18. Assembly types are listed by reactor type (BWR, PWR) and are then further identified by fabricator, cladding material, initial uranium content, size, and number discharged (Appendix B, Table B5). There are 124 different reactor fuel assembly types which are, have been, or will be in use by nuclear power reactors. Table B5 also shows that BWR fuel assemblies range from 4.28 to 6.52 inches in width, from 84.0 to 176.2 inches in length, and have a uranium component of 70 to 195 kilograms (kg). PWR fuel assemblies range from 6.27 to 8.54 inches in width, from 111.8 to 199.0 inches in length, and have a uranium component of 191 to $542 \mathrm{~kg}$. Tables B6 and $B 7$ present assembly type summaries by reactor unit and by assembly type, respectively.

The Characteristics Data Base (CDB) developed by ORNL contains a more complete physical description of each type of assembly. That data base includes the characteristics of the fuel pellets in each assembly, the material composition of each component of the hardware, and related information. It also contains detailed mechanical drawings for each assembly type.

\section{Fabricator Summary}

Spent fuel assemblies summarized by fabricator and reactor type show the percentage of BWR and PWR assemblies produced by each fabricator in Table 17 . General Electric fabricated 91 percent of the BWR discharged fuel assemblies, most of which are 176.2 inches long. Approximately 58 percent of the discharged PWR fuel assemblies were manufactured by Westinghouse. The majority of Westinghouse PWR fuel assemblies have a length of 159.8 inches.

\section{Assembly Distribution}

The distribution of assembly transverse dimensions is presented in Table 18. Assemblies utilized in BWRs vary from 84.0 to 176.2 inches long. However, 
Table 17. Assemblies by Fuel Fabricating Company and Reactor Type

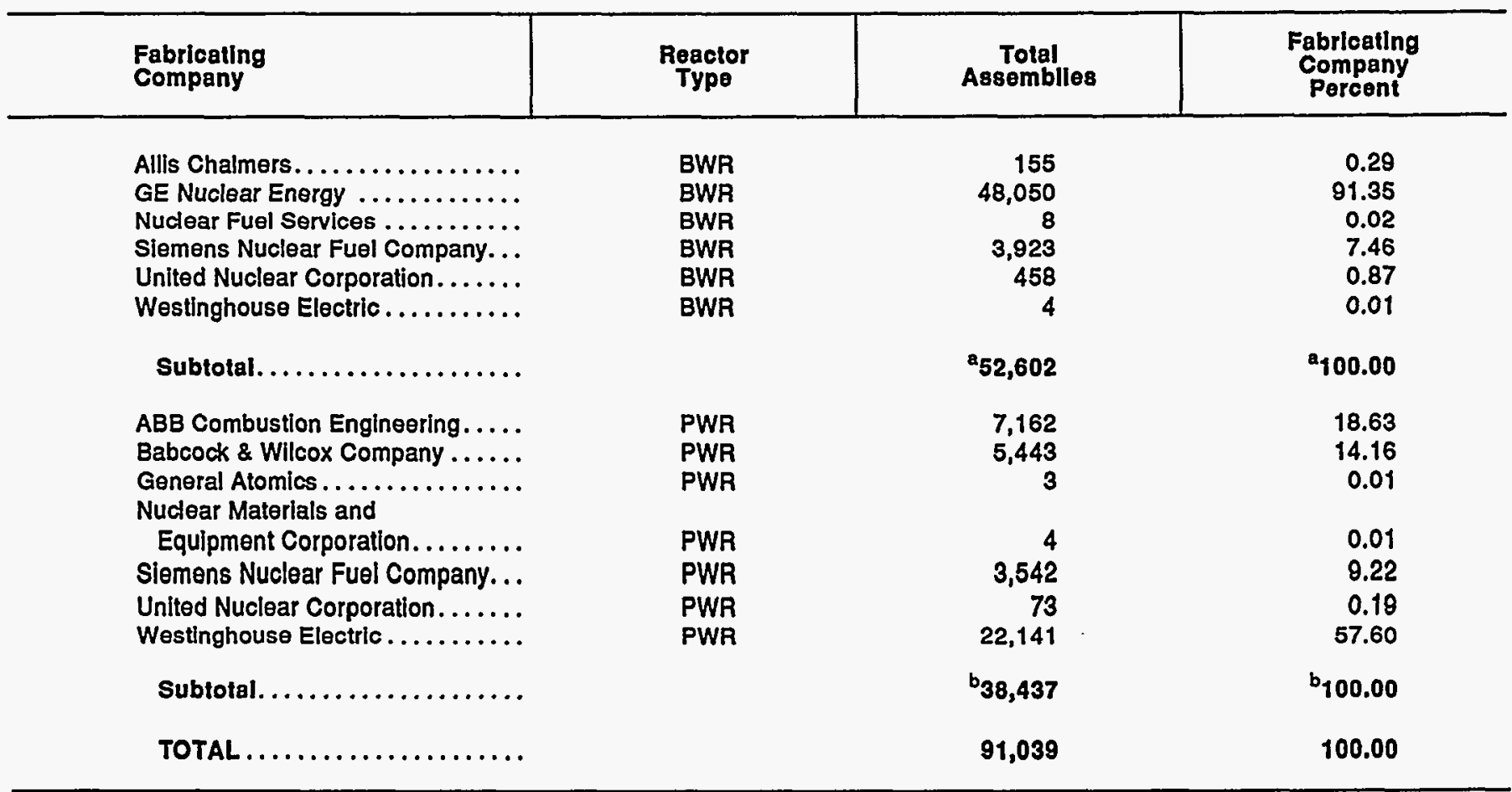

${ }^{a} A$ total of 4 temporarily discharged BWR assemblies that have not been assigned an assembly type code are included in the Subtotal columns. Without an assembly type code, the fuel fabricating company cannot be Identified. See Technical Note 10 in Appendix E.

${ }_{A} A$ total of 69 temporarily discharged PWR assemblies that have not been assigned an assembly type code are included in the Subtotal columns. Without an assembly type code, the fuel fabricating company cannot be identified. See Technical Note 10 in Appendix E.

BWR = Boiling-water reactor; PWR = Pressurized-water reactor.

Note: A total of 2,208 high-temperature, gas-cooled reactor (HTGR) fuel elements, with inltial uranlum content equal to 25.0 metric bns of uranium (MTU), were discharged. These HTGR fuel elements are not included in the above table. See Technical Note 6 in Appendix E. Source: Energy Information Administration, Form RW-859, "Nuclear Fuel Data" (1992). 
Table 18. Distribution of Assembly Transverse Dimensions

\begin{tabular}{|c|c|c|c|}
\hline $\begin{array}{c}\text { Assembly } \\
\text { Length } \\
\text { (Inohes) }\end{array}$ & $\begin{array}{c}\text { Assembly } \\
\text { Width } \\
\text { (inches) }\end{array}$ & $\begin{array}{c}\text { Number } \\
\text { of } \\
\text { Assemblies }\end{array}$ & $\begin{array}{l}\text { Assembly } \\
\text { Percent }\end{array}$ \\
\hline \multicolumn{4}{|c|}{ Bolling-Water Reactors (BWR) } \\
\hline $\begin{array}{r}84.0 \\
95.0 \\
102.5 \\
134.4 \\
171.2 \\
176.2\end{array}$ & $\begin{array}{l}6.52 \\
4.67 \\
5.62 \\
4.28 \\
5.44 \\
5.44\end{array}$ & $\begin{array}{r}379 \\
390 \\
333 \\
892 \\
17,205 \\
33,399\end{array}$ & $\begin{array}{r}0.72 \\
0.74 \\
0.63 \\
1.70 \\
32.71 \\
63.49\end{array}$ \\
\hline TOTAL & & $a_{52,602}$ & $a_{100.00}$ \\
\hline \multicolumn{4}{|c|}{ Pressurlzed-Water Reactors (PWR) } \\
\hline 111.8 & 7.62 & 533 & 1.39 \\
\hline 137.1 & 7.76 & 665 & 1.73 \\
\hline 137.1 & 8.42 & 840 & 2.19 \\
\hline 138.8 & 6.27 & 160 & 0.42 \\
\hline 146.0 & 8.10 & 529 & 1.38 \\
\hline 147.5 & 8.20 & 733 & 1.91 \\
\hline 157.0 & 8.10 & 4,069 & 10.59 \\
\hline 158.2 & 8.10 & 464 & 1.21 \\
\hline 159.8 & 7.76 & 3,740 & 9.73 \\
\hline 159.8 & 8.44 & 18,608 & 48.41 \\
\hline 165.7 & 8.54 & 4,870 & 12.67 \\
\hline 176.8 & 8.10 & 1,960 & 5.10 \\
\hline 178.3 & 8.10 & 848 & 2.21 \\
\hline 199.0 & 8.43 & 349 & 0.91 \\
\hline TOTAL & & $b_{38,437}$ & $b_{100.00}$ \\
\hline
\end{tabular}

A total of 4 temporarily discharged BWR assemblies that have not been assigned an assembly type code are included in the Total colymns. See Technical Note 10 in Appendix E.

${ }_{A}$ total of 69 temporarily discharged PWR assemblies that have not been assigned an assembly type code are included in the Total columns. Seo Technical Note 10 in Appendix E.

Source: Energy Information Administration, Form RW-859, "Nuclear Fuel Data" (1992). 
96 percent of all these assemblies are greater than or equal to 171.2 inches in length. Assembly lengths used in PWRs are from 111.8 to 199.0 inches; 79 percent of all PWR assemblies are greater than or equal to 159.8 inches in length. Assemblies used in BWRs vary in maximum as built assembly width from 4.28 to 6.52 inches. Maximum as built assembly widths used in PWRs are from 6.27 to 8.54 inches. More than 97 percent of the BWR assemblies are greater than or equal to 5.44 inches in width, and approximately 61 percent of PWR assemblies are greater than or equal to 8.44 inches in width.

\section{Assembly Identifiers}

Domestic LWR fuel assemblies are identified by a serial number that is stamped on each assembly. These serial numbers are used as identifiers throughout the life of the fuel. The uniqueness of assembly serial numbers is important in determining their effectiveness as unambiguous identifiers. The ORNL report Analysis of Assembly Serial Number Usage in Domestic Light-Water Reactors (ORNL/TM11841, May 1991), studied serial numbering schemes, the effectiveness of these schemes, and how many duplicate serial numbers occur on domestic LWR fuel assemblies. The serial numbering scheme adopted by the
American National Standards Institute (ANSI) ensures uniqueness of assembly serial numbers. Westinghouse, Babcock \& Wilcox, and Siemens use the ANSI Standard for serial numbers. Combustion Engineering and General Electric both use serial numbers which are unique but do not follow the ANSI Standard. Some assemblies fabricated prior to the establishment of the ANSI Standard in 1974 may have their own serial number schemes which are unique only at the reactor where they were originally irradiated.

Most of the numbering schemes used by electric utilities are not inherently unique, and errors within such schemes account for all of the identified serial number duplication except for three isolated instances. Three duplicate serial numbers were found when analysis focused on duplication within the individual fuel inventory at each reactor site, but these were traced back to data response errors. There were also three instances where the serial numbers used to identify assemblies used for hot cell studies differed from the serial numbers reported to EIA. As a result of the study, EIA is requesting that utilities provide the stamped serial number on the assembly when reporting these data on the Form RW-859 survey. 


\section{Appendix A}

\section{Survey Methodology}

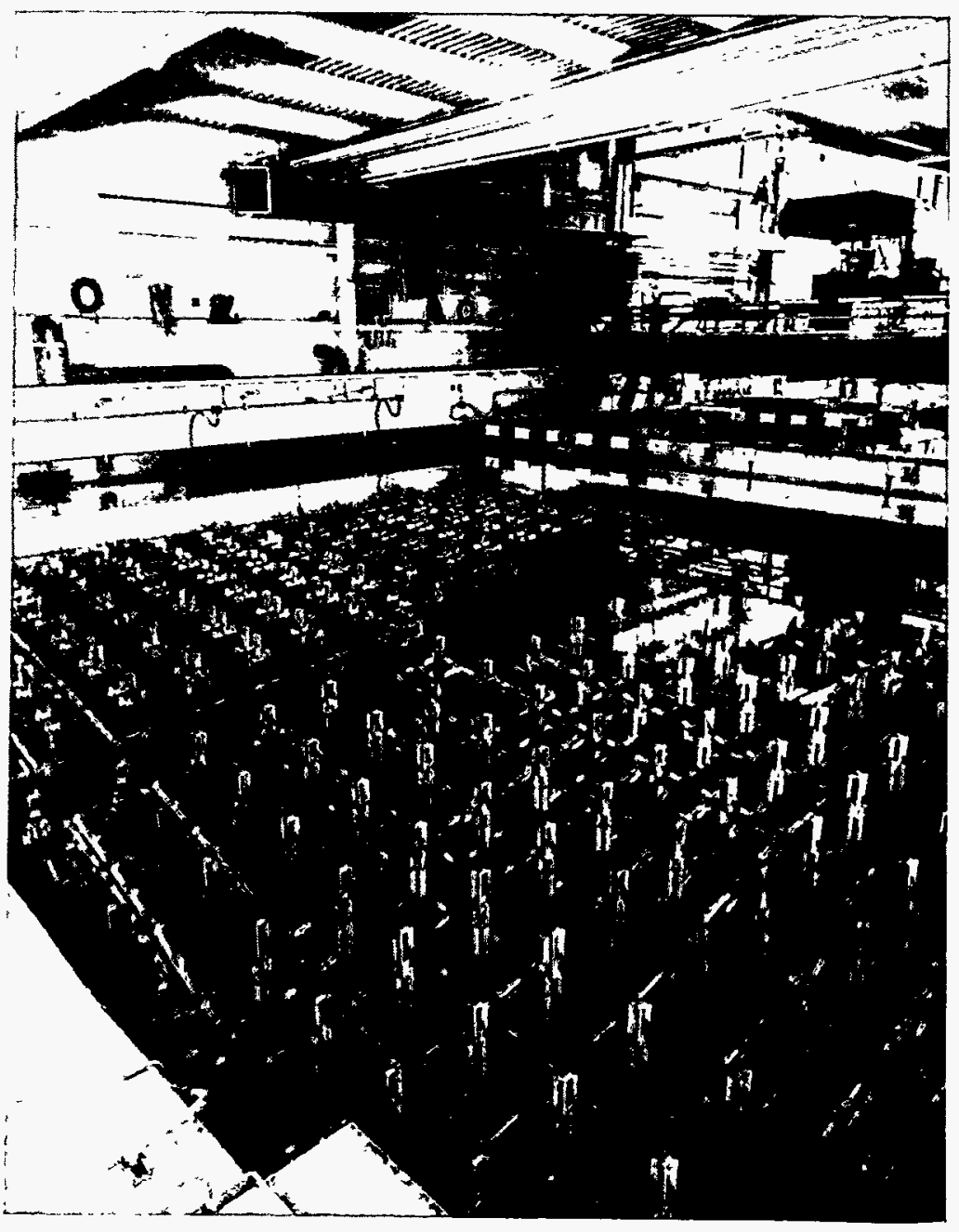




\section{Appendix A}

\section{Survey Methodology}

\section{Survey Design}

The Energy Information Administration (EIA) administers the Nuclear Fuel Data survey, Form RW-859, under a Memorandum of Understanding with the Office of Civilian Radioactive Waste Management (OCRWM). Form RW-859 is used to collect data from all commercial utilities that operate nuclear power plants and from all other owners of commercial spent nuclear fuel. Form RW-859 is a mandatory data collection form authorized under the Federal Energy Administration Act of 1974 (15 USC 761 et seq.) and the Nuclear Waste Policy Act of 1982, as amended (42 USC 10101 et seq.)

EIA normally revises the Nuclear Fuel Data survey form every 3 years. The current version of the survey form, which has been used since the 1991 survey year, is used to collect more detailed data on dry storage, consolidated assemblies, assembly identifier codes, failed fuel, and nonfuel components. A number of reporting inconsistencies have appeared on these revised sections of the survey form. EIA is currently undertaking an extensive form redesign effort to correct the current deficiencies, to collect additional data elements, and to delete no longer needed data elements. This next version of the form will be used beginning with the 1994 survey year.

Respondents to the Nuclear Fuel Data survey are required to report data on every fuel assembly discharged from domestic commercial nuclear reactors, spent fuel projected to be discharged, and spent fuel storage pool inventories and capacities. Form RW-859 respondents list all assemblies permanently discharged at the end of the reporting cycle, assemblies declared to be temporarily discharged at the end of the last cycle, assemblies to be inserted during the next cycle, previously irradiated assemblies to be inserted in the next cycle, and assemblies shipped to another storage site or to dry storage.

For all assemblies permanently discharged during the most recent refueling interval, the utilities submit the following data on Form RW-859:
- Discharge cycle

- End-of-cycle date

- Average initial loading weight for each group of assemblies, in kilograms of uranium (kg)

- Average nominal initial enrichment assay for each group of assemblies, in percent weight

- Average discharge burnup for each group of assemblies, in gigawattdays thermal per initial metric ton of uranium (GWDt/MTU)

- Assembly model type (Table B4) and rod array configuration for each group of assemblies

- Current storage location of each assembly

- Standard disposal contract number applicable to each assembly

- Number of assemblies in each group

- Specific unique assembly identifiers for all assemblies in each group, including Form RW-859, American National Standards Institute (ANSI), and fuel fabricator identifiers.

The above spent fuel discharge data are reported at the batch level, where a batch is characterized by the number of assemblies in it, the nominal initial enrichment, and the batch average discharge burnup. The utilities also provide spent fuel discharge projections for their next five fueling cycles, along with the projected cycle shutdown dates and the cycle burnup in terms of effective full-power days. Spent fuel storage data include the amount of fuel stored at each pool site, or in dry storage, current, licensed and maximum storage site capacities, data on planned increases in pool storage capacity, maximum cask weights that can be handled at each site, and scheduled shipments of assemblies to other locations.

This appendix includes microfiche containing the Nuclear Fuel Data survey, Form RW-859, and the instructions for completing the survey. 


\section{Survey Universe and Frame}

Form RW-859 is a total universe survey of all owners of commercial spent nuclear fuel. The Form RW- 859 survey frame, therefore, consists of all commercial electric utility nuclear power plants and all storage facilities possessing irradiated nuclear fuel from commercial power plants. The respondent population for Form RW-859 is stable. The frame contains operating reactors, storage facilities, reactors that have been permanently shut down, and reactors that have been constructed but are not yet operating. Shut down reactors are maintained on the survey frame for their historical data. Currently, 54 utilities report for 120 reactors. Four storage facilities are also required to report.

\section{Data Collection Procedures}

EIA sends survey packages in December to an official of each company, via certified mail to ensure their receipt and to ascertain the correct mailing address. Survey packages include a diskette containing current data, a software diskette, a paper copy of the survey form, a set of instructions, a pictogram showing current inventory and storage data, a cover letter, and a return envelope. Most Form RW-859 respondents submit their data on diskette.

The microcomputer software system used is referred to as the Automated Nuclear Fuel Data Collection System (ANFDCS). This is a CLIPPER-compiled program with a dBASE file structure. The microcomputer software is designed to run on any IBM-compatible microcomputer.

In order to reduce the respondent burden, EIA provides the organizations with their current data and requests them to update these data using the ANFDCS and the instructions provided. EIA provides assistance in completing the survey by telephone when requested. Most respondents successfully and accurately update their data diskettes with little or no assistance and file their completed surveys by the requested deadlines.

\section{Data Editing, Analysis, and Processing}

As EIA receives survey packages from Form RW-859 respondents, they are logged in and reviewed. The preliminary review is to check for obvious errors or omissions and to verify respondent name, address, and contact information. Data from the completed Form RW-859 surveys are then uploaded to the EIA mainframe computer and maintained in two files. The first is an assembly-level SAS file, which contains data on all individual assemblies at all reactors. These detailed assembly data are aggregated to the batch level. Batch level data are maintained in the second file, a partitioned data set of flat transaction files, with one member for each reactor.

Computer programs review individual assembly data and report the data in well-organized formats that are useful to both analysts and management. A number of the tables and graphs presented in this Report were produced from these programs. These tables are also available through the Nuclear Data Information System (NUCDIS), a microcomputerbased, menu-driven information retrieval system. Additional tables, which may be of interest to analysts requiring greater detail, are available upon request from OCRWM or EIA.

In addition to updating the historical Form RW-859 data files, EIA also produces spent fuel projections, using a set of assumptions concerning the future operation of the Nation's nuclear power plants. While the projected data submitted by the utilities generally forecast a more optimistic operating environment, in aggregate, than has actually been achieved, both EIA and utility forecasts track each other reasonably well in the short term. EIA developed the Disaggregate Forecasting System to bring the aggregated EIA forecasts in line with comparable utility projections. This process preserves, as much as possible, the information that the reactor operators provide in their spent fuel projections. The disaggregated forecasts are published annually in EIA's World Nuclear Capacity and Fuel Cycle Requirements report.

The historical data submitted on Form RW-859 aggregated to the batch-level, along with the projections of spent fuel discharges from EIA's disaggregated forecasts, constitute the official DOE spent fuel data base. EIA distributes this data base in computer files on magnetic tape to the OCRWM and its contractors, and to the DOE national laboratories for their review and analysis.

\section{Response Rates}

Respondents to Form RW-859 are required to submit forms under one of two options, chosen by the utility. 
Option A requires a utility to submit its data within 90 days of cycle startup after refueling. Data reflect the utility's status at cycle startup. Option B requires a utility to submit data once each year by February 15. Data reflect the utility's status as of December 31 of the previous year. Storage-only facilities are required to report annually by February 15 for the previous year.

A total of 27 Option A reactors submitted data reflecting a cycle startup after refueling in 1992. Data for the remaining 19 Option A reactors were simply "rolled over" from the previous year. All 74 Option B reactors and 4 storage facilities also reported. The data presented in this report, therefore, represent an accurate account as of December 31, 1992.

\section{Missing Data and Imputation}

EIA encourages utility companies to complete all elements on Form RW-859. There is no imputing of missing values. If there are omissions, or if incorrect data are reported, the EIA Survey Manager telephones the utility to obtain missing or corrected data.

A limited number of data items were identified as missing or omitted from Form RW-859 data files. Most missing items represented data that were not available to the companies in their data files. Respondents were telephoned regarding the missing items to verify that these items could not be reported. Only confirmed company-reported data are contained in the data base and included in this report.

\section{EIA Quality Assurance Procedures}

To ensure the quality and accuracy of data collected on Form RW-859, EIA has developed extensive quality assurance (QA) procedures as part of the Form RW-859 survey processing system. At the core of the process is a mainframe Pascal QA program that produces a matrix for each reactor. The EIA QA report is run to identify possible errors throughout the processing cycle and to ensure that data submitted on diskettes have been correctly uploaded from the PC to the mainframe. After the Survey Manager and other data users have reviewed the EIA QA matrix for a particular reactor, data are corrected and an iterative process of running additional reports is performed until all data for that reactor are clean. A description of the EIA QA reports and a more detailed discussion of EIA QA procedures is included in Appendix D of this Service Report.

The data certainty of all spent fuel assembly weights and discharge dates has been verified through reconciliation with two separate but related efforts: the spent fuel fee paid by utilities for assemblies discharged prior to April 7, 1983, and the Acceptance Priority Ranking (APR) process which designates the order in which spent fuel will be picked up by DOE on an oldest-fuel-first basis. 


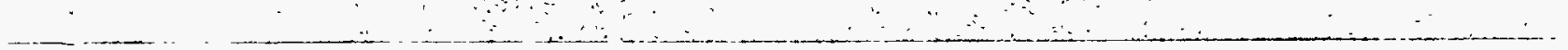


Appendix B

\section{Detailed Assembly Type Statistics}

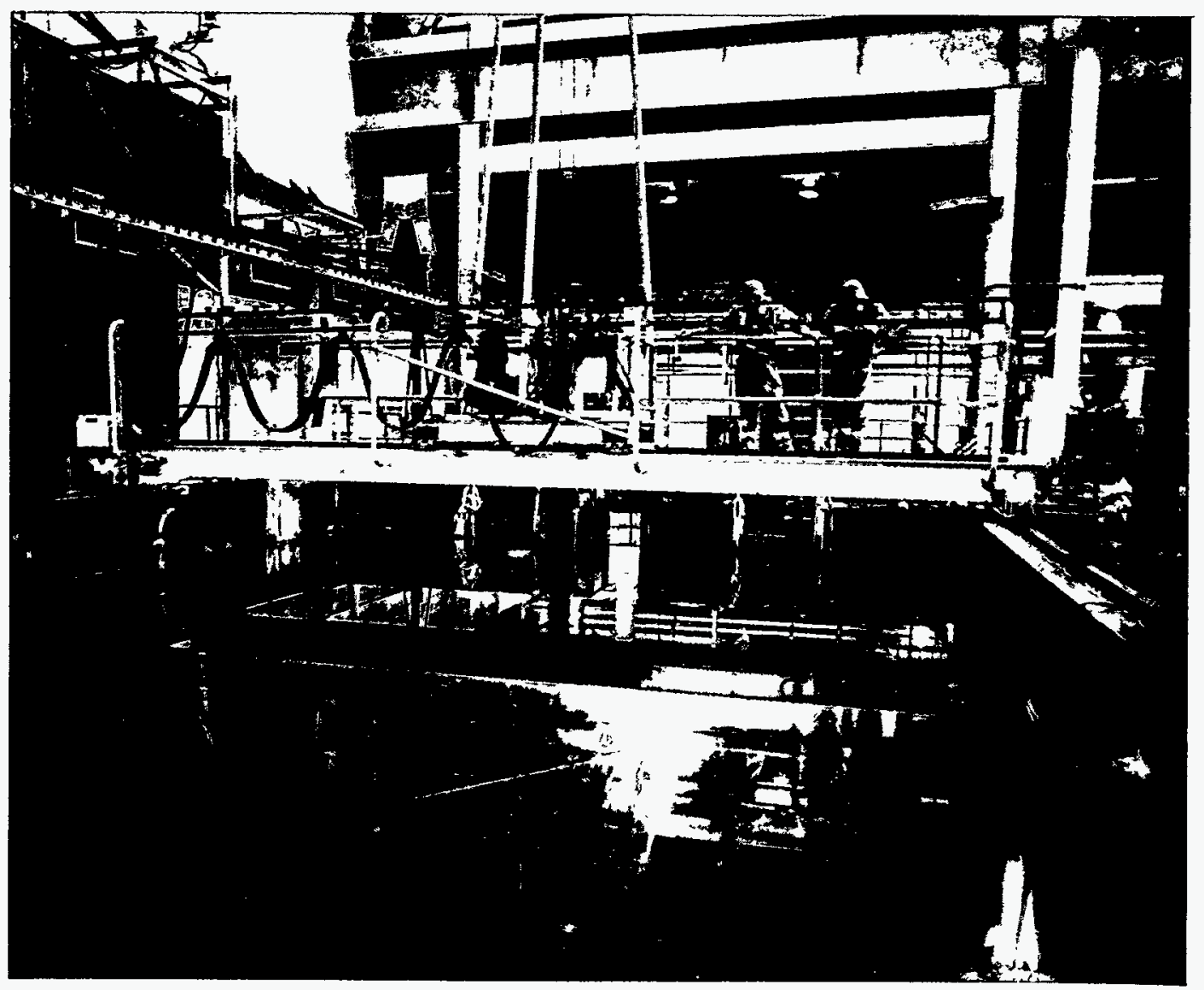


$\therefore$ 


\section{Detailed Assembly Type Statistics}

The spent fuel Characteristics Data Base (CDB) developed by Oak Ridge National Laboratory (ORNL) includes detailed information on physical, radiological, and quantitative characteristics of spent nuclear fuel. The physical characteristics are organized by assembly class and, within assembly class, by assembly type codes (developed jointly by ORNL and EIA) to provide detailed information about each type, including the length and width of the overall assembly and of the individual fuel rods, the number of fuel rods, the type of material used in the cladding and other parts of the assembly, and detailed assembly drawings. An eight-character assembly type code links Form RW-859 data with the CDB.

The characteristics of fuel assemblies are important in many areas of radioactive waste management. Assembly dimensions are clearly a major concern for shipping cask and waste package designers. If the fuel is to be consolidated, knowledge of the individual assembly types is essential, from the rod array configuration to the location of the bolts and welds for disassembly. The composition of the assembly hardware and cladding is also important. For example, cobalt- 60 , one of the major activation products, is more concentrated in stainless steel than in zircaloy. Assembly type characteristics are summarized by assembly type and reactor and are further amplified by a CDB module referred to as "LWR Fuel Assemblies Data Base," as described in the four-volume publication, Characteristics of Potential Repository Wastes (DOE/RW-0184-R1).

Whereas the physical characteristics of assemblies are identified through the assembly type codes, the radiological data in the $C D B$ are calculated based on the reactor type, discharge burnup, initial enrichment, and age from time of discharge. All these data are obtainable from Form RW-859. The radiological characteristics of the discharged assemblies are timedependent and generally subject to the laws of exponential decay. The radiological characteristics in the CDB include grams, curies, and watts for more than 350 isotopes; photons for 18 gamma energy ranges; and neutrons.
This appendix provides detailed information on assembly type characteristics that is not included in the body of the report. These data are presented in table format and include the following:

- Table B1, Fabricator Identifiers

- Table B2, Assembly Class and Design Type

- Table B3, Reactors by Assembly Class and Design Type

- Table B4, Descriptions of Assembly Types

- Table B5, Assembly Characteristics by Assembly Type

- Table B6, Assembly Type Summary by Reactor

- Table B7, Assembly Type Summary by Assembly Type

- Table B8, Spent Fuel Assemblies, by Type, Discharged by Year

- Table B9, Initial Uranium Content of Spent Fuel Assemblies, by Type, Discharged by Year

- Table B10, Burnup of Spent Fuel Assemblies, by Type, Discharged by Year.

Each assembly is characterized by fabricator, array size, and model type. Table B1 assigns an identification code for each fabricator. Table B2 lists reactors by assembly class and design type. Table B3 lists each reactor by array size and assembly class. Descriptions of assembly types are presented in Table B4.

The information provided for each assembly type in Table B5 includes fabricator, cladding material, assembly dimensions and initial uranium content, and number discharged. Additionally, number of rods, length, and rod position data are also presented. Table B6 is sorted by reactor. The data elements are assembly type, number of assemblies, initial uranium content, average burnup, and enrichment (weight percent). Table B7 presents the same information, sorted by assembly type. The numbers of assemblies discharged in 2-year periods are shown in Table B8. An eight-character alphanumeric code is used to identify the assembly type. The same information, in metric tons of uranium, appears in Table B9. The average discharge burnups of spent fuel assemblies by 2-year group and assembly type are presented in Table B10. 
The fuel assembly types throughout Appendix B have been listed in the following manner:

- The primary ordering criterion is assembly class -only assembly types within a reactor's particular assembly class are used at that reactor.

- Within an assembly class, the secondary ordering criterion is fabricator. All fuel assembly types fabricated by the primary fuel fabricator (typically the reactor vendor) are listed first, followed by other fabricators in a generally alphabetical manner.

- Within a fabricator in an assembly class, the primary ordering criterion is chronology. Earlier versions of fuels are listed first, more recent versions are listed later. This ordering is not absolute, but is based on the progression of designs over time.

The detailed assembly type table statistics presented in Appendix B use the eight-character assembly type code. The eight-character code is used exclusively for Form RW-859 submissions and in the CDB.

The code will be presented as follows: 8-Character Code W1414ATR

The first three characters of the code identify the assembly class and are fully compatible with the CDB scheme. The W14 in the example indicates WE $14 \times 14$ assembly class. All reactor-specific assembly type codes have an " $\mathrm{X}$ " as the first characteristic of the eight-character code (e.g., XHN indicates Haddam Neck). The next two characters identify the array size $(14=14 \times 14)$. The sixth character represents the fuel vendor (e.g., A for Advanced Nuclear Fuel (formerly Exxon Nuclear)). The last two characters are a discriminator code that is designed to distinguish two fuel assembly types that are otherwise the same. The TR in the example indicates a top rod version.

Some examples of discriminator codes that are used as the last two characters are:

Discriminator

- L

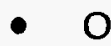

- 5

- $\mathrm{P}$ $\underline{\text { Meaning }}$

Low parasitic fuel

(LOPAR) zircaloy guide tubes

Optimized Fuel Assembly

(OFA) zircaloy spacers,

smaller fuel rods

GE-5 fuel design (original

retrofit fuel)

Prepressurized BWR fuel rods
Assembly Type Code

W1414WL

W1515WL

W1414WO

W1515WO

W1717WO

G2308G5

G4608G5

G2308GP

G4608GP 
A fuel rod, used in a nuclear power reactor, consists of uranium dioxide pellets encapsulated in a metal tube that is sealed at both ends. A fuel assembly is fabricated from a number of individual fuel rods arranged in arrays. The arrays are generally square, and they have been made in many different dimensions. There are 124 reactor fuel assembly types which have been, or will be used. Each assembly type has certain distinguishing characteristics, such as the number of rods per assembly, fuel rod diameter, cladding type, materials used in fabrication, and other design features. The fuel assembly fabricator, the specific reactor and fuel models are characteristics often used to describe or name assembly types.
Assembly classes generally refer to generic groupings of assembly types designed for a particular class of reactor (for example, WE $14 \times 14$ class reactors). Assembly classes can also be reactor-specific, because in some cases fuel assembly arrays are configured so that they fit only one reactor (for example, the Big Rock Point reactor).

The fabricator of an assembly for a given reactor is not necessarily related to the reactor vendor or to the original fuel fabricator. The following fuel fabricator codes are used:

\begin{tabular}{|c|c|c|c|}
\hline Current Fabrlcator Name & Other Names Used & $\begin{array}{l}\text { Short } \\
\text { Name }\end{array}$ & Code \\
\hline ABB Combustion Engineering... & $\begin{array}{l}\text { Combustion Engineering } \\
\text { ABB Atom (ABB) (BWR Only) }\end{array}$ & CE & c \\
\hline Allis Chalmers............................. & & $A C$ & $\mathbf{L}$ \\
\hline Babcock \& Wilcox Company .... & & $B \& W$ & B \\
\hline GE Nuclear Energy..$\ldots \ldots \ldots \ldots \ldots \ldots \ldots \ldots$ & General Electric & GE & G \\
\hline General Atomics ........... & $\begin{array}{l}\text { Gulf General Atomics (GGA) } \\
\text { Gulf/United Nuclear Fuels (GULF) }\end{array}$ & GA & $H$ \\
\hline Nuclear Fuel Services ..... & & NFS & N \\
\hline $\begin{array}{l}\text { Nuclear Materlals and Equipment } \\
\text { Corporation } \ldots \ldots \ldots \ldots \ldots \ldots \ldots \ldots \text {. }\end{array}$ & NUMEC & NU & M \\
\hline Slemens Nuclear Fuel Company.... & $\begin{array}{l}\text { Advanced Nuclear Fuel Corporation (ANF) } \\
\text { Exxon Nuclear Corporatlon (EXA) } \\
\text { Jersey Nuclear }\end{array}$ & SIEM & A \\
\hline Unlted Nuclear Corporation.................. & & UNC & U \\
\hline Westlnghouse Electric ..................... & & WE & w \\
\hline
\end{tabular}

BWR = Bolling-water reactor.

Source: Energy Information Administration, Form RW-859, "Nuclear Fuel Data" (1992). 
Table B2. Assembly Class and Design Type

\begin{tabular}{|c|c|c|c|c|c|}
\hline Electrlc Uttllty Name & $\begin{array}{l}\text { Reactor } \\
\text { Name }\end{array}$ & $\begin{array}{l}\text { Reactor } \\
\text { ID }\end{array}$ & $\begin{array}{l}\text { Assombly } \\
\text { Class }\end{array}$ & $\begin{array}{l}\text { Nuclear Steam } \\
\text { System Supplier }\end{array}$ & Design Typeb \\
\hline $\begin{array}{l}\text { Arizona Public Servic } \\
\text { Company } \ldots \ldots \ldots \ldots \ldots \ldots\end{array}$ & $\begin{array}{l}\text { Palo Verde } 1 \\
\text { Palo Verde } 2 \\
\text { Palo Verde } 3\end{array}$ & $\begin{array}{l}0301 \\
0302 \\
0303\end{array}$ & $\begin{array}{l}\text { CE System } 80 \\
\text { CE System } 80 \\
\text { CE System } 80\end{array}$ & $\begin{array}{l}\text { COMB } \\
\text { COMB } \\
\text { COMB }\end{array}$ & $\begin{array}{l}\text { CE80 } \\
\text { CE80 } \\
\text { CE80 }\end{array}$ \\
\hline $\begin{array}{l}\text { Arkansas Power and } \\
\text { Light Company........... }\end{array}$ & $\begin{array}{l}\text { Arkansas Nuclear } 1 \\
\text { Arkansas Nuclear } 2\end{array}$ & $\begin{array}{l}0401 \\
0402\end{array}$ & $\begin{array}{l}\text { BW } 15 \times 15 \\
C E 16 \times 16\end{array}$ & $\begin{array}{l}\text { B\&W } \\
\text { COMB }\end{array}$ & $\begin{array}{l}\text { LLP } \\
\text { CE }\end{array}$ \\
\hline $\begin{array}{l}\text { Baltimore Gas and } \\
\text { Electric Company } \ldots \ldots \ldots \ldots\end{array}$ & $\begin{array}{l}\text { Calvert Cliffs } 1 \\
\text { Calvert Cliffs } 2\end{array}$ & $\begin{array}{l}0501 \\
0502\end{array}$ & $\begin{array}{l}\text { CE } 14 \times 14 \\
\text { CE } 14 \times 14\end{array}$ & $\begin{array}{l}\text { COMB } \\
\text { COMB }\end{array}$ & $\begin{array}{l}\mathrm{CE} \\
\mathrm{CE}\end{array}$ \\
\hline $\begin{array}{l}\text { Carolina Power and Light } \\
\text { Company .............. }\end{array}$ & $\begin{array}{l}\text { Brunswick } 1 \\
\text { Brunswick } 2 \\
\text { Harris } 1 \\
\text { Robinson } 2\end{array}$ & $\begin{array}{l}0701 \\
0702 \\
0703 \\
0705\end{array}$ & $\begin{array}{l}\text { GE BWR/4-6 } \\
\text { GE BWR/4-6 } \\
\text { WE } 17 \times 17 \\
\text { WE } 15 \times 15\end{array}$ & $\begin{array}{l}\text { GE } \\
\text { GE } \\
\text { WEST } \\
\text { WEST }\end{array}$ & $\begin{array}{l}4 \\
4 \\
3 L P \\
3 L P\end{array}$ \\
\hline $\begin{array}{l}\text { Cleveland Electric } \\
\text { Illuminating Company ....... }\end{array}$ & Perry 1 & 0901 & GE BWR/4-6 & GE & 6 \\
\hline $\begin{array}{l}\text { Commonwealth Edison } \\
\text { Company } \ldots \ldots \ldots \ldots \ldots \ldots\end{array}$ & $\begin{array}{l}\text { Braidwood } 1 \\
\text { Braidwood } 2 \\
\text { Byron } 1 \\
\text { Byron } 2 \\
\text { Dresden } 1 \\
\text { Dresden } 2 \\
\text { Dresden } 3 \\
\text { LaSalle County } 1 \\
\text { LaSalle County } 2 \\
\text { Quad Cities } 1 \\
\text { Quad Cities } 2 \\
\text { Zion } 1 \\
\text { Zion } 2\end{array}$ & $\begin{array}{l}1001 \\
1002 \\
1003 \\
1004 \\
1005 \\
1006 \\
1007 \\
1008 \\
1009 \\
1010 \\
1011 \\
1012 \\
1013\end{array}$ & $\begin{array}{l}\text { WE } 17 \times 17 \\
\text { WE } 17 \times 17 \\
\text { WE } 17 \times 17 \\
\text { WE } 17 \times 17 \\
\text { Dresden } 1 \\
\text { GE BWR/2,3 } \\
\text { GE BWR/2,3 } \\
\text { GE BWR/4-6 } \\
\text { GE BWR/4-6 } \\
\text { GE BWR/2,3 } \\
\text { GE BWR/2,3 } \\
\text { WE } 15 \times 15 \\
\text { WE } 15 \times 15\end{array}$ & $\begin{array}{l}\text { WEST } \\
\text { WEST } \\
\text { WEST } \\
\text { WEST } \\
\text { GE } \\
\text { GE } \\
\text { GE } \\
\text { GE } \\
\text { GE } \\
\text { GE } \\
\text { GE } \\
\text { WEST } \\
\text { WEST }\end{array}$ & $\begin{array}{l}4 L P \\
4 L P \\
4 L P \\
4 L P \\
1 \\
3 \\
3 \\
5 \\
5 \\
3 \\
3 \\
4 L P \\
4 L P\end{array}$ \\
\hline $\begin{array}{l}\text { Consolldated Edison } \\
\text { Company of New York ....... }\end{array}$ & $\begin{array}{l}\text { Indian Point } 1 \\
\text { Indian Point } 2\end{array}$ & $\begin{array}{l}1101 \\
1102\end{array}$ & $\begin{array}{l}\text { Indian Point } 1 \\
\text { WE } 15 \times 15\end{array}$ & $\begin{array}{l}\text { B\&W } \\
\text { WEST }\end{array}$ & $\overline{4 L P}$ \\
\hline $\begin{array}{l}\text { Consumers Power } \\
\text { Company } \ldots \ldots \ldots \ldots \ldots\end{array}$ & $\begin{array}{l}\text { Big Rock Point } \\
\text { Pallsades }\end{array}$ & $\begin{array}{l}1201 \\
1204\end{array}$ & $\begin{array}{l}\text { Big Rock Point } \\
\text { Pallsades }\end{array}$ & $\begin{array}{l}\text { GE } \\
\text { COMB }\end{array}$ & $\begin{array}{l}1 \\
\mathrm{CE}\end{array}$ \\
\hline $\begin{array}{l}\text { Dairyland Power } \\
\text { Cooperative } \ldots \ldots \ldots \ldots \ldots\end{array}$ & Lacrosse & 1301 & LaCrosse & $A C$ & -- \\
\hline Detrolt Edison Comapny ...... & Enrico Fermi 2 & 1402 & GE BWR/4-6 & GE & 4 \\
\hline
\end{tabular}

See footnotes at end of table. 
Table B2. Assembly Class and Design Type (Continued)

\begin{tabular}{|c|c|c|c|c|c|}
\hline Duquesne Light Company ..... & $\begin{array}{l}\text { Beaver Valley } 1 \\
\text { Beaver Valley } 2\end{array}$ & $\begin{array}{l}1601 \\
1602\end{array}$ & $\begin{array}{l}\text { WE } 17 \times 17 \\
\text { WE } 17 \times 17\end{array}$ & $\begin{array}{l}\text { WEST } \\
\text { WEST }\end{array}$ & $\begin{array}{l}\text { 3LP } \\
\text { 3LP }\end{array}$ \\
\hline Florida Power Corporation..... & Crystal River 3 & 1701 & BW $15 \times 15$ & B\&W & LLP \\
\hline GPU Nuclear Corporation ..... & $\begin{array}{l}\text { Three Mile Island } 1 \\
\text { Oyster Creek }\end{array}$ & $\begin{array}{l}1801 \\
1903\end{array}$ & $\begin{array}{l}\text { BW } 15 \times 15 \\
\text { GE BWR/2,3 }\end{array}$ & $\begin{array}{l}\text { B\&W } \\
\text { GE }\end{array}$ & $\begin{array}{l}\text { LLP } \\
2\end{array}$ \\
\hline Georgla Power Company...... & $\begin{array}{l}\text { Hatch } 1 \\
\text { Hatch } 2 \\
\text { Vogtle } 1 \\
\text { Vogtle } 2\end{array}$ & $\begin{array}{l}2001 \\
2002 \\
2003 \\
2004\end{array}$ & $\begin{array}{l}\text { GE BWR/4-6 } \\
\text { GE BWR/4-6 } \\
\text { WE } 17 \times 17 \\
\text { WE } 17 \times 17\end{array}$ & $\begin{array}{l}\text { GE } \\
\text { GE } \\
\text { WEST } \\
\text { WEST }\end{array}$ & $\begin{array}{l}4 \\
4 \\
4 L P \\
4 L P\end{array}$ \\
\hline $\begin{array}{l}\text { Gulf States Utilltes } \\
\text { Company } \ldots \ldots \ldots \ldots \ldots \ldots\end{array}$ & River Bend 1 & 2101 & GE BWR/4-6 & GE & 6 \\
\hline $\begin{array}{l}\text { Indlana Mlchlgan } \\
\text { Power Company .......... }\end{array}$ & $\begin{array}{l}\text { Cook } 1 \\
\text { Cook } 2\end{array}$ & $\begin{array}{l}5801 \\
5802\end{array}$ & $\begin{array}{l}\text { WE } 15 \times 15 \\
\text { WE } 17 \times 17\end{array}$ & $\begin{array}{l}\text { WEST } \\
\text { WEST }\end{array}$ & $\begin{array}{l}\text { 4LP } \\
4 L P\end{array}$ \\
\hline $\begin{array}{l}\text { lowa Electric Light and } \\
\text { Power Company ........... }\end{array}$ & Duane Arnold & 2401 & GE BWR/4-6 & GE & 4 \\
\hline $\begin{array}{l}\text { Kansas Gas and Electrlc } \\
\text { Company } . \ldots \ldots \ldots \ldots \ldots \ldots\end{array}$ & Wolf Creek 1 & 2501 & WE $17 \times 17$ & WEST & 4LP \\
\hline $\begin{array}{l}\text { Long Island Lighting } \\
\text { Company } \ldots \ldots \ldots \ldots \ldots \ldots\end{array}$ & Shoreham & 2601 & GE BWR/4-6 & GE & 4 \\
\hline $\begin{array}{l}\text { Louisana Power and } \\
\text { Light Company............ }\end{array}$ & Waterford 3 & 2701 & CE $16 \times 16$ & COMB & CE \\
\hline $\begin{array}{l}\text { Maine Yankee Atomlc } \\
\text { Power Company .......... }\end{array}$ & Maine Yankee & 2801 & CE $14 \times 14$ & COMB & CE \\
\hline
\end{tabular}

See footnotes at end of table. 
Table B2. Assembly Class and Design Type (Continued')

\begin{tabular}{|c|c|c|c|c|c|}
\hline Electric Utllity Name & $\begin{array}{l}\text { Reactor } \\
\text { Name }\end{array}$ & $\begin{array}{l}\text { Reactor } \\
\text { ID }\end{array}$ & $\begin{array}{c}\text { Assembly } \\
\text { Class }\end{array}$ & \begin{tabular}{|} 
Nuclear Steam \\
System Supplier
\end{tabular} & Deslgn Typob \\
\hline \multicolumn{6}{|l|}{ Nebraska Publlc Power } \\
\hline District...$\ldots \ldots \ldots \ldots$ & Cooper Station & 3001 & GE BWR/4-6 & GE & 4 \\
\hline \multicolumn{6}{|l|}{ New York Power } \\
\hline Authority....... & $\begin{array}{l}\text { Fitzpatrick } \\
\text { Indian Point } 3\end{array}$ & $\begin{array}{l}3901 \\
3902\end{array}$ & $\begin{array}{l}\text { GE BWR/4-6 } \\
\text { WE } 15 \times 15\end{array}$ & $\begin{array}{l}\text { GE } \\
\text { WEST }\end{array}$ & $\begin{array}{l}4 \\
4 L P\end{array}$ \\
\hline \multicolumn{6}{|l|}{ Niagara Mohawk Power } \\
\hline Corporation .......... & $\begin{array}{l}\text { Nine Mile Point } 1 \\
\text { Nine Mile Point } 2\end{array}$ & $\begin{array}{l}3101 \\
3102\end{array}$ & $\begin{array}{l}\text { GE BWR/2,3 } \\
\text { GE BWR/4-6 }\end{array}$ & $\begin{array}{l}\mathrm{GE} \\
\mathrm{GE}\end{array}$ & $\begin{array}{l}2 \\
5\end{array}$ \\
\hline \multicolumn{6}{|l|}{ North Atlantic Energy } \\
\hline Service Corporation & Seabrook & 5901 & WE $17 \times 17$ & WEST & 4LP \\
\hline \multicolumn{6}{|l|}{ Northeast Utilities } \\
\hline Service Company . & Millstone 1 & 3201 & GE BWR/2,3 & GE & 3 \\
\hline & Millstone 2 & 3202 & CE $14 \times 14$ & COMB & $\mathrm{CE}$ \\
\hline & Millstone 3 & 3203 & WE $17 \times 17$ & WEST & 4LP \\
\hline & Haddam Neck & 5701 & Haddam Neck & WEST & 4LP \\
\hline \multicolumn{6}{|l|}{ Northern States Power } \\
\hline Company ........... & Monticello & 3301 & GE BWR/2,3 & GE & 3 \\
\hline & $\begin{array}{l}\text { Prairie Island } 1 \\
\text { Prairie Island } 2\end{array}$ & $\begin{array}{l}3302 \\
3303\end{array}$ & WE $14 \times 14$ & WEST & 2LP \\
\hline & 2 & & $3014 \times 8$ & & \\
\hline \multicolumn{6}{|l|}{ Omaha Public Power } \\
\hline District...$\ldots \ldots$ & Fort Calhoun & 3401 & Fort Calhoun & COMB & CE \\
\hline \multicolumn{6}{|l|}{ Pacific Gas and Electric } \\
\hline Company $\ldots \ldots \ldots \ldots$ & Diablo Canyon 1 & 3501 & WE $17 \times 17$ & WEST & 4LP \\
\hline & Diablo Canyon 2 & 3502 & WE $17 \times 17$ & WEST & $4 L P$ \\
\hline \multicolumn{6}{|l|}{ Pennsylvania Power and } \\
\hline Light Company......... & Susquehanna 1 & 3601 & GE BWR/4-6 & GE & 4 \\
\hline & Susquehanna 2 & 3602 & GE BWR/4-6 & GE & 4 \\
\hline \multicolumn{6}{|l|}{ Philadelphia Electric } \\
\hline Company ........ & Limerick 1 & 3701 & GE BWR/4-6 & GE & 4 \\
\hline & Limerick 2 & 3702 & GE BWR/4-6 & GE & 4 \\
\hline & Peach Bottom 2 & 3704 & GE BWR/4-6 & $\mathrm{GE}$ & 4 \\
\hline & Peach Bottom 3 & 3705 & GE BWR/4-6 & GE & 4 \\
\hline \multicolumn{6}{|l|}{ Portland General Electrle } \\
\hline Company ............. & Trojan & 3801 & WE $17 \times 17$ & WEST & 4LP \\
\hline \multicolumn{6}{|l|}{ Public Service Electric } \\
\hline and Gas Company... & Hope Creek & 4201 & GE BWR/4-6 & GE & 4 \\
\hline & Salem 1 & 4202 & WE $17 \times 17$ & WEST & 4LP \\
\hline & Salem 2 & 4203 & WE $17 \times 17$ & WEST & 4LP \\
\hline \multicolumn{6}{|l|}{ Rochester Gas and } \\
\hline Electric Corporation.... & Ginna & 4401 & WE $14 \times 14$ & WEST & 2LP \\
\hline \multicolumn{6}{|l|}{ Sacramento Municipal } \\
\hline Utillty District ........ & Rancho Seco & 4501 & BW $15 \times 15$ & B\&W & LLP \\
\hline
\end{tabular}

See footnotes at end of table. 
Table B2. Assembly Class and Design Type (Continued)

\begin{tabular}{|c|c|c|c|c|c|}
\hline Electrlc Utllity Name & $\begin{array}{l}\text { Reactor } \\
\text { Name }\end{array}$ & $\underset{\text { ID }}{\text { Reactor }}$ & $\begin{array}{c}\text { Assembly } \\
\text { Class }\end{array}$ & $\begin{array}{l}\text { Nuclear Steam } \\
\text { System Supplier }\end{array}$ & Design Type \\
\hline $\begin{array}{l}\text { South Carolina Electric } \\
\text { and Gas Company.......... }\end{array}$ & Summer & 4601 & WE $17 \times 17$ & WEST & 3LP \\
\hline $\begin{array}{l}\text { Southern Callfornla } \\
\text { Edison Company } . . . \ldots \ldots \ldots\end{array}$ & $\begin{array}{l}\text { San Onofre } 1 \\
\text { San Onofre } 2 \\
\text { San Onofre } 3\end{array}$ & $\begin{array}{l}4701 \\
4702 \\
4703\end{array}$ & $\begin{array}{l}\text { San Onofre } 1 \\
\text { CE } 16 \times 16 \\
\text { CE } 16 \times 16\end{array}$ & $\begin{array}{l}\text { WEST } \\
\text { COMB } \\
\text { COMB }\end{array}$ & $\begin{array}{l}\text { BLP } \\
C E \\
C E\end{array}$ \\
\hline $\begin{array}{l}\text { System Energy Resources, } \\
\text { Inc. } \ldots \ldots \ldots \ldots \ldots \ldots \ldots \ldots\end{array}$ & Grand Gulf 1 & 2901 & GE BWR/4-6 & GE & 6 \\
\hline $\begin{array}{l}\text { Tennessee Valley } \\
\text { Authority } . . \ldots \ldots \ldots \ldots \ldots\end{array}$ & $\begin{array}{l}\text { Browns Forry } 1 \\
\text { Browns Ferry } 2 \\
\text { Browns Forry } 3 \\
\text { Sequoyah } 1 \\
\text { Soquoyah } 2 \\
\text { Watts Bar } 1 \\
\text { Watts Bar } 2\end{array}$ & $\begin{array}{l}4803 \\
4804 \\
4805 \\
4808 \\
4809 \\
4810 \\
4811\end{array}$ & $\begin{array}{l}\text { GE BWR/4-6 } \\
\text { GE BWR/4-6 } \\
\text { GE BWR/4-6 } \\
\text { WE } 17 \times 17 \\
\text { WE } 17 \times 17 \\
\text { WE } 17 \times 17 \\
\text { WE } 17 \times 17\end{array}$ & $\begin{array}{l}\text { GE } \\
\text { GE } \\
\text { GE } \\
\text { WEST } \\
\text { WEST } \\
\text { WEST } \\
\text { WEST }\end{array}$ & $\begin{array}{l}4 \\
4 \\
4 \\
4 L P \\
4 L P \\
4 L P \\
4 L P\end{array}$ \\
\hline TU Electric................ & $\begin{array}{l}\text { Comanche Peak } 1 \\
\text { Comanche Peak } 2\end{array}$ & $\begin{array}{l}4901 \\
4902\end{array}$ & $\begin{array}{l}\text { WE } 17 \times 17 \\
\text { WE } 17 \times 17\end{array}$ & $\begin{array}{l}\text { WEST } \\
\text { WEST }\end{array}$ & $\begin{array}{l}4 L P \\
4 L P\end{array}$ \\
\hline Tolodo Edison Company ...... & Davis-Besse & 5001 & BW $15 \times 15$ & $B \& W$ & LLP \\
\hline Union Electrlo Company....... & Callaway & 5101 & WE $17 \times 17$ & WEST & 4LP \\
\hline $\begin{array}{l}\text { Vermont Yankee Nuclear } \\
\text { Power Corporation.......... }\end{array}$ & Vermont Yankee & 6001 & GE BWR/4-6 & GE & 4 \\
\hline Virginia Power............... & $\begin{array}{l}\text { North Anna } 1 \\
\text { North Anna } 2 \\
\text { Surry } 1 \\
\text { Surry } 2\end{array}$ & $\begin{array}{l}5201 \\
5202 \\
5203 \\
5204\end{array}$ & $\begin{array}{l}\text { WE } 17 \times 17 \\
\text { WE } 17 \times 17 \\
\text { WE } 15 \times 15 \\
\text { WE } 15 \times 15\end{array}$ & $\begin{array}{l}\text { WEST } \\
\text { WEST } \\
\text { WEST } \\
\text { WEST }\end{array}$ & $\begin{array}{l}\text { 3LP } \\
\text { 3LP } \\
\text { 3LP } \\
\text { 3LP }\end{array}$ \\
\hline $\begin{array}{l}\text { Washington Publlc Power } \\
\text { Supply System........... }\end{array}$ & Washington Nuclear 2 & 5302 & GE BWR/4-6 & GE & 5 \\
\hline $\begin{array}{l}\text { Wlsconsin Electrlc Power } \\
\text { Company } \ldots \ldots \ldots \ldots \ldots \ldots\end{array}$ & $\begin{array}{l}\text { Point Beach } 1 \\
\text { Point Beach } 2\end{array}$ & $\begin{array}{l}5401 \\
5402\end{array}$ & $\begin{array}{l}\text { WE } 14 \times 14 \\
\text { WE } 14 \times 14\end{array}$ & $\begin{array}{l}\text { WEST } \\
\text { WEST }\end{array}$ & $\begin{array}{l}2 L P \\
2 L P\end{array}$ \\
\hline $\begin{array}{l}\text { Wisconsin Publlc Servico } \\
\text { Corporation ............. }\end{array}$ & Kewaunee & 5501 & WE $14 \times 14$ & WEST & 2LP \\
\hline $\begin{array}{l}\text { Yankee Atomic Electrlc } \\
\text { Company ............... }\end{array}$ & Yanke日 Rowe & 5601 & Yankee Rowe & WEST & 4LP \\
\hline
\end{tabular}

aAuclear Steam System Supplier (NSSS) codes are as follows: AC = Allis Chalmers; B\&W = Babcock \& Wilcox Company; COMB = Combustion Engineering; GE = GE Nuclear Energy; WEST = Westinghouse Electric. Data obtained from Nuclear Regulatory Commission publication Informatlon Dlgest 1993, Appendix A, NSSS column.

Design types are as follows: 1 = GE Type $1 ; 2=$ GE Type 2; $3=$ GE Type 3; 4 = GE Type 4; 5 = GE Type 5;6 = GE Type 6; 2LP = Westinghouse Two-Loop; 3LP - Westinghouse Three-Loop; $4 \mathrm{LP}=$ Westinghouse Four-Loop; CE = Combustion Englneering; CE80 = CE Standard Design; LLP = B\&W Lowered Loop. Data obtained from Nuclear Regulatory Commission publication Information Digest 1993, Appendix A, NSSS column.

$\ldots=$ Not applicable.

Source: Energy Information Administration, Form RW-859, "Nuclear Fuel Data" (1992). 
Table B3. Reactors by Assembly Class and Design Type

B\&W $15 \times 15$ Assembly Class

Arkansas Nuclear 1 (LLP)

Oconee 1 (LLP)

Crystal River 3 (LLP)

Davis-Besse (LLP)

Rancho Seco (LLP)*

Oconee 2 (LLP)

Three Mile Island 1 (LLP)

Oconee 3 (LLP)

B\&W $17 \times 17$ Assembly Class

(Four test assemblies irradiated at Oconee $1,2, \& 3$ )

CE $14 \times 14$ Assembly Class

Calvert Gliffs 1 (CE)

Calvert Cliffs 2 (CE)

Millstone 2 (CE)

St. Lucie 1 (CE)

Maine Yankee (CE)

CE $16 \times 16$ Assembly Class

Arkansas Nuclear 2 (CE)

Waterford 3 (CE)

San Onofre 2 (CE)

San Onofre 3 (CE)

CE $16 \times 16$ System 80 Assembly Class

Palo Verde 1 (CE80)

Palo Verde 2 (CE80)

Palo Verde 3 (CE80)

GE BWR/2,3 Assembly Class

Dresden 2 (3)

Monticello (3)

Pilgrim 1 (3)

Dresden 3 (3)

Nine Mile.Point 1 (2)

Quad Cities 1 (3)

Browns Ferry 2 (4)

Brunswick 2 (4)

Duane Arnold (4)

Grand Gulf 1 (6)

Hope Creek (4)

Limerick 1 (4)

Peach Bottom 2 (4)

River Bend 1 (6)

Susquehanna 2 (4)
Millstone 1 (3)

Oyster Creek (2)

Quad Cities 2 (3)
Fitzpatrick (4)

Hatch 2 (4)

LaSalle County 2 (5)

Nine Mile Point 2 (5)

Perry 1 (6)

Susquehanna 1 (4)

Washington Nuclear 2 (5)

WE $14 \times 14$ Assembly Class

Ginna (2LP)

Point Beach 2 (2LP)
Kewaunee (2LP)

Prairie Island 1 (2LP)
Browns Ferry 3 (4)

Clinton 1 (6)

Enrico Fermi 2 (4)

Hatch 1 (4)

LaSalle County 1 (5)

Limerick 2 (4)

Peach Bottom 3 (4)

Shoreham (4)*

Vermont Yankee (4)

See footnotes at end of table.

Point Beach 1 (2LP)

Prairie Island 2 (2LP) 
Table B3. Reactors by Assembly Class and Design Type (Continued)

WE $15 \times 15$ Assembly Class

Cook 1 (4LP)

Robinson 2 (3LP)

Turkey Point 3 (3LP)

Zlon 2 (4LP)

WE $17 \times 17$ Assembly Class

Beaver Valley 1 (3LP)

Braidwood 2 (4LP)

Callaway (4LP)

Comanche Peak 1 (4LP)

Diablo Canyon 1 (4LP)

Farley 2 (3LP)

McGuire 2 (4LP)

North Anna 2 (3LP)

Seabrook (4LP)

Summer (3LP)

Vogtle 2 (4LP)

Wolf Creek 1 (4LP)

South Texas Assembly Class

South Texas 1 (4LP)

Single Beactor Assembly Classes

Big Rock Point (1)

Humboldt Bay (1)*

LaCrosse (-.)*

San Onofre 1 (3LP)*
Indian Point 2 (4LP)

Surry 1 (3LP)

Turkey Point 4 (3LP)

Indian Point 3 (4LP)

Surry 2 (3LP)

Zion 1 (4LP)
Beaver Valley 2 (3LP)

Byron 1 (4LP)

Catawba 1 (4LP)

Comanche Peak 2 (4LP)

Diablo Canyon 2 (4LP)

Harris 1 (3LP)

Millstone 3 (4LP)

Salem 1 (4LP)

Sequoyah 1 (4LP)

Trojan (4LP)*

Watts Bar 1 (4LP)
Braidwood 1 (4LP)

Byron 2 (4LP)

Catawba 2 (4LP)

Cook 2 (4LP)

Farley 1 (3LP)

McGuire 1 (4LP)

North Anna 1 (3LP)

Salem 2 (4LP)

Sequoyah 2 (4LP)

Vogtle 1 (4LP)

Watts Bar 2 (4LP)

South Texas 2 (4LP)

-. - Not appllcable.

Notes: Design types are as follows: 1 - GE Type 1;2 = GE Type 2; $3=$ GE Type 3; 4 = GE Type 4; $5=$ GE Type 5; 6 = GE Type 6; $2 L P=$ Westinghouse Two-Loop; $3 L P=$ Westinghouse Three-Loop; 4LP = Westinghouse Four-Loop; CE = Combustion Engineering; CE80 - CE Standard Design; LLP = B\&W Lowered Loop. Data obtained from Nuclear Regulatory Commission publication Information Digest 1993, Appendix A, NSSS column. * = Reactors are permanently shut down.

Source: Energy Information Administration, Form RW-859, "Nuclear Fuel Data" (1992).

Dresden $1(1)^{\star}$

Haddam Neck (4LP)

Palisades (CE)

Yankee Rowe (4LP)*
Fort Calhoun (CE) Indian Point $1(--)^{*}$ St. Lucie 2 (CE) 
Table B4. Descriptions of Assembly Types

\begin{tabular}{|c|c|c|}
\hline Assembly Type & Description & $\begin{array}{l}\text { Assembly } \\
\text { Type Code }\end{array}$ \\
\hline \multicolumn{3}{|c|}{$\begin{array}{l}\text { B\&W } 15 \times 15 \text { Assembly Class } \\
\text { (Reactor Type: PWR/Length: } 165.7 \text { in./Width: } 8.54 \text { in.) }\end{array}$} \\
\hline B\&W 15 X 15 B\&W Mark B2 & $\begin{array}{l}\text { B\&W-manufactured fuel for B\&W } 15 \times 15 \text { reactors; } \\
\text { Mark B2 fuel uses a corrugated flexible grid spacer } \\
\text { and a zirconium dioxide solid spacer between the fuel } \\
\text { column and the fuel rod end plug; } \sim 464 \mathrm{~kg} \mathrm{U} \text {. }\end{array}$ & B1515B2 \\
\hline B\&W 15 X 15 B\&W Mark B3 & $\begin{array}{l}\text { B\&W-manufactured fuel for } B \& W 15 \times 15 \text { reactors; } \\
\text { Mark B3 characteristics are not well defined because it } \\
\text { is an early fuel design; } \sim 464 \mathrm{~kg} \mathrm{U.}\end{array}$ & B1515B3 \\
\hline B\&W 15 X 15 B\&W Mark B4 & $\begin{array}{l}\text { B\&W-manufactured fuel for } B \& W 15 \times 15 \text { reactors; } \\
\text { standard fuel from } B \& W \text { for many years; } \\
\text { inconel spacer grids; } \sim 464 \mathrm{~kg} \mathrm{U.}\end{array}$ & B1515B4 \\
\hline B\&W $15 \times 15$ B\&W Mark B4Z & $\begin{array}{l}\text { B\&W-manufactured fuel for B\&W } 15 \times 15 \text { reactors; } \\
\text { features } 6 \text { Zircaloy grid spacers in the core zone; } \\
\sim 464 \mathrm{~kg} \mathrm{U} \text {. }\end{array}$ & $\mathrm{B} 1515 \mathrm{~B} 4 \mathrm{Z}$ \\
\hline B\&W 15 X 15 B\&W Mark B5 & $\begin{array}{l}\text { B\&W-manufactured fuel for B\&W } 15 \times 15 \text { reactors; } \\
\text { redesigned upper end fitting eliminates retainers } \\
\text { for Burnable Poison Rod Assembly holddown; } \\
\text { redesigned holddown spring made of Inconel } 718 \\
\text { rather than Inconel X-750; } 464 \mathrm{~kg} \mathrm{U.}\end{array}$ & B1515B5 \\
\hline B\&W 15 X 15 B\&W Mark B5Z & $\begin{array}{l}\text { B\&W-manufactured fuel for B\&W } 15 \times 15 \text { reactors; } \\
\text { assemblies feature } 6 \text { Zircaloy acid spacers in the core } \\
\text { zone, a redesigned upper end fitting which eliminates } \\
\text { the retainers for Burnable Poison Rod Assembly } \\
\text { holddown, and a redesigned holddown spring made of } \\
\text { Inconel } 718 \text { rather than Inconel } X-750 ; \sim 464 \mathrm{~kg} \mathrm{U.}\end{array}$ & B1515B5Z \\
\hline B\&W 15 X 15 B\&W Mark B6 & $\begin{array}{l}\text { B\&W-manufactured fuel for B\&W } 15 \times 15 \text { reactors; } \\
\text { assemblies feature } 6 \text { Zircaloy grid spacers in the core } \\
\text { zone and a skirtless and removable upper end fitting; } \\
\sim 464 \mathrm{~kg} \mathrm{U.}\end{array}$ & B1515B6 \\
\hline B\&W 15 X 15 B\&W Mark B7 & $\begin{array}{l}\text { B\&W-manufactured fuel for B\&W } 15 \times 15 \text { reactors; } \\
\text { in addition to the Mark B6 fuel, the Mark B7 features } \\
\text { slightly longer fuel rods and a shorter lower end fitting; } \\
\text { these features increase the plenum volume and fuel } \\
\text { rod-to-nozzle gap, allowing for increased discharge } \\
\text { burnups; } \sim 464 \mathrm{~kg} \mathrm{U.}\end{array}$ & $\mathrm{B} 1515 \mathrm{~B} 7$ \\
\hline B\&W 15 X 15 B\&W Mark B8 & $\begin{array}{l}\text { B\&W-manufactured fuel for B\&W } 15 \times 15 \text { reactors; } \\
\text { in addition to the features of Mark B7 fuel, the Mark } \\
\text { B8 fuel has a debris fretting resistant fuel rod design; } \\
\sim 464 \mathrm{~kg} \mathrm{U.}\end{array}$ & B1515B8 \\
\hline
\end{tabular}


Table B4. Descriptions of Assembly Types (Continued)

\begin{tabular}{c|c|c}
\hline Assembly Type & Description & $\begin{array}{l}\text { Assembly } \\
\text { Type Code }\end{array}$ \\
\hline
\end{tabular}

B\&W $15 \times 15$ Assembly Class (Continued)

(Reactor Type: PWR/Length: 165.7 in./Width: 8.54 in.)

B\&W $15 \times 15$ B\&W Mark B

B\&W 15 X 15 B\&W Zirc Grids

$B \& W 15 \times 15$ B\&W Mark BEB

$B \& W 15 \times 15$ B\&W Mark BGd

$B \& W 15 \times 15$ WE
B\&W-manufactured fuel for $B \& W 15 \times 15$ reactors; Mark $B$ is a generic designation and is used when the specific assembly type is unknown; $464 \mathrm{~kg} \mathrm{U}$.

$B \& W$-manufactured fuel for $B \& W 15 \times 15$ reactors; generic designation for fuels with Zircaloy grid spaces which encompasses Mark B4Z, B5Z, B6, B7 and $B 8$ fuels when specific assembly type has not been determined; $464 \mathrm{~kg} \mathrm{U}$.

B\&W-manufactured fuel for B\&W $15 \times 15$ reactors; designed to test extended burnup features, this fuel had a shorter active fuel length and was used only as lead test assemblies at Arkansas Nuclear 1; $\sim 464 \mathrm{~kg} \mathrm{U}$.

B\&W-manufactured fuel for B\&W $15 \times 15$ reactors; used as lead test assemblies at Oconee 1 ; this fuel utilized gadolinia in the fuel rods as a neutron absorber; apparenty had removable upper end fittings, the forerunner of the skirtless (Mark B6) upper end fitting; $430 \mathrm{~kg}$ 'U.

WE-manufactured fuel for B\&W $15 \times 15$ reactors; scheduled for lead test assembly use at Three Mile Island $1 ; \sim 462 \mathrm{~kg} \mathrm{U}$.
B1515B

$B 1515 B Z$

B1515BEB

B1515BGD

B1515W

\section{B\&W 17 X 17 Assembly Class}

(Reactor Type: PWR/Length: 165.7 in.Width: 8.54 in.)

B\&W $17 \times 17$ B\&W Mark C

$B \& W$-manufactured fuel for $B \& W 17 \times 17$ reactors; test assemblies used at Oconee 1, 2, \& 3; 456 kg U.

B1717B

CE 14 X14 Assembly Class

(Reactor Type: PWR/Length: 157 in./Width: 8.1 in.)

CE $14 \times 14 \mathrm{CE}$

CE-manufactured fuel for CE $14 \times 14$ reactors; $\sim 382 \mathrm{~kg} \mathrm{U}$.

C1414C

ANF-manufactured fuel for CE $14 \times 14$ reactors;

C1414A $\sim 370 \mathrm{~kg} \mathrm{U}$.

WE-manufactured fuel for CE $14 \times 14$ reactors;

C1414W

CE $14 \times 14$ WE $\sim 407 \mathrm{~kg} \mathrm{U}$. 
Table B4. Descriptions of Assembly Types (Continued)

\begin{tabular}{|c|c|c|}
\hline Assembly Type & Description & $\begin{array}{l}\text { Assembly } \\
\text { Type Code }\end{array}$ \\
\hline \multicolumn{3}{|c|}{$\begin{array}{l}\text { CE } 16 \times 16 \text { Assembly Class } \\
\text { (Reactor Type: PWR/Length: } 176.8 \text { in./Width: } 8.1 \text { in.) }\end{array}$} \\
\hline CE $16 \times 16 \mathrm{CE}$ & $\begin{array}{l}\text { CE-manufactured fuel for CE } 16 \times 16 \text { reactors; } \\
\sim 416 \mathrm{~kg} \mathrm{U} .\end{array}$ & C1616CSD \\
\hline \multicolumn{3}{|c|}{$\begin{array}{l}\text { CE } 16 \times 16 \text { System } 80 \text { Assembly Class } \\
\text { (Reactor Type: PWR/Length: } 178.3 \text { in./Width: } 8.1 \text { in.) }\end{array}$} \\
\hline CE $16 \times 16$ CE System 80 & $\begin{array}{l}\text { CE-manufactured fuel for CE System } 80 \text { reactors; } \\
\sim 413 \mathrm{~kg} \mathrm{U} \text {. }\end{array}$ & C8016C \\
\hline \multicolumn{3}{|c|}{$\begin{array}{l}\text { GE BWR/2,3 Assembly Class } \\
\text { (Reactor Type: BWR/Length: } 171.2 \text { in./Width: } 5.44 \text { in.) }\end{array}$} \\
\hline GE BWR/2,3 $7 \times 7$ GE-2a & $\begin{array}{l}\text { GE-manufactured fuel for GE BWR/2,3 reactors; } \\
\text { original core fuel at Oyster Creek, Nine Mile Point 1, } \\
\text { and Millstone 1; fuel rod diameter of } 0.570 " ; \\
\sim 195 \mathrm{~kg} \mathrm{U.}\end{array}$ & G2307G2A \\
\hline GE BWR/2,3 $7 \times 7$ GE-2b & $\begin{array}{l}\text { GE-manufactured fuel for GE BWR/2,3 reactors; } \\
\text { original core fuel at other reactors; fuel } \\
\text { rod diameter of } 0.563^{\prime \prime} \text {; cladding thickness } \\
\text { of } 0.032 " ; \sim 193 \mathrm{~kg} \mathrm{U} \text {. }\end{array}$ & G2307G2B \\
\hline GE BWR/2,3 $7 \times 7$ GE-3 & $\begin{array}{l}\text { GE-manufactured fuel for GE BWR/2,3 reactors; } \\
\text { "Improved" fuel; cladding thickness of } 0.037 \text {; } \\
\text { hydrogen getter introduced; } \sim 188 \mathrm{~kg} \mathrm{U} \text {. }\end{array}$ & G2307G3 \\
\hline GE BWR/2,3 8 X 8 GE-4 & $\begin{array}{l}\text { GE-manufactured fuel for GE BWR/2,3 reactors; } \\
\text { first } 8 \times 8 \text { fuel; } 1 \text { water rod; } ~ 184 \mathrm{~kg} \text { U. }\end{array}$ & G2308G4 \\
\hline GE BWR/2,3 8 X 8 GE-5 & $\begin{array}{l}\text { GE-manufactured fuel for GE BWR/2,3 reactors; } \\
\text { "retrofit" fuel; } 2 \text { water rods; natural uranium axial } \\
\text { blankets; } \sim 177 \mathrm{~kg} \mathrm{U.}\end{array}$ & G2308G5 \\
\hline GE BWR/2,3 8 X 8 Prepres. & $\begin{array}{l}\text { GE-manufactured fuel for GE BWR/2,3 reactors; } \\
\text { fuel rods prepressurized to } 3 \text { Atm He; } 2 \text { water rods; } \\
\sim 177 \mathrm{~kg} \mathrm{U.}\end{array}$ & G2308GP \\
\hline GE BWR/2,3 8 X 8 Barrier & $\begin{array}{l}\text { GE-manufactured fuel for GE BWR/2,3 reactors; } \\
\text { pure zirconium "barrier" on inside of cladding to } \\
\text { reduce pellet-clad interaction; } \sim 177 \mathrm{~kg} \mathrm{U} \text {. }\end{array}$ & G2308GB \\
\hline GE BWR/2,3 $8 \times 8$ GE- $8 a$ & $\begin{array}{l}\text { GE-manufactured fuel for GE BWR/2,3 reactors; } \\
\text { features of GE BWR/2,3 } 8 \times 8 \text { fuel with only } 2 \text { water } \\
\text { rods; } 177 \mathrm{~kg} \mathrm{U} \text {. }\end{array}$ & G2308G8A \\
\hline
\end{tabular}


Table B4. Descriptions of Assembly Types (Continued)

\begin{tabular}{|c|c|c|}
\hline Assembly Type & Description & $\begin{array}{l}\text { Assembly } \\
\text { Type Code }\end{array}$ \\
\hline \multicolumn{3}{|c|}{$\begin{array}{l}\text { GE BWR/2,3 Assembly Class (Continued) } \\
\text { (Reactor Type: BWR/Length: } 171.2 \text { in./Width: } 5.44 \text { in.) }\end{array}$} \\
\hline GE BWR/2,3 8 X 8 GE-8b & $\begin{array}{l}\text { GE-manufactured fuel for GE BWR/2,3 reactors; } \\
4 \text { water rods; introduces axially zoned enrichments } \\
\text { and burnable absorbers; fuel rod prepressurization } \\
\text { increased to } 5 \text { Atms; } \sim 172 \mathrm{~kg} \mathrm{U} \text {. }\end{array}$ & G2308G8B \\
\hline GE BWR/2,3 $8 \times 8$ GE-9 & $\begin{array}{l}\text { GE-manufactured fuel for GE BWR/2,3 reactors; } \\
\text { ferule-type spacer grids; large diameter water rod } \\
\text { which displaced } 4 \text { fuel rod positions; axially zoned } \\
\text { enrichment and burnable absorbers; fuel rod pre- } \\
\text { pressurization of } 5 \text { Atm; "barrier" cladding; } \sim 172 \mathrm{~kg} \mathrm{U} \text {. }\end{array}$ & G2308G9 \\
\hline GE BWR/2,3 8 X 8 GE-10 & $\begin{array}{l}\text { GE-manufactured fuel for GE BWR/2,3 reactors; } \\
\text { fuel channel is } 100 \text {-mils thick at corners and } 65-\text { mils } \\
\text { thick on sides reducing the parasitic material in core; } \\
\text { uses flow directors on the inside of the channel thus } \\
\text { redirecting the flow of water away from the channel } \\
\text { wall towards the center of the fuel bundle; no weight } \\
\text { available. }\end{array}$ & G2308G10 \\
\hline GE BWR/2,3 $9 \times 9$ GE-11 & $\begin{array}{l}\text { GE-manufactured fuel for GE BWR/2,3 reactors; } \\
\text { uses } 2 \text { medium diameter water rods which replace } \\
7 \text { fuel rods; } 8 \text { of the remaining fuel rods extend only to } \\
\text { the top of the fifth spacer grid; no weight available. }\end{array}$ & G2309G11 \\
\hline GE BWR/2,3 $7 \times 7$ ANF & $\begin{array}{l}\text { ANF-manufactured fuel for GE BWR/2,3 reactors; } \\
7 \times 7 \text { fuel rod array; used only at Oyster Creek; } \\
\sim 182 \mathrm{~kg} \mathrm{U} \text {. }\end{array}$ & G2307A \\
\hline GE BWR/2,3 $8 \times 8$ ANF & $\begin{array}{l}\text { ANF-manufactured fuel for GE BWR/2,3 reactors; } \\
8 \times 8 \text { fuel rod array; } 1 \text { water rod; estimated } 175 \mathrm{~kg} \mathrm{U} \text {. }\end{array}$ & G2308A \\
\hline GE BWR/2,3 8 X 8 ANF Prepres. & $\begin{array}{l}\text { ANF-manufactured fuel for GE BWR/2,3 reactors; } \\
\text { rods prepressurized with He to several Atms; } \\
\sim 175 \mathrm{~kg} \mathrm{U} \text {. }\end{array}$ & G2308AP \\
\hline GE BWR/2,39 99 ANF & $\begin{array}{l}\text { ANF-manufactured fuel for GE BWR/2,3 reactors; } \\
9 \times 9 \text { fuel rod array; } 2 \text { water rods; } \sim 168 \mathrm{~kg} \mathrm{U} \text {. }\end{array}$ & G2309A \\
\hline GE BWR/2,3 $9 \times 9$ ANF 9-5 & $\begin{array}{l}\text { ANF-manufactured fuel for GE BWR/2,3 reactors; } \\
5 \text { water rods per assembly; estimated } 161 \mathrm{~kg} \mathrm{U} \text {. }\end{array}$ & G2309A5 \\
\hline GE BWR/2,3 $9 \times 9$ ANF $9 X$ & $\begin{array}{l}\text { ANF-manufactured fuel for GE BWR/2,3 reactors; } \\
\text { uses a central water channel that replaces } \\
9 \text { water rods; estimated } 153 \mathrm{~kg} \mathrm{U} \text {. }\end{array}$ & G2309A9X \\
\hline
\end{tabular}


Table B4. Descriptions of Assembly Types (Continued)

\begin{tabular}{|c|c|c|}
\hline Assembly Type & Description & $\begin{array}{l}\text { Assembly } \\
\text { Type Code }\end{array}$ \\
\hline \multicolumn{3}{|c|}{$\begin{array}{l}\text { GE BWR/2,3 Assembly Class (Continued) } \\
\text { (Reactor Type: BWR/Length: } 171.2 \text { in./Width: } 5.44 \text { in.) }\end{array}$} \\
\hline GE BWR/2,3 $9 \times 9$ ANF IX & $\begin{array}{l}\text { ANF-manufactured fuel for GE BWR/2,3 reactors; } \\
\text { uses a central water channel that replaces } \\
9 \text { rods; the regular fuel rods in the IX version utilize } \\
\text { an internal cladding liner of pure zirconium; } \\
\text { estimated } 153 \mathrm{~kg} U \text {. }\end{array}$ & G2309AIX \\
\hline \multicolumn{3}{|c|}{$\begin{array}{l}\text { GE BWR/4-6 Assembly Class } \\
\text { (Reactor Type: BWR/Length: } 176.2 \text { in./Width: } 5.44 \text { in.) }\end{array}$} \\
\hline GE BWR/4-6 $7 \times 7$ GE-2 & $\begin{array}{l}\text { GE-manufactured fuel for GE BWR/4-6 reactors; } \\
\text { original core fuel for several BWR/4 plants; high } \\
\text { failure rate instigated introduction of GE-3 and GE-4 } \\
\text { fuels; } 195 \mathrm{~kg} \mathrm{U} \text {. }\end{array}$ & G4607G2 \\
\hline GE BWR/4-6 7 X 7 GE-3a & $\begin{array}{l}\text { GE-manufactured fuel for GE BWR/4-6 reactors; } \\
\text { "Improved" fuel; cladding thickness of } 0.037 \text { "; } \\
\text { hydrogen getter introduced; } 144 \text { inch active fuel } \\
\text { length; } \sim 187 \mathrm{~kg} \mathrm{U.}\end{array}$ & G4607G3A \\
\hline GE BWR/4-6 $7 \times 7$ GE-3b & $\begin{array}{l}\text { GE-manufactured fuel for GE BWR/4-6 reactors; } \\
\text { "Improved" fuel; cladding thickness of } 0.037 \text { "; } \\
\text { hydrogen getter introduced; } 146 \text { active } \\
\text { length; 190 kg U. }\end{array}$ & G4607G3B \\
\hline GE BWR/4-6 8 X 8 GE-4a & $\begin{array}{l}\text { GE-manufactured fuel for GE BWR/4-6 reactors; } \\
\text { first } 8 \text { X } 8 \text { fuel; } 1 \text { water rod; } 144 \text { inch active } \\
\text { fuel length; } \sim 184 \mathrm{~kg} \mathrm{U.}\end{array}$ & G4608G4A \\
\hline GE BWR/4-6 $8 \times 8$ GE-4b & $\begin{array}{l}\text { GE-manufactured fuel for GE BWR } / 4-6 \text { reactors; } \\
\text { first } 8 \times 8 \text { fuel; } 1 \text { water rod; } 146 \text { inch active } \\
\text { fuel length; } \sim 187 \mathrm{~kg} \mathrm{U.}\end{array}$ & G4608G4B \\
\hline GE BWR/4-6 8 X 8 GE-5 & $\begin{array}{l}\text { GE-manufactured fuel for GE BWR } / 4-6 \text { reactors; } \\
\text { "retrofit" fuel; } 2 \text { water rods; natural uranium axial } \\
\text { blankets; } \sim 183 \mathrm{~kg} \mathrm{U} \text {. }\end{array}$ & G4608G5 \\
\hline GE BWR/4-6 8 X 8 GE Prepres. & $\begin{array}{l}\text { GE-manufactured fuel for GE BWR } / 4-6 \text { reactors; } \\
\text { fuel rods prepressureized to } 3 \text { Atm He; } 2 \text { water rods; } \\
\sim 183 \mathrm{~kg} \mathrm{U.}\end{array}$ & G4608GP \\
\hline GE BWR/4-6 8 × 8 Barrier & $\begin{array}{l}\text { GE-manufactured fuel for GE BWR/4-6 reactors; } \\
\text { pure zirconium "barrier" on inside of cladding to } \\
\text { reduce pellet-clad interaction; } \sim 185 \mathrm{~kg} \mathrm{U} \text {. }\end{array}$ & G4608GB \\
\hline
\end{tabular}


Table B4. Descriptions of Assembly Types (Continued)

\begin{tabular}{l|l|l}
\hline Assembly Type & Description & $\begin{array}{l}\text { Assembly } \\
\text { Type Code }\end{array}$ \\
\hline
\end{tabular}

GE BWR/4-6 Assembly Class (Continued)

(Reactor Type: BWR/Length: 176.2 in./Width: 5.44 in.)

GE BWR/4-6 8 X 8 GE-8

GE BWR/4-6 8 X 8 GE-9

GE BWR/4-6 8 X 8 GE-10

GE BWR/4-6 $9 \times 9$ GE-11

GE BWR/4-6 $8 \times 8$ ANF

GE BWR/4-6 $8 \times 8$ ANF Prepres.

GE BWR/4-6 $8 \times 8$ WE

GE BWR/4-6 $9 \times 9$ ANF

GE BWR/4-6 $9 \times 9$ ANF 9-5

GE BWR/4-6 $9 \times 9$ ANF $9 X$
GE-manufactured fuel for GE BWR/4-6 reactors;

4 water rods; axially zoned enrichment and burnable absorbers; fuel rod prepressurization increased to $5 \mathrm{Atm}$; other "barrier" fuel features; $\sim 179 \mathrm{~kg} \mathrm{U}$.

GE-manufactured fuel for GE BWR/4-6 reactors; ferule-type spacer grids; large diameter water rod G4608G9 which displaced 4 fuel rod positions; axially zoned enrichment and burnable absorbers; fuel rod prepressurization of $5 \mathrm{Atm}$; "barrier" cladding; $\sim 172 \mathrm{~kg} \mathrm{U}$.

GE-manufactured fuel for GE BWR/4-6 reactors; fuel channel is 100 mils thick at the corners and 65 mils thick on the sides, reducing the parasitic material in core; uses flow directors on the inside of the channel thus redirecting the flow of water away from the channel wall towards the center of the fuel bundle; estimated $177 \mathrm{~kg} \mathrm{U}$.

GE-manufactured fuel for GE BWR/4-6 reactors; uses 2 medium diameter water rods which replace 7

G4609G11 fuel rods; 8 of the rods remaining extend to only the fifth spacer grid; estimated $170 \mathrm{~kg} \mathrm{U}$.

ANF-manufactured fuel for GE BWR/4-6 reactors; $8 \times 8$ fuel rod array; 1 water rod; $176 \mathrm{~kg} \mathrm{U}$.

G4608A

ANF-manufactured fuel for GE BWR/4-6 reactors; prepressurized fuel rods; $\sim 176 \mathrm{~kg} \mathrm{U}$.

WE-manufactured fuel for GE BWR/4-6 reactors; reactors; "QUAD + fuel"; design of fuel is lead test assembly use only; a licensed version of $A B B$ Atom water cross fuel; $\sim 174 \mathrm{~kg} \mathrm{U}$.

ANF-manufactured fuel for GE BWR/4-6 reactors; $9 \times 9$ fuel rod array; 2 water rods; $\sim 173 \mathrm{~kg} \mathrm{U}$.

G4609A

ANF-manufactured fuel for GE BWR/4-6 reactors; 5 water rods per assembly; estimated $168 \mathrm{~kg} \mathrm{U}$.

G4609A5

ANF-manufactured fuel for GE BWR/4-6 reactors; central water channel replaces 9 fuel rods;

G4609A9X estimated $168 \mathrm{~kg} \mathrm{U}$. 
Table B4. Descriptions of Assembly Types (Continued)

\begin{tabular}{c|c|c}
\hline Assembly Type & Description & $\begin{array}{l}\text { Assembly } \\
\text { Type Code }\end{array}$ \\
\hline
\end{tabular}

GE BWR/4 - 6 Assembly Class (Continued)

(Reactor Type: BWR/Length: 176.2 in./Width: 5.44 in.)

GE BWR/4-6 9 × 9 ANF IX

GE BWR/4-6 $9 \times 9$ ANF IX+

GE BWR/4-610 $\times 10$ ABB

WE $14 \times 14$ WE LOPAR

WE $14 \times 14$ WE OFA

WE $14 \times 14$ ANF

WE $14 \times 14$ ANF Top Rod

WE $14 \times 14$ B\&W
ANF-manufactured fuel for GE BWR/4-6 reactors; central water channel replaces 9 fuel rods; regular fuel rods utilize an internal cladding liner of pure zirconium; estimated $168 \mathrm{~kg} \mathrm{U}$.

ANF-manufactured fuel for GE BWR/4-6 reactors; central water channel replaces 9 fuel rods; high-performance thermal spacers; estimated $168 \mathrm{~kg} \mathrm{U}$.

ABB Atom-manufactured fuel for GE BWR/4-6 reactors; fuel has $4,5 \times 5$ minibundles, each with G4610C 24 fuel rods; fuel bundles are separated by a water cross and a centralized water channel; estimated $176 \mathrm{~kg} \mathrm{U}$.

WE $14 \times 14$ Assembly Class

(Reactor Type: PWR/Length: 159.8 in./Width: 7.76 in.)

WE-manufactured fuel for WE $14 \times 14$ reactors; Zircaloy cladding; stainless steel guide tubes; $\sim 394 \mathrm{~kg} \mathrm{U}$.

WE-manufactured fuel for WE $14 \times 14$ reactors; 'Low Parasitic (LOPAR) fuel"; Zircaloy guide tubes; often referred to as "Standard" fuel; $\sim 399 \mathrm{~kg} \mathrm{U}$.

WE-manufactured fuel for WE $14 \times 14$ reactors; "Optimized Fuel Assemblies"; Zircaloy spacer grids; smaller fuel rod diameter; $358 \mathrm{~kg} \mathrm{U}$.

ANF-manufactured fuel for WE $14 \times 14$ reactors; W1414A shorter, larger diameter fuel rod than WE 14 X 14 ANF Top Rod fuel; $377 \mathrm{~kg} \mathrm{U}$.

ANF-manufactured fuel for WE $14 \times 14$ reactors; longer, smaller diameter fuel rod than

G4609AX+

G4609AIX

\section{G4609AXt}

G46100

W1414W

W1414WL

W1414WO

WE $14 \times 14$ ANF fuel; $~ 361 \mathrm{~kg} \mathrm{U}$.

B\&W-manufactured fuel for WE $14 \times 14$ reactors; only 2 lead test assemblies used at Ginna; $383 \mathrm{~kg} \mathrm{U.}$
W1414ATR

W1414B 
Table B4. Descriptions of Assembly Types (Continued)

\begin{tabular}{c|c|c}
\hline Assembly Type & Description & $\begin{array}{l}\text { Assembly } \\
\text { Type Code }\end{array}$ \\
\hline
\end{tabular}

WE $15 \times 15$ Assembly Class

(Reactor Type: PWR/Length: 159.8 in./Width: 8.44 in.)

WE $15 \times 15$ WE Standard

WE $15 \times 15$ WE LOPAR

WE $15 \times 15$ WE OFA

WE $15 \times 15$ ANF

WE $15 \times 15$ ANF Part Length

WE $15 \times 15$ B\&W Mark BW

WE $17 \times 17$ WE OFA

WE $17 \times 17$ WE Vantage 5

WE $17 \times 17$ WE LOPAR
WE-manufactured fuel for WE $15 \times 15$ reactors; Zircaloy cladding; stainless steel guide tubes; $\sim 454 \mathrm{~kg} \mathrm{U}$.

WE-manufactured fuel for WE $15 \times 15$ reactors; "'Low Parasitic (LOPAR) fuel"; Zircaloy guide tubes; often referred to as "Standard" fuel; $\sim 455 \mathrm{~kg} \mathrm{U}$.

WE-manufactured fuel for WE $15 \times 15$ reactors; "Optimized Fuel Assemblies"; Zircaloy spacer grids; $\sim 460 \mathrm{~kg} \mathrm{U}$.

ANF-manufactured fuel for WE $15 \times 15$ reactors; $\sim 429 \mathrm{~kg} \mathrm{U}$.

W1515A

ANF-manufactured fuel for WE $15 \times 15$ reactors; bottom 42 inches of fuel rods replaced by inserts made from stainless steel 304; assemblies placed in positions on the core periphery to provide shielding for core support structure; estimated $305 \mathrm{~kg} \mathrm{U}$.

B\&W-manufactured fuel for WE $15 \times 15$ reactors; no weight available.

W1515B

WE $17 \times 17$ Assembly Class

(Reactor Type: PWR/Length: 159.8 in./Width: 8.44 in.)

WE-manufactured fuel for WE $17 \times 17$ reactors; "Low Parasitic (LOPAR) fuel"; Zircaloy guide tubes; inconel Spacer grids; often referred to as "Standard" fuel; $\sim 460 \mathrm{~kg} \mathrm{U}$.

WE-manufactured fuel for WE $17 \times 17$ reactors; "Optimized Fuel Assemblies"; Zircaloy spacer grids; $\sim 425 \mathrm{~kg} \mathrm{U}$.

WE-manufactured fuel for WE $17 \times 17$ reactors; integral zirconium dioboride neutron absorbers in fuel; natural uranium axial blankets, intermediated flow mixers, removable top nozzle, increased discharged burnup; other OFA features; $426 \mathrm{~kg} \mathrm{U}$.

W1717WL

W1717WO

W1717WV5 
Table B4. Descriptions of Assembly Types (Continued)

\begin{tabular}{c|c|c}
\hline Assembly Type & Description & $\begin{array}{l}\text { Assembly } \\
\text { Type Code }\end{array}$ \\
\hline
\end{tabular}

WE 17 X 17 Assembly Class (Continued)

(Reactor Type: PWR/Length: 159.8 in./Width: 8.44 in.)

WE $17 \times 17$ WE Vantage +

WE-manufactured fuel for WE $17 \times 17$ reactors;

$W 1717 W V_{+}$ combines a new cladding material - ZIRLO

(a zirconium-niobium alloy) with the advanced neutronic characteristics of Vantage 5 fuel; estimated $426 \mathrm{~kg} \mathrm{U}$.

WE $17 \times 17$ WE Vantage $5 \mathrm{H}$

WE-manufactured fuel for WE $17 \times 17$ reactors;

W1717WVH hybrid fuel combining the advanced neutronic characteristics of Vantage 5 fuel with the larger fuel rod diameter associated with LOPAR fuel; $\sim 464 \mathrm{~kg} \mathrm{U}$.

WE $17 \times 17$ ANF

ANF-manufactured fuel for WE $17 \times 17$ reactors; $\sim 402 \mathrm{~kg} \mathrm{U}$.

W1717A

B\&W-manufactured fuel for WE $17 \times 17$ reactors; $\sim 425 \mathrm{~kg} \mathrm{U}$.

W1717B

South Texas Assembly Class

(Reactor Type: PWR/Length: 199 in./Width: 8.43 in.)

South Texas $17 \times 17$ WE

WE-manufactured fuel for use at South Texas reactors; initial core fuel; $\sim 542 \mathrm{~kg} \mathrm{U}$.

WST17W

Big Rock Point Assembly Class

(Reactor Type: BWR/Length: 84 in./Width: 6.52 in.)

Big Rock Point $12 \times 12$ GE

GE-manufactured îuel for use at Big Rock Point;

XBR12G stainless steel clad fuel; all assemblies reprocessed at West Valley.

Big Rock Point $11 \times 11 \mathrm{GE}$

GE-manufactured fuel for use at Big Rock Point; Zircaloy cladding; generic designation which encompasses "B," "C," "D1," and "D2" assembly types; most assemblies reprocessed at West Valley; $\sim 124 \mathrm{~kg} \mathrm{U}$.

Big Rock Point 7 X 7 GE

GE-manufactured fuel for use at Big Rock Point developmental center-melt fuel; test assembly use XBR07G only; $\sim 131 \mathrm{~kg} \mathrm{U}$.

XBR11G

GE-manufactured fuel for use at Big Rock Point developmental center-melt fuel; test assembly use XBR08G

Big Rock Point $8 \times 8 \mathrm{GE}$ only; $112 \mathrm{~kg} \mathrm{U}$. 
Table B4. Descriptions of Assembly Types (Continued)

\begin{tabular}{l|l|l}
\hline Assembly Type & Description & $\begin{array}{l}\text { Assembly } \\
\text { Type Code }\end{array}$ \\
\hline
\end{tabular}

Big Rock Point Assembly Class (Continued)

(Reactor Type: BWR/Length: 84 in.Width: 6.52 in.)

Big Rock Point $9 \times 9$ GE

GE-manufactured fuel for use at Big Rock Point;

XBR09G

Zircaloy cladding; generic designation which encompasses "E," "EG," "F," "MEG," and "PEG" assembly types; $\sim 137 \mathrm{~kg} \mathrm{U}$.

Big Rock Point $9 \times 9$ ANF

ANF-manufactured fuel for use at Big Rock Point;

XBR09A lead test assembly use only; $\sim 127 \mathrm{~kg} \mathrm{U}$.

Big Rock Point $11 \times 11$ ANF

ANF-manufactured fuel for use at Big Rock Point; $\sim 128 \mathrm{~kg} \mathrm{U}$.

XBR11A

NFS-manufactured fuel for use at Big Rock Point;

XBR11N

Big Rock Point $11 \times 11$ NFS lead test assembly use only; $\sim 129 \mathrm{~kg} \mathrm{U}$.

\section{Dresden 1 Assembly Class}

(Reactor Type: BWR/Length: 134.4 in./Width: 4.28 in.)

Dresden 16 X 6 GE Type 1

Dresden $17 \times 7 \mathrm{GE}$

Dresden 16 X 6 GE Type III-B

Dresden 16 X6 GE Type III-F

Dresden.1 6 X 6 GE Type V

Dresden $17 \times 7$ GE SA-1
GE-manufactured fuel for use at Dresden 1; all but one assembly reprocessed at West Valley; $\sim 111 \mathrm{~kg} \mathrm{U}$.

GE-manufactured fuel for use at Dresden 1; stainless steel clad fuel; 9 thorium oxide corner rods; XDR07G all reprocessed at West Valley except for the corner rods, which were shipped to the Savannah River Plant.

GE-manufactured fuel for use at Dresden 1; erbium oxide as burnable absorber in all 36 fuel rods; some assemblies reprocessed at West Valley; $\sim 102 \mathrm{~kg} \mathrm{U}$.

GE-manufactured fuel for use at Dresden 1; gadolinium oxide as a burnable absorber in a single, nonfueled rod; some assemblies reprocessed at West Valley; $102 \mathrm{~kg} \mathrm{U}$.

GE-manufactured fuel for use at Dresden 1; gadolinium oxide as a burnable absorber in selected fuel rods; $106 \mathrm{~kg} \mathrm{U}$.

GE-manufactured fuel for use 'at Dresden 1; a single prototype fuel assembly manufactured and owned by GE.
XDR06G

XDR06G3B

XDR06G3F

XDR06G5

XDR07GS 
Table B4. Descriptions of Assembly Types (Continued)

\begin{tabular}{|c|c|c|}
\hline Assembly Type & Description & $\begin{array}{l}\text { Assembly } \\
\text { Type Code }\end{array}$ \\
\hline \multicolumn{3}{|c|}{$\begin{array}{l}\text { Dresden } 1 \text { Assembly Class (Continued) } \\
\text { (Reactor Type: BWR/Length: } 134.4 \text { in./Width: } 4.28 \text { in.) }\end{array}$} \\
\hline Dresden $18 \times 8$ GE PF Fuels & $\begin{array}{l}\text { GE-manufactured fuel for use at Dresden } 1 \text {; } \\
\text { prototype fuel assemblies with } 6 \times 6,7 \times 7 \text {, and } \\
8 \times 8 \text { fuel rod arrays; all have been reprocessed } \\
\text { except for one } 8 \times 8 \text { assembly; } \sim 100 \mathrm{~kg} \mathrm{U} \text {. }\end{array}$ & XDR08G \\
\hline Dresden $16 \times 6$ UNC & $\begin{array}{l}\text { UNC-manufactured fuel for use at Dresden 1; } \\
\sim 102 \mathrm{~kg} \mathrm{U.}\end{array}$ & XDR06U \\
\hline Dresden $16 \times 6$ ANF & $\begin{array}{l}\text { ANF-manufactured fuel for use at Dresden 1; } \\
\sim 95 \mathrm{~kg} \mathrm{U} \text {. }\end{array}$ & XDR06A \\
\hline \multicolumn{3}{|c|}{$\begin{array}{c}\text { Fort Calhoun Assembly Class } \\
\text { (Reactor Type: PWR/Length: } 146 \text { in./Width: } 8.1 \text { in.) }\end{array}$} \\
\hline Fort Calhoun $14 \times 14 \mathrm{CE}$ & $\begin{array}{l}\text { CE-manufactured fuel for use at Fort Calhoun; } \\
\text { similar to CE } 14 \times 14 \text { class fuels except for shorter } \\
\text { length; } \sim 366 \mathrm{~kg} \mathrm{U} \text {. }\end{array}$ & $\mathrm{XFC14C}$ \\
\hline Fort Calhoun $14 \times 14$ ANF & $\begin{array}{l}\text { ANF-manufactured fuel for use at Fort Calhoun; } \\
\text { similar to CE } 14 \times 14 \text { class fuels except for shorter } \\
\text { length; } \sim 353 \mathrm{~kg} \mathrm{U} \text {. }\end{array}$ & XFC14A \\
\hline Fort Calhoun $14 \times 14$ WE & $\begin{array}{l}\text { WE-manufactured fuel for use at Fort Calhoun; } \\
\text { similar to CE } 14 \times 14 \text { class fuels except for shorter } \\
\text { length; } \sim 374 \mathrm{~kg} \mathrm{U} \text {. }\end{array}$ & XFC14W \\
\hline \multicolumn{3}{|c|}{$\begin{array}{l}\text { Humboldt Bay Assembly Class } \\
\text { (Reactor Type: BWR/Length: } 95 \text { in./Width: } 4.67 \text { in.) }\end{array}$} \\
\hline Humboldt Bay 7 X 7 GE Type 1 & $\begin{array}{l}\text { GE-manufactured fuel for use at Humboldt Bay; } \\
\text { stainless steel clad fuel; all assemblies reprocessed } \\
\text { at West Valley; } \sim 80 \mathrm{~kg} \text { U. }\end{array}$ & XHB07G \\
\hline Humboldt Bay 7 X 7 GE Type II & $\begin{array}{l}\text { GE-manufactured fuel for use at Humboldt Bay; } \\
\text { Zircaloy cladding; some assemblies reprocessed at } \\
\text { West Valley; } 76 \mathrm{~kg} \mathrm{U} \text {. }\end{array}$ & XHB07G2 \\
\hline Humboldt Bay 6 X $6 \mathrm{GE}$ & $\begin{array}{l}\text { GE-manufactured fuel for use at Humboldt Bay; } \\
\text { Zircaloy cladding; } \sim 76 \mathrm{~kg} \mathrm{U.}\end{array}$ & XHB06G \\
\hline Humboldt Bay $6 \times 6$ ANF & $\begin{array}{l}\text { ANF-manufactured fuel for use at Humboldt Bay; } \\
\text { Zircaloy cladding; } 70 \mathrm{~kg} U \text {. }\end{array}$ & XHB06A \\
\hline
\end{tabular}


Table B4. Descriptions of Assembly Types (Continued)

\begin{tabular}{l|l|l}
\hline Assembly Type & Description & $\begin{array}{l}\text { Assembly } \\
\text { Type Code }\end{array}$ \\
\hline
\end{tabular}

Haddam Neck Assembly Class

(Reactor Type: PWR/Length: 137.1 in./Width: 8.42 in.)

Haddam Neck $15 \times 15$ WE

WE-manufactured fuel for use at Haddam Neck; stainless steel clad; $\sim 416 \mathrm{~kg} \mathrm{U}$.

XHN15W

Haddam Neck $15 \times 15$ NUMEC SS NU-manufactured fuel for use at Haddam Neck; stainless steel clad; lead test assembly use only;

XHN15MS $\sim 406 \mathrm{~kg} \mathrm{U}$.

Haddam Neck $15 \times 15$ NUMEC Zr NU-manufactured fuel for use at Haddam Neck; Zircaloy clad; lead test assembly use only;

$\sim 371 \mathrm{~kg} \mathrm{U}$.

Haddam Neck $15 \times 15$ GULF SS GULF-manufactured fuel for use at Haddam Neck; stainless steel clad; lead test assembly use only; $\sim 406 \mathrm{~kg} \mathrm{U}$.

Haddam Neck $15 \times 15$ GULF Zr GULF-manufactured fuel for use at Haddam Neck; Zircaloy clad; lead test assembly use only;

$\sim 363 \mathrm{~kg} \mathrm{U}$.

XHN15MZ

B\&W-manufactured fuel for use at Haddam Neck; stainless steel clad; $\sim 412 \mathrm{~kg} \mathrm{U}$.

XHN15HS

Haddam Neck $15 \times 15$ B\&W SS

B\&W-manufactured fuel for use at Haddam Neck; Zircaloy clad; $\sim 412 \mathrm{~kg} \mathrm{U}$.

$\mathrm{XHN15HZ}$

Haddam Neck $15 \times 15$ B\&W Zr

XHN15B

XHN15BZ

Indian Point 1 Assembly Class

(Reactor Type: PWR/Length: 138.8 in./Width: 6.27 in.)

Indian Point $114 \times 15$ B\&W

B\&W-manufactured fuel for use at Indian Point 1;

XIP14B a thorium/uranium oxide fuel pellet; stainless steel clad; assemblies were used with the initial core configuration and were not the same assembly dimensions as later fuels; all assemblies reprocessed at West Valley; $\sim 152 \mathrm{~kg} \mathrm{U}$, thorium/uranium.

Indian Point $113 \times 14$ WE

WE-manufactured fuel for use at Indian Point 1;

XIP14W stainless steel clad; dimensions are as specified above for Indian Point 1 assembly class; some assemblies reprocessed at West Valley; $\sim 191 \mathrm{~kg} \mathrm{U}$. 
Table B4. Descriptions of Assembly Types (Continued)

\begin{tabular}{|c|c|c|}
\hline Assembly Type & Description & $\begin{array}{l}\text { Assembly } \\
\text { Type Code }\end{array}$ \\
\hline \multicolumn{3}{|c|}{$\begin{array}{l}\text { LaCrosse Assembly Class } \\
\text { (Reactor Type: BWR/Length: } 102.5 \text { in./Width: } 5.62 \text { in.) }\end{array}$} \\
\hline LaCrosse $10 \times 10 \mathrm{AC}$ & $\begin{array}{l}\text { AC-manufactured fuel for use at LaCrosse; } \\
\text { stainless steel cladding; } \sim 120 \mathrm{~kg} \mathrm{U} \text {. }\end{array}$ & XLC10L \\
\hline LaCrosse $10 \times 10 \mathrm{ANF}$ & $\begin{array}{l}\text { ANF-manufactured fuel for use at LaCrosse; } \\
\text { stainless steel cladding; } \sim 109 \mathrm{~kg} \mathrm{U} \text {. }\end{array}$ & XLC10A \\
\hline \multicolumn{3}{|c|}{$\begin{array}{l}\text { Palisades Assembly Class } \\
\text { (Reactor Type: PWR/Length: } 147.5 \text { in./Width: } 8.2 \text { in.) }\end{array}$} \\
\hline Palisades $15 \times 15 \mathrm{CE}$ & $\begin{array}{l}\text { CE-manufactured fuel for use at Palisades; } \\
\sim 412 \mathrm{~kg} \mathrm{U} \text {. }\end{array}$ & XPA15C \\
\hline Palisades $15 \times 15$ ANF & $\begin{array}{l}\text { ANF-manufactured fuel for use at Palisades; } \\
\sim 391 \mathrm{~kg} \mathrm{U} \text {. }\end{array}$ & XPA15A \\
\hline \multicolumn{3}{|c|}{$\begin{array}{l}\text { St. Lucie } 2 \text { Assembly Class } \\
\text { (Reactor Type: PWR/Length: } 158.2 \text { in./Width: } 8.1 \text { in.) }\end{array}$} \\
\hline St. Lucio $216 \times 16 \mathrm{CE}$ & $\begin{array}{l}\text { CE-manufactured fuel for use at St. Lucie 2; } \\
\sim 377 \mathrm{~kg} \mathrm{U} \text {. }\end{array}$ & XSL16C \\
\hline \multicolumn{3}{|c|}{$\begin{array}{l}\text { San Onofre } 1 \text { Assembly Class } \\
\text { (Reactor Type: PWR/Length: } 137.1 \text { in./Width: } 7.76 \text { in.) }\end{array}$} \\
\hline San Onofre $114 \times 14$ WE & $\begin{array}{l}\text { WE-manufactured fuel for use at San Onofre 1; } \\
\text { stainless steel clad; } \sim 366 \mathrm{~kg} \mathrm{U.}\end{array}$ & XS014W \\
\hline San Onofre $114 \times 14$ WE Zr & $\begin{array}{l}\text { WE-manufactured fuel for use at San Onofre 1; } \\
\text { Zircaloy clad demonstration fuel; no weight } \\
\text { available. }\end{array}$ & XSO14WZ \\
\hline \multicolumn{3}{|c|}{$\begin{array}{l}\text { Yankee Rowe Assembly Class } \\
\text { (Reactor Type: PWR/Length: } 111.8 \text { in./Width: } 7.62 \text { in.) }\end{array}$} \\
\hline Yankee Rowe $17 \times 18$ WE & $\begin{array}{l}\text { WE-manufactured fuel for use at Yankee Rowe; } \\
\text { stainless steel clad; nonsquare array designed to } \\
\text { allow space for cruciform control blades; most } \\
\text { assemblies reprocessed at West Valley; } 273 \mathrm{~kg} \mathrm{U} \text {. }\end{array}$ & XYR18W \\
\hline Yankee Rowe $15 \times 16$ UNC & $\begin{array}{l}\text { UNC-manufactured fuel for use at Yankee Rowe; } \\
\text { nonsquare array designed to allow for space for } \\
\text { cruciform control blades; 239 kg U. }\end{array}$ & XYR16U \\
\hline
\end{tabular}


Table B4. Descriptions of Assembly Types (Continued)

\begin{tabular}{|c|c|c|}
\hline Assembly Type & Description & $\begin{array}{l}\text { Assembly } \\
\text { Type Code }\end{array}$ \\
\hline \multicolumn{3}{|c|}{$\begin{array}{c}\text { Yankee Rowe Assembly Class (Continued) } \\
\text { (Reactor Type: PWR/Length: } 111.8 \text { in./Width: } 7.62 \text { in.) }\end{array}$} \\
\hline Yankee Rowe $15 \times 16$ ANF & $\begin{array}{l}\text { ANF-manufactured fuel for use at Yankee Rowe; } \\
\text { nonsquare array designed to allow space for } \\
\text { cruciform control blades; } 2234 \mathrm{~kg} \mathrm{U} \text {. }\end{array}$ & XYR16A \\
\hline Yankee Rowe $15 \times 16$ CE & $\begin{array}{l}\text { CE-manufactured fuel for use at Yankee Rowe; } \\
\text { nonsquare array designed to allow space for } \\
\text { cruciform control blades; } \sim 229 \mathrm{~kg} \mathrm{U} \text {. }\end{array}$ & XYR16C \\
\hline
\end{tabular}

Source: Energy Information Administration, Form RW-859, "Nuclear Fuel Data" (1992). 
Table B5. Assembly Characteristics by Assembly Type

\begin{tabular}{|c|c|c|c|c|c|c|c|c|c|}
\hline \multicolumn{7}{|c|}{ Assemblles } & \multicolumn{3}{|c|}{ Rods } \\
\hline $\begin{array}{c}\text { Assembly } \\
\text { Typo } \\
\text { Code }\end{array}$ & $\begin{array}{c}\text { Current } \\
\text { Fabricator }\end{array}$ & Cladding & $\begin{array}{l}\text { Avg. Initial } \\
\text { Loading } \\
\text { Wolght } \\
\text { of Uranlum } \\
\text { (kg) }\end{array}$ & $\begin{array}{l}\text { Width } \\
\text { gs Bullt } \\
\text { (inches) }\end{array}$ & $\begin{array}{l}\text { Length } \\
\text { as Bullt } \\
\text { (inches) }\end{array}$ & $\begin{array}{c}\text { Number } \\
\text { Discharged }\end{array}$ & $\begin{array}{l}\text { Length } \\
\text { (inches) }\end{array}$ & $\begin{array}{l}\text { Number of } \\
\text { Positlons }\end{array}$ & $\begin{array}{l}\text { Number of } \\
\text { Fuel Rods }\end{array}$ \\
\hline \multicolumn{10}{|c|}{ Bolling-Water Reactors (BWR) } \\
\hline 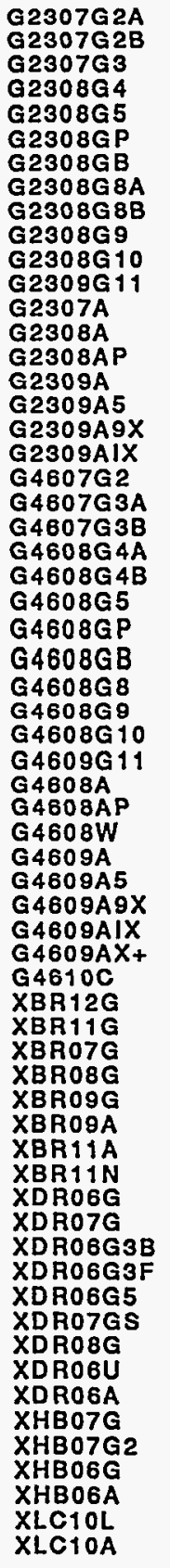 & $\begin{array}{l}\text { GE } \\
\text { GE } \\
\text { GE } \\
\text { GE } \\
\text { GE } \\
\text { GE } \\
\text { GE } \\
\text { GE } \\
\text { GE } \\
\text { GE } \\
\text { GE } \\
\text { GE } \\
\text { SIEM } \\
\text { SIEM } \\
\text { SIEM } \\
\text { SIEM } \\
\text { SIEM } \\
\text { SIEM } \\
\text { SIEM } \\
\text { GE } \\
\text { GE } \\
\text { GE } \\
\text { GE } \\
\text { GE } \\
\text { GE } \\
\text { GE } \\
\text { GE } \\
\text { GE } \\
\text { GE } \\
\text { GE } \\
\text { GE } \\
\text { SIEM } \\
\text { SIEM } \\
\text { WE } \\
\text { SIEM } \\
\text { SIEM } \\
\text { SIEM } \\
\text { SIEM } \\
\text { SIEM } \\
\text { GE } \\
\text { GE } \\
\text { GE } \\
\text { GEE } \\
\text { GE } \\
\text { GE } \\
\text { SIEM } \\
\text { SIEM } \\
\text { NFS } \\
\text { GE } \\
\text { GE } \\
\text { GE } \\
\text { GE } \\
\text { GE } \\
\text { GE } \\
\text { GE } \\
\text { UNC } \\
\text { SIEM } \\
\text { GE } \\
\text { GE } \\
\text { GE } \\
\text { SIEM } \\
\text { AC } \\
\text { SIEM }\end{array}$ & 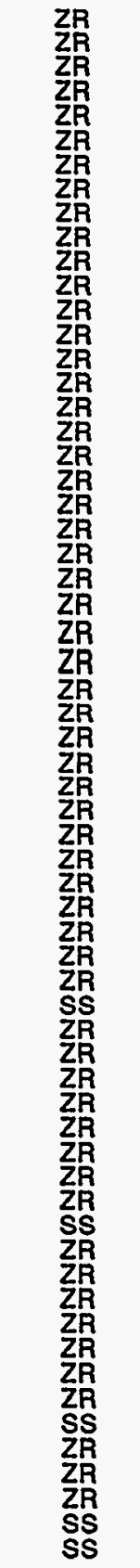 & 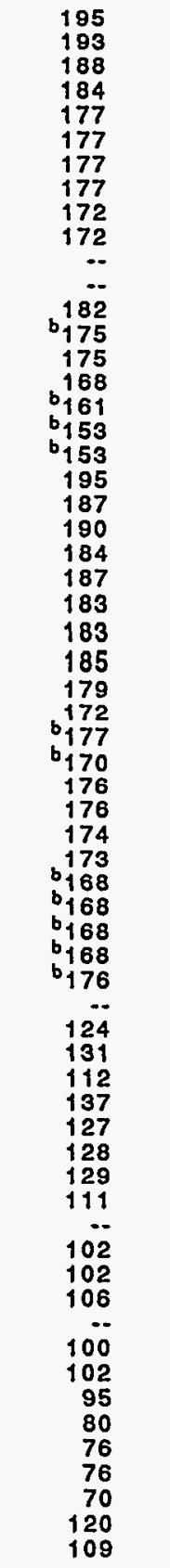 & 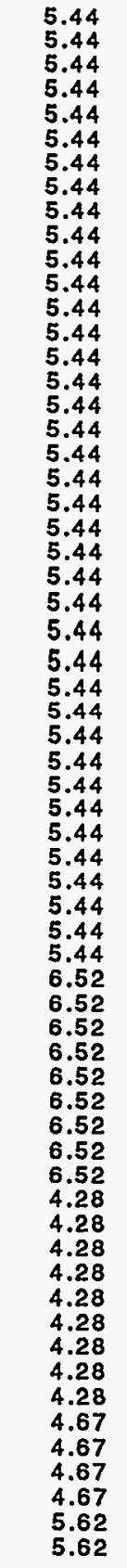 & 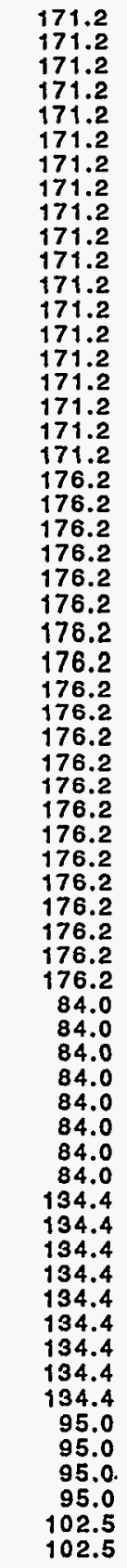 & 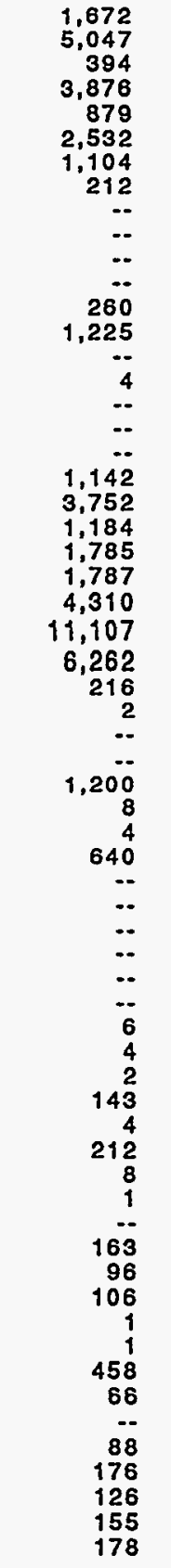 & 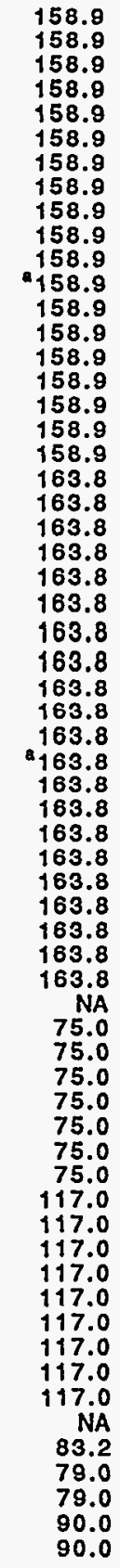 & 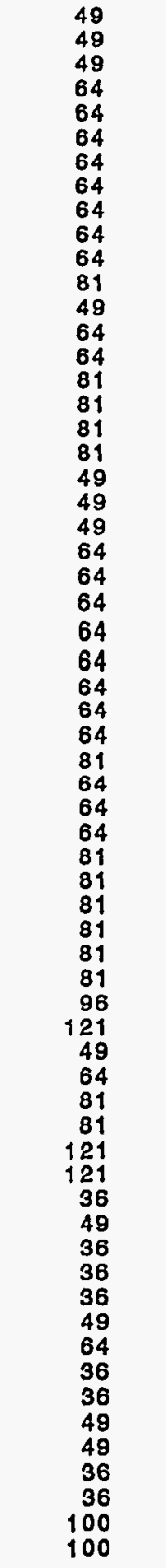 & 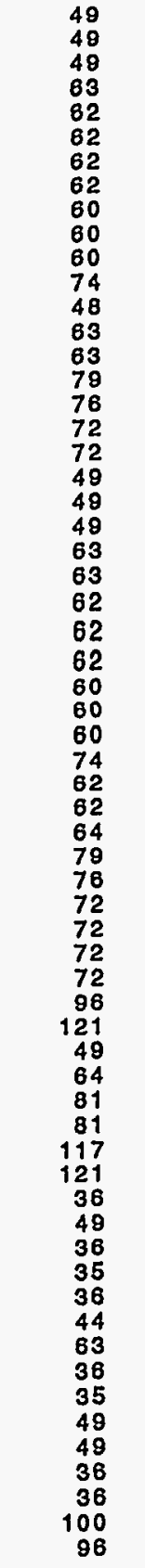 \\
\hline
\end{tabular}

See footnotes at end of table. 
Table B5. Assembly Characteristics by Assembly Type (Continued)

\begin{tabular}{c|c|c|c|c|c|c|c|c}
\hline \multicolumn{3}{c|}{ Assemblles } & \multicolumn{3}{c}{ Rods } \\
\hline $\begin{array}{c}\text { Assembly } \\
\text { Type } \\
\text { Code }\end{array}$ & $\begin{array}{c}\text { Current } \\
\text { Fabricator }\end{array}$ & $\begin{array}{c}\text { Avg. Initial } \\
\text { Loading } \\
\text { Weighi } \\
\text { of Uranlum } \\
\text { (kg) }\end{array}$ & $\begin{array}{c}\text { Widith } \\
\text { as Built } \\
\text { (inches) }\end{array}$ & $\begin{array}{c}\text { Length } \\
\text { as Bullit } \\
\text { (inches) }\end{array}$ & $\begin{array}{c}\text { Number } \\
\text { Discharged }\end{array}$ & $\begin{array}{c}\text { Length } \\
\text { (inches) }\end{array}$ & $\begin{array}{c}\text { Number of } \\
\text { Positions }\end{array}$ & $\begin{array}{l}\text { Number of } \\
\text { Fuel Rods }\end{array}$ \\
\hline
\end{tabular}

Pressurlzed-Water Reactors (PWR)

\begin{tabular}{|c|c|c|c|c|c|c|c|c|c|}
\hline 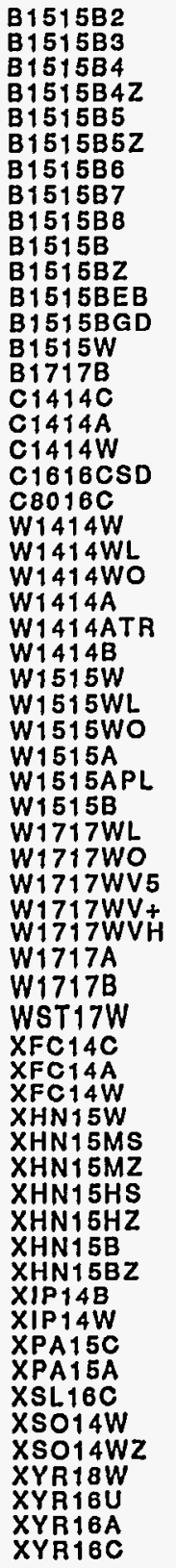 & 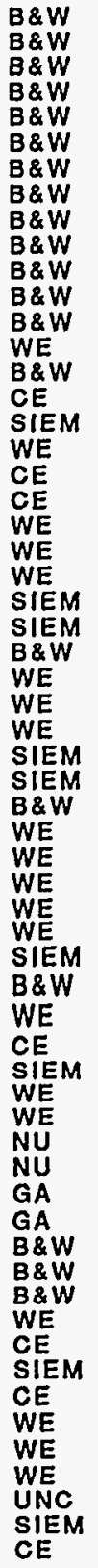 & 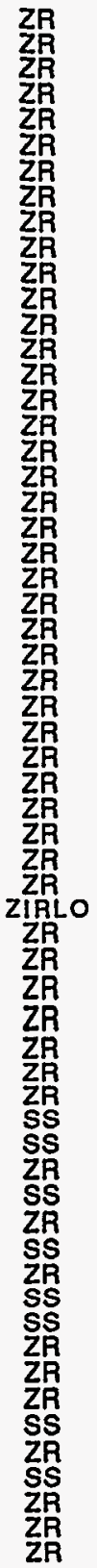 & 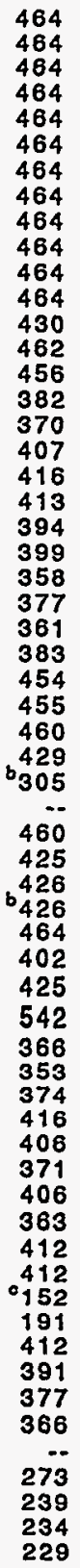 & $\begin{array}{l}8.54 \\
8.54 \\
8.54 \\
8.54 \\
8.54 \\
8.54 \\
8.54 \\
8.54 \\
8.54 \\
8.54 \\
8.54 \\
8.54 \\
8.54 \\
8.54 \\
8.54 \\
8.10 \\
8.10 \\
8.10 \\
8.10 \\
8.10 \\
7.76 \\
7.76 \\
7.76 \\
7.76 \\
7.76 \\
7.76 \\
8.44 \\
8.44 \\
8.44 \\
8.44 \\
8.44 \\
8.44 \\
8.44 \\
8.44 \\
8.44 \\
8.44 \\
8.44 \\
8.44 \\
8.44 \\
8.43 \\
8.10 \\
8.10 \\
8.10 \\
8.42 \\
8.42 \\
8.42 \\
8.42 \\
8.42 \\
8.42 \\
8.42 \\
6.14 \\
6.27 \\
8.20 \\
8.20 \\
8.10 \\
7.76 \\
7.76 \\
7.62 \\
7.62 \\
7.62 \\
7.62\end{array}$ & $\begin{array}{l}165.7 \\
165.7 \\
165.7 \\
165.7 \\
165.7 \\
165.7 \\
165.7 \\
165.7 \\
165.7 \\
165.7 \\
165.7 \\
165.7 \\
165.7 \\
165.7 \\
165.7 \\
157.0 \\
157.0 \\
157.0 \\
176.8 \\
178.3 \\
159.8 \\
159.8 \\
159.8 \\
159.8 \\
159.8 \\
159.8 \\
159.8 \\
1477.5 \\
149.8 \\
1111.8 \\
137.2 \\
137.1 \\
111.8 \\
159.8 \\
159.8 \\
159.8 \\
159.8 \\
159.8 \\
159.8 \\
159.8 \\
159.8 \\
159.8 \\
159.8 \\
159.8 \\
199.0 \\
137.1 \\
146.0 \\
146.0 \\
136.0 \\
137.1 \\
137.1 \\
137.1 \\
137.1\end{array}$ & $\begin{array}{r}0 \\
4,106 \\
4 \\
-. \\
20 \\
109 \\
43 \\
-- \\
-. \\
580 \\
-- \\
4 \\
-- \\
4 \\
3,124 \\
522 \\
423 \\
1,960 \\
848 \\
619 \\
1,411 \\
698 \\
722 \\
288 \\
2 \\
1,577 \\
3,349 \\
1,139 \\
840 \\
-- \\
-. \\
8,627 \\
2,349 \\
278 \\
733 \\
112 \\
156\end{array}$ & 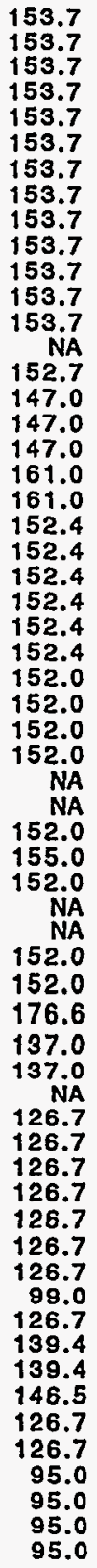 & $\begin{array}{l}225 \\
225 \\
225 \\
225 \\
225 \\
225 \\
225 \\
225 \\
225 \\
225 \\
225 \\
225 \\
225 \\
225 \\
289 \\
176 \\
196 \\
176 \\
236 \\
231 \\
196 \\
196 \\
196 \\
196 \\
196 \\
196 \\
225 \\
225 \\
225 \\
225 \\
225 \\
225 \\
289 \\
289 \\
289 \\
289 \\
289 \\
289 \\
289 \\
289 \\
196 \\
196 \\
196 \\
225 \\
225 \\
225 \\
225 \\
225 \\
225 \\
225 \\
206 \\
196 \\
216 \\
225 \\
236 \\
196 \\
240 \\
240\end{array}$ & $\begin{array}{l}208 \\
208 \\
208 \\
208 \\
208 \\
208 \\
208 \\
208 \\
208 \\
208 \\
208 \\
208 \\
208 \\
208 \\
264 \\
164 \\
176 \\
176 \\
224 \\
220 \\
179 \\
179 \\
179 \\
179 \\
179 \\
179 \\
204 \\
204 \\
204 \\
204 \\
204 \\
204 \\
264 \\
264 \\
264 \\
260 \\
264 \\
264 \\
264 \\
264 \\
168 \\
176 \\
178 \\
204 \\
204 \\
204 \\
204 \\
204 \\
204 \\
208 \\
208 \\
180 \\
204 \\
216 \\
224 \\
180 \\
179 \\
305 \\
231 \\
231 \\
231\end{array}$ \\
\hline
\end{tabular}

Elght part-length fuel rods.

Estimated welght based on most current data.

'Average Inltlal loading welght of thorlum/uranlum (kg).

- = Not appllcable.

NA = Not avallable.

Note: There are 4 temporarly dlscharged BWR assemblies and 69 temporarily dlscharged PWR assemblles that have not been assigned an assembly type code. These assemblles are not Included in the above table. See Technical Note 10 in Appendix E.

Source: Energy Information Adminlstratlon, Form AW-858, "Nuclear Fuel Data" (1992). 
Table B6. Assembly Type Summary by Reactor

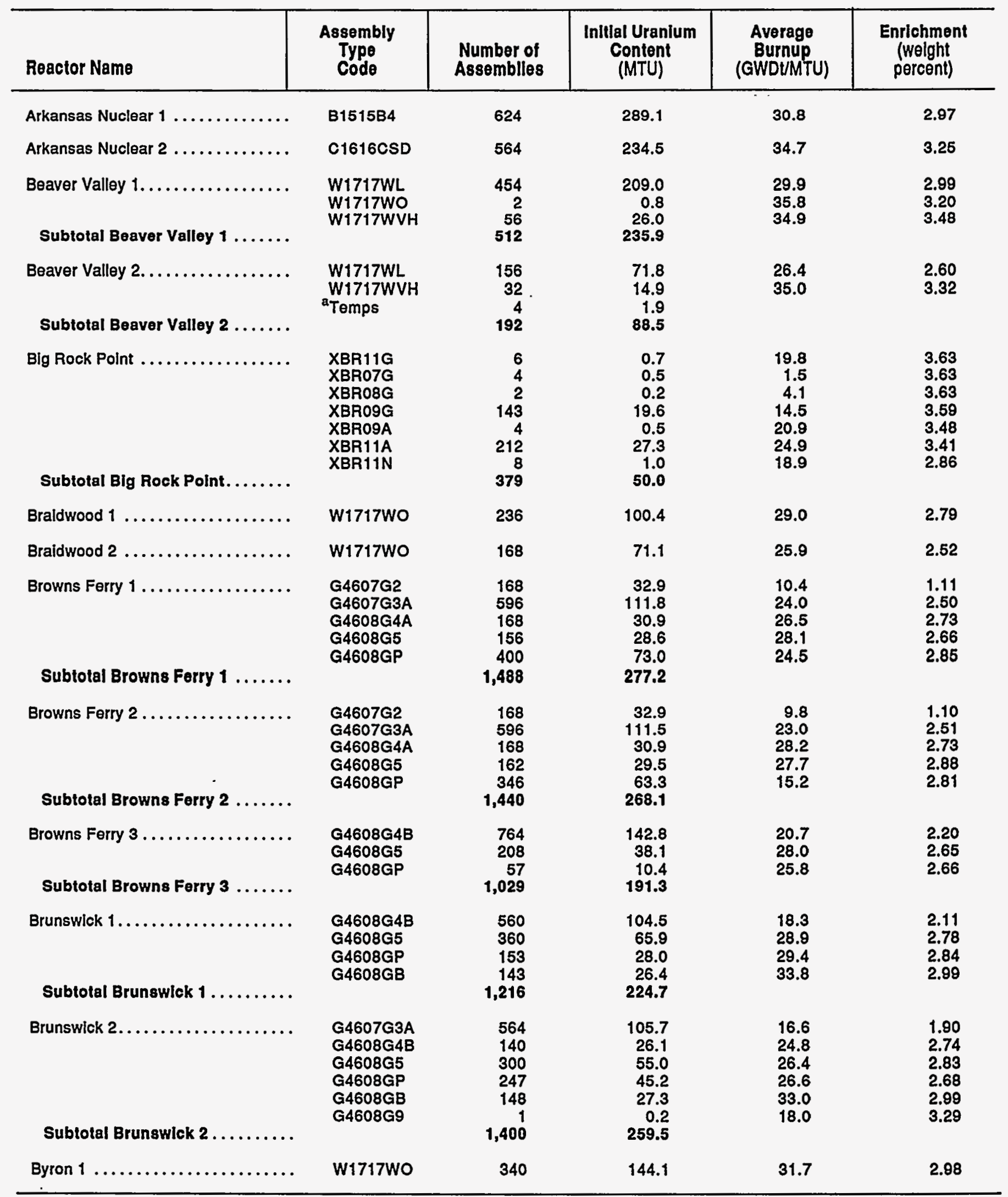

See footnotes at end of table. 
Table B6. Assembly Type Summary by Reactor (Continued)

\begin{tabular}{|c|c|c|c|c|c|}
\hline Reactor Namo & $\begin{array}{l}\text { Assembly } \\
\text { Type } \\
\text { Code }\end{array}$ & $\begin{array}{l}\text { Number of } \\
\text { Assemblles }\end{array}$ & $\begin{array}{l}\text { Inltlal Uranium } \\
\text { Content } \\
\text { (MTU) }\end{array}$ & $\begin{array}{l}\text { Average } \\
\text { Burnup } \\
\text { (GWDt/MTU) }\end{array}$ & $\begin{array}{c}\text { Enrlchment } \\
\text { (weight } \\
\text { percent) }\end{array}$ \\
\hline Byron $2 \ldots \ldots \ldots \ldots \ldots \ldots$ & W1717Wo & 256 & 109.0 & 31.5 & 2.82 \\
\hline $\begin{array}{l}\text { Callaway } \ldots \ldots \ldots \ldots \ldots \ldots \ldots \ldots \\
\text { Subtotal Callaway } \ldots \ldots \ldots \ldots \ldots\end{array}$ & $\begin{array}{l}\text { W1717WL } \\
\text { W1717Wo } \\
\text { W1717WV5 }\end{array}$ & $\begin{array}{r}193 \\
84 \\
175 \\
452\end{array}$ & $\begin{array}{r}89.0 \\
35.9 \\
74.4 \\
199.2\end{array}$ & $\begin{array}{l}24.4 \\
39.7 \\
39.2\end{array}$ & $\begin{array}{l}2.60 \\
3.61 \\
3.81\end{array}$ \\
\hline Calvert Cliffs $1 \ldots \ldots \ldots \ldots \ldots$ & C1414C & 800 & 307.6 & 32.1 & 3.10 \\
\hline Calvert Cliffs $2 \ldots \ldots \ldots \ldots \ldots \ldots$ & $\mathrm{C} 1414 \mathrm{C}$ & 612 & 235.0 & 32.8 & 3.08 \\
\hline Catawba $1 \ldots \ldots \ldots \ldots \ldots \ldots$ & W1717Wo & 408 & 173.1 & 30.1 & 2.87 \\
\hline Catawba $2 \ldots \ldots \ldots \ldots \ldots \ldots$ & W1717Wo & 280 & $\$ 18.9$ & 28.3 & 2.67 \\
\hline Cllnton $1 \ldots \ldots \ldots \ldots \ldots \ldots$ & G4608GP & 568 & 104.3 & 15.7 & 1.68 \\
\hline Comanche Peak $1 \ldots \ldots \ldots \ldots \ldots$ & W1717WL & 117 & 53.7 & 18.6 & 2.01 \\
\hline $\begin{array}{l}\text { Cook } 1, \ldots \ldots \ldots \ldots \ldots \ldots \ldots \ldots \\
\text { Subtotal Cook } 1, \ldots \ldots \ldots \ldots \ldots\end{array}$ & $\begin{array}{l}\text { W1515WL } \\
\text { W1515WO } \\
\text { W1515A }\end{array}$ & $\begin{array}{l}193 \\
287 \\
386 \\
866\end{array}$ & $\begin{array}{r}87.5 \\
132.1 \\
165.0 \\
384.7\end{array}$ & $\begin{array}{l}27.5 \\
37.5 \\
31.5\end{array}$ & $\begin{array}{l}2.78 \\
3.42 \\
2.91\end{array}$ \\
\hline $\begin{array}{l}\text { Cook } 2 \ldots \ldots \ldots \ldots \ldots \ldots \ldots \ldots \\
\text { Subtotal Cook } 2 \ldots \ldots \ldots \ldots \ldots\end{array}$ & $\begin{array}{l}\text { W1717WL } \\
\text { W1717WV5 } \\
\text { W1717A }\end{array}$ & $\begin{array}{r}365 \\
2 \\
290 \\
657\end{array}$ & $\begin{array}{r}167.6 \\
0.9 \\
116.6 \\
285.1\end{array}$ & $\begin{array}{l}28.8 \\
21.0 \\
39.3\end{array}$ & $\begin{array}{l}2.98 \\
4.20 \\
3.76\end{array}$ \\
\hline Cooper Station $\ldots \ldots \ldots \ldots \ldots \ldots$ & $\begin{array}{l}\text { G4607G2 } \\
\text { G4607G3B } \\
\text { G4608G4A } \\
\text { G4608G4B } \\
\text { G4608G5 } \\
\text { G4608GP } \\
\text { G4608GB }\end{array}$ & $\begin{array}{r}128 \\
420 \\
72 \\
136 \\
192 \\
636 \\
92 \\
1,676\end{array}$ & $\begin{array}{r}25.1 \\
79.8 \\
13.2 \\
25.4 \\
35.1 \\
116.5 \\
17.0 \\
312.1\end{array}$ & $\begin{array}{l}10.1 \\
25.2 \\
25.0 \\
30.5 \\
30.8 \\
29.6 \\
31.2\end{array}$ & $\begin{array}{l}1.09 \\
2.51 \\
2.51 \\
2.73 \\
2.82 \\
2.75 \\
2.79\end{array}$ \\
\hline $\begin{array}{l}\text { Crystal River } 3 \ldots \ldots \ldots \ldots \ldots \ldots \\
\text { Subtotal Crystal Rlver } 3 \ldots \ldots \ldots\end{array}$ & $\begin{array}{l}\text { B1515B4 } \\
\text { B1515BZ }\end{array}$ & $\begin{array}{r}496 \\
40 \\
536\end{array}$ & $\begin{array}{r}230.3 \\
18.5 \\
248.9\end{array}$ & $\begin{array}{l}26.7 \\
38.0\end{array}$ & $\begin{array}{l}2.77 \\
3.85\end{array}$ \\
\hline $\begin{array}{l}\text { Davis-Besse } \ldots \ldots \ldots \ldots \ldots \ldots \ldots \\
\text { Subtotal Davls-Besse ......... }\end{array}$ & $\begin{array}{l}\mathrm{B} 1515 \mathrm{BA} \\
\mathrm{a} \text { Temps }\end{array}$ & $\begin{array}{r}386 \\
6 \\
392\end{array}$ & $\begin{array}{r}181.5 \\
b_{0.0} \\
181.5\end{array}$ & 30.0 & 2.82 \\
\hline $\begin{array}{l}\text { Dlablo Canyon } 1 \ldots \ldots \ldots \ldots \ldots \ldots \\
\text { Subtotal Dlablo Canyon } 1 \ldots \ldots\end{array}$ & $\begin{array}{l}\text { W1717WL } \\
\text { W1717WV5 }\end{array}$ & $\begin{array}{r}337 \\
39 \\
376\end{array}$ & $\begin{array}{r}155.4 \\
16.6 \\
171.9\end{array}$ & $\begin{array}{l}33.5 \\
39.7\end{array}$ & $\begin{array}{l}3.06 \\
4.01\end{array}$ \\
\hline Dlablo Canyon $2 \ldots \ldots \ldots \ldots \ldots$ & W1717WL & 308 & 141.9 & 31.8 & 3.05 \\
\hline Subtotal Dresden $1 \ldots \ldots \ldots \ldots$ & $\begin{array}{l}\text { XDR06G } \\
\text { XDR06G3B } \\
\text { XDR06G3F } \\
\text { XDR06G5 } \\
\text { XDR07GS } \\
\text { XDR08G } \\
\text { XDR06U } \\
\text { XDR06A }\end{array}$ & $\begin{array}{r}1 \\
163 \\
96 \\
106 \\
1 \\
1 \\
458 \\
66 \\
892\end{array}$ & $\begin{array}{r}0.1 \\
16.6 \\
9.8 \\
11.2 \\
0.1 \\
0.1 \\
46.7 \\
6.3 \\
90.9\end{array}$ & $\begin{array}{r}21.3 \\
16.9 \\
20.0 \\
19.0 \\
29.0 \\
23.3 \\
16.1 \\
4.5\end{array}$ & $\begin{array}{l}1.47 \\
1.83 \\
2.25 \\
2.26 \\
3.10 \\
1.95 \\
2.24 \\
2.23\end{array}$ \\
\hline
\end{tabular}

See footnotes at end of table. 
Table B6. Assembly Type Summary by Reactor (Continued)

\begin{tabular}{|c|c|c|c|c|c|}
\hline Reactor Name & $\begin{array}{c}\text { Assembly } \\
\text { Type } \\
\text { Code }\end{array}$ & $\begin{array}{l}\text { Number of } \\
\text { Assemblles }\end{array}$ & $\begin{array}{l}\text { Initlal Uranlum } \\
\text { Content } \\
\text { (MTU) }\end{array}$ & $\begin{array}{c}\text { Average } \\
\text { Burnup } \\
\text { (GWDt/MTU) }\end{array}$ & $\begin{array}{c}\text { Enrlchment } \\
\text { (weight } \\
\text { percent) }\end{array}$ \\
\hline Subtotal Dresden $2 . . \ldots \ldots \ldots$ & $\begin{array}{l}\text { G2307G2B } \\
\text { G2307G3 } \\
\text { G2308G4 } \\
\text { G2308G5 } \\
\text { G2308GP } \\
\text { G2308A } \\
\text { G2309A }\end{array}$ & $\begin{array}{r}1,477 \\
32 \\
476 \\
160 \\
228 \\
228 \\
4 \\
4 \\
2,605\end{array}$ & $\begin{array}{r}284.1 \\
6.0 \\
87.8 \\
28.3 \\
40.4 \\
39.5 \\
0.7 \\
486.7\end{array}$ & $\begin{array}{l}10.4 \\
23.7 \\
25.1 \\
28.7 \\
28.4 \\
30.8 \\
36.0\end{array}$ & $\begin{array}{l}2.08 \\
2.29 \\
2.52 \\
2.67 \\
2.64 \\
2.82 \\
2.77\end{array}$ \\
\hline $\begin{array}{l}\text { Dresden } 3 \ldots \ldots \ldots \ldots \ldots \ldots \ldots \\
\text { Subtotal Dresden } 3 \ldots \ldots \ldots \ldots \text {. }\end{array}$ & $\begin{array}{l}\text { G2307G2B } \\
\text { G2307G3 } \\
\text { G2308G4 } \\
\text { G2308GP } \\
\text { G2308A }\end{array}$ & $\begin{array}{r}724 \\
52 \\
508 \\
200 \\
380 \\
1,864\end{array}$ & $\begin{array}{r}140.2 \\
9.8 \\
93.4 \\
35.4 \\
66.0 \\
344.8\end{array}$ & $\begin{array}{l}17.3 \\
24.0 \\
25.7 \\
28.0 \\
31.9\end{array}$ & $\begin{array}{l}2.13 \\
2.30 \\
2.56 \\
2.65 \\
2.74\end{array}$ \\
\hline Duane Arnold $\ldots \ldots \ldots \ldots \ldots \ldots$ & $\begin{array}{l}\text { G4607G3A } \\
\text { G4608G4A } \\
\text { G4608GP } \\
\text { G4608GB } \\
\text { G4608G8 }\end{array}$ & $\begin{array}{r}372 \\
185 \\
388 \\
.119 \\
88 \\
1,152\end{array}$ & $\begin{array}{r}69.8 \\
34.1 \\
71.0 \\
21.9 \\
15.7 \\
212.5\end{array}$ & $\begin{array}{l}15.9 \\
26.3 \\
28.6 \\
28.0 \\
33.7\end{array}$ & $\begin{array}{l}1.90 \\
2.74 \\
2.86 \\
3.01 \\
3.01\end{array}$ \\
\hline Enrico Fermi $2 . \ldots \ldots \ldots \ldots \ldots$ & G4608GP & 672 & 121.8 & 19.0 & 1.86 \\
\hline $\begin{array}{l}\text { Farley } 1, \ldots \ldots \ldots \ldots \ldots \ldots \ldots \ldots \\
\text { Subtotal Farley } 1 \ldots \ldots \ldots \ldots \ldots\end{array}$ & $\begin{array}{l}\text { W1717WL } \\
\text { W1717WO }\end{array}$ & $\begin{array}{r}653 \\
2 \\
655\end{array}$ & $\begin{array}{r}301.0 \\
0.8 \\
301.8\end{array}$ & $\begin{array}{l}31.5 \\
46.3\end{array}$ & $\begin{array}{l}3.18 \\
3.11\end{array}$ \\
\hline Farley $2 \ldots \ldots \ldots \ldots \ldots \ldots \ldots$ & W1717WL & 498 & 229.7 & 32.2 & 3.22 \\
\hline Subtotal Fitzpatrick. ......... & $\begin{array}{l}\text { G4607G2 } \\
\text { G4607G3A } \\
\text { G4608G4A } \\
\text { G4608G5 } \\
\text { G4608GP } \\
\text { G4608GB } \\
\text { G4608G8 } \\
\text { G4608W }\end{array}$ & $\begin{array}{r}132 \\
428 \\
132 \\
136 \\
552 \\
196 \\
104 \\
4 \\
1,684\end{array}$ & $\begin{array}{r}25.8 \\
80.2 \\
24.3 \\
24.8 \\
100.8 \\
36.1 \\
18.5 \\
0.6 \\
311.3\end{array}$ & $\begin{array}{r}9.1 \\
22.8 \\
26.0 \\
26.8 \\
27.9 \\
29.8 \\
32.0 \\
23.0\end{array}$ & $\begin{array}{l}1.11 \\
2.50 \\
2.74 \\
2.78 \\
2.90 \\
2.99 \\
3.19 \\
3.01\end{array}$ \\
\hline $\begin{array}{l}\text { Fort Calhoun } \ldots \ldots \ldots \ldots \ldots \ldots \ldots \\
\text { Subtotal Fort Calhoun } \ldots \ldots \ldots \ldots\end{array}$ & $\begin{array}{l}\text { XFC14C } \\
\text { XFC14A }\end{array}$ & $\begin{array}{l}337 \\
192 \\
529\end{array}$ & $\begin{array}{r}122.7 \\
67.8 \\
190.5\end{array}$ & $\begin{array}{l}29.1 \\
37.3\end{array}$ & $\begin{array}{l}2.78 \\
3.57\end{array}$ \\
\hline Subtotal Ginna............. & $\begin{array}{l}\text { W1414W } \\
\text { W1414WO } \\
\text { W1414A } \\
\text { W1414B }\end{array}$ & $\begin{array}{r}362 \\
143 \\
194 \\
2 \\
701\end{array}$ & $\begin{array}{r}141.8 \\
50.4 \\
72.3 \\
0.8 \\
265.3\end{array}$ & $\begin{array}{l}24.4 \\
39.6 \\
37.1 \\
24.4\end{array}$ & $\begin{array}{l}3.03 \\
3.49 \\
3.22 \\
3.22\end{array}$ \\
\hline $\begin{array}{l}\text { Grand Gulf } 1 \ldots \ldots \ldots \ldots \ldots \ldots \\
\text { Subtotal Grand Gulf } 1 \ldots \ldots \ldots\end{array}$ & $\begin{array}{l}\text { G4608GP } \\
\text { G4608A }\end{array}$ & $\begin{array}{r}800 \\
584 \\
1,384\end{array}$ & $\begin{array}{l}146.9 \\
103.0 \\
249.9\end{array}$ & $\begin{array}{l}15.5 \\
30.3\end{array}$ & $\begin{array}{l}1.72 \\
2.94\end{array}$ \\
\hline
\end{tabular}

See footnotes at end of table. 
Table B6. Assembly Type Summary by Reactor (Continued)

\begin{tabular}{|c|c|c|c|c|c|}
\hline Reactor Name & $\begin{array}{c}\text { Assembly } \\
\text { Typo } \\
\text { Code }\end{array}$ & $\begin{array}{l}\text { Number of } \\
\text { Assemblles }\end{array}$ & $\begin{array}{l}\text { Initlal Uranlum } \\
\text { Content } \\
\text { (MTU) }\end{array}$ & $\begin{array}{c}\text { Average } \\
\text { Burnup } \\
\text { (GWDt/MTU) }\end{array}$ & $\begin{array}{c}\text { Enrichment } \\
\text { (wolght } \\
\text { percent) }\end{array}$ \\
\hline $\begin{array}{l}\text { Haddam Neck ................. } \\
\text { Subtotal Haddam Neck. ........ }\end{array}$ & $\begin{array}{l}\text { XHN15W } \\
\text { XHN15MS } \\
\text { XHN15MZ } \\
\text { XHN15HS } \\
\text { XHN15HZ } \\
\text { XHN15B }\end{array}$ & $\begin{array}{r}309 \\
2 \\
2 \\
1 \\
2 \\
524 \\
840\end{array}$ & $\begin{array}{r}128.4 \\
0.8 \\
0.7 \\
0.4 \\
0.7 \\
215.6 \\
346.7\end{array}$ & $\begin{array}{l}27.9 \\
28.3 \\
25.6 \\
32.2 \\
18.5 \\
33.7\end{array}$ & $\begin{array}{l}3.58 \\
3.66 \\
2.95 \\
3.89 \\
3.26 \\
3.99\end{array}$ \\
\hline $\begin{array}{l}\text { Harris } 1 \ldots \ldots \ldots \ldots \ldots \ldots \ldots \\
\text { Subtotal Harris } 1 \ldots \ldots \ldots \ldots \ldots\end{array}$ & $\begin{array}{l}\text { W1717WL } \\
\text { W1717WV5 }\end{array}$ & $\begin{array}{r}205 \\
19 \\
224\end{array}$ & $\begin{array}{r}95.0 \\
8.1 \\
103.1\end{array}$ & $\begin{array}{l}29.5 \\
40.4\end{array}$ & $\begin{array}{l}2.83 \\
3.87\end{array}$ \\
\hline Hatch $1 \ldots \ldots \ldots \ldots \ldots \ldots \ldots \ldots$ & $\begin{array}{l}\text { G4607G3A } \\
\text { G4608G4A } \\
\text { G4608G5 } \\
\text { G4608GP } \\
\text { G4608GB }\end{array}$ & $\begin{array}{r}560 \\
92 \\
384 \\
328 \\
506 \\
1,870\end{array}$ & $\begin{array}{r}104.9 \\
16.9 \\
70.3 \\
60.1 \\
93.3 \\
345.5\end{array}$ & $\begin{array}{l}20.9 \\
23.1 \\
22.9 \\
25.2 \\
25.5\end{array}$ & $\begin{array}{l}2.33 \\
2.51 \\
2.43 \\
2.82 \\
2.69\end{array}$ \\
\hline $\begin{array}{l}\text { Hatch } 2 \ldots \ldots \ldots \ldots \ldots \ldots \ldots \ldots \\
\text { Subtotal Hatch } 2 \ldots \ldots \ldots \ldots \ldots\end{array}$ & $\begin{array}{l}\text { G4608G5 } \\
\text { G4608GP } \\
\text { G4608GB } \\
\text { G4608G9 } \\
\text { G4609A }\end{array}$ & $\begin{array}{r}509 \\
407 \\
585 \\
1 \\
4 \\
1,506\end{array}$ & $\begin{array}{r}93.4 \\
74.5 \\
108.2 \\
0.2 \\
0.7 \\
276.9\end{array}$ & $\begin{array}{l}19.2 \\
25.0 \\
27.0 \\
14.0 \\
36.0\end{array}$ & $\begin{array}{l}1.97 \\
2.74 \\
2.74 \\
3.14 \\
2.79\end{array}$ \\
\hline $\begin{array}{l}\text { Hope Creok.................. } \\
\text { Subtotal Hope Creok......... }\end{array}$ & $\begin{array}{l}\text { G4608GP } \\
\text { G4608GB }\end{array}$ & $\begin{array}{r}92 \\
916 \\
1,008\end{array}$ & $\begin{array}{r}17.0 \\
169.5 \\
186.6\end{array}$ & $\begin{array}{r}3.0 \\
22.7\end{array}$ & $\begin{array}{l}0.73 \\
2.27\end{array}$ \\
\hline $\begin{array}{l}\text { Humboldt Bay ................... } \\
\text { Subtotal Humboldt Bay ........ }\end{array}$ & $\begin{array}{l}\text { XHB07G2 } \\
\text { XHB06G } \\
\text { XHB06A }\end{array}$ & $\begin{array}{r}88 \\
176 \\
126 \\
390\end{array}$ & $\begin{array}{r}6.7 \\
13.4 \\
8.8 \\
28.9\end{array}$ & $\begin{array}{r}18.2 \\
17.2 \\
9.0\end{array}$ & $\begin{array}{l}2.11 \\
2.43 \\
2.40\end{array}$ \\
\hline Indlan Polnt 1 ................. & XIP14W & 160 & 30.6 & 16.7 & 4.11 \\
\hline $\begin{array}{l}\text { Indlan Polnt } 2 \ldots \ldots \ldots \ldots \ldots \ldots \\
\text { Subtotal Indlan Point } 2 \ldots \ldots \ldots\end{array}$ & $\begin{array}{l}\text { W1515W } \\
\text { W1515WL } \\
\text { aTemps }\end{array}$ & $\begin{array}{r}393 \\
267 \\
8 \\
668\end{array}$ & $\begin{array}{r}177.3 \\
122.3 \\
0.0 \\
299.6\end{array}$ & $\begin{array}{l}29.3 \\
36.0\end{array}$ & $\begin{array}{l}3.00 \\
3.32\end{array}$ \\
\hline $\begin{array}{l}\text { Indlan Point } 3 \ldots \ldots \ldots \ldots \ldots \ldots \\
\text { Subtolal Indlan Polnt } 3 \ldots \ldots \ldots\end{array}$ & $\begin{array}{l}\text { W1515WL } \\
\text { W1515WO } \\
\text { aTemps }\end{array}$ & $\begin{array}{r}409 \\
173 \\
2 \\
584\end{array}$ & $\begin{array}{r}186.8 \\
79.9 \\
0.0 \\
266.7\end{array}$ & $\begin{array}{l}31.5 \\
36.6\end{array}$ & $\begin{array}{l}3.03 \\
3.35\end{array}$ \\
\hline $\begin{array}{l}\text { Kewaunee } \ldots \ldots \ldots \ldots \ldots \ldots \ldots \\
\text { Subtotal Kewauneo........... }\end{array}$ & $\begin{array}{l}\text { W1414W } \\
\text { W1414A } \\
\text { aTemps }\end{array}$ & $\begin{array}{r}241 \\
363 \\
12 \\
616\end{array}$ & $\begin{array}{r}95.7 \\
137.4 \\
b_{0.0} \\
233.1\end{array}$ & $\begin{array}{l}31.7 \\
35.2\end{array}$ & $\begin{array}{l}3.06 \\
3.27\end{array}$ \\
\hline $\begin{array}{l}\text { LaCrosse } \ldots \ldots \ldots \ldots \ldots \ldots \ldots \ldots \\
\text { Subtotal LaCrosse............ }\end{array}$ & $\begin{array}{l}\text { XLC10L } \\
\text { XLC10A }\end{array}$ & $\begin{array}{l}155 \\
178 \\
333\end{array}$ & $\begin{array}{l}18.6 \\
19.3 \\
38.0\end{array}$ & $\begin{array}{l}14.5 \\
14.9\end{array}$ & $\begin{array}{l}3.77 \\
3.69\end{array}$ \\
\hline $\begin{array}{l}\text { LaSalle County } 1 \ldots \ldots \ldots \ldots \ldots \\
\text { Subtotal LaSalle County } 1 \ldots \ldots\end{array}$ & $\begin{array}{l}\text { G4608G5 } \\
\text { G4608GP } \\
\text { G4608GB }\end{array}$ & $\begin{array}{r}627 \\
192 \\
201 \\
1,020\end{array}$ & $\begin{array}{r}114.9 \\
35.0 \\
37.2 \\
187.1\end{array}$ & $\begin{array}{l}17.9 \\
28.9 \\
33.5\end{array}$ & $\begin{array}{l}1.81 \\
2.42 \\
3.04\end{array}$ \\
\hline
\end{tabular}

See footnotes at end of table. 
Table B6. Assembly Type Summary by Reactor (Continued)

\begin{tabular}{|c|c|c|c|c|c|}
\hline Reactor Name & $\begin{array}{l}\text { Assembly } \\
\text { Type } \\
\text { Code }\end{array}$ & $\begin{array}{l}\text { Number of } \\
\text { Assemblles }\end{array}$ & $\begin{array}{l}\text { Initial Uranium } \\
\text { Content } \\
\text { (MTU) }\end{array}$ & $\begin{array}{c}\text { Average } \\
\text { Burnup } \\
\text { (GWDt/MTU) }\end{array}$ & $\begin{array}{l}\text { Enrichment } \\
\text { (welght } \\
\text { percent) }\end{array}$ \\
\hline Limerick $2 . . . \ldots \ldots \ldots \ldots \ldots$ & G4608GB & 224 & 41.7 & 5.9 & 0.85 \\
\hline $\begin{array}{l}\text { Maine Yankee................ } \\
\text { Subtotal Malne Yankee........ }\end{array}$ & $\begin{array}{l}C 1414 C \\
C 1414 A\end{array}$ & $\begin{array}{r}854 \\
223 \\
1,077\end{array}$ & $\begin{array}{r}318.3 \\
82.5 \\
400.8\end{array}$ & $\begin{array}{l}25.6 \\
36.8\end{array}$ & $\begin{array}{l}2.66 \\
3.20\end{array}$ \\
\hline $\begin{array}{l}\text { McGuire } 2 \ldots \ldots \ldots \ldots \ldots \ldots \ldots \\
\text { Subtotal McGulre } 2 \ldots \ldots \ldots \ldots\end{array}$ & $\begin{array}{l}\text { W1717WL } \\
\text { W1717WO }\end{array}$ & $\begin{array}{l}193 \\
283 \\
476\end{array}$ & $\begin{array}{r}88.8 \\
120.2 \\
209.1\end{array}$ & $\begin{array}{l}26.1 \\
35.2\end{array}$ & $\begin{array}{l}2.58 \\
3.28\end{array}$ \\
\hline Subtotal Millstone $1 \ldots \ldots \ldots$ & $\begin{array}{l}\text { G2307G2A } \\
\text { G2307G2B } \\
\text { G2307G3 } \\
\text { G2308G4 } \\
\text { G2308G5 } \\
\text { G2308GP } \\
\text { G2308GB } \\
\text { G2308G8A }\end{array}$ & $\begin{array}{r}580 \\
82 \\
154 \\
392 \\
148 \\
360 \\
212 \\
188 \\
2,116\end{array}$ & $\begin{array}{r}113.4 \\
15.9 \\
28.9 \\
72.2 \\
26.2 \\
63.8 \\
37.7 \\
33.5 \\
391.6\end{array}$ & $\begin{array}{l}16.8 \\
21.0 \\
24.1 \\
26.9 \\
26.4 \\
27.9 \\
27.8 \\
32.0\end{array}$ & $\begin{array}{l}2.08 \\
2.30 \\
2.45 \\
2.69 \\
2.66 \\
2.79 \\
3.00 \\
2.88\end{array}$ \\
\hline Subtotal Nine Mlle Polnt $1 . . .$. . & $\begin{array}{l}\text { G2307G2A } \\
\text { G2307G2B } \\
\text { G2307G3 } \\
\text { G2308G4 } \\
\text { G2308G5 } \\
\text { G2308GP }\end{array}$ & $\begin{array}{r}532 \\
96 \\
108 \\
456 \\
184 \\
244 \\
1,620\end{array}$ & $\begin{array}{r}103.2 \\
18.6 \\
20.2 \\
83.9 \\
32.5 \\
43.0 \\
301.4\end{array}$ & $\begin{array}{l}16.2 \\
20.1 \\
26.0 \\
25.7 \\
27.9 \\
24.0\end{array}$ & $\begin{array}{l}2.11 \\
2.42 \\
2.60 \\
2.60 \\
2.77 \\
2.77\end{array}$ \\
\hline $\begin{array}{l}\text { Nine Mile Point } 2 \ldots \ldots \ldots \ldots \ldots \ldots \\
\text { Subtotal Nine Mile Polnt } 2 \ldots \ldots\end{array}$ & $\begin{array}{l}\text { G4608GP } \\
\text { G4608GB }\end{array}$ & $\begin{array}{l}332 \\
112 \\
444\end{array}$ & $\begin{array}{l}61.4 \\
20.7 \\
82.0\end{array}$ & $\begin{array}{l}12.5 \\
22.0\end{array}$ & $\begin{array}{l}1.47 \\
2.19\end{array}$ \\
\hline
\end{tabular}

See footnotes at end of table. 
Table B6. Assembly Type Summary by Reactor (Continued)

\begin{tabular}{|c|c|c|c|c|c|}
\hline Reactor Name & $\begin{array}{l}\text { Assembly } \\
\text { Type } \\
\text { Code }\end{array}$ & $\begin{array}{l}\text { Number of } \\
\text { Assemblies }\end{array}$ & $\begin{array}{l}\text { Inltlal Uranium } \\
\text { Content } \\
\text { (MTU) }\end{array}$ & $\begin{array}{c}\text { Averago } \\
\text { Burnup } \\
\text { (GWDUMTU) }\end{array}$ & $\begin{array}{c}\text { Enrichment } \\
\text { (woight } \\
\text { percent) }\end{array}$ \\
\hline $\begin{array}{l}\text { North Anna } 1, \ldots \ldots \ldots \ldots \ldots \ldots \\
\text { Subtotal North Anna } 1 \ldots \ldots \ldots\end{array}$ & $\begin{array}{l}\text { W1717WL } \\
\text { aTemps }\end{array}$ & $\begin{array}{r}492 \\
8 \\
500\end{array}$ & $\begin{array}{r}226.5 \\
0.0 \\
226.5\end{array}$ & 32.2 & 3.26 \\
\hline $\begin{array}{l}\text { North Anna } 2 \ldots \ldots \ldots \ldots \ldots \ldots \\
\text { Subtotal North Anna } 2 \ldots \ldots \ldots\end{array}$ & $\begin{array}{l}\text { W1717WL } \\
\text { W1717WVH } \\
\text { aTemps }\end{array}$ & $\begin{array}{r}487 \\
4 \\
2 \\
493\end{array}$ & $\begin{array}{r}224.7 \\
1.9 \\
60.0 \\
226.6\end{array}$ & $\begin{array}{l}34.8 \\
23.0\end{array}$ & $\begin{array}{l}3.33 \\
4.21\end{array}$ \\
\hline $\begin{array}{l}\text { Ocones } 1 \ldots \ldots \ldots \ldots \ldots \ldots \ldots \\
\text { Subtotal Oconee } 1 \ldots \ldots \ldots \ldots \ldots\end{array}$ & $\begin{array}{l}\mathrm{B} 1515 \mathrm{~B} 4 \\
\mathrm{~B} 1515 \mathrm{~B} 4 \mathrm{Z} \\
\mathrm{B} 1515 \mathrm{~B} 6 \\
\mathrm{~B} 1515 \mathrm{BZ} \\
\mathrm{B} 1515 \mathrm{BGD}\end{array}$ & $\begin{array}{r}569 \\
4 \\
43 \\
206 \\
4 \\
826\end{array}$ & $\begin{array}{r}264.5 \\
1.9 \\
19.9 \\
95.5 \\
1.7 \\
383.5\end{array}$ & $\begin{array}{l}26.7 \\
42.0 \\
42.6 \\
37.9 \\
43.0\end{array}$ & $\begin{array}{l}2.75 \\
3.30 \\
3.56 \\
3.33 \\
3.92\end{array}$ \\
\hline Subtotal Oconer 2.......... & $\begin{array}{l}\text { B1515B4 } \\
\text { B1515B5Z } \\
B 1515 B 6 \\
B 1515 B Z \\
B 1717 B\end{array}$ & $\begin{array}{r}568 \\
20 \\
53 \\
91 \\
4 \\
736\end{array}$ & $\begin{array}{r}263.5 \\
9.3 \\
24.5 \\
42.2 \\
1.8 \\
341.3\end{array}$ & $\begin{array}{l}29.1 \\
36.0 \\
42.0 \\
36.2 \\
29.5\end{array}$ & $\begin{array}{l}2.89 \\
3.20 \\
3.40 \\
3.22 \\
2.84\end{array}$ \\
\hline $\begin{array}{l}\text { Ocones } 3 . \ldots \ldots \ldots \ldots \ldots \ldots \ldots \\
\text { Subtotal Oconer } 3 \ldots \ldots \ldots \ldots\end{array}$ & $\begin{array}{l}\mathrm{B} 1515 \mathrm{~B} 4 \\
\mathrm{~B} 1515 \mathrm{~B} 6 \\
\mathrm{~B} 1515 \mathrm{~B} 7 \\
\mathrm{~B} 1515 \mathrm{BZ}\end{array}$ & $\begin{array}{r}505 \\
13 \\
43 \\
187 \\
748\end{array}$ & $\begin{array}{r}233.9 \\
6.0 \\
19.9 \\
86.7 \\
346.4\end{array}$ & $\begin{array}{l}28.3 \\
37.0 \\
41.0 \\
34.1\end{array}$ & $\begin{array}{l}2.83 \\
3.20 \\
3.50 \\
3.24\end{array}$ \\
\hline Oyster Creek................. & $\begin{array}{l}\text { G2307G2A } \\
\text { G2307G2B } \\
\text { G2308G5 } \\
\text { G2308GP } \\
\text { G2308GB } \\
\text { G2307A } \\
\text { G2308A }\end{array}$ & $\begin{array}{r}560 \\
156 \\
87 \\
156 \\
40 \\
260 \\
617 \\
1,876\end{array}$ & $\begin{array}{r}109.3 \\
30.2 \\
15.4 \\
27.5 \\
7.1 \\
47.4 \\
108.0 \\
344.8\end{array}$ & $\begin{array}{l}17.3 \\
22.7 \\
26.0 \\
29.2 \\
25.6 \\
23.5 \\
25.0\end{array}$ & $\begin{array}{l}2.11 \\
2.62 \\
2.39 \\
2.84 \\
2.68 \\
2.64 \\
2.50\end{array}$ \\
\hline $\begin{array}{l}\text { Pallsades................... } \\
\text { Subtotal Pallsades } \ldots \ldots \ldots \ldots \ldots\end{array}$ & $\begin{array}{l}\text { XPA15C } \\
\text { XPA15A }\end{array}$ & $\begin{array}{l}273 \\
460 \\
733\end{array}$ & $\begin{array}{l}112.6 \\
179.9 \\
292.5\end{array}$ & $\begin{array}{l}16.0 \\
30.4\end{array}$ & $\begin{array}{l}2.47 \\
2.92\end{array}$ \\
\hline Palo Verde $1 . \ldots \ldots \ldots \ldots \ldots$ & C8016C & 276 & 113.6 & 28.1 & 2.73 \\
\hline Palo Verde $2 \ldots \ldots \ldots \ldots \ldots \ldots$ & C8016C & 288 & 118.1 & 29.2 & 2.85 \\
\hline Palo Verde $3 \ldots \ldots \ldots \ldots \ldots$ & $\mathrm{C} 8016 \mathrm{C}$ & 284 & 115.9 & 27.2 & 2.79 \\
\hline Subtotal Peach Bottom $2 \ldots .$. & $\begin{array}{l}\text { G4607G2 } \\
\text { G4607G3A } \\
\text { G4608G4A } \\
\text { G4608G5 } \\
\text { G4608GP } \\
\text { G4608GB }\end{array}$ & $\begin{array}{r}168 \\
596 \\
360 \\
260 \\
552 \\
228 \\
2,164\end{array}$ & $\begin{array}{r}32.9 \\
111.7 \\
66.3 \\
47.5 \\
100.8 \\
41.7 \\
400.8\end{array}$ & $\begin{array}{r}9.5 \\
22.7 \\
25.5 \\
29.2 \\
28.0 \\
31.4\end{array}$ & $\begin{array}{l}1.10 \\
2.51 \\
2.74 \\
2.86 \\
2.89 \\
2.99\end{array}$ \\
\hline
\end{tabular}

See footnotes at end of table. 
Table B6. Assembly Type Summary by Reactor (Continued)

\begin{tabular}{|c|c|c|c|c|c|}
\hline Reactor Name & $\begin{array}{l}\text { Assembly } \\
\text { Type } \\
\text { Code }\end{array}$ & $\begin{array}{l}\text { Number of } \\
\text { Assemblles }\end{array}$ & $\begin{array}{l}\text { Inlital Uranium } \\
\text { Content } \\
\text { (MTU) }\end{array}$ & $\begin{array}{c}\text { Average } \\
\text { Burnup } \\
\text { (GWDt/MTU) }\end{array}$ & $\begin{array}{l}\text { Enrlchment } \\
\text { (welght } \\
\text { percent) }\end{array}$ \\
\hline $\begin{array}{l}\text { Peach Bottom } 3 \ldots \ldots \ldots \ldots \ldots \\
\text { Subtotal Peach Bottom } 3 \ldots \ldots\end{array}$ & $\begin{array}{l}\text { G4607G3B } \\
\text { G4608G4B } \\
\text { G4608G5 } \\
\text { G4608GP }\end{array}$ & $\begin{array}{r}764 \\
187 \\
252 \\
741 \\
1,944\end{array}$ & $\begin{array}{r}145.0 \\
34.9 \\
46.1 \\
135.1 \\
361.2\end{array}$ & $\begin{array}{l}20.1 \\
24.1 \\
29.7 \\
29.7\end{array}$ & $\begin{array}{l}2.19 \\
2.74 \\
2.83 \\
2.94\end{array}$ \\
\hline $\begin{array}{l}\text { Perry } 1 \ldots \ldots \ldots \ldots \ldots \ldots \ldots \\
\text { Subtotal Perry } 1 \ldots \ldots \ldots \ldots \ldots\end{array}$ & $\begin{array}{l}\text { G4608GB } \\
{ }^{8} \text { Temps }\end{array}$ & $\begin{array}{r}744 \\
4 \\
748\end{array}$ & $\begin{array}{r}137.1 \\
0.0 \\
137.1\end{array}$ & 17.4 & 1.88 \\
\hline $\begin{array}{l}\text { Pilgrim } 1, \ldots \ldots \ldots \ldots \ldots \ldots \ldots \\
\text { Subtotal Pllgrim } 1 \ldots \ldots \ldots \ldots \ldots\end{array}$ & $\begin{array}{l}\text { G2307G2B } \\
\text { G2308G4 } \\
\text { G2308GP }\end{array}$ & $\begin{array}{r}580 \\
580 \\
328 \\
1,488\end{array}$ & $\begin{array}{r}111.9 \\
106.7 \\
58.1 \\
276.7\end{array}$ & $\begin{array}{l}14.9 \\
20.7 \\
26.9\end{array}$ & $\begin{array}{l}2.19 \\
2.30 \\
2.73\end{array}$ \\
\hline $\begin{array}{l}\text { Point Beach } 1, \ldots \ldots \ldots \ldots \ldots \ldots \\
\text { Subtotal Point Beach } 1 . \ldots \ldots \ldots\end{array}$ & $\begin{array}{l}\text { W1414W } \\
\text { W1414WL } \\
\text { W1414WO }\end{array}$ & $\begin{array}{r}10 \\
517 \\
109 \\
636\end{array}$ & $\begin{array}{r}4.0 \\
206.0 \\
39.2 \\
249.2\end{array}$ & $\begin{array}{l}38.0 \\
31.3 \\
41.4\end{array}$ & $\begin{array}{l}3.21 \\
3.06 \\
3.45\end{array}$ \\
\hline $\begin{array}{l}\text { Point Beach } 2 \ldots \ldots \ldots \ldots \ldots \ldots \\
\text { Subtotal Point Beach 2. ....... }\end{array}$ & $\begin{array}{l}\text { W1414W } \\
\text { W1414WL } \\
\text { W1414WO }\end{array}$ & $\begin{array}{r}6 \\
412 \\
148 \\
566\end{array}$ & $\begin{array}{r}2.4 \\
164.5 \\
53.1 \\
220.0\end{array}$ & $\begin{array}{l}41.8 \\
33.3 \\
40.5\end{array}$ & $\begin{array}{l}3.20 \\
3.09 \\
3.42\end{array}$ \\
\hline $\begin{array}{l}\text { Prairie Island } 1 \ldots \ldots \ldots \ldots \ldots \ldots \\
\text { Subtotal Prairle Island } 1 \ldots \ldots \ldots\end{array}$ & $\begin{array}{l}\text { W1414WL } \\
\text { W1414WO } \\
\text { W1414A } \\
\text { W1414ATR }\end{array}$ & $\begin{array}{r}241 \\
149 \\
89 \\
145 \\
624\end{array}$ & $\begin{array}{r}95.9 \\
53.5 \\
33.6 \\
52.9 \\
235.8\end{array}$ & $\begin{array}{l}30.6 \\
40.3 \\
37.5 \\
37.7\end{array}$ & $\begin{array}{l}3.01 \\
3.60 \\
3.44 \\
3.33\end{array}$ \\
\hline $\begin{array}{l}\text { Prairie Island } 2 \ldots \ldots \ldots \ldots \ldots \ldots \\
\text { Subtotal Prairie Island } 2 \ldots \ldots \ldots\end{array}$ & $\begin{array}{l}\text { W1414WL } \\
\text { W1414WO } \\
\text { W1414A } \\
\text { W1414ATR }\end{array}$ & $\begin{array}{r}241 \\
149 \\
76 \\
143 \\
609\end{array}$ & $\begin{array}{r}96.8 \\
53.6 \\
28.8 \\
51.6 \\
230.7\end{array}$ & $\begin{array}{l}31.7 \\
39.4 \\
37.3 \\
38.6\end{array}$ & $\begin{array}{l}3.11 \\
3.57 \\
3.45 \\
3.43\end{array}$ \\
\hline Quad Citles $1 \ldots \ldots \ldots \ldots \ldots$ & $\begin{array}{l}\text { G2307G2B } \\
\text { G2307G3 } \\
\text { G2308G4 } \\
\text { G2308G5 } \\
\text { G2308GP } \\
\text { G2308GB }\end{array}$ & $\begin{array}{r}693 \\
28 \\
376 \\
188 \\
336 \\
432 \\
2,053\end{array}$ & $\begin{array}{r}133.5 \\
5.2 \\
69.2 \\
33.3 \\
59.5 \\
76.6 \\
377.3\end{array}$ & $\begin{array}{l}20.1 \\
29.0 \\
28.0 \\
30.1 \\
29.1 \\
31.5\end{array}$ & $\begin{array}{l}2.13 \\
2.24 \\
2.52 \\
2.63 \\
2.76 \\
2.75\end{array}$ \\
\hline Quad Cities $2 \ldots \ldots \ldots \ldots \ldots \ldots$ & $\begin{array}{l}\text { G2307G2B } \\
\text { G2308G4 } \\
\text { G2308GP } \\
\text { G2308GB }\end{array}$ & $\begin{array}{r}755 \\
492 \\
276 \\
420 \\
1,943\end{array}$ & $\begin{array}{r}144.8 \\
90.6 \\
48.8 \\
74.3 \\
358.6\end{array}$ & $\begin{array}{l}19.8 \\
28.2 \\
29.1 \\
31.5\end{array}$ & $\begin{array}{l}2.14 \\
2.55 \\
2.66 \\
2.73\end{array}$ \\
\hline $\begin{array}{l}\text { Rancho Seco. } \ldots \ldots \ldots \ldots \ldots \ldots \ldots \\
\text { Subtotal Rancho Seco } \ldots \ldots \ldots \ldots\end{array}$ & $\begin{array}{l}\mathrm{B} 151584 \\
\mathrm{~B} 1515 \mathrm{BZ}\end{array}$ & $\begin{array}{r}437 \\
56 \\
493\end{array}$ & $\begin{array}{r}202.5 \\
25.9 \\
228.4\end{array}$ & $\begin{array}{l}28.2 \\
10.0\end{array}$ & $\begin{array}{l}2.92 \\
3.06\end{array}$ \\
\hline River Bend 1 ................ & G4608GB & 764 & 141.6 & 20.8 & 2.07 \\
\hline $\begin{array}{l}\text { Robinson } 2 \ldots \ldots \ldots \ldots \ldots \ldots \ldots \\
\text { Subtotal Robinson } 2 \ldots \ldots \ldots \ldots\end{array}$ & $\begin{array}{l}\text { W1515WL } \\
\text { W1515A }\end{array}$ & $\begin{array}{l}314 \\
454 \\
768\end{array}$ & $\begin{array}{l}141.5 \\
195.1 \\
336.6\end{array}$ & $\begin{array}{l}23.7 \\
33.5\end{array}$ & $\begin{array}{l}2.63 \\
3.03\end{array}$ \\
\hline
\end{tabular}

See footnotes at end of table. 
Table B6. Assembly Type Summary by Reactor (Continued)

\begin{tabular}{|c|c|c|c|c|c|}
\hline Reactor Name & $\begin{array}{l}\text { Assembly } \\
\text { Type } \\
\text { Code }\end{array}$ & $\begin{array}{l}\text { Number of } \\
\text { Assemblles }\end{array}$ & $\begin{array}{l}\text { Initial Uranium } \\
\text { Content } \\
\text { (MTU) }\end{array}$ & $\begin{array}{c}\text { Average } \\
\text { Burnup } \\
\text { (GWDt/MTU) }\end{array}$ & $\begin{array}{l}\text { Enrichment } \\
\text { (welght } \\
\text { percent) }\end{array}$ \\
\hline $\begin{array}{l}\text { St. Lucie } 1 \ldots \ldots \ldots \ldots \ldots \ldots \ldots \ldots \\
\text { Subtotal St. Lucle } 1 . \ldots \ldots \ldots \ldots\end{array}$ & $\begin{array}{l}C 1414 C \\
C 1414 A\end{array}$ & $\begin{array}{l}497 \\
299 \\
796\end{array}$ & $\begin{array}{l}189.6 \\
110.7 \\
300.3\end{array}$ & $\begin{array}{l}28.9 \\
37.1\end{array}$ & $\begin{array}{l}2.81 \\
3.47\end{array}$ \\
\hline St, Lucle $2, \ldots \ldots \ldots \ldots \ldots \ldots \ldots$ & XSL16C & 464 & 175.7 & 32.1 & 2.93 \\
\hline $\begin{array}{l}\text { Salem } 1 \ldots \ldots \ldots \ldots \ldots \ldots \ldots \ldots \\
\text { Subtotal Salem } 1 \ldots \ldots \ldots \ldots \ldots\end{array}$ & $\begin{array}{l}\text { W1717WL } \\
\text { W1717WO } \\
\text { W1717WVH } \\
\text { aTemps }\end{array}$ & $\begin{array}{r}611 \\
2 \\
19 \\
24 \\
656\end{array}$ & $\begin{array}{r}281.5 \\
0.8 \\
8.8 \\
b_{0.0} \\
291.2\end{array}$ & $\begin{array}{l}32.5 \\
34.7 \\
33.2\end{array}$ & $\begin{array}{l}3.23 \\
2.79 \\
3.66\end{array}$ \\
\hline Salem $2 \ldots \ldots \ldots \ldots \ldots \ldots$ & W1717WL & 436 & 200.9 & 31.3 & 3.21 \\
\hline San Onofre $1 . \ldots \ldots \ldots \ldots \ldots \ldots$ & XSO14W & 665 & 244.3 & 27.3 & 3.86 \\
\hline San Onofre $2 . . . \ldots \ldots \ldots \ldots \ldots$ & C1616CSD & 484 & 200.4 & 30.4 & 3.10 \\
\hline San Onofre $3 . . . \ldots \ldots \ldots \ldots \ldots$ & C1616CSD & 484 & 200.3 & 30.6 & 3.11 \\
\hline 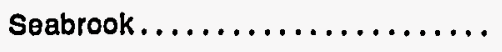 & W1717WL & 136 & 63.0 & 20.2 & 2.06 \\
\hline $\begin{array}{l}\text { Sequoyah } 1 . \ldots \ldots \ldots \ldots \ldots \ldots \ldots \\
\text { Subtotal Sequoyah } 1 . \ldots \ldots \ldots \ldots\end{array}$ & $\begin{array}{l}\text { W1717WL } \\
\text { W1717WVH }\end{array}$ & $\begin{array}{r}349 \\
1 \\
350\end{array}$ & $\begin{array}{r}160.6 \\
0.5 \\
161.1\end{array}$ & $\begin{array}{l}31.6 \\
20.0\end{array}$ & $\begin{array}{l}3.06 \\
3.55\end{array}$ \\
\hline Sequoyah $2 \ldots \ldots \ldots \ldots \ldots \ldots$ & W1717WL & 387 & 178.2 & 31.6 & 3.06 \\
\hline Shoreham ................... & G4608GP & 560 & 102.5 & 0.5 & 1.88 \\
\hline $\begin{array}{l}\text { South Texas } 1 \ldots \ldots \ldots \ldots \ldots \ldots \\
\text { Subtotal South Texas } 1 \ldots \ldots \ldots\end{array}$ & $\begin{array}{l}\text { WST17W } \\
\text { aTemps }\end{array}$ & $\begin{array}{r}233 \\
3 \\
236\end{array}$ & $\begin{array}{r}126.2 \\
0.0 \\
126.2\end{array}$ & 21.3 & 2.37 \\
\hline South Texas $2, \ldots . . . . . . . . .$. & WST17W & 116 & 62.6 & 19.8 & 2.32 \\
\hline 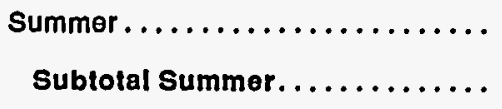 & $\begin{array}{l}\text { W1717WL } \\
\text { W1717WV5 }\end{array}$ & $\begin{array}{r}329 \\
43 \\
372\end{array}$ & $\begin{array}{r}151.5 \\
18.2 \\
169.7\end{array}$ & $\begin{array}{l}31.6 \\
37.7\end{array}$ & $\begin{array}{l}3.16 \\
3.52\end{array}$ \\
\hline surry $1, \ldots \ldots \ldots \ldots \ldots \ldots$ & $\begin{array}{l}\text { W1515W } \\
\text { W1515WL } \\
\text { W1515WO } \\
\text { W1717WL }\end{array}$ & $\begin{array}{r}614 \\
57 \\
8 \\
2 \\
681\end{array}$ & $\begin{array}{r}279.5 \\
26.2 \\
3.7 \\
0.9 \\
310.3\end{array}$ & $\begin{array}{l}29.6 \\
43.6 \\
37.0 \\
16.1\end{array}$ & $\begin{array}{l}2.98 \\
3.64 \\
3.80 \\
1.86\end{array}$ \\
\hline $\begin{array}{l}\text { Surry } 2 \ldots \ldots \ldots \ldots \ldots \ldots \ldots \ldots \\
\text { Subtotal Surry } 2, \ldots \ldots \ldots \ldots \ldots\end{array}$ & $\begin{array}{l}\text { W1515W } \\
\text { W1515WL } \\
\text { W1717WL }\end{array}$ & $\begin{array}{r}570 \\
20 \\
2 \\
592\end{array}$ & $\begin{array}{r}259.6 \\
9.2 \\
0.9 \\
269.8\end{array}$ & $\begin{array}{l}31.3 \\
36.0 \\
35.3\end{array}$ & $\begin{array}{l}3.12 \\
3.80 \\
3.10\end{array}$ \\
\hline Susquehanna $1 \ldots \ldots \ldots \ldots \ldots \ldots$ & $\begin{array}{l}\text { G4608GP } \\
\text { G4608A } \\
\text { G4609A }\end{array}$ & $\begin{array}{r}764 \\
488 \\
152 \\
1,404\end{array}$ & $\begin{array}{r}140.2 \\
85.8 \\
26.3 \\
252.3\end{array}$ & $\begin{array}{l}18.3 \\
30.4 \\
36.0\end{array}$ & $\begin{array}{l}1.88 \\
2.83 \\
3.31\end{array}$ \\
\hline $\begin{array}{l}\text { Susquehanna } 2, \ldots \ldots \ldots \ldots \ldots \ldots \\
\text { Subtotal Susquehanna } 2 \ldots \ldots \ldots\end{array}$ & $\begin{array}{l}\text { G4608GP } \\
\text { G4609A }\end{array}$ & $\begin{array}{r}764 \\
472 \\
1,236\end{array}$ & $\begin{array}{r}140.1 \\
81.7 \\
221.8\end{array}$ & $\begin{array}{l}18.9 \\
34.7\end{array}$ & $\begin{array}{l}1.89 \\
3.29\end{array}$ \\
\hline
\end{tabular}

See footnotes at end of table. 
Table B6. Assembly Type Summary by Reactor (Continued)

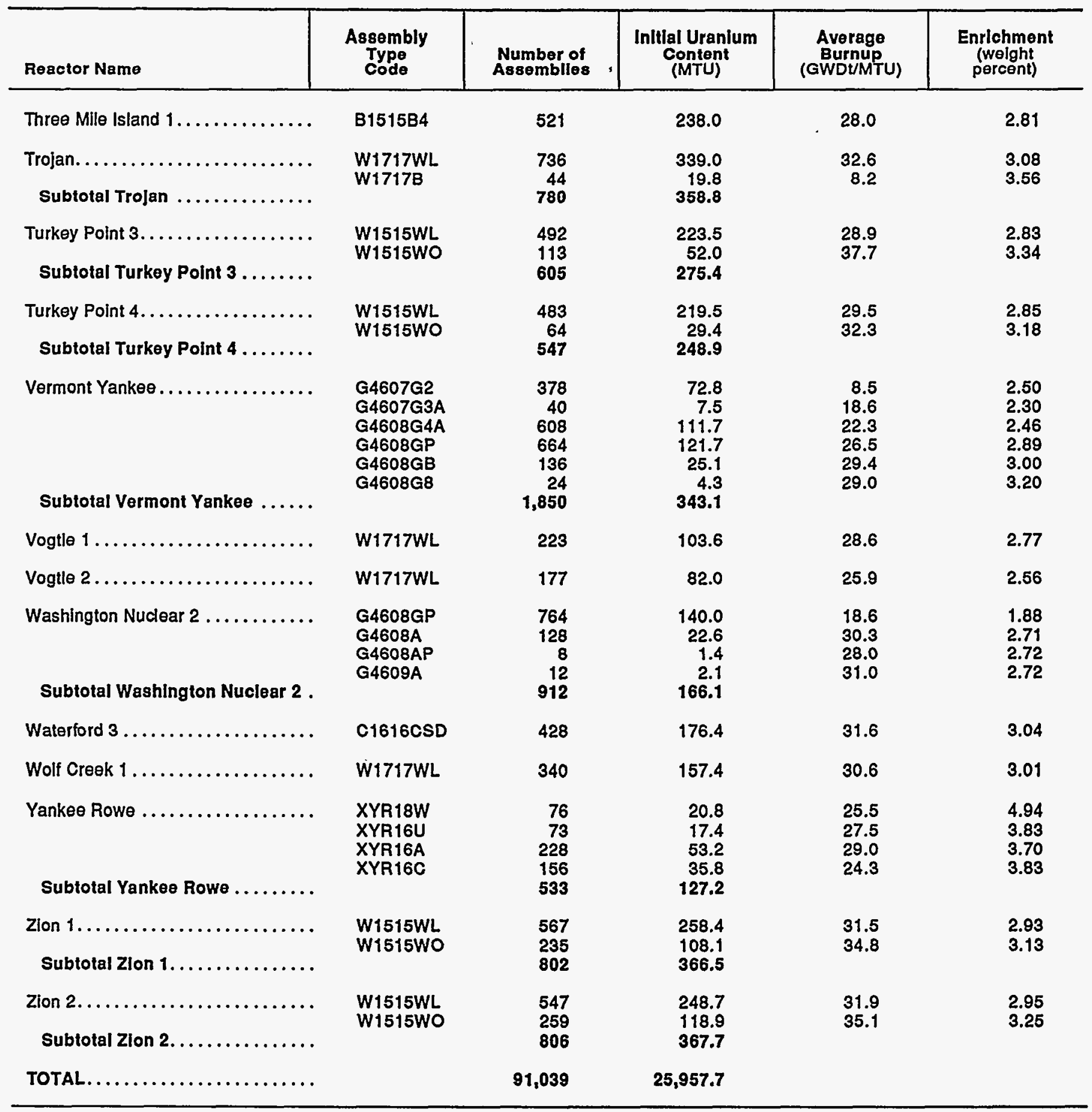

${ }^{a}$ Temps represent temporarily discharged assemblies with no assigned assembly type.

No weight reported for temporarily discharged assemblies with no assigned assembly type.

MTU = Metric tons of uranium; GWDVMTU = Gigawattdays thermal per metric ton of uranium.

Note: Totals may not equal sum of components because of independent rounding. See Technical Note 11 in Appendix E.

Source: Energy Information Administration, Form RW-859, "Nuclear Fuel Data" (1992). 
Table B7. Assembly Type Summary by Assembly Type

\begin{tabular}{|c|c|c|c|c|c|}
\hline $\begin{array}{l}\text { B1515B4 } \ldots \ldots \ldots \ldots \ldots \ldots \\
\text { Subtotal B1515B4 } \ldots \ldots \ldots\end{array}$ & $\begin{array}{l}\text { Arkansas Nuclear } 1 \\
\text { Crystal Rlver } 3 \\
\text { Davis-Besse } \\
\text { Oconee } 1 \\
\text { Oconee } 2 \\
\text { Oconee } 3 \\
\text { Rancho Seco } \\
\text { Three Mile Island } 1\end{array}$ & $\begin{array}{r}624 \\
496 \\
386 \\
569 \\
568 \\
505 \\
437 \\
521 \\
4,106\end{array}$ & $\begin{array}{r}289.1 \\
230.3 \\
181.5 \\
264.5 \\
263.5 \\
233.9 \\
202.5 \\
238.0 \\
1,903.3\end{array}$ & $\begin{array}{l}30.8 \\
26.7 \\
30.0 \\
26.7 \\
29.1 \\
28.3 \\
28.2 \\
28.0\end{array}$ & $\begin{array}{l}2.97 \\
2.77 \\
2.82 \\
2.75 \\
2.89 \\
2.83 \\
2.92 \\
2.81\end{array}$ \\
\hline B1515B4Z ............. & Oconee 1 & 4 & 1.9 & 42.0 & 3.30 \\
\hline B1515B7 $\ldots \ldots \ldots \ldots \ldots \ldots$ & Oconee 3 & 43 & 19.9 & 41.0 & 3.50 \\
\hline Subtotal B1515BZ ........ & $\begin{array}{l}\text { Crystal Rlver } 3 \\
\text { Oconee } 1 \\
\text { Oconee } 2 \\
\text { Oconee } 3 \\
\text { Rancho Seco }\end{array}$ & $\begin{array}{r}40 \\
206 \\
91 \\
187 \\
56 \\
580\end{array}$ & $\begin{array}{r}18.5 \\
95.5 \\
42.2 \\
86.7 \\
25.9 \\
268.8\end{array}$ & $\begin{array}{l}38.0 \\
37.9 \\
36.2 \\
34.1 \\
10.0\end{array}$ & $\begin{array}{l}3.85 \\
3.33 \\
3.22 \\
3.24 \\
3.06\end{array}$ \\
\hline B1515BGD $\ldots \ldots \ldots \ldots \ldots$ & Oconee 1 & 4 & 1.7 & 43.0 & 3.92 \\
\hline $\begin{array}{l}\text { C1414A } \ldots \ldots \ldots \ldots \ldots \ldots \ldots \\
\text { subtotal C1414A } \ldots \ldots \ldots \ldots\end{array}$ & $\begin{array}{l}\text { Maine Yankee } \\
\text { St. Lucle } 1\end{array}$ & $\begin{array}{l}223 \\
299 \\
522\end{array}$ & $\begin{array}{r}82.5 \\
110.7 \\
193.2\end{array}$ & $\begin{array}{l}36.8 \\
37.1\end{array}$ & $\begin{array}{l}3.20 \\
3.47\end{array}$ \\
\hline C1414W................. & Millstone 2 & 423 & 172.3 & 32.9 & 3.05 \\
\hline C1616CsD $\ldots \ldots \ldots \ldots \ldots$ & $\begin{array}{l}\text { Arkansas Nuclear } 2 \\
\text { San Onofre } 2 \\
\text { San Onofre } 3 \\
\text { Waterford } 3\end{array}$ & $\begin{array}{r}564 \\
484 \\
484 \\
428 \\
1,960\end{array}$ & $\begin{array}{l}234.5 \\
200.4 \\
200.3 \\
176.4 \\
811.6\end{array}$ & $\begin{array}{l}34.7 \\
30.4 \\
30.6 \\
31.6\end{array}$ & $\begin{array}{l}3.25 \\
3.10 \\
3.11 \\
3.04\end{array}$ \\
\hline Subtotal C8016C ......... & $\begin{array}{l}\text { Palo Verde } 1 \\
\text { Palo Verde } 2 \\
\text { Palo Verde } 3\end{array}$ & $\begin{array}{l}276 \\
288 \\
284 \\
848\end{array}$ & $\begin{array}{l}113.6 \\
118.1 \\
115.9 \\
347.7\end{array}$ & $\begin{array}{l}28.1 \\
29.2 \\
27.2\end{array}$ & $\begin{array}{l}2.73 \\
2.85 \\
2.79\end{array}$ \\
\hline Subtotal G2307G2A. ....... & $\begin{array}{l}\text { Millstone } 1 \\
\text { Nine Mile Point } 1 \\
\text { Oyster Creek }\end{array}$ & $\begin{array}{r}580 \\
532 \\
560 \\
1,672\end{array}$ & $\begin{array}{l}113.4 \\
103.2 \\
109.3 \\
325.9\end{array}$ & $\begin{array}{l}16.8 \\
16.2 \\
17.3\end{array}$ & $\begin{array}{l}2.08 \\
2.11 \\
2.11\end{array}$ \\
\hline
\end{tabular}

See footnotes at end of table. 
Table B7. Assembly Type Summary by Assembly Type (Continued)

\begin{tabular}{|c|c|c|c|c|c|}
\hline Assembly Type Code & Reactor Name & $\begin{array}{l}\text { Number of } \\
\text { Assemblles }\end{array}$ & $\begin{array}{l}\text { Initial Uranium } \\
\text { Content } \\
\text { (MTU) }\end{array}$ & $\begin{array}{c}\text { Average } \\
\text { Burnup } \\
\text { (GWDt/MTU) }\end{array}$ & $\begin{array}{c}\text { Enrlchment } \\
\text { (weight } \\
\text { percent) }\end{array}$ \\
\hline $\begin{array}{l}\text { G2307G3 } \ldots \ldots \ldots \ldots \ldots \ldots \\
\text { Subtotal G2307G3 } \ldots \ldots \ldots .\end{array}$ & $\begin{array}{l}\text { Dresden } 2 \\
\text { Dresden } 3 \\
\text { Millstone } 1 \\
\text { Monticello } \\
\text { Nine Mile Point } 1 \\
\text { Quad Cities } 1\end{array}$ & $\begin{array}{r}32 \\
52 \\
154 \\
20 \\
108 \\
28 \\
394\end{array}$ & $\begin{array}{r}6.0 \\
9.8 \\
28.9 \\
3.7 \\
20.2 \\
5.2 \\
73.9\end{array}$ & $\begin{array}{l}23.7 \\
24.0 \\
24.1 \\
23.8 \\
26.0 \\
29.0\end{array}$ & $\begin{array}{l}2.29 \\
2.30 \\
2.45 \\
2.30 \\
2.50 \\
2.24\end{array}$ \\
\hline Subtotal G2308G4 ........ & $\begin{array}{l}\text { Dresden } 2 \\
\text { Dresden } 3 \\
\text { Millstone } 1 \\
\text { Monticello } \\
\text { Nine Mile Point } 1 \\
\text { Pilgrim 1 } \\
\text { Quad Citles } 1 \\
\text { Quad Cltles } 2\end{array}$ & $\begin{array}{r}476 \\
508 \\
392 \\
596 \\
456 \\
580 \\
376 \\
492 \\
3,876\end{array}$ & $\begin{array}{r}87.8 \\
93.4 \\
72.2 \\
109.8 \\
83.9 \\
106.7 \\
69.2 \\
90.6 \\
713.7\end{array}$ & $\begin{array}{l}25.1 \\
25.7 \\
26.9 \\
24.6 \\
25.7 \\
20.7 \\
28.0 \\
28.2\end{array}$ & $\begin{array}{l}2.52 \\
2.56 \\
2.69 \\
2.41 \\
2.60 \\
2.30 \\
2.52 \\
2.55\end{array}$ \\
\hline Subtotal G2308G5 ........ & $\begin{array}{l}\text { Dresden } 2 \\
\text { Millstone } 1 \\
\text { Monticello } \\
\text { Nine Mile Point } 1 \\
\text { Oyster Creek } \\
\text { Quad Cities } 1\end{array}$ & $\begin{array}{r}160 \\
148 \\
112 \\
184 \\
87 \\
188 \\
879\end{array}$ & $\begin{array}{r}28.3 \\
26.2 \\
19.8 \\
32.5 \\
15.4 \\
33.3 \\
155.5\end{array}$ & $\begin{array}{l}28.7 \\
26.4 \\
30.8 \\
27.9 \\
26.0 \\
30.1\end{array}$ & $\begin{array}{l}2.67 \\
2.66 \\
2.74 \\
2.77 \\
2.39 \\
2.63\end{array}$ \\
\hline Subtotal G2308GB....... & $\begin{array}{l}\text { Millstone } 1 \\
\text { Oyster Creek } \\
\text { Quad Cities } 1 \\
\text { Quad Cities } 2\end{array}$ & $\begin{array}{r}212 \\
40 \\
432 \\
420 \\
1,104\end{array}$ & $\begin{array}{r}37.7 \\
7.1 \\
76.6 \\
74.3 \\
195.7\end{array}$ & $\begin{array}{l}27.8 \\
25.6 \\
31.5 \\
31.5\end{array}$ & $\begin{array}{l}3.00 \\
2.68 \\
2.75 \\
2.73\end{array}$ \\
\hline $\begin{array}{l}\text { G2308G8A. . . . . . . . . . } \\
\text { Subtotal G2308G8A. . . . }\end{array}$ & $\begin{array}{l}\text { Millstone } 1 \\
\text { Monticello }\end{array}$ & $\begin{array}{r}188 \\
24 \\
212\end{array}$ & $\begin{array}{r}33.5 \\
4.3 \\
37.8\end{array}$ & $\begin{array}{l}32.0 \\
34.5\end{array}$ & $\begin{array}{l}2.98 \\
2.98\end{array}$ \\
\hline G2307A ................ & Oyster Creek & 260 & 47.4 & 23.5 & 2.64 \\
\hline
\end{tabular}

See footnotes at end of table. 
Table B7. Assembly Type Summary by Assembly Type (Continued)

\begin{tabular}{|c|c|c|c|c|c|}
\hline Assembly Type Code & Reactor Name & $\begin{array}{l}\text { Number of } \\
\text { Assomblles }\end{array}$ & $\begin{array}{l}\text { Initial Uranium } \\
\text { Content } \\
\text { (MTU) }\end{array}$ & $\begin{array}{c}\text { Average } \\
\text { Burnup } \\
\text { (GWDU/MTU) }\end{array}$ & $\begin{array}{c}\text { Enrlchment } \\
\text { (weight } \\
\text { percent) }\end{array}$ \\
\hline $\begin{array}{l}\text { G2308A } \ldots \ldots \ldots \ldots \ldots \ldots \ldots \\
\text { Subtotal Q2308A } \ldots \ldots \ldots \ldots\end{array}$ & $\begin{array}{l}\text { Dresden } 2 \\
\text { Dresden } 3 \\
\text { Oyster Creok }\end{array}$ & $\begin{array}{r}228 \\
380 \\
617 \\
1,225\end{array}$ & $\begin{array}{r}39.5 \\
66.0 \\
108.0 \\
213.5\end{array}$ & $\begin{array}{l}30.8 \\
31.9 \\
25.0\end{array}$ & $\begin{array}{l}2.82 \\
2.74 \\
2.50\end{array}$ \\
\hline $\begin{array}{c}\text { G4607G2............. } \\
\text { Subtotal G4607G2 ....... }\end{array}$ & $\begin{array}{l}\text { Browns Ferry } 1 \\
\text { Browns Ferry } 2 \\
\text { Cooper Station } \\
\text { Fitzpatrick } \\
\text { Peach Bottom } 2 \\
\text { Vermont Yankeo }\end{array}$ & $\begin{array}{r}168 \\
168 \\
128 \\
132 \\
168 \\
378 \\
1,142\end{array}$ & $\begin{array}{r}32.9 \\
32.9 \\
25.1 \\
25.8 \\
32.9 \\
72.8 \\
222.4\end{array}$ & $\begin{array}{r}10.4 \\
9.8 \\
10.1 \\
9.1 \\
9.5 \\
8.5\end{array}$ & $\begin{array}{l}1.11 \\
1.10 \\
1.09 \\
1.11 \\
1.10 \\
2.50\end{array}$ \\
\hline $\begin{array}{l}\text { G4607G3B. } \ldots \ldots \ldots \ldots \ldots \ldots \\
\text { subtolal G4807G3B...... }\end{array}$ & $\begin{array}{l}\text { Cooper Station } \\
\text { Peach Bottom } 3\end{array}$ & $\begin{array}{r}420 \\
764 \\
1,184\end{array}$ & $\begin{array}{r}79.8 \\
145.0 \\
224.9\end{array}$ & $\begin{array}{l}25.2 \\
20.1\end{array}$ & $\begin{array}{l}2.51 \\
2.19\end{array}$ \\
\hline G4608G4A. ............. & $\begin{array}{l}\text { Browns Ferry } 1 \\
\text { Browns Ferry } 2 \\
\text { Cooper Station } \\
\text { Duane Arnold } \\
\text { Fltzpatrick } \\
\text { Hatch } 1 \\
\text { Peach Bottom } 2 \\
\text { Vermont Yankee }\end{array}$ & $\begin{array}{r}168 \\
168 \\
72 \\
185 \\
132 \\
92 \\
360 \\
608 \\
1,785\end{array}$ & $\begin{array}{r}30.9 \\
30.9 \\
13.2 \\
34.1 \\
24.3 \\
16.9 \\
66.3 \\
111.7 \\
328.3\end{array}$ & $\begin{array}{l}26.5 \\
28.2 \\
25.0 \\
26.3 \\
26.0 \\
23.1 \\
25.5 \\
22.3\end{array}$ & $\begin{array}{l}2.73 \\
2.73 \\
2.51 \\
2.74 \\
2.74 \\
2.51 \\
2.74 \\
2.46\end{array}$ \\
\hline G4608G4B, ............ & $\begin{array}{l}\text { Browns Ferry } 3 \\
\text { Brunswick } 1 \\
\text { Brunswick } 2 \\
\text { Cooper Station } \\
\text { Peach Bottom } 3\end{array}$ & $\begin{array}{r}764 \\
560 \\
140 \\
136 \\
187 \\
1,787\end{array}$ & $\begin{array}{r}142.8 \\
104.5 \\
26.1 \\
25.4 \\
34.9 \\
333.7\end{array}$ & $\begin{array}{l}20.7 \\
18.3 \\
24.8 \\
30.5 \\
24.1\end{array}$ & $\begin{array}{l}2.20 \\
2.11 \\
2.74 \\
2.73 \\
2.74\end{array}$ \\
\hline Subtotal G4608G5 ........ & $\begin{array}{l}\text { Browns Ferry } 1 \\
\text { Browns Ferry } 2 \\
\text { Browns Ferry } 3 \\
\text { Brunswick } 1 \\
\text { Brunswick } 2 \\
\text { Cooper Station } \\
\text { Fitzpatrick } \\
\text { Hatch } 1 \\
\text { Hatch } 2 \\
\text { LaSalle County } 1 \\
\text { LaSalle County } 2 \\
\text { Peach Bottom } 2 \\
\text { Peach Bottom } 3\end{array}$ & $\begin{array}{r}156 \\
162 \\
208 \\
360 \\
300 \\
192 \\
136 \\
384 \\
509 \\
627 \\
764 \\
260 \\
252 \\
4,310\end{array}$ & $\begin{array}{r}28.6 \\
29.5 \\
38.1 \\
65.9 \\
55.0 \\
35.1 \\
24.8 \\
70.3 \\
93.4 \\
114.9 \\
139.6 \\
47.5 \\
46.1 \\
788.7\end{array}$ & $\begin{array}{l}28.1 \\
27.7 \\
28.0 \\
28.9 \\
26.4 \\
30.8 \\
26.8 \\
22.9 \\
19.2 \\
17.9 \\
19.7 \\
29.2 \\
29.7\end{array}$ & $\begin{array}{l}2.66 \\
2.88 \\
2.65 \\
2.78 \\
2.83 \\
2.82 \\
2.78 \\
2.43 \\
1.97 \\
1.81 \\
1.89 \\
2.86 \\
2.83\end{array}$ \\
\hline
\end{tabular}

See footnotes at end of table. 
Table B7. Assembly Type Summary by Assembly Type (Continued)

\begin{tabular}{|c|c|c|c|c|c|}
\hline Assembly Type Code & Reactor Name & $\begin{array}{l}\text { Number of } \\
\text { Assemblies }\end{array}$ & $\begin{array}{l}\text { Initlal Uranium } \\
\text { Content } \\
\text { (MTU) }\end{array}$ & $\begin{array}{c}\text { Average } \\
\text { Burnup } \\
\text { (GWDtMTU) }\end{array}$ & $\begin{array}{c}\text { Enrlchment } \\
\text { (weight } \\
\text { percent) }\end{array}$ \\
\hline G4608GB.............. & $\begin{array}{l}\text { Brunswick } 1 \\
\text { Brunswick } 2 \\
\text { Cooper Station } \\
\text { Duane Arnold } \\
\text { Fitzpatrick } \\
\text { Hatch } 1 \\
\text { Hatch } 2 \\
\text { Hope Creek } \\
\text { LaSalle County } 1 \\
\text { LaSalle County } 2 \\
\text { Limerick } 1 \\
\text { Limerick } 2 \\
\text { Nine Mile Point } 2 \\
\text { Peach Bottom } 2 \\
\text { Perry } 1 \\
\text { River Bend } 1 \\
\text { Vermont Yankee }\end{array}$ & $\begin{array}{r}143 \\
148 \\
92 \\
119 \\
196 \\
506 \\
585 \\
916 \\
201 \\
136 \\
1,012 \\
224 \\
112 \\
228 \\
744 \\
764 \\
136 \\
6,262\end{array}$ & $\begin{array}{r}26.4 \\
27.3 \\
17.0 \\
21.9 \\
36.1 \\
93.3 \\
108.2 \\
169.5 \\
37.2 \\
25.3 \\
187.2 \\
41.7 \\
20.7 \\
41.7 \\
137.1 \\
141.6 \\
25.1 \\
1,157.1\end{array}$ & $\begin{array}{r}33.8 \\
33.0 \\
31.2 \\
28.0 \\
29.8 \\
25.5 \\
27.0 \\
22.7 \\
33.5 \\
33.0 \\
18.0 \\
5.9 \\
22.0 \\
31.4 \\
17.4 \\
20.8 \\
29.4\end{array}$ & $\begin{array}{l}2.99 \\
2.99 \\
2.79 \\
3.01 \\
2.99 \\
2.69 \\
2.74 \\
2.27 \\
3.04 \\
3.00 \\
2.22 \\
0.85 \\
2.19 \\
2.99 \\
1.88 \\
2.07 \\
3.00\end{array}$ \\
\hline $\begin{array}{l}\text { G4608G8............... } \\
\text { Subtotal G4608G8 } \ldots \ldots \ldots \ldots\end{array}$ & $\begin{array}{l}\text { Duane Arnold } \\
\text { Fitzpatrick } \\
\text { Vermont Yankee }\end{array}$ & $\begin{array}{r}88 \\
104 \\
24 \\
216\end{array}$ & $\begin{array}{r}15.7 \\
18.5 \\
4.3 \\
38.5\end{array}$ & $\begin{array}{l}33.7 \\
32.0 \\
29.0\end{array}$ & $\begin{array}{l}3.01 \\
3.19 \\
3.20\end{array}$ \\
\hline $\begin{array}{l}\text { G4608G9. } \ldots \ldots \ldots \ldots \ldots \ldots \\
\text { Subtotal G4608G9 } \ldots \ldots \ldots\end{array}$ & $\begin{array}{l}\text { Brunswick } 2 \\
\text { Hatch } 2\end{array}$ & $\begin{array}{l}1 \\
1 \\
2\end{array}$ & $\begin{array}{l}0.2 \\
0.2 \\
0.4\end{array}$ & $\begin{array}{l}18.0 \\
14.0\end{array}$ & $\begin{array}{l}3.29 \\
3.14\end{array}$ \\
\hline G4608A $\ldots \ldots \ldots \ldots \ldots$ & $\begin{array}{l}\text { Grand Gulf } 1 \\
\text { Susquehanna } 1 \\
\text { Washington Nuclear } 2\end{array}$ & $\begin{array}{r}584 \\
488 \\
128 \\
1,200\end{array}$ & $\begin{array}{r}103.0 \\
85.8 \\
22.6 \\
211.3\end{array}$ & $\begin{array}{l}30.3 \\
30.4 \\
30.3\end{array}$ & $\begin{array}{l}2.94 \\
2.83 \\
2.71\end{array}$ \\
\hline G4608AP $\ldots \ldots \ldots \ldots \ldots \ldots$ & Washington Nuclear 2 & 8 & 1.4 & 28.0 & 2.72 \\
\hline
\end{tabular}

See footnotes at end of table. 
Table B7. Assembly Type Summary by Assembly Type (Continued)

\begin{tabular}{|c|c|c|c|c|c|}
\hline Assembly Type Code & Reactor Name & $\begin{array}{l}\text { Number of } \\
\text { Assemblles }\end{array}$ & $\begin{array}{l}\text { Initial Uranlum } \\
\text { Content } \\
\text { (MTU) }\end{array}$ & $\begin{array}{c}\text { Average } \\
\text { Burnup } \\
\text { (GWDUMTU) }\end{array}$ & $\begin{array}{l}\text { Enrlchment } \\
\text { (weight } \\
\text { percent) }\end{array}$ \\
\hline G4608W................. & Fitzpatrick & 4 & 0.6 & 23.0 & 3.01 \\
\hline $\begin{array}{l}\text { G4608A } \ldots \ldots \ldots \ldots \ldots \ldots \ldots \\
\text { Subtotal G4608A } \ldots \ldots \ldots \ldots\end{array}$ & $\begin{array}{l}\text { Hatch } 2 \\
\text { Susquehanna } 1 \\
\text { Susquehanna } 2 \\
\text { Washington Nuclear } 2\end{array}$ & $\begin{array}{r}4 \\
152 \\
472 \\
12 \\
640\end{array}$ & $\begin{array}{r}0.7 \\
26.3 \\
81.7 \\
2.1 \\
110.8\end{array}$ & $\begin{array}{l}36.0 \\
36.0 \\
34.7 \\
31.0\end{array}$ & $\begin{array}{l}2.79 \\
3.31 \\
3.29 \\
2.72\end{array}$ \\
\hline $\begin{array}{l}\text { W1414W } \ldots \ldots \ldots \ldots \ldots \ldots \\
\text { Subtotal W1414W......... }\end{array}$ & $\begin{array}{l}\text { Ginna } \\
\text { Kowaunee } \\
\text { Point Beach } 1 \\
\text { Point Beach } 2\end{array}$ & $\begin{array}{r}362 \\
241 \\
10 \\
6 \\
619\end{array}$ & $\begin{array}{r}141.8 \\
95.7 \\
4.0 \\
2.4 \\
243.9\end{array}$ & $\begin{array}{l}24.4 \\
31.7 \\
38.0 \\
41.8\end{array}$ & $\begin{array}{l}3.03 \\
3.06 \\
3.21 \\
3.20\end{array}$ \\
\hline $\begin{array}{l}\text { W1414WL } \ldots \ldots \ldots \ldots \ldots \ldots \\
\text { Subtotal W1414WL } \ldots \ldots \ldots\end{array}$ & $\begin{array}{l}\text { Point Beach } 1 \\
\text { Point Beach } 2 \\
\text { Praírie Island } 1 \\
\text { Prairio Island } 2\end{array}$ & $\begin{array}{r}517 \\
412 \\
241 \\
241 \\
1,411\end{array}$ & $\begin{array}{r}206.0 \\
164.5 \\
95.9 \\
96.8 \\
563.1\end{array}$ & $\begin{array}{l}31.3 \\
33.3 \\
30.6 \\
31.7\end{array}$ & $\begin{array}{l}3.06 \\
3.09 \\
3.01 \\
3.11\end{array}$ \\
\hline W1414wo $\ldots \ldots \ldots \ldots \ldots$ & $\begin{array}{l}\text { Ginna } \\
\text { Point Beach } 1 \\
\text { Point Beach } 2 \\
\text { Prairie Island } 1 \\
\text { Prairie Island } 2\end{array}$ & $\begin{array}{l}143 \\
109 \\
148 \\
149 \\
149 \\
698\end{array}$ & $\begin{array}{r}50.4 \\
39.2 \\
53.1 \\
53.5 \\
53.6 \\
249.8\end{array}$ & $\begin{array}{l}39.6 \\
41.4 \\
40.5 \\
40.3 \\
39.4\end{array}$ & $\begin{array}{l}3.49 \\
3.45 \\
3.42 \\
3.60 \\
3.57\end{array}$ \\
\hline $\begin{array}{l}\text { W1414A................ } \\
\text { Subtotal W1414A........ }\end{array}$ & $\begin{array}{l}\text { Ginna } \\
\text { Kewaunee } \\
\text { Prairle Island } 1 \\
\text { Prairle Island } 2\end{array}$ & $\begin{array}{r}194 \\
363 \\
89 \\
76 \\
722\end{array}$ & $\begin{array}{r}72.3 \\
137.4 \\
33.6 \\
28.8 \\
272.0\end{array}$ & $\begin{array}{l}37.1 \\
35.2 \\
37.5 \\
37.3\end{array}$ & $\begin{array}{l}3.22 \\
3.27 \\
3.44 \\
3.45\end{array}$ \\
\hline $\begin{array}{l}\text { W1414ATR ............. } \\
\text { Subtotal W1414ATR ...... }\end{array}$ & $\begin{array}{l}\text { Pralrie Island } 1 \\
\text { Prairie Island } 2\end{array}$ & $\begin{array}{l}145 \\
143 \\
288\end{array}$ & $\begin{array}{r}52.9 \\
51.6 \\
104.5\end{array}$ & $\begin{array}{l}37.7 \\
38.6\end{array}$ & $\begin{array}{l}3.33 \\
3.43\end{array}$ \\
\hline$w 14148 \ldots \ldots \ldots \ldots \ldots$ & Ginna & 2 & 0.8 & 24.4 & 3.22 \\
\hline $\begin{array}{l}\text { W1515W } \ldots \ldots \ldots \ldots \ldots \ldots \\
\text { subtotal W1515W. . . . } \ldots \ldots\end{array}$ & $\begin{array}{l}\text { Indian Point } 2 \\
\text { Surry } 1 \\
\text { Surry } 2\end{array}$ & $\begin{array}{r}393 \\
614 \\
570 \\
1,577\end{array}$ & $\begin{array}{l}177.3 \\
279.5 \\
259.6 \\
716.4\end{array}$ & $\begin{array}{l}29.3 \\
29.6 \\
31.3\end{array}$ & $\begin{array}{l}3.00 \\
2.99 \\
3.12\end{array}$ \\
\hline Subtotal W1515WL ........ & $\begin{array}{l}\text { Cook } 1 \\
\text { Indlan Point } 2 \\
\text { Indlan Point } 3 \\
\text { Robinson } 2 \\
\text { Surry } 1 \\
\text { Surry } 2 \\
\text { Turkey Point } 3 \\
\text { Turkey Point } 4 \\
\text { Zlon } 1 \\
\text { Zlon } 2\end{array}$ & $\begin{array}{r}193 \\
267 \\
409 \\
314 \\
57 \\
20 \\
492 \\
483 \\
567 \\
547 \\
3,349\end{array}$ & $\begin{array}{r}87.5 \\
122.3 \\
186.8 \\
141.5 \\
26.2 \\
9.2 \\
223.5 \\
219.5 \\
258.4 \\
248.7 \\
1,523.7\end{array}$ & $\begin{array}{l}27.5 \\
36.0 \\
31.5 \\
23.7 \\
43.6 \\
36.0 \\
28.9 \\
29.5 \\
31.5 \\
31.9\end{array}$ & $\begin{array}{l}2.78 \\
3.32 \\
3.03 \\
2.63 \\
3.64 \\
3.80 \\
2.83 \\
2.85 \\
2.93 \\
2.95\end{array}$ \\
\hline
\end{tabular}

See footnotes at end of table. 
Table B7. Assembly Type Summary by Assembly Type (Continued)

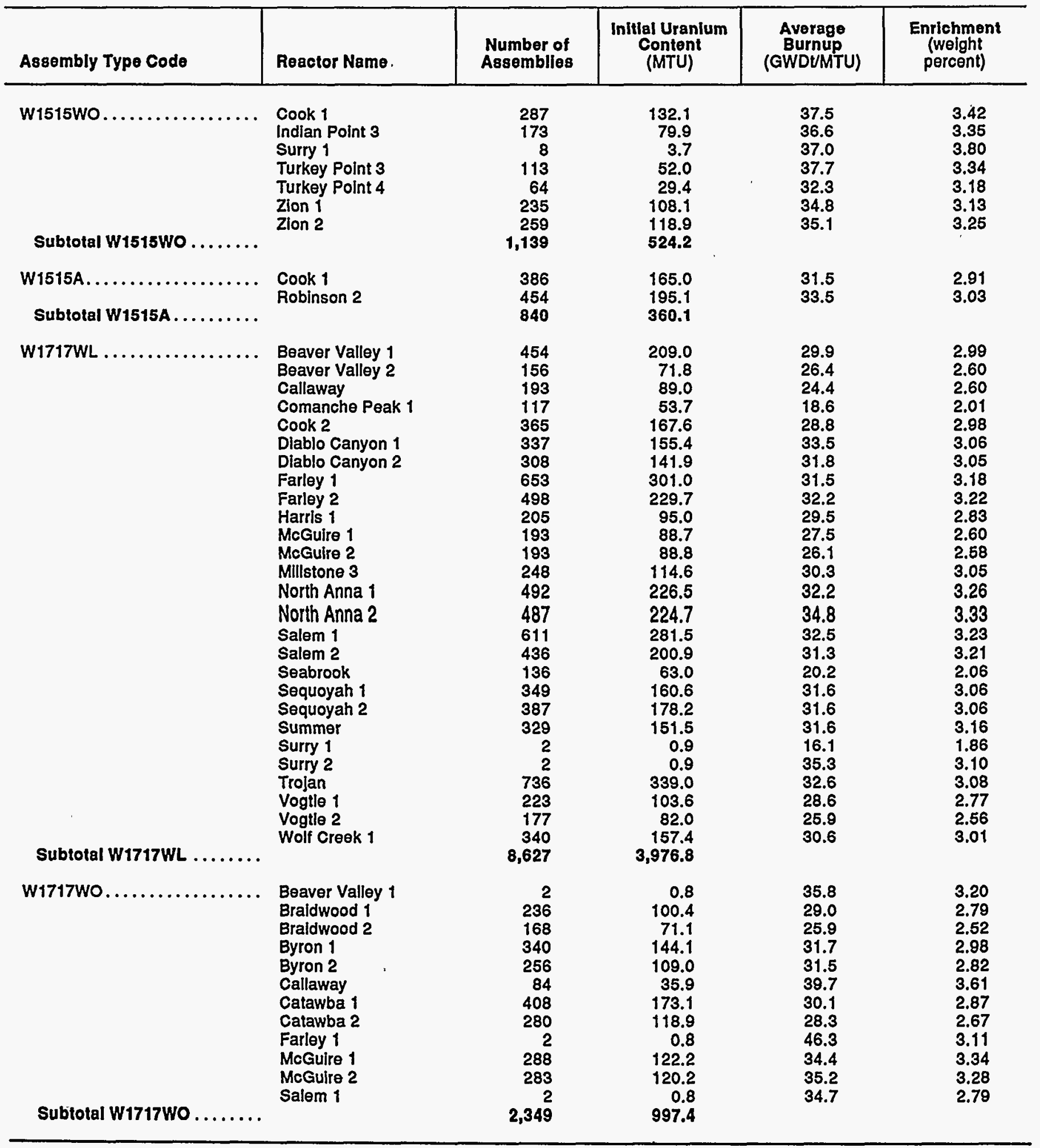

See footnotes at end of table. 
Table B7. Assembly Type Summary by Assembly Type (Continued)

\begin{tabular}{|c|c|c|c|c|c|}
\hline Assembly Type Code & Reactor Name & $\begin{array}{l}\text { Number of } \\
\text { Assemblies }\end{array}$ & $\begin{array}{l}\text { Initlal Uranlum } \\
\text { Content } \\
\text { (MTU) }\end{array}$ & $\begin{array}{c}\text { Average } \\
\text { Burnup } \\
\text { (GWDUMTU) }\end{array}$ & $\begin{array}{l}\text { Enrlchment } \\
\text { (weight } \\
\text { percent) }\end{array}$ \\
\hline $\begin{array}{l}\text { W1717WV5 } \ldots \ldots \ldots \ldots \ldots \ldots \\
\text { Subtotal W1717WV5 } \ldots \ldots \ldots\end{array}$ & $\begin{array}{l}\text { Callaway } \\
\text { Cook } 2 \\
\text { Diablo Canyon } 1 \\
\text { Harris } 1 \\
\text { Summer }\end{array}$ & $\begin{array}{r}175 \\
2 \\
39 \\
19 \\
43 \\
278\end{array}$ & $\begin{array}{r}74.4 \\
0.9 \\
16.6 \\
8.1 \\
18.2 \\
118.1\end{array}$ & $\begin{array}{l}39.2 \\
21.0 \\
39.7 \\
40.4 \\
37.7\end{array}$ & $\begin{array}{l}3.81 \\
4.20 \\
4.01 \\
3.87 \\
3.52\end{array}$ \\
\hline $\begin{array}{l}\text { W1717WVH.............. } \\
\text { Subtotal W1717WVH....... }\end{array}$ & $\begin{array}{l}\text { Beaver Valley } 1 \\
\text { Beaver Valley } 2 \\
\text { North Anna } 2 \\
\text { Salem } 1 \\
\text { Sequoyah } 1\end{array}$ & $\begin{array}{r}56 \\
32 \\
4 \\
19 \\
1 \\
112\end{array}$ & $\begin{array}{r}26.0 \\
14.9 \\
1.9 \\
8.8 \\
0.5 \\
52.0\end{array}$ & $\begin{array}{l}34.9 \\
35.0 \\
23.0 \\
33.2 \\
20.0\end{array}$ & $\begin{array}{l}3.48 \\
3.32 \\
4.21 \\
3.66 \\
3.55\end{array}$ \\
\hline W1717A.................. & Cook 2 & 290 & 116.6 & 39.3 & 3.76 \\
\hline $\begin{array}{l}\text { W1717B................. } \\
\text { Subtotal W1717B ......... }\end{array}$ & $\begin{array}{l}\text { McGuire } 1 \\
\text { Trojan }\end{array}$ & $\begin{array}{r}3 \\
44 \\
47\end{array}$ & $\begin{array}{r}1.3 \\
19.8 \\
21.1\end{array}$ & $\begin{array}{r}42.0 \\
8.2\end{array}$ & $\begin{array}{l}2.90 \\
3.56\end{array}$ \\
\hline $\begin{array}{l}\text { Wstitw } \ldots \ldots \ldots \ldots \ldots \ldots \\
\text { subtotal wst17w ......... }\end{array}$ & $\begin{array}{l}\text { South Texas } 1 \\
\text { South Texas } 2\end{array}$ & $\begin{array}{l}233 \\
116 \\
349\end{array}$ & $\begin{array}{r}126.2 \\
62.6 \\
188.8\end{array}$ & $\begin{array}{l}21.3 \\
19.8\end{array}$ & $\begin{array}{l}2.37 \\
2.32\end{array}$ \\
\hline ............. & Big Rock Point & 6 & 0.7 & 19.8 & 3.63 \\
\hline XBRO7G.... & Big Rock Point & 4 & 0.5 & 1.5 & 3.63 \\
\hline XBROBG.... & Big Rock Point & 2 & 0.2 & 4.1 & 3.63 \\
\hline XBROgG.... & Big Rock Point & 143 & 19.6 & 14.5 & 3.59 \\
\hline XBROOA... & Big Rock Point & 4 & 0.5 & 20.9 & 3.48 \\
\hline XBR11A................ & Big Rock Point & 212 & 27.3 & 24.9 & 3.41 \\
\hline XBR11N.... & Big Rock Point & 8 & 1.0 & 18.9 & 2.86 \\
\hline XDROGG $\ldots \ldots \ldots \ldots \ldots \ldots$ & Dresden 1 & 1 & 0.1 & 21.3 & 1.47 \\
\hline XDRO6G3B .. & Dresden 1 & 163 & 16.6 & 16.9 & 1.83 \\
\hline XDROGG3F & Dresden 1 & 96 & 9.8 & 20.0 & 2.25 \\
\hline XDR06G5 $\ldots \ldots \ldots \ldots \ldots$ & Dresden 1 & 106 & 11.2 & 19.0 & 2.26 \\
\hline XDR07GS. & Dresden 1 & 1 & 0.1 & 29.0 & 3.10 \\
\hline XDR08G & Dresden 1 & 1 & 0.1 & 23.3 & 1.95 \\
\hline XDR06U.... & Dresden 1 & 458 & 46.7 & 16.1 & 2.24 \\
\hline XDR06A.................. & Dresden 1 & 66 & 6.3 & 4.5 & 2.23 \\
\hline XFC14C... & Fort Calhoun & 337 & 122.7 & 29.1 & 2.78 \\
\hline XFC14A... & Fort Calhoun & 192 & 67.8 & 37.3 & 3.57 \\
\hline XHB07G2,............... & Humboldt Bay & 88 & 6.7 & 18.2 & 2.11 \\
\hline
\end{tabular}

See footnotes at end of table. 
Table B7. Assembly Type Summary by Assembly Type (Continued)

\begin{tabular}{|c|c|c|c|c|c|}
\hline Assembly Type Code & Reactor Name & $\begin{array}{l}\text { Number of } \\
\text { Assemblles }\end{array}$ & $\begin{array}{l}\text { Initial Uranium } \\
\text { Content } \\
\text { (MTU) }\end{array}$ & $\begin{array}{c}\text { Average } \\
\text { Burnup } \\
\text { (GWDt/MTU) }\end{array}$ & $\begin{array}{c}\text { Enrlchment } \\
\text { (welght } \\
\text { percent) }\end{array}$ \\
\hline 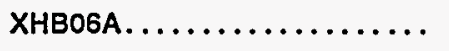 & Humboldt Bay & 126 & 8.8 & 9.0 & 2.40 \\
\hline XHN15MS $\ldots \ldots \ldots \ldots \ldots$ & Haddam Neck & 2 & 0.8 & 28.3 & 3.66 \\
\hline XHN15MZ $\ldots \ldots \ldots \ldots \ldots$ & Haddam Neck & 2 & 0.7 & 25.6 & 2.95 \\
\hline XHN15HS $\ldots \ldots \ldots \ldots \ldots \ldots$ & Haddam Neck & 1 & 0.4 & 32.2 & 3.99 \\
\hline $\mathrm{XHN} 15 \mathrm{HZ} \ldots \ldots \ldots \ldots \ldots$ & Haddam Neck & 2 & 0.7 & 18.5 & 3.26 \\
\hline XLC10L $\ldots \ldots \ldots \ldots \ldots \ldots$ & LaCrosse & 155 & 18.6 & 14.5 & 3.77 \\
\hline XLC10A................. & LaCrosse & 178 & 19.3 & 14.9 & 3.69 \\
\hline XPA15C. & Palisades & 273 & 112.6 & 16.0 & 2.47 \\
\hline XPA15A $\ldots \ldots \ldots \ldots \ldots \ldots$ & Palisades & 460 & 179.9 & 30.4 & 2.92 \\
\hline$x s L 16 C \ldots \ldots \ldots \ldots \ldots \ldots$ & St. Lucie 2 & 464 & 175.7 & 32.1 & 2.93 \\
\hline xso14w $\ldots \ldots \ldots \ldots \ldots \ldots$ & San Onofre 1 & 665 & 244.3 & 27.3 & 3.86 \\
\hline XYR18W $\ldots \ldots \ldots \ldots \ldots \ldots$ & Yankee Rowe & 76 & 20.8 & 25.5 & 4.94 \\
\hline
\end{tabular}

ancludes 4 temporarliy discharged bolling-water reactor (BWR) assemblles and 69 temporarily discharged pressurized-water reactor (PWR) assemblles with no assigned assembly type. Seo Technical Note 10 in Appendix E.

Includes 1.9 metric tons of uranium (MTU) for temporarily discharged PWR assemblies with no assigned assembly type. See Technical Note 10 in Appendix E.

GWDVMTU = Gigawattdays thermal per metric ton of uranium.

Note: Totals may not equal sum of components because of independent rounding. See Technical Note 11 in Appendix E.

Source: Energy Information Administration, Form RW-859, "Nuclear Fuel Data" (1992). 
Table B8. Spent Fuel Assemblies, by Type, Discharged by Year

\begin{tabular}{|c|c|c|c|c|c|c|c|c|c|c|c|c|c|c|c|}
\hline $\begin{array}{l}\text { Assembly } \\
\text { Type } \\
\text { Code }\end{array}$ & $\begin{array}{r}1968- \\
1969\end{array}$ & $\begin{array}{c}1970 \\
1971\end{array}$ & $\begin{array}{r}1972- \\
1973\end{array}$ & $\begin{array}{c}1974- \\
1975\end{array}$ & $\begin{array}{r}1976- \\
1977\end{array}$ & $\begin{array}{c}1978 \\
1979\end{array}$ & $\begin{array}{c}1980 \\
1981\end{array}$ & $\begin{array}{c}1982- \\
1983\end{array}$ & $\begin{array}{r}1984- \\
1985\end{array}$ & $\begin{array}{c}1986- \\
1987\end{array}$ & $\begin{array}{c}1988- \\
1989\end{array}$ & $\begin{array}{c}1990- \\
1991\end{array}$ & $1992^{b}$ & Temps $^{\circ}$ & Tolal \\
\hline \multicolumn{16}{|c|}{ Boilling-Water Reactors (BWR) } \\
\hline G2307G2A & - & 41 & 447 & 917 & 174 & 93 & - & $\cdots$ & - & -- & - & -. & -. & -- & 1,672 \\
\hline G2307G2B & -- & 244 & 593 & 1,045 & 1,765 & 993 & 407 & $\cdots$ & - & -- & -. & -- & -- & -- & 5,047 \\
\hline G2307G3 &.- &.- & -- & 2 & 52 & 191 & 119 & 30 & -- & -- & -. & -- & -- &.- & 394 \\
\hline G2308G4 & -- & $\cdots$ & -- & - & $\ldots$ & 102 & 1,268 & 1,636 & 730 & 140 &.- & $\ddot{n}$ & -- & -- & 3,876 \\
\hline G2308G5 & -- & -- & -. & - & -- & -. & -. & 2 & 518 & 272 & -- & 87 & - & $\cdots$ & 879 \\
\hline G2308GP & -- & -. & -- & -- & -- & -- & -- & - & 300 & 1,184 & 544 & 348 & 156 & -- & 2,532 \\
\hline G2308GB & -- & -- & $=$ & -- & -- & -- & -- & - & -- & 164 & 348 & 288 & 304 & $\cdots$ & 1,104 \\
\hline G2308G8A & -- & - & -- & -- & - & -- & -- & -- & - & - & - & 212 & .. & - & 212 \\
\hline G2307A & -- & -- & -. & -- & 4 & 124 & 108 & 24 & -- & -- & - & -- & - & 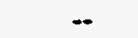 & 260 \\
\hline G2308A & -- & -- & -- & -- & -- & -- & 45 & 183 & - & 184 & 408 & 393 & 12 & -. & 1,225 \\
\hline G2309A & .- & $\cdots$ & -- & - & - & -- & - & - & -- & -. & - & 4 & $\ldots$ & -. & 4 \\
\hline G4607G2 & -- & - & 50 & 328 & 589 & 175 & -- & - & -- & -- & -- & -- & - & -- & 1,142 \\
\hline G4607G3A & .- & - & $\ldots$ & 2 & 501 & 1,037 & 1,723 & 349 & 140 & -- & -- & -- & - & -- & 3,752 \\
\hline G4607G3B & -- & -- & -- & -- & 192 & 747 & 209 & 36 & - & -- & -- & -- & $\cdots$ & -- & 1,184 \\
\hline G4608G4A & -- & -- & -- & -- & 112 & 250 & 647 & 571 & 204 & -- & -- & -- & 1 & -- & 1,785 \\
\hline G4608G4B & -- & -- & -- & -. & -. & 140 & 1,100 & 353 & 194 & -- & -- & -- & $\cdots$ & - & 1,787 \\
\hline G4608G5 & -- & -. & $\cdots$ & -- & -- & -- & 111 & 1,170 & 1,426 & 312 & 987 & 216 & 88 & -- & 4,310 \\
\hline G4608GP & .- & -- & -- & - & -- & -- & 26 & 199 & 1,994 & 3,814 & 2,592 & 1,749 & 733 & - & 11,107 \\
\hline G4608GB & -- & -- & $\cdots$ & - & -- & -- & -. & -. & 1 & 233 & 1,738 & 2,436 & 1,853 & 1 & 6,262 \\
\hline G4608G8 & -- & -- & - & -- & - & -- & - & -- & - & - & - & 104 & 112 & -- & 216 \\
\hline G4608G9 & -- & -- & -- & -- & -- & -- & -- & -- & -- & -- & -- & 1 & 1 & -- & 2 \\
\hline G4608A &.- & -- & - & -- & -- & -- & -- & -- & -- & -- & 220 & 632 & 348 & -- & 1,200 \\
\hline G4608AP & -- & -- & -- & -. & -- & -- & -- & -- & -- & -- & - & -. & 8 & -- & 8 \\
\hline G4608W & -. & -- & -- & -- & -. &.- & - & -. & - & -- & -- & 4 & - & - & 4 \\
\hline G4609A & -. & -- & -- & -. & -. & -- & -- & .. & -- & -- & 1 & 231 & 408 & -- & 640 \\
\hline XBR11G & $\ldots$ & - & 6 & -- & -. & -- & -. & -- & -- & $\cdots$ & .. & $\cdots$ & -. & -- & 6 \\
\hline XBR07G & 4 & - & -- & - & -- & -- & - & - & - & - & - & -- & - & - & 4 \\
\hline XBROBG & 2 & -- & -- & - & -- & -- & - & -- & - & -- & -- & -- & -- & - & 2 \\
\hline XBRO9G &.- & -. & 37 & 52 & 32 & 18 & 4 & $\cdots$ & -- & -- & - & -- & -- & - & $14 \overline{3}$ \\
\hline XBRO9A & -. & -- & -- & - & 4 & -- & - & - & - & -- & - & -- & - & - & 4 \\
\hline XBA11A & -. & -- & -- & 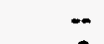 & $\ddot{\theta}$ & 8 & 18 & 44 & 36 & 24 & 42 & 40 & $\cdots$ & $\cdots$ & 212 \\
\hline XBR11N & $\cdots$ & - & -- & 2 & 6 & -. & -- & $\cdots$ & -- & -- & - & - & - & $\cdots$ & 8 \\
\hline XDR06G & 1 &.- & - & - & -- & $\cdots$ & $\cdots$ & -- & -- & -- & -- & -. & $\cdots$ & $\cdots$ & 1 \\
\hline XDR06G3B & 68 & 69 & 5 & 22 & 9 & -- & -- & -- & -- & -- & -- & -- & - & - & 163 \\
\hline XDR06G3F & 24 & 20 & 3 & 36 & 13 & -- & -- & -- & -- & -- & -- & -. & -- & -- & 96 \\
\hline XDR06G5 & 9 & 15 & 11 & 2 & 15 & 54 & -- & -- & -- & -- & -- & -- & -- & -- & 106 \\
\hline XDR07GS & 1 & - & $\cdots$ & $\cdots$ & - & - & -- & -- & -- & -- & -- & -- & -- & - & 1 \\
\hline XDR08G & 1 & -- & $-\cdot$ & $-\infty$ & - & -- & -- & -- & -- & -- & -- & -- &.- & $\cdots$ & 1 \\
\hline XDR06U & 1 & 8 & 33 & 43 & 29 & 344 & -- & -- & -- & -- & -- & -- & -- & -. & 458 \\
\hline XDRO6A &.- & -- & -- & - & -- & 66 & -. & -- & -- & $\cdots$ & -- & -- & -- & -- & 66 \\
\hline XHB07G2 & -- & 33 & 55 & -- & -- & -- & -- & -- & -- & -. & -- & -- & -- & -- & 88 \\
\hline XHB06G & -- & 7 & 51 & 56 & 62 & -- & -. & - & $\cdots$ & $\cdots$ & $\cdots$ & $\cdots$ & - & -- & 176 \\
\hline XHBO6A & -- & - & $\ldots$ & 4 & 122 & -- & -. & -. & - & $\cdots$ & -- & - & - & $\cdots$ & 126 \\
\hline XLC1OL & -. & -- & 56 & 25 & 32 & 28 & 12 & 2 & - & $\cdots$ & - & $\ldots$ & -- & $\cdots$ & 155 \\
\hline XLC10A & -- & -- & -- & -- & -- & - & - & 50 & 28 & 100 & -- & -- & -- & $\cdots$ & 178 \\
\hline Total BWR & 101 & 437 & 1,347 & 2,536 & 3,713 & 4,370 & 5,797 & 4,649 & 5,571 & 6,427 & 6,880 & 6,745 & 4,024 & 5 & $d_{52,602}$ \\
\hline
\end{tabular}

See footnotes at end of table. 
Table B8. Spent Fuel Assemblies, by Type, Dlscharged by Year (Continued)

\begin{tabular}{|c|c|c|c|c|c|c|c|c|c|c|c|c|c|c|c|}
\hline $\begin{array}{l}\text { Assembly } \\
\text { Type } \\
\text { Code }^{a}\end{array}$ & \begin{tabular}{|r}
$1968-$ \\
1969
\end{tabular} & $\begin{array}{l}1970- \\
1971\end{array}$ & $\begin{array}{c}1972- \\
1973\end{array}$ & $\begin{array}{r}1974- \\
1975\end{array}$ & $\begin{array}{l}1976- \\
1977\end{array}$ & $\begin{array}{c}1978- \\
1979\end{array}$ & $\begin{array}{c}1980- \\
1981\end{array}$ & $\begin{array}{r}1982- \\
1983\end{array}$ & $\begin{array}{l}1984- \\
1985\end{array}$ & $\begin{array}{c}1986- \\
1987\end{array}$ & $\begin{array}{l}1988- \\
1989\end{array}$ & $\begin{array}{l}1990- \\
1991\end{array}$ & $1992^{b}$ & Temps ${ }^{\circ}$ & Total \\
\hline \multicolumn{16}{|c|}{ Pressurlzed-Water Reactors (PWR) } \\
\hline B1515B4 & $\cdots$ & $\cdots$ & $\cdots$ & 53 & 450 & 684 & 555 & 546 & 499 & 380 & 382 & 438 & 106 & 13 & 4,106 \\
\hline B1515B4Z & $\cdots$ & $\cdots$ & -- & -- & - & - & - & - & - & - & - & - & 4 & $\ldots$ & 4 \\
\hline B1515B5Z & -- & -- & -- & - & $\ldots$ & $\cdots$ & - & .- & -- & -- & - & .. & 20 & .- & 20 \\
\hline B1515B6 & -- & - & -- & - & - & .. & -. & -- & .. & -- & $\cdots$ & -- & 109 & - & 100 \\
\hline B1515B7 & -- & -- & -- & -- & - & -. & - & -- & -- & -- & - & -- & 43 & $=$ & 43 \\
\hline B1515BZ & $\cdots$ & $\cdots$ & $\cdots$ & -- & -- & -- & - & -- & -- & 72 & 269 & 199 & 40 & -. & 580 \\
\hline B1515BGD & 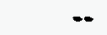 & -. & - & -- & - & -- & -- & $\cdots$ & -- & 3 & 1 & -. & -- & -- & 4 \\
\hline B1717B & -- & -- & -- & -- & - & $\cdots$ & 4 & -- & -- & -- & $\cdots$ & -- & -- & $\cdots$ & 4 \\
\hline C1414C & 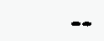 & -- & -. & 224 & 147 & 645 & 588 & 467 & 426 & 221 & 189 & 64 & 141 & 12 & 3,124 \\
\hline C1414A & -- & -- & -- & - & -. & -- & - & - & 72 & 97 & 147 & 194 & 1 & 11 & 522 \\
\hline C1414W & -- & -- & -- & -- & -- & -- & - & 3 & 59 & 130 & 73 & 85 & 73 & - & 423 \\
\hline C1616CSD & -- & -. & -- & - & - & -. & 35 & 125 & 196 & 443 & 520 & 371 & 270 & $\cdots$ & 1,960 \\
\hline $\mathrm{coc}$ & -- & -- & -- & -- & -. & -. & -- & -- & -- & 74 & 260 & 299 & 214 & 1 & 848 \\
\hline W1414W & -. & 12 & 109 & 37 & 130 & 135. & 123 & 42 & 14 & 1 & - & 14 & 2 & $\cdots$ & 619 \\
\hline W1414WL & -- & - & 44 & 129 & 301 & 252 & 261 & 158 & 74 & 130 & 51 & 11 & - & -- & 1,411 \\
\hline W1414Wo & -- & -- & - & -- & -- & - & -- & 1 & 3 & 12 & 184 & 300 & 198 & -. & 698 \\
\hline W1414A & $\cdots$ & -. & .. & $\cdots$ & 2 & -- & 2 & 178 & 177 & 130 & 91 & 97 & 45 & -- & 722 \\
\hline W1414ATR & -- & -- & .. & -- & -- & -- & -- & -- & 64 & 137 & 83 & 3 & 1 & -- & 288 \\
\hline W1414B & -- & -- & -- & -- & 2 & -. & -- & -- & -- & $\cdots$ & -- & -. & -- & -- & 2 \\
\hline W1515W & - & -- & -- & 117 & $31 \overline{4}$ & 217 & 178 & 198 & 189 & 104 & 137 & 116 & - & 7 & 1,577 \\
\hline W1515WL & -- & -. & 53 & 283 & 407 & 781 & 331 & 348 & 407 & 439 & 167 & 76 & 57 & -- & 3,349 \\
\hline W1515WO & -- & -- & - & - & -. &.- & -. & - & 1 & 80 & 324 & 349 & 385 & -- & 1,139 \\
\hline$W 1515 A$ & -- & -- & -- & -- & .. & 50 & 180 & 176 & 154 & 133 & 49 & 49 & 49 & $\ldots$ & 840 \\
\hline W1717WL & -. & -. & .. & -- & 2 & 243 & 442 & 850 & 1,232 & 1,568 & 1,424 & 1,966 & 856 & 44 & 8,627 \\
\hline W1717WO & -- & -- & -. & -- & -- & -- & .. & -- & 5 & 344 & 666 & 1,005 & 329 & -. & 2,349 \\
\hline W1717WV5 & -. & .. & .• & -- & -. & -- & .. & -- & $\ldots$ & .- & 4 & 113 & 161 & - & 278 \\
\hline W1717WVH & -- & - & -- & - & -- & -- & $\cdots$ & -- & -- & -- & -- & 57 & 55 & -- & 112 \\
\hline W1717A & -- & -- & -. & -- & -- & -- & .. & -- & - & 60 & 71 & 85 & 74 & .. & 290 \\
\hline$W_{1}$ & -- & -. & -. & .- & -- & -- & -. & -- & -- & -- & -- & 3 & 44 & -. & 47 \\
\hline $17 W$ & - & -- & -- & -- & - & -- & -- & -- & -. & -- & 36 & 227 & 82 & 4 & 349 \\
\hline XFC14C & .- & -. & -. & 25 & 88 & 44 & 80 & 20 & 21 & 12 & -- & 2 & 45 & -- & 337 \\
\hline XFC14A & -. & - & .. & $\ldots$ & - & - & -- & $\cdots$ & 69 & 34 & 44 & 37 & 8 & -- & 192 \\
\hline XHN15W & -- & 103 & 102 & 48 & 56 & -- & -. & -- & - & -- & -. & -- & -- & - & 309 \\
\hline XHN15MS & -- & $\cdots$ & 2 & $\cdots$ & - & -- & -- & -. & - & $\ldots$ & -- & -. & - & -- & 2 \\
\hline XHN15MZ & -- & -- & 2 & .- & -- & -- & -- & -- & -- & -- & .. & -- & -- & -- & 2 \\
\hline XHN15HS & -- & -- & -- & 1 & -- & - & -- & -- & -- & .. & -- & -. & -. & - & 1 \\
\hline XHN15HZ & $\cdots$ & -- & 2 & -- & -- & -- & -- & -- & - & -- & -- & -- & -. & -. & 2 \\
\hline XHN15B & -. & -- & -- & -- & 50 & 49 & 106 & 49 & 53 & 109 & 54 & 53 & - & 1 & 524 \\
\hline XIP14W & - & - & 40 & 120 & -. & -- & - & -. & - & -. & -. & $\ldots$ & - & -. & 160 \\
\hline XPA15C & $\ldots$ & - & -. & 205 & -- & 8 & 60 & -- & - & -- & -. & -- & $\ldots$ & -- & 273 \\
\hline XPA15A & $\cdots$ & - & -- & - & -. & 128 & 8 & 68 & 52 & - & 52 & 75 & 76 & 1 & 460 \\
\hline XSL16C & - & - & -- & -- & -- & -- & -- & -- & 80 & 156 & 84 & 76 & 68 & -- & 464 \\
\hline XSO14W & - & 97 & 57 & 53 & 53 & 52 & 52 & .. & 52 & - & 52 & 40 & 157 & -- & 665 \\
\hline XYR18W & 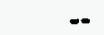 & -. & 36 & 40 & -. & -- & -- & -. & -. & -. & .. & .. & -- & -. & 76 \\
\hline XYA16U & -- & -. & -. & 37 & 36 & -. & -. & -- & .. & -. & .. & -- & -. & -- & 73 \\
\hline XYR16A & -. & -. & -. & -. & - & 40 & 36 & 40 & 76 & 36 & .- & -- & -- & -- & 228 \\
\hline XYA16C & -. & -. & -. & - & -- & - & - & -. & -. & -. & 40 & 116 & -- & - & 156 \\
\hline Total PWR & 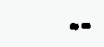 & 212 & 447 & 1,372 & 2,038 & 3,328 & 3,041 & 3,269 & 3,975 & 4,905 & 5,454 & 6,520 & 3,713 & 163 & • 38,437 \\
\hline
\end{tabular}

\footnotetext{
Assembly types are defined in Table B4.

'Some data for prevlous years have been revised. Current-year data may be revised in future publicatlons. When utllitles reinsert assemblies dlscharged In prevlous years, historical totals change. See Technical Note 12 In Appendlx $E$.

Tomps are temporarily discharged assemblles, as of December 31, 1992, as reported on Form RW-859.

No assigned assembly type for 4 temporarily dlscharged BWR assemblies. These BWR assemblies are Included In the Total column. See Technlcal Note 10 in Appendix E.

'No assigned assembly type for 69 temporarlly dlscharged PWR assemblles. These PWR assemblles are included in the Total column. See Technical Note 10 In Appendlx E.

-. Not applicable.

Source: Energy Information Adminlstration, Form RW-859, "Nuclear Fuel Data" (1992).
} 
Table B9. Initial Uranium Content of Spent Fuel Assemblies, by Type, Discharged by Year (Metric tons of uranium)

\begin{tabular}{|c|c|c|c|c|c|c|c|c|c|c|c|c|c|c|c|}
\hline $\begin{array}{l}\text { Assembly } \\
\text { Type } \\
\text { Code }\end{array}$ & $\begin{array}{c}1968- \\
1969\end{array}$ & $\begin{array}{c}1970- \\
1971\end{array}$ & $\begin{array}{c}1972- \\
1973\end{array}$ & $\begin{array}{r}1974- \\
1975\end{array}$ & $\begin{array}{c}1976 . \\
1977\end{array}$ & $\begin{array}{r}1978- \\
1979\end{array}$ & $\begin{array}{r}1980- \\
1981\end{array}$ & $\begin{array}{c}1982- \\
1983\end{array}$ & $\begin{array}{l}1984- \\
1985\end{array}$ & $\begin{array}{c}1986- \\
1987\end{array}$ & $\begin{array}{c}1988- \\
1989\end{array}$ & $\begin{array}{c}1990- \\
1991\end{array}$ & $1992^{6}$ & Tomps ${ }^{\circ}$ & Total \\
\hline \multicolumn{16}{|c|}{ Bolling-Water Reactors (BWR) } \\
\hline G2307G2A & - & 7.8 & 87.0 & 178.8 & 33.9 & 18.1 & -- & $\therefore$ & - & -. & -- & 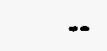 & -- & -- & 325.9 \\
\hline G2307G2B & .. & 7.1 & 114.1 & 201.8 & 339.8 & 191.6 & 78.5 & $\because$ & -- & -. & -. & .. & -. & -- & 972.9 \\
\hline G2307G3 & -. & -. & .. & 0.4 & 9.7 & 35.8 & 22.3 & 5.6 & -- & -- & -- & -- & -. & -- & 73.9 \\
\hline G2308G4 & -- & -- & -- & - & -. & 18.7 & 233.4 & 301.3 & 134.4 & 25.8 & -. & -. & .. & .. & 713.7 \\
\hline G2308G5 & -- & -- & -. & -- & -. & -- & 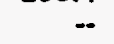 & 0.4 & 91.6 & 48.1 & -- & 15.4 & -. & -. & 156.5 \\
\hline G2308GP & -- & -- & -- & .- & -- & -- & -- & - & 53.1 & 209.4 & 96.4 & 61.7 & 27.5 & -. & 448.1 \\
\hline G2308GB & -- & -- & -- & -- & -- & - & -- & -- & .. & 29.1 & 61.9 & 51.2 & 53.6 & .. & 195.7 \\
\hline G2308G8A & -. & -- & -- & -- & -. & - & -- & -- & - & -. & - & 37.8 & -. & .- & 37.8 \\
\hline G2307A & -. & -- & -- & .. & 0.7 & 22.6 & 19.7 & 4.4 & -- & -- & -- & -- & -- & -- & 47.4 \\
\hline G2308A & .. & -- & -- & -. & -- & -- & 7.9 & 32.0 & -- & 32.2 & 71.0 & 68.3 & 2.1 & -. & 213.5 \\
\hline G2309A & -- & -- & -- & .. & .- & -- & -- & -. & - & -. & -. & 0.7 & .. & -- & 0.7 \\
\hline G4607G2 & -- & -- & 9.5 & 63.3 & 115.3 & 34.2 & - & -- & -- & -- & $-\cdot$ & -- & -- & .. & 222.4 \\
\hline G4607G3A & -. & -- & -- & 0.4 & 93.9 & 194.4 & 323.1 & 65.3 & 26.2 & -- & -- & - & -- & .. & 703.3 \\
\hline G4607G3B & .. & -. & -- & - & 36.6 & 141.7 & 39.7 & 6.8 & -- & -- & -- & -- & -. & .. & 224.9 \\
\hline G4608G4A & -. & -- & -- & -- & 20.6 & 45.8 & 119.1 & 105.1 & 37.4 & -. & -. & -- & 0.2 & -. & 328.3 \\
\hline G4608G4B & .. & -- & .. & -- & .. & 26.1 & 205.4 & 65.9 & 36.2 & -- & -- & -- & -- & -. & 333.7 \\
\hline G4608G5 & -. & -- & -. & -- & -- & -. & 20.3 & 213.9 & 261.1 & 57.1 & 180.7 & 39.5 & 16.0 & -- & 788.7 \\
\hline G4608GP & -. & -. & -. & -- & -- & .. & 4.8 & 36.4 & 364.6 & 698.9 & 475.2 & 320.3 & 133.5 & -. & $2,033.7$ \\
\hline G4608GB & -- & -- & .. & -- & -. & -- & -. & -- & 0.2 & 43.2 & 320.9 & 450.0 & 342.7 & 0.2 & $1,167.1$ \\
\hline G4608G8 & .- & -- & .. & -- & -. & -- & -. & -. & - & -- & . & 18.5 & 20.0 & -. & 38.5 \\
\hline G4608G9 & -- & -- & -. & -- & -. & -- & -- & -- & -. & -. & -- & 0.2 & 0.2 & -. & 0.4 \\
\hline G4608A & -- & -- & -. & -- & -- & -- & -- & -- & -- & -- & 38.8 & 111.5 & 61.0 & -- & 211.3 \\
\hline G4608AP & .- & -- & -. & -- & -. & -- & -- & -- & -- & -- & -- & -- & 1.4 & -- & 1.4 \\
\hline G4608W & .- & -- & -. & -- & -- & -- & .- & -- & -. & -- & -- & 0.6 & - & - & 0.6 \\
\hline G4609A & -- & -. & -- & -. & -- & -. & -- & -- & -- & -. & 0.2 & 40.0 & 70.6 & -- & 110.8 \\
\hline XBR11G & -- & -. & 0.7 & -. & -- & -. & -- & -. & -. & -- & -. & -. & -- & -. & 0.7 \\
\hline XBR07G & 0.5 & -. & -- & -. & -- & - & -- & -- & -- & -- & -- & -. & -. & - & 0.5 \\
\hline XBRO8G & 0.2 & -. & -. & -- & -- & -- & -- & -. &.- & -- & -- & $\cdots$ & -- & - & 0.2 \\
\hline XBRO9G & -- & -- & 5.1 & 7.1 & 4.4 & 2.5 & 0.6 & -. & .. & -. & -. & -. & -- & -. & 19.6 \\
\hline XBROQA & -. & -. &.. & .. & 0.5 & -. & .. & -- & -. & 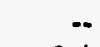 & .- & .. & -. & .. & 0.5 \\
\hline XBR11A & .. & .. & -. & .. & -. & 1.0 & 2.3 & 5.6 & 4.6 & 3.1 & 5.5 & 5.3 & -- &.- & 27.3 \\
\hline XBR11N & -. & -- & .. & 0.3 & 0.8 & - & - & -- & -. & -. & - & .. & -. & -. & 1.0 \\
\hline XDRO6G & 0.1 & -- &.- & -. & - & -- & -. & -- & -- & -- & -. & -. & -- & - & 0.1 \\
\hline XDRO6G3B & 5.9 & 7.0 & 0.5 & 2.2 & 0.9 & -- & -- & -- & -- & -. & -- & -- & -- & -- & 16.6 \\
\hline XDROGG3F & 2.4 & 2.0 & 0.3 & 3.7 & 1.3 & -- & -. &.- & -- & -- & -- & -- & -. & -- & 9.8 \\
\hline XDRO6G5 & 1.0 & 1.6 & 1.2 & 0.2 & 1.6 & 5.7 & -- & -- & -- & -. & -. & -. & -. & .. & 11.2 \\
\hline XDR07GS & 0.1 & $-\cdot$ & -- & -- & -. & -- & -- & -. & -- & -. & -- & -- & -- & -- & 0.1 \\
\hline XDROBG & 0.1 & -. & -- & .. & -- & -- & -- & -. & -- & .- & -- & -. & .- & -- & 0.1 \\
\hline XDROGU & 0.1 & 0.8 & 3.4 & 4.4 & 3.0 & 35.0 & -- & .- & -- & -- & -- & -- & -. & -. & 46.7 \\
\hline XDRO6A & -. & - & -- & -. & - & 6.3 & -- & -- & -- & -- & -. & -. & -- & -- & 6.3 \\
\hline XHB07G2 & -. & 2.5 & 4.2 & -- & -. & -. & -- & .. & -- & -- & -- & -- & - & -- & 6.7 \\
\hline XHBO6G & .- & 0.5 & 3.9 & 4.3 & 4.8 & -. & -- & -- & .. & .- & .. & -. & -- & -- & 13.4 \\
\hline XHB06A & .- & $\cdot$. & .. & 0.3 & 8.5 & .. & -- & -- & -- & - & -- & -- & -. & .. & 8.8 \\
\hline XLC1OL & -- & -. & 6.7 & 3.0 & 3.9 & 3.4 & 1.4 & 0.2 & -. & -- & .. & -. & -. & -. & 18.6 \\
\hline XLC1OA & -- & $\cdots$ & -. & .. & .. & $\cdots$ & - & 5.4 & 3.0 & 10.8 & -. & -- & -- & -- & 19.3 \\
\hline Total BWR & 10.4 & 69.6 & 236.6 & 470.2 & 680.3 & 783.0 & $1,078.6$ & 848.5 & $1,012.6$ & $1,157.6$ & $1,250.5$ & $1,220.8$ & 728.7 & 0.2 & $9,547.6$ \\
\hline
\end{tabular}

See footnotes at end of table. 
Table B9. Initial Uranium Content of Spent Fuel Assemblies, by Type, Discharged by Year (Continued) (Metric tons of uranium)

\begin{tabular}{|c|c|c|c|c|c|c|c|c|c|c|c|c|c|c|c|}
\hline $\begin{array}{l}\text { Assembly } \\
\text { Type } \\
\text { Code }^{a}\end{array}$ & $\begin{array}{l}1968- \\
1969\end{array}$ & $\begin{array}{r}1970- \\
1971\end{array}$ & $\begin{array}{r}1972- \\
1973\end{array}$ & $\begin{array}{r}1974- \\
1975\end{array}$ & $\begin{array}{r}1976- \\
1977\end{array}$ & $\begin{array}{r}1978- \\
1979\end{array}$ & $\begin{array}{r}1980- \\
1981\end{array}$ & $\begin{array}{r}1982- \\
1983\end{array}$ & $\begin{array}{r}1984- \\
1985\end{array}$ & $\begin{array}{r}1986 \\
1987\end{array}$ & $\begin{array}{r}1988- \\
1989\end{array}$ & $\begin{array}{r}1990- \\
1991\end{array}$ & $1992^{b}$ & Temps ${ }^{\circ}$ & Total \\
\hline
\end{tabular}

Pressurlzed-Water Reactors (PWR)

\begin{tabular}{|c|c|c|c|c|c|c|c|c|c|c|c|c|c|c|c|}
\hline B1515B4 & -- & -- & -- & 24.8 & 209.2 & 317.0 & 257.2 & 254.8 & 231.7 & 176.0 & 177.4 & 203.7 & 49.1 & 2.3 & $1,903.3$ \\
\hline B1515B4Z & $\cdots$ & $-\cdot$ & -- & -- & -- & -. & -- & - & $\cdots$ & -- & - & -. & 1.9 & -- & 1.9 \\
\hline B1515B5Z & -- & -- & -- & - & -- & -- & -- & -- & -- & -. & -- & - & 9.3 & -- & 9.3 \\
\hline B1515B6 & -- & -- & -- & $\cdots$ & $\cdots$ & -- & -- & $-\cdot$ & .. & -- & -- & -. & 50 & -- & 50 \\
\hline B1515B7 & -- & -- & -- & -- & - & -- & -- & - & - & - & - & -- & 19. & -- & 18.9 \\
\hline B1515BZ & -- & -- & -- & -- & -- & -. & $\cdots$ & - & - & 33.4 & 124.6 & 92.3 & 18.5 & -. & 268.8 \\
\hline B1515BGD & -- & -- & -- & -- & -- & -- & - & - & -- & 1.3 & 0.4 & -. & -- & -- & 1.7 \\
\hline B1717B & -- & -- & -- & -- & -- & -- & 1.8 & -- & -- & -- & -- & -. & -- & $\cdots$ & 1.8 \\
\hline $\mathrm{C} 1414 \mathrm{C}$ & -- & - & -- & 84.2 & 57.6 & 245.1 & 224.3 & 179.6 & 162.1 & 84.8 & 73.5 & 24.0 & 54.3 & -- & $1,189,4$ \\
\hline C1414A & -- & -- & -- & - & -- & -- & -- & -- & 27.0 & 35.0 & 54.3 & 72.5 & 0.4 & 4.0 & 193.2 \\
\hline C1414W & -- & -- & - & 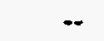 & -- & -- & $-\cdot$ & 1.2 & 24.0 & 52.8 & 29.8 & 34.7 & 29.8 & -. & 172.3 \\
\hline CSD & -- & -- & -- & 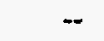 & -- & -- & 14.9 & 51.3 & 83.7 & 182.8 & 216.1 & 152.8 & 110.0 & -- & 1.6 \\
\hline C8 & -- & -- & -- & -- & -- & -- & -- & -- & -- & 31.5 & 107.4 & 120.5 & 87.9 & 0.4 & 47.7 \\
\hline W1414W & -- & 4.6 & 42.7 & 14.4 & 51.1 & 53.0 & 48.9 & 16.8 & 5.6 & 0.4 & -- & 5.6 & 0.8 & -- & 243.9 \\
\hline W1414WL & -- & -- & 17.5 & 50.5 & 119.4 & 100.6 & 104.8 & 63.5 & 29.7 & 52.2 & 20.5 & 4.4 & -- & - & 563.1 \\
\hline W1414WO & -- & -- & -- & -- & -- & - & $\cdots$ & 0.4 & 1.1 & 4.3 & 65.8 & 107.5 & 70.8 & -. & 249.8 \\
\hline W1414A & -- & -- & -- & -- & 0.7 & -- & 0.7 & 67.3 & 66.7 & 48.8 & 34.3 & 36.4 & 17.0 & -- & 272.0 \\
\hline W1414ATR & -- & -- & -- & -- & -- & -- & -- & -- & 23.3 & 50.0 & 29.7 & 1.1 & 0.4 & -- & 104.5 \\
\hline W1414B & $\cdots$ & -- & -- & -- & 0.8 & -- & -. & -- & -. & -- & -- & -- & -- & -. & 0.8 \\
\hline W1! & -- & -- & - & 53.0 & 141.2 & 98.3 & 81.1 & 90.1 & 86.0 & 47.6 & 62.8 & 53.3 & -- & 3.2 & 716.4 \\
\hline W1515WL & -- & - & 24.2 & 126.8 & 183.9 & 354.2 & 151.3 & 159.0 & 186.0 & 200.6 & 76.5 & 35.0 & 26.2 & -- & $1,523.7$ \\
\hline 5WO & -- & -- & - & -- & -- & -- & - & -- & 0.5 & 36.8 & 149.0 & 160.6 & 177.2 & -- & 524.2 \\
\hline W1 & $-\cdot$ & -- & - & - & -- & 21.4 & 77.1 & 75.3 & 66.0 & 57.2 & 21.1 & 21.2 & 20.8 & -- & 360.1 \\
\hline NL & -- & -- & -- & -- & 0.9 & 111.7 & 203.2 & 390.3 & 565.4 & 721.9 & 657.7 & 909.2 & 396.2 & 20.3 & $3,976.8$ \\
\hline Wo & -- & -- & -- & -- & -- & -- & -- & -- & 2.1 & 145.9 & 283.3 & 426.4 & 139.7 & -- & 997.4 \\
\hline WV5 & - & -- & - & -- & -- & -- & - & -- & -- & -- & 1.7 & 48.0 & 68.4 & -- & 118.1 \\
\hline W1717WVH & -- & -. & - & -- & -- & -- & - & -- & -- & -- & .- & 26.5 & 25.5 & -- & 52.0 \\
\hline W1717A & -- & -- & -- & -- & -- & -- & - & -- & -- & 24.1 & 28.5 & 34.2 & 28.8 & -. & 116.6 \\
\hline W1717B & -- & - & -- & -- & -- & -- & -- & -- & -- & -- & -- & 1.3 & 19.8 & -- & 21.1 \\
\hline Ws & $-\cdot$ & -- & -- & -- & -- & -- & 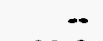 & -- & -- & -- & 19.6 & 122.7 & 44.4 & 2.2 & 188.8 \\
\hline $4 C$ & $-\cdot$ & $-\cdot$ & -- & 9.3 & 31.7 & 16.4 & 29.3 & 7.3 & 7.6 & 4.4 & -. & 0.7 & 16.0 & - & 122.7 \\
\hline XF & -- & -- & -- & -. & -- & -- & -- & -- & 24.7 & 12.1 & 15.2 & 13.0 & 2.9 & -- & 67.8 \\
\hline $\mathrm{XH}$ & -. & 43.3 & 42.3 & 19.8 & 23.0 & -- & -. & -- & -- & -- & -. & -. & -- & 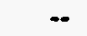 & 128.4 \\
\hline XHN15MS & -- & -- & 0.8 & -- & -- & -- & - & $-\cdot$ & - & -- & -- & -- & $\cdots$ & -- & 0.8 \\
\hline XHN15MZ & -- & -- & 0.7 & -- & -- & -- & -- & -- & -- & 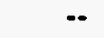 & -• & -- & -- & -- & 0.7 \\
\hline XHN15HS & $-\cdot$ & -- & $\cdots$ & 0.4 & -. & $-\cdot$ & -- & $\cdots$ & $-\cdot$ & -- & -. & -- & -- & -- & 0.4 \\
\hline XHN15HZ & -- & - & 0.7 & -- & -- & - & -- & -- & -- & -- & -- & -- & -- & -- & 0.7 \\
\hline XHN15B & -- & -- & -- & -- & 20.6 & 20.2 & 43.6 & 20.2 & 21.8 & 44.9 & 22.1 & 21.8 & -- & 0.4 & 215.6 \\
\hline XIP14W & -. & -- & 7.8 & 22.8 & - & - & - & - & - & - & - & - & -- & - & 30.6 \\
\hline XPA15C & -- & -- & -- & 84.3 & -- & 3.3 & 24.9 & -- & -- & - & -- & - & -- & -- & 112.6 \\
\hline XPA15A & -- & -- & -. & -- & -- & 50.5 & 3.1 & 26.3 & 20.2 & - & 20.4 & 29.3 & 29.7 & 0.4 & 179.9 \\
\hline XSL16C & -- & -. & -- & - & -- & -- & - & $\cdots$ & 31.0 & 57.9 & 31.8 & 29.3 & 25.8 & -- & 175.7 \\
\hline XS014W & -- & 35.6 & 20.5 & 19.3 & 19.2 & 19.2 & 19.2 & -- & 19.3 & -- & 19.2 & 14.8 & 58.1 & -- & 244.3 \\
\hline$X Y$ & -- & -- & 9.8 & 10.9 & -- & -. & - & -- & -- & -- & $-\cdots$ & -- & -- & -- & 20.8 \\
\hline XYR16U & -- & $\cdots$ & $\cdots$ & 8.8 & 8.6 & -- & -- & -- &.- & -- & -- & -. & -- & -- & 17.4 \\
\hline XYR16A & -- & -- & $\cdots$ & - & -- & 9.4 & 8.5 & 9.4 & 17.7 & 8.3 & - & - & .. & -- & 53.2 \\
\hline XYR16C & -- & -- & -- & -- & - & - & -- & - & -. & -- & 9.2 & 26.6 & -- & -- & 35.8 \\
\hline Total PWR & -. & 83.5 & 167.0 & 529.5 & 867.9 & $1,420.2$ & $1,294.0$ & $1,412.6$ & $1,703.0$ & $2,114.7$ & $2,351.9$ & $2,829.6$ & $1,601.0$ & $d_{35.1}$ & $16,410.1$ \\
\hline
\end{tabular}

\footnotetext{
Assembly types are Identfled in Table B4.

bSome data for prevlous years have been revised. Current-y ear data may be revised in future publlcations. When utlities relnsert assemblies discharged In previous years, historical totals change. See Technical Note 12 in Appendlx E.

Temps are temporarlly discharged assemblles, as of December 31, 1992, as reported on Form RW-859.

Includes 1.9 metric tons of uranium (MTU) for temporarily discharged PWR assemblles with no assigned assembly type. See Technical Note 10 In Appendlx E.

$--=$ Not appllcable.

Note: Totals may not equal sum of components because of independent rounding. See Technical Note 11 in Appendlx E.

Source: Energy Informatlon Administration, Form RW-859, "Nuclear Fuel Data" (1992).
} 
Table B10. Burnup of Spent Fuel Assemblies, by Type, Discharged by Year (Gigawattdays thermal per metric ton of uranium)

\begin{tabular}{|c|c|c|c|c|c|c|c|c|c|c|c|c|c|c|}
\hline $\begin{array}{l}\text { Assembly } \\
\text { Typo } \\
\text { Code }\end{array}$ & $\begin{array}{r}1968 . \\
1969\end{array}$ & $\begin{array}{c}1970- \\
1971\end{array}$ & $\begin{array}{c}1972- \\
1973\end{array}$ & $\begin{array}{r}1974- \\
1975\end{array}$ & $\begin{array}{r}1976- \\
1977\end{array}$ & $\begin{array}{c}1978- \\
1979\end{array}$ & $\begin{array}{c}1980- \\
1981\end{array}$ & $\begin{array}{c}1982- \\
1983\end{array}$ & $\begin{array}{r}1984- \\
1985\end{array}$ & $\begin{array}{c}1986- \\
1987\end{array}$ & $\begin{array}{l}1988- \\
1989\end{array}$ & $\begin{array}{c}1990 . \\
1991\end{array}$ & $1992^{b}$ & Tempo ${ }^{\circ}$ \\
\hline \multicolumn{15}{|c|}{ Bolling-Water Reactors (BWR) } \\
\hline G2307G2A & -. & 7.7 & 13.2 & 17.8 & 20.0 & 21.7 & $\cdots$ & -. & -. & -- & -. & .. & -. & -. \\
\hline G2307G2B & -. & 1.4 & 4.4 & 13.4 & 17.3 & 23.4 & 23.0 & -. & -. & -- & - & -- & -- & $\cdots$ \\
\hline G2307G3 & -- & -. & -. & 9.0 & 22.2 & 25.6 & 25.1 & 25.4 & -- & -- & - & $\ldots$ & .. & $\ldots$ \\
\hline G2308G4 & -- & - & -- & .. & - & 23.9 & 24.0 & 25.8 & 27.0 & 24.7 & $\cdots$ & -. & $\cdots$ & $\cdots$ \\
\hline G2308G6 & -- & -- & .. & -- & -. & -- & -. & 22.5 & 28.2 & 29.8 & $\cdots$ & 26.0 & -- & - \\
\hline G2308GP & - & -. & - & $\cdots$ & -. & $\ldots$ & $\ldots$ & .. & 27.8 & 27.6 & 29.5 & 30.5 & 29.2 & -. \\
\hline G2308GB & $\cdots$ & -- & -- & .. & -- & -- & -- & -- & -- & 27.6 & 29.0 & 30.2 & 34.3 & -. \\
\hline G2308G8A & - & -- & -- & -. & - & -- & -- & -- & -- & -. & - & 32.3 & -- & -. \\
\hline G2307A & - & - & -. &.- & 25.2 & 24.7 & 22.6 & 21.8 & $\cdots$ & $\cdots$ & - & -. & -- & $\cdots$ \\
\hline G2308A & $\cdots$ & -- & .. & - & -. & -. & 25.1 & 27.3 & -. & 23.9 & 27.3 & 32.2 & 22.7 & $\ldots$ \\
\hline G2309A & -- & -- & -- & - &.- & - & -- & -- & -- & -- & -- & 36.0 & 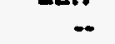 & $\cdots$ \\
\hline G4607G2 & -- & -- & 3.7 & 9.2 & 9.8 & 9.9 & -. &.- & -. & -. & -. & -. & - & $\cdots$ \\
\hline G4607G3A & -- & -- & -- & 4.7 & 12.8 & 19.9 & 23.6 & 23.1 & 22.8 & .. & .- & -- & -- & - \\
\hline G4607G3B & -- & - & -- & -. & 10.6 & 23.5 & 25.9 & 26.7 & -- & -- & -- & -. & -- & $\cdots$ \\
\hline G4608G4A & - & - & -- & - & 18.9 & 19.6 & 24.6 & 26.9 & 28.5 & -- & -- & - & 33.0 & $\cdots$ \\
\hline G4608G4B & -- & $\ldots$ & -- & -- & -- & 17.1 & 20.7 & 24.2 & 23.0 & -. & -- & -- & - & - \\
\hline G4608G5 & -- & .. & -- & -- & -- & -- & 7.9 & 26.6 & 23.6 & 14.9 & 25.0 & 23.4 & 29.0 & -- \\
\hline G4608GP & -- & -- & -. & $\cdots$ & -- & -- & 23.2 & 26.7 & 22.4 & 17.4 & 22.6 & 24.1 & 22.4 & - \\
\hline G4608GB & -- & $\ldots$ & -- & $\ldots$ & -- & -- & -. & -. & 5.5 & 9.2 & 17.6 & 24.7 & 27.9 & 23.0 \\
\hline G4608G8 & -- & $-\cdots$ & -- & -- & -- & -- & -- & - & - & - & -- & 32.0 & 32.7 & -. \\
\hline G4608G9 & -. & -. & .. & -. & -- & -- & -- & - & -- & - & -- & 18.0 & 14.0 & -- \\
\hline G4608A & $\ldots$ & -- & -- & -. & -. & - & $\cdots$ & -. & -. & -. & 26.7 & 29.8 & 33.6 & $-\infty$ \\
\hline G4608AP & - & -- & -- & -. & -- & -- &.- & -. & -- & -- & $\therefore$ & -- & 28.0 & - \\
\hline G4608W & $\ldots$ & -. & -- & - & - & - & -. & .. & -- & -- & - & 23.0 & -. & - \\
\hline G4609A & -. & - & -- & -- & $\therefore$ & - & -. & -- & -- & -. & 24.0 & 33.5 & 35.9 & .. \\
\hline XBRIIG & -. & -- & 19.8 & - & - & - & - & - & -- & -. & -. & 0.0 & .. & $\ldots$ \\
\hline XBRO7G & 1.5 & -- & -. & - & -- & - & - & - & -- & - & - & -- & .. & - \\
\hline XBRO8G & 4.1 & - & - & -- & -- & - & -- & - & -- & -- & - & -- & - & - \\
\hline XBR09G & $\ldots$ & -. & 10.9 & 12.3 & 17.7 & 20.5 & 21.3 & -- & -- & -- & -- & -- & -- & -- \\
\hline XBRO9A & $-\cdot$ & - & - & -. & 20.9 & -- & -- & -- & - & - & - & -- & -- & -- \\
\hline XBA11A & -. & -- & -- & -- & $\ldots$ & 25.7 & 25.5 & 25.3 & 23.4 & 27.3 & 24.4 & 24.4 & -• & $\cdots$ \\
\hline XBR11N & -- & -- & -- & 16.3 & 19.8 & -- & - & - &.- & - & - & -. & -- & $\cdots$ \\
\hline XDROGG & 21.3 & - & -- & -. & - & -- & -- & - & -- & - & -- & - & - & - \\
\hline XDR06G3B & 16.3 & 16.6 & 22.7 & 18.3 & 16.7 & - & -- & -- & -- & -- & -- & $\therefore$ & -. & -- \\
\hline XDRO6G3F & 14.7 & 21.3 & 21.5 & 22.3 & 21.3 & - & -. & -- & -- & -. & -- & $\cdots$ & - & -. \\
\hline XDR06G5 & 9.3 & 13.5 & 17.8 & 19.5 & 22.2 & 21.5 & -. & - & -- & - & -- & - & -- & - \\
\hline XDR07GS & 29.0 & - & - & - & - & -. & -- & -- & -- & -- & -. & $\cdots$ & $-\bullet$ & 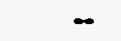 \\
\hline XDRO8G & 23.3 & -- & - & - & -- & -- & -- & - & -- & -- & $-\cdot$ & $\cdots$ & -- & - \\
\hline XDR06U & 5.2 & 12.5 & 16.4 & 17.3 & 22.4 & 15.5 & -- & -- & -- & -- & -- & - & - & -- \\
\hline XDROGA & - & -- & -- & - & -. & 4.5 & -- & -- &.- & -. & -. & -. & -• & $\cdots$ \\
\hline XHB07G2 & - & 17.7 & 18.5 & $\cdots$ & - & - & -- & -. & -. & -. & -. & -. & -- & - \\
\hline XHB06G & -. & 10.0 & 17.1 & 18.1 & 17.3 & - & -. & -- & -- & -- & - & $\cdots$ & -. & -. \\
\hline XHB06A & -- & .. & $\ldots$ & 17.5 & 8.8 & -- & -- & -- & - & -. & -- & -. & -- & -- \\
\hline XLC1OL & -. & - & 13.0 & 15.5 & 16.5 & 14.0 & 15.9 & 11.0 & -. & - & -- & - & .- & -. \\
\hline XLC1OA & -- & - & -- & - & -. & $\ldots$ & -. & 16.9 & 18.0 & 13.0 & -. & -. & $\cdots$ & -. \\
\hline
\end{tabular}

See footnotes at end of table. 
Table B10. Burnup of Spent Fuel Assemblles, by Type, Discharged by Year (Continued) (Gigawattdays thermal per metric ton of uranium)

\begin{tabular}{|c|c|c|c|c|c|c|c|c|c|c|c|c|c|c|}
\hline $\begin{array}{l}\text { Assembly } \\
\text { Type } \\
\text { Code }^{\text {a }}\end{array}$ & $\begin{array}{c}1968- \\
1969\end{array}$ & $\begin{array}{c}1970- \\
1971\end{array}$ & $\begin{array}{c}1972- \\
1973\end{array}$ & $\begin{array}{l}1974- \\
1975\end{array}$ & $\begin{array}{c}1976- \\
1977\end{array}$ & $\begin{array}{c}1978- \\
1979\end{array}$ & $\begin{array}{c}1980- \\
1981\end{array}$ & $\begin{array}{c}1982- \\
1983\end{array}$ & $\begin{array}{l}1984- \\
1985\end{array}$ & $\begin{array}{c}1986- \\
1987\end{array}$ & $\begin{array}{c}1988- \\
1989\end{array}$ & $\begin{array}{c}1990- \\
1991\end{array}$ & $1992^{b}$ & Temps $^{\circ}$ \\
\hline \multicolumn{15}{|c|}{ Pressurized-Water Reactors (PWR) } \\
\hline B1515B4 & -- & -- & -- & 11.6 & 21.1 & 25.7 & 27.7 & 28.3 & 31.0 & 32.2 & 31.7 & 32.9 & 36.9 & 20.8 \\
\hline B1515B4Z & -- & -- & -- & -- & -- & -- & -- & -- & -. & -- & -- & -- & 42.0 & -. \\
\hline B1515B5Z & -- & -- & -- & -- & -. & -- & -- & -- & -- & -- & -- & -. & 36.0 & -. \\
\hline $\mathrm{B} 1515 \mathrm{~B} 6$ & -- & -- & -. & -- & -- & -- & -- & -- & -- & -- & -- & -- & 41.6 & -- \\
\hline B1515B7 & -. & -. & -- & -- & -. & -- & -- & -- & -- & - & -- & -- & 41.0 & -- \\
\hline B1515BZ & -. & -. & -. & -- & -- & -. & $-\infty$ & -. & -- & 34.9 & 28.8 & 38.9 & 38.0 & -- \\
\hline B1515BGD & -- & -- & -- & .- & -- & -- & -- & -- & -- & 38.0 & 58.0 & -. & - & -- \\
\hline B1717B & -. & -- & -- & -- & -- & -- & 29.5 & -. & -- & - & .. & -- & -- & -. \\
\hline C1414C & -. & -- & -- & 12.9 & 17.4 & 22.8 & 30.8 & 32.2 & 34.3 & 37.6 & 39.1 & 40.8 & 41.5 & -- \\
\hline C1414A & -- & -- & -- & - & - & - & .. & -- & 35.7 & 37.0 & 38.6 & 36.6 & 47.0 & 26.9 \\
\hline C1414W & -. & -- & .. & -- & -- & -- & -- & 22.1 & 33.8 & 33.7 & 32.3 & 31.3 & 33.6 & - \\
\hline C1616CSD & -. & -- & -- & -. & -- & -- & 13.6 & 26.9 & 21.0 & 25.8 & 33.4 & 39.4 & 42.2 & -- \\
\hline C8016C & $-\infty$ & -- & -- & -- & -- & -- & -- & - & - & 17.1 & 21.1 & 32.6 & 34.6 & 18.0 \\
\hline W1414W & -- & 7.7 & 17.6 & 24.4 & 26.6 & 31.0 & 32.1 & 34.3 & 35.2 & 30.5 & -. & 39.5 & 39.0 & $\ldots$ \\
\hline W1414WL & -- & -- & 18.4 & 24.3 & 27.2 & 32.8 & 34.3 & 35.5 & 36.2 & 36.4 & 40.0 & 43.0 & & -. \\
\hline W1414WO & -. & -. & -. & - & 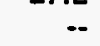 & .. & -. & 19.4 & 40.7 & 36.0 & 39.5 & 39.7 & 41.8 & -. \\
\hline W1414A & -- & -- & -. & -- & 25.3 & -- & 28.6 & 36.2 & 35.2 & 37.2 & 35.9 & 36.9 & 36.8 & -. \\
\hline W1414ATR & -- & -- & -- & -- & & -- & -- & - & 37.4 & 38.0 & 38.5 & 45.0 & 44.0 & -- \\
\hline W1414B & -. & -- & -- & -- & 24.4 & - & -- & -. & -- & -- & -. & - & - & -- \\
\hline W1515W & -- & -- & -- & 19.3 & 21.2 & 30.7 & 29.0 & 33.1 & 35.2 & 35.5 & 37.2 & 39.2 & -- & 25.1 \\
\hline W1515WL & -- & -- & 15.9 & 21.1 & 24.3 & 30.2 & 31.5 & 33.3 & 32.9 & 35.0 & 35.4 & 36.4 & 43.6 & - \\
\hline W1515WO & -- & -- & -- & - & -. & -- & -. & - & 18.3 & 32.1 & 35.9 & 35.6 & 37.2 & -- \\
\hline W1515A & -- & -- & -- & -- & -- & 30.3 & 31.6 & 31.4 & 30.6 & 32.6 & 35.7 & 38.0 & 39.9 & -. \\
\hline W1717WL & .. & - & -- & -- & 16.1 & 17.0 & 28.0 & 25.2 & 28.4 & 29.6 & 32.7 & 34.5 & 34.8 & 28.8 \\
\hline W1717Wo & .- & .- & -- & -- & -- & - & -- & - & 36.1 & 20.4 & 30.2 & 34.0 & 37.3 & -- \\
\hline W1717WV5 & -- & -. & -. & -- & -. & -- & -. & -- & - &.. & 46.0 & 38.3 & 39.3 & -- \\
\hline W1717WVH & -- & -- & -- & -- & -- & -- & -- & -- & -- & -- & - & 34.7 & 33.5 & -- \\
\hline W1717A & -- & -- & -- & -. & -- & -. & -- & -- & -. & 37.0 & 38.6 & 38.9 & 42.1 & -- \\
\hline W1717B & -- & -- & -- & -- & -- & --. & -- & -- & -- & -. & -- & 42.0 & 8.2 & -- \\
\hline WST17W & -- & -- & -- & .- & -. & $\therefore$ & -- & -- & .. & - & 12.0 & 19.4 & 29.7 & -- \\
\hline $\mathrm{XFC14C}$ & -- & -- & -- & 8.6 & 25.5 & 24.0 & 30.9 & 36.1 & 39.2 & 39.0 & -. & 31.0 & 39.1 & -- \\
\hline XFC14A & -- & -- & -- & -- & -- & -. & -- & -- & 34.8 & 36.3 & 38.9 & 41.0 & 38.0 & -- \\
\hline XHN15W & -- & 22.5 & 29.3 & 32.8 & 31.2 & -- & -- & -- & -. & -. & -. & -. &. & -. \\
\hline XHN15MS & -- & -- & 28.3 & -- & -- & -- & -- & -- & -- & -- & -- & -- & -- & -- \\
\hline XHN15MZ & -- & -- & 25.6 & -- & -- & -- & -- & -- & -- & -. & -- & -. & .. & .. \\
\hline XHN15HS & -. & - & -. & 32.2 & -- & .- & -- & -. & -- & -. & .- & -- & -. & -- \\
\hline XHN15HZ & -. & -- & 18.5 & -- & -- & -- & -- & - & -- & .- & -- & .. & -. & .. \\
\hline XHN15B & -- & -- & -. & -. & 33.5 & 32.7 & 33.8 & 33.8 & 35.2 & 33.9 & 32.0 & 34.3 & -- & 22.4 \\
\hline XIP14W & -- & -- & 25.2 & 13.8 & - & -. & -. & -- & -. & -. & -. & -. & -- & - \\
\hline XPA15C & -- & -- & -. & 11.3 & -- & 26.7 & 30.3 & -- & -. & -. & -- & .. & .. & .. \\
\hline XPA15A & -- & -- & -- & - & -- & 20.2 & 35.3 & 33.3 & 34.8 & -- & 35.0 & 36.7 & 32.4 & 23.5 \\
\hline XSL16C & -. & -. & -. & .- & -- & .. & -. & -- & 12.6 & 29.5 & 39.1 & 39.8 & 43.6 & .. \\
\hline XSO14W & -- & 21.7 & 29.0 & 28.9 & 31.9 & 31.9 & 30.5 & -- & 29.7 & .. & 34.9 & 30.0 & 21.7 & .. \\
\hline XYR18W & -- & -- & 23.9 & 27.0 & -. & -- & -. & -- & -. & .- & .- & -- & -. & -. \\
\hline XYR16U & -. & - & -. & 26.9 & 28.2 & -- & -. & .. & -- & .- & .. & -- & .. & .. \\
\hline XYR16A & -- & -- & -- & -- & -. & 27.3 & 29.8 & 29.0 & 28.5 & 31.0 & .- & -. & .- & -. \\
\hline XYR16C & -- & -- & -. & -- & -. & -. & .. & -. & .. & -. & 32.0 & 21.6 & -- & .. \\
\hline
\end{tabular}

\footnotetext{
Assembly types are Identifled in Table B4.

bome data for prevlous years have been revlsed. Current-y ear data may be revised in future publicatlons. When utilttles relnsert assemblles discharged In prevlous years, hlstorlcal totals change. See Technical Note 12 in Appendlx E.

TTemps are temporarlity discharged assemblles, as of December 31, 1992, as reported on Form RW-859.

$-=$ Not applicable.

Source: Energy Information Adminlstration, Form RW-859, "Nuclear Fuel Data" (1992).
} 
Appendix C

Pictograms Showing Reactor Storage Pool Configurations, Pool Capacities, and Inventories at Nuclear Power Plant Sites and Storage Facilities

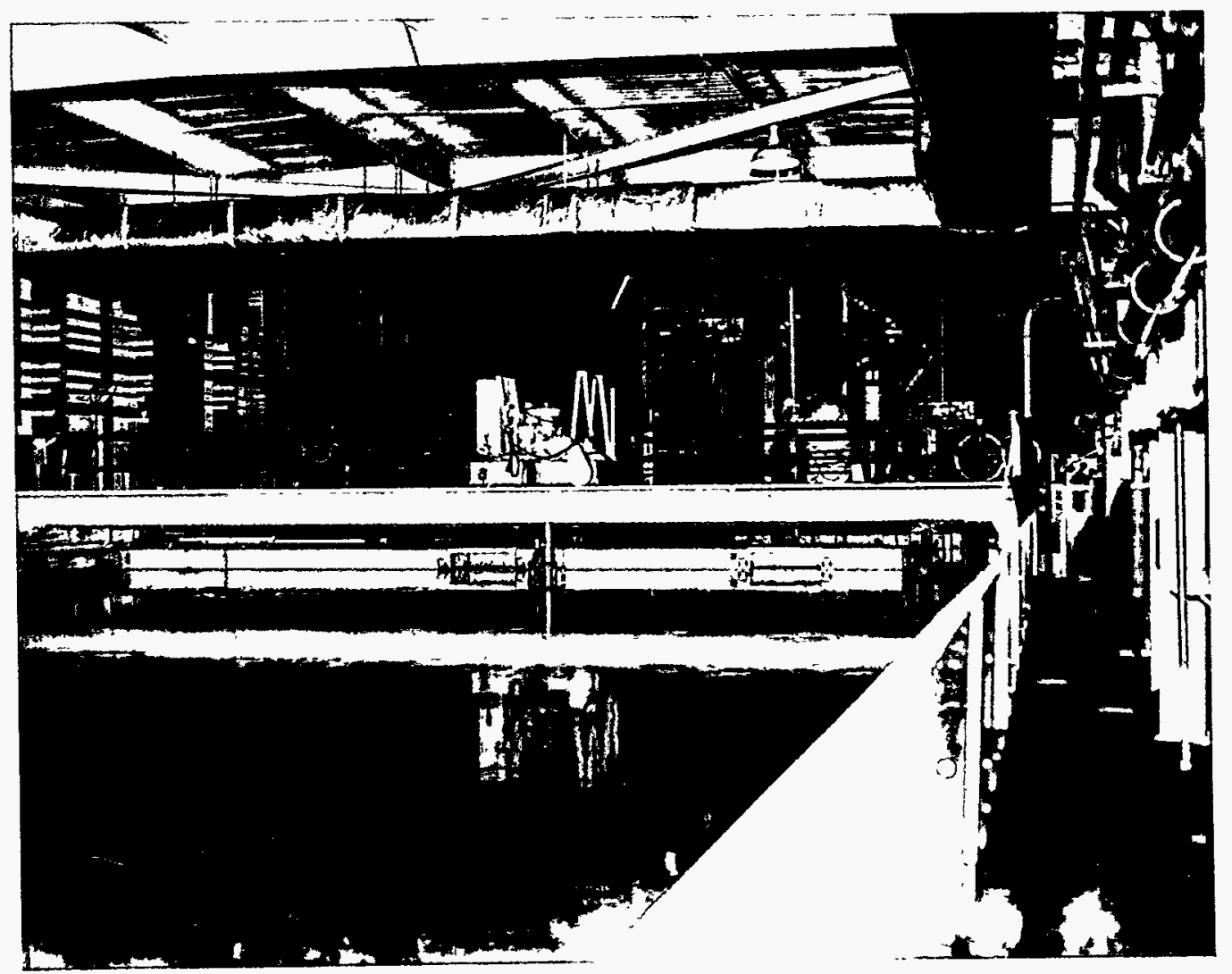


$\ldots$ 


\section{Pictograms Showing Reactor Storage Pool Configurations, Pool Capacities, and Inventories at Nuclear Power Plant Sites and Storage Facilities}

The pool site pictograms contained in this appendix graphically compare the maximum storage capacity of each spent fuel storage pool site with its inventory as of December 31, 1992. (A pool site is a configuration of pools having a single cask loading area.) EIA defines maximum established spent fuel capacity for the site as the number of intact assemblies that will be able to be stored at some point in the future (between the reporting date and the reactor's end of life) considering any established or current studies or engineering evaluations, at the time of submittal for licensing approval from the U.S. Nuclear Regulatory Commission. (See Technical Note 13).

The pictograms are grouped by utility and highlight the various reactor/pool site configurations, such as the absence or presence of transfer canals, multiple pools servicing single reactors, and reactors sharing one pool site. Multiple storage pools linked by transfer canals have one pool site ID number because they share a common cask loading dock. In the pictograms, temporarily discharged assemblies are included in the present inventory totals. The movement of fuel between the storage pools at an individual site is represented by arrows. Two different designs of Independent Spent Fuel Storage Installations (ISFSIs) are also shown. Additional information on the ISFSIs is reported in Chapter 2.

The definition of an assembly has been extended to include assembly skeletons occupying the same space (slot) as a single assembly. A slot holds the equivalent of one nuclear fuel assembly in a matrix within a spent fuel storage rack. 


\section{Legend}

In Appendix $C$ the pictograms show selected data from Tables 13 and 14. Data in the pictograms include Utility and Reactor Names, Pool Site ID Number, Maximum Established Storage Capacity, and Current Inventory.

Each pictogram illustrates reactor/pool site configurations, such as the absence or presence of transfer canals, multiple pools servicing single reactors, and reactors sharing one pool site. Multiple storage pools linked by transfer canals have one pool site ID number. Two different designs of Independent Spent Fuel Storage Installations (ISFSIs) are depicted. A pie chart indicating the reactor's percentage of storage capacity remaining is shown in each pictogram. A State map has been included indicating the location of reactor(s) within that State.
Maximum established storage capacity and current inventory in number of assemblies.

\section{Utility Name}

\section{Reactor Name}

\section{POOL SITE ID \#}

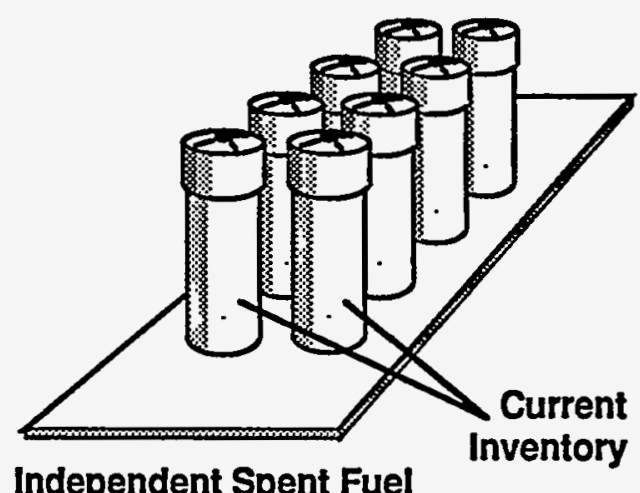

The arrow depicts movement of discharged assemblies from a reactor to a pool or storage module, or from one pool to another.

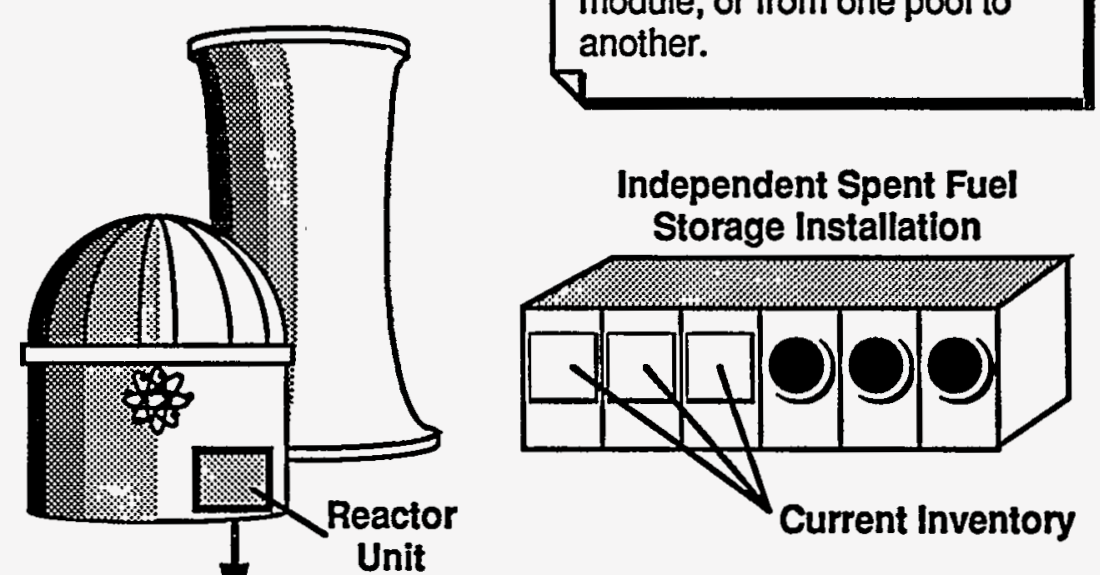

Storage Installation

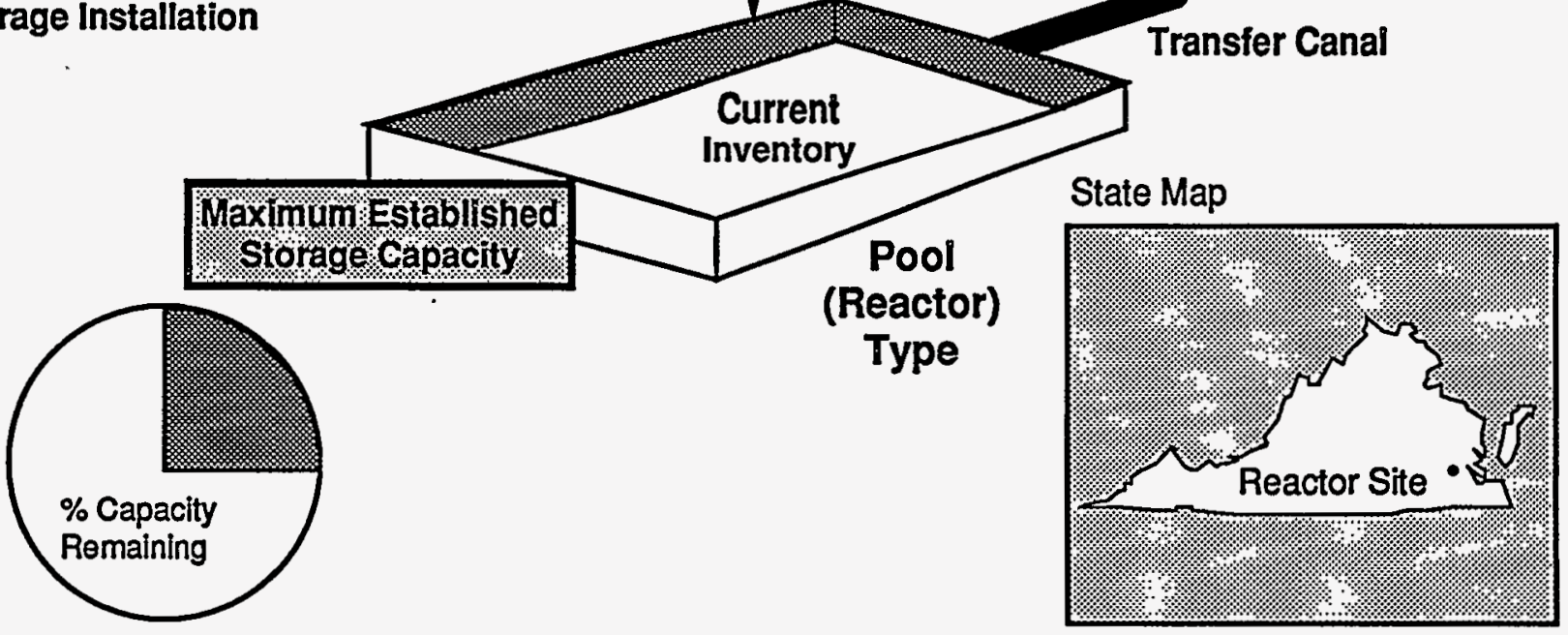




\section{Alabama Power Company Farley}
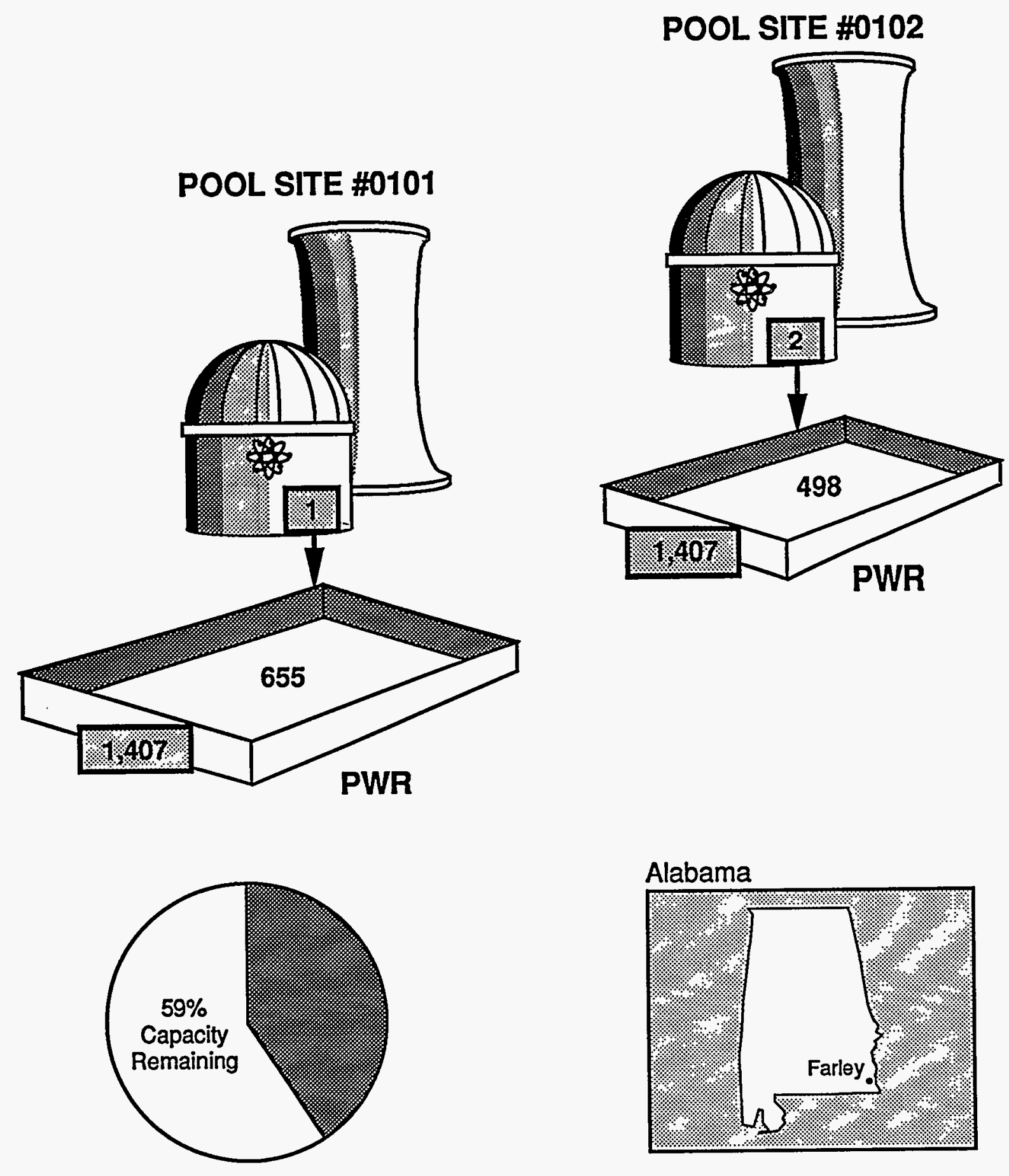


\section{Arizona Public Service Company Palo Verde}
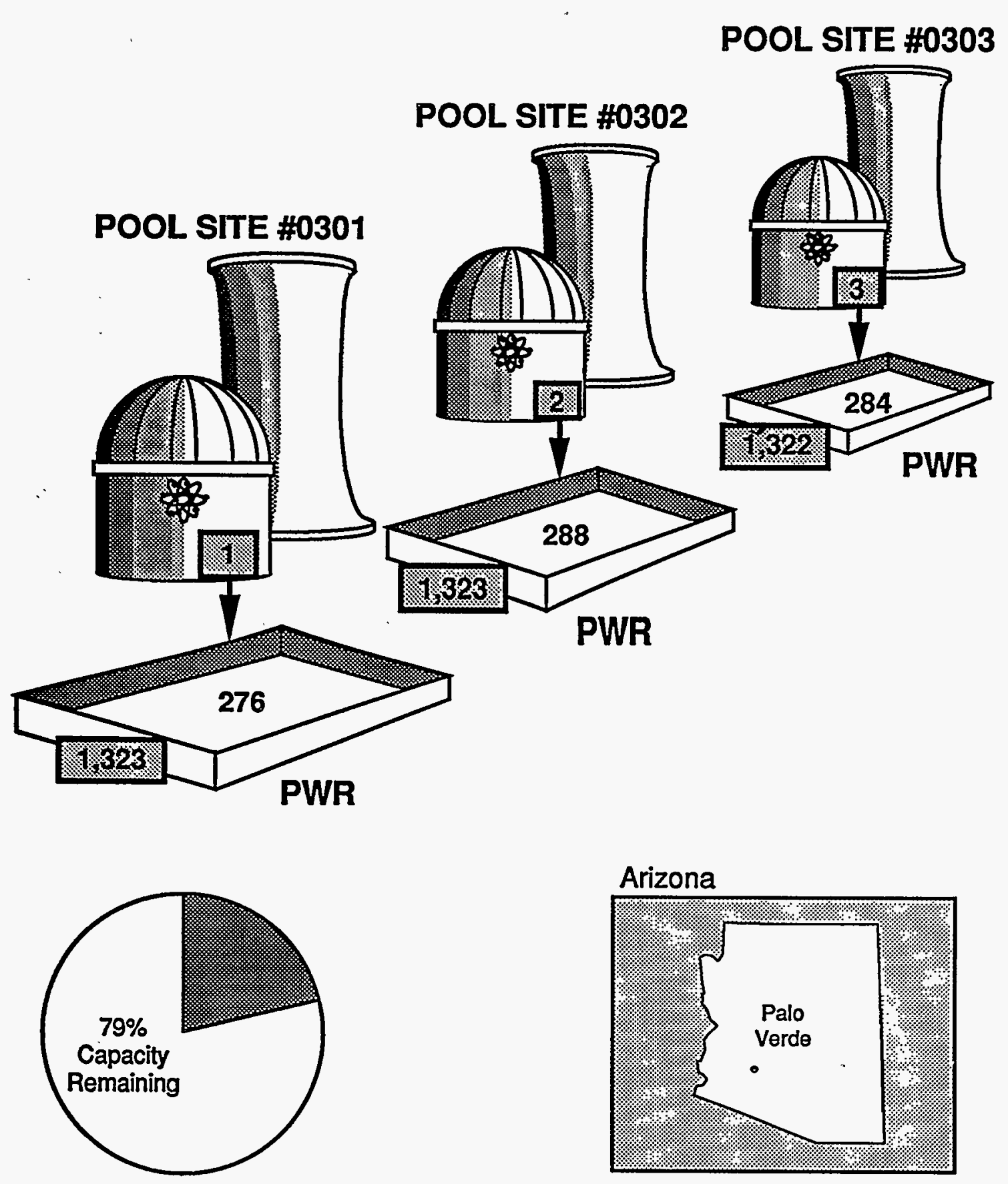


\section{Arkansas Power and Light Company Arkansas Nuclear}

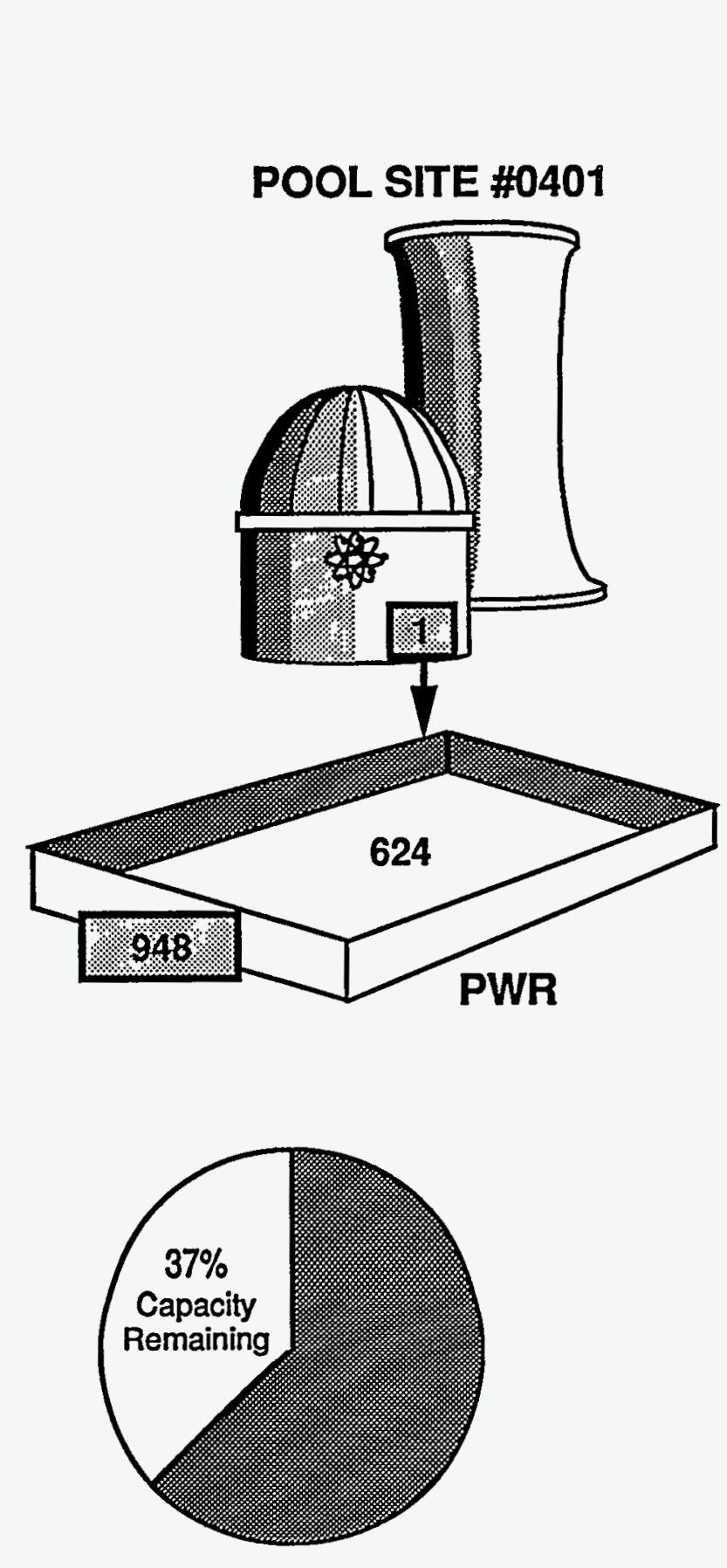

POOL SITE \#0402

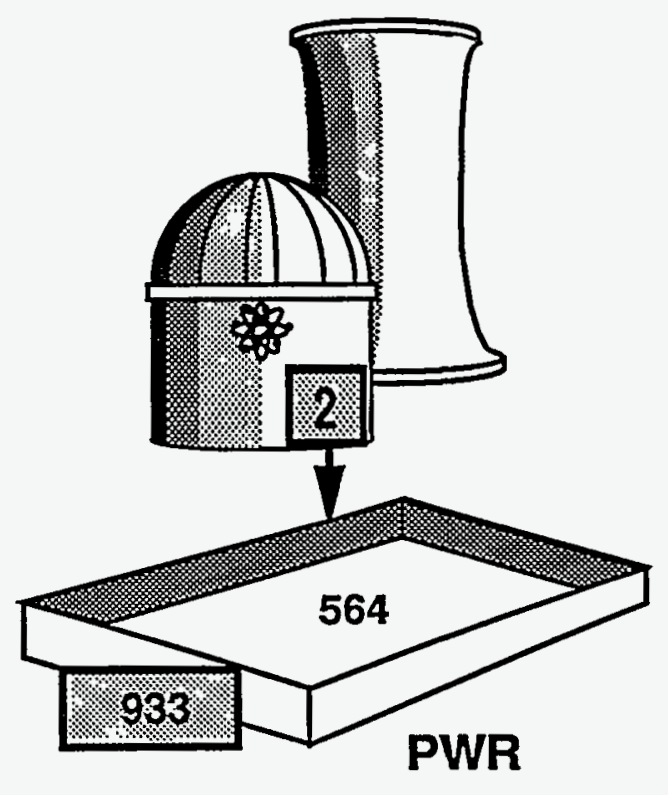




\section{Baltimore Gas and Electric Company \\ Calvert Cliffs}
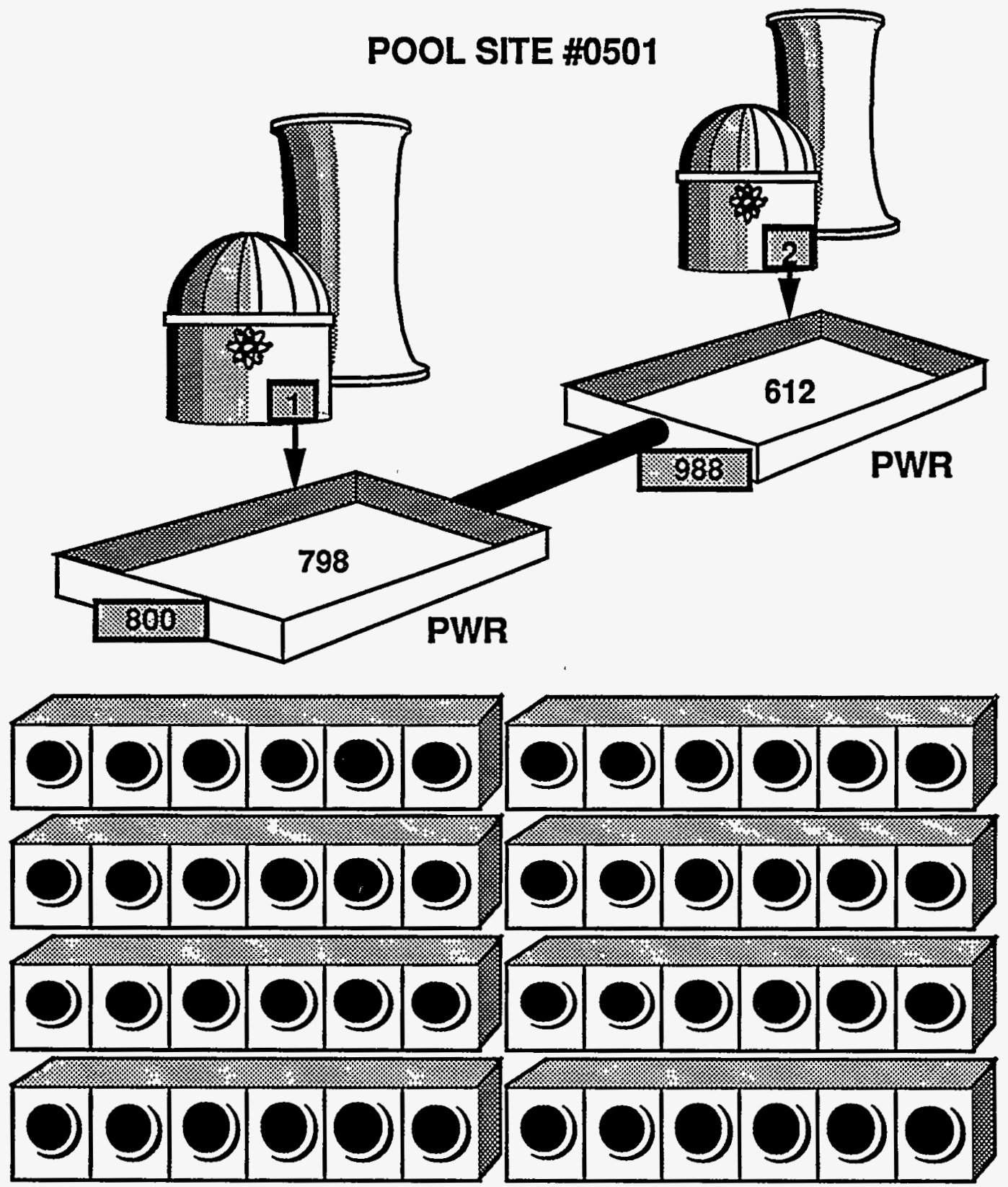

Independent Spent Fuel Storage Installation
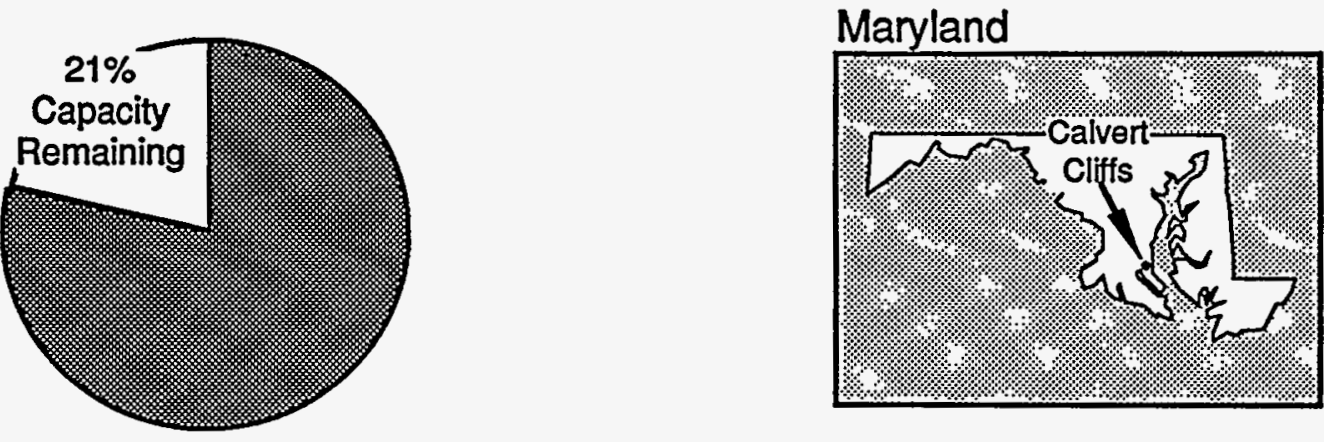


\section{Boston Edison Company Pilgrim}

POOL SITE \#0601
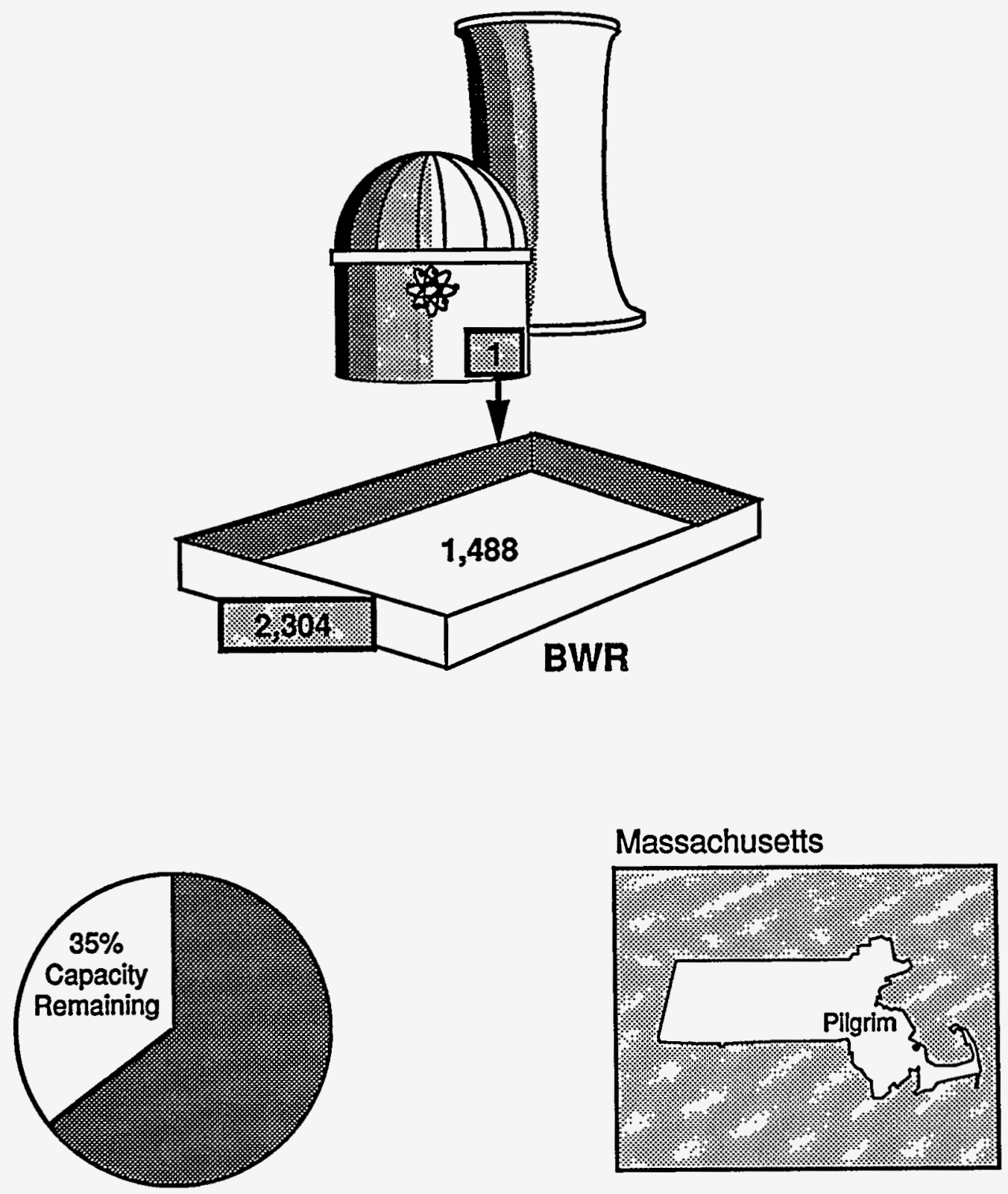


\section{Carolina Power and Light Company}

Brunswick

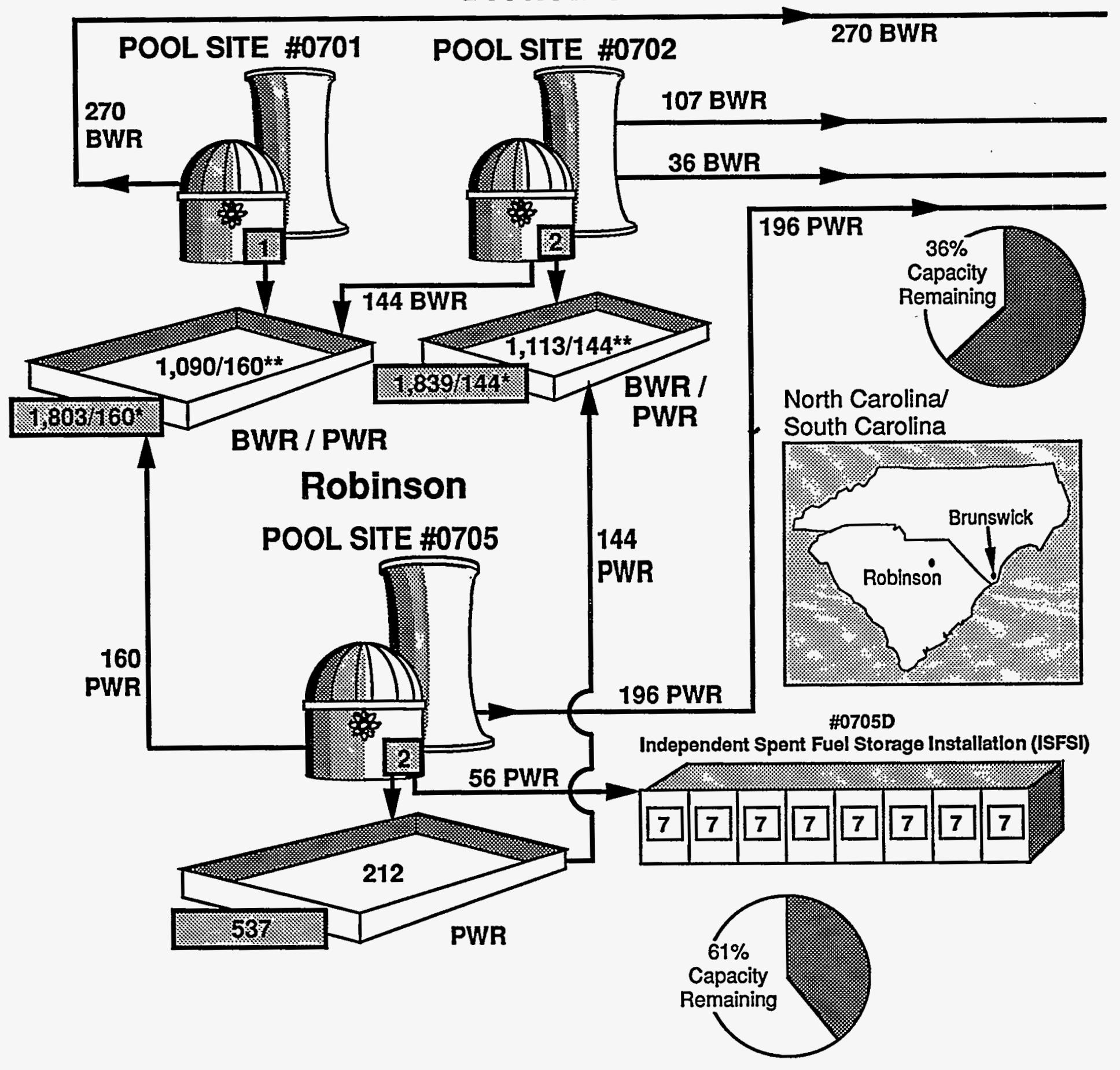

-The first number in the ratio denotes the maximum established storage capacity for assemblles in the BWR portion of the Brunswick pools. The second number is the maximum established storage capacity for assemblies in the PWR portion of the Brunswick pools.

*n The first number in the ratio denotes the current inventory of assemblies stored in the BWR portion of the Brunswiek pools. The second number is the current inventory of assemblies stored in the PWR portion of the Brunswick pools.

Notes: The arrows depict movement of assemblies from a storage pool site to another storage pool site or ISFSI. The numbers beside the arrows indicate the number of assemblies moved. 


\section{Carolina Power and Light Company \\ (Continued)}

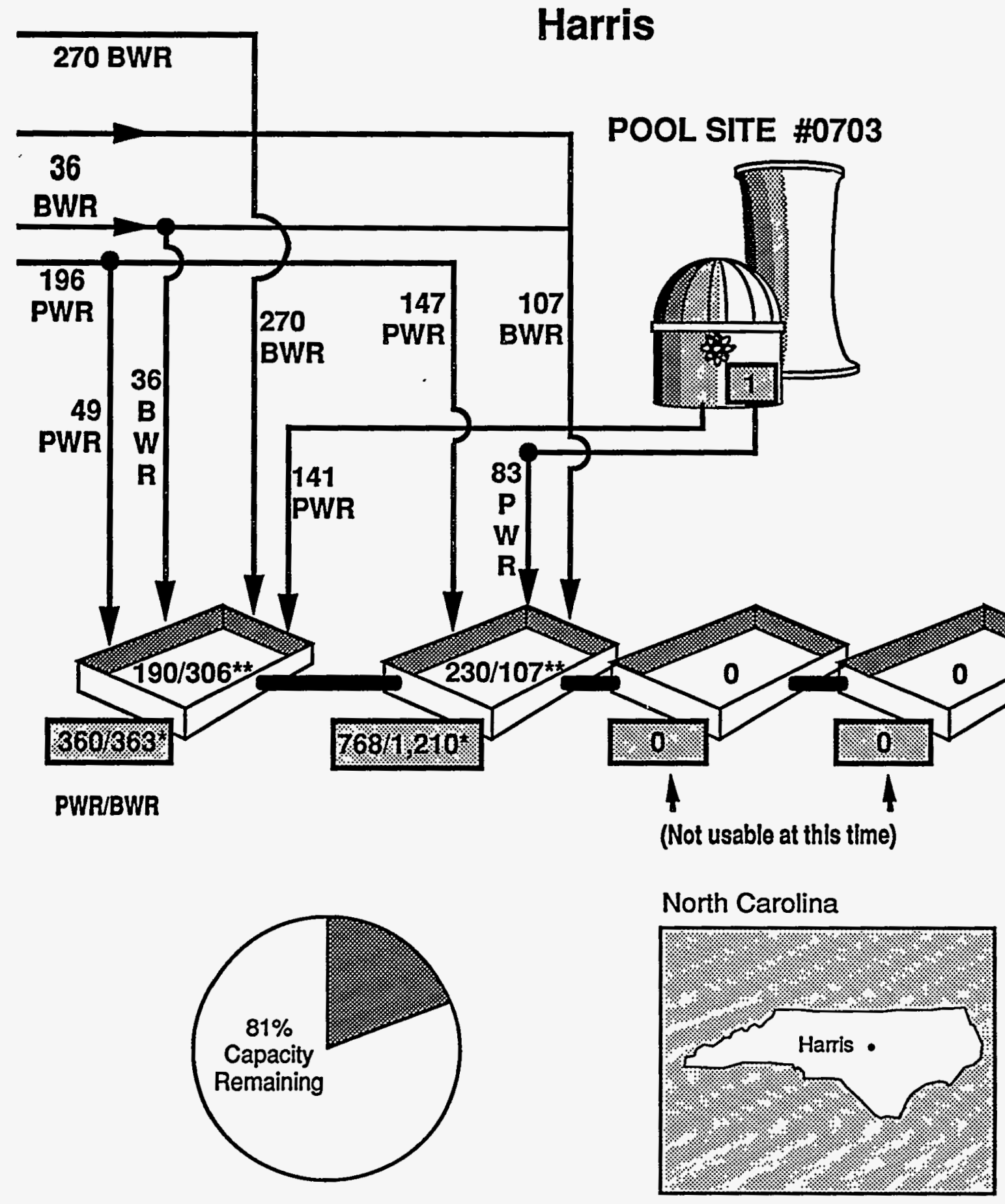

-The first number in the ratio denotes the maximum established storage capacity for assemblies in the PWR portion of the Harris pool. The second number is the maximum established storage capacity for assemblies in the BWR portion of the Harris pool.

The first number in the ratio denotes the current inventory of assemblies stored in the PWR portion of the Harris pool. The second number is the current inventory of assemblies stored in the BWR portion of the Harris pool.

Notes: The Harris pools are licensed to hold up to 4,184 PWR or 5,808 BWR assemblies or a combination of each. In addition to the capaclites shown, 704 PWR and 968 BWR racks are available as needed from site storage. These capacities reflect the maximum storage unili additional storage racks are purchased. 


\section{Cleveland Electric Illuminating Company \\ Perry}

POOL SITES \#0901 and \#0902
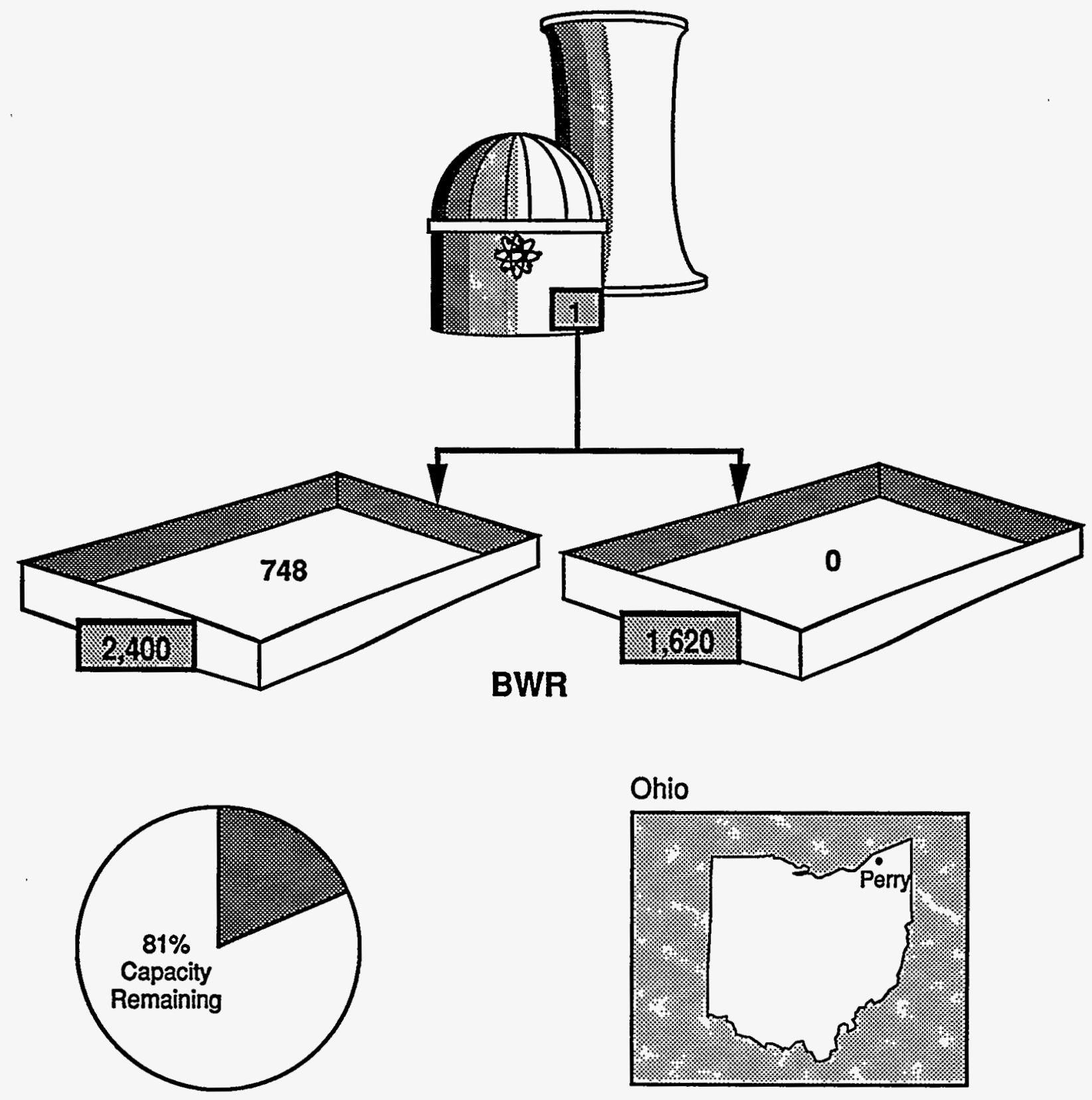

Ohio

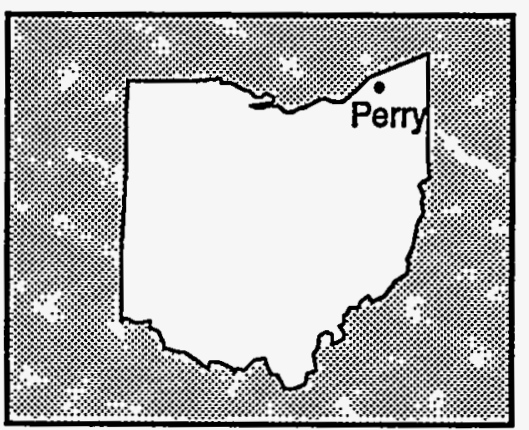




\section{Commonwealth Edison Company}

\section{Braidwood}

\section{POOL SITE \#1001}

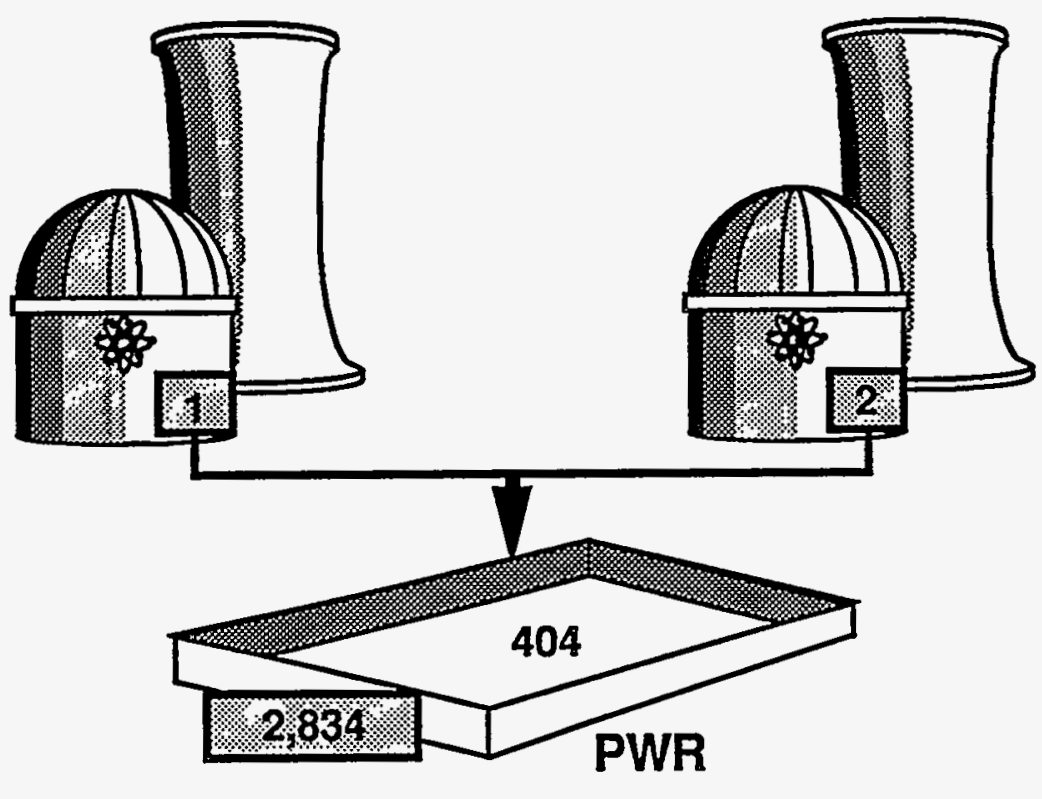

\section{Byron}

POOL SITE \#1003
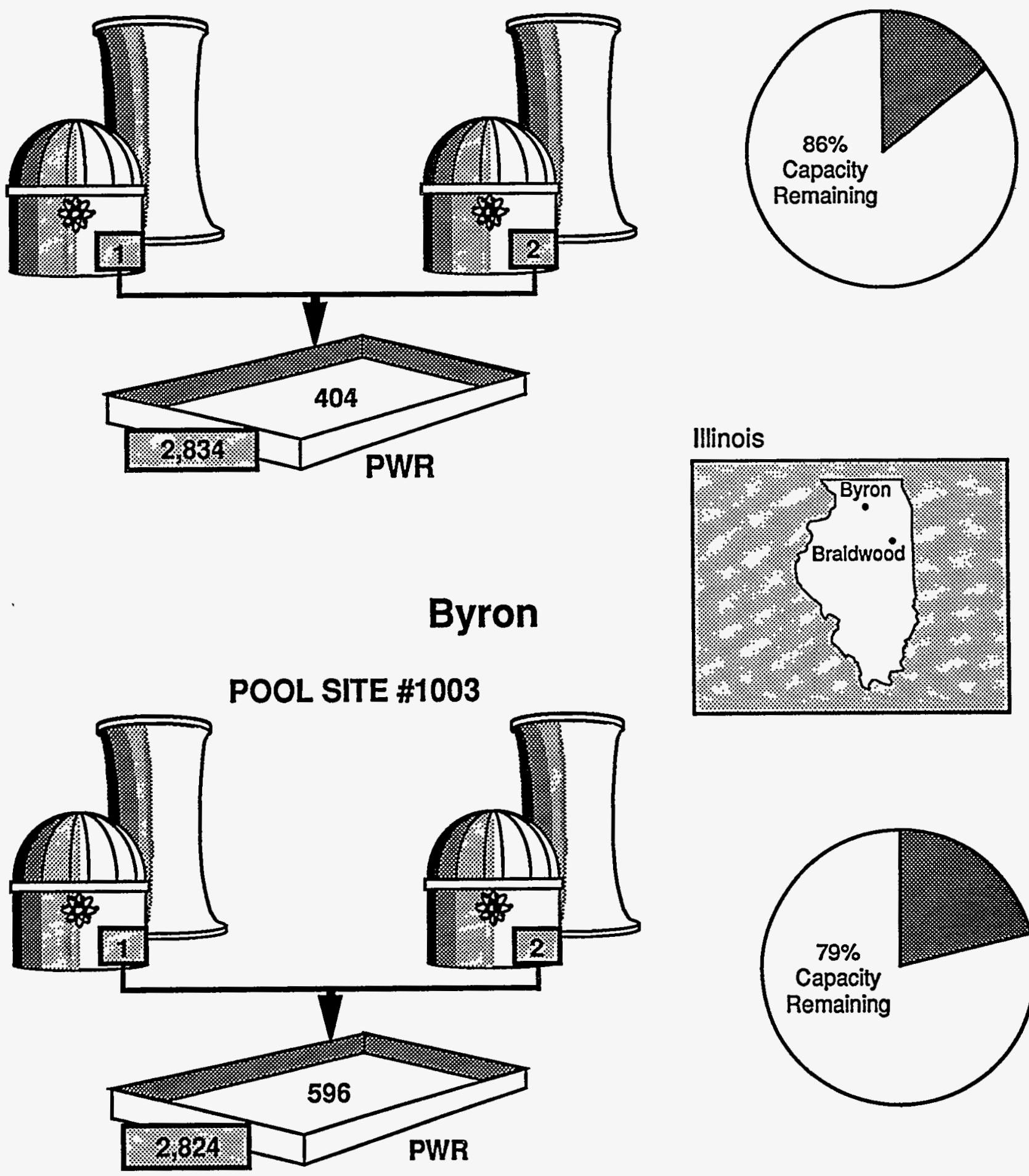

Illinois

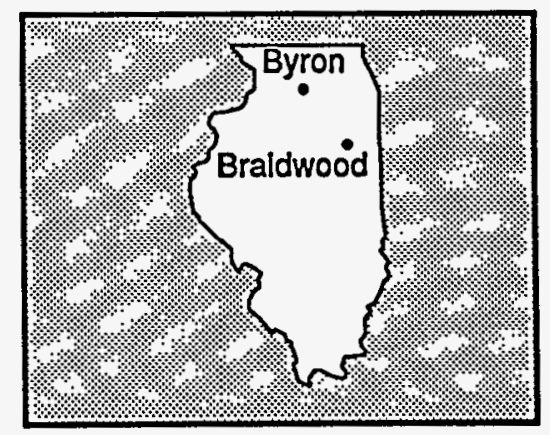




\section{Commonwealth Edison Company (Continued) \\ Dresden}

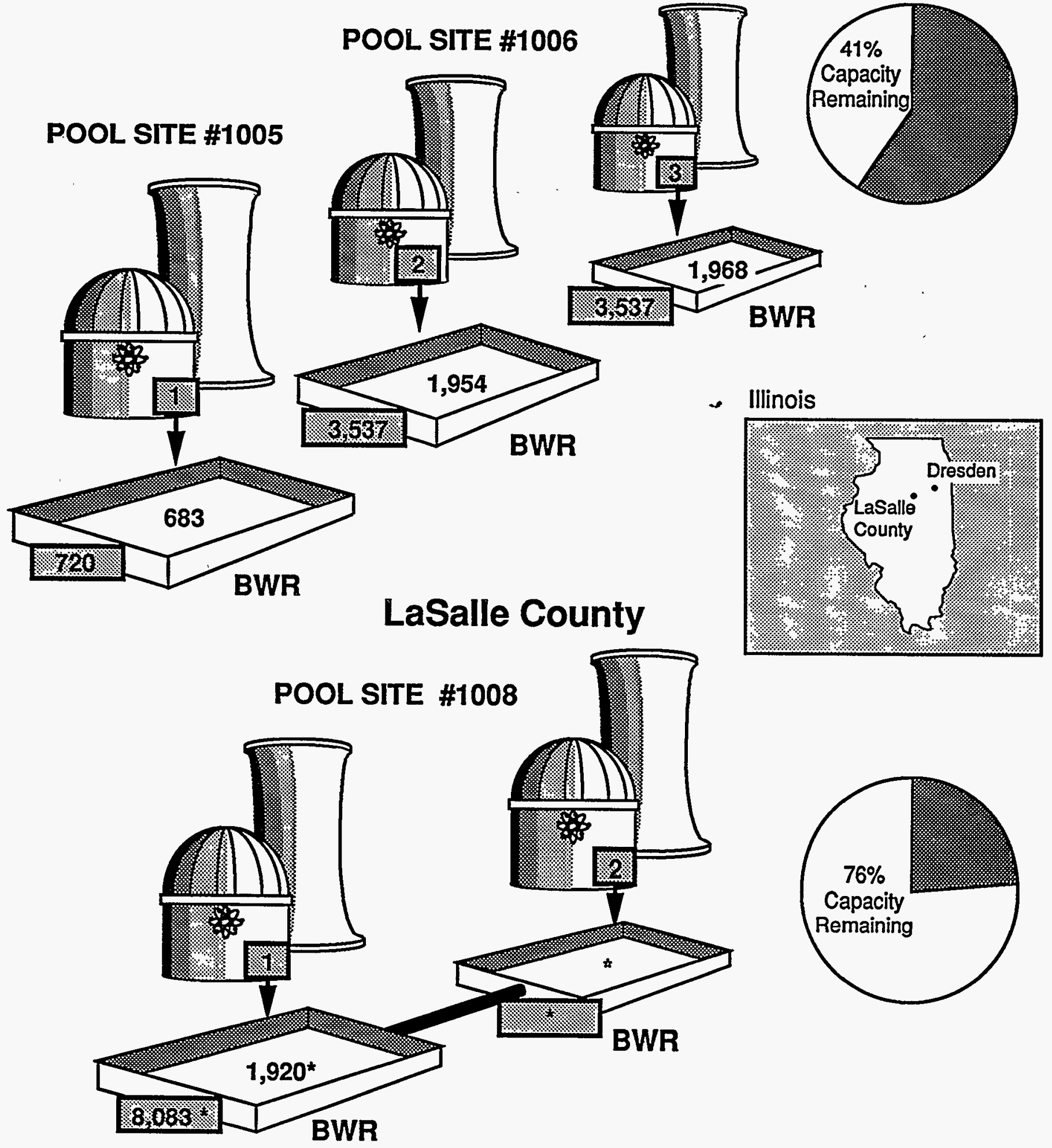

- Reported by utility as a single pool. 


\section{Commonwealth Edison Company (Continued) \\ Quad Cities}

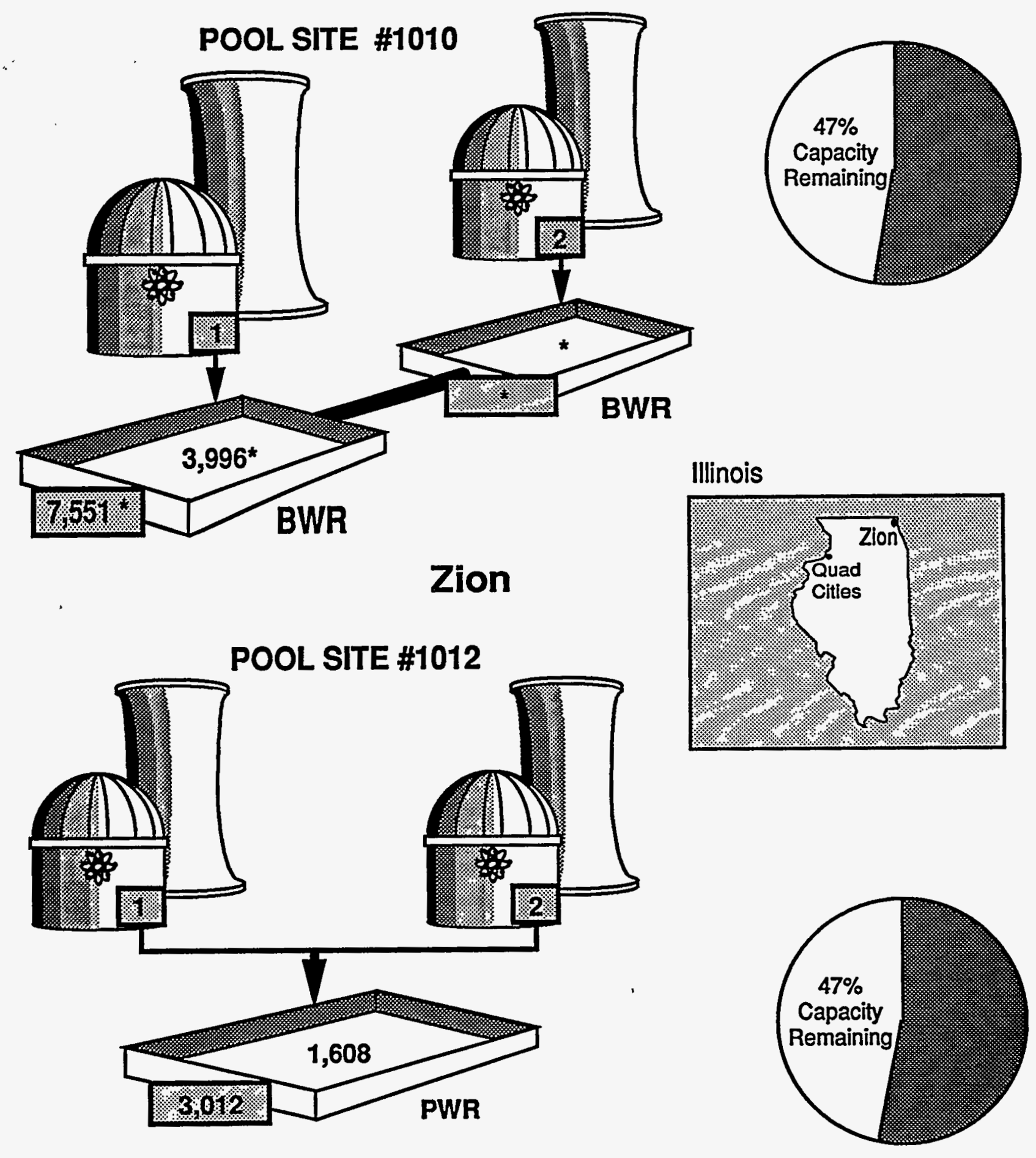

- Reported by utility as a single pool. 


\section{Consolidated Edison Company of New York \\ Indian Point*}

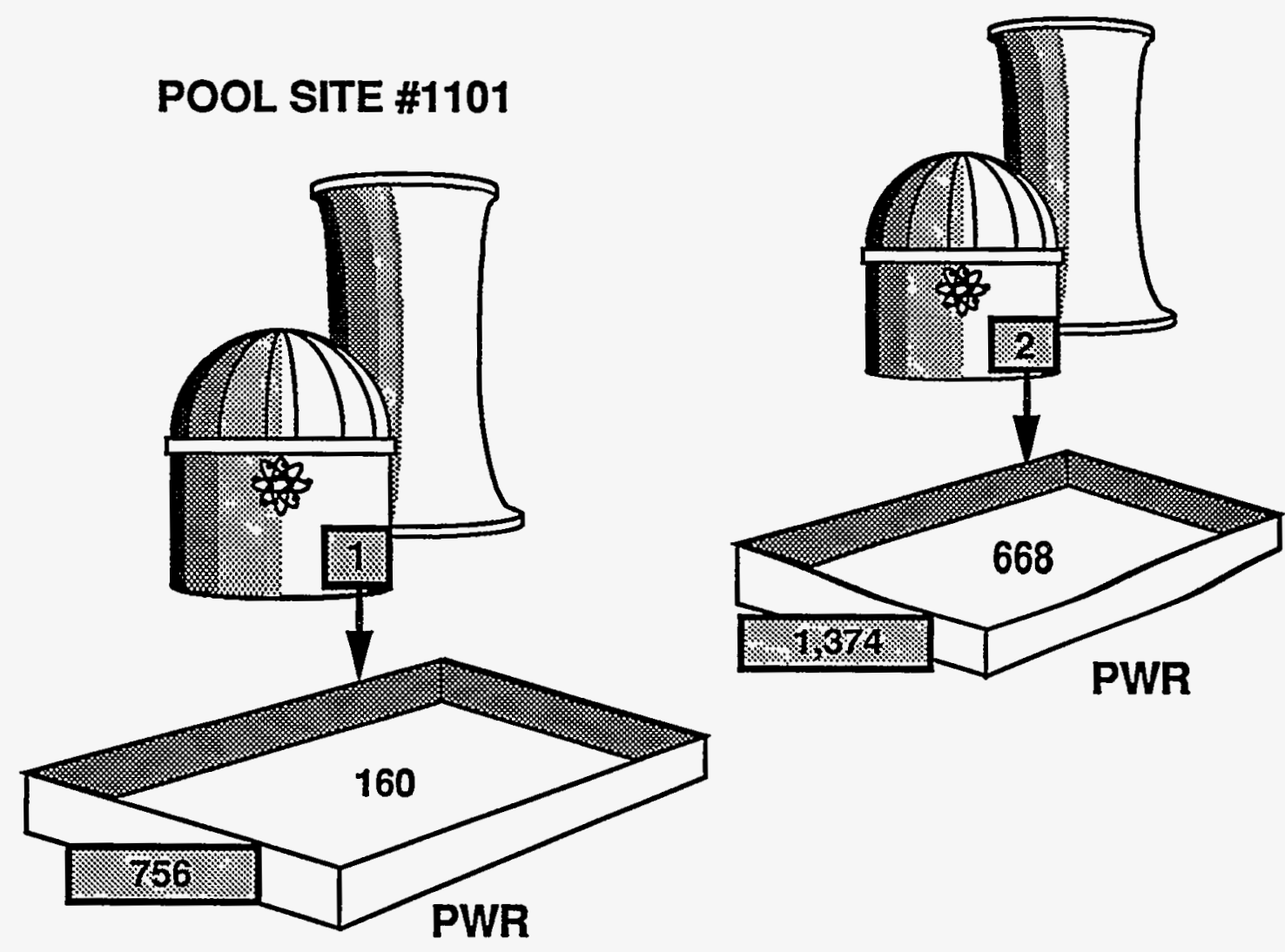

POOL SITE \#1102
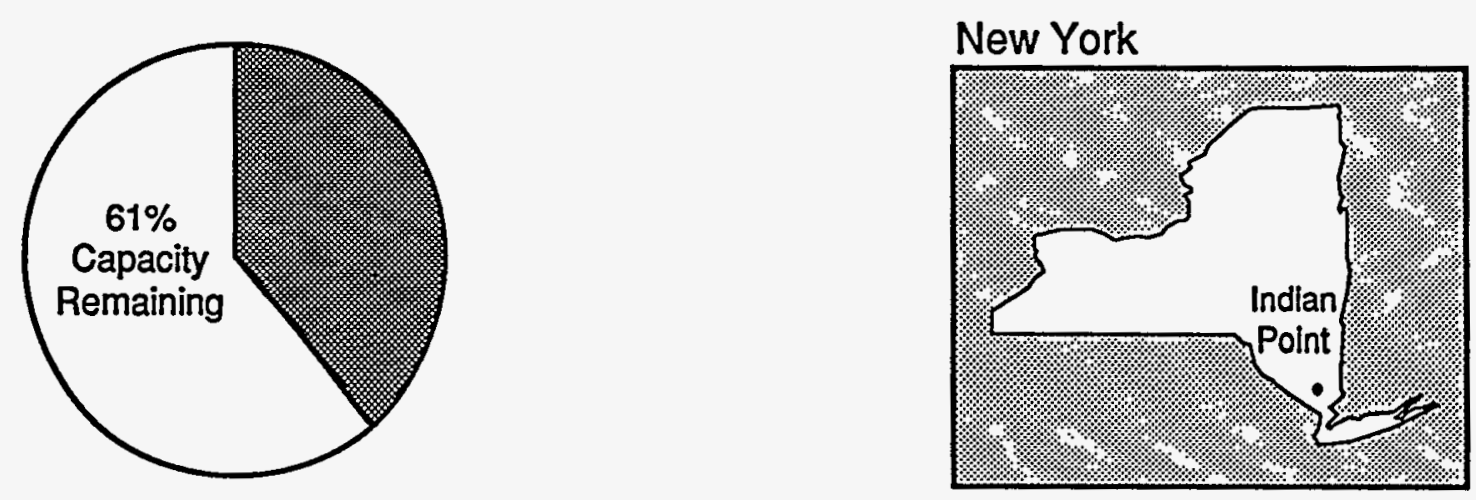

- Reactors at this site are Indian Point 1 \& 2. 


\section{Consumers Power Company Big Rock Point}

POOL SITE \#1201
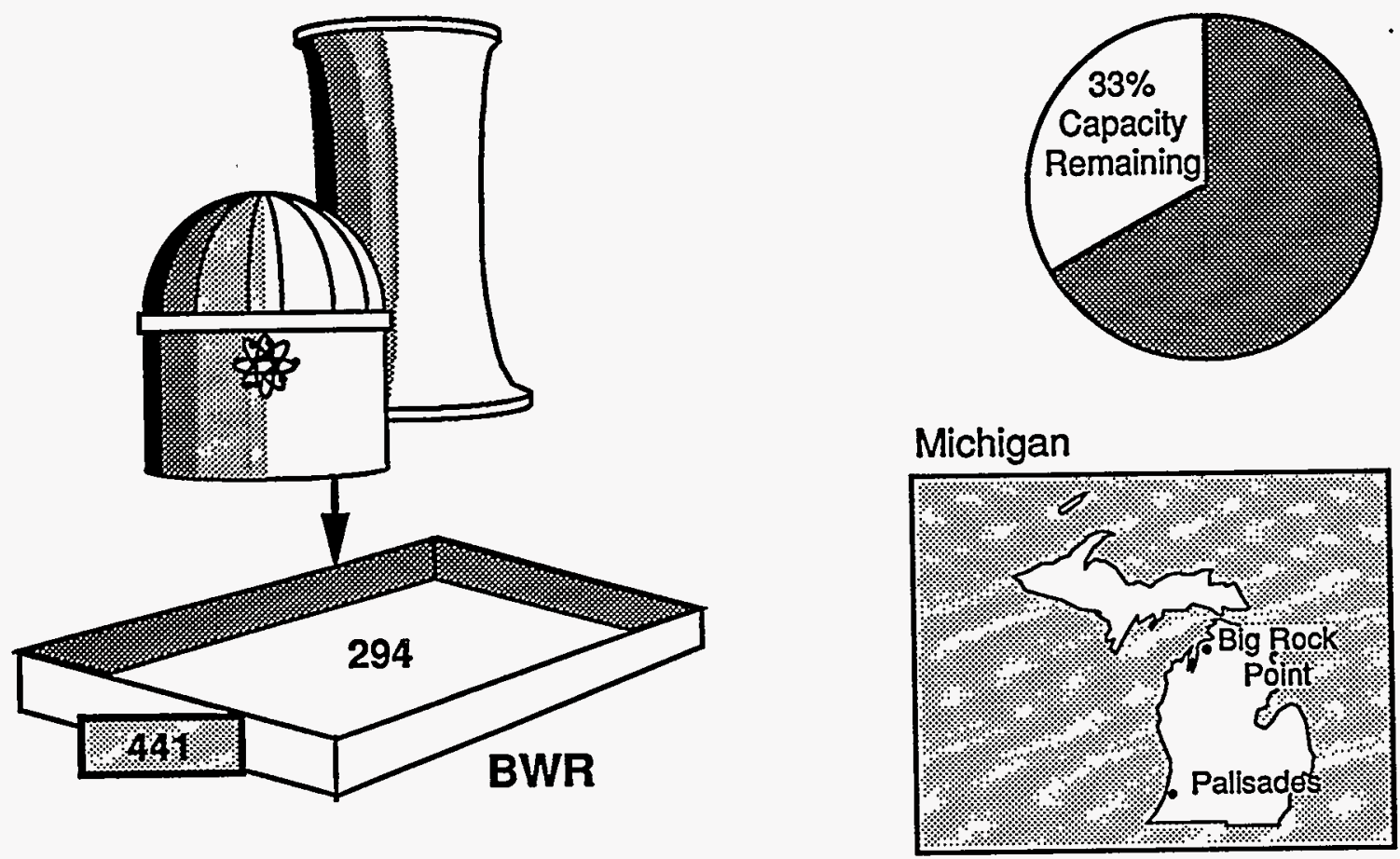

\section{Palisades}

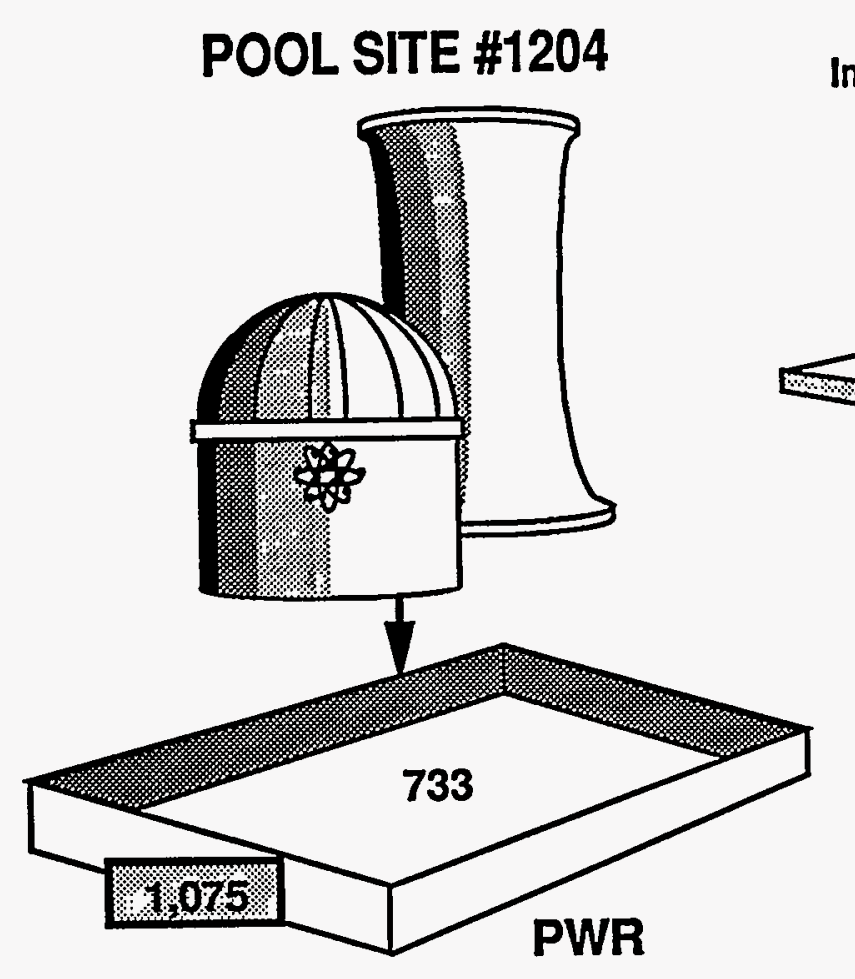

Independent Spent Fuel Storage Installation

\#1204D

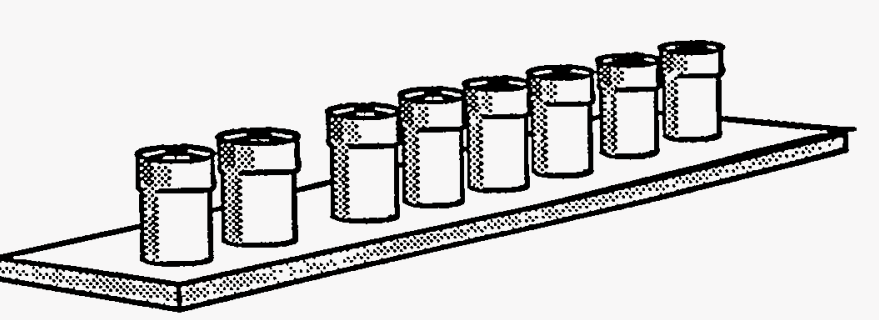




\section{Dairyland Power Cooperative \\ LaCrosse}
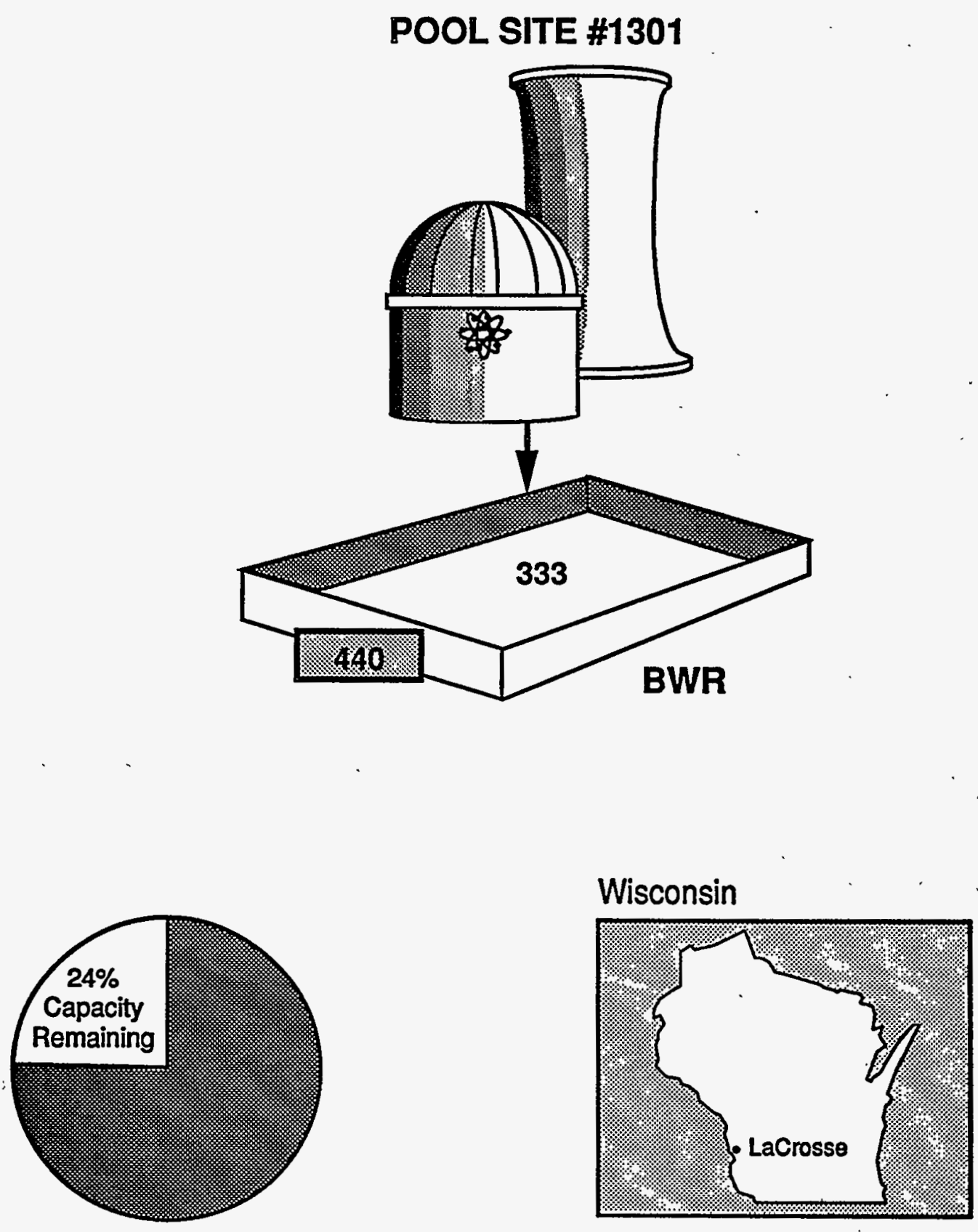


\section{Detroit Edison Company Enrico Fermi}
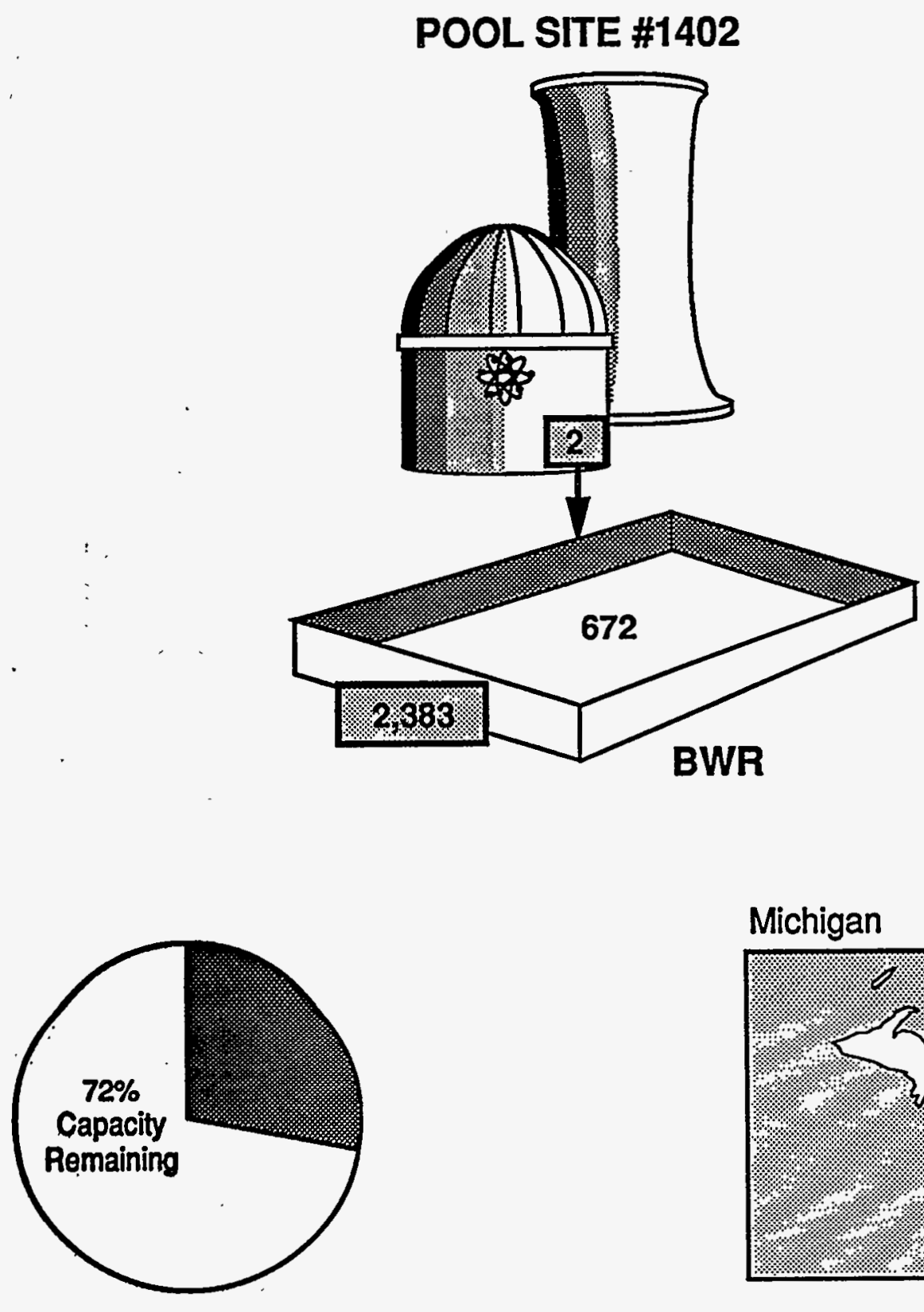

Michigan

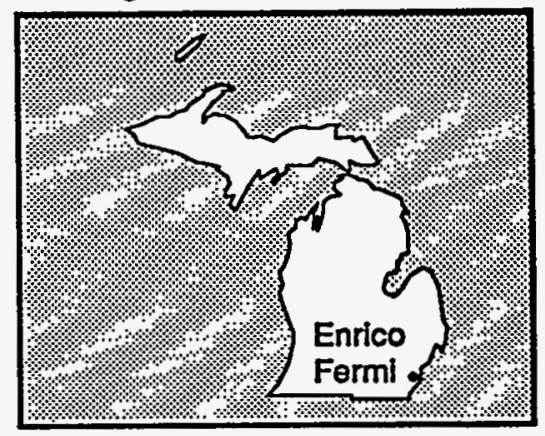




\section{Duke Power Company \\ Catawba}

POOL SITE \#1502
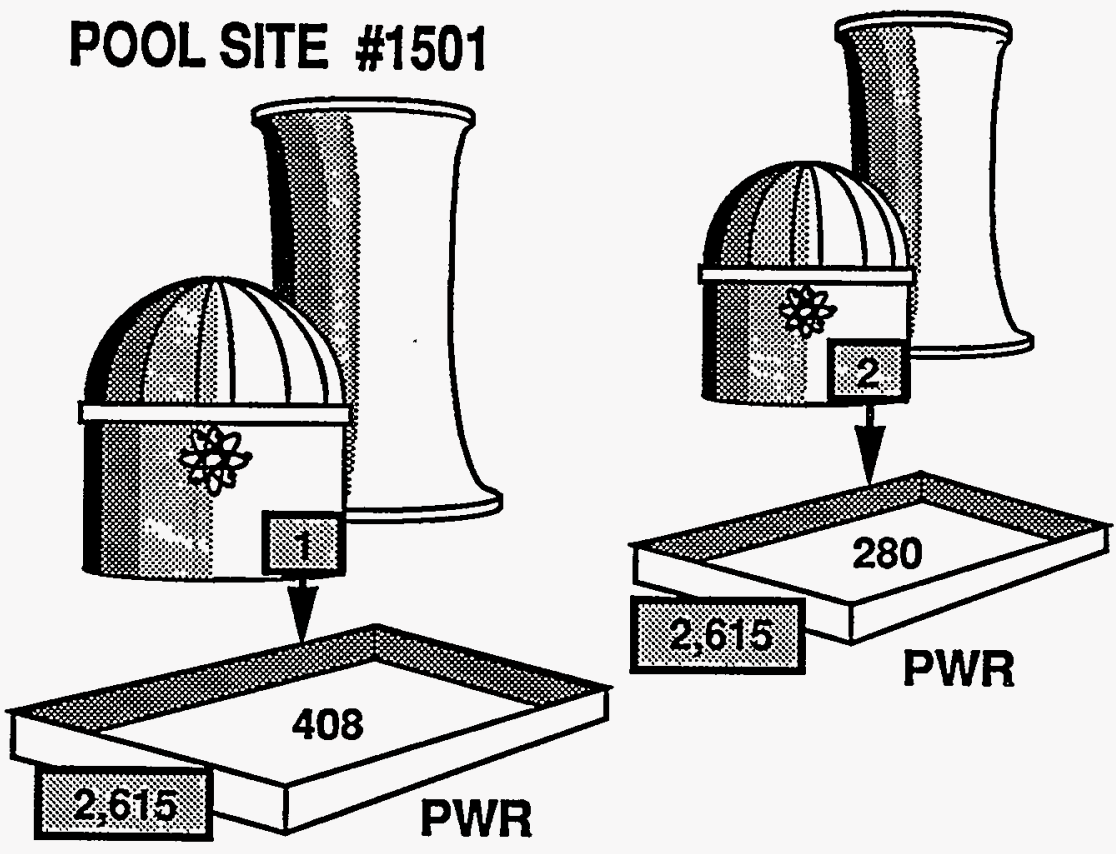

\section{McGuire}

POOL SITE \#1505

POOL SITE \#1504
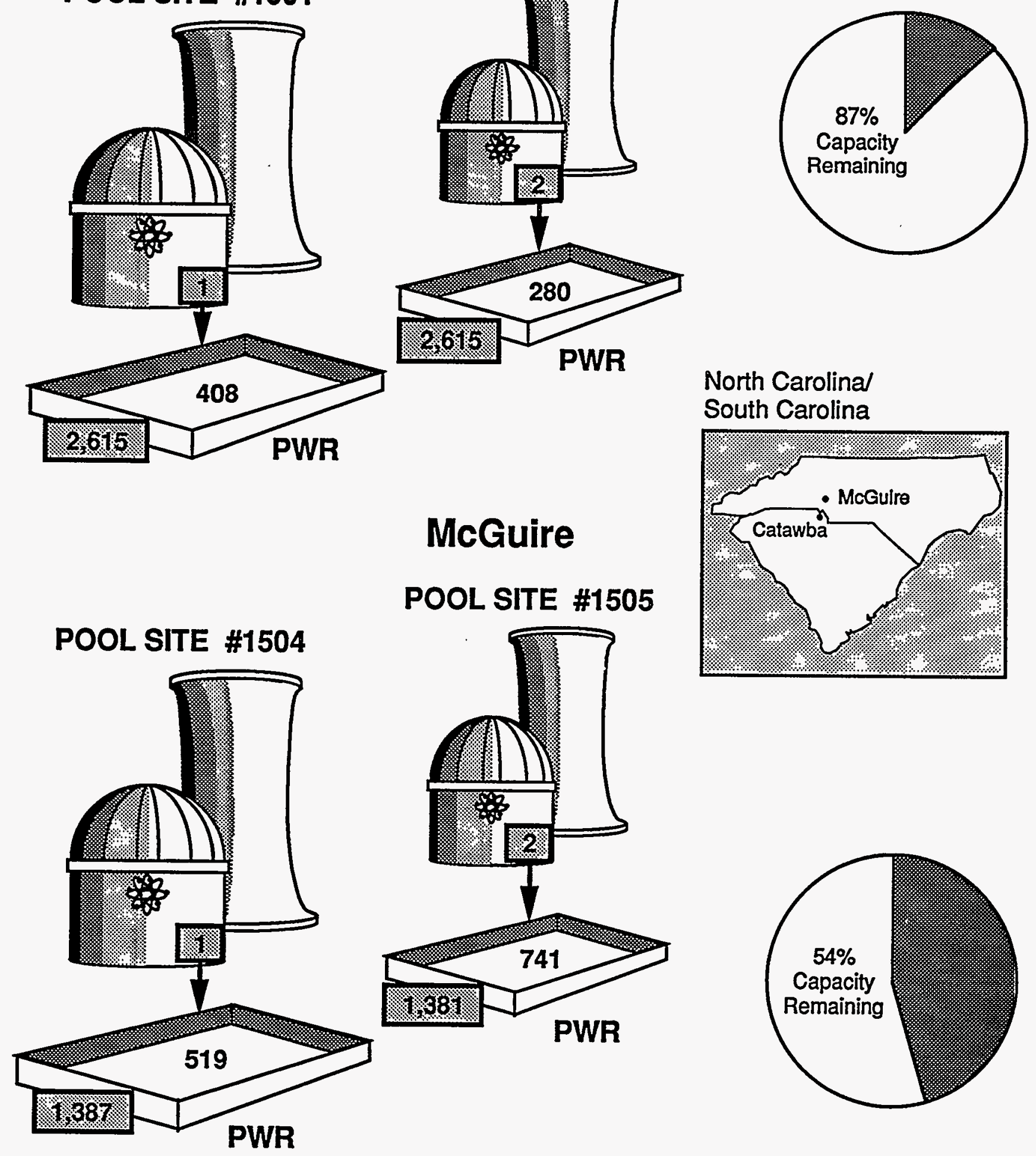

North Carolina/ South Carolina
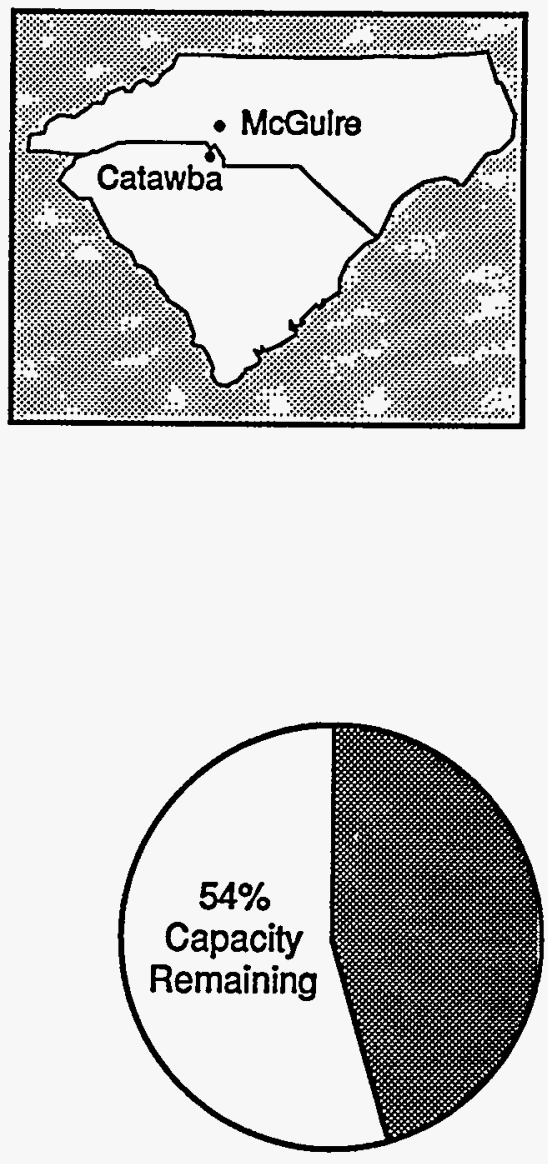


\section{Duke Power Company \\ (Continued) \\ Oconee}

POOL SITE \#1506
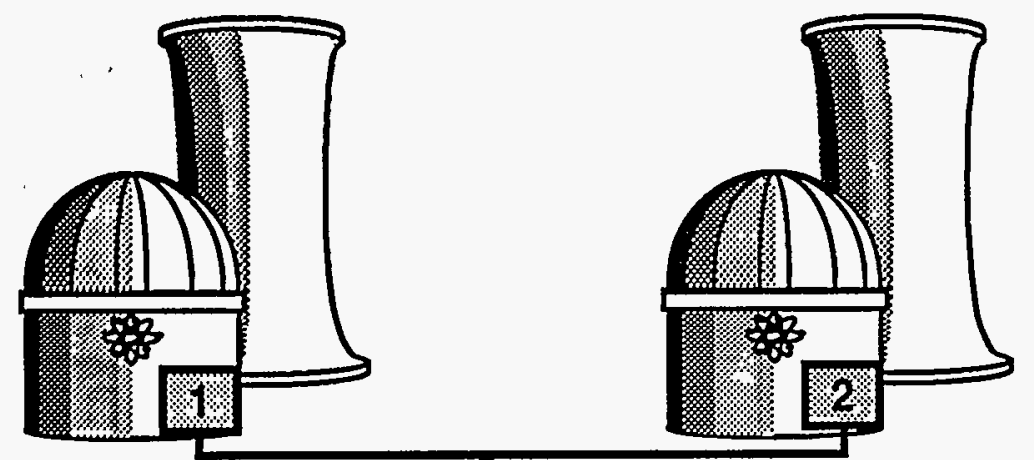

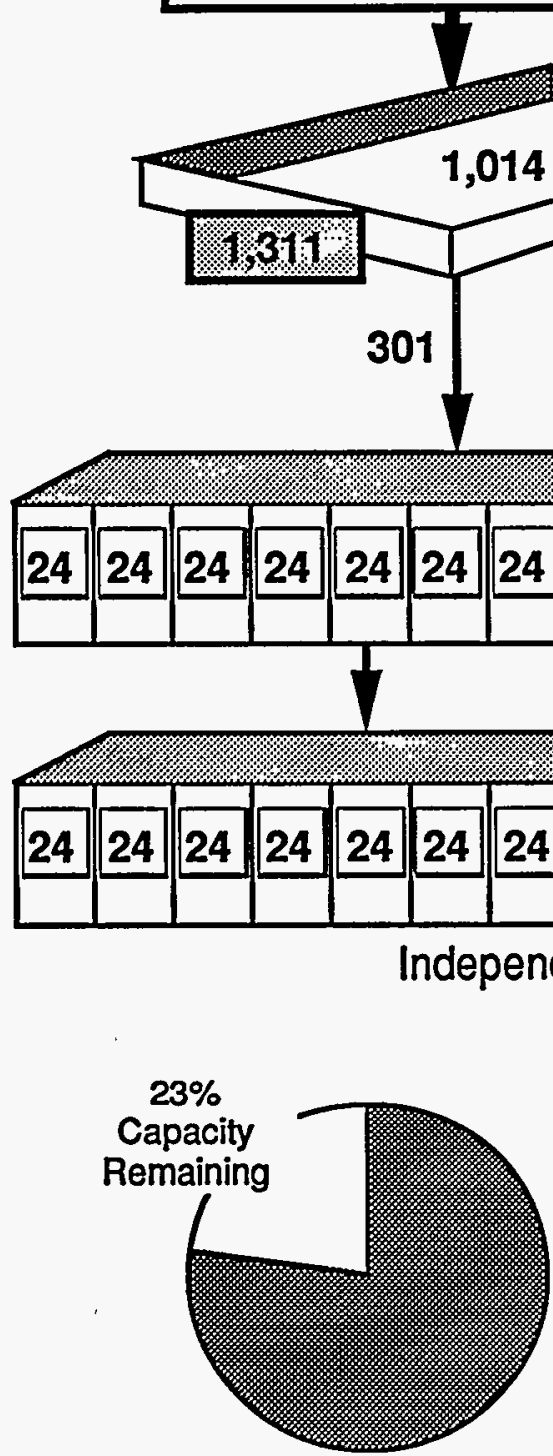

Oconee 1 \& 2

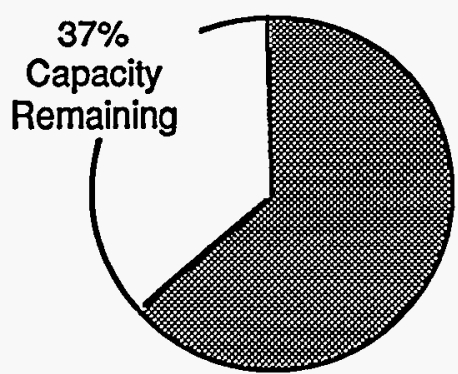

Oconee 3
POOL SITE \#1508

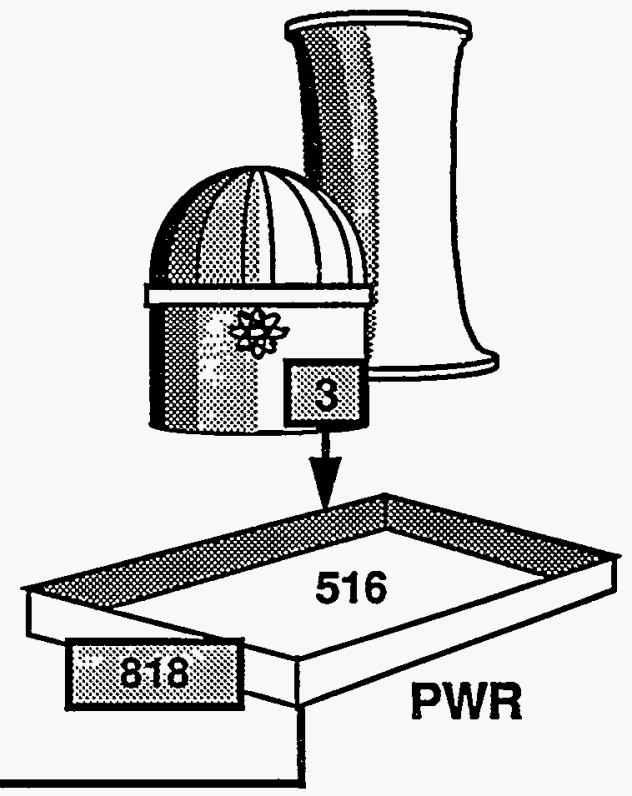

\section{\#1506D}

\section{\#1506D}

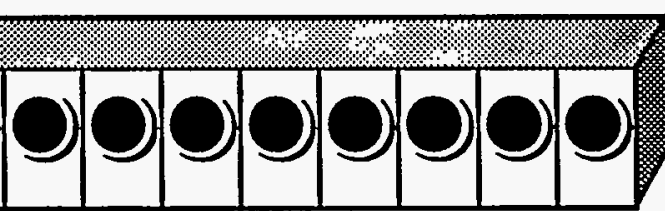

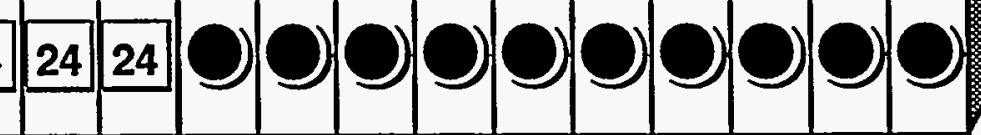




\section{Duquesne Light Company Beaver Valley}
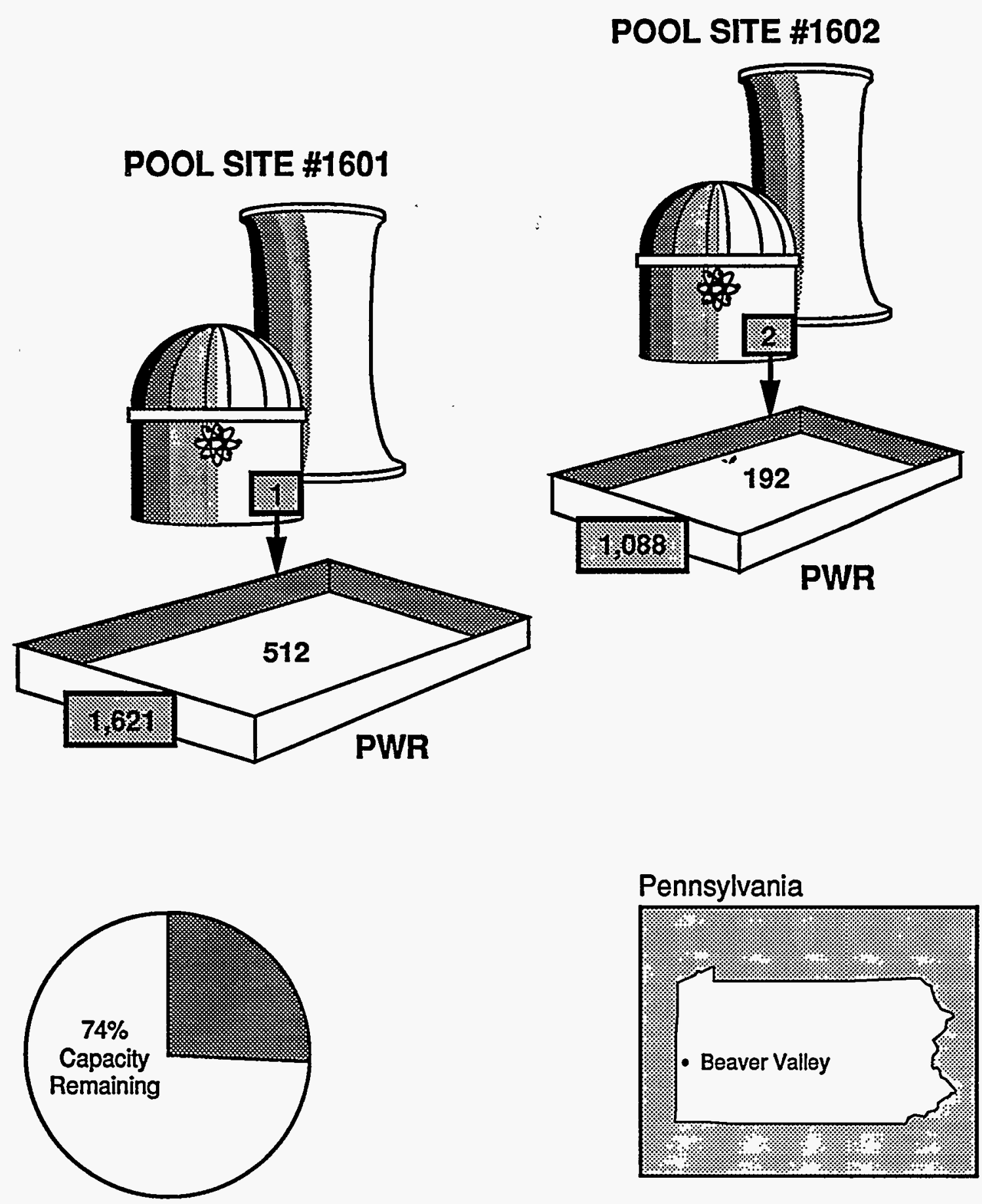


\section{Florida Power Corporation \\ Crystal River}

\section{POOL SITE \#1701}
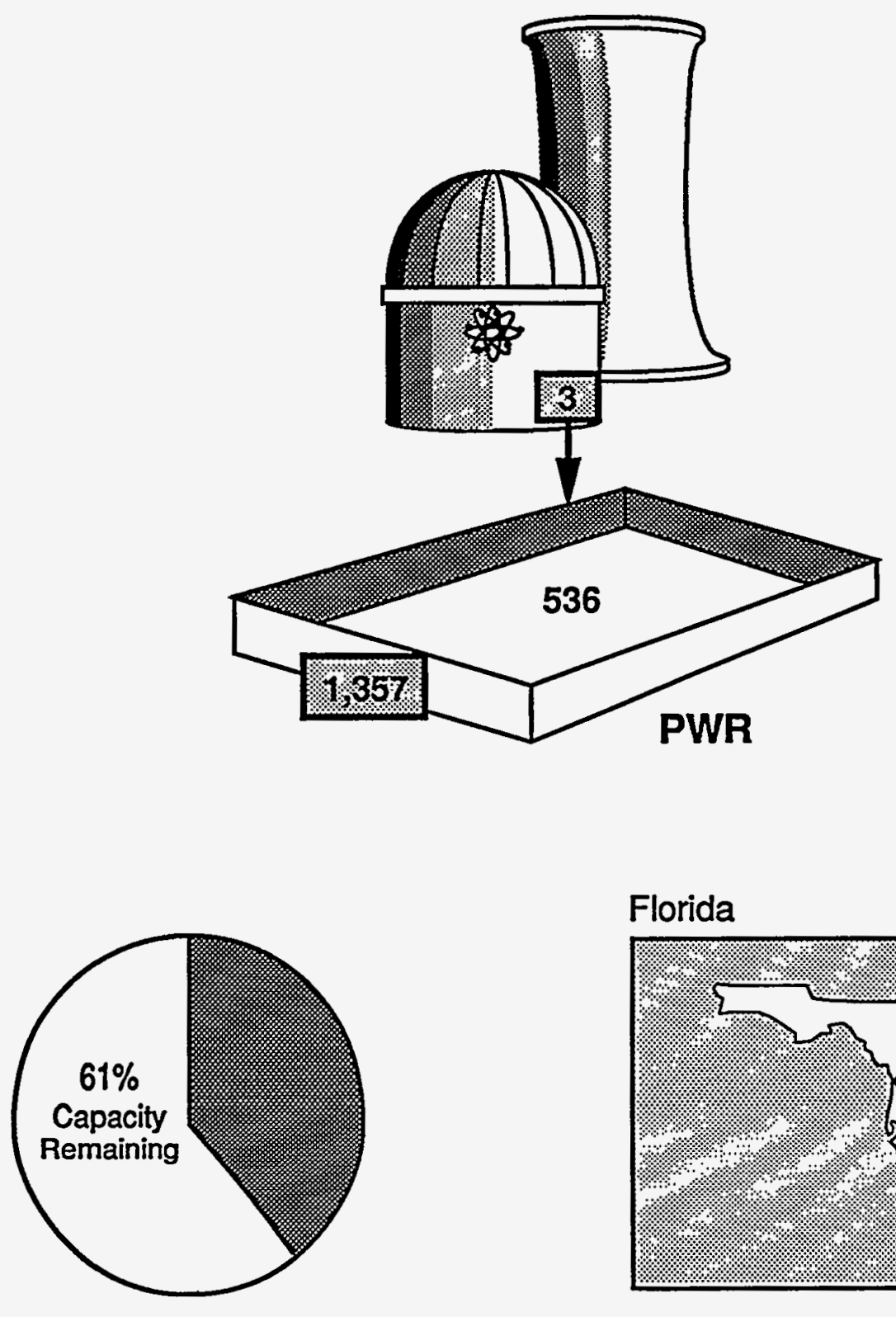

Florida

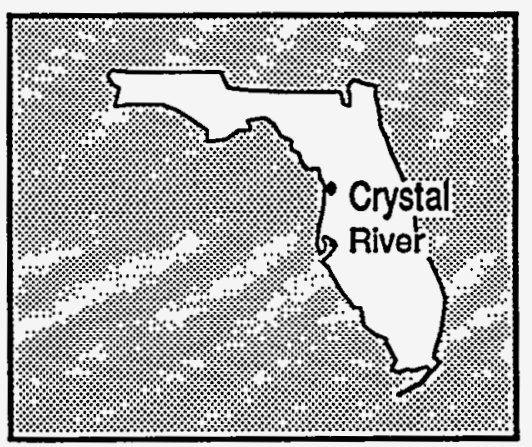




\section{Florida Power and Light Company St. Lucie}

POOL SITE \#1802
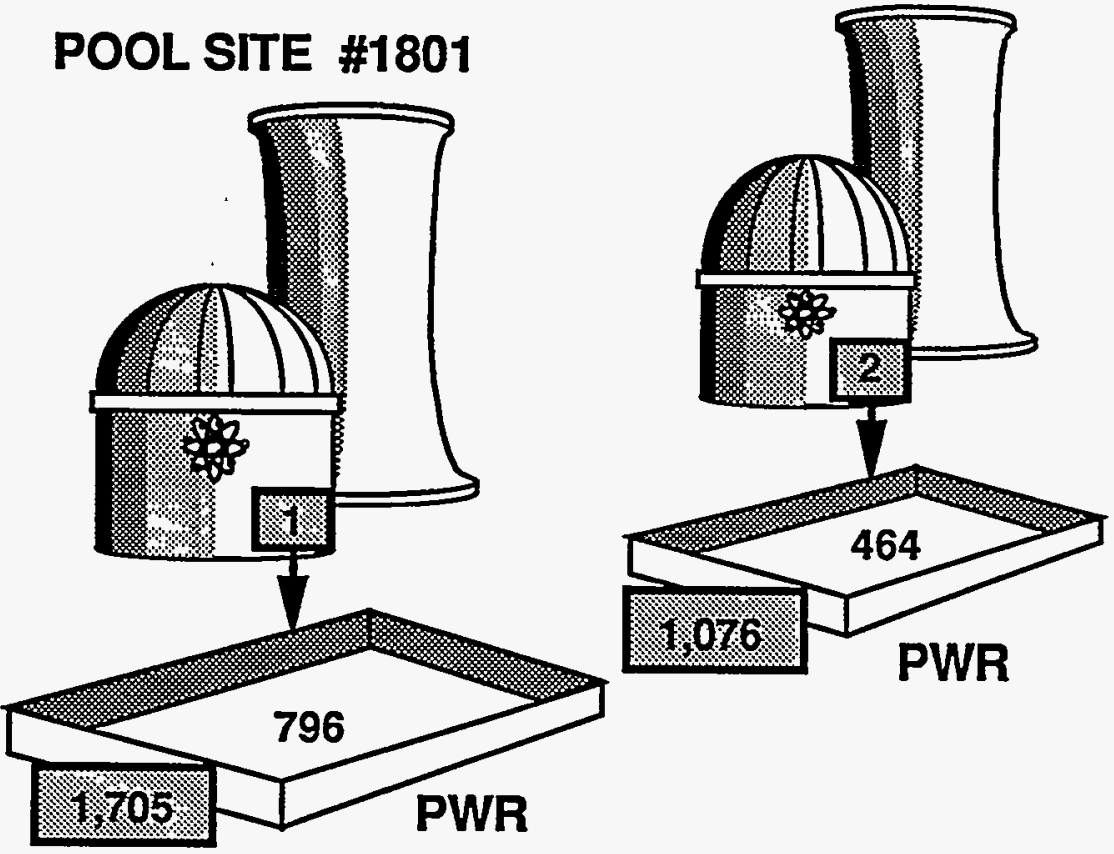

\section{Turkey Point}

POOL SITE \#1804

\section{POOL SITE \#1803}
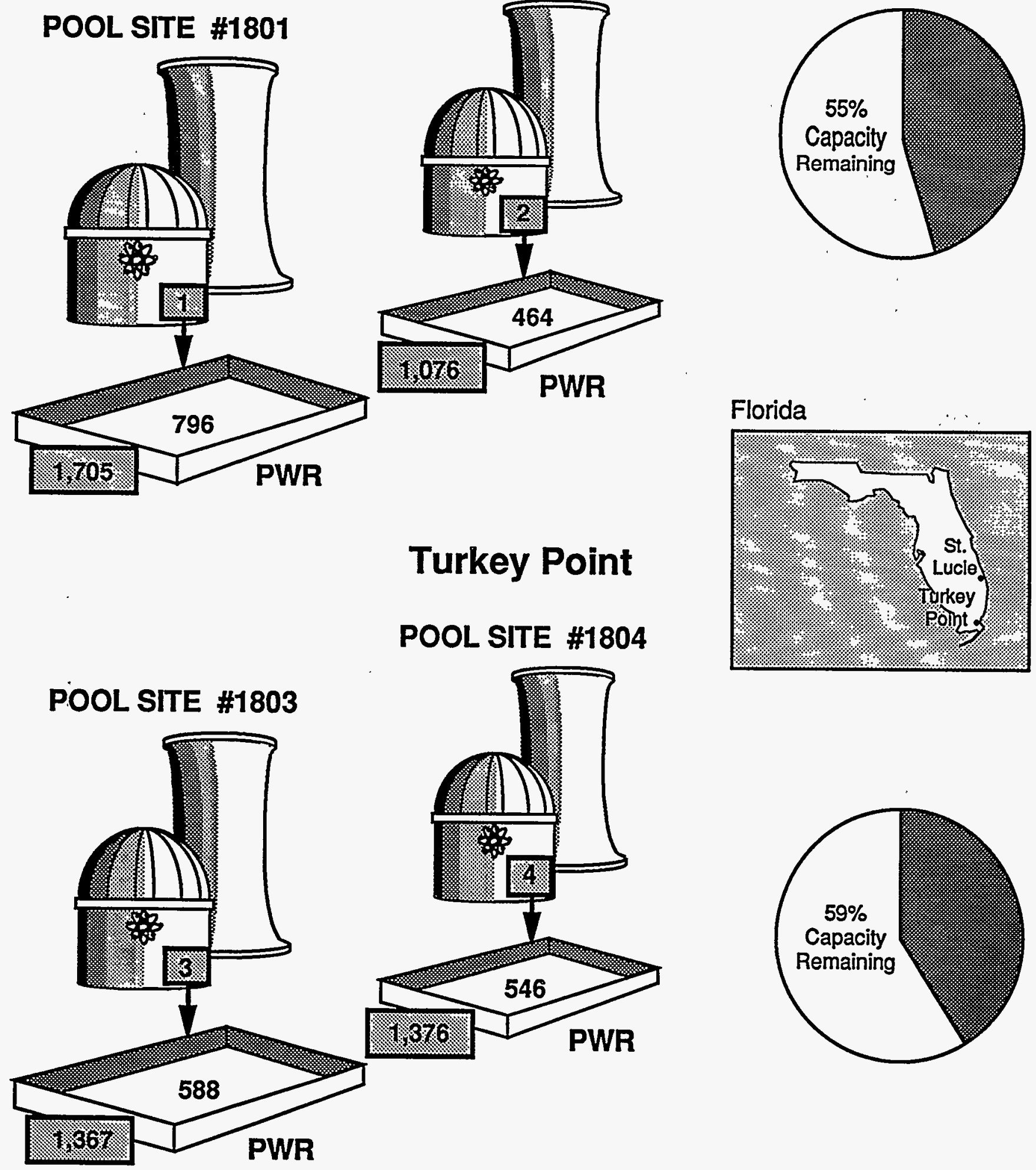


\section{GPU Nuclear Corporation Three Mile Island}

\section{POOL SITE \#1901}
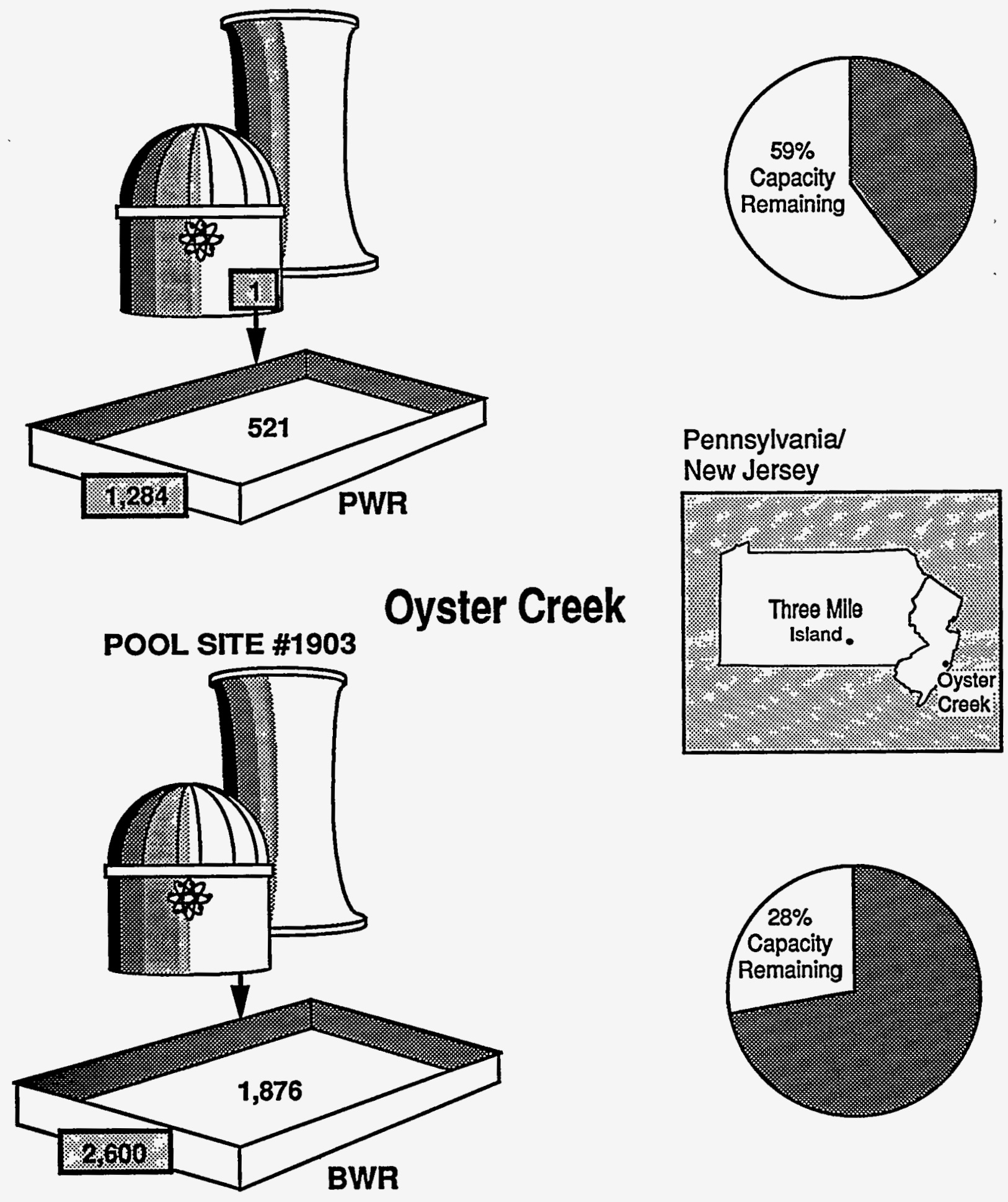

Pennsylvanial New Jersey 


\section{Georgia Power Company \\ Hatch}

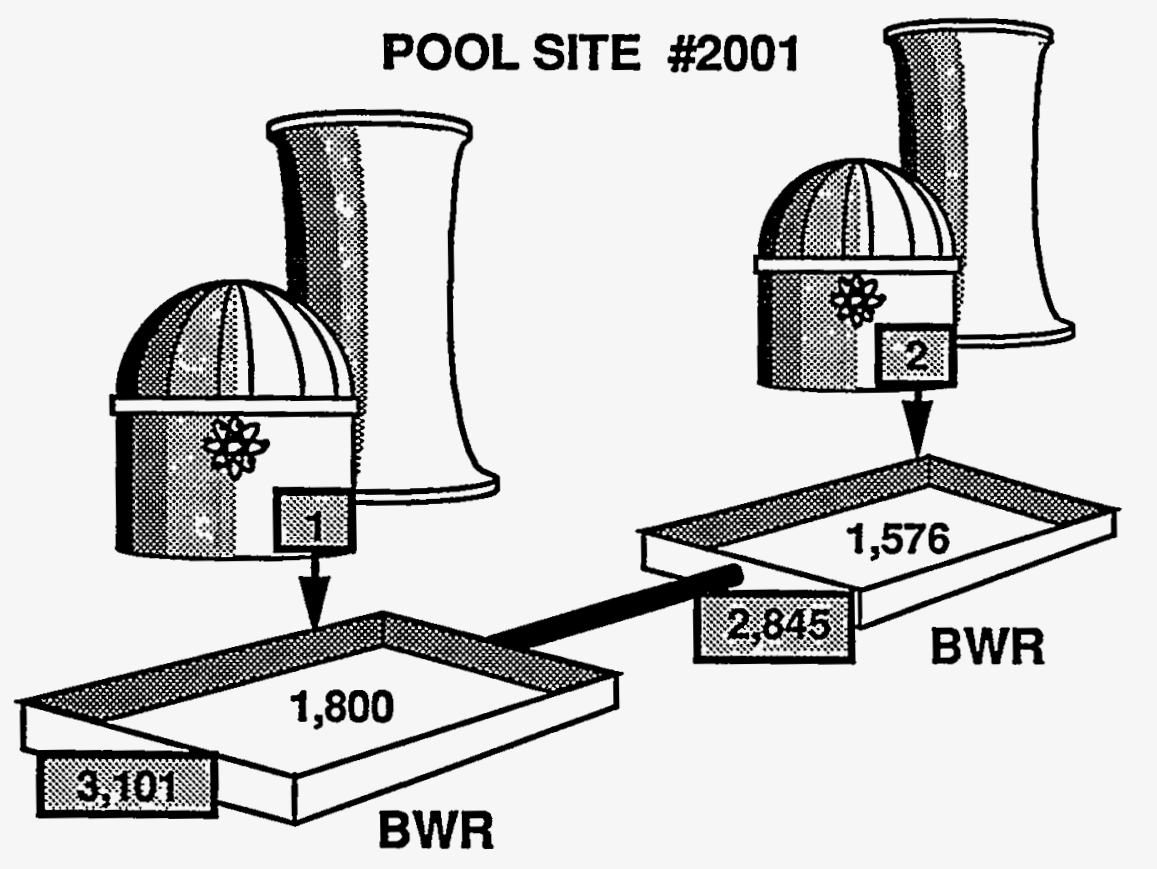

Vogtle

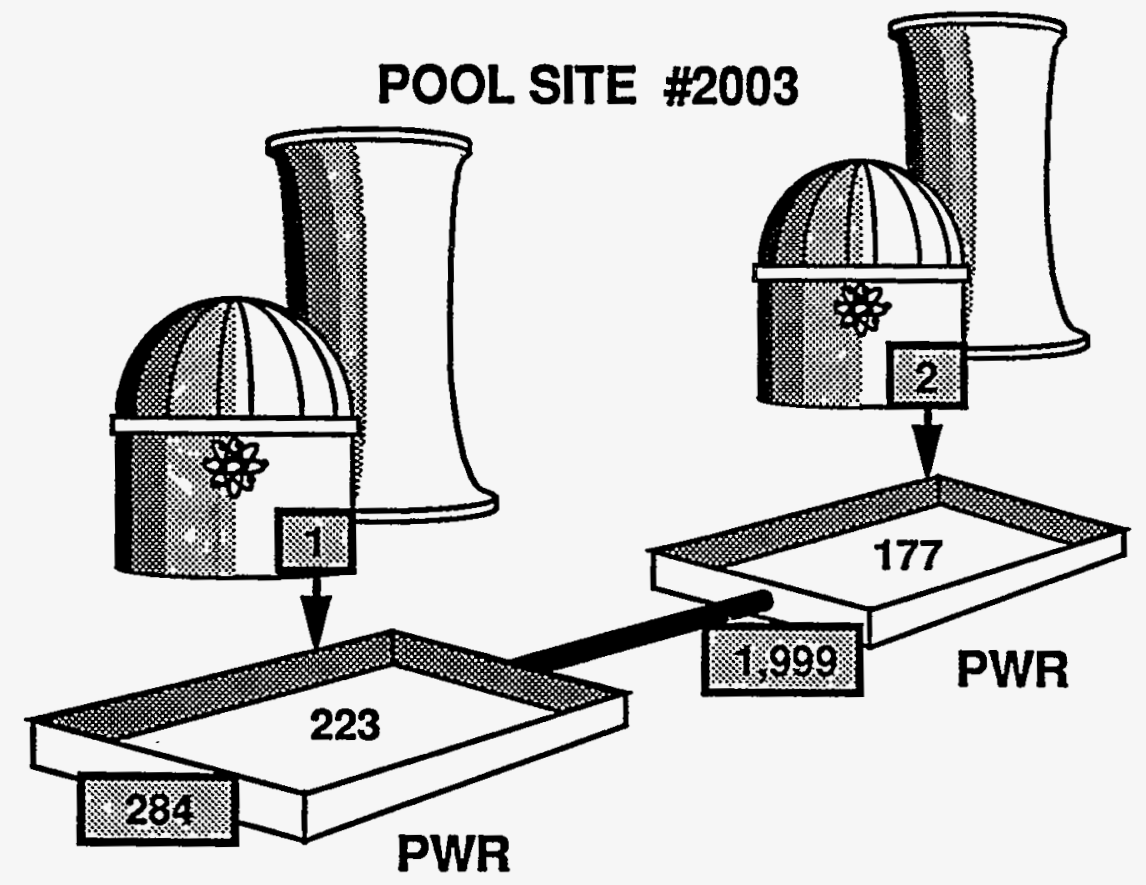

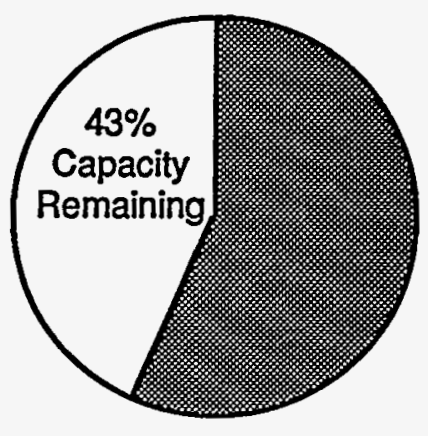

Georgia
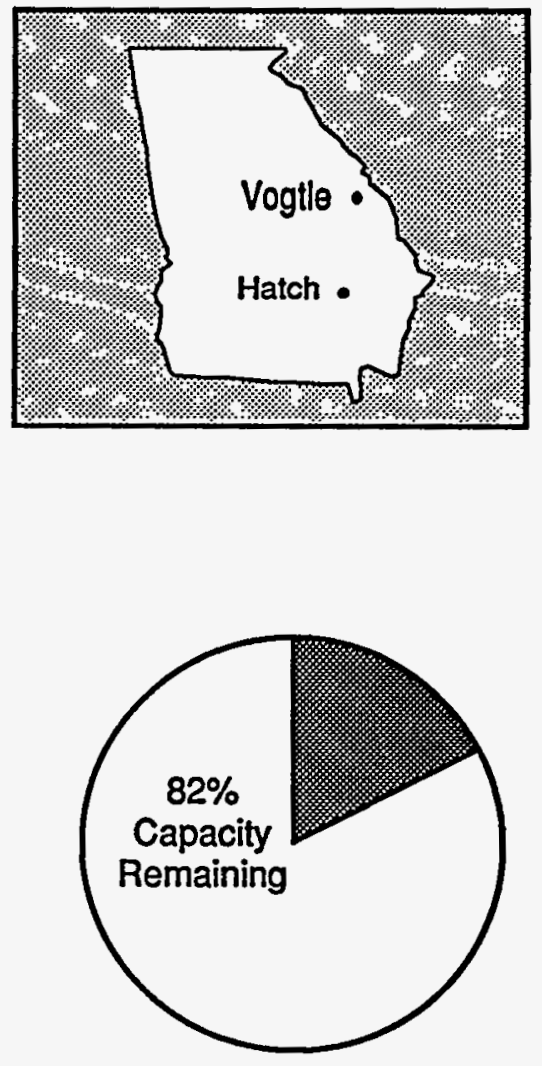


\section{Gulf States Utilities Company River Bend}
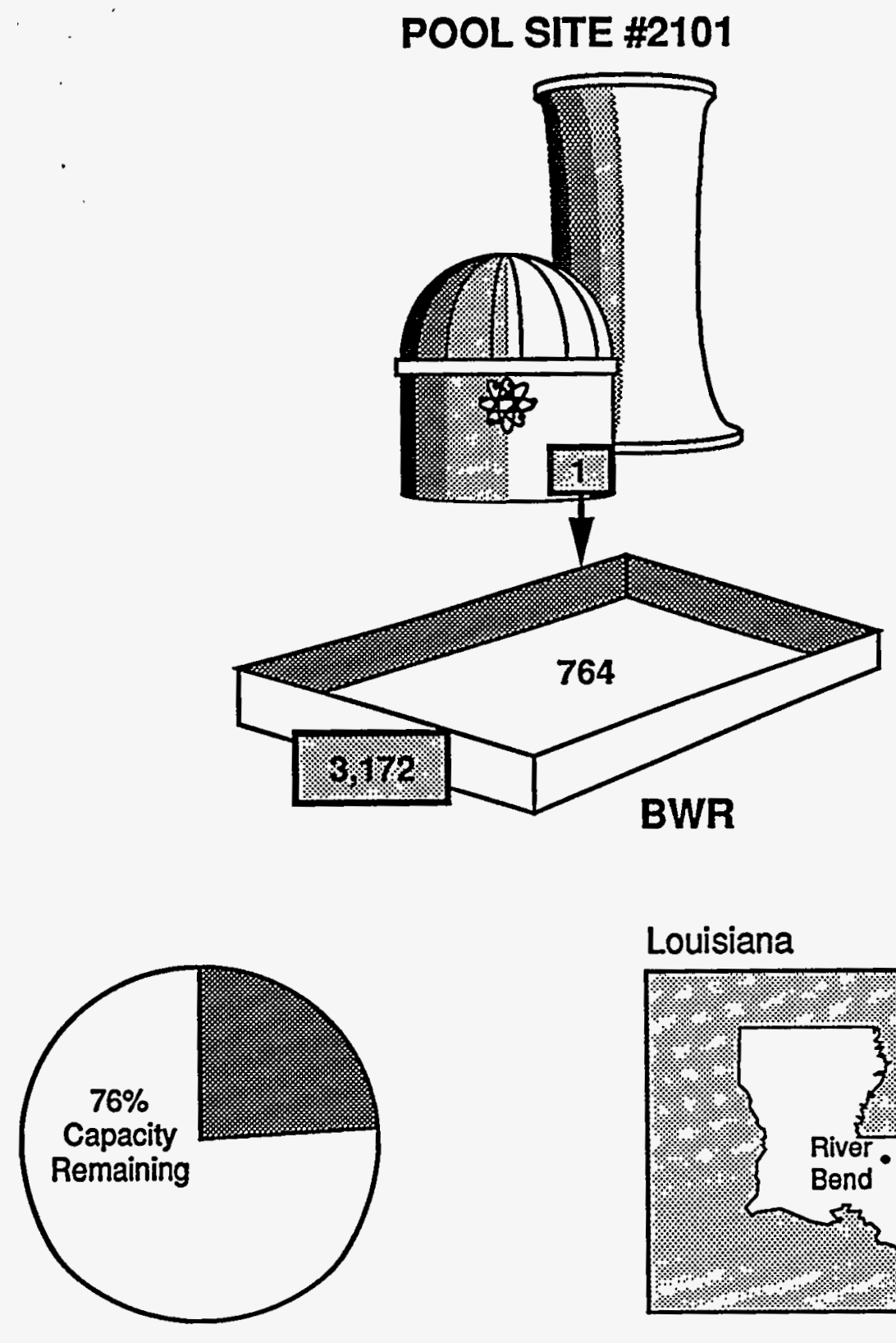

Louisiana

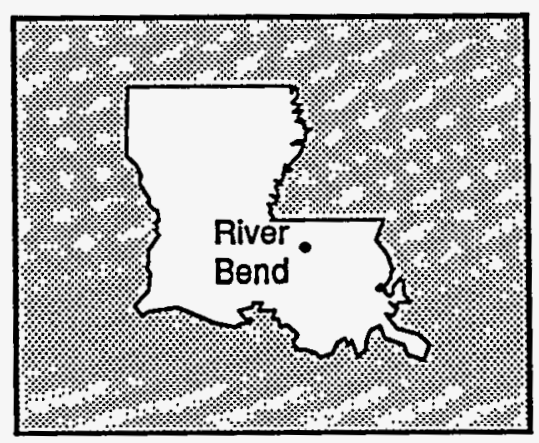




\section{Houston Lighting and Power Company South Texas}
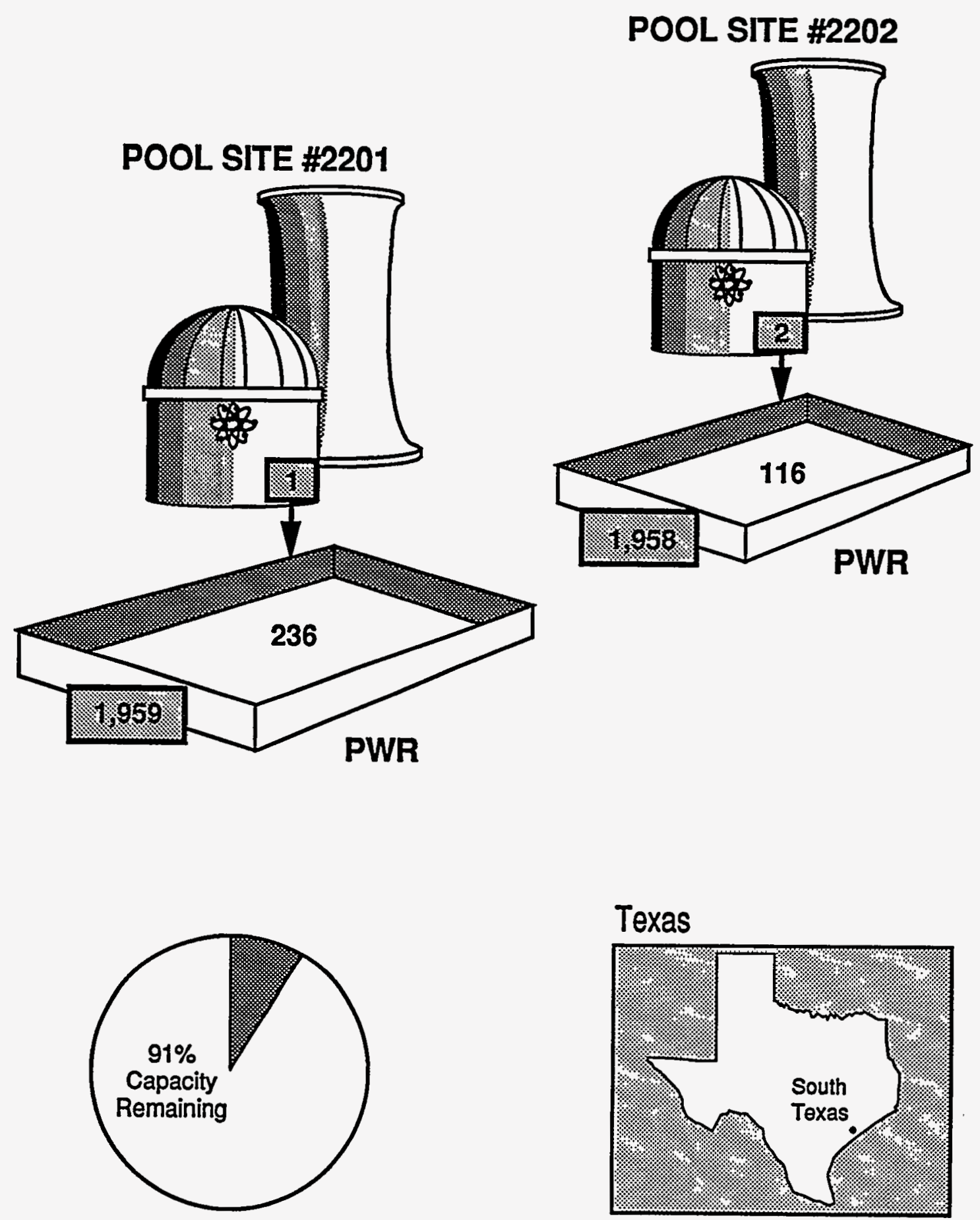


\section{Illinois Power Company \\ Clinton}
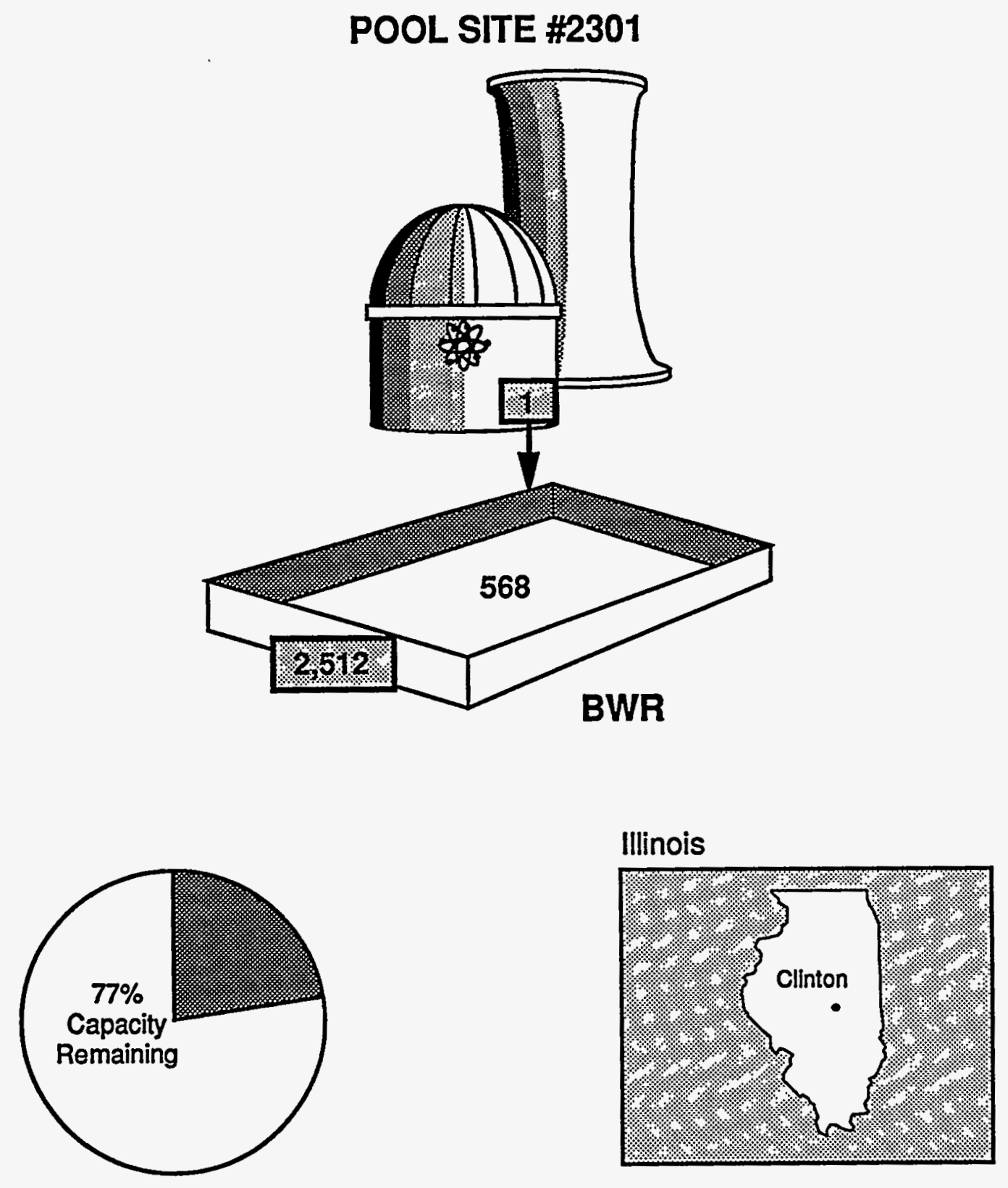


\section{Indiana Michigan Power Company}

Cook
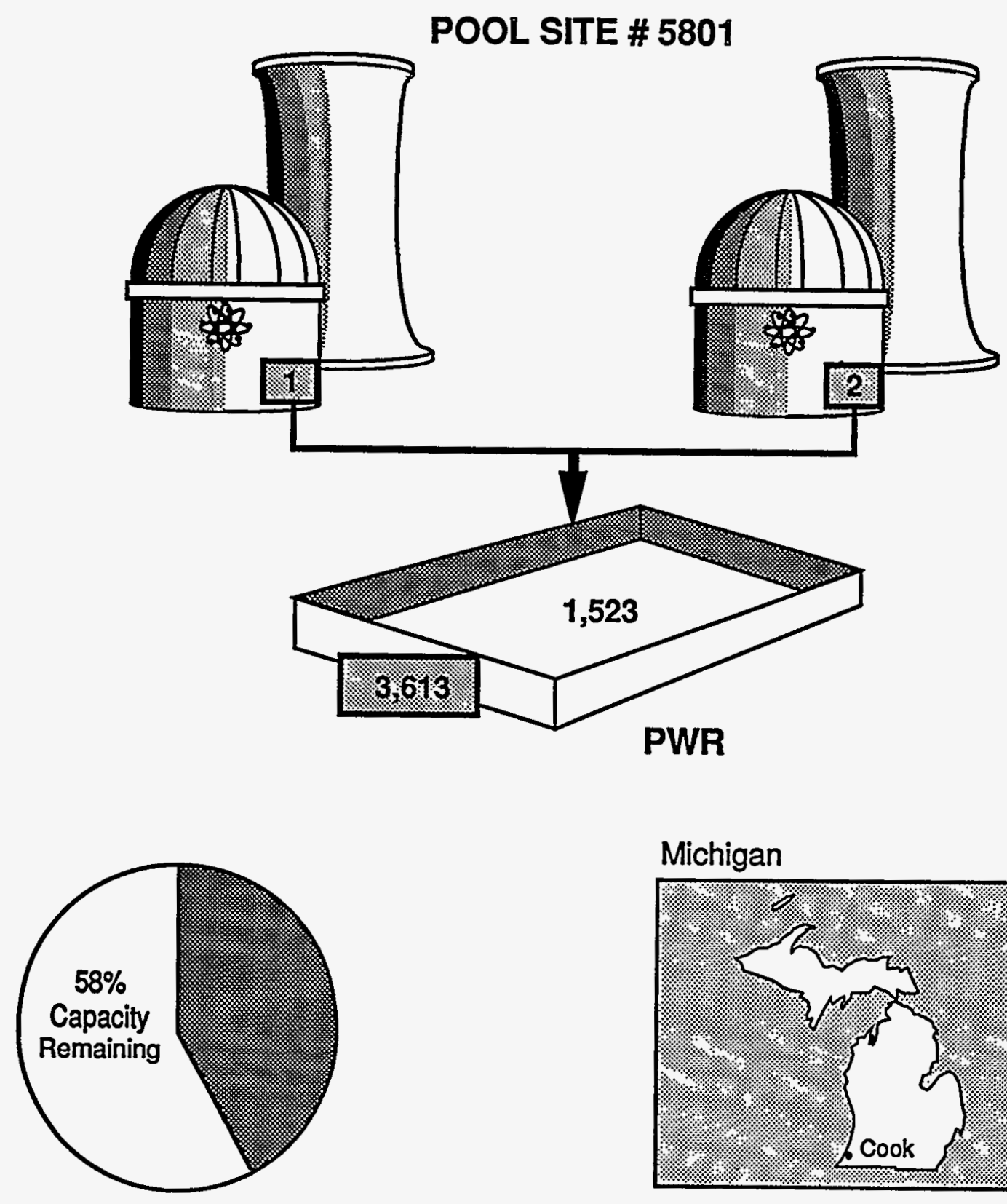

Michigan

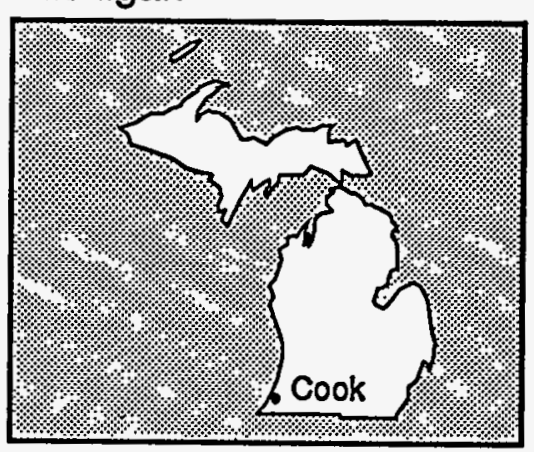




\title{
lowa Electric Light and Power Company
}

\author{
Duane Arnold
}
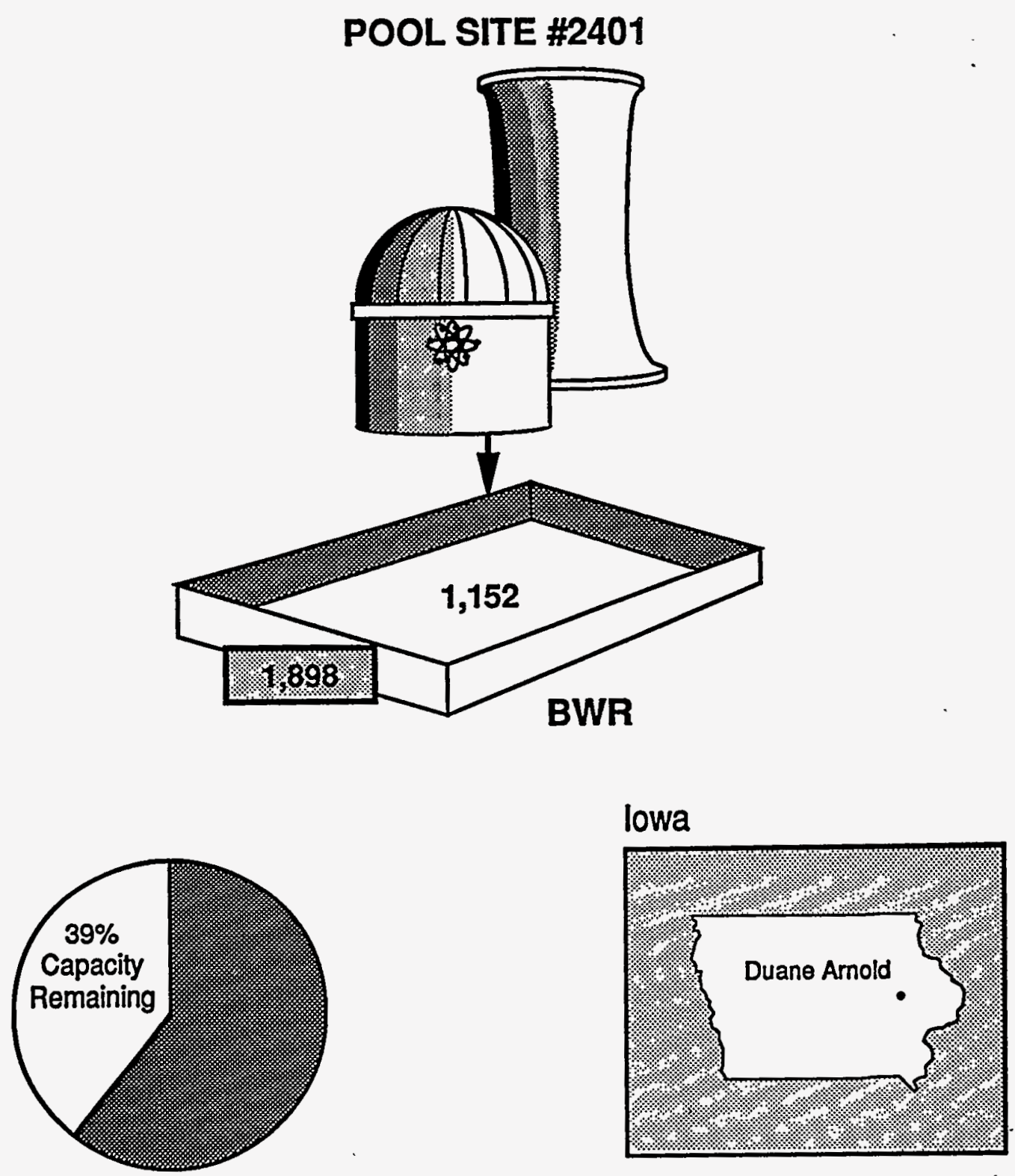


\section{Kansas Gas and Electric Company Wolf Creek}
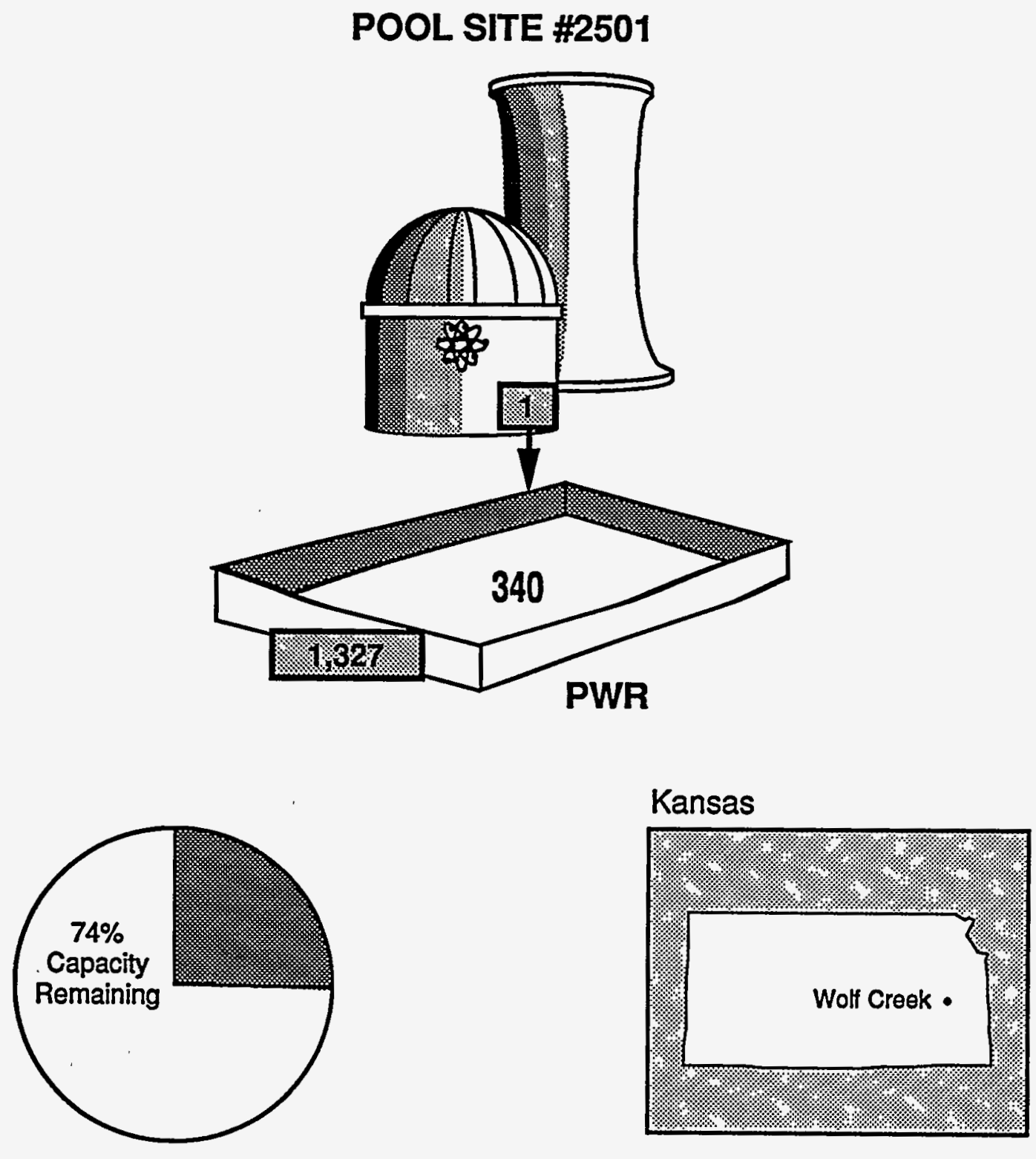


\section{Long Island Lighting Company Shoreham}
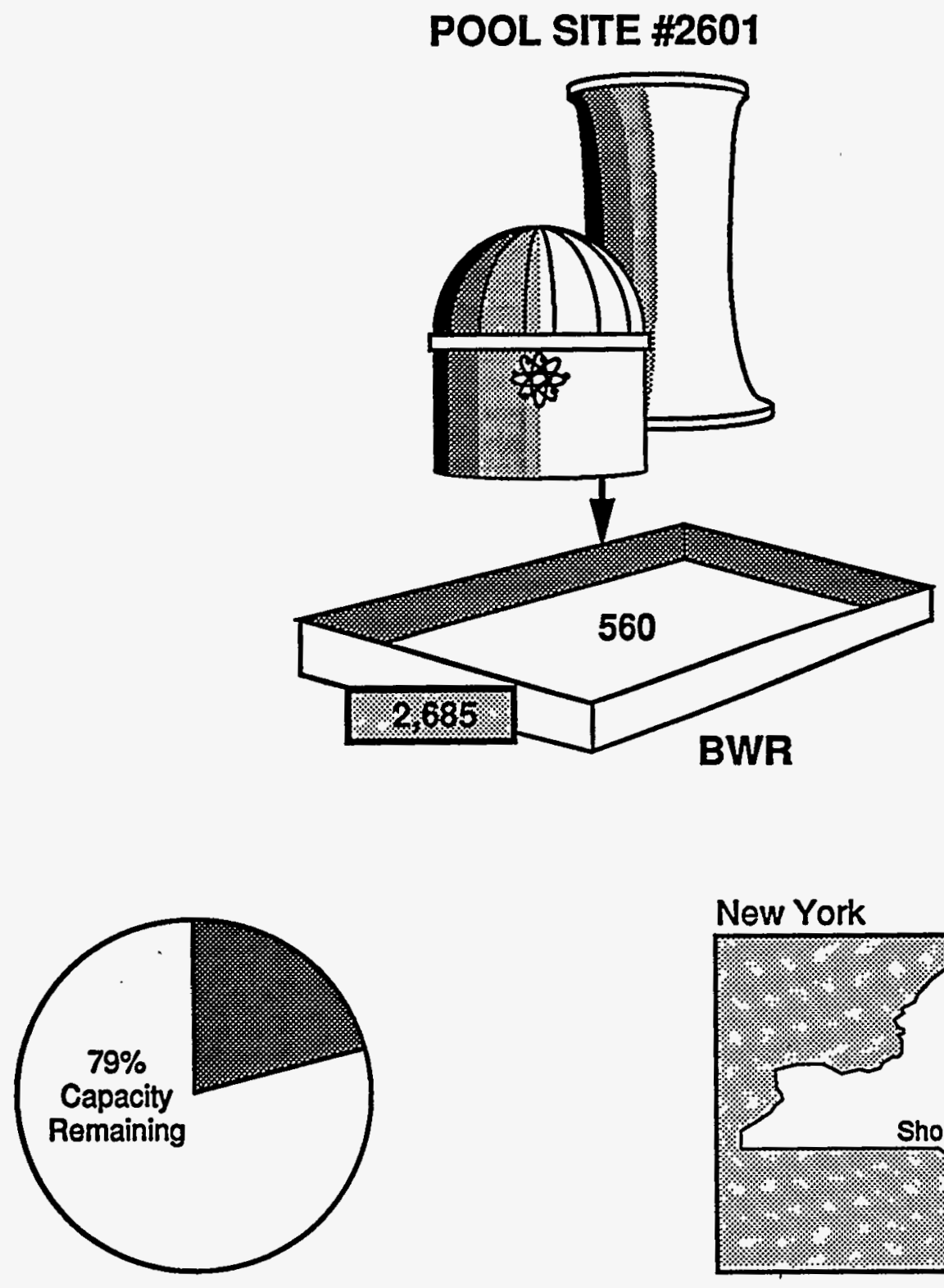

New York

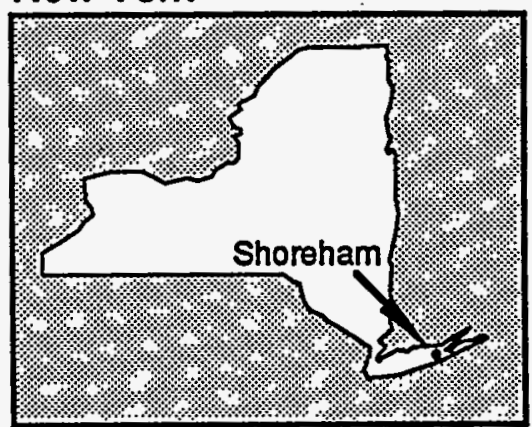




\title{
Louisiana Power and Light Company
}

\author{
Waterford
}

POOL SITE \#2701
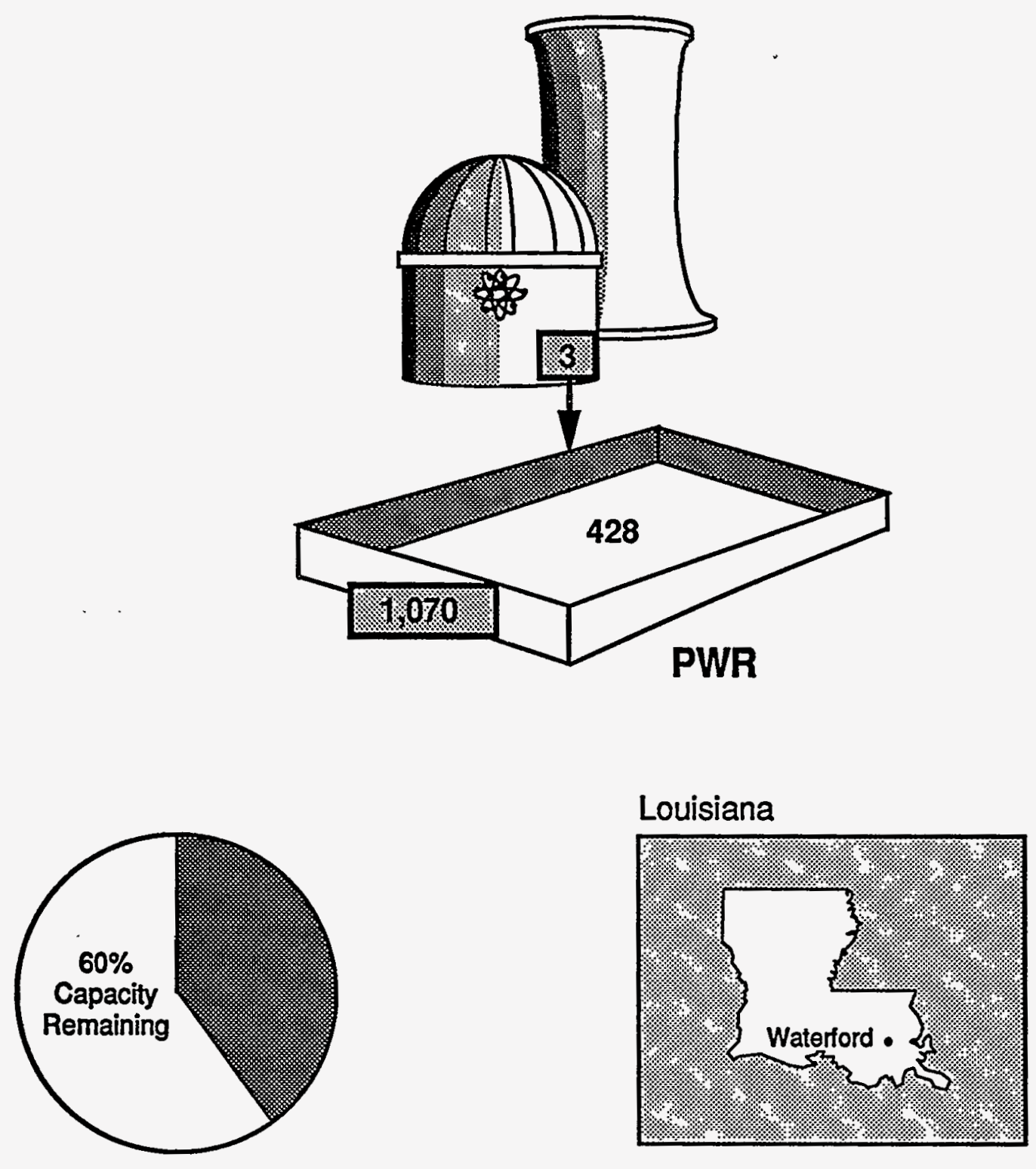


\title{
Maine Yankee Atomic Power Company
}

\author{
Maine Yankee
}

POOL SITE \#2801
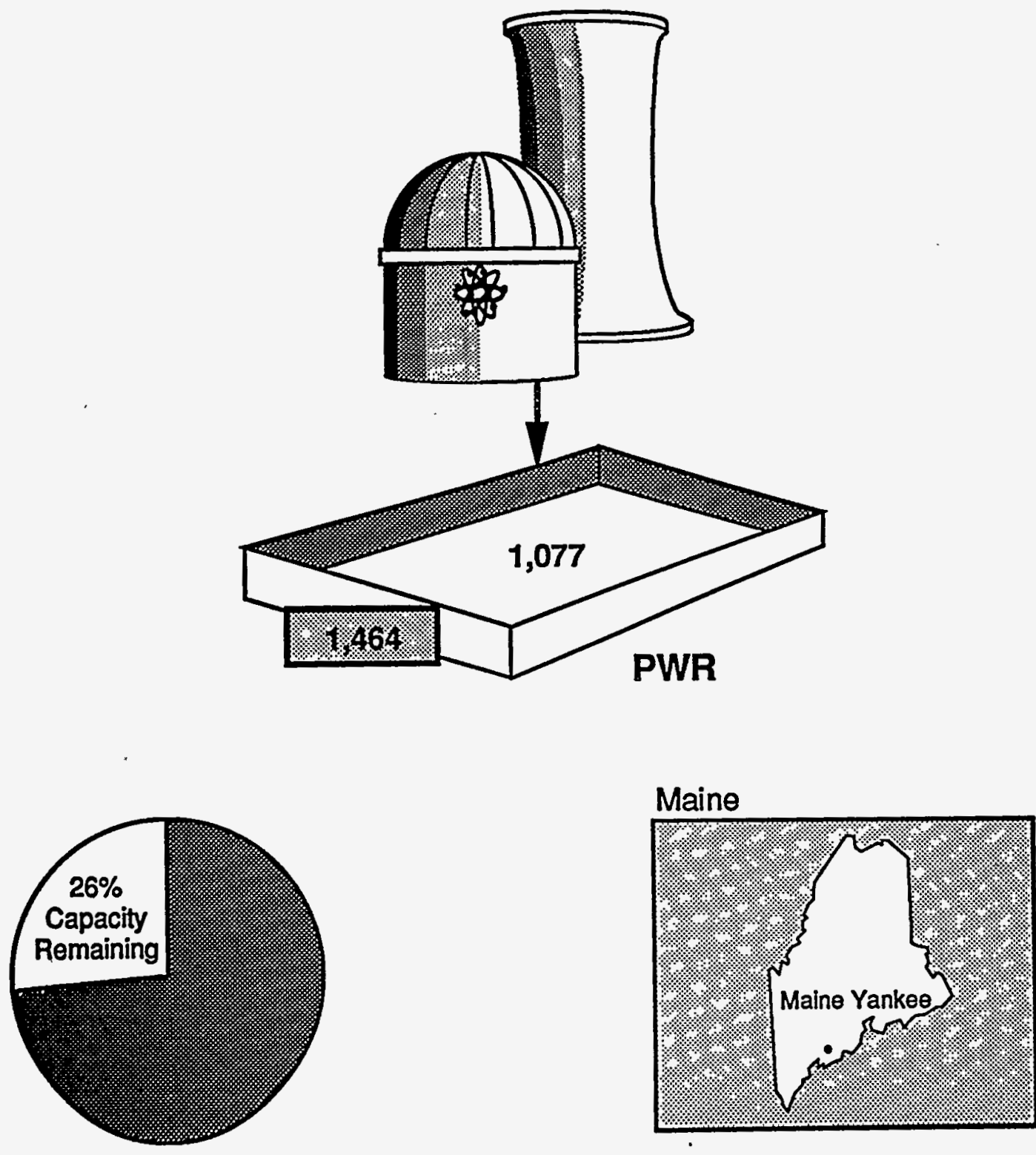


\section{Nebraska Public Power District Cooper Station}
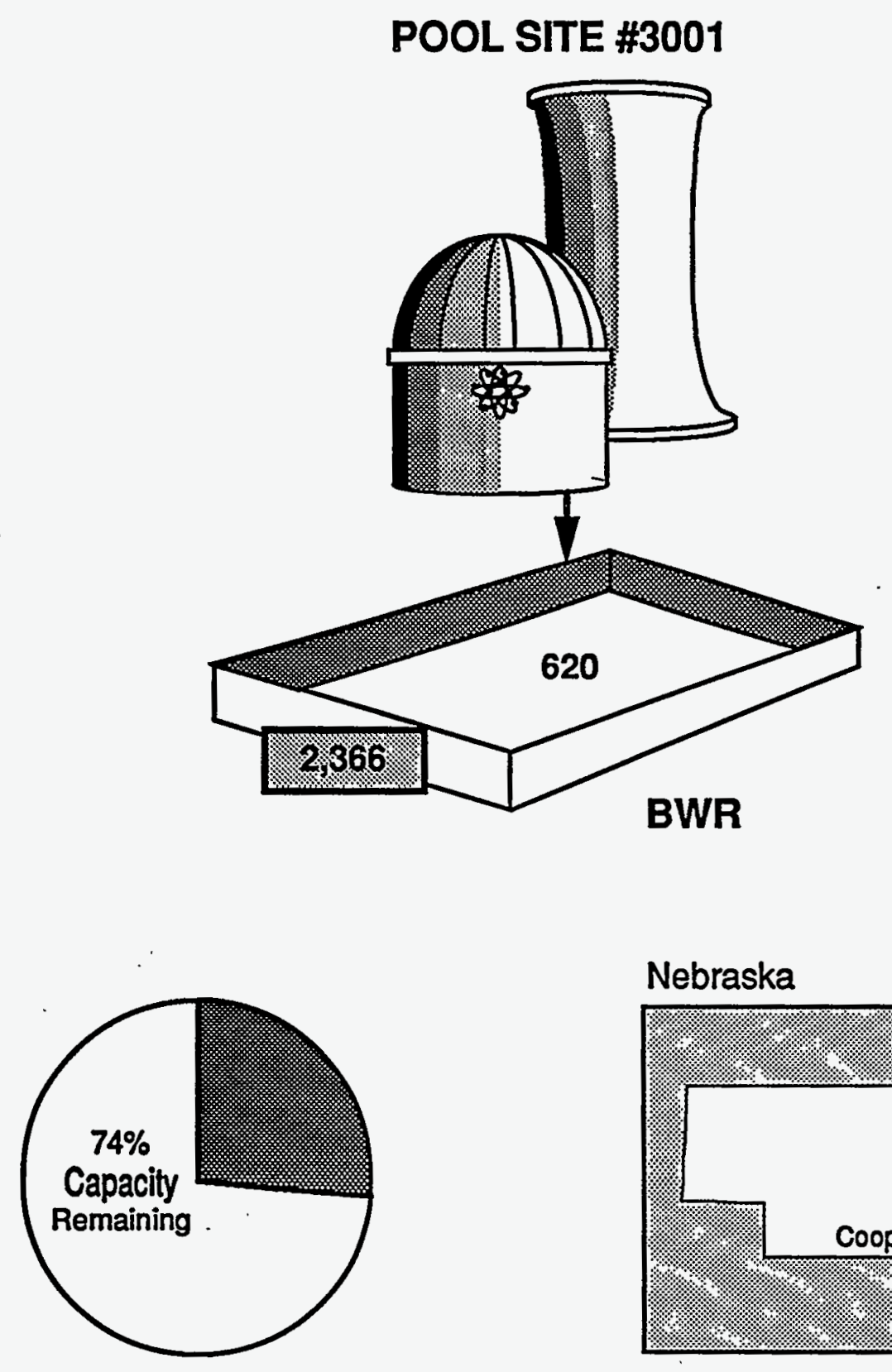

Nebraska

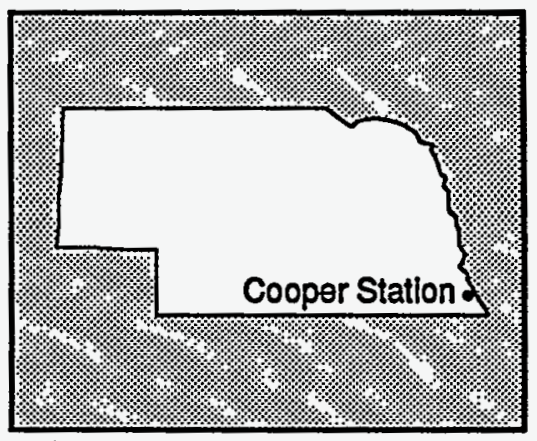




\section{New York Power Authority}

\section{Fitzpatrick}
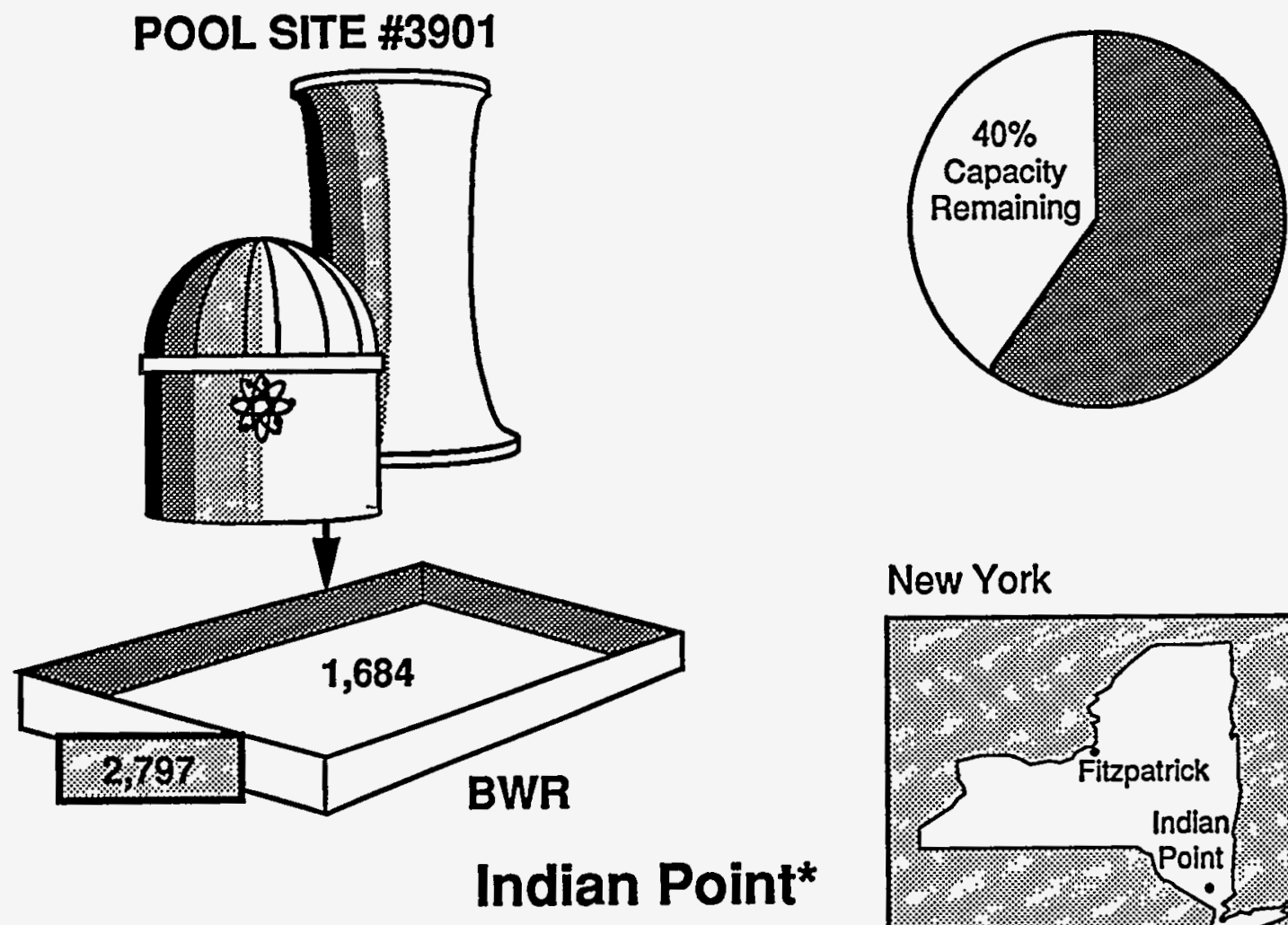

POOL SITE \#3902

New York
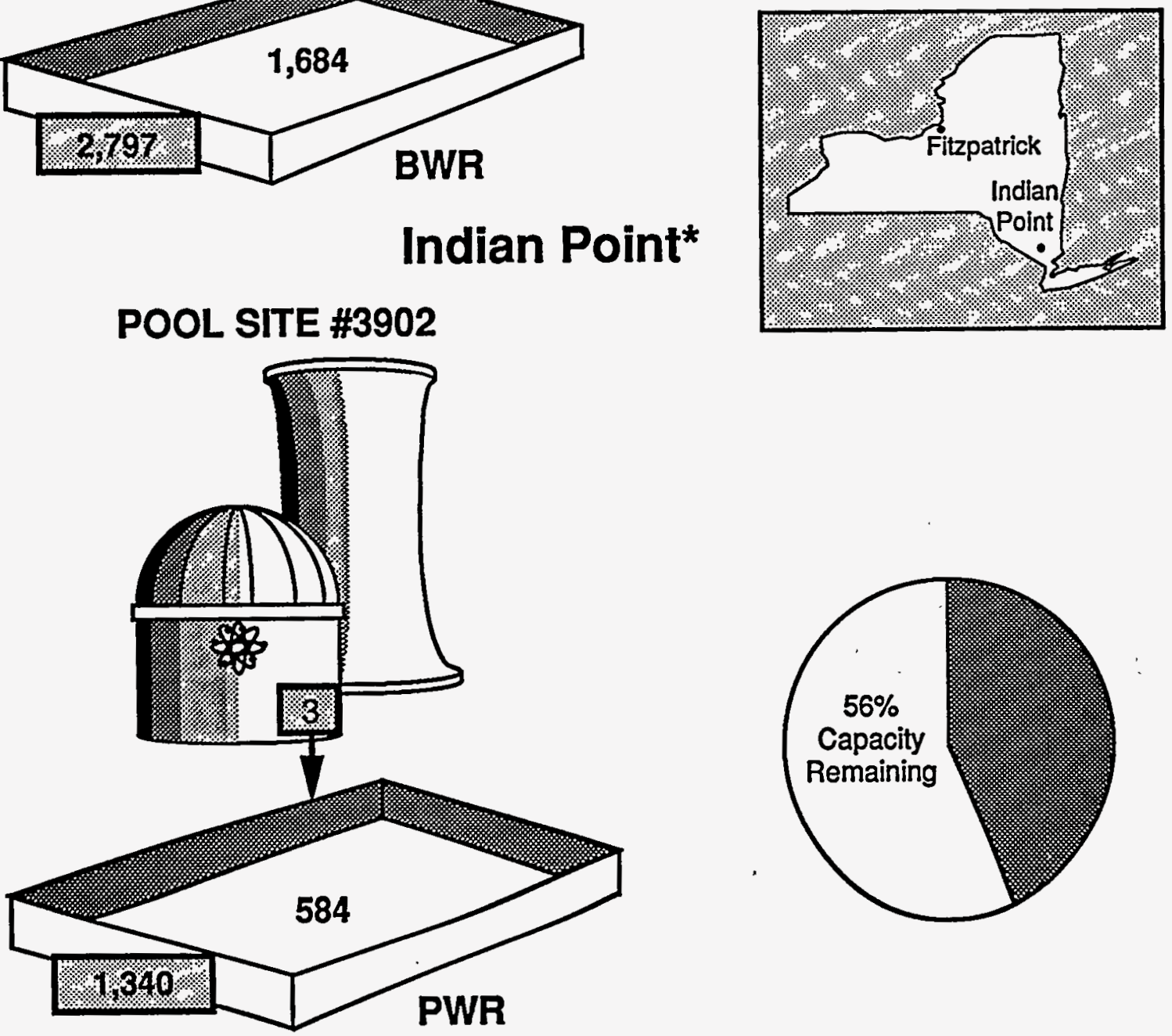

- Reactor at this site is Indian Point 3.

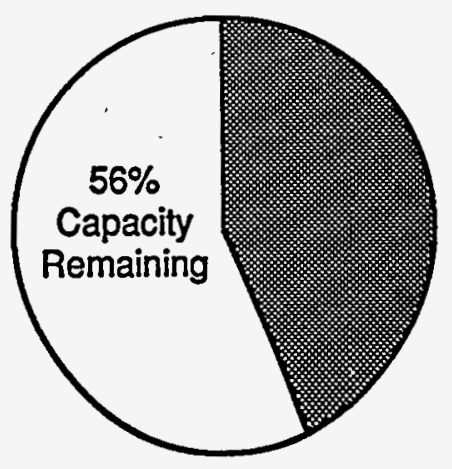




\title{
Niagara Mohawk Power Corporation
}

\author{
Nine Mile Point
}
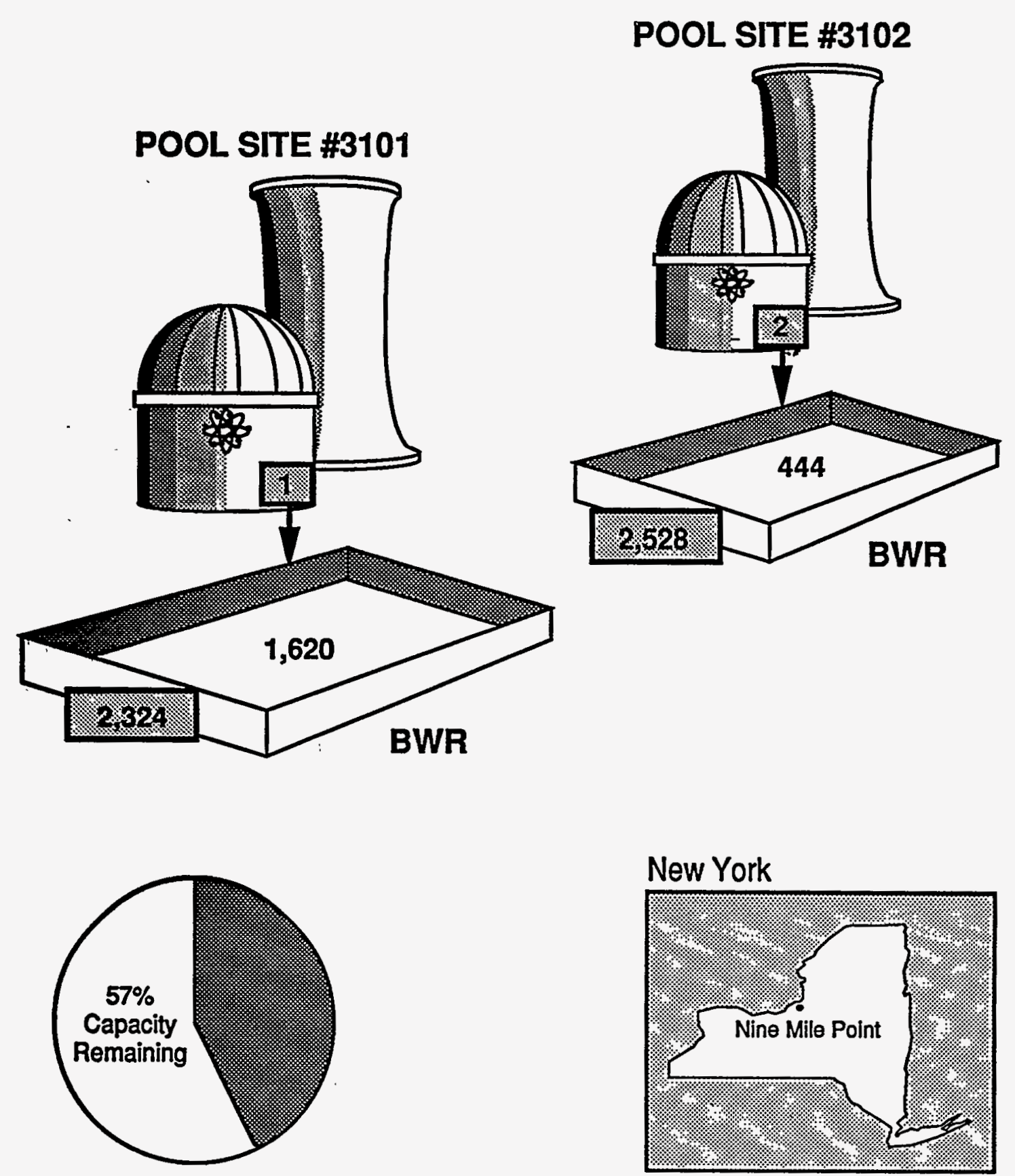


\section{North Atlantic Energy Service Corporation}

Seabrook
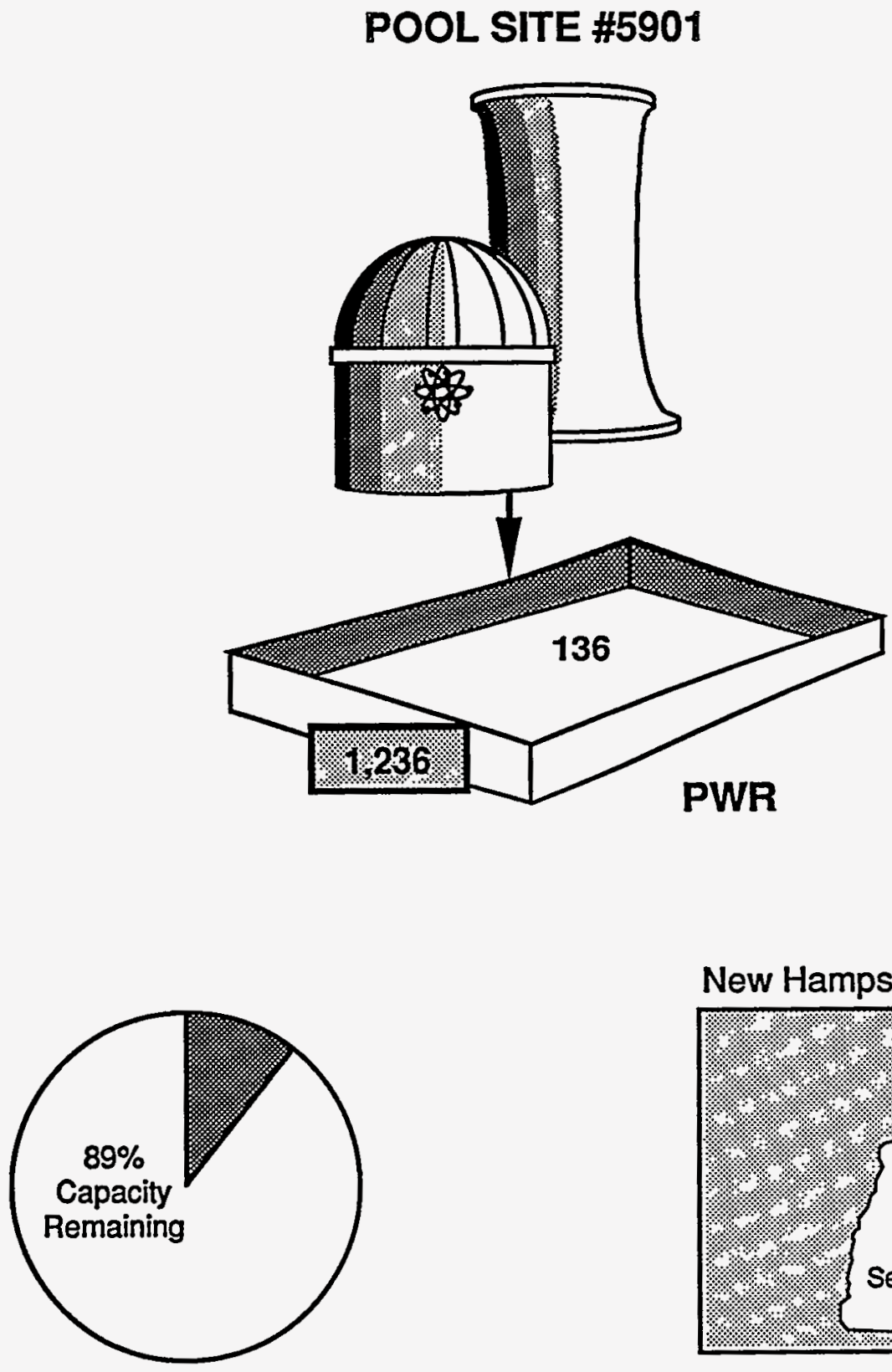

New Hampshire

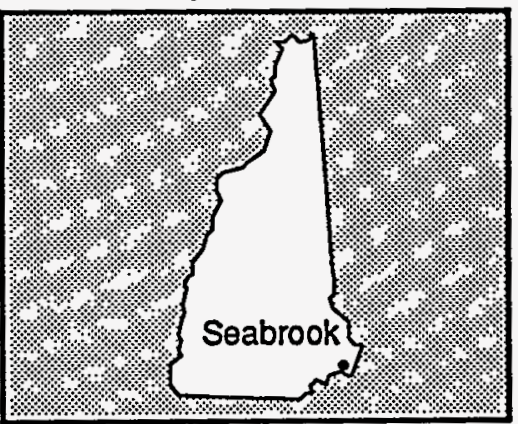




\section{Northeast Utilities Service Company}

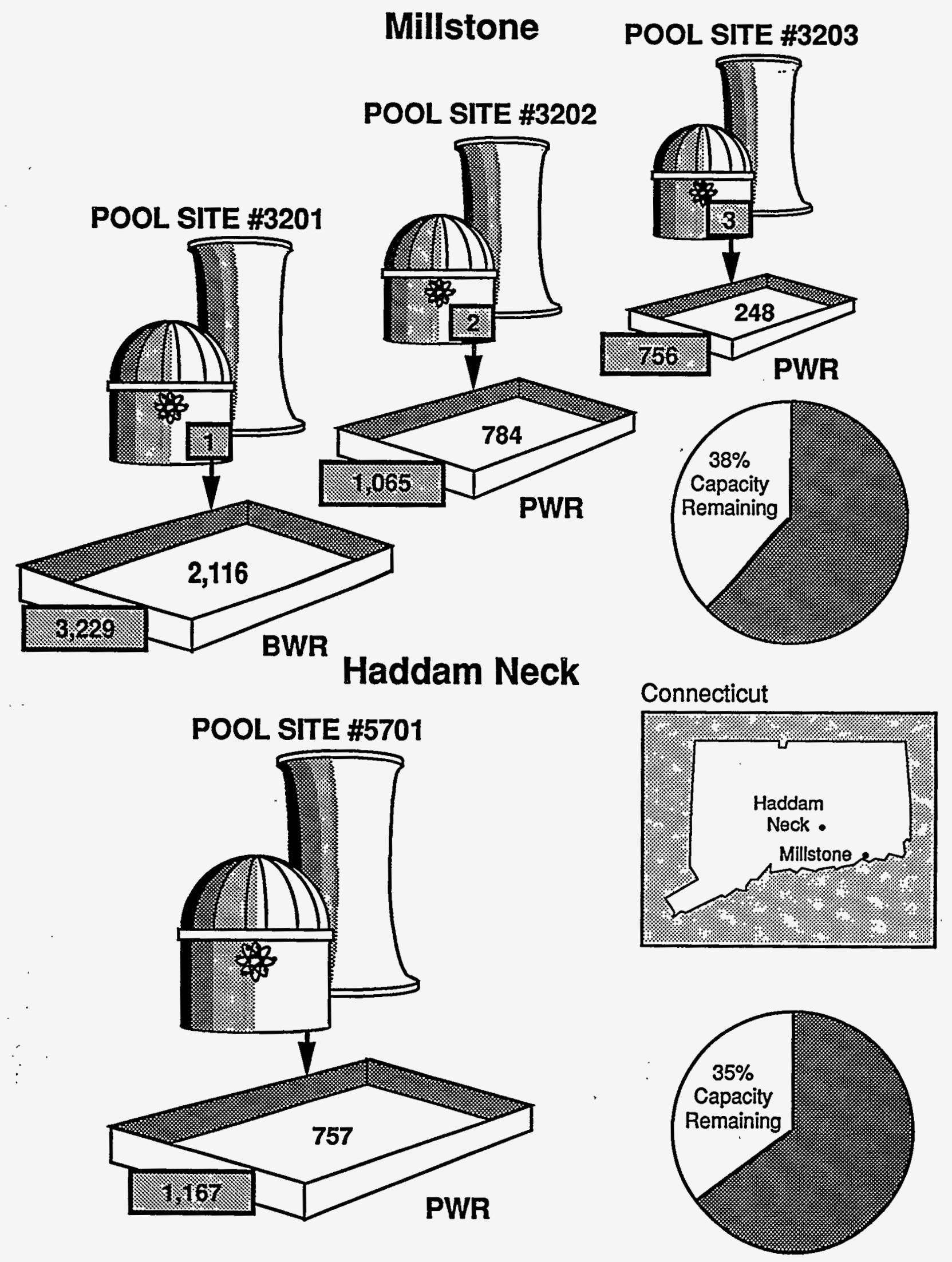




\section{Northern States Power Company}

\section{Monticello}

\section{POOL SITE \#3301}

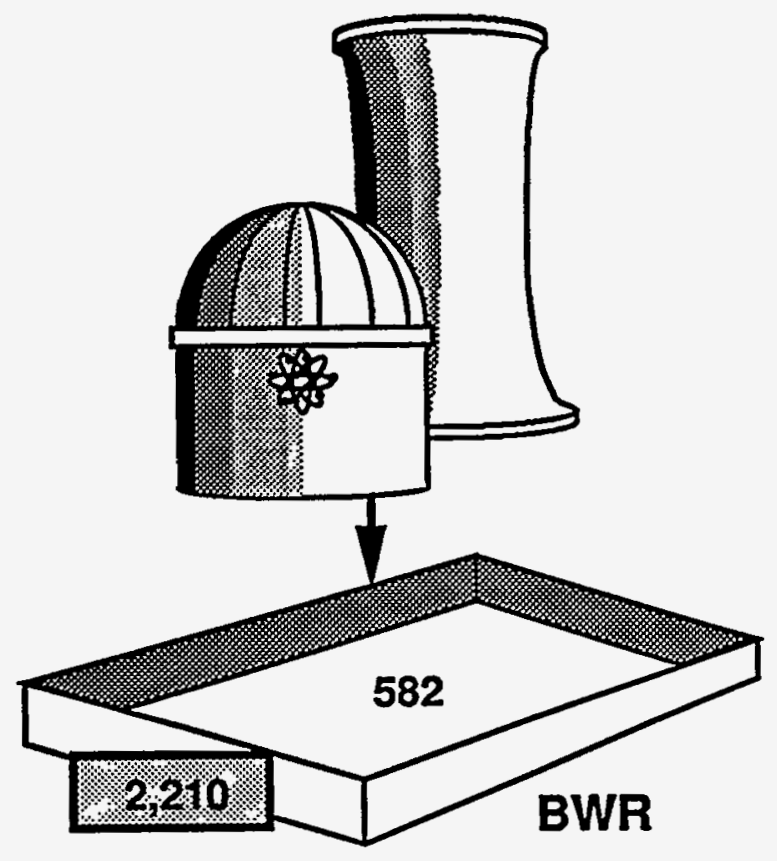

Prairie Island
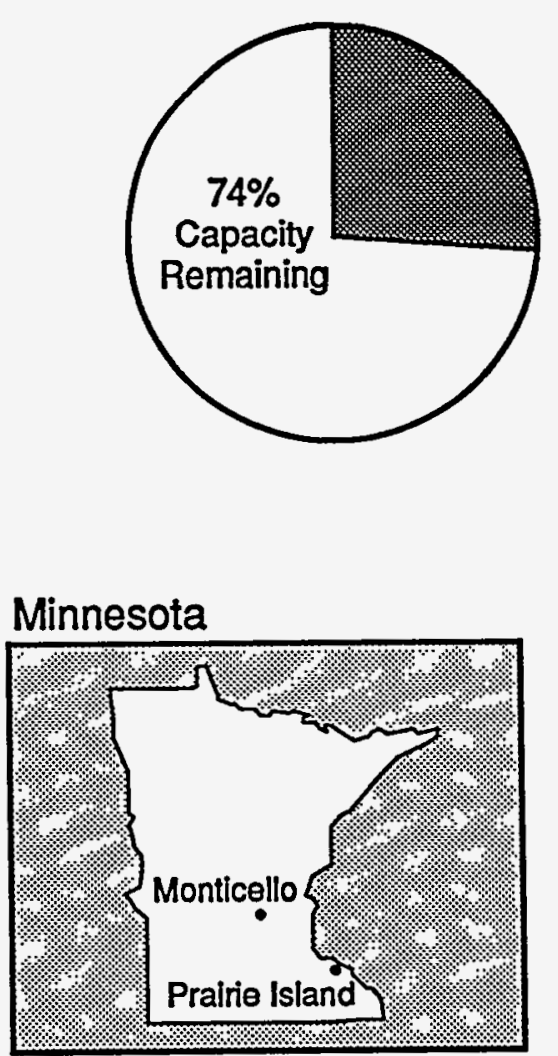

POOL SITE \#3302
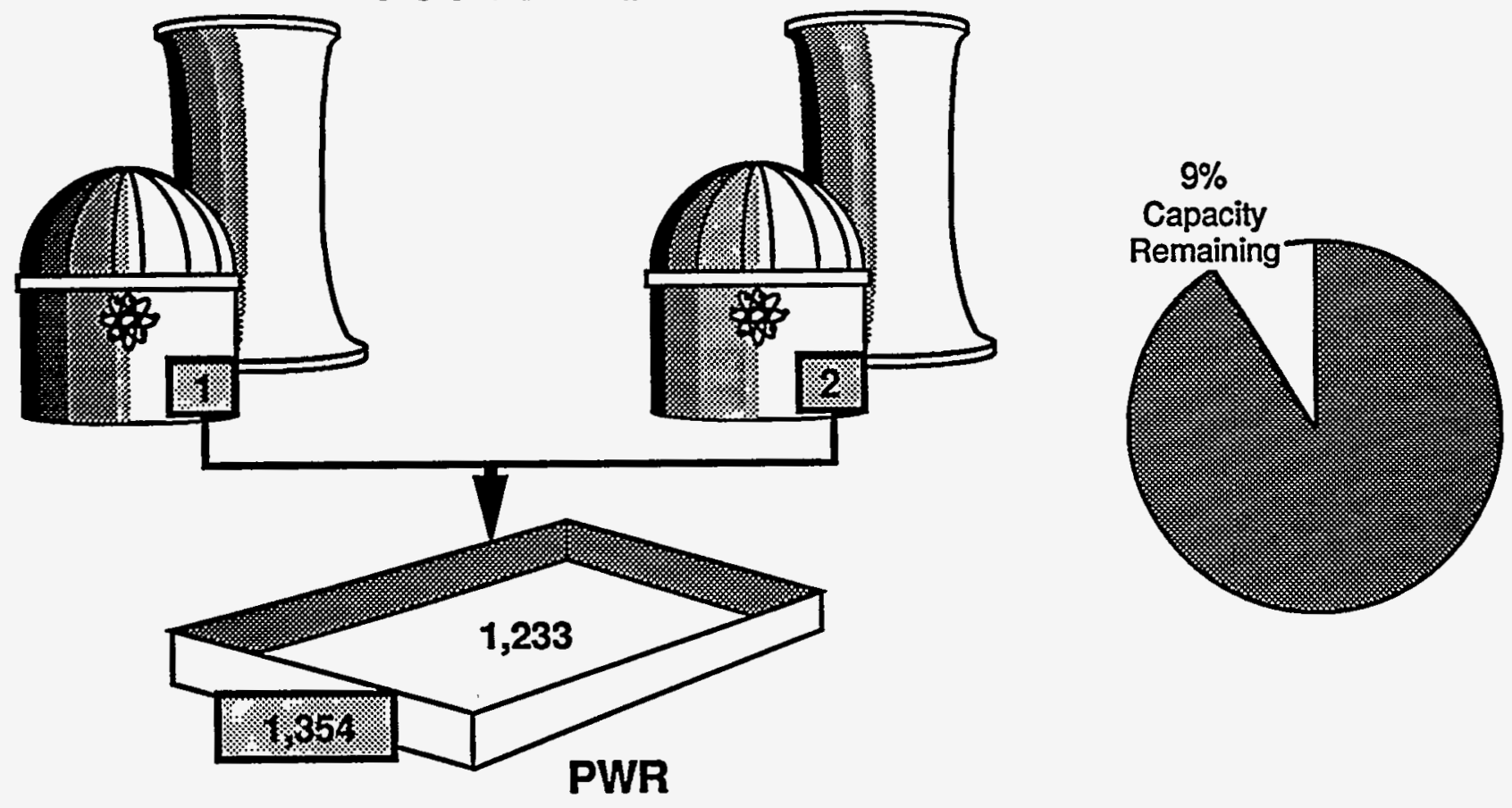


\title{
Omaha Public Power District
}

\author{
Fort Calhoun
}

POOL SITE \#3401
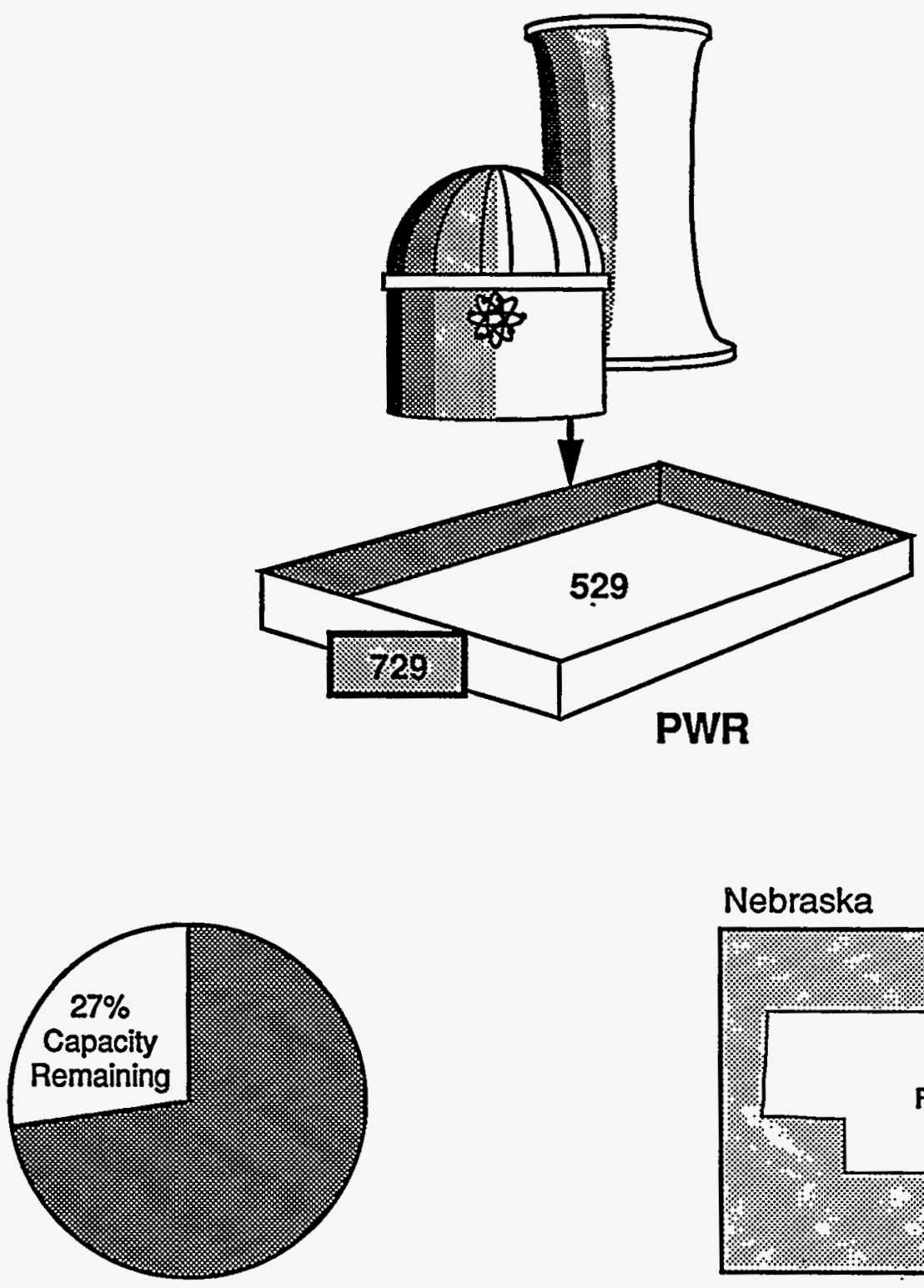

Nebraska

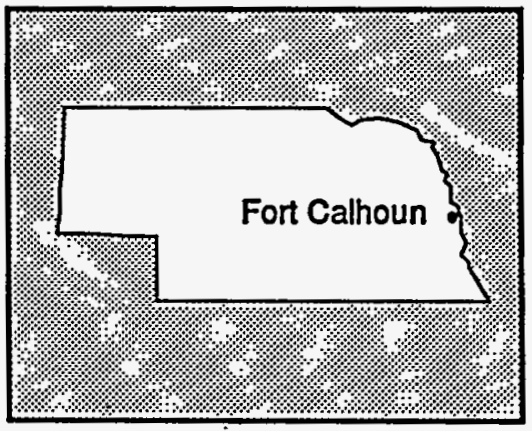




\section{Pacific Gas and Electric Company \\ Diablo Canyon}

POOL SITE \#3502

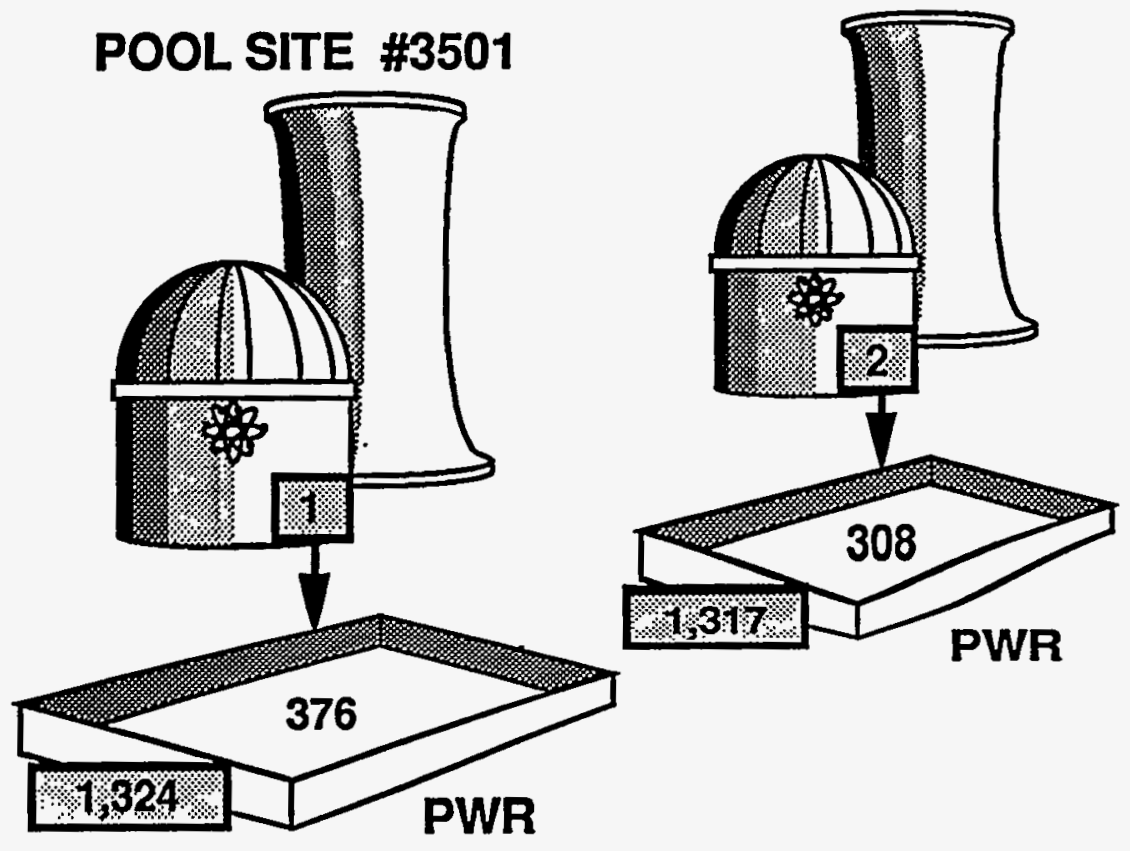

Humboldt Bay

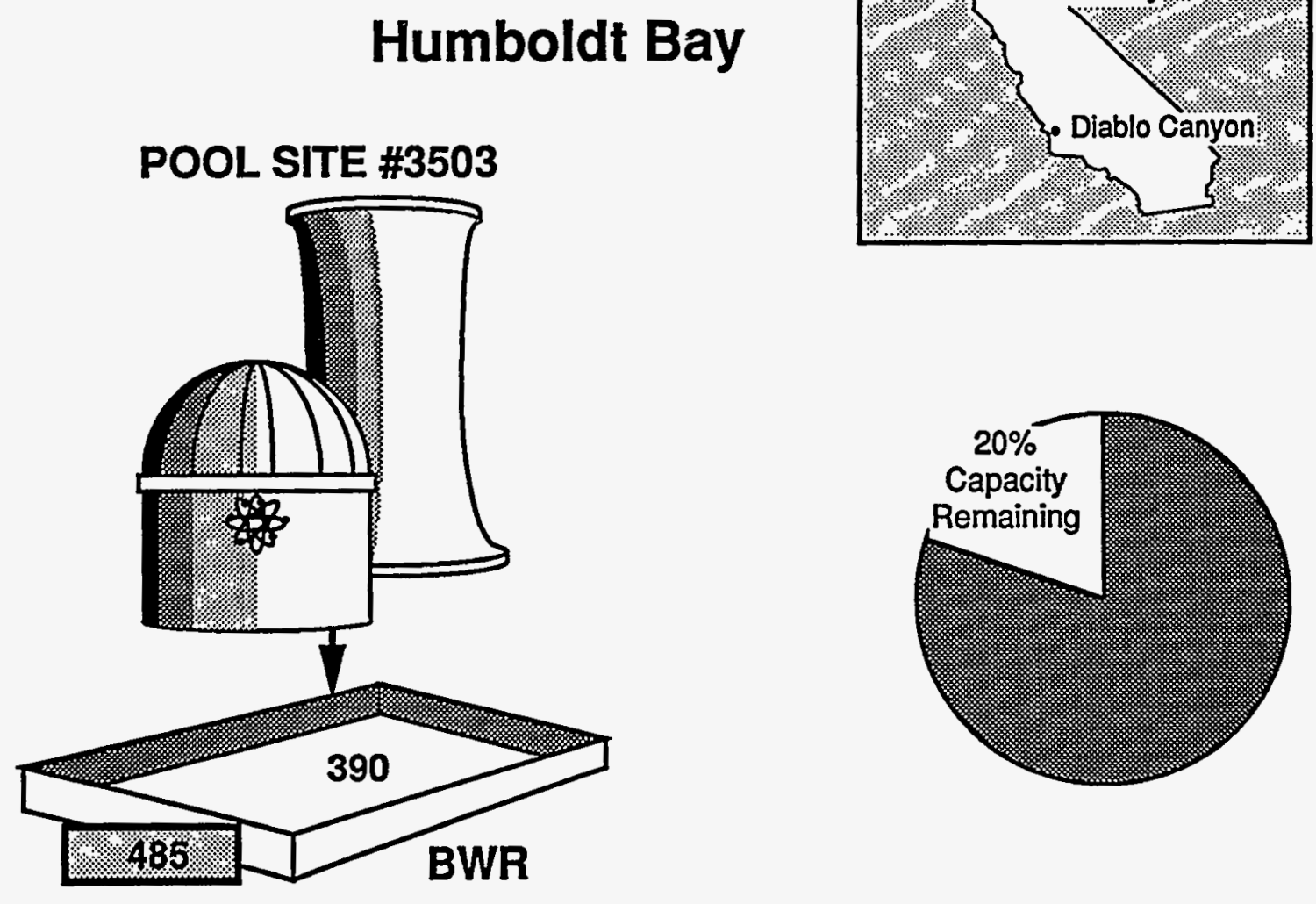

California

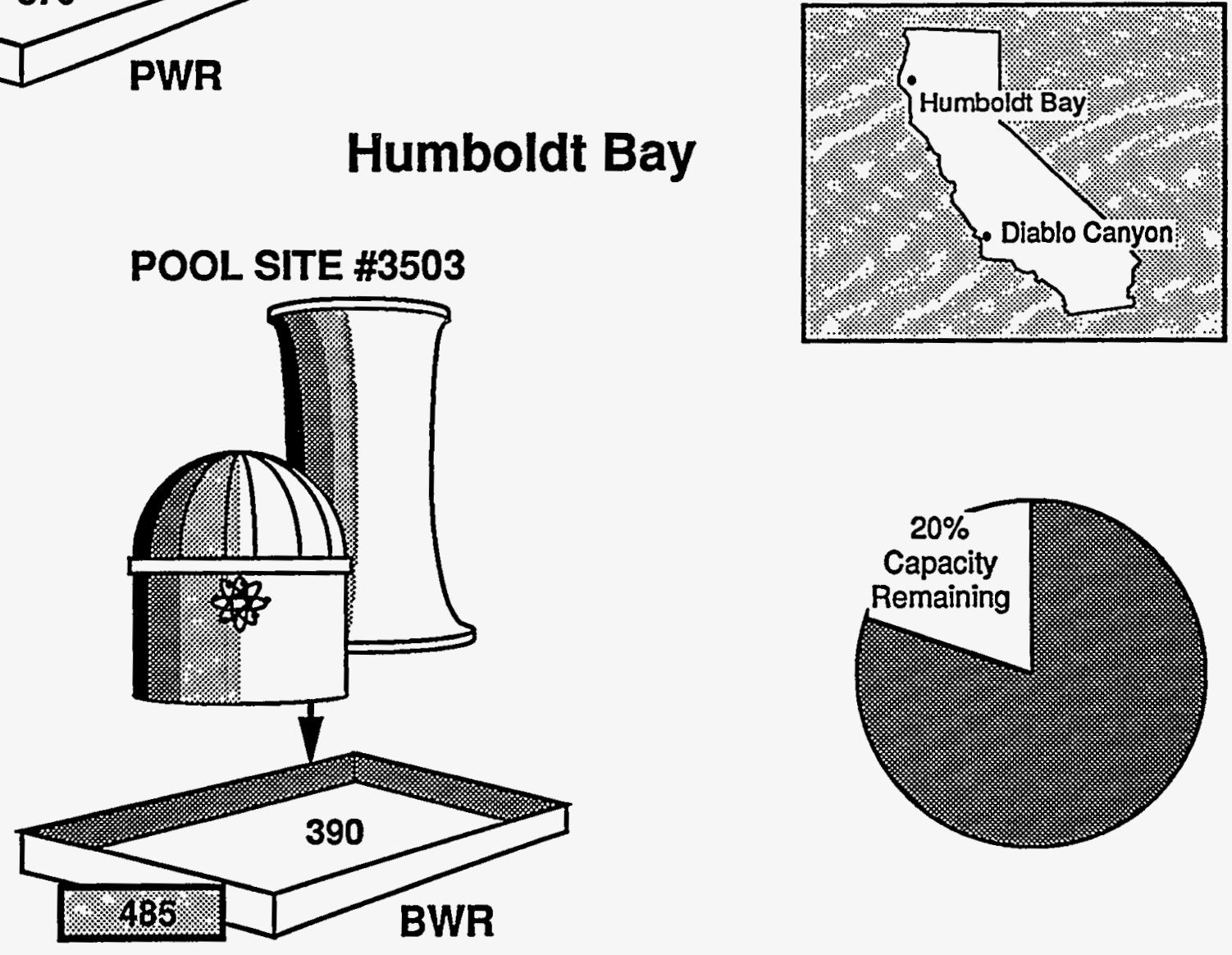

Capacity

Remaining

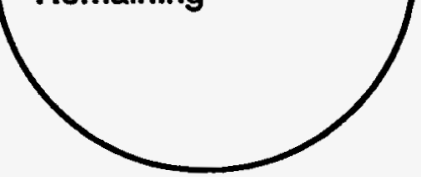




\section{Pennsylvania Power and Light Company}

\section{Susquehanna}
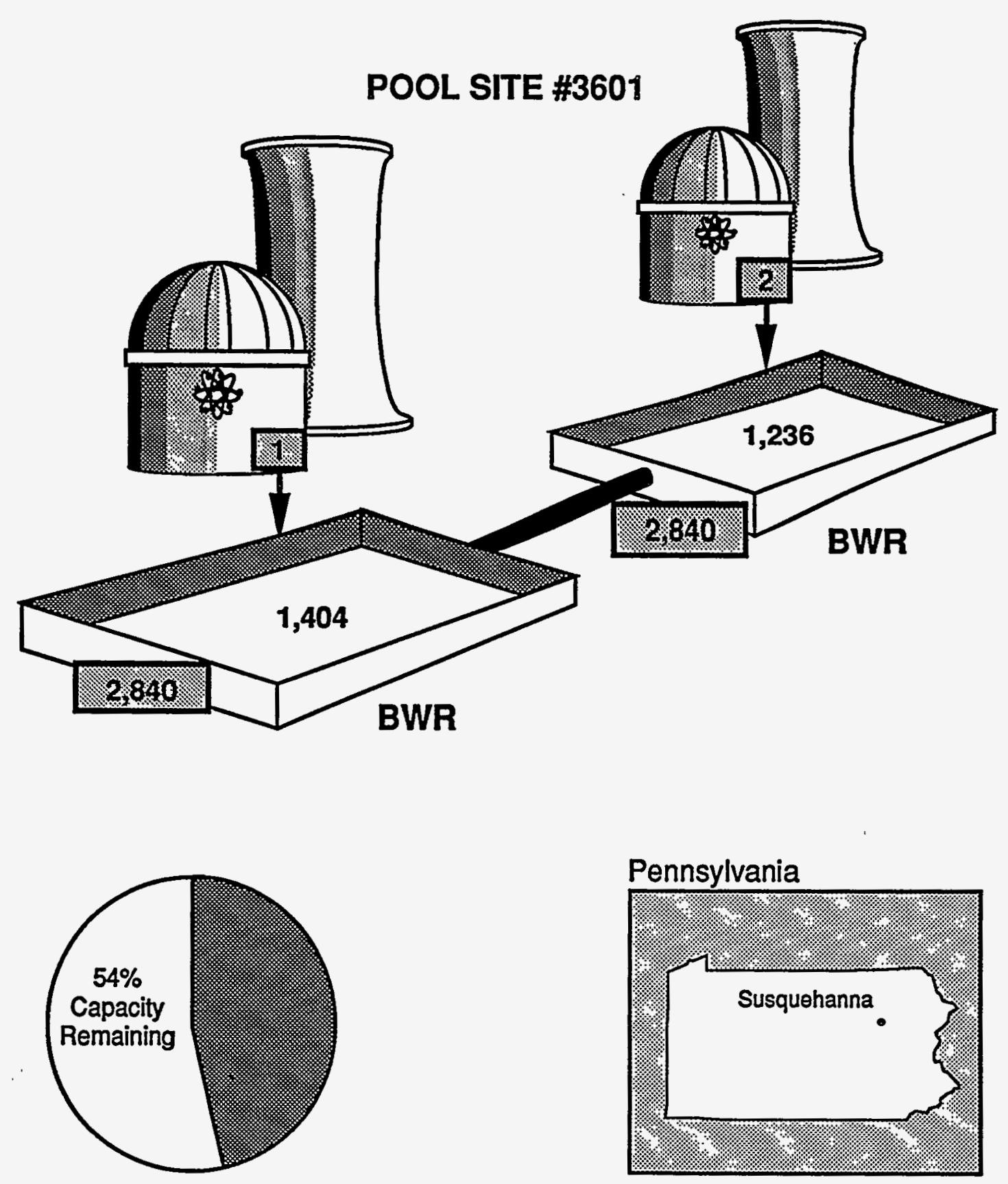


\section{Philadelphia Electric Company}

\section{Limerick}

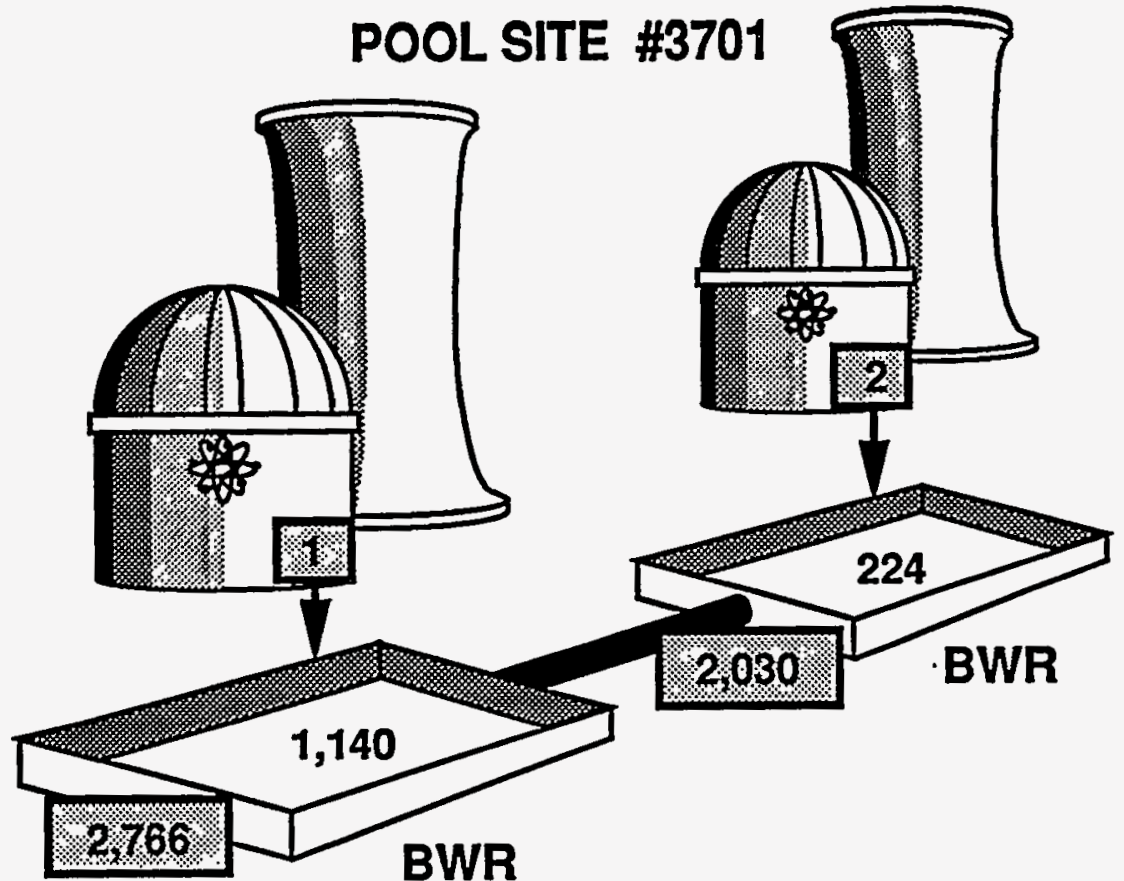

Peach Bottom

POOL SITE \#3705

POOL SITE \#3704

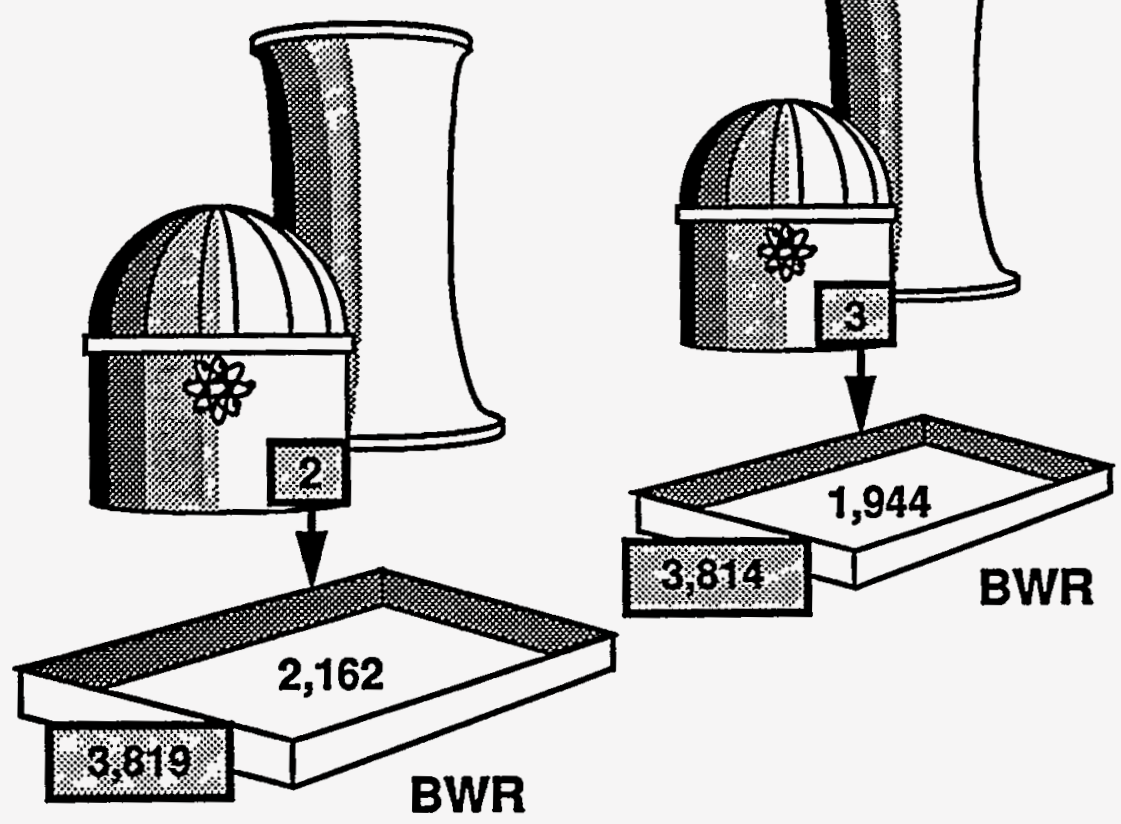

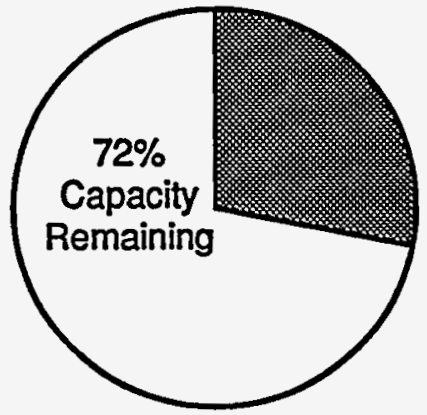

Pennsylvania
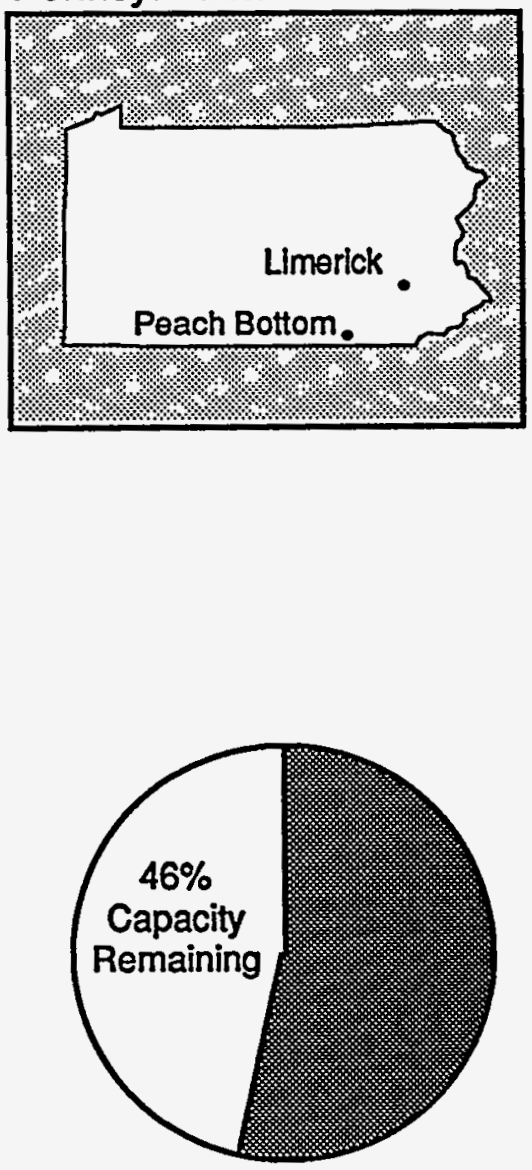


\title{
Portland General Electric Company
}

\author{
Trojan
}

POOL SITE \#3801
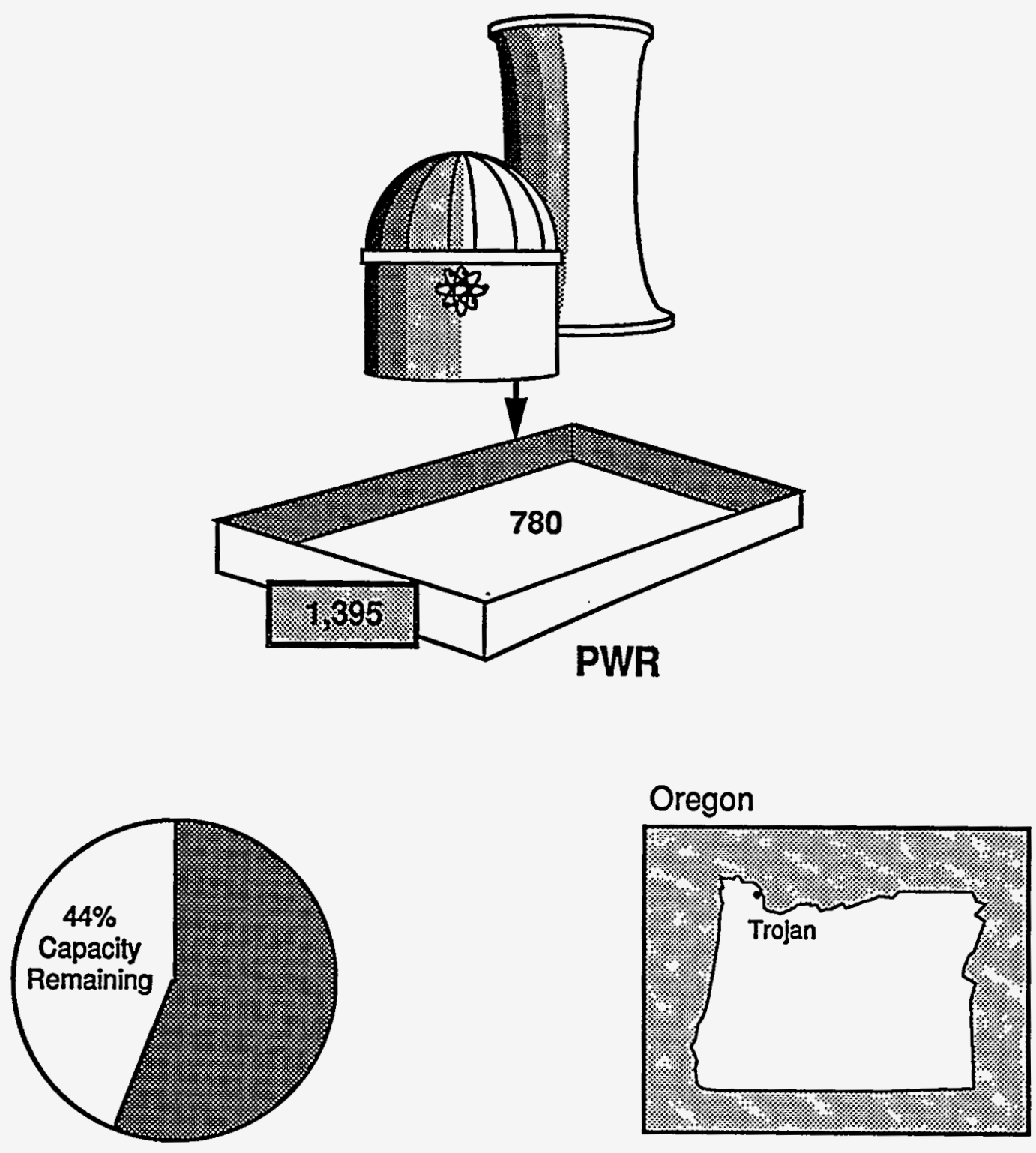


\section{Public Service Electric and Gas Company}

POOL SITE \#4201

\section{Hope Creek}
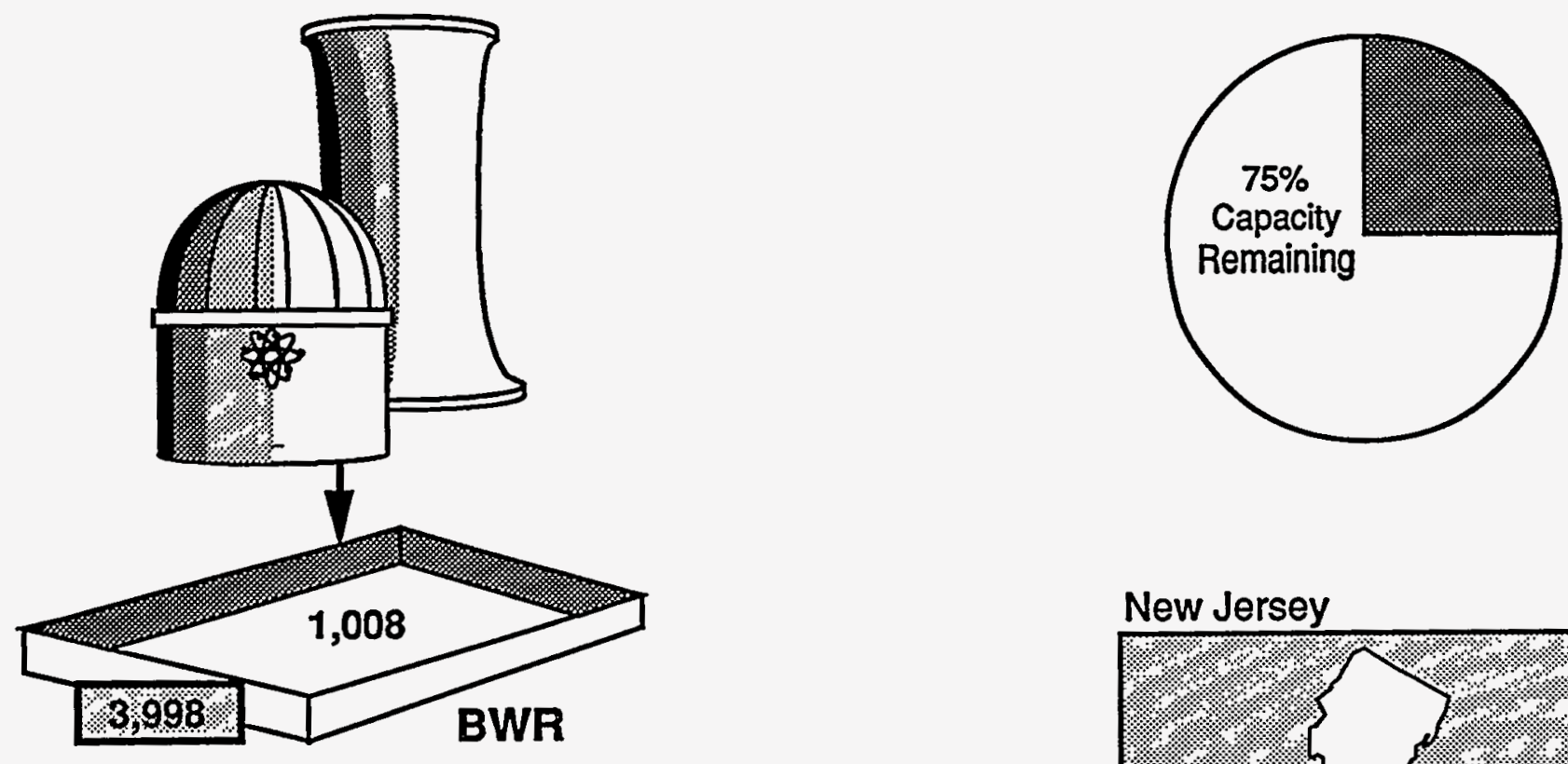

Salem

POOL SITE \#4202

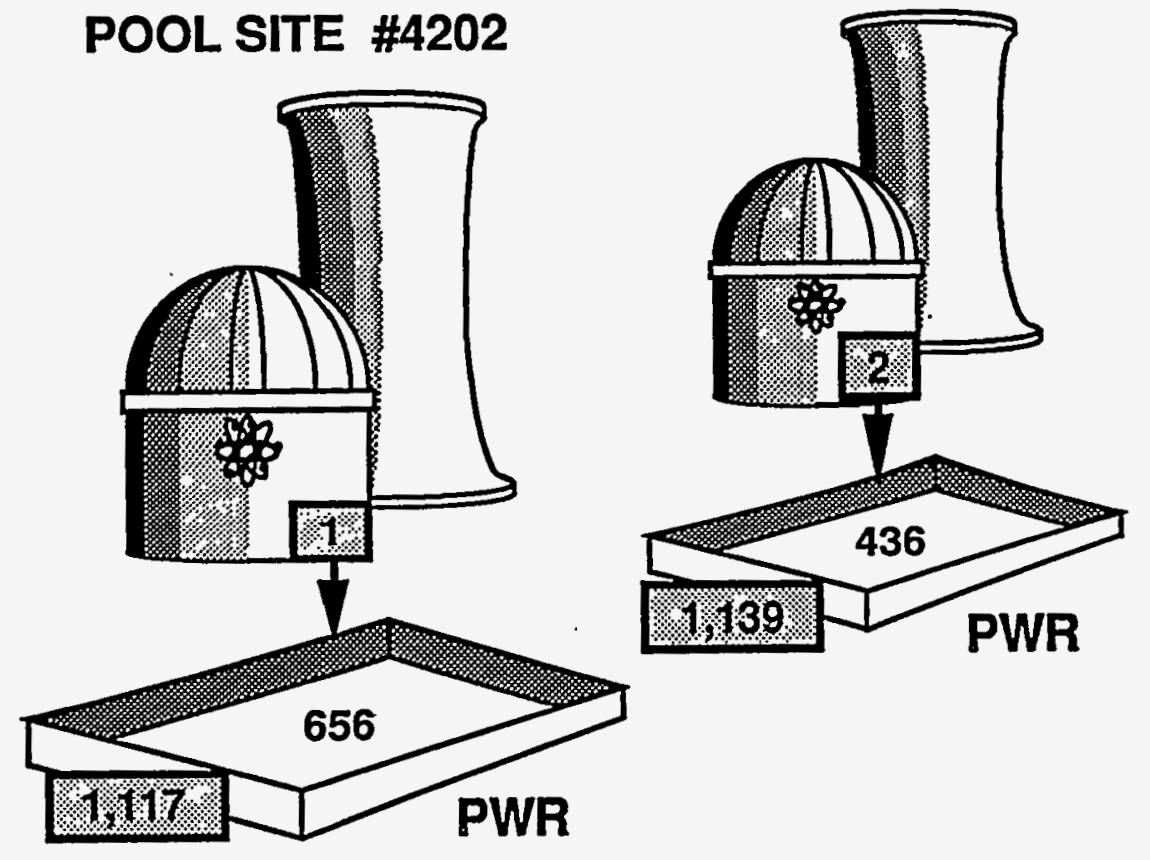

POOL SITE \#4203

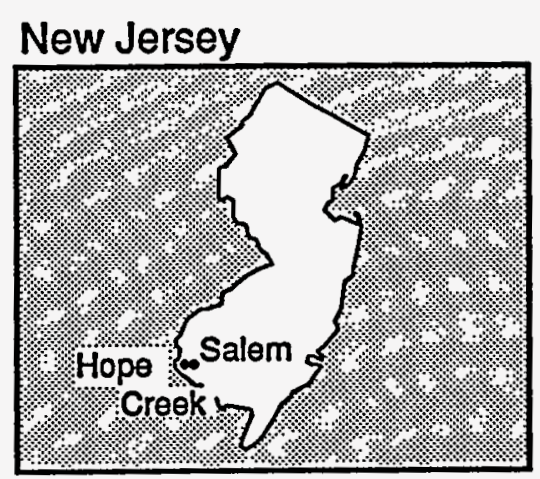




\title{
Rochester Gas and Electric Corporation
}

\author{
Ginna
}

\section{POOL SITE \#4401}
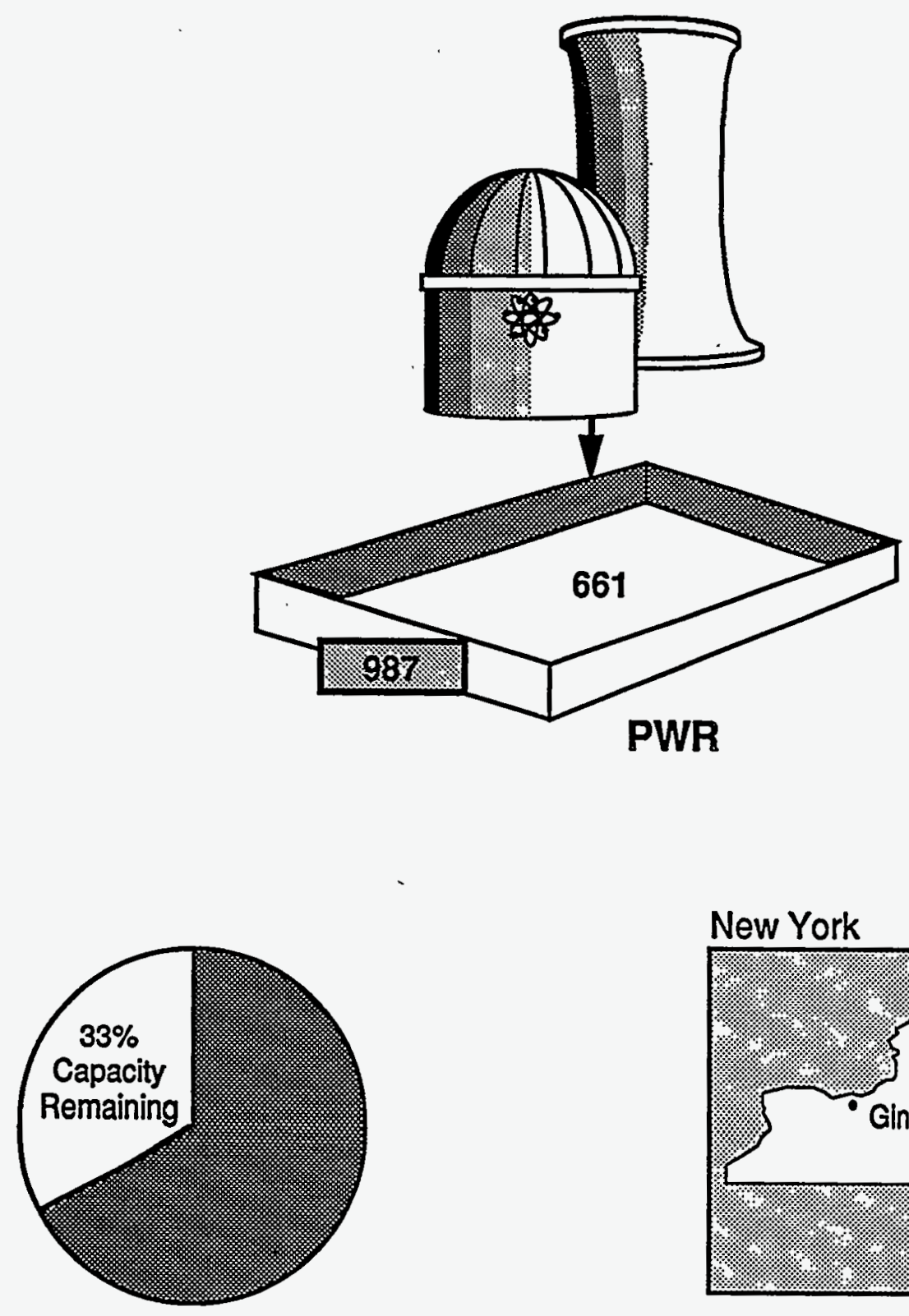

New York

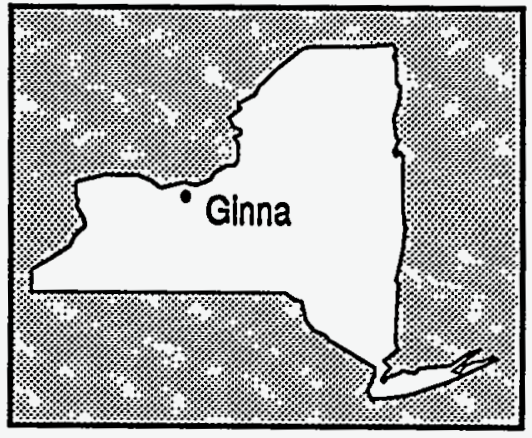




\title{
Sacramento Municipal Utility District Rancho Seco
}

\author{
POOL SITE \#4501
}
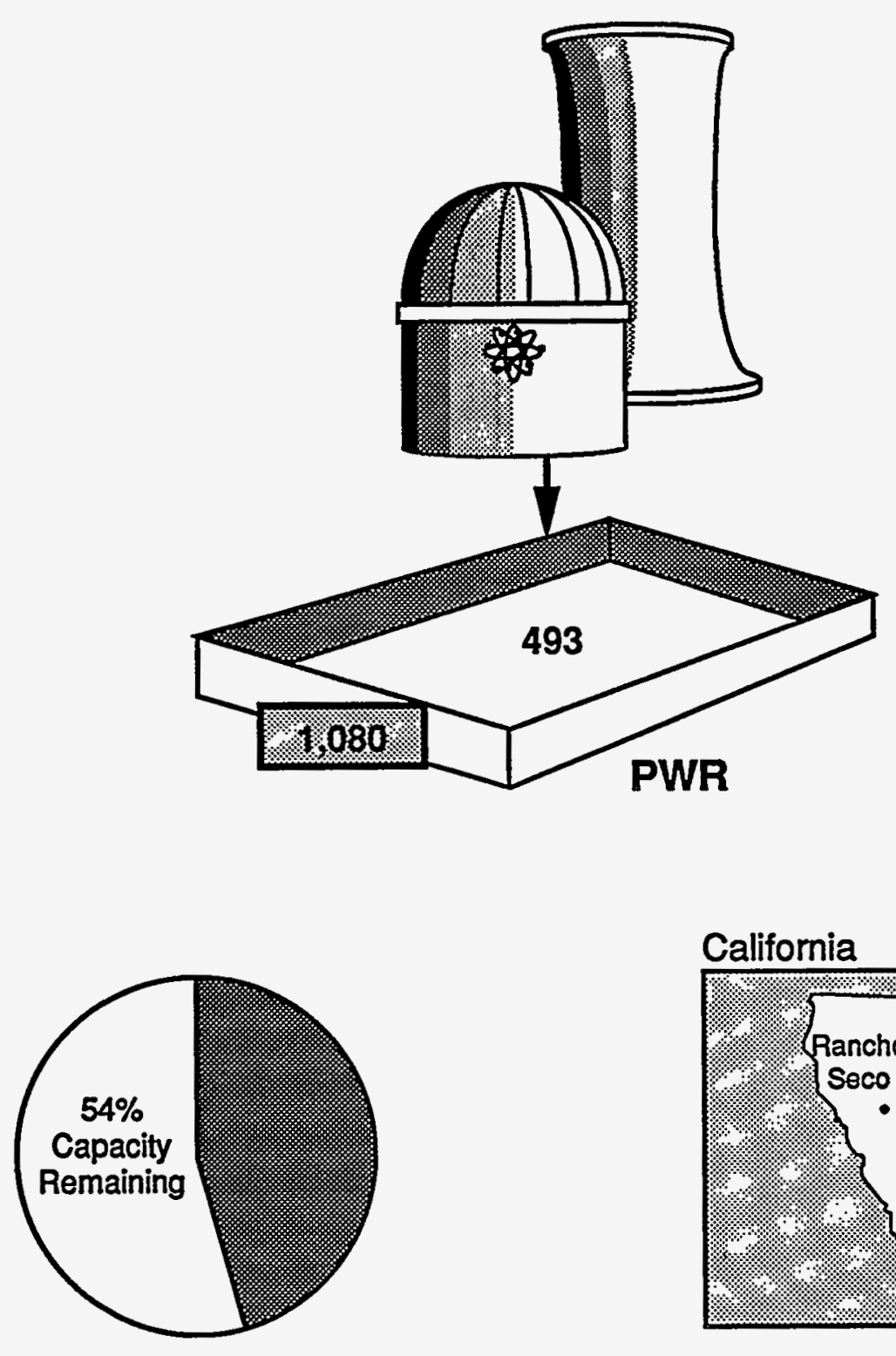

California

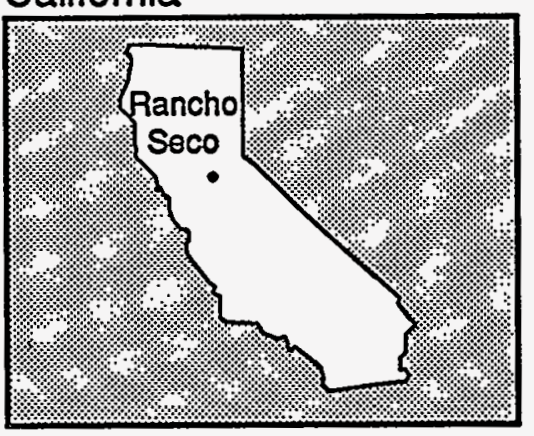




\section{South Carolina Electric and Gas Company

\author{
Summer
}
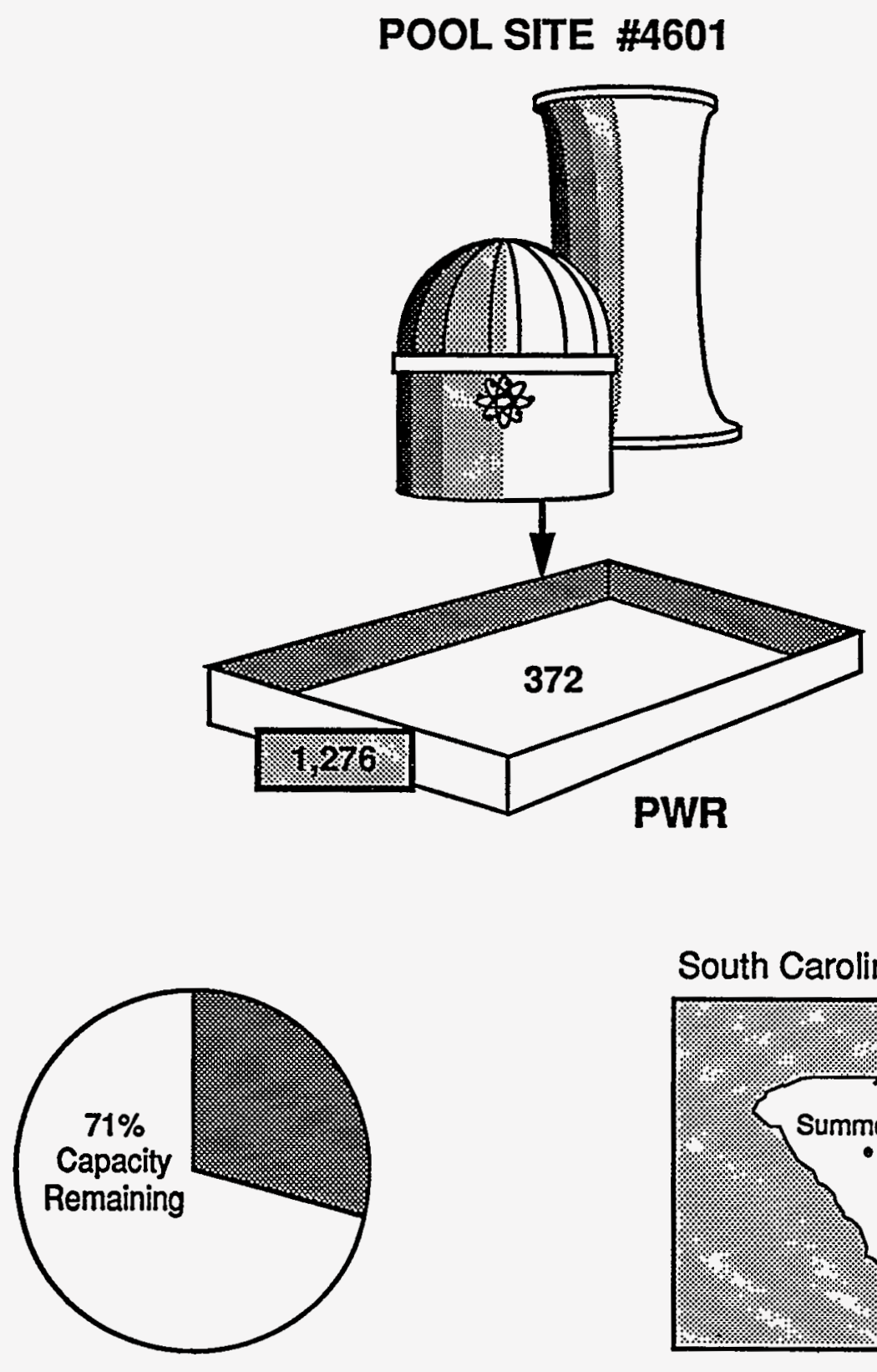

South Carolina

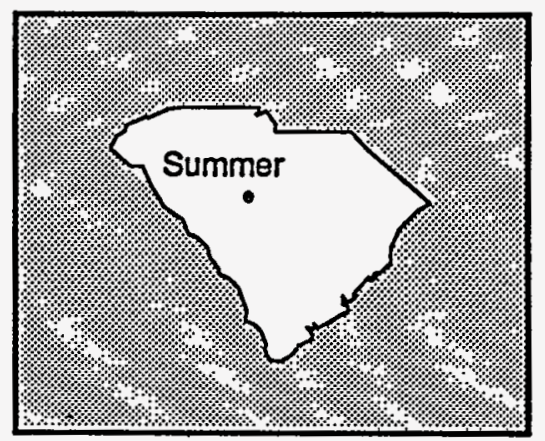




\section{Southern California Edison Company}

\section{San Onofre}
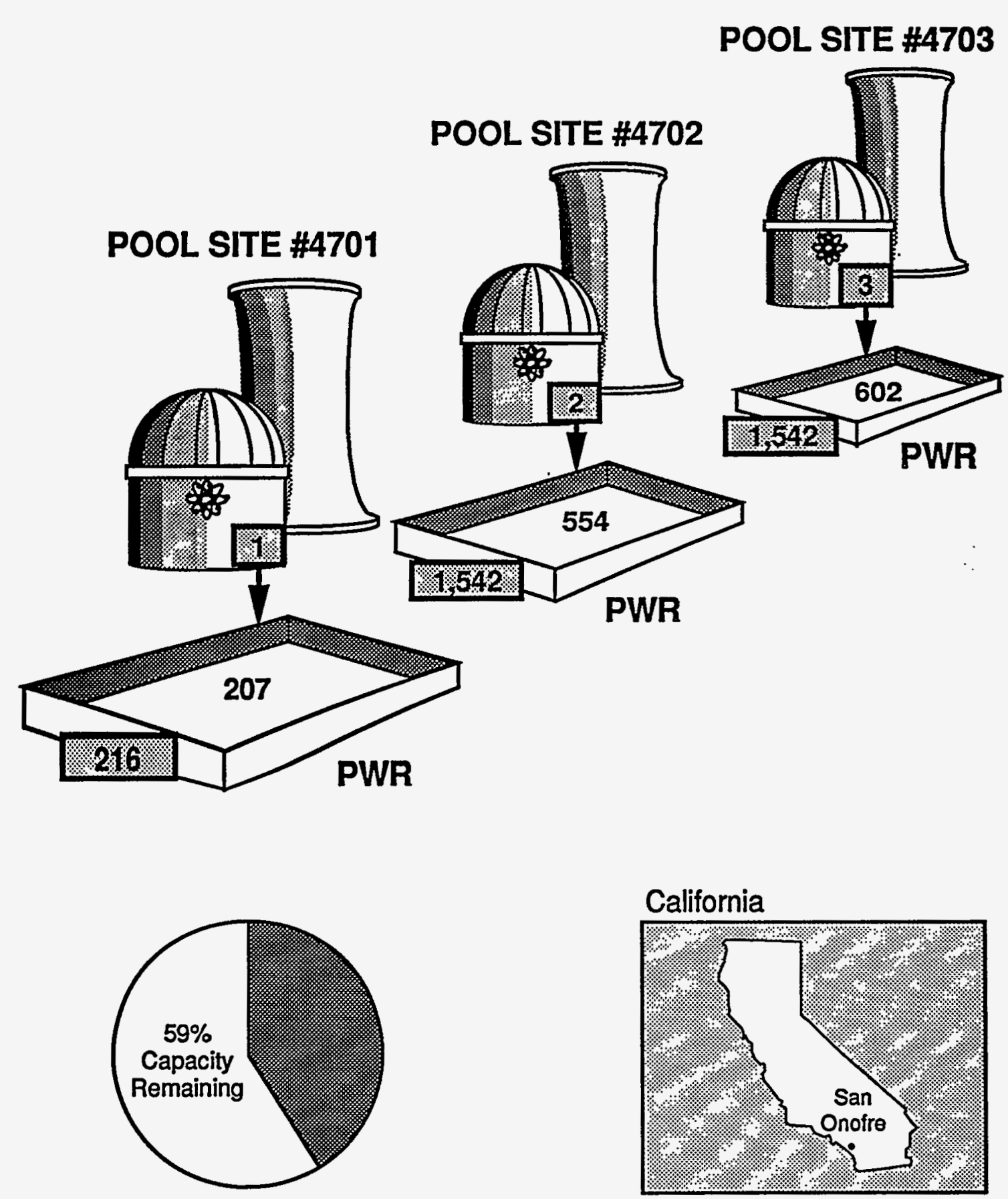


\title{
System Energy Resources, Inc.
}

\author{
Grand Gulf
}
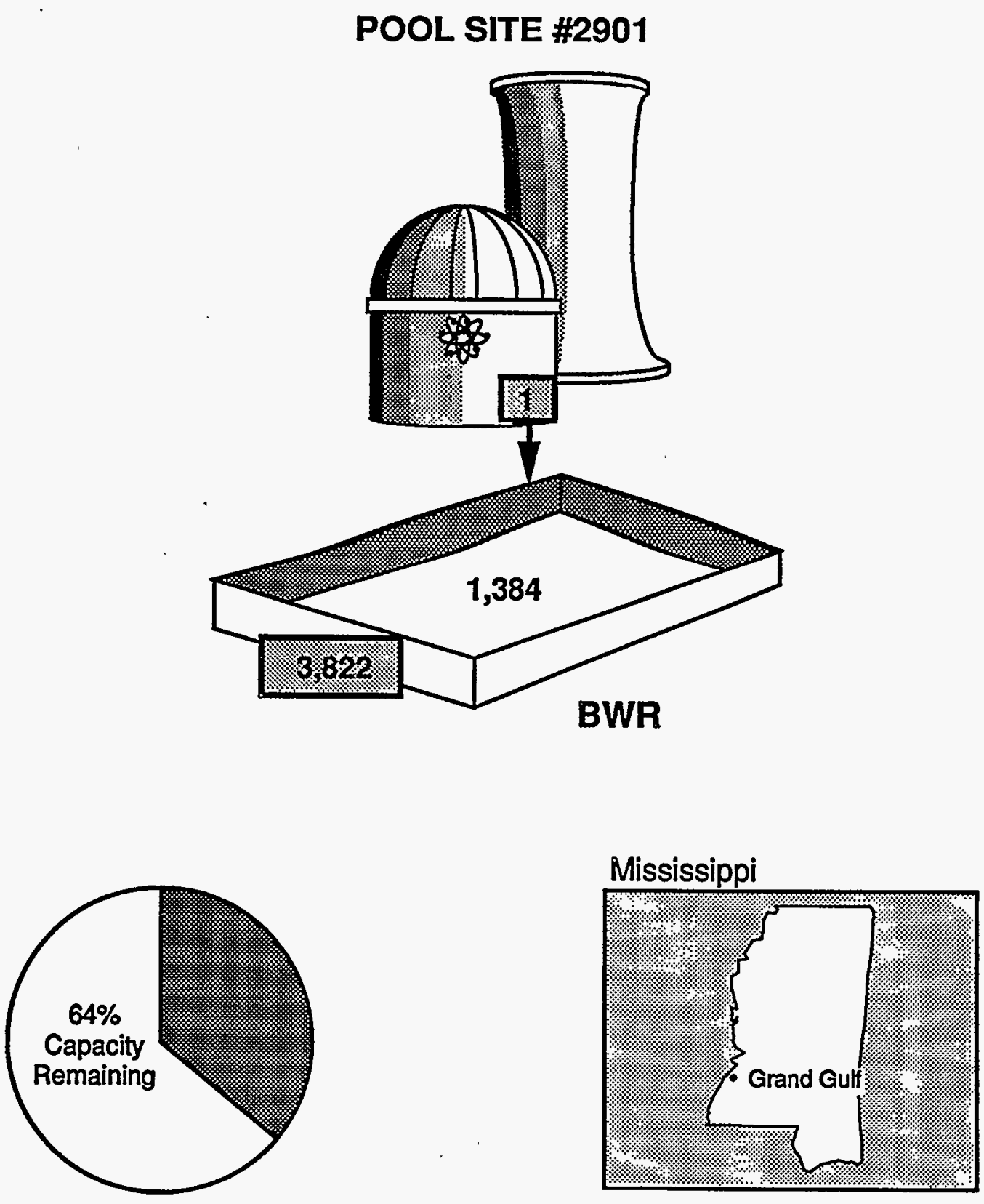


\section{Tennessee Valley Authority Browns Ferry}

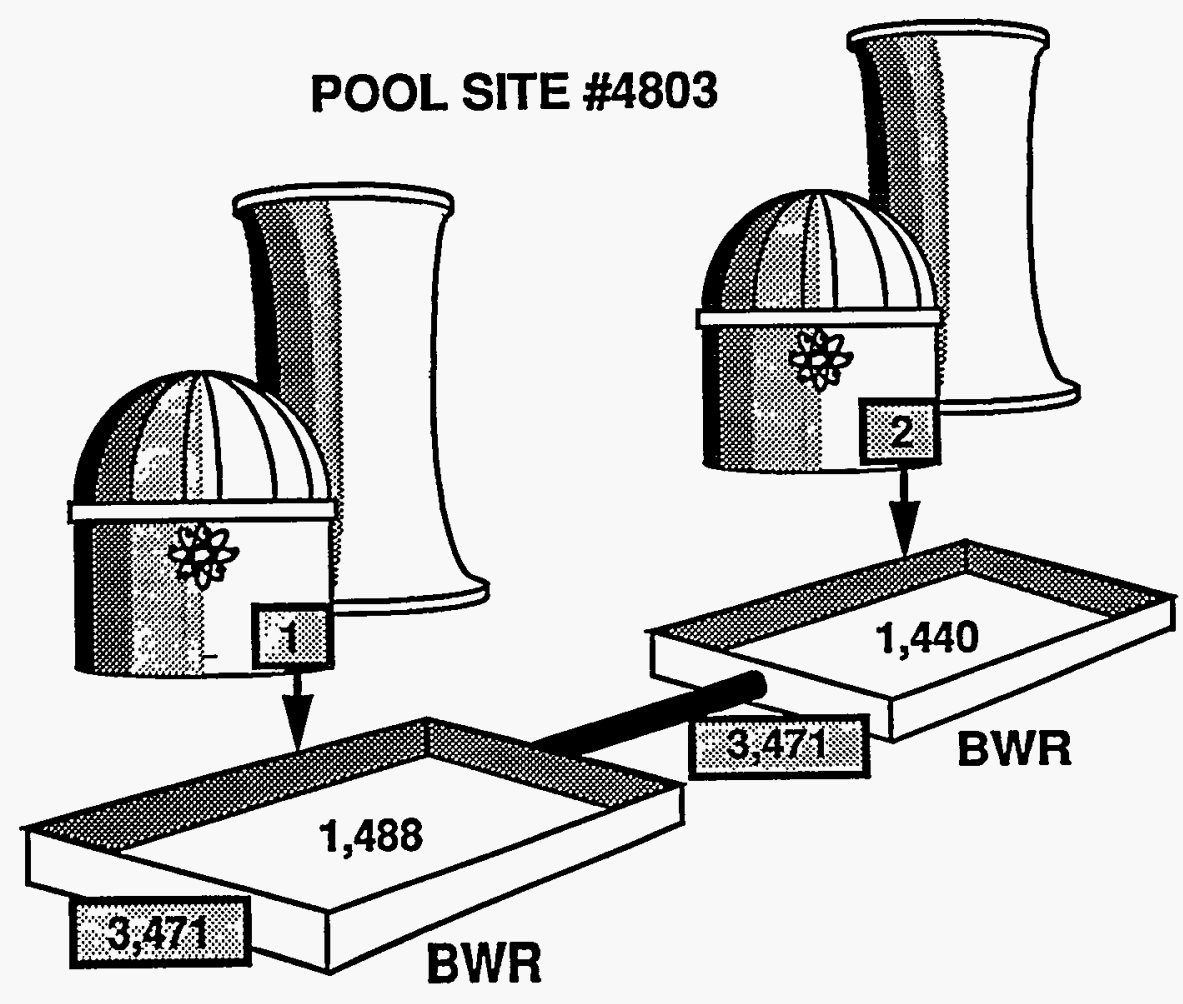

POOL SITE \#4805

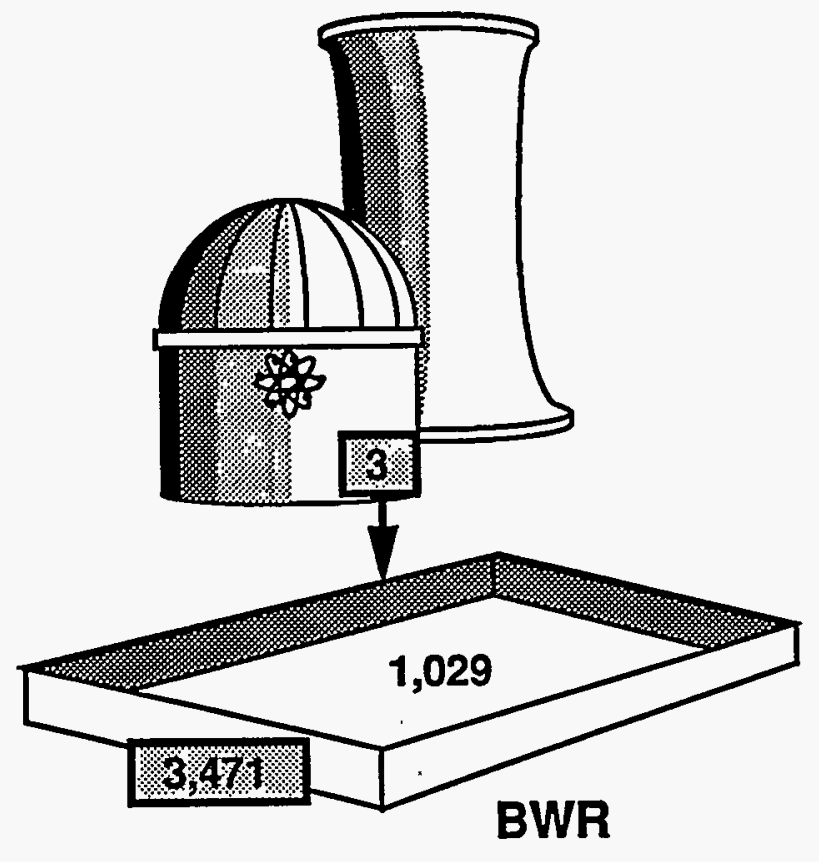

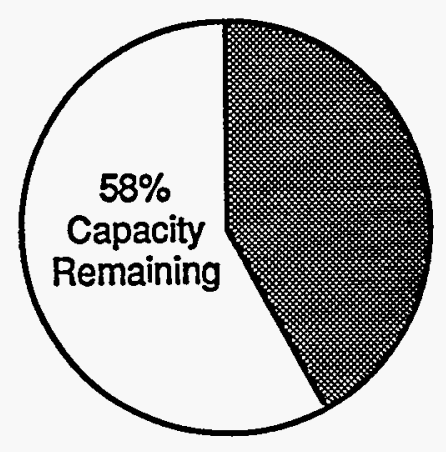

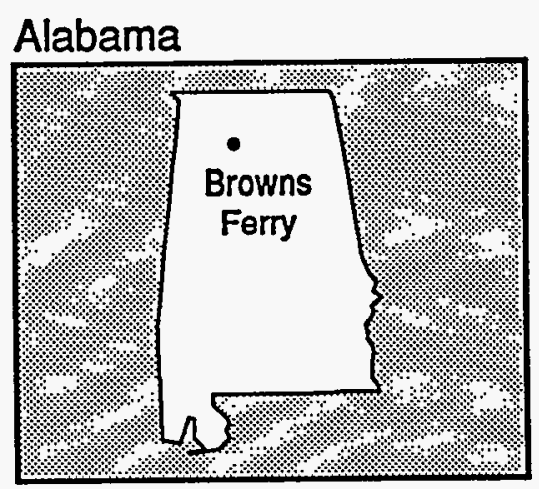




\section{Tennessee Valley Authority (Continued)}

Sequoyah

POOL SITE \#4808

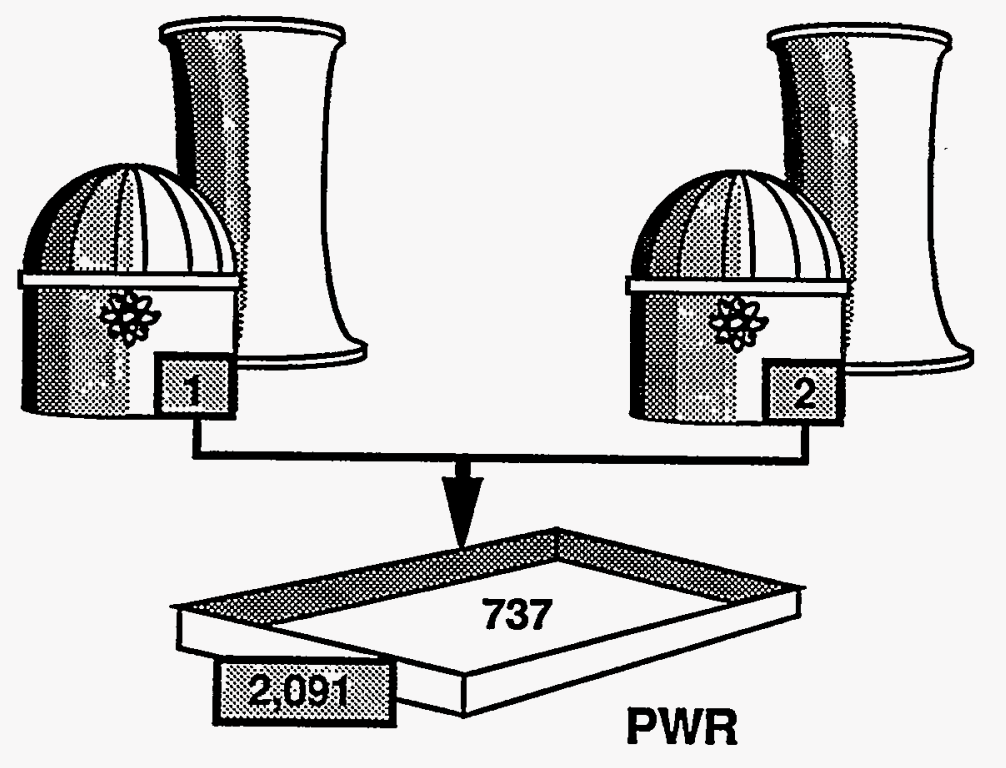

\section{Watts Bar}
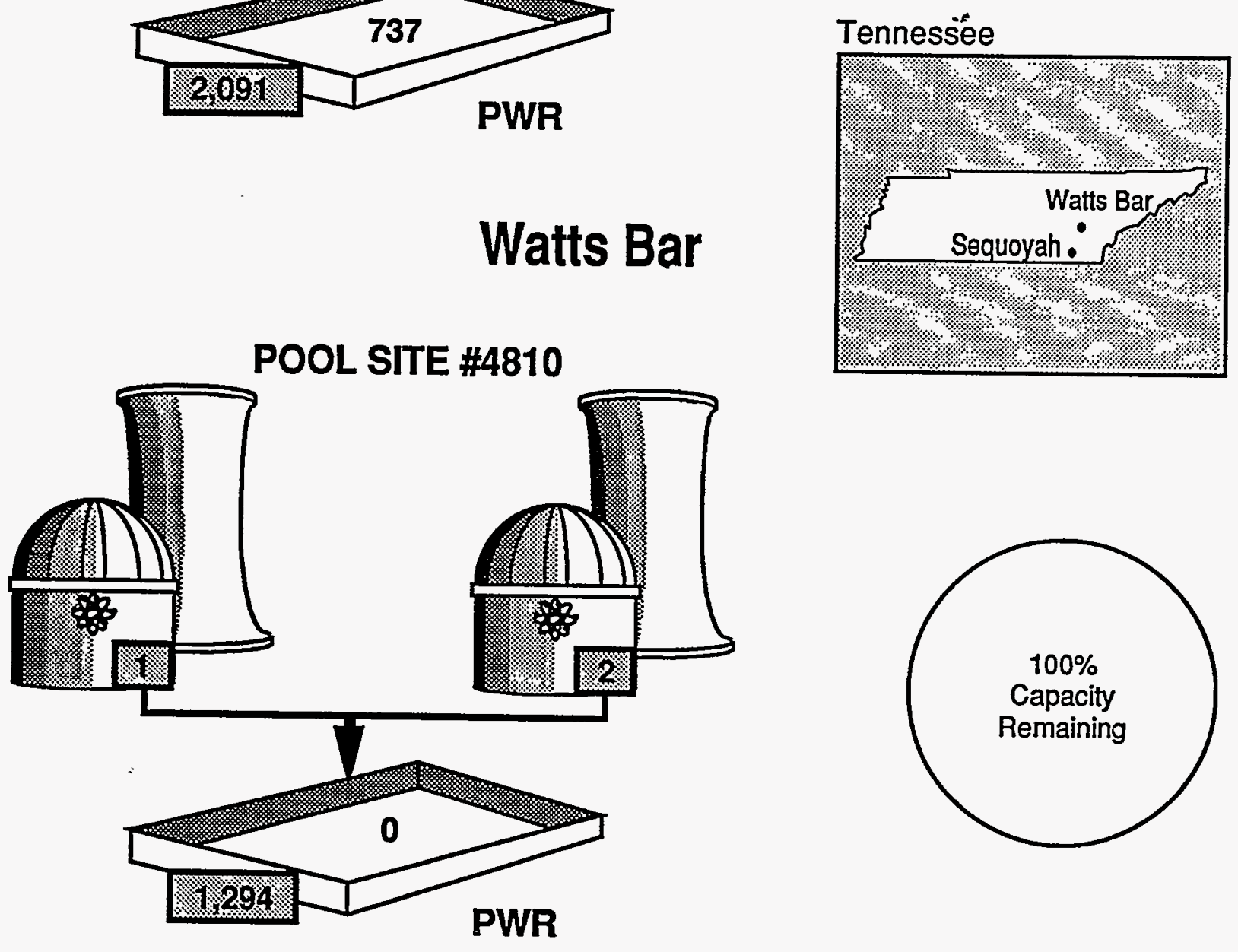


\section{TU Electric \\ Comanche Peak}
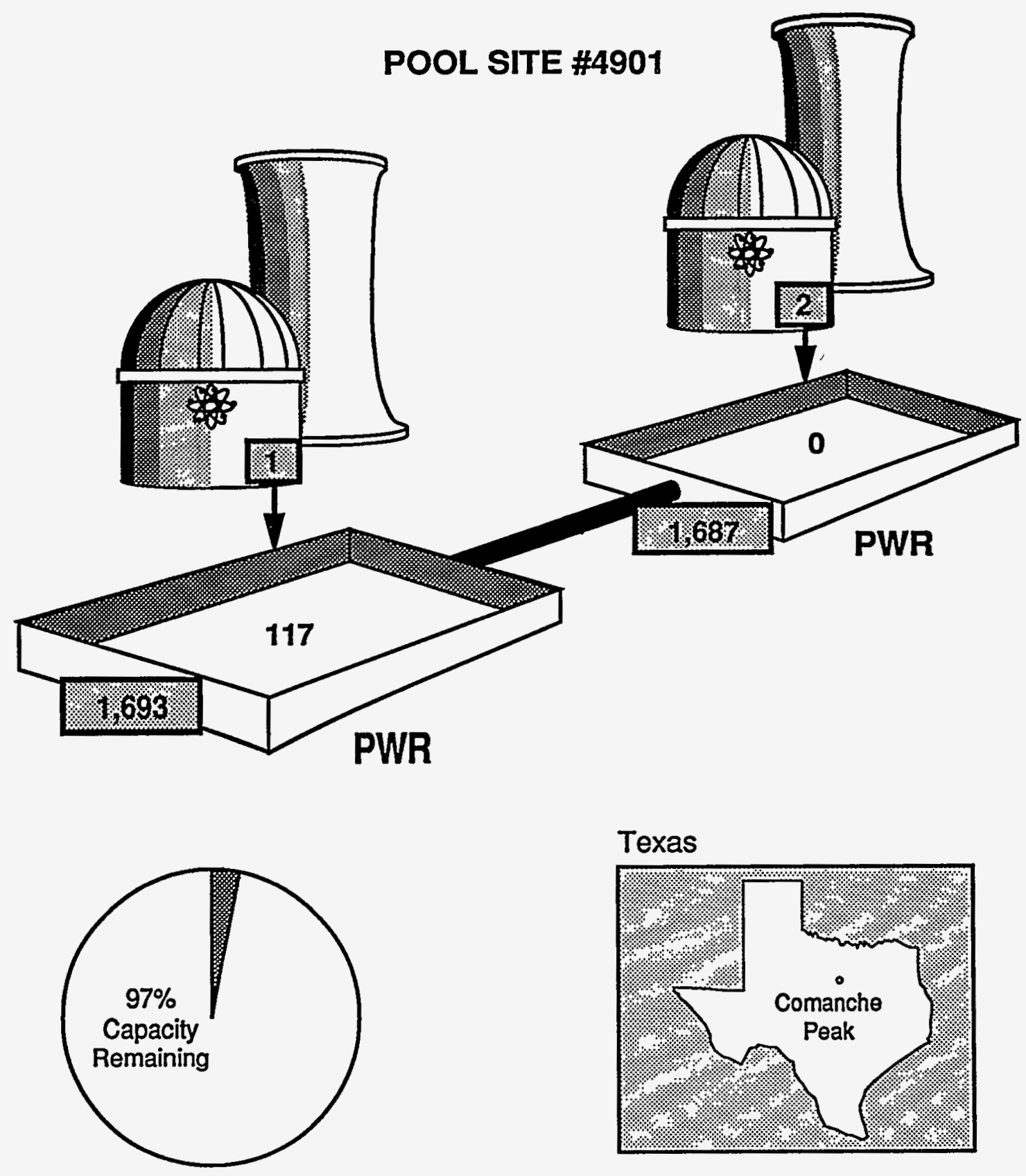

Texas

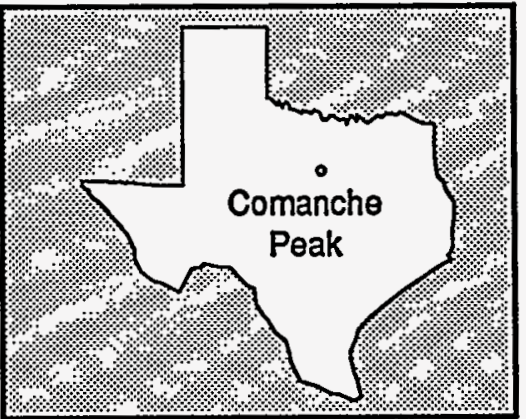




\section{Toledo Edison Company Davis-Besse}
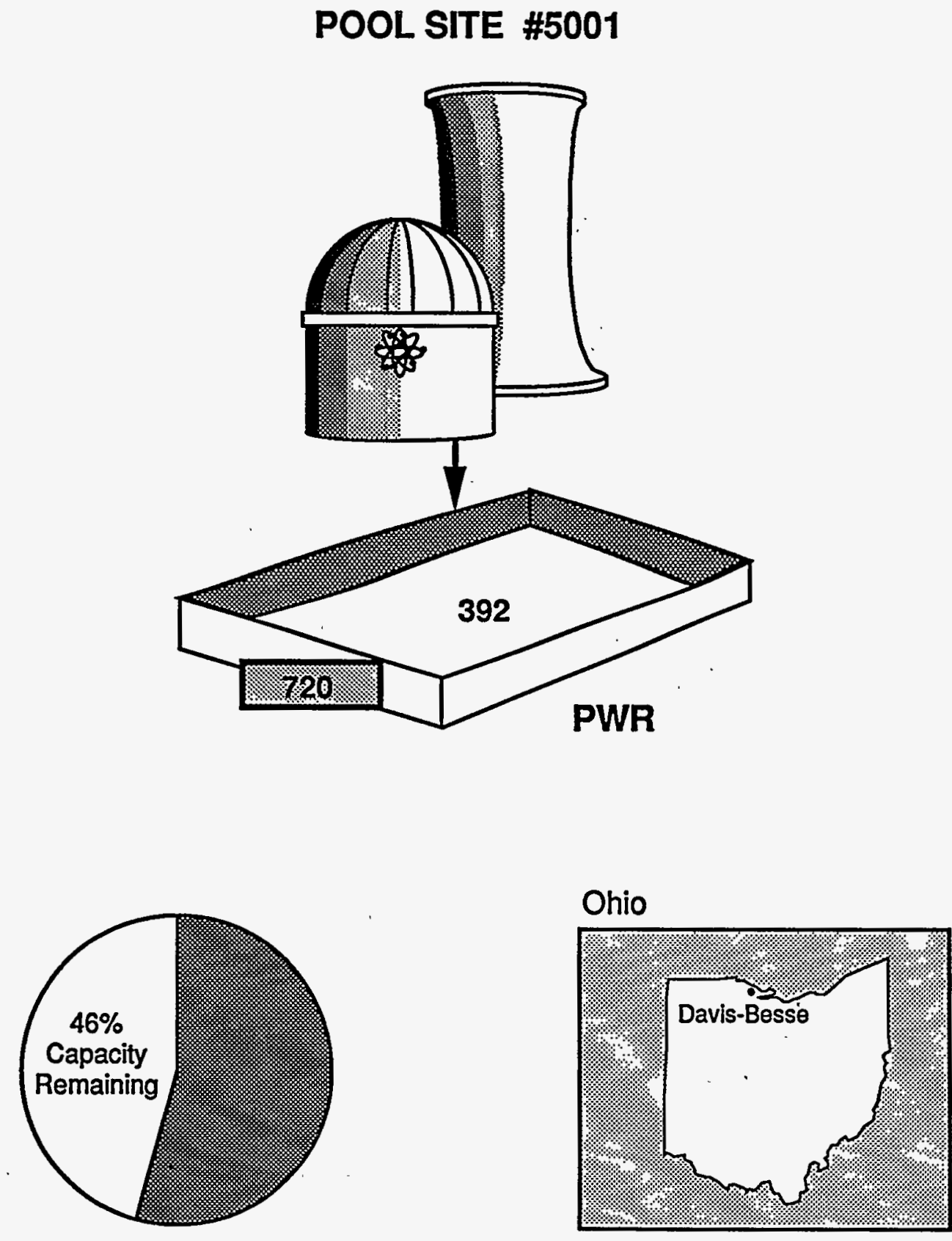


\section{Union Electric Company Callaway}
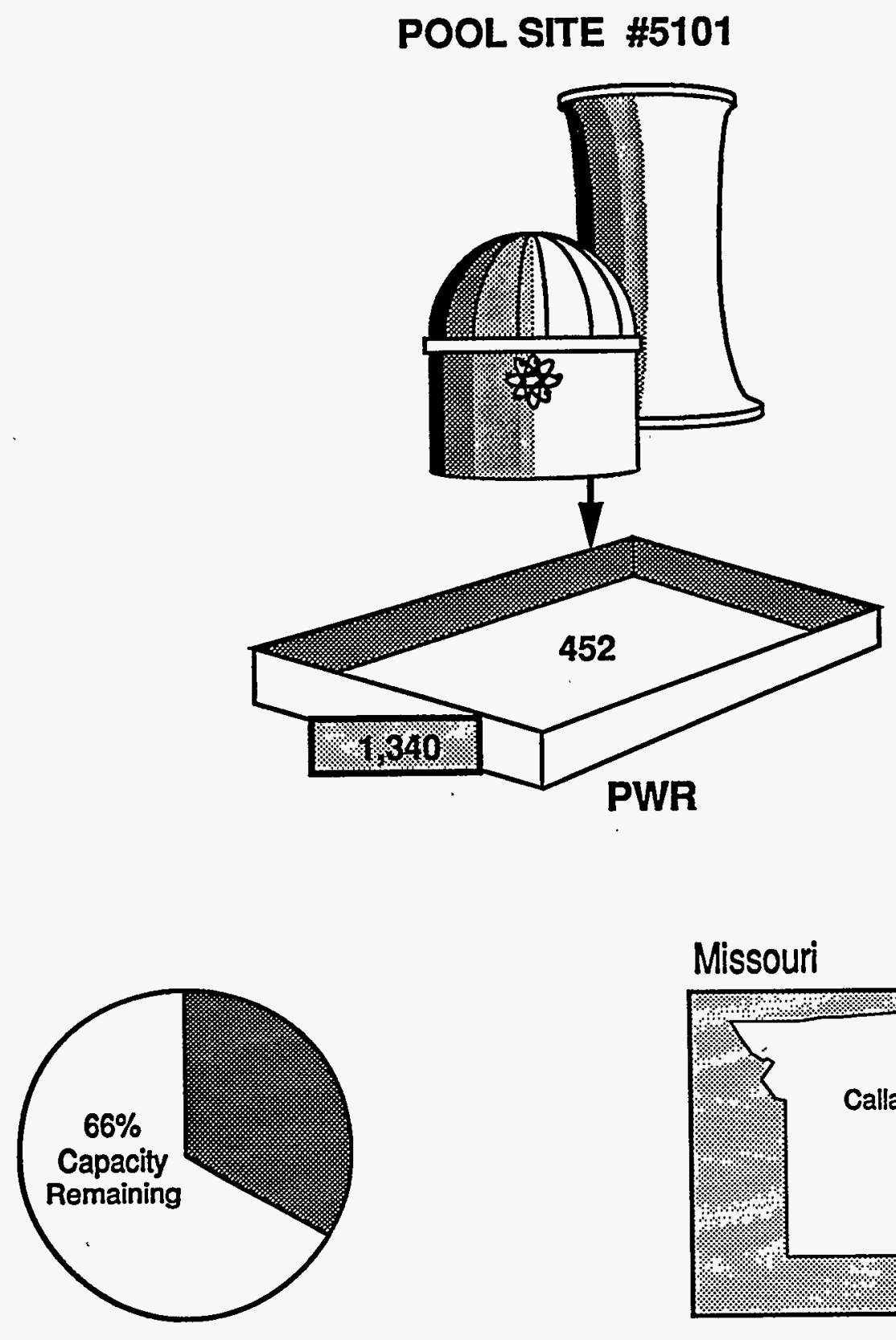

Missouri

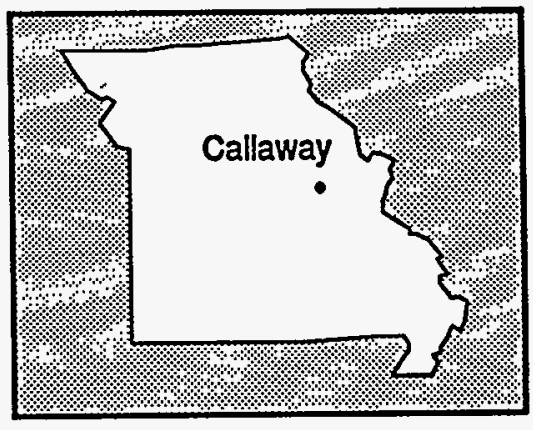




\title{
Vermont Yankee Nuclear Power Corporation
}

\author{
Vermont Yankee
}
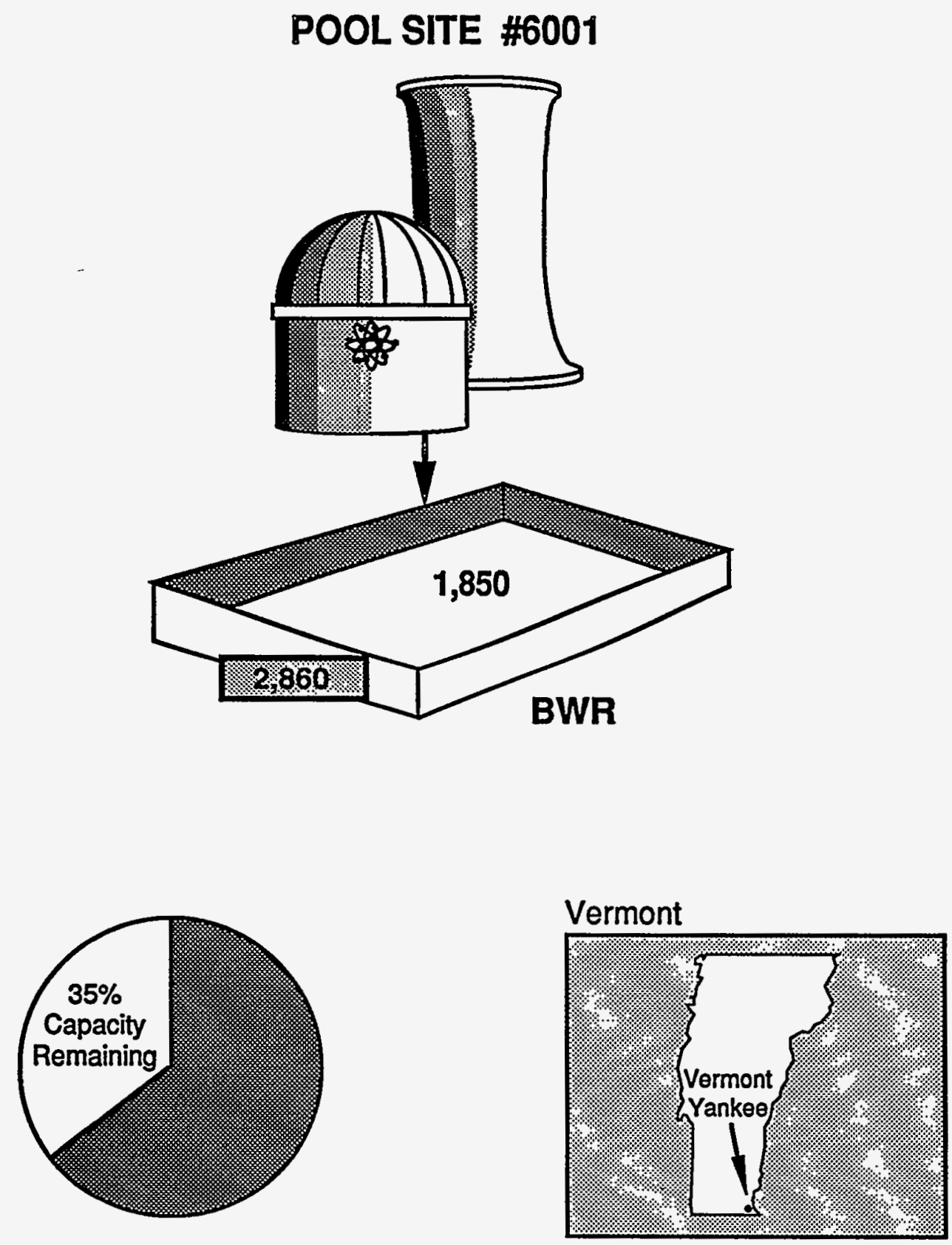
This page is intentionally blank. 


\title{
Virginia Power
}

\author{
North Anna
}

POOL SITE \#5201
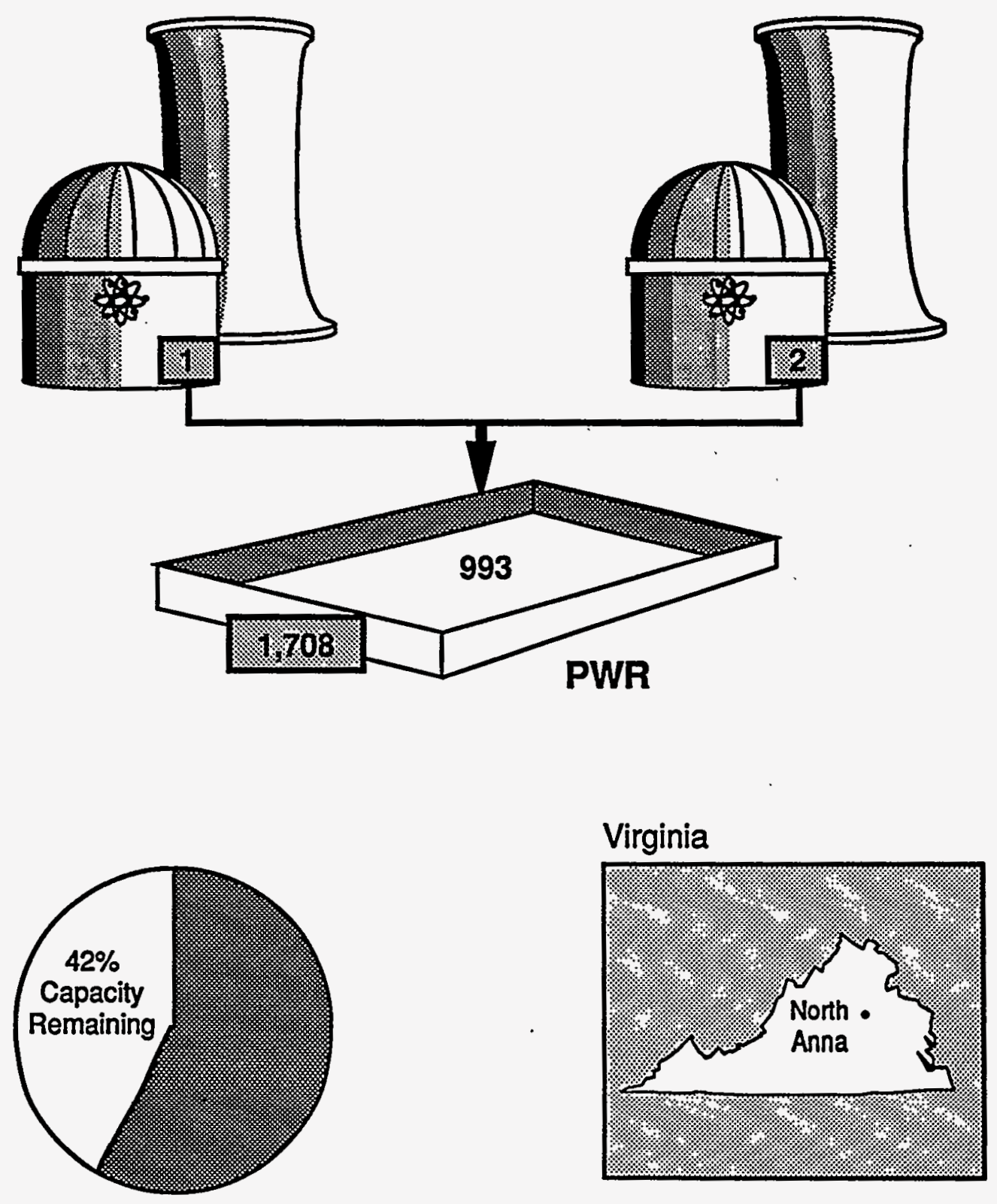


\section{Virginia Power \\ (Continued) \\ Surry}

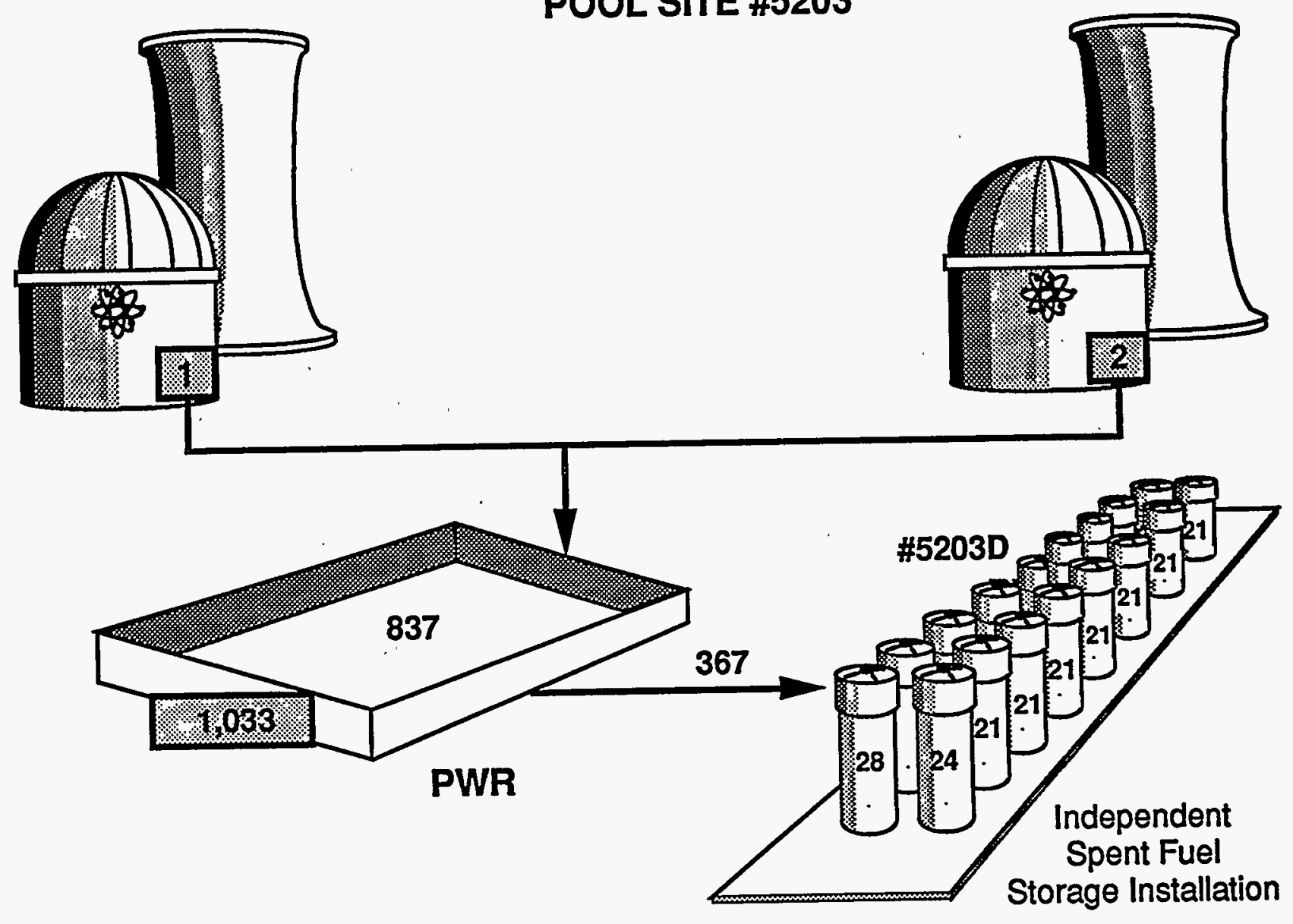

POOL SITE \#5203

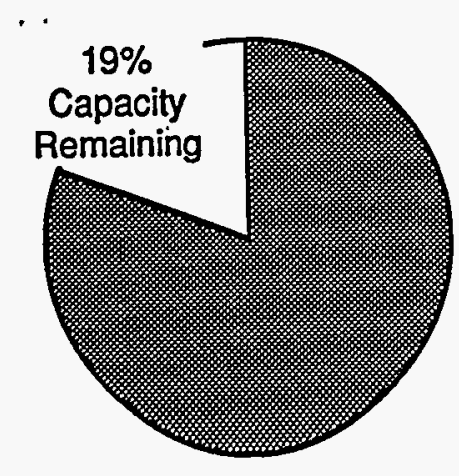

Virginia

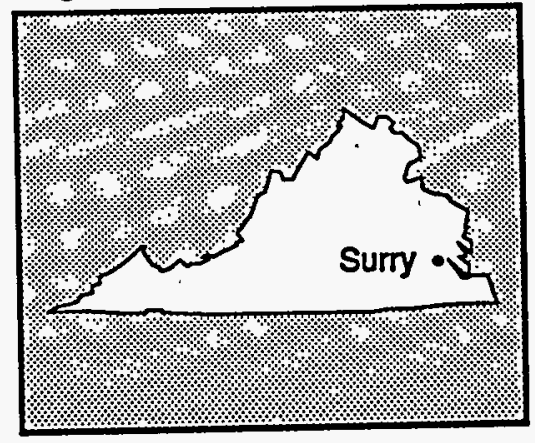




\title{
Washington Public Power Supply System
}

\author{
Washington Nuclear
}
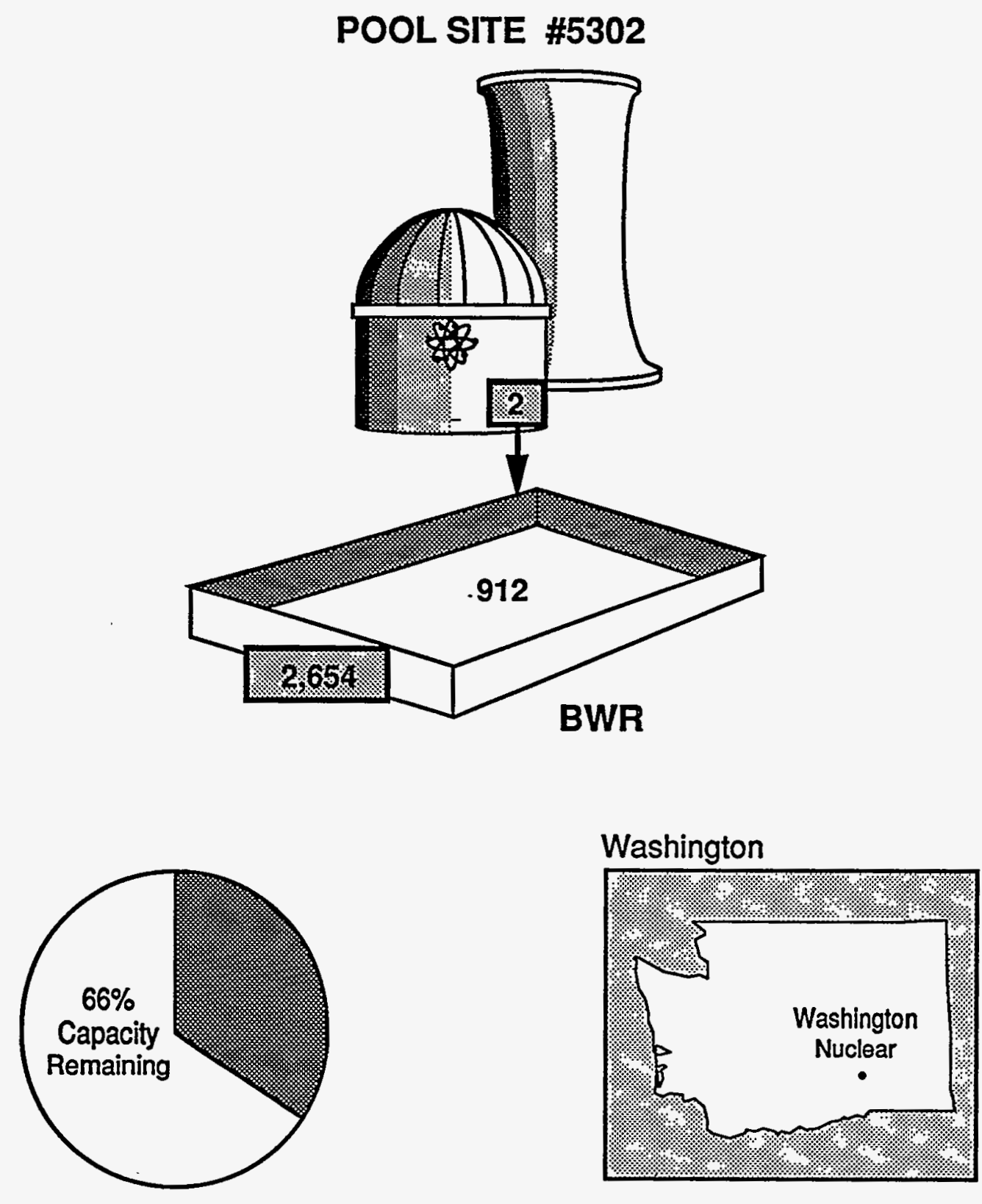


\section{Wisconsin Electric Power Company Point Beach}
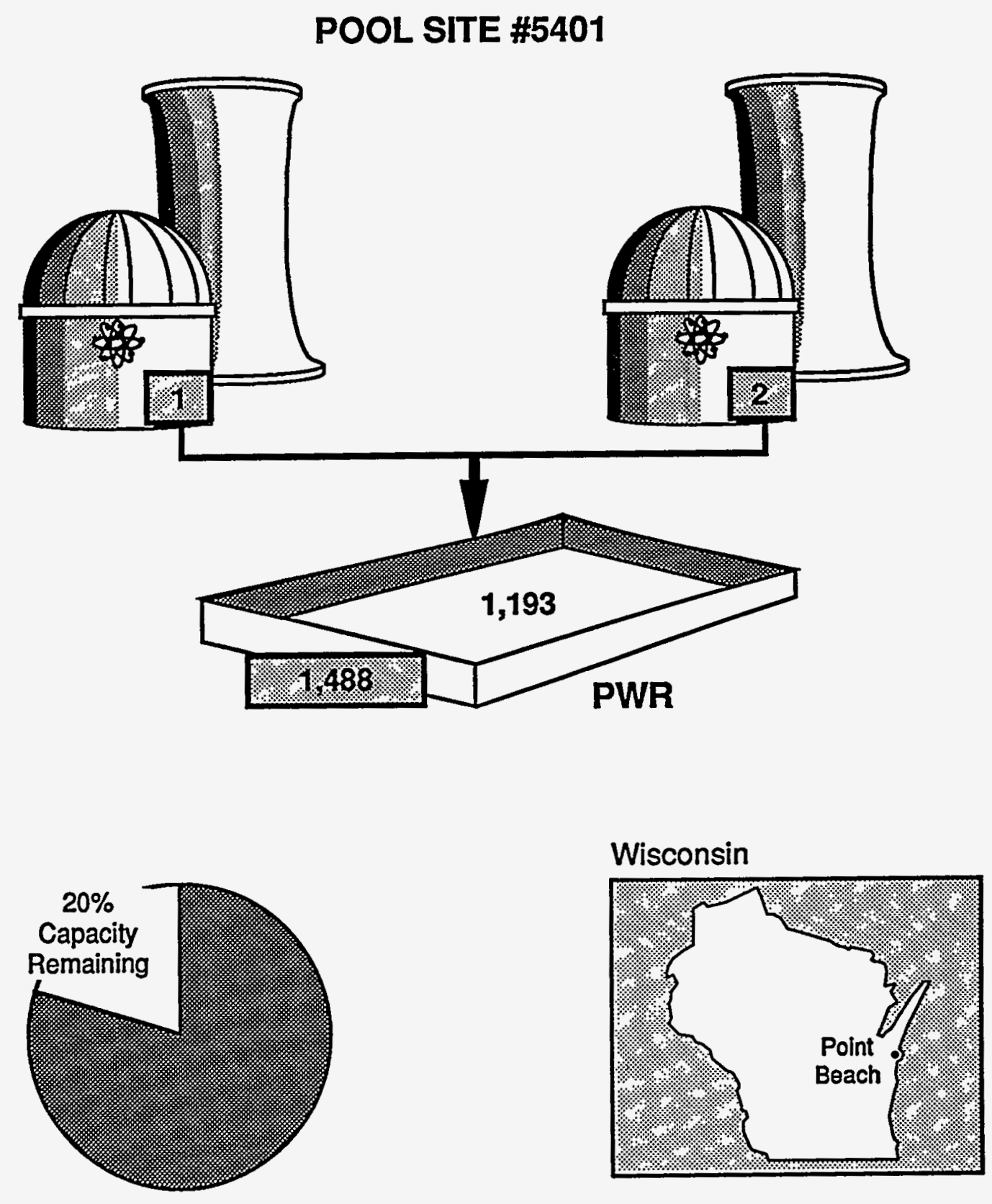


\section{Wisconsin Public Service Corporation Kewaunee}
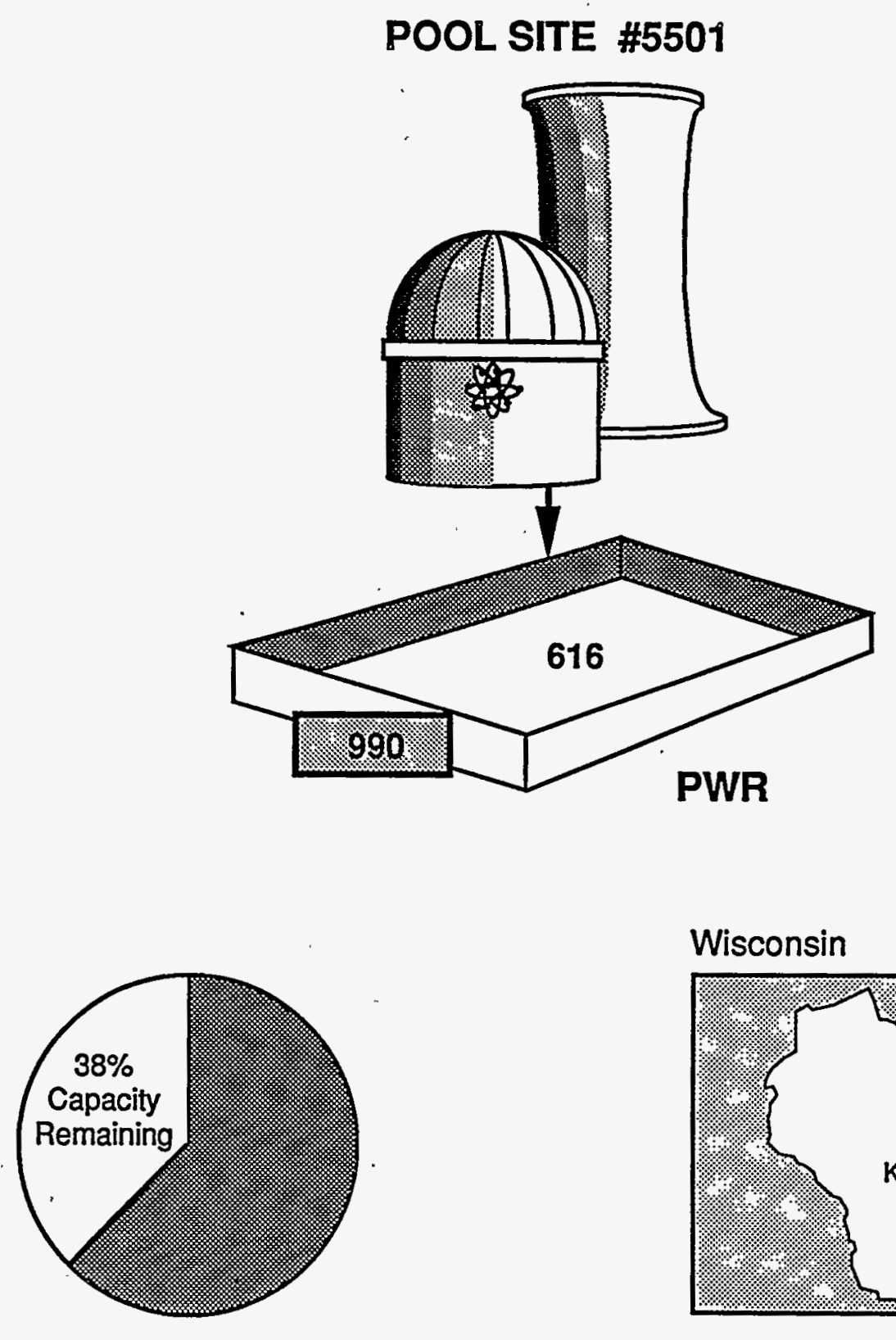

Wisconsin

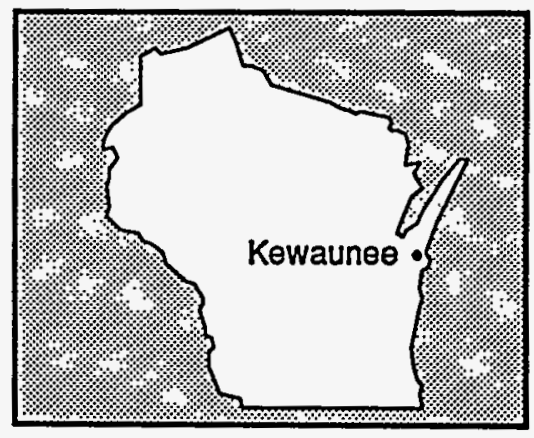




\section{Yankee Atomic Electric Company Yankee Rowe}
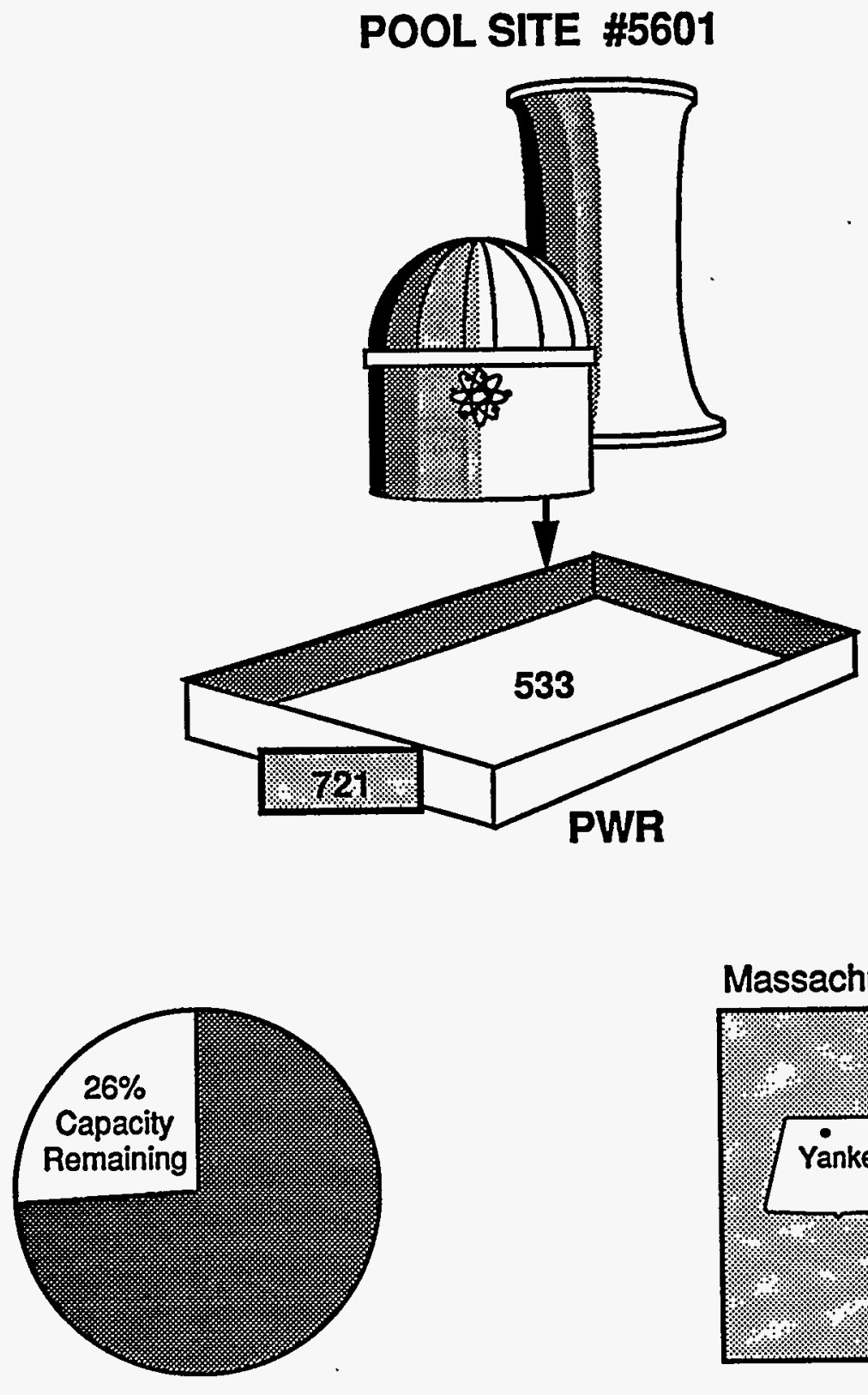

Massachusetts

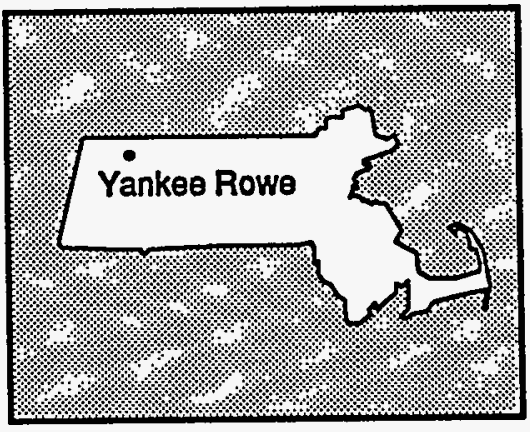




\section{General Electric Company}

\section{Morris Operation \\ Away-from-reactor Spent Fuel Storage Facility}

\section{POOL SITE \#6601}

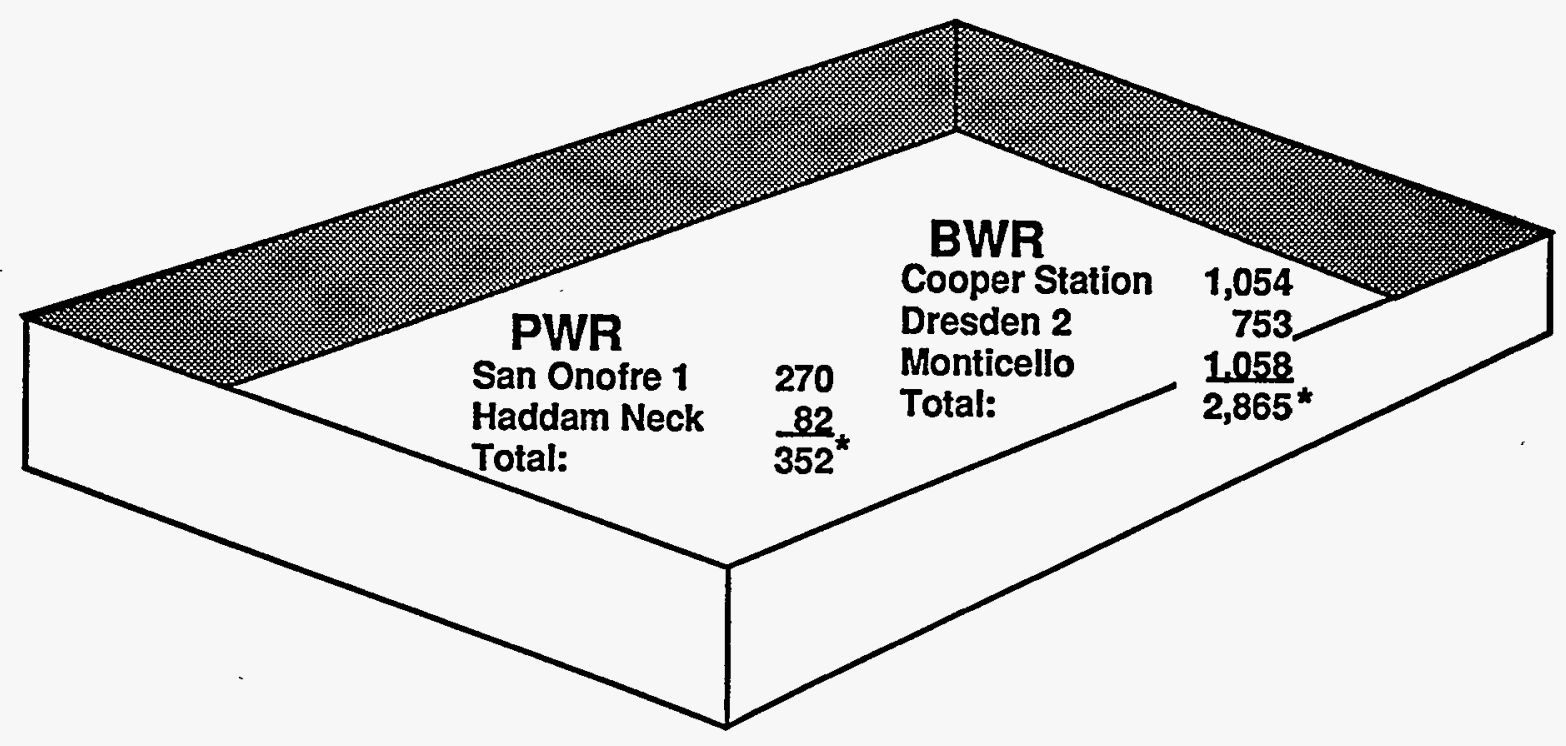

Illinois

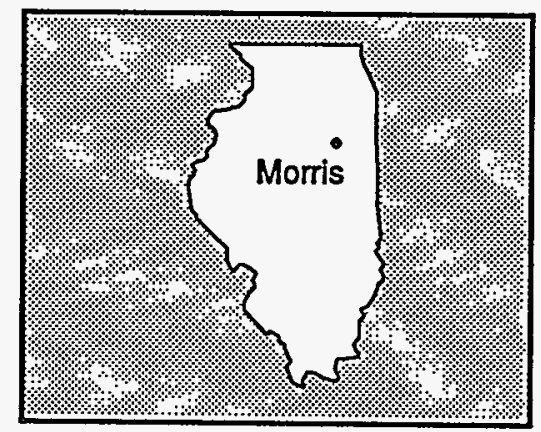

There are 7 slots still available for use. If PWR assemblies are stored, 4 assemblies will fit in each of the 7 slots totaling 28 assemblies. If BWR assemblies are stored, 9 assemblies will fit in each of the 7 slots totaling 63 assemblies. 


\section{West Valley Demonstration Project Away-from-reactor Spent Fuel Storage Facility}
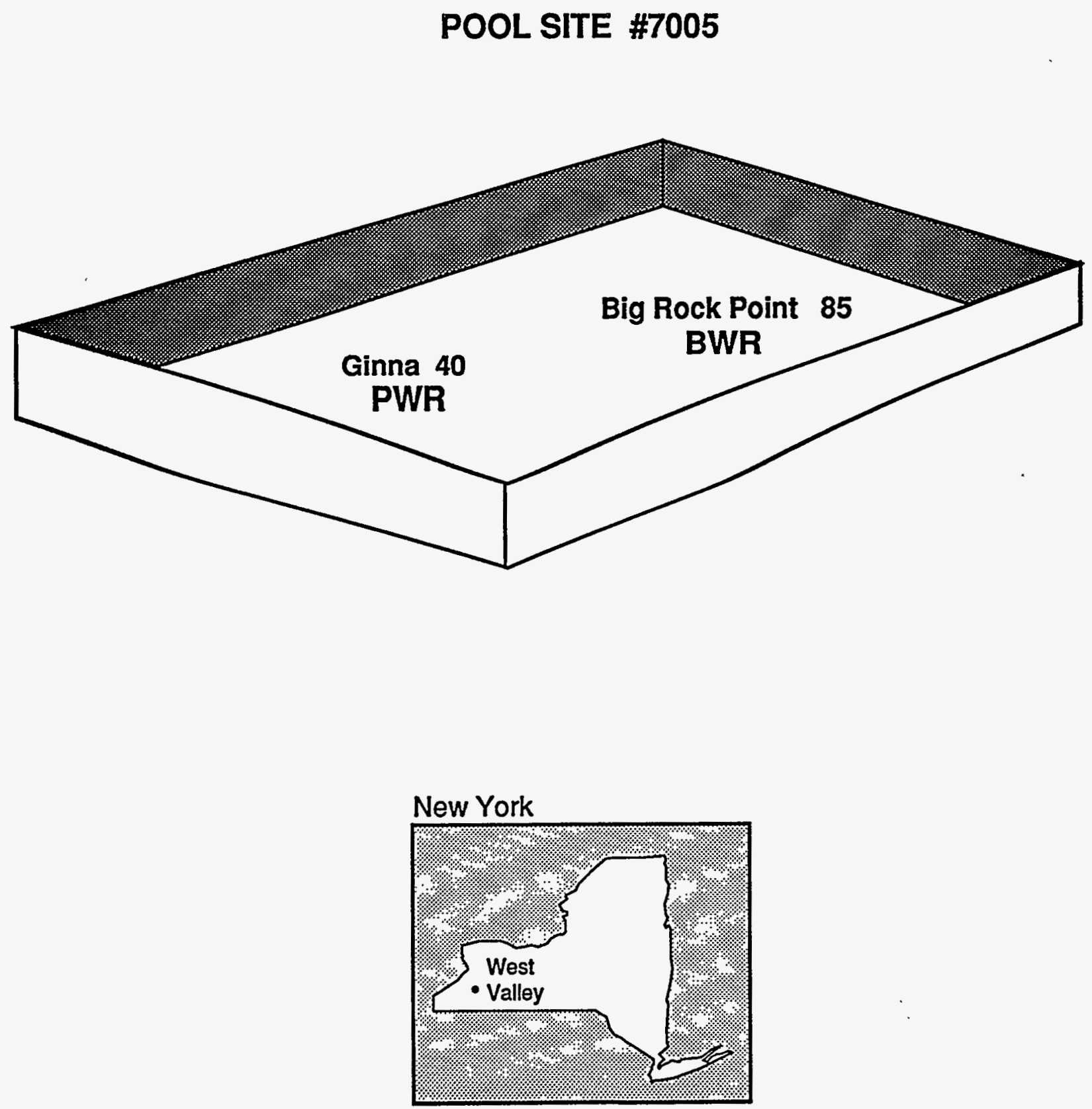


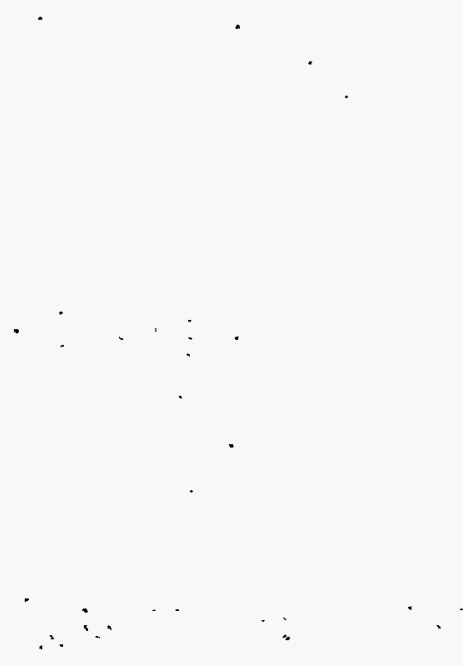




\author{
Appendix D
}

\title{
Quality Assurance
}

Virginia Power's Surry Independent Spent Fuel Storage Installation site.

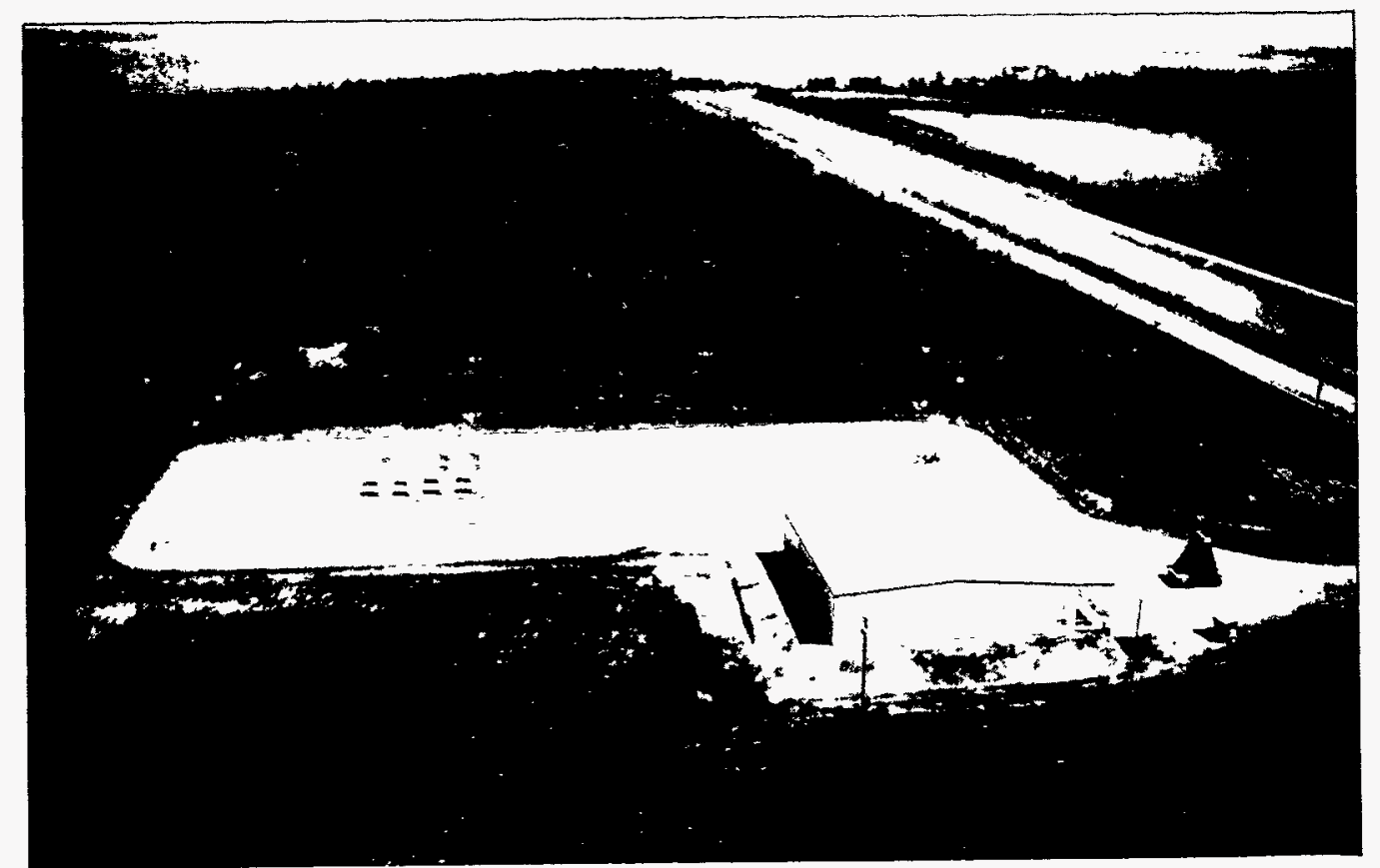




\section{Appendix D}

\section{Quality Assurance}

Data submitted by respondents to Form RW-859 Nuclear Fuel Data survey are subject to extensive Energy Information Administration (EIA) quality assurance $(\mathrm{QA})$ procedures to ensure their accuracy and completeness. A mainframe EIA QA program, written in Pascal, generates a nuclear fuel cycle matrix and summary reports for each reactor. These reports identify and highlight errors in assembly and fuel batch data submitted by nuclear fuel utilities on Form RW-859. Analysts review the EIA QA matrix report produced by the program to check that the data submitted are consistent with data obtained from previous surveys.

Figure D1 presents a sample EIA QA report for one reactor on the Form RW-859 data base. The first part of the report is composed of a nuclear fuel cycle matrix generated from assembly data aggregated to the batch level, which presents the fuel batches through their cycle history. The format helps to identify and highlight corrections needed for data on assemblies and batches of irradiated and unirradiated fuel. The sum of the number of assemblies in the fuel batches in the reactor core during each fuel cycle are compared with the reactor's full core capacity. In addition, assemblies permanently or temporarily discharged (as indicated by their status codes) are aggregated and compared with totals of assemblies discharged and currently not in cycle. The matrix extends over all reactor cycles from reactor startup to the fifth cycle past the current reporting period. Other key data displayed in the matrix include utility ID, cycle discharge dates, batch status, initial uraniumcontent, burnup, assay (enrichment percent), assembly type codes, and an indicator for defective fuel.

The second part of the report contains a discharge/storage summary page, which is composed of three groups of data. Group 1 represents a count of the assemblies discharged at the end of each cycle. Group 2 represents the net cumulative total number of assemblies in storage during each cycle. Group 3 represents net cumulative totals of initial uranium content for assemblies stored during each cycle. The cycle number and end date for the reactor's next $(n+1)$ cycle also appears on the page.

The final part of the report, the storage pool inventory report, lists storage pool inventories (and reactors contributing to the pool) as submitted at the end of the survey report period. Inventories are subdivided into permanent and temporary discharges. Storage pool inventories by pool ID, based on assembly status codes, are compared with those based on the reactor's reported total inventory. The number of canisters in storage and the number of assemblies contained in these canisters are also reporied. Assembly type and pool type (wet or dry) are also indicated. 
Figure D1. Energy Information Administration Quality Assurance Report

\section{Nuclear Fuel Matrix}

Utility ID $=23 \quad$ Utility Name: Illinois Power Company

Reactor ID $=01 \quad$ Reactor Name: Clinton 1

Entries in cycle matrix are number of assemblies.

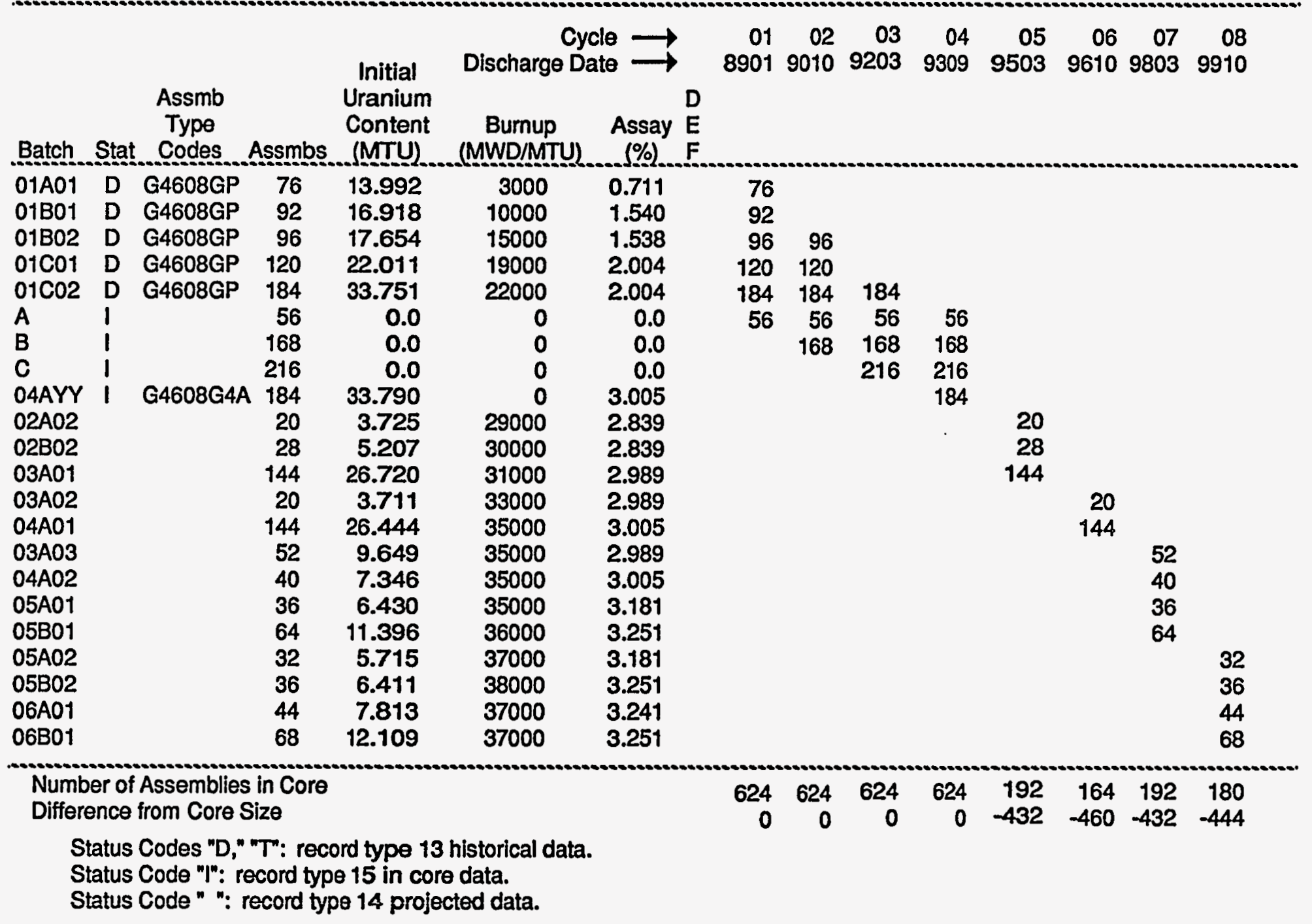




\section{Figure D1. Energy Information Administration Quality Assurance Report (Continued)}

\section{Discharge/Storage Summary}

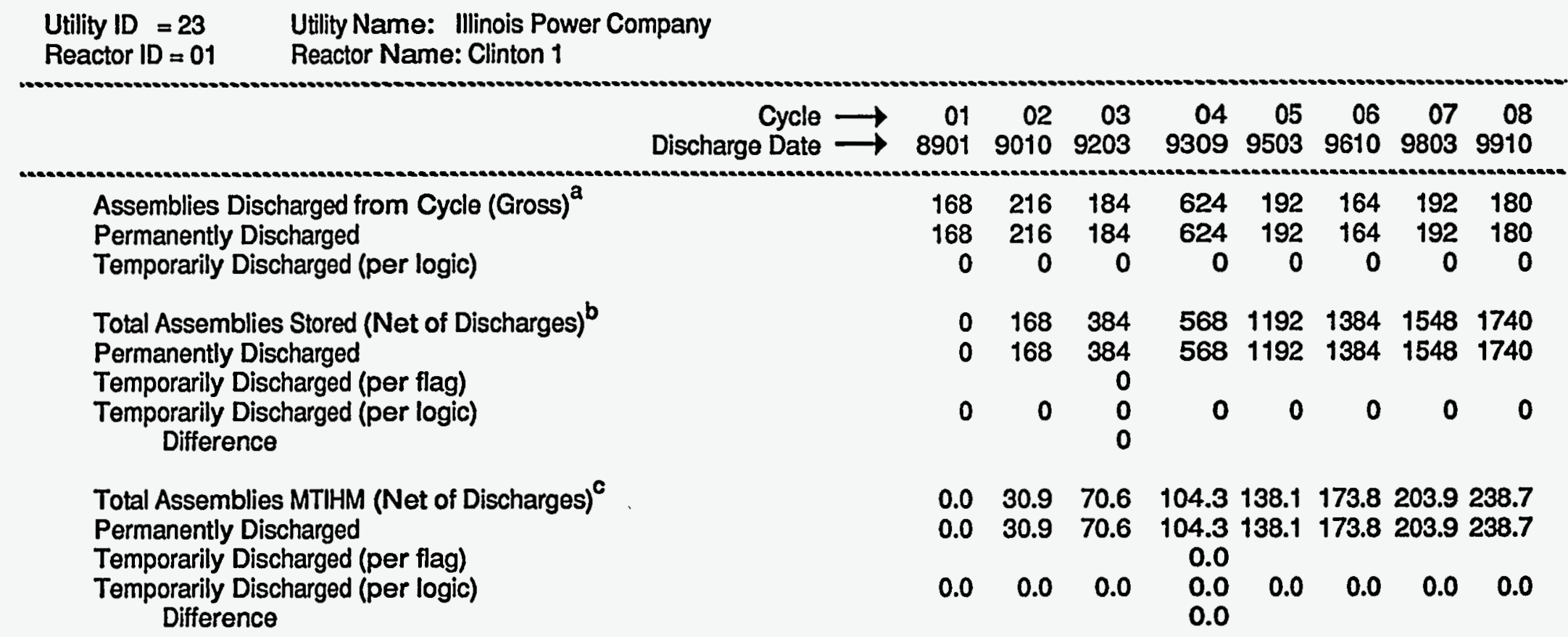

a Counts of assemblies that were discharged after the end of each cycle. The numbers can be understood by inserting them into the sentence, "After cycle _ there were _ assemblies permanently/temporarily discharged."

b Net cumulative totals of assemblies that were in storage during each cycle. The numbers can be understood by inserting them into the sentence, "During cycle _ there were a cumulative total of __ permanently/temporarily stored irradiated assemblies."

c Net cumulative totals of weights of assemblies that were stored during each cycle. The numbers can be understood by inserting them into the sentence, "During cycle _ the cumulative weight of the permanently/temporarily stored assemblies was

\section{Cycle $N+1=04$}

End of cycle date $=$ SEP-26-1993 


\section{Storage Pool Inventory}

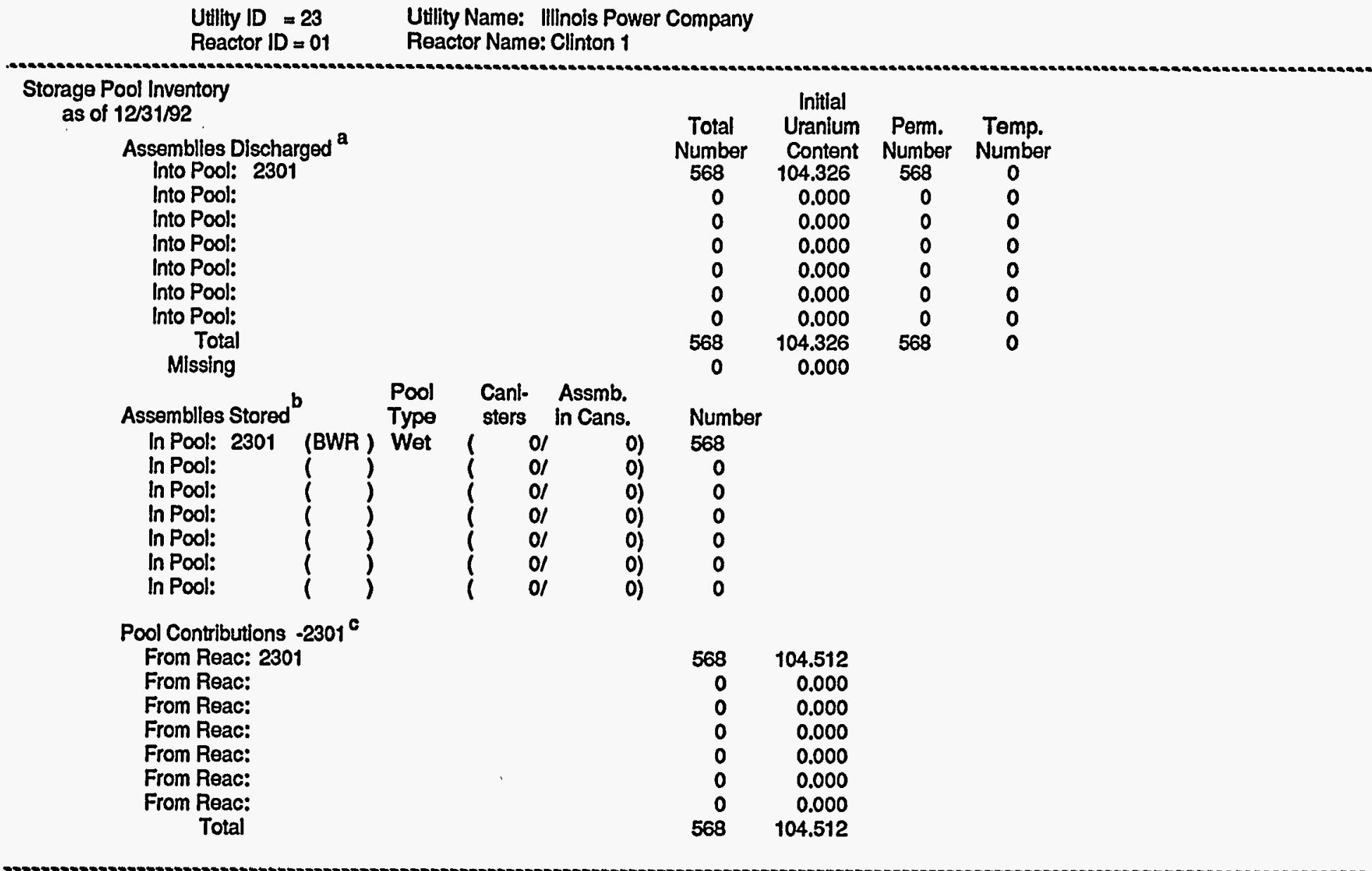

a The number of assemblies discharged and their total weight for the life of the reactor ordered by the storage pools at which these assemblies are stored. The total number of assemblies equals the number of permanently discharged assemblies (status code $=\mathrm{D}$ ) plus the number of temporarily discharged assemblies (status code $=\mathrm{T}$ ).

b The number of assemblies currently stored in each storage pool. Reactor type (BWR = Bolling-water reactor, PWR $=$ Pressurized-water reactor) Is indicated in parentheses. Pool type indicates Wet (storage pool) or Dry (dry cask) storage. The number of assemblies contained in canisters are shown in parentheses (canisters/assemblies).

The number of assemblies stored in the pool indicated along with their total weight ordered by discharging reactor.

Source: Energy Information Administration, Form RW-859, "Nuclear Fuel Data" (1992). 
Appendix E

Technical Notes

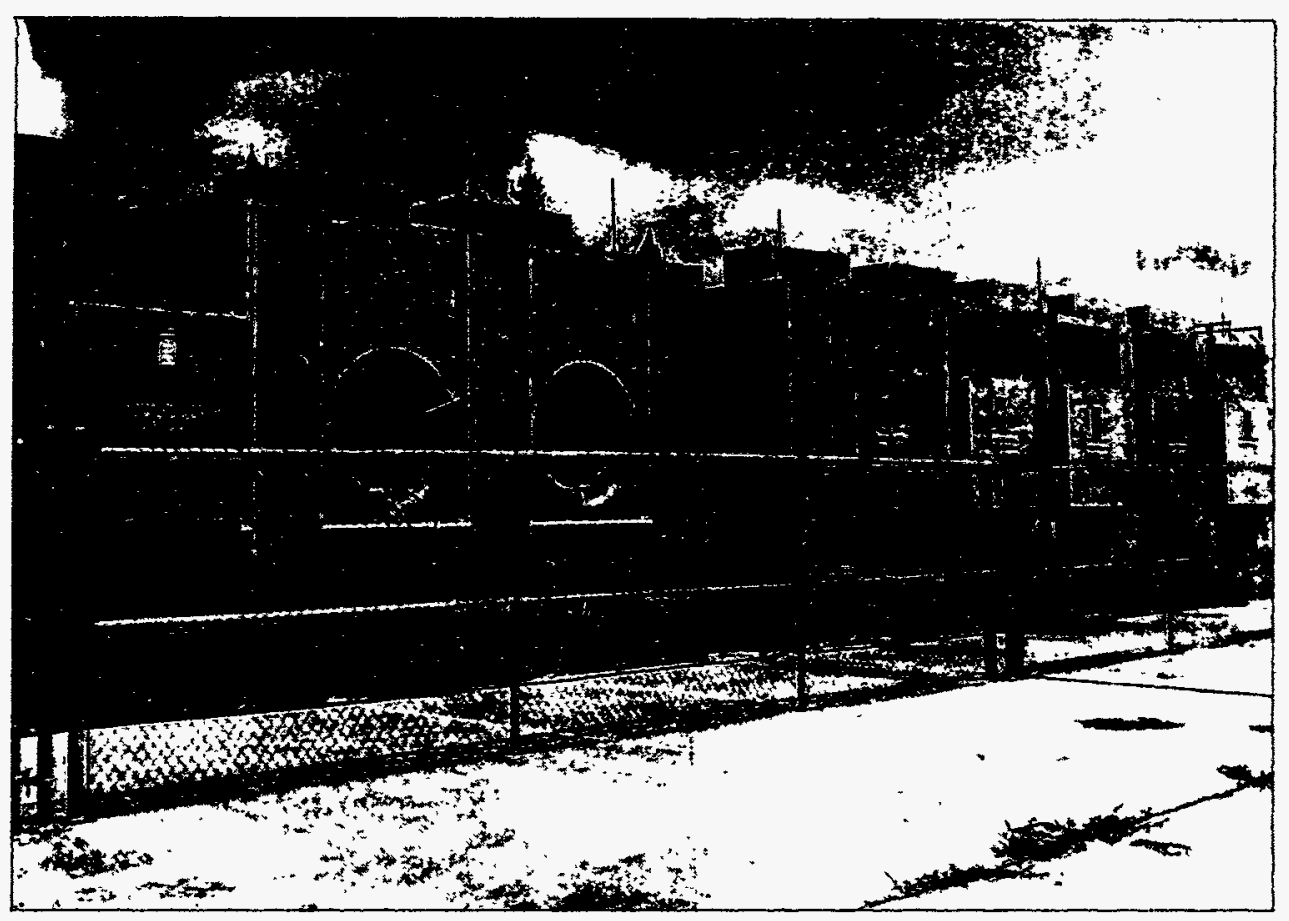




\section{Appendix E}

\section{Technical Notes}

\section{Note 1: Reactor Status}

Data for a total of 120 commercial light-water reactors have been reported on the Form RW-859 survey. The total includes 117 reactors which have been or are discharging and/or storing nuclear fuel assemblies. Reports are also required for three reactors that have not discharged spent nuclear fuel (Watts Bar 1 and 2, and Comanche Peak 2). For three reactors that were reported on previous surveys (Bellefonte 1 and 2, which have been indefinitely deferred, and Fort St. Vrain, which has been shut down and is not a lightwater reactor), reports are no longer required. Nine reactors that have either shut down or been retired (Dresden 1, Humboldt Bay, Indian Point 1, LaCrosse, Shoreham, Rancho Seco, San Onofre 1, Trojan, and Yankee Rowe) are maintained on the data base for their historical information.

\begin{tabular}{lrr} 
& 1991 & 1992 \\
Operating Reactors & 110 & 108 \\
Shut Down Reactors & 7 & 9 \\
Under Construction & 3 & 3 \\
\cline { 2 - 3 } & & \\
Total & 120 & 120
\end{tabular}

Note: Totals do not include Bellefonte 1 and 2, which are Indefinitely deferred, and Fort St. Vrain, which is an HTGR and was shut down in 1989. Totals also do not include the four storage-only facilities.

Two reactors that have discharged spent nuclear fuel are not reported on Form RW-859, and their data are not included in this report. Hanford- $\mathrm{N}$ was placed in cold standby status by the DOE in 1988 . Hanford-N spent fuel is used in DOE's Defense Programs. Three Mile Island 2 was shut down in 1979 because of an accident. Three Mile Island 2 spent fuel now belongs to a DOE experimental research program. Five other reactors have begun construction but have been indefinitely deferred. These reactors are Perry 2, Bellefonte 1 and 2, and Washington Nuclear Power 1 and 3.

\section{Note 2: Storage Facilities}

In addition to the spent nuclear fuel assemblies stored at reactor sites, a number of assemblies are stored at away-from-reactor storage facilities throughout the United States. Four of these facilities (General Electric Company (Morris Operation), West Valley Demonstration Project, Babcock and Wilcox Company (Lynchburg), and General Electric Vallecitos Nuclear) report on the Form RW-859 survey. Data for these facilities are obtained directly from their survey submissions and verified by checking shipment data from the contributing reactors (Table 14). Data for an additional four storage facilities (Idaho National Engineering Laboratory, Ohio (Battelle), South Carolina, and Washington (Hanford)) are compiled from shipment data submitted by the contributing reactors.

Table 14 contains complete data on assemblies stored at away-from-reactor storage facilities. These totals are also included in Table ES1, Figures ES1 and ES2, and the State totals in Table 15. Table 12 presents a compilation of data on shipments from utilities to away-from-reactor storage facilities. See Technical Note 7 on reprocessed assemblies and Technical Note 12 on data adjustments for more information.

\section{Note 3: Pool Site Configurations}

The format for presenting data in Table 13 is dependent upon the individual reactor/pool site configurations. The configurations are shown graphically in the pictograms in Appendix $C$ and include the presence of transfer canals, multiple pools servicing a single reactor, and multiple reactors sharing a single pool site. Each case is presented below.

- When two pools are joined by a transfer canal, only one pool site ID is assigned since there is 
only one loading area. Such is the case at Baltimore Gas and Electric Company's Calvert Cliffs 1 and 2. In Table 13 only one pool site ID (0501) is listed, but separate capacity and inventory data are listed for each reactor.

- Only two reactors have multiple pools. Cleveland Electric Illuminating Company's Perry 1 is assigned two pool site ID's (0901 and 0902) and separate capacity and inventory data for each. Carolina Power and Light Company's Harris 1 has four pools which share the same pool site ID (0703). Of these four pools, two are currently licensed and two are not usable at this time. Harris 1 is a PWR reactor with the capability of storing both PWR and BWR fuel assemblies in its storage pools. In Table 13, separate capacity and inventory data are listed for each reactor type.

- In the case where two or more reactors share a single pool, as with Commonwealth Edison Company's Byron 1 and 2, only one pool site ID (1003) is listed, and the multiple reactors' data are combined and listed with that pool site.

\section{Note 4: Intact Assemblies in Canisters}

Prior to the collection of 1991 survey data, Form RW-859 respondents were required to report the number of canisters stored at a pool site separate from the number of assemblies stored at the site. A few of the canisters reported contained either intact spent fuel assemblies or rods from consolidated assemblies. This resulted in a difference between the number of spent fuel assemblies discharged and the number of spent fuel assemblies stored.

More detailed data on the contents of canisters were collected on the revised Form RW-859 survey. This alleviated some of the reporting inconsistencies. However, since the collection of these data are relatively new, they should be considered as preliminary and subject to change.

\section{Note 5: Alternative Data Sources}

The majority of data in this report come directly from a compilation of data on the Form RW-859 survey. Alternative data were obtained from the EIA publication, World Nuclear Capacity and Fuel Cycle
Requirements 1993 (Table 4), and the Nuclear Regulatory Commission publication, Information Digest 1993 (Tables B2 and B3). The following data were obtained from these publications:

- World Nuclear Capacity and Fuel Cycle Requirements 1993

-Summer capability rating (net MWe) for all reactors

-Startup year for Watts Bar 1 and 2, and Comanche Peak 2

-License expiration year for Watts Bar 1 and 2, and Comanche Peak 2.

- Information Digest 1993

-Nuclear steam system supplier and design type for all reactors.

\section{Note 6: HTGR Fuel Elements}

One Form RW-859 reactor, Fort St. Vrain, is a hightemperature, gas-cooled reactor (HTGR). An HTGR uses an inert gas (helium) as the primary coolant and a graphite moderator. Highly enriched uranium is used as initial fuel and thorium as a source of new fuel.

This HTGR has spent fuel wells instead of a pool. Data for Fort St. Vrain are listed only in Tables 15 and 16 , and are not included in totals on those tables.

Fort St. Vrain shut down in 1988 for repairs and will not restart. A total of 726 spent fuel elements (including 6 test elements) were discharged prior to this shutdown and shipped to Idaho National Engineering Laboratory (INEL). An additional 18 spent fuel elements were shipped to INEL in October 1991. Ultimately, all the irradiated fuel elements will be shipped to INEL for storage and reprocessing. Until all legal issues with the State of Idaho regarding transportation of irradiated spent fuel are resolved, contingency plans have been developed to hold the spent fuel in an Independent Spent Fuel Storage Installation (ISFSI). The ISFSI was licensed on November 4, 1991, by the U.S. Nuclear Regulatory Commission. The license authorizes storage in the ISFSI of up to 1,482 spent fuel elements, 37 reflector control rod elements, and 6 neutron source elements. In December 1991, the Public Service Company of Colorado transferred 18 elements to its ISFSI. By June of 1992, 
1,446 elements were transferred to the ISFSI, for a total of 1,464 which now reside in the ISFSI.

\section{Note 7: Reprocessed Assemblies}

Spent fuel discharged from light-water reactors contains appreciable quantities of fissile (U-235, $\mathrm{Pu}-239)$, fertile (U-238), and other radioactive materials. These fissile and fertile materials can be chemically separated and recovered from the spent nuclear fuel. The recovered uranium and plutonium can, if economic and institutional conditions permit, be recycled for use as nuclear fuel.

A number of assemblies were discharged and reprocessed prior to 1972. Data on these reprocessed assemblies are not included in this report, except for 962 reprocessed assemblies reflected in Table 12 as shipments. Other reprocessed assemblies have not been included on Form RW-859 submissions.

\section{Note 8: Reactor Dates}

Key dates in the life of a reactor are presented in Table 4. While the majority of the information presented in this table was obtained from Form RW-859 data, other data sources were used in cases where Form RW-859 data were not available (See Technical Note 5: Alternative Data Sources). Definitions for each of the key dates are as follows:

- Startup date-year in which electricity was first generated from the reactor.

- License expiration date-year listed on the reactor's Nuclear Regulatory Commission operating license.

- Loss of ability to operate-year estimated by the utility on Form RW-859 in which the utility would not continue reactor operation because of a lack of storage space for discharged fuel, absent spent fuel pickup by DOE. This also takes into consideration the current licensed capacity (slots) and the total maximum established site capacity.

- Projected retirement year-utility estimate, either the license expiration date or a date based on a license extension.

\section{Note 9: Burnup Ranges}

Burnup is defined as the amount of thermal energy produced per unit weight of fuel irradiated or "burned." Burnup levels are generally expressed in units of gigawattdays thermal per metric ton of uranium (GWDt/MTU). Average burnups are presented in Tables 5, 6, 7, 8, and 11, Figure 2, and Appendix B. Burnup ranges reflect associated batch average discharge burnups. A burnup range may have a given assembly in one of the following: 0-4.999 GWDt/MTU; 5.000-9.999 GWDt/MTU; 10.000-14.999 GWDt/MTU; etc.

\section{Note 10: Missing Data}

Most Form RW-859 respondents submit complete and accurate data. All Form RW-859 data are subject to extensive EIA quality assurance procedures. As of the final frozen file date of September 1, 1993, a limited number of data items were missing. In these instances, the missing data items involved temporarily discharged assemblies. Utilities are not required to report complete data until these assemblies are permanently discharged. These items, listed below, are omitted from the tables in this report.

- The following 73 assemblies, all temporarily discharged, are missing assembly type codes, burnups, and enrichment (weight percents).

$\begin{array}{llr}\text { Beaver Valley 2 } & \text { PWR } & 4 \\ \text {-Davis-Besse } & \text { PWR } & 6 \\ \text { Indian Point 2 } & \text { PWR } & 8 \\ \text { Indian Point 3 } & \text { PWR } & 2 \\ \text { Kewaunee } & \text { PWR } & 12 \\ \text { North Anna 1 } & \text { PWR } & 8 \\ \text { North Anna 2 } & \text { PWR } & 2 \\ \text { Perry 1 } & \text { BWR } & 4 \\ \text { Salem 1 } & \text { PWR } & 24 \\ \text { South Texas 1 } & \text { PWR } & 3\end{array}$

- The following 26 assemblies, all temporarily discharged, are missing burnups.

$\begin{array}{llr}\text { Maine Yankee } & \text { PWR } & 12 \\ \text { North Anna 2 } & \text { PWR } & 1 \\ \text { Salem 1 } & \text { PWR } & 1 \\ \text { South Texas 1 } & \text { PWR } & 4 \\ \text { Three Mile Island } & \text { PWR } & 8\end{array}$




\section{Note 11: Rounding}

All initial uranium contents presented in the tables of this Service Report are presented in metric tons of uranium (MTU) and are rounded to the nearest one-tenth. Totals may not exactly equal sum of components because of independent rounding. All average burnups in the tables of the Service Report are presented in gigawattdays thermal per initial metric ton of uranium (GWDt/MTU) and are rounded to the nearest one-tenth. Average burnup totals are weighted averages and, as such, are not averages of individual table entries.

\section{Note 12: Data Adjustments}

The Form RW-859 survey is a total universe survey of all commercial nuclear reactors. Respondents usually submit complete data. As such, data adjustments are not usually necessary. In cases where errors or omissions are identified during the quality assurance process, EIA adjusts the data only after the respondent has been contacted to verify the change. A footnote has been added to tables where data for previous years have been revised. The note also states that current-year data may be revised in future publications. When utilities reinsert assem- blies discharged in previous years, historical totals change.

\section{Note 13: Storage Capacities}

Form RW-859 respondents are required to report their licensed storage capacity and their maximum established spent fuel storage capacity.

- Licensed storage capacity represents the number of spent nuclear fuel assemblies and canisters to be stored at a given site or spent nuclear fuel pool, as licensed by the Nuclear Regulatory Commission.

- Maximum established spent fuel storage capacity represents the maximum number of intact assemblies that will be able to be stored at some point in the future (between the reporting date and the reactor's end of life) considering any established or current studies or engineering evaluations at the time of submittal for licensing approval from the U.S. Nuclear Regulatory Commission. Utilities adjust their maximum established spent fuel storage capacity by adding capacity based on reracking or other engineering improvements or decreasing capacity by subtracting unusable pool locations. 


\section{Glossary}

Transfer Cask loading spent fuel into Oconee's Independent Spent Fuel Storage Installation.

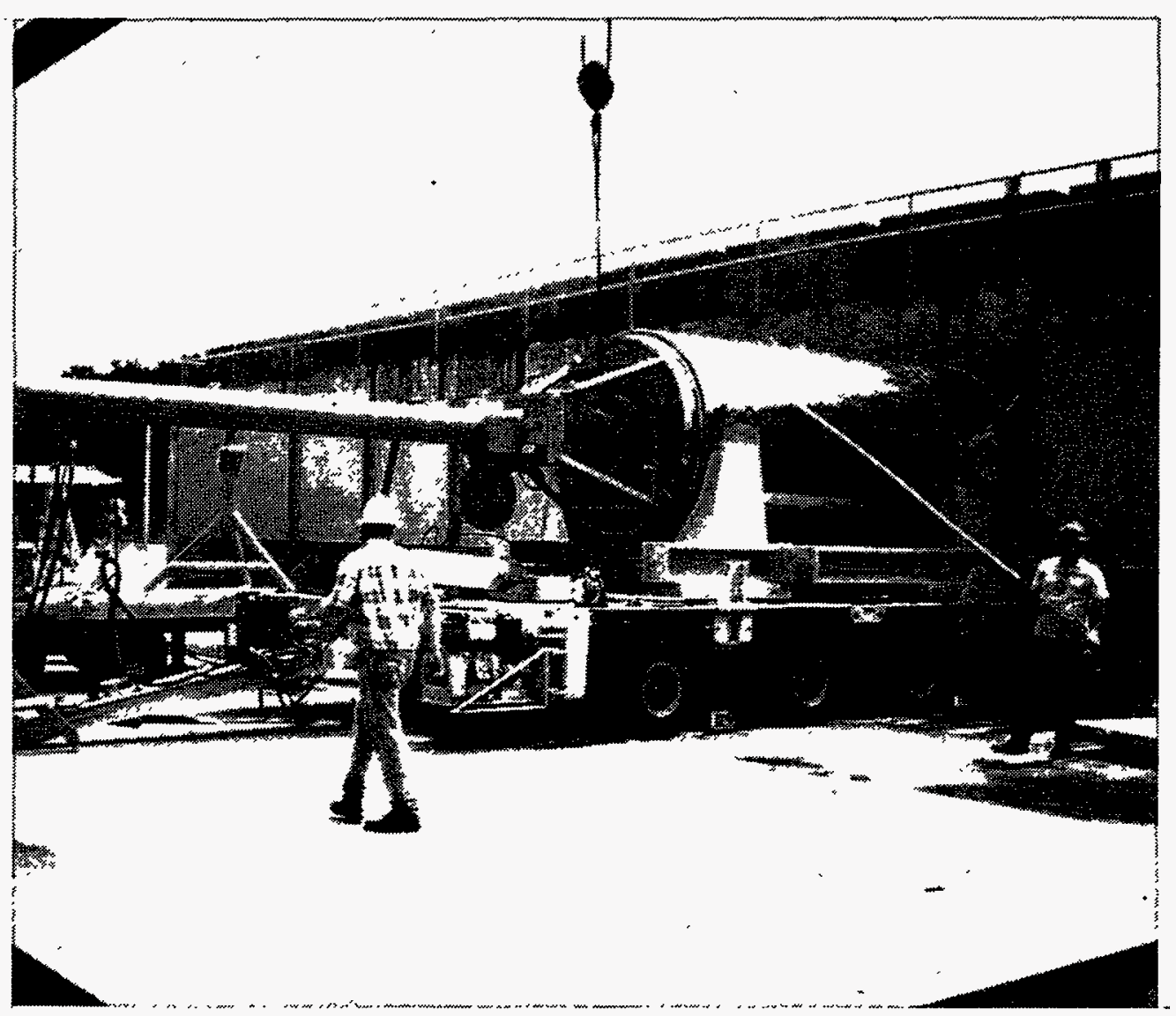




\section{Glossary}

Activity or Radioactivity: The rate at which radioactive material emits radiation, stated in terms of the number of nuclear disintegrations occurring in a unit of time; the common unit of radioactivity is the curie.

Assembly: A group of fuel rods fastened together with top and bottom tie plates, guide tubes, and grids. For boiling water reactors, they are sometimes called a bundle without the channels.

Assembly Class: A generic grouping for individual reactors based on the core configuration. The assembly class for a particular reactor is primarily a function of overall assembly size (length and transverse width). For pressurized water reactors, assembly class is also a function of fuel rod array configuration. A key attribute of the assembly class scheme is that fuel assemblies in an assembly class are only used in reactors belonging to the same assembly class. Codes representing the assembly class are used as the first three characters of the eightcharacter assembly type codes. Codes for multiplereactor assembly classes consist of a single-character code representing the reactor vendor and a twocharacter code identifying the particular assembly class. Codes for single-reactor assembly classes consist of the letter " $\mathrm{X}$ " and a two-character code representing the specific reactor. The assembly class also serves as the basis for the characterization of nonfuel components.

Assembly Identifier: A unique string of alphanumeric characters which identifies an assembly, bundle, or canister from a specific reactor in which it has been irradiated. Must be consistent with other submissions to the Department of Energy (DOE)/ Nuclear Regulatory Commission (NRC); that is, Annex B, previous Form RW-859, and DOE/NRC Form 741.

Assembly Type: Describes a group of fuel assemblies with similar physical and design characteristics. In general, a breakdown of the assembly class into various fuel designs. Assemblies of the same assembly type are always of the same assembly class, fuel design, and manufacturer. Typically, they have identical lengths, widths, cladding materials, active fuel lengths, hardware (both parts and materials), and weight of heavy metal.
Average Discharge Burnup: The average amount of energy produced by each assembly in a batch of spent fuel assemblies that have been discharged from a nuclear reactor, reported in gigawattdays thermal per metric ton of uranium (GWDt/MTU).

Average Initial Enrichment Assay: Average enrichment for a fresh fuel assembly as specified and ordered in fuel cycle planning. This average includes axial blankets, and axially and radially zoned enrichments.

Average Initial Uranium Content: Average weight in kilograms (kg) of uranium of fresh fuel assemblies in a batch before they are initially inserted into the reactor core. To be included in a batch the initial uranium content of each assembly must be within 3 $\mathrm{kg}$ of the average for boiling water reactor assemblies and within $5 \mathrm{~kg}$ of the average for pressurized water reactor assemblies.

Axial Power Shaping Assemblies: Assemblies used at Babcock and Wilcox-designed pressurized-water reactors to provide control over the power level of the reactor core during operation. They are very similar to control rod assemblies (CRAs), but have less neutron absorbing material. Gray axial power shaping assemblies use a weaker neutron absorber, but serve an identical function.

Batch: A logical grouping of assemblies with similar characteristics. All assemblies in a batch have the same vendor model number, the same approximate initial loading weight, the same initial average loading enrichment, and approximately the same burnup after final discharge.

Boiling-Water Reactor (BWR): A light-water reactor in which water, used as both coolant and moderator, is allowed to boil in the core. The resulting steam can be used directly to drive a turbine.

Burnable Poison Rod Assemblies (BPRAs): Assemblies are inserted and locked into the guide tubes of pressurized-water reactor fuel assemblies in Babcock and Wilcox- and Westinghouse Electricdesigned reactors to provide additional reactivity control. Both borosilicate glass and a boron carbide/aluminum oxide mixture have been used as the 
absorber for these assemblies. Because these absorbing materials are rapidly depleted, BPRAs are useful in limiting power output in the higher enriched assemblies that are now being placed into service. The use of burnable poison rod assemblies varies greatly from utility to utility.

Burnup: A measure of the amount of energy obtained from fuel in a reactor. Typically, burnup is expressed as the amount of energy produced per unit weight of fuel irradiated or "burned." Burnup levels are generally measured in units of gigawattdays thermal per initial loading weight of uranium, in metric tons (GWDt/MTU).

Burnup Range: A data structure that represents assemblies, once and only once, by their associated batch average discharge burnup. As a batch is defined as all assemblies having identical reactor and cycle histories, identical nominal initial enrichments, and a burnup within 10 percent of the final mean average burnup, some assemblies placed within a range may not actually fall within that range. A burnup range may have a given assembly in one of the following: 0-4.999 gigawattdays thermal per inital loading weight of uranium, in metric tons (GWDt/MTU); 5.000-9.999 GWDt/MTU; 10.00014.999 GWDt/MTU; etc.

Canister: The outermost container into which vitrified high-level waste, intact spent fuel assemblies, consolidated spent fuel, or spent fuel rods are to be placed. Made of stainless steel or an inert alloy.

Caretaker: Spent fuel storage facility that stores assemblies, subassemblies, or batches but does not irradiate them in a reactor.

Characteristic Waste: A set of qualitative and quantitative criteria used to describe the features of a particular category of radioactive waste. Typical quantitative criteria used for most radioactive wastes include volume $\left(\mathrm{m}^{3}\right)$, activity $(\mathrm{Ci})$, and thermal power (W).

Cladding: Protective alloy tube shielding in which fissionable fuel is inserted. Cladding is relatively resistant to radiation and the physical and chemical conditions in a reactor core. The cladding may be of stainless steel or some alloy, such as zircaloy.

Commercial Operation: The phase of reactor operation that begins when power ascension ends and the operating utility formally declares to the Nuclear Regulatory Commission that the nuclear power plant is available for the regular production of electricity. This declaration is usually related to the satisfactory completion of qualification tests on critical components of the unit.

Consolidation: The process whereby fuel rods are removed from an assembly and placed into a container in which a minimum of space is left unoccupied by the rods.

Control Blades: Blades (often called cruciforms) that control the nuclear chain reactor in boiling-water reactors by limiting the number of neutrons in the core. Made of strongly neutron-absorbing materials, control blades are inserted into the reactor core at planned shutdowns and during scrams. Particular groups of control blades are also used to control the power level of the reactor. Several pressurized-water reactors (Indian Point 1, Palisades, and Yankee Rowe) use(d) control blades.

Control Element Assemblies (CEAs): Assemblies used at Combustion Engineering (CE)-designed pressurized-water reactors to provide control over the nuclear chain reaction by limiting the number of neutrons in the core. Made of strongly neutronabsorbing materials, CEAs are inserted into the reactor core at planned shutdowns and during scrams. At most $\mathrm{CE}$-designed reactors, they consist of 4 or 5 control elements connected together by a control rod spider and fit into the guide tubes of a single assembly. At CE-designed System 80 reactors, they consist of either 4 or 12 control elements connected together by a control rod spider. The 4-element CEAs fit into the guide tubes of a single assembly, but the 12-element CEAs fit across 5 fuel assemblies in the core of the reactor.

Control Element Assembly Flow Plugs: Plugs used at some Combustion Engineering-designed reactors in the same manner that thimble plug assemblies and orifice rod assemblies are used at Westinghouse Electric- and Babcock and Wilcox-designed reactors, respectively.

Control Rod Assemblies (CRAs): Assemblies used at Babcock and Wilcox-designed pressurized-water reactors to provide control over the nuclear chain reaction by limiting the number of neutrons in the core. Made of strongly neutron-absorbing materials, CRAs are inserted into the reactor core at planned shutdowns and during scrams. They consist of 16 control rods connected together by a control rod spider and fit into each guide tube of a single assembly. 
Coolant: Material used to conduct heat away from the reactor core to the steam cycle of a nuclear power plant. Liquids, gases, and liquid metals can be used as coolants.

Core: The place in the reactor in which the nuclear fuel is irradiated and thermal energy is generated.

Core Size: The fixed number of fuel assemblies that can be irradiated at any one time in the reactor core.

Current Inventory: Number of spent nuclear fuel . assemblies stored at a given site or spent nuclear fuel pool, at a given point in time.

Cycle: The period of time from reactor fuel loading to subsequent refueling.

Cycle End Date: The date of reactor shutdown for fuel discharge and refueling.

Decay or Radioactive Decay: The transition of a nucleus from one energy state to a lower one, usually involving the emission of a photon, electron, or neutron.

Design Electrical Rating (Capacity), Net: The nominal net electrical output of a nuclear unit, as specified by the utility for the purpose of plant design.

Discharge Cycle: The sequential numbering of a reactor's refueling, from the start of reactor fuel loading to subsequent refueling. Initial loading, first operation, and first refueling is cycle 01.

Discharged Fuel: Irradiated fuel removed from a reactor during refueling. (See Spent Nuclear Fuel.)

Disposal Contract: See Standard Contract.

Dry Shielded Canister (DSC): The dry shielded canister is a cylindrical stainless steel pressureretaining component which contains an internal basket assembly to maintain the spent fuel assemblies in a safe geometry during loading, transfer, storage, and postulated cask drop events.

Dry Storage Facilities: Shielded mobile or stationary containers, silos, modules, vaults, or dry wells filled with an inert gas or with air, as appropriate, in which spent fuel assemblies or canisters of highly radioactive material may be stored.

End of Cycle Date: See Cycle End Date.
Enrichment: A nuclear fuel cycle process in which the concentration of fissionable uranium is increased above its natural level of 0.71 percent. Enrichment is the process that changes the isotopic ratio in a material. The process currently utilized in the United States to increase uranium-235 to uranium-238 is gaseous diffusion of uranium hexafluoride $\left(\mathrm{UF}_{6}\right)$.

Enrichment Weight Percent: A value determined on : the basis of burnup calculations for the original fresh . fuel assembly.

Equilibrium Cycle: An analytical term that refers to fuel cycles that occur after the initial one or two cycles of a reactor's operation. For a given reactor, equilibrium cycles have similar fuel characteristics.

Fabricator: The manufacturer or supplier of a particular nuclear fuel assembly. The fabricator of an assembly for a given reactor is not necessarily related to the reactor vendor or to the original fuel fabricator.

Fuel Assembly: Nuclear fuel is fabricated into small pellets. These pellets are encased into long cylindrical rods. An assembly is a group of these rods fastened together. Referred to as a "bundle" for some boiling water reactors.

Fuel Assembly Identifier: A unique string of alphanumeric characters that identifies an assembly, bundle, or canister for a specific reactor in which it. has been irradiated.

Fuel Channiels: Metal (usually zircaloy) box-like items which are used in boiling-water reactors (BWR) to control the flow of water through the core. BWR fuel bundles are inserted into the fuel channels to form the fuel assembly.

Fuel Cycle: The length of time a reactor is operated between refuelings (typically 14 to 24 months), including the refueling time, usually measured from the startup of one cycle to the startup of the following cycle.

Fuel Rods: Tubes of high-grade zircaloy or stainless steel that contain pellets of ceramic-grade uranium dioxide nuclear fuel.

Gigawattday: A unit of measure equal to 1 billion wattdays.

Graphite: A crystalline form of carbon used to moderate nuclear reactions. 
High-Level Waste (HLW): Either of the following are considered to be high-level waste: (1) the highly radioactive material resulting from the reprocessing of spent nuclear fuel, including liquid waste produced directly in reprocessing and any solid material derived from such liquid waste that contains fission products in sufficient concentrations; and (2) other highly radioactive material that the Nuclear Regulatory Commission, consistent with existing law, determines by rule to require permanent isolation.

High-Temperature, Gas-Cooled Reactor (HTGR): A reactor that is cooled by helium and moderated by graphite. The reactor is fueled with enriched uranium and with thorium, which can be transformed into useful fuel when irradiated.

Horizontal Storage Module (HSM): The horizontal storage module is a steel reinforced concrete structure. The HSM may be constructed in-place at the Independent Spent Fuel Storage Installation (ISFSI) location or it may be prefabricated off-site and positioned on a reinforced concrete pad at the ISFSI. The principal design functions of the HSM are to provide radiation shielding for the fuel/dry shielded canister during storage and to provide a passive mechanism for the removal of decay heat by natural circulation of ambient air.

Incore Instrumentation: Instrumentation used to monitor the neutron flux within the core. In pressurized-water reactors, this instrumentation is inserted into an instrument tube in the fuel assembly.

Independent Spent Fuel Storage Installation (ISFSI): Dry storage facility located at reactor site in which spent fuel assemblies may be stored.

Integral Fuel Burnable Absorbers (IFBAs): Westinghouse Electric-design neutron absorbers that are integral to the fuel assembly (i.e., not a nonfuel component). IFBAs consist of a zirconium diboride coating on the exterior of selected fuel pellets.

Interim Storage: The temporary holding of wastes on or away from the generator's site when disposal space is not available. Monitoring and human control are provided, and subsequent action involving treatment, transportation, or final disposition is expected.

Irradiated Nuclear Fuel: Nuclear fuel which has undergone a self-sustaining chain reaction. (See Burnup and Spent Nuclear Fuel.)
Joule: The International System unit of energy, equal to the work done when a current of 1 ampere is passed through a resistance of 1 ohm for 1 second.

Licensed Storage Capacity: Maximum number of spent nuclear fuel assemblies and canisters to be stored at a given site or spent nuclear fuel pool, as licensed by the Nuclear Regulatory Commission.

Licensed Thermal Power: The maximum thermal power of the reactor as authorized by the Nuclear Regulatory Commission, expressed in gigawatts thermal (GWt).

Licensing: The guidelines and procedures required to obtain a permit to build or operate a facility.

Light-Water Reactor (LWR): A nuclear reactor that uses ordinary water as the primary coolant and moderator, with slightly enriched uranium as fuel. There are two types of commercial light-water reactors--the boiling-water reactor (BWR) and the pressurized-water reactor (PWR).

Local Power Range Monitors: Incore instrumentation used to monitor the power level of General Electric-designed reactors during regular operation (at or near full power).

Low-Level Waste (LLW): Radioactive waste not classified as high-level waste, spent nuclear fuel, or by-product material specified as uranium or thorium tailing and waste.

Maximum Established Storage Capacity: The maximum established spent fuel capacity for a storage site, defined by the Department of Energy as the maximum number of intact assemblies that will be able to be stored at some point in the future (between the reporting date and the reactor's end of life) taking into account any established or current studies or engineering evaluations, at the time of submittal for licensing approval from the Nuclear Regulatory Commission.

Maximum Initial Pellet Enrichment Assay: The maximum enrichment in uranium-235 weight percent of all pellets contained in the fuel rods in a nuclear fuel assembly.

MTIHM (Metric Tons of Initial Heavy Metal): The weight of the initial fuel loading (in metric tons) used in an assembly and other fissile and fertile materials that are loaded into an assembly during fabrication of 
the assembly, also expressed in MTU (metric tons of uranium) and MTHM (metric tons of heavy metal).

MTU (Metric Tons of Uranium): A measure of weight equivalent to $2,204.6$ pounds of uranium.

Net Summer Capability: The steady hourly output which generating equipment is expected to supply to a system load exclusive of auxiliary power as demonstrated by testing at the time of summer peak demand.

Neutron Poison: A material (e.g., boron or cadmium) that absorbs neutrons readily. Insertion of one of these poisons results in a decrease in the reactivity (or neutron multiplying property) of the core, and consequently in a decrease of the neutron density.

Neutron Sources: A base source of neutrons that initiate the nuclear chain reaction within the reactor core. Primary neutron sources are typically polonium- or plutonium-beryllium alloys; secondary neutron sources are typically antimony-beryllium alloys.

Nonfuel Components: Nuclear reactor core parts and hardware, excluding the nuclear fuel itself. Such components include shrouds, control rods, fuel channels, in-core chambers, support tubes, and dummy fuel rods.

Nuclear Fuel: Fissionable materials that have been enriched to such a composition that when placed in a nuclear reactor they will support a self-sustained chain reaction, producing heat in a controlled manner for process use.

Nuclear Fuel Cycle: The complete series of steps involved in supplying fuel for nuclear reactors, including mining, refining, enrichment, the original fabrication of fuel elements, their use in a reactor, and management of spent fuel and radioactive wastes. A closed fuel cycle includes chemical reprocessing to recover the fissionable material remaining in the spent fuel; an open fuel cycle does not.

Nuclear Fuel Cycle "Front End": Locating and mining ore bodies containing uranium; milling the uranium-bearing ore to extract the uranium oxide $\left(\mathrm{U}_{3} \mathrm{O}_{8}\right)$, called uranium concentrate or'yellowcake'; chemically converting the $\mathrm{U}_{3} \mathrm{O}_{8}$ to uranium hexafluoride (UF $)_{6}$, which is a solid at room tempera- ture but changes to a gas at slightly higher temperatures; enriching, that is, increasing the concentration of the uranium-235 isotope in the $U F_{6}$, so that a nuclear chain reaction can be sustained; converting the enriched $\mathrm{UF}_{6}$ into fuel pellets of uranium dioxide $\left(\mathrm{UO}_{2}\right)$; and fabricating rods containing the pellets into assemblies for loading into a reactor. (See Fuel Cycle.)

Nuclear Power: Electricity generated by an electric power plant whose turbines are driven by steam generated in a reactor by heat from the fissioning of nuclear fuel.

Nuclear Power Plant: A single- or multi-unit facility in which heat produced in a reactor(s) by the fissioning of nuclear fuel is used to drive a steam turbine(s).

Nuclear Reactor: An apparatus in which the nuclear fission chain reaction can be initiated, maintained, and controlled so that energy is released at a specific rate. The reactor apparatus includes fissionable material (fuel) such as uranium or plutonium; fertile material; moderating material (unless it is a fast reactor); a heavy-walled pressure vessel; shielding to protect personnel; provision for heat removal; and control elements and instrumentation.

Nuclear Regulatory Commission (NRC): The U.S. Government organization that regulates the nuclear industry, under the Atomic Energy Act of 1954, et seq., to insure public safety.

Nuclear Waste Policy Act of 1982, as Amended: The Act mandates the establishment of repositories for the long-term storage and disposal of high-level radioactive waste and the collection of fees from utilities to finance these repositories. Under this Act the Department of Energy (DOE) was given the responsibility for providing permanent disposal of spent fuel from domestic nuclear power reactors.

Operable: A nuclear unit in the United States is "operable" after it completes low power testing and is issued a full power license by the Nuclear Regulatory Commission.

Orifice Rod Assemblies: Short (typically 8-10") metal plugs that are inserted into the guide tubes of fuel assemblies in Babcock and Wilcox-designed reactors to block the flow of coolant through guide tubes. They consist of 20 orifice rods connected together by a spider or similar holddown device. Each orifice rod assembly fits into the guide tubes of a single assembly. 
Other Instrumentation: Incore instrumentation, such as source range monitors (SRMs), intermediate. range monitors (IRMs), and transverse incore probes (TIPs), that are used to monitor the power level of General Electric-designed reactors.

Pool Crane: A machine for raising assemblies and depositing them into or out of a spent fuel storage pool.

Pool Site: A configuration of pools having a single cask loading area.

Pressurized-Water Reactor (PWR): A nuclear reactor in which heat is transferred from the core to a heat exchanger via water kept under high pressure, so that high temperatures can be maintained in the primary system without boiling the water. Steam is generated in a secondary circuit.

Radiation: Particles or waves from atomic or nuclear processes and the process in which energy is emitted as particles or waves.

Radioactivity: See Activity.

Reactor, Commercial Power: Any unit of a commercial nuclear facility, licensed by the Nuclear Regulatory Commission, which generates electrical power.

Refueling: The process of shutting down a reactor and replacing the spent nuclear fuel assemblies. The refueling process usually takes several months to accomplish.

Reinserted Fuel: Irradiated fuel that is discharged in one cycle and inserted in the same reactor after sitting in the storage pool for at least one subsequent cycle. In a few cases, fuel discharged from one reactor has been used to fuel a different reactor.

Repository: A permanent disposal facility for highlevel or transuranic wastes and spent fuel.

Reprocessing: The process by which spent fuel is separated into waste material for disposal and material such as uranium and plutonium for reuse.

Reracking: The installation of high-density racks to increase the storage capacity of a spent fuel pool.

Rod Array Configuration: Fuel assemblies are constructed from a number of individual fuel rods arranged together. These arrays have been of many different sizes, generally in square arrays. The number of fuel rods on each side of these arrays is referred to as the rod array configuration. Thus, an assembly with a rod array configuration of $14 \times 14$ has a total of 196 positions in a $14 \times 14$ array (most positions contain fuel rods, although some are guide tube locations, etc.). Pressurized-water reactor (PWR) rod array configurations are typically $14 \times 14$, $15 \times 15,16 \times 16$, and $17 \times 17$. A very few nonsquare arrays (13 X 14, $15 \times 16,17 \times 18)$ have been used in PWRs which use cruciform control blades (Indian Point 1 and Yankee Rowe). Boiling-water reactor (BWR) rod array configurations have typically been either $7 \times 7$ or $8 \times 8$, but newer fuel assembly types are in use in reactors with $9 \times 9$ and $10 \times 10$ rod array configurations.

Rod Cluster Control Assemblies (RCCAs): Assemblies used at Westinghouse Electric-designed pressurized-water reactors to provide control over the nuclear chain reaction by limiting the number of neutrons in the core. Made of strongly neutronabsorbing materials, RCCAs are inserted into the reactor core at planned shutdowns and during scrams. They consist of 16,20 , or 24 control rods connected together by a control rod spider. Each RCCA fits into the guide tubes of a single assembly.

Shipment: A generic term meant to cover all the diverse forms of shipments away from the discharging reactor's spent fuel pool. As in: shipped down the transfer canal to an adjoining spent fuel pool; shipped in a transport cask to an on-site storage facility; shipped to an off-site storage facility; or shipped in a storage cask to a dry storage facility. Excludes round-trip and intermediate shipments that do not affect inventories of spent nuclear fuel.

Shutdown Date: Month and year of reactor shutdown for fuel discharge and refueling. The date is the point at which the reactor becomes subcritical.

Site: Any location under institutional control within which radioactive wastes are handled, stored, or disposed.

Slot: A physical position in a rack in a spent fuel pool which is intended to be occupied by an intact assembly or equivalent (that is, a canister or an assembly skeleton).

Spent Fuel Storage Pool: A borated water-filled pool in which assemblies may be stored. 
Spent Nuclear Fuel (SNF): Fuel that has been withdrawn from a nuclear reactor following irradiation, the constituent elements of which have not been separated by reprocessing. Spent or irradiated fuel is usually discharged from reactors because of chemical, physical, and nuclear changes that make the fuel no longer efficient for the production of heat, rather than because of the complete depletion of fissionable material. (Uranium metal is susceptible to radiation damage, which limits its operating life in a reactor.)

Standard Contract: A contract between the Department of Energy (DOE) and each commercial owner and/or generator of irradiated nuclear fuel. The contract stipulates the terms and method of payment, how fees are to be calculated, and how spent nuclear fuel is to be transferred to DOE custody.

Startup: The first date electrical energy is generated by a reactor.

Storage: Operations designed to provide isolation and easy recovery of spent fuel (and other radioactive materials), which rely on continuous human monitoring, maintenance, and protection from human intrusion for a specified period of time.

Thimble Plug Assemblies: Short (typically 8-10") metal plugs that are inserted into the guide tubes.of fuel assemblies in Westinghouse Electric-designed reactors to block the flow of coolant through guide tubes. Thimble plug assemblies consist of 16,20 , or 24 thimble plugs connected together by a spider or similar holddown device. Each thimble plug assembly fits into the guide tubes of a single assembly.

Transport Cask: Container that provides shielding during transportation of canisters of radioactive materials, available in several sizes for truck and rail use.

Transshipment: The shipment of spent nuclear fuel assemblies and canisters from one storage site to another using railroads or trucks as the primary mode of transportation.

Uranium (U): A heavy, naturally radioactive, metallic element (atomic number 92). Its two principally occurring isotopes are uranium-235 and uranium-238. Uranium-235 is indispensable to the nuclear industry because it is the only isotope existing in nature to any appreciable extent that is fissionable by thermal neutrons. Uranium-238 is also important because it absorbs neutrons to produce a radioactive isotope that subsequently decays to plutonium-239, an isotope that also is fissionable by thermal neutrons.

Waste Disposal: A current concern in the nuclear power field is the safe disposal and isolation of spent fuel. These materials must be isolated from the biosphere until the radioactivity contained in them has diminished to a safe level. Under the Nuclear Waste Policy Act of 1982, as amended, the Department of Energy has been given responsibility for the long-term management of spent fuel and highlevel radioactive waste. Current plans call for the ultimate disposal of the wastes in solid form in licensed deep, stable geologic structures.

Waste Stream: The processing sequence for waste materials that isolates particular types of waste and prevents the mixing of undesirable materials during handling and disposition.

Watt: A unit of power. The rate of energy transfer equivalent to 1 joule per second. 


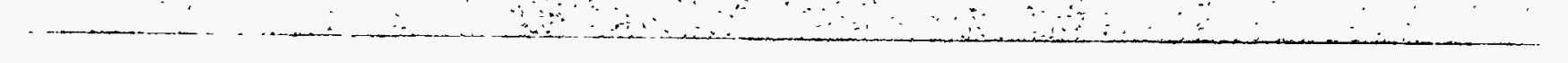




\section{Bibliography and Notes}

Diablo Canyon nuclear power plant, operated by Pacific Gas and Electric, located in Avila Beach, Claifornia.

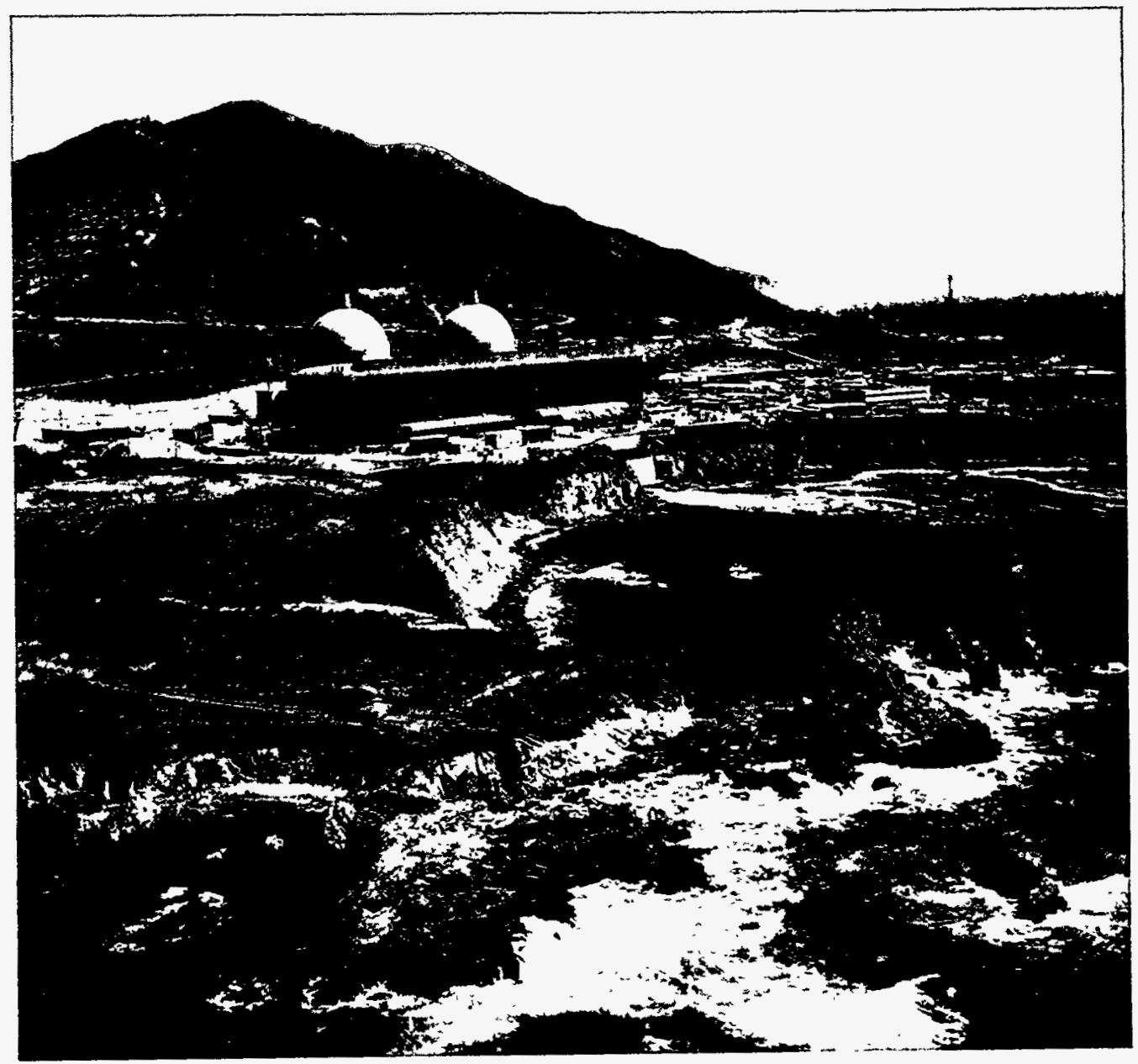




\section{Bibliography and Notes}

This section presents a listing of other publications containing information about nuclear waste along with a brief abstract of each. The most recent version of each report is listed.

Integrated Data Base for 1993, U.S. Spent Fuel and Radioactive Waste Inventories, Projections, and Characteristics, Office of Civilian Radioactive Waste Management (DOE/RW-0009).

The Integrated Data Base (IDB) Program contains current data on inventories and characteristics of commercial spent fuel and both commercial and U.S. Government-owned radioactive waste through December 31, 1992. The radioactive materials considered are spent fuel, high-level waste, transuranic waste, low-level waste, commercial uranium mill tailings, remedial action program wastes, commercial reactor and fuel cycle facility decommissioning wastes, highly radioactive materials that may require geologic disposal, and mixed (hazardous and radioactive) low-level waste. For these categories, current and projected inventories are given through the year 2020, and the radioactivity and thermal power are calculated based on reported or estimated isotopic compositions.

Characteristics of Potential Repository Wastes, Office of Civilian Radioactive Waste Management (DOE/RW-0184-R1, Volumes 1-4).

The Characteristics Data Base (CDB) provides the detailed technical characteristics of those wastes that will, or may, eventually be disposed of in a deep geologic repository. This includes spent fuel from commercial light-water reactors (LWRs) and immobilized high-level waste (HLW) from reprocessing. Other sources include spent fuel from non-LWRs such as test and experimental reactors, and various miscellaneous sources such as greater-than-class-C low-level wastes and radioisotope capsules. The technical characteristics of relevant interest are physical descriptions and measurements, chemical compositions, radiological properties, inventories, and projected quantities. These data are collected, tabulated, organized, placed into computer data files, and made available to users in a range of formats, including hard-copy reports and menu-driven PC data bases.

Spent Fuel Storage Requirements: 1992 to 2036, Office of Civilian Radioactive Waste Management (DOE/RW-0431).

The source data contained in this report are based on individual reactor information supplied by the reactor owners on the Nuclear Fuel Data survey, Form RW-859. Historical inventories of spent fuel from existing, planned, and projected pressurizedwater reactors (PWRs) and boiling-water reactors (BWRs) and one existing high-temperature, gascooled reactor (HTGR), and utility estimates of future discharges, are presented through the year 2036. The ultimate needs for additional storage capacity are estimated. These estimates are based on the maximum capacities within current and planned at-reactor facilities and on any planned transshipments of fuel to other reactors or facilities.

World Nuclear Capacity and Fuel Cycle Requirements 1993, Energy Information Administration (DOE/EIA-0436-93).

The nuclear fuel cycle consists of mining and milling uranium ore, processing the uranium into a form suitable for generating electricity, "burning" the fuel in nuclear reactors, and managing the resulting spent nuclear fuel. This report presents the current status and projections of nuclear capacity, generation, and fuel cycle requirements for all countries in the world with commercial nuclear power programs. Also included are projections of domestic and foreign requirements for natural uranium and enrichment services as well as projections of discharges of spent nuclear fuel.

1993 Acceptance Priority Ranking, Office of Civilian Radioactive Waste Management (DOE/RW-0328P).

This document is based on spent nuclear fuel (SNF) discharges as of December 31, 1992. The 1993 Acceptance Priority Ranking (APR) report is the basis for allocating projected spent nuclear fuel acceptance capacity to each owner in the 1993 Annual Capacity Report. 
1993 Annual Capacity Report, Office of Civilian Radioactive Waste Management (DOE/ RW-0331P).

The Standard Contract for Disposal of Spent Nuclear Fuel and/or High-Level Radioactive Waste (10 CFR Part 961) requires the U.S. Department of Energy to issue an Annual Capacity Report (ACR) for planning purposes. As required by the Standard Contract, the acceptance priority ranking is based on the date the spent nuclear fuel was permanently discharged, with the owners of the oldest spent nuclear fuel, on an industry-wide basis, given the highest priority. The 1993 ACR applies the projected waste acceptance rates in Table 2.1 to the 1993 Acceptance Priority Ranking (APR), resulting in individual allocations for the owners and generators of the spent nuclear fuel. These allocations are listed in detail in the Appendix, and summarized in Table 3.1 .

\section{Uranium Industry Annual 1992, Energy Information Administration (DOE/EIA-0478-92).}

This report presents data on U.S. uranium raw materials and marketing activities of the domestic uranium industry and electric utilities. It contains aggregated data reported by U.S. companies on the "Uranium Industry Annual" survey, Form EIA-858, and historical data from prior data collections and other pertinent sources.

Analysis of Assembly Serial Number Usage in Domestic Light-Water Reactors, Oak Ridge National Laboratory (ORNL/TM-11841, May 1991, authors W.J. Reich and R.S. Moore).

This report is an analysis of the domestic light-water reactor (LWR) fuel assembly serial number identification that is placed on each assembly. The study was made to determine what serial numbering schemes are used, the effectiveness of these schemes, and how many duplicate serial numbers occur on domestic LWR fuel assemblies.

\section{Information Digest 1993 Edition, Nuclear Regula- tory Commission (NUREG-1350, Volume 5).}

This digest is a compilation of nuclear and NRC related data and is designed to provide a quick reference to major facts about the agency and the industry it regulates. In general, the data cover 1975 through 1992, with exceptions noted. Information on generating capacity and average capacity factor for operating U.S. commercial nuclear power reactors is obtained from monthly operating reports that are submitted directly to the NRC by the licensee. This information is reviewed by the NRC for consistency only. No independent validation and/or verification is performed.

Form RW-859 Canisters, Energy Information Administration Contractor Report (, EIA-9306601), Z, Inc., Silver Spring, Maryland.

The Nuclear Fuel Data survey, Form RW-859 is now collecting detailed data on canisters and their contents. A canister is a container made of stainless steel or an inert alloy into which radioactive or highlevel waste may be placed. The report presents detailed data on the size and contents of canisters stored in pools at commercial utilities that operate nuclear reactors. Each canister may contain an intact spent fuel assembly, consolidated spent fuel assemblies, spent fuel rods or pieces, nonfuel components, nonfuel hardware, or debris.

Form RW-859 Nonfuel Components, Energy Information Administration Contractor Report (ASG/EIA9302), Automated Science Group, Inc., Oak Ridge, Tennessee.

The Nuclear Fuel Data survey, Form RW-859, is now collecting detailed data on nonfuel components. According to the Standard Contract between the Department of Energy and the nuclear utilities (10 CFR 961), nonfuel components that are contained within the fuel assembly, or which are an integral part of the assembly, may be included as part of the spent nuclear fuel delivered for disposal. Other nonfuel components, generally used within or between assemblies and usually retired from service on a schedule that is different from that of the fuel assemblies are called nonfuel assembly (NFA) hardware. This report focuses on the specific NFA components which are listed in the contract, including control spiders, burnable absorber assemblies, control rod elements, thimble plugs, fission chambers, primary and secondary neutron sources, and fuel channels. 


\title{
COMMENTS CARD AND ORDER FORM FOR SERVICE REPORI
}

The ElA solicits your comments on the Service Report, Spent Nuclear Fuel Discharges from U.S. Reactors 1992. Corrections of errors and suggestions for improvement are desirable. Forward a copy of this form or an equivalent memorandum to:

\author{
Ms. Kathleen Gibbard \\ Survey Management Division \\ Office of Coal, Nuclear, Electric and Alternate Fuels \\ Energy Information Administration \\ U.S. Department of Energy \\ 1707 H Street, N.W., Room 821 \\ Washington, DC 20006
}

Category (check all that apply)

口 Order $\operatorname{copy}(s)$

Error

口 Improvement

Is there more information attached?

口Yes. Number of sheets:

aNo

Comments:

Contact Information

Name: Telephone:

Address: 


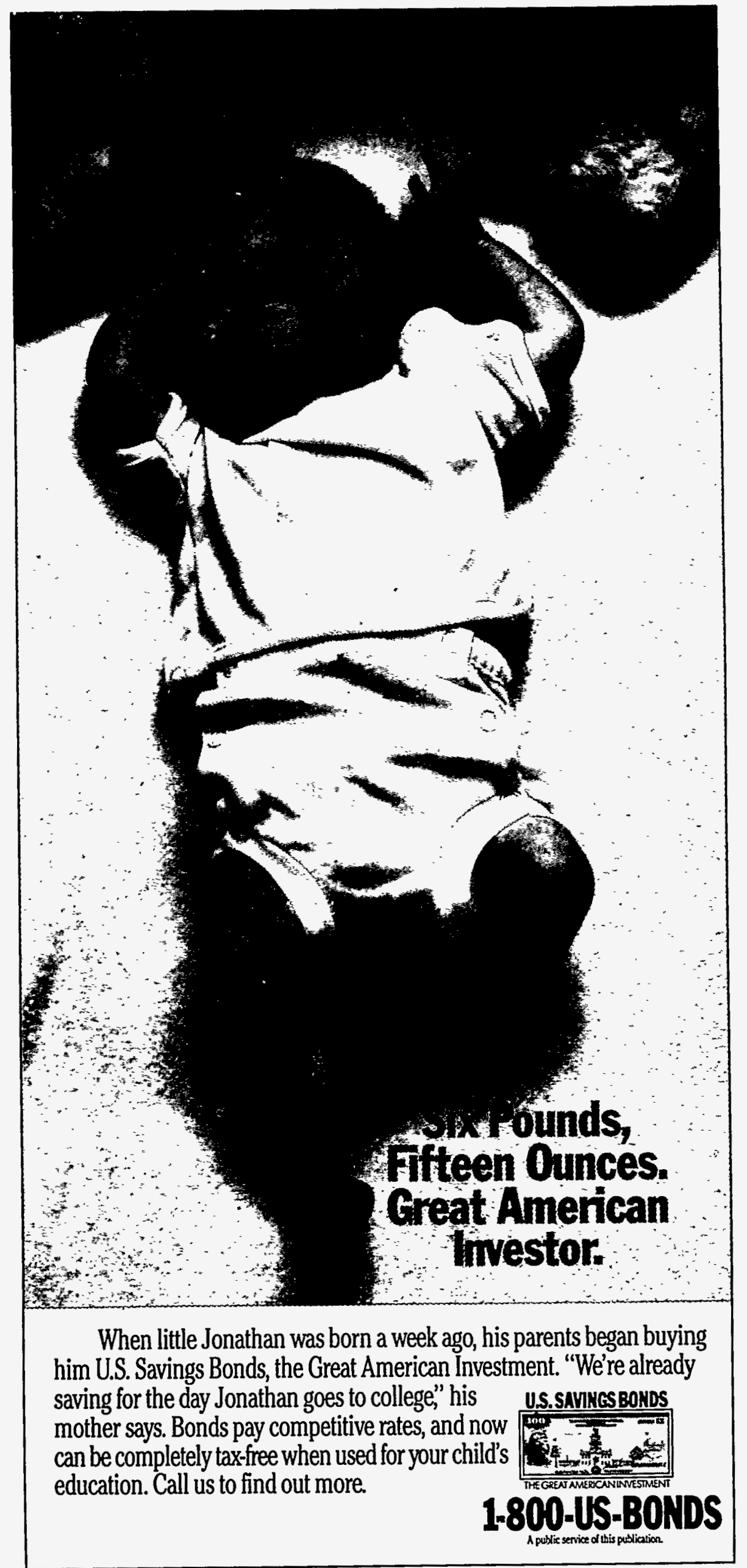

